



$$
\frac{20}{2,141}
$$

9kW 5076

$$
\begin{aligned}
& \text { oer siblivthelt bes Silofers } \\
& \text { chleinneihun }
\end{aligned}
$$

95 Bh esfehlon:

hage $b^{2+2+2+2}$
$c 3^{2+2}$
$d_{1}^{2+2} d_{2}^{2+2}$

$$
\overline{x v} . \mp .711
$$




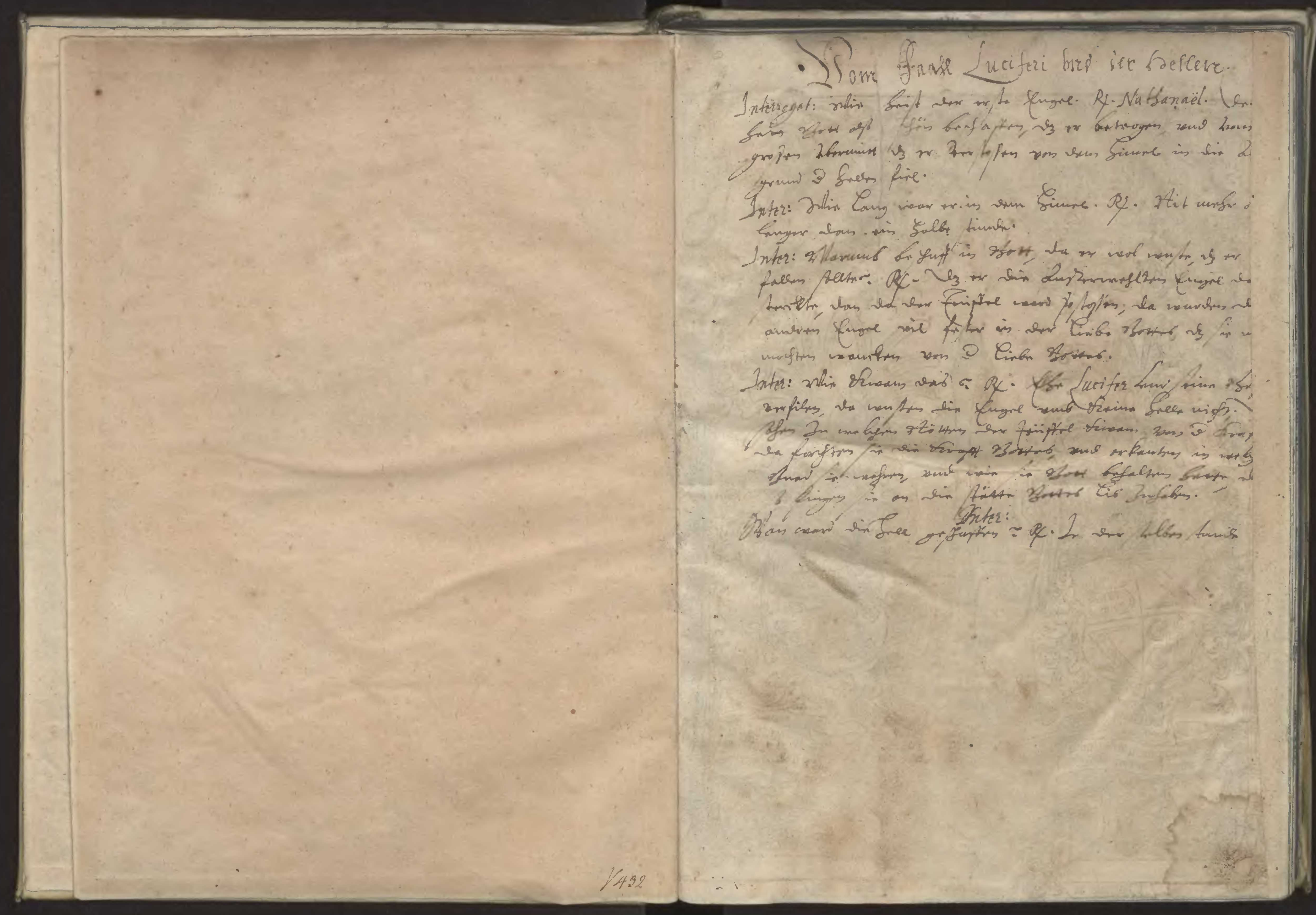




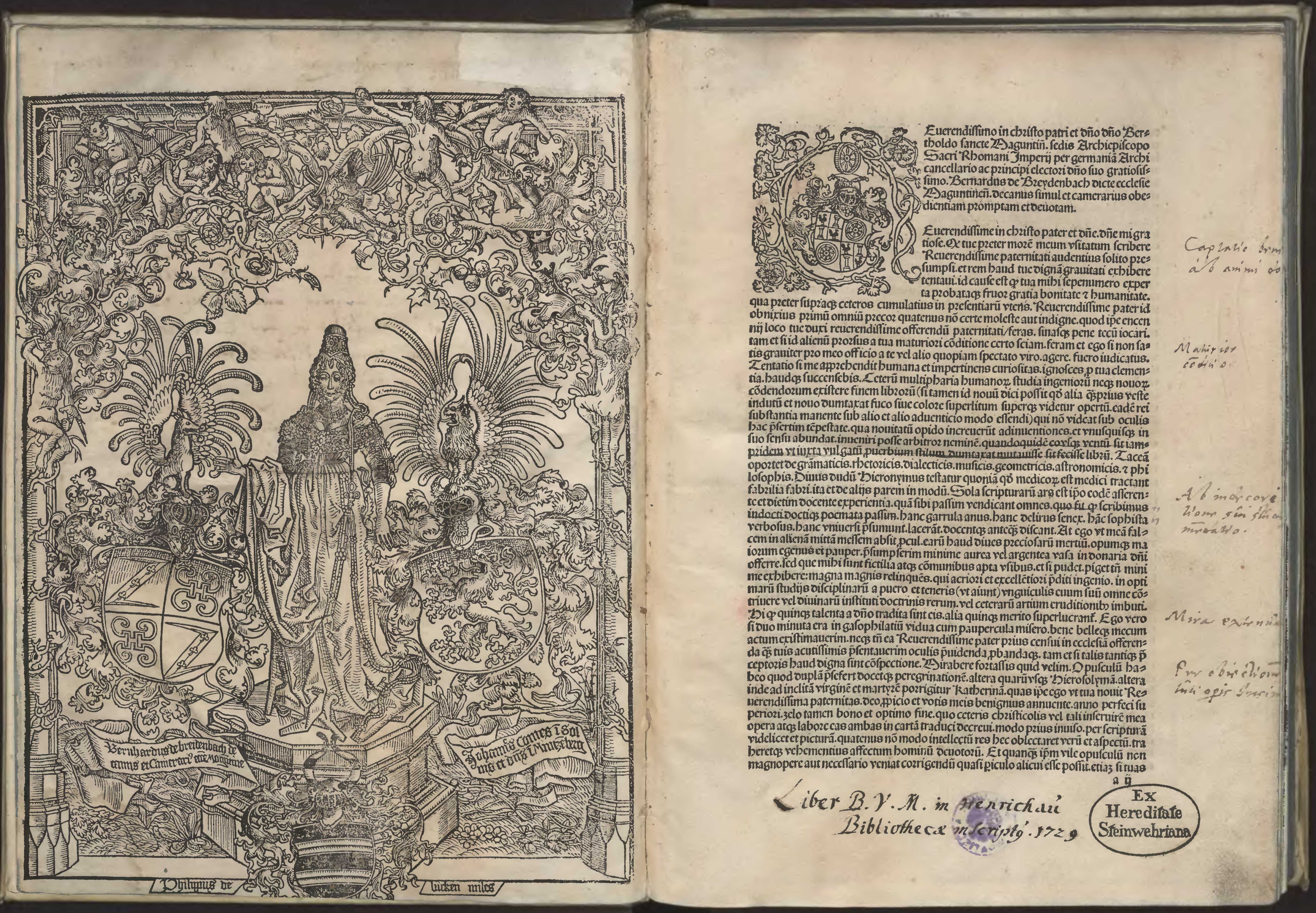




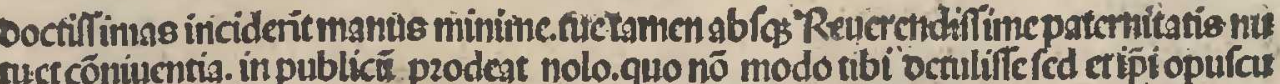

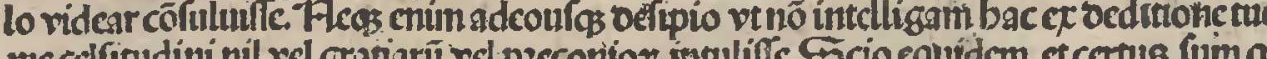

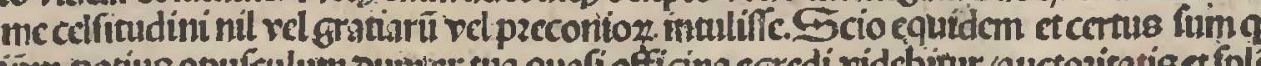

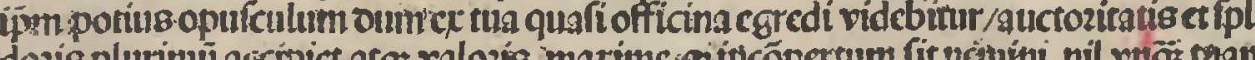

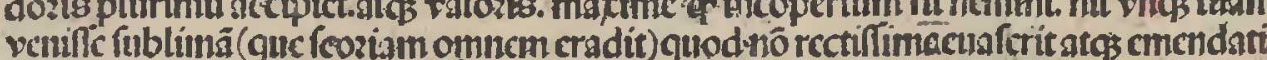

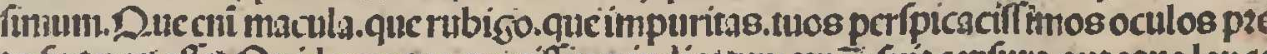

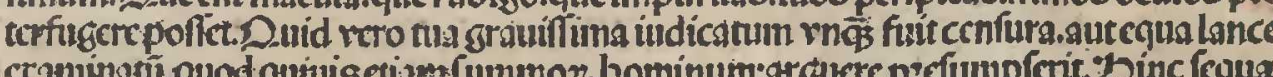

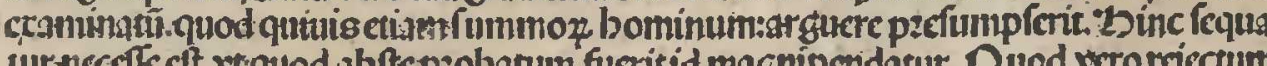

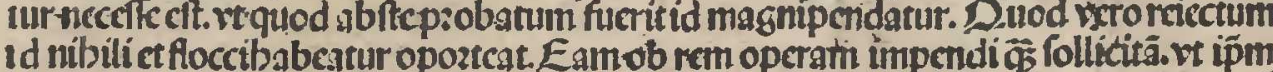

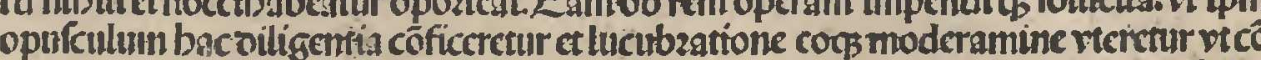

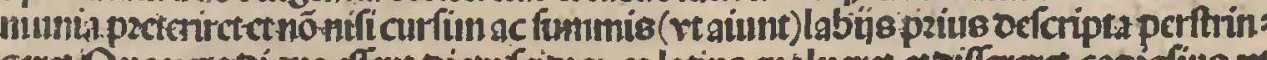

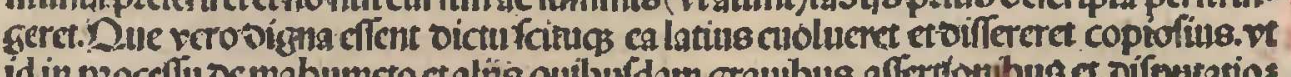
nibus baudgs consennendis o nibus baud ds contchncndis oagtionibue fict cuidens magis. To:ro quoniam

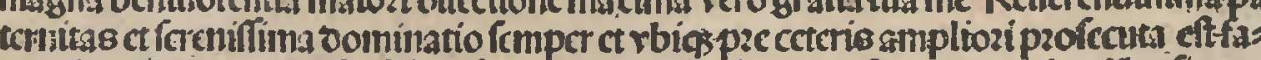

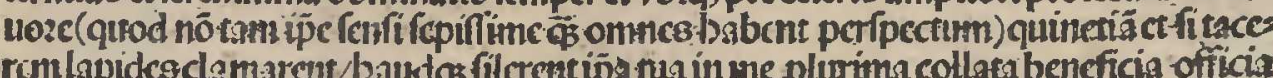

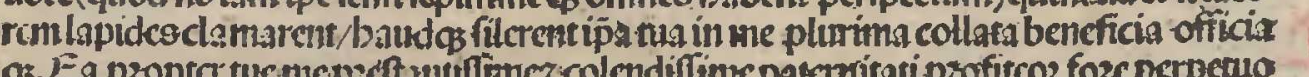

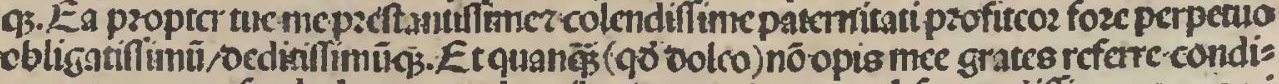

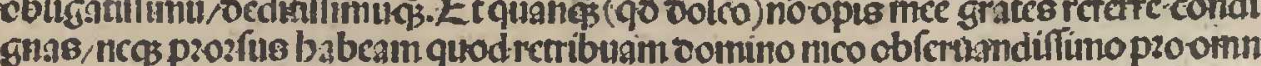

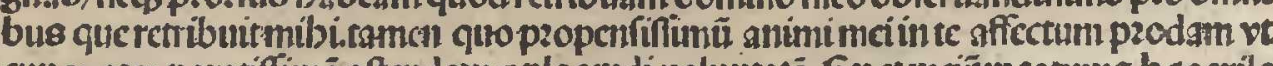

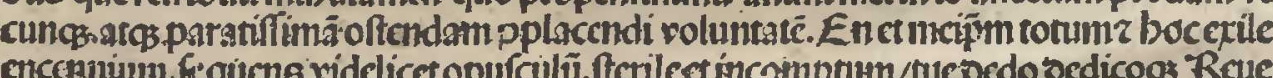

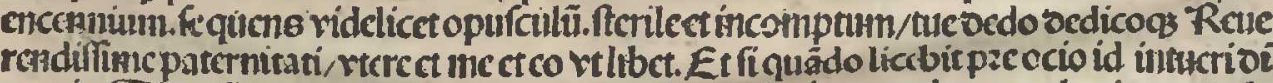

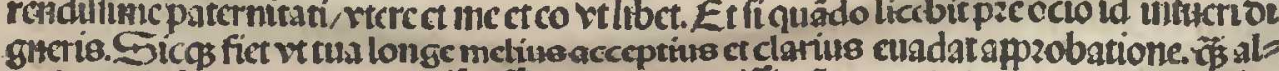
teriuscuiurpiam velexacta oifcurfionce.

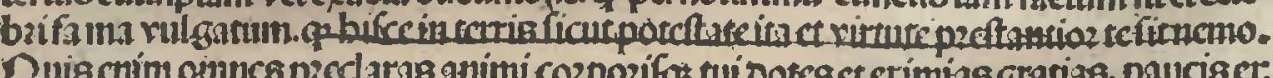

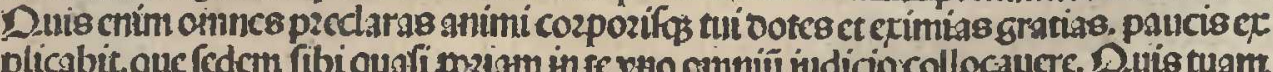

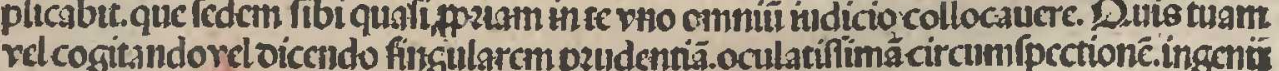

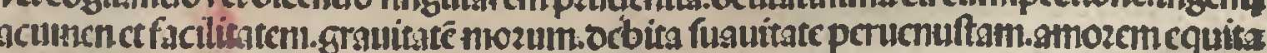

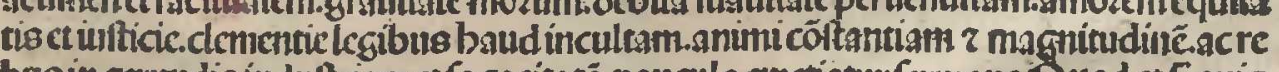

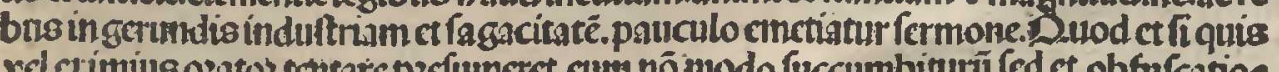

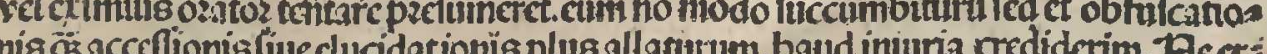

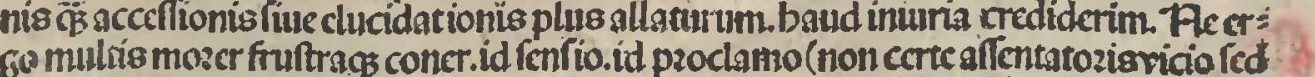

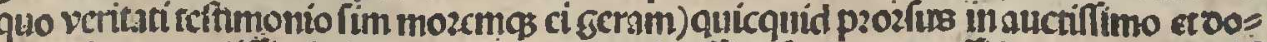

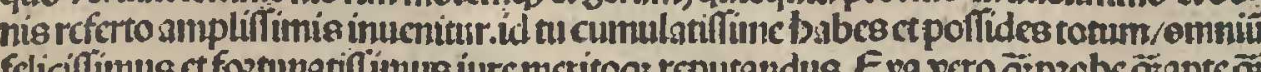

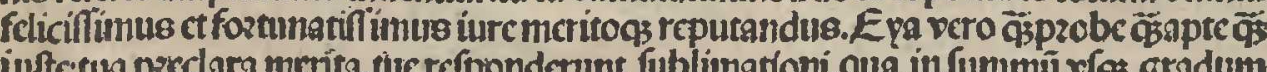

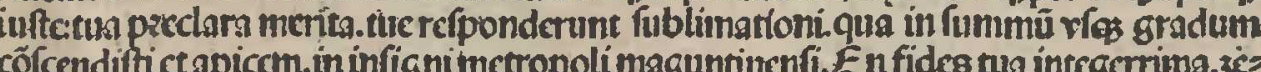

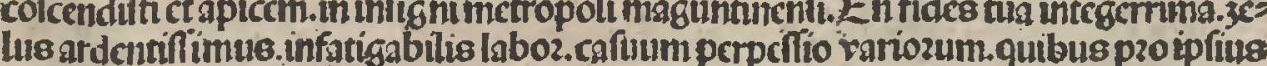

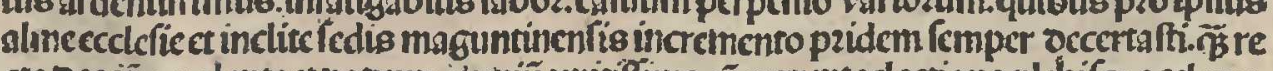

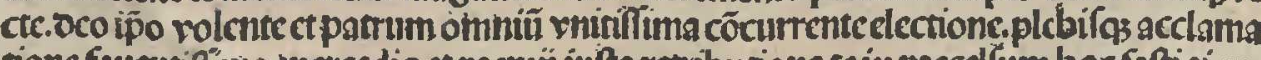

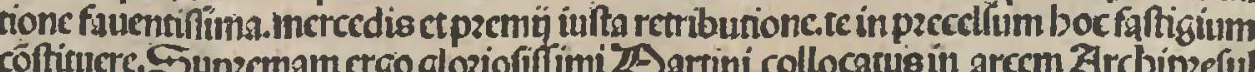
fauftifime oivo por charam pomotamig babuifithǘc facopcrofius ates accurative recepiftimagnificces

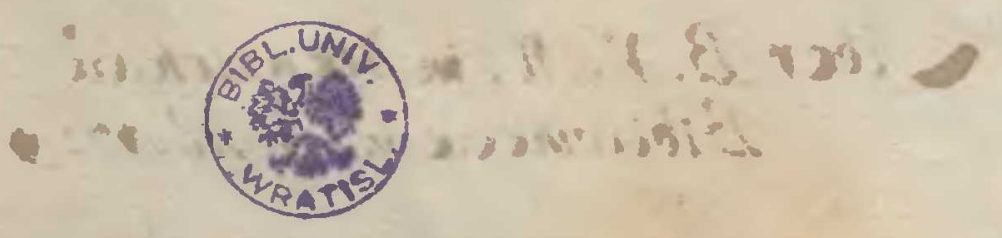

amplificcs.ctilluftres in Dics.quarenus feterectorcteduce.tepaftozciuttis ct optimis

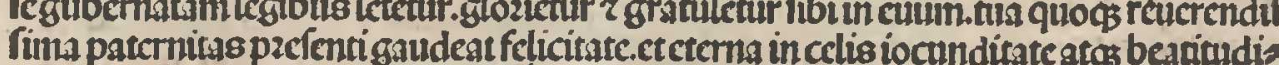

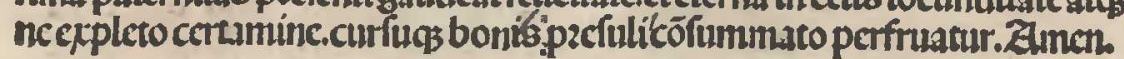

$$
\text { Continentiam buius operis fubiccta monftrabunt. }
$$

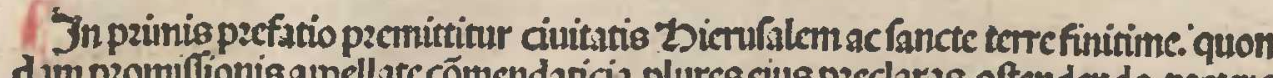
d.im pzonimas

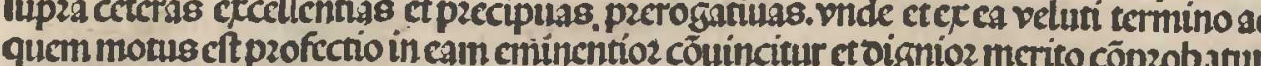

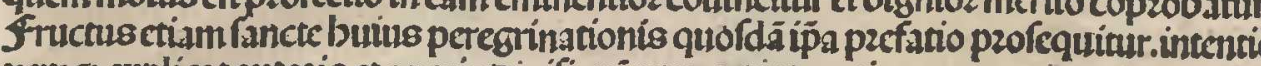

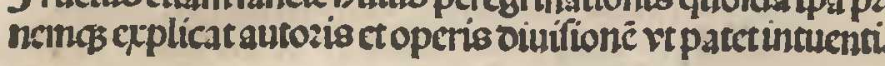

DDeinde proceffis to bierofolpmitane peregrinationis oe loco ad locum osdinatee

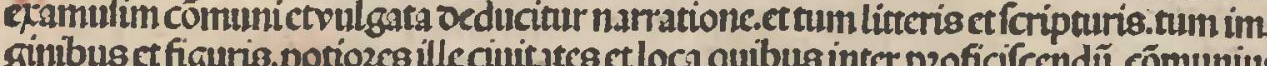
anplicatur terra marigj,ad intellectum et fenfum oefcribuntur videlicet.

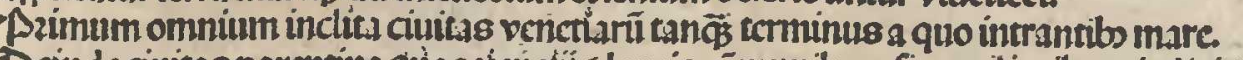

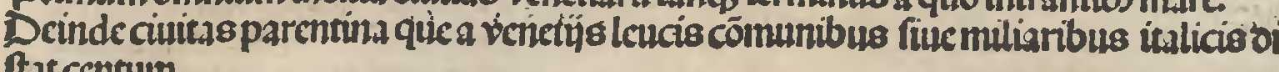

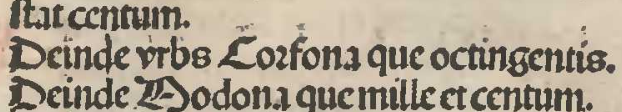

Drinde Joppen fure Japba que bis millenis. Zabiperegrini ad terram oc man egre

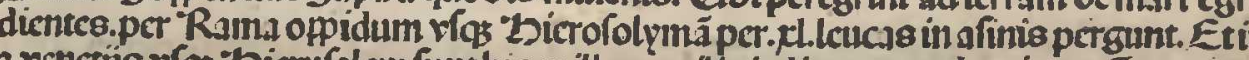

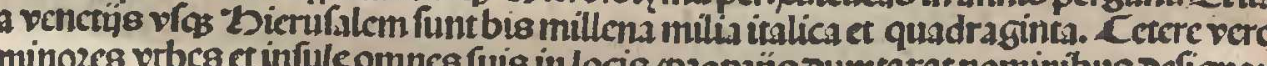

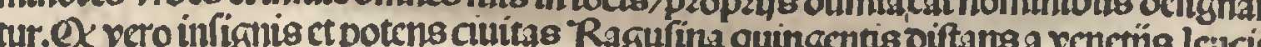

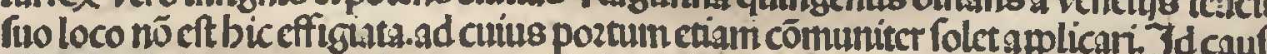

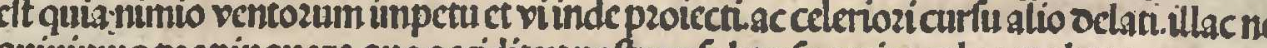
quitumus p2opinquare.quo accidit vetnoftros fubterfugerit oculos ct obcutus.

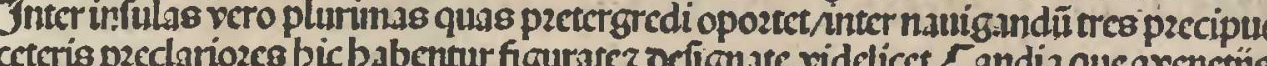

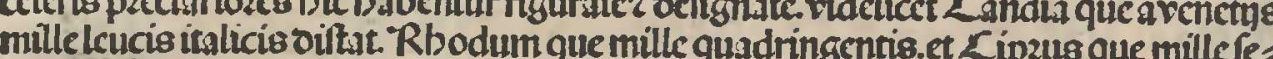
ptingentis.' Relique oumtarat numerantur etnominantur fuis in locis. Porro loc

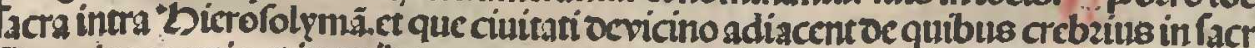

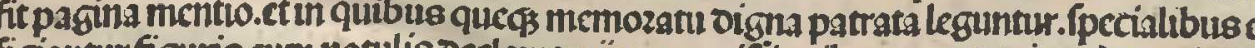
set remil tonbus opoztunis ad veteris vect

perme buius partis paincipalis.octerminato oc locis. agitur ozd

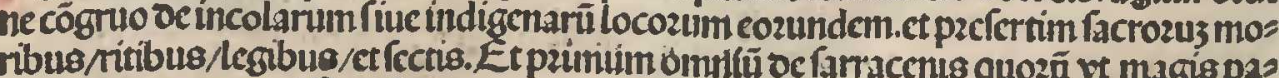
teaterToz ct damnatiffima fect.

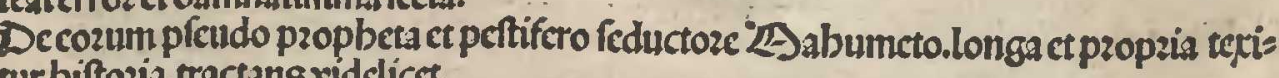

.

Secius infelici moztect indubia oamnationc. Ettandem côpendiofa quedam fed no cabilis et matura annectitur mabumetice fectect perfidici improbatio. vbi multa bauld

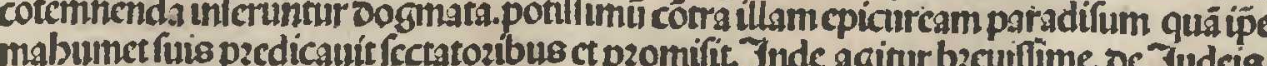

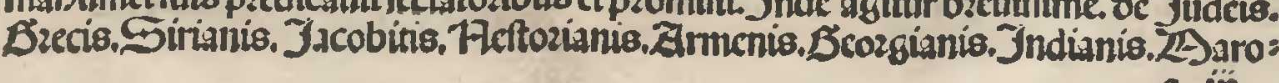




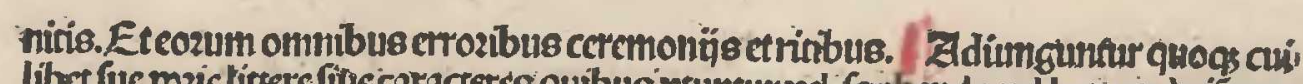

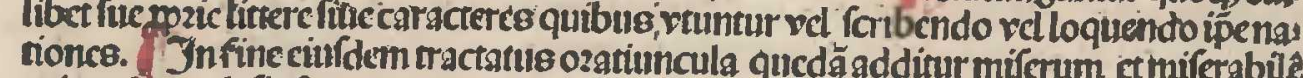

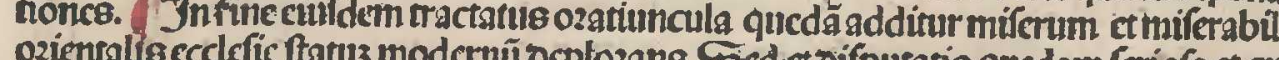

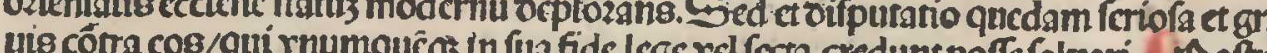
mo de veris carbolicis latinis quiperpatri funt "bicrofótymis fit memozis cum lam

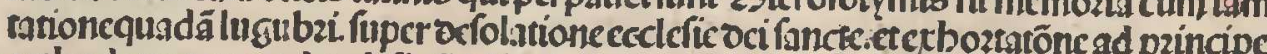

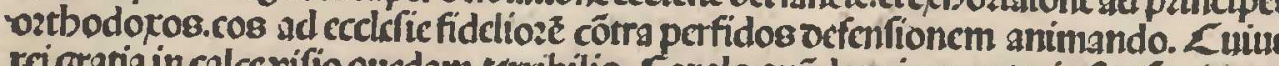

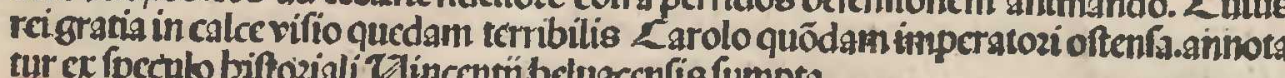

政

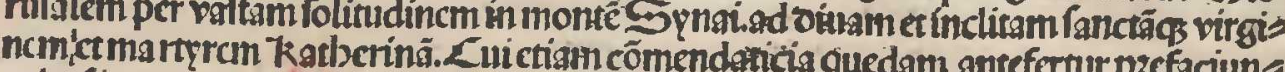

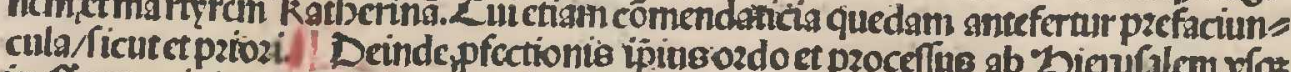

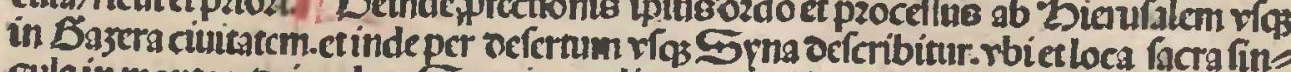

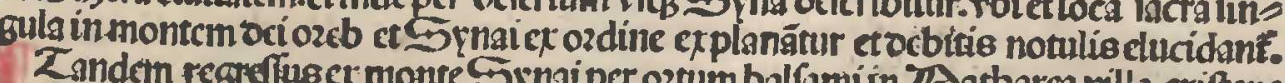

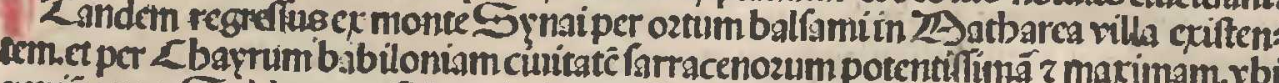

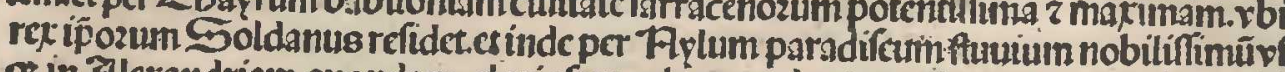

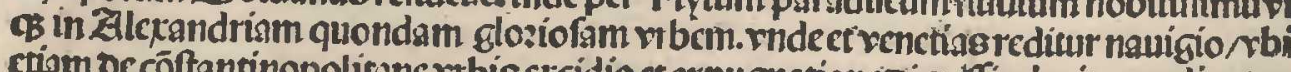
opoztuna explicatur narration c. Ex rin fine omnium quedó

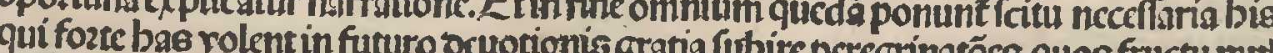

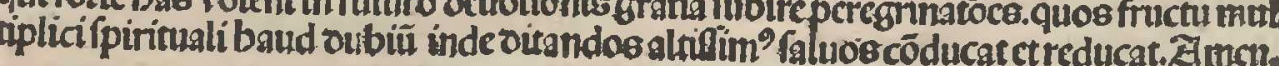

10 2efatio mopus tranfimarine peregri, nationisad Denerandum et gloziofum res pulcbzumi oominicum un tsierufalem cius tum laudes tum fructuspaucis attollens. intentionemqzexpzimens auctozls 7 ope ris ounfionem. sncipitfelicuter.

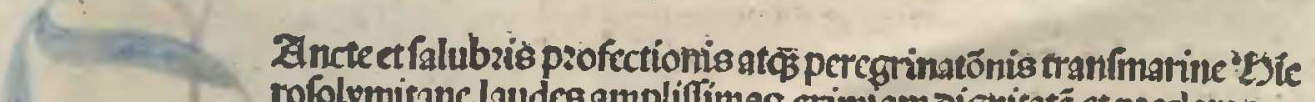

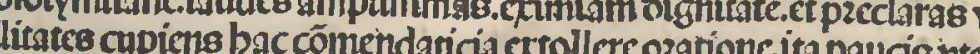
dum cenfui quo mozcm geram lectozi.rt babeam nibiloninus rat

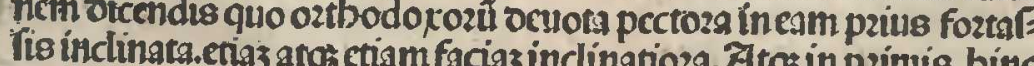

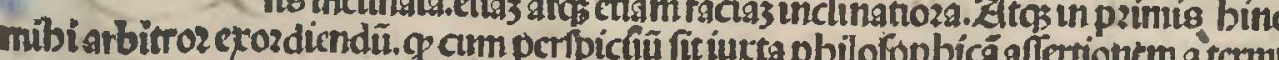

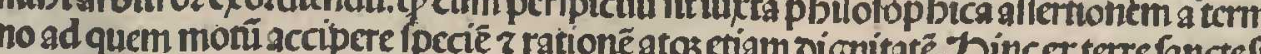
per cunctas pzectninctitra/buius pegrinationis laus et cốditio nobilis tenit. baud ou (rempecha pron. rem pzecetcris pzeftantiam/maximã p2erogatiuam fancte illius cinicutis "bicrufalco mam in eam ren fecife

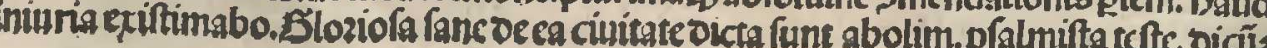

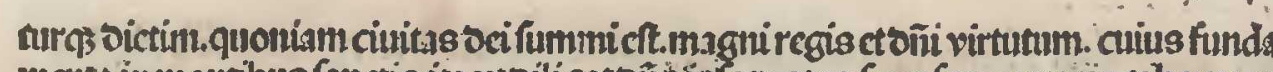

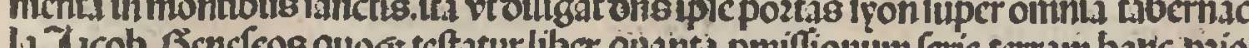
Feculo per

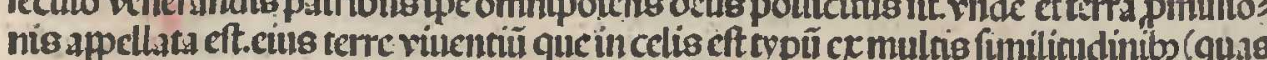

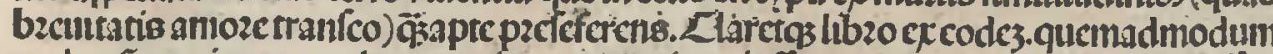

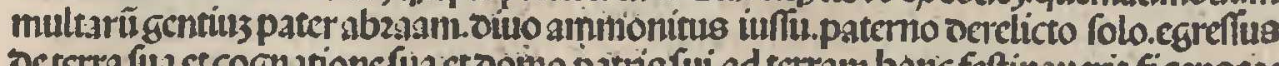

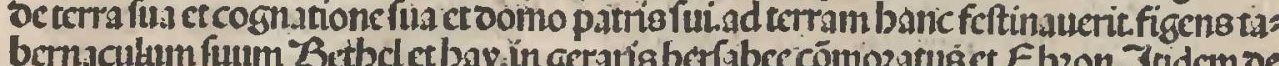

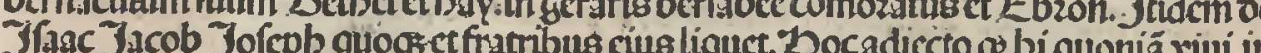

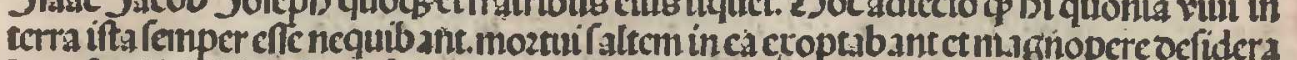

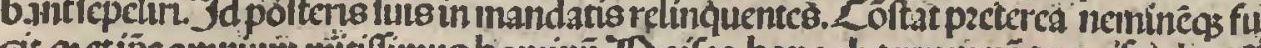

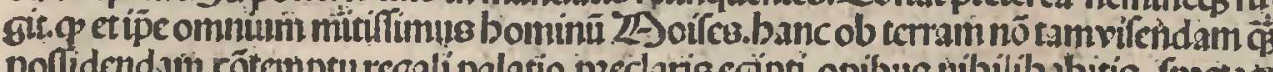

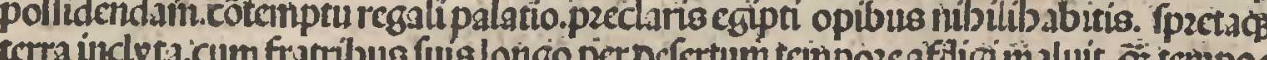

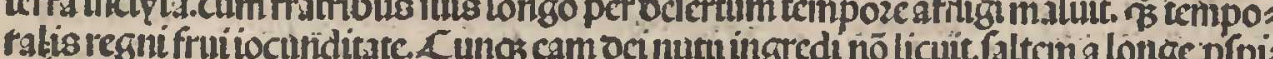

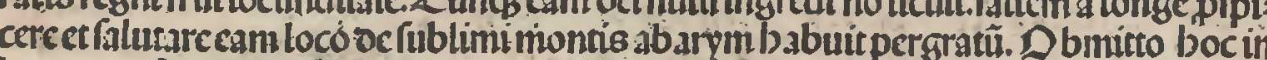

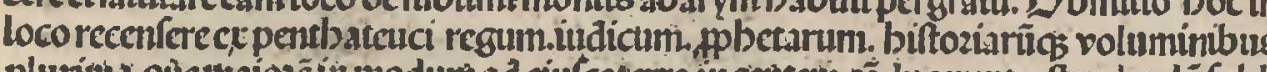

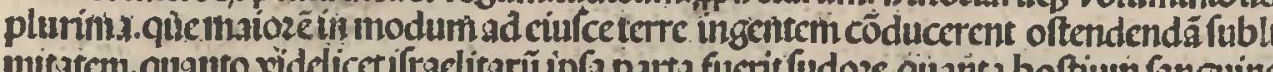

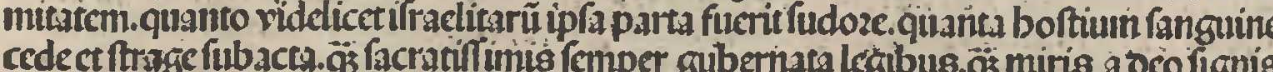

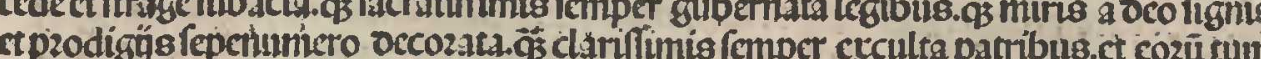

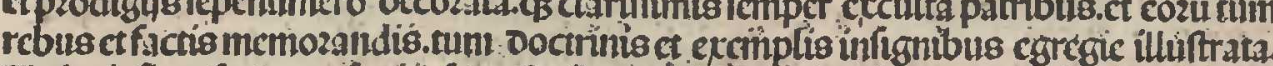
g̈gloziofiar referta perfonis. Takerdotobus videlicetet regibus et ptopbetis. quozum

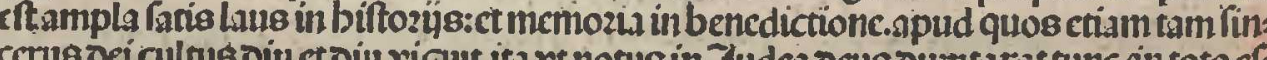

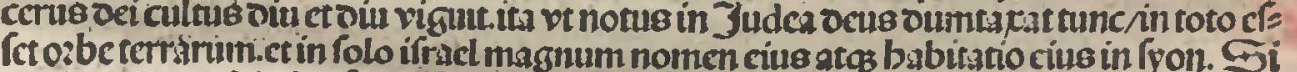

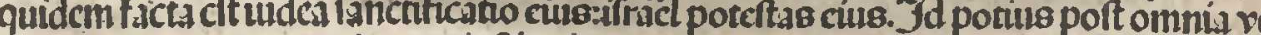

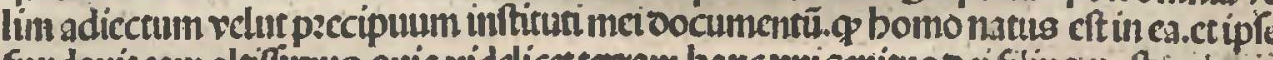

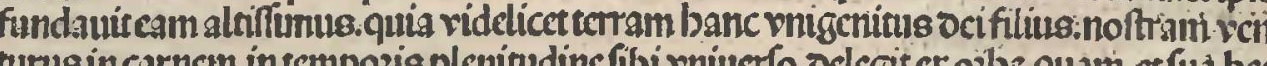
nedic cón

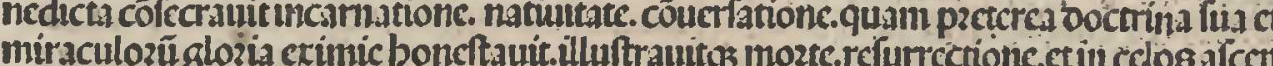

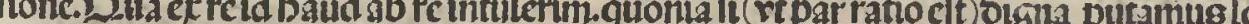

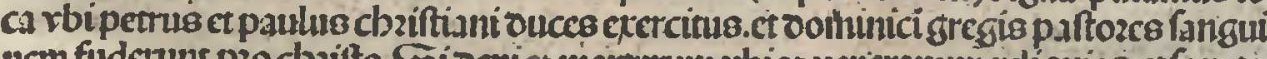

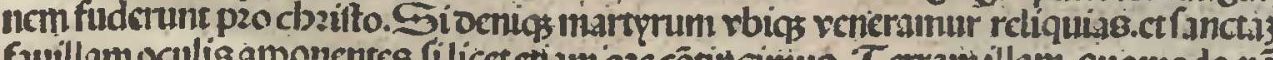
faullamocalts aponentes.

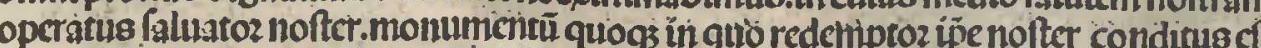
quomodo ho magghifaciemus thagle.quomo ho pluris reputabunus.irimi rcro que

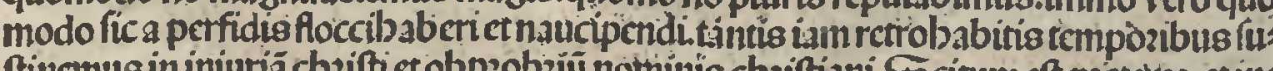

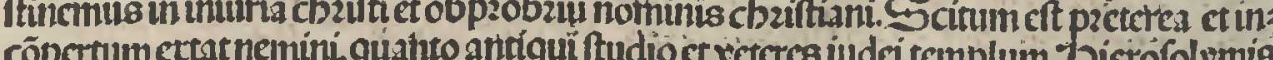

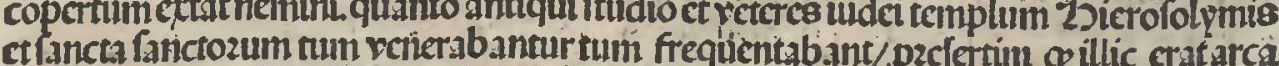

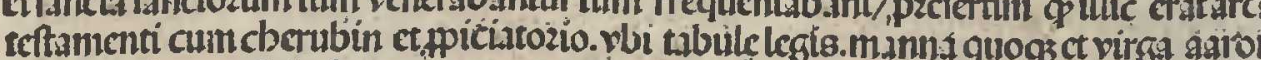

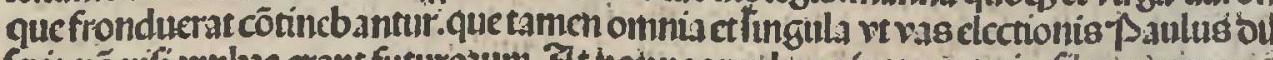

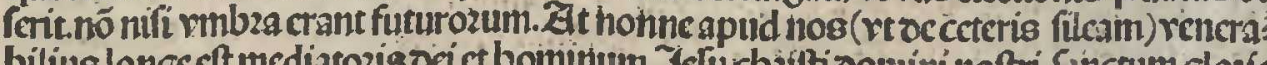

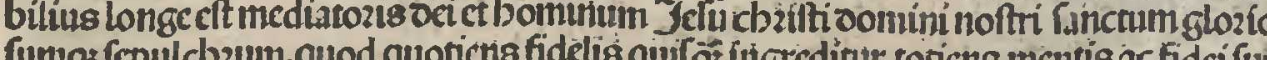

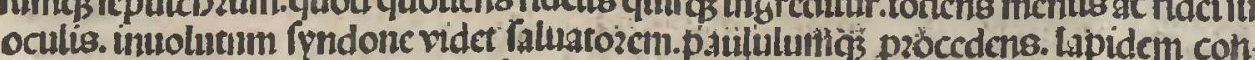
(1)

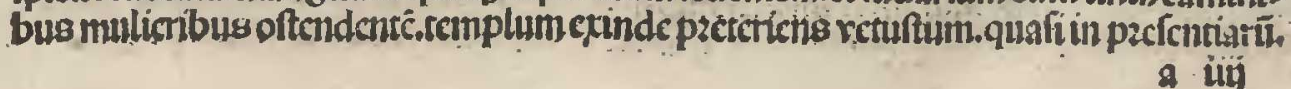

Promifiem Promifiam 2) importick 3 in Mromoria si bur Arsatertarat insures fintions 


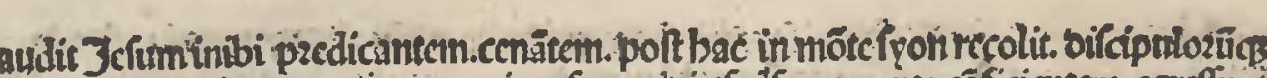

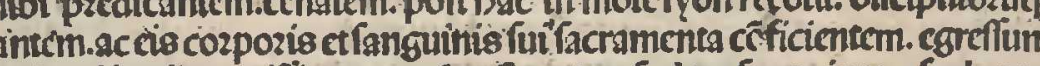

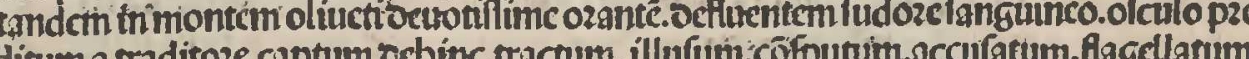
dituma thind

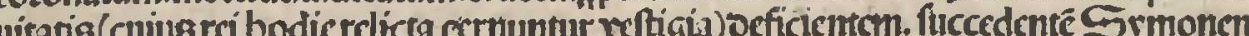

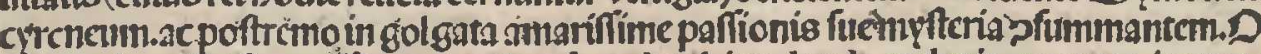

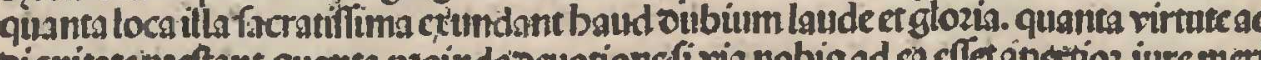

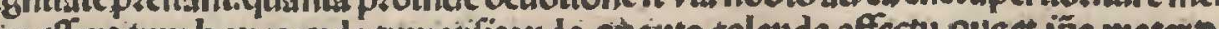
mini ta ani vo

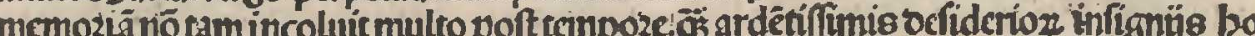

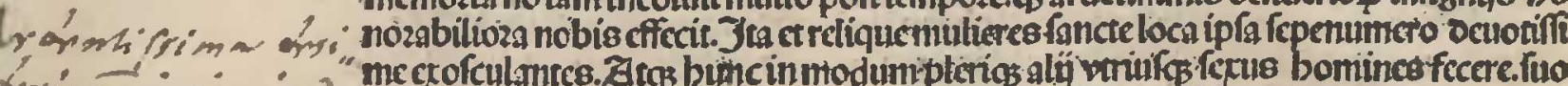
mes.

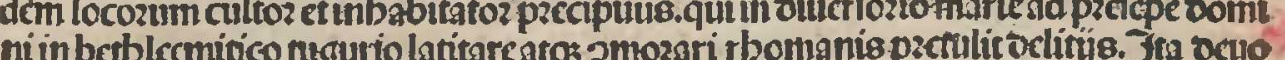
(cin

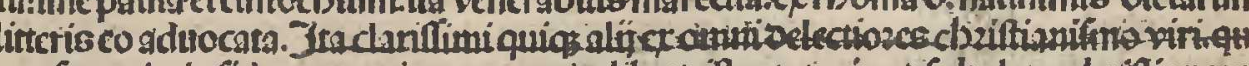

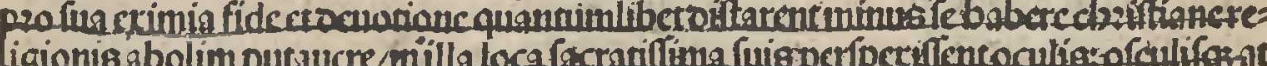

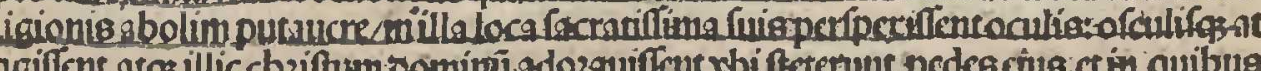

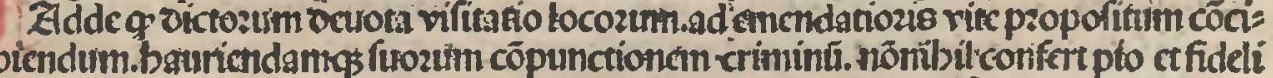

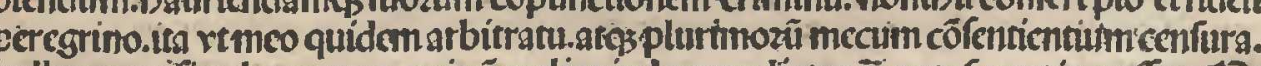

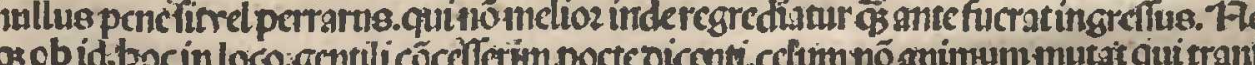

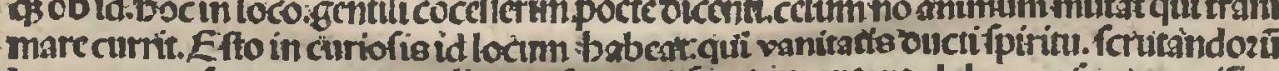

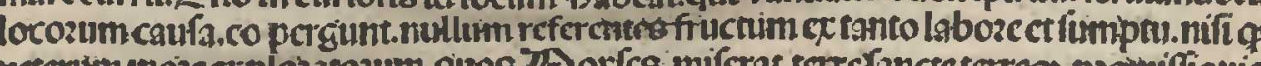
rectrtum mozectoplos

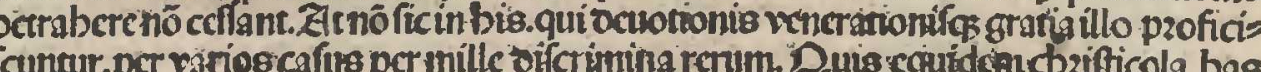

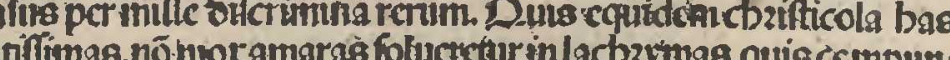

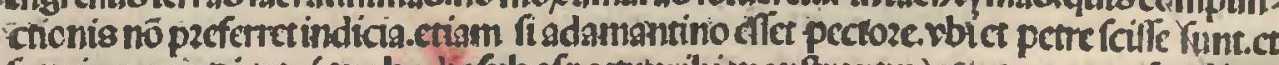

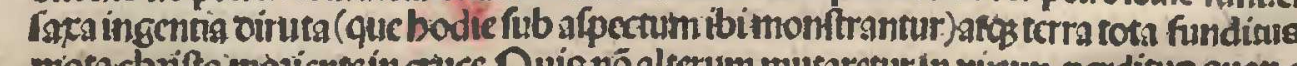

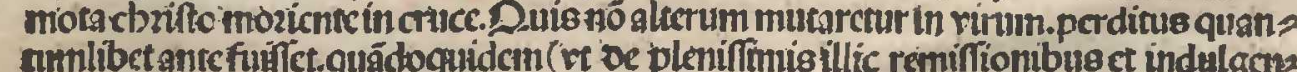

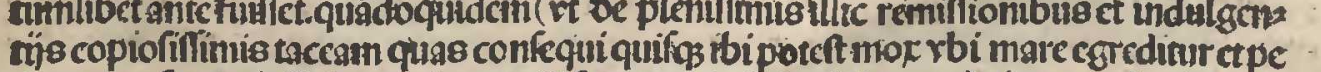

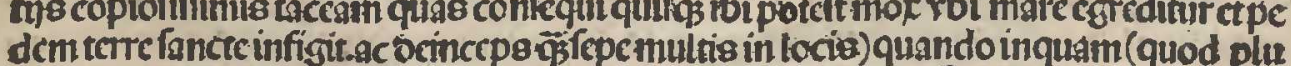

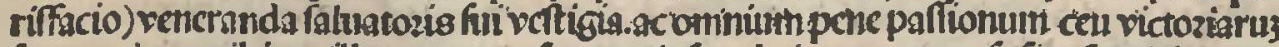

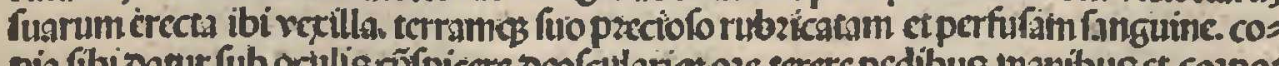

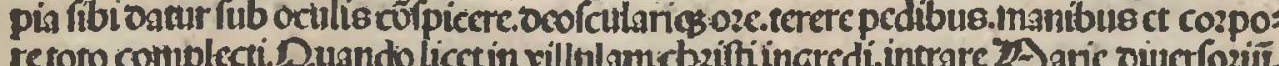

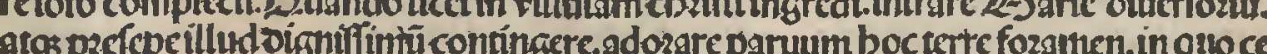
losum conditoz natris eft.in quo occus immenfue infans facrus vaguit.in quo inuoltus

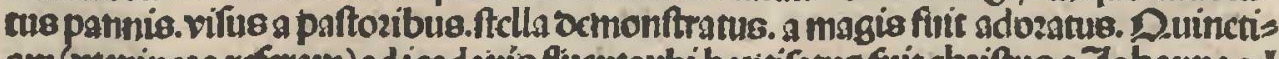

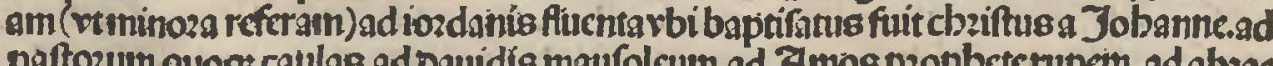
ocmiox íacc

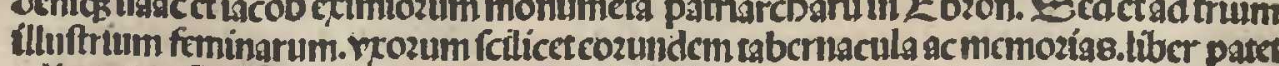

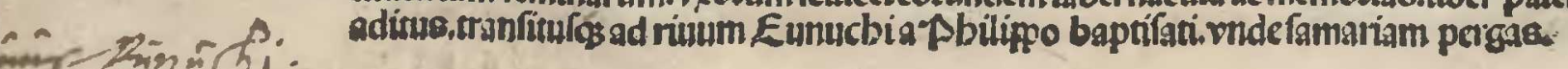

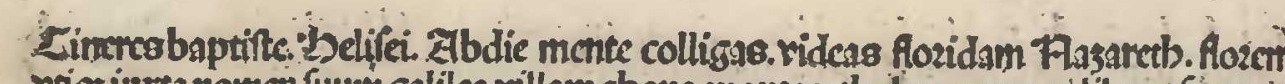

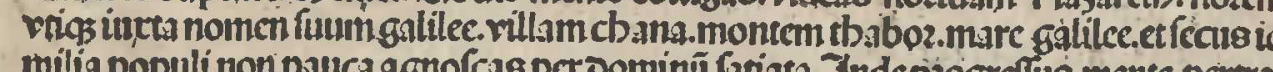

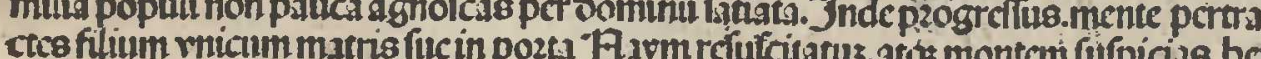

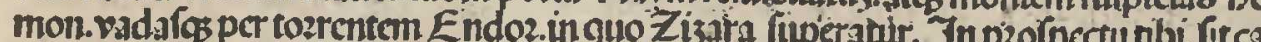

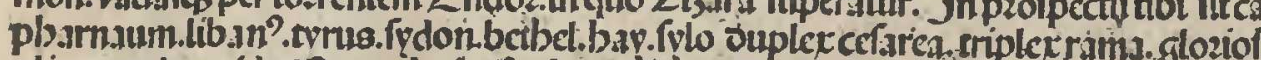
olim ptolemcida. Ramathain Topbprm. Jope. I ydia. Jamnitia. Fobcac pbilifitin -

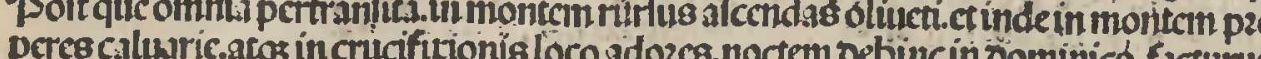

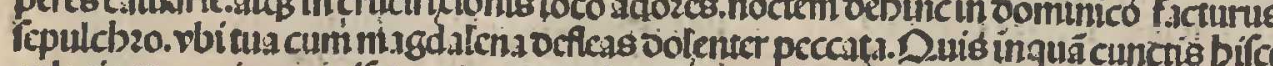

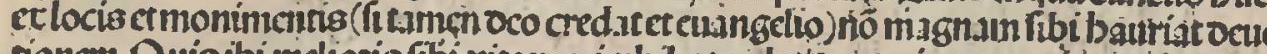

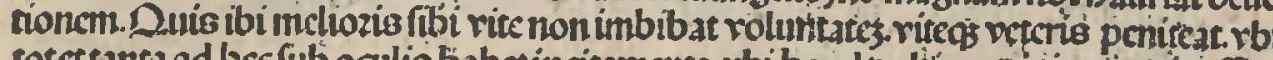

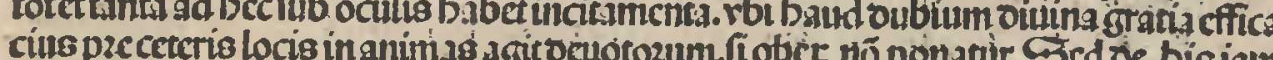
fatis fiperog fortafio.

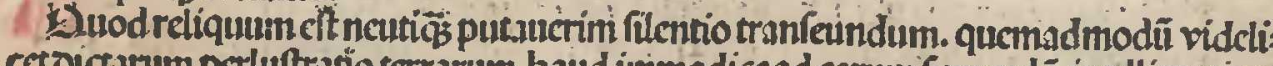
cet oichanum pertuftratro terrarum. baud intimodice ad carum fumendā intelligentiam

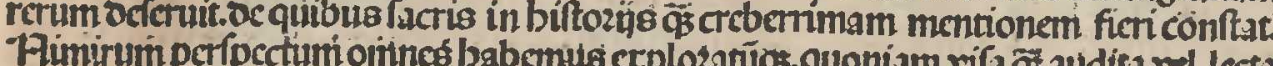

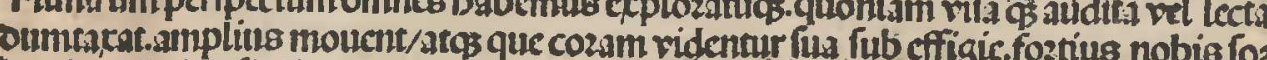

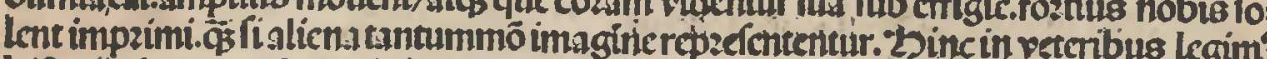

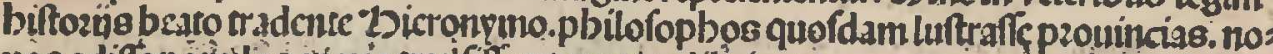

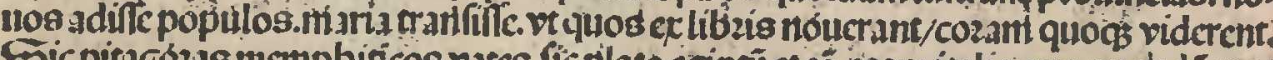

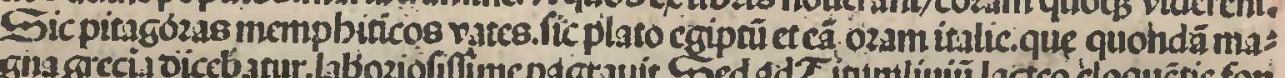

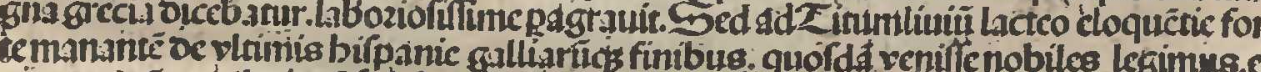

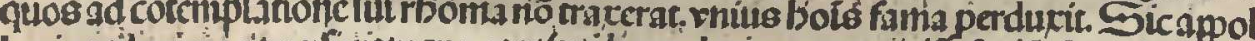

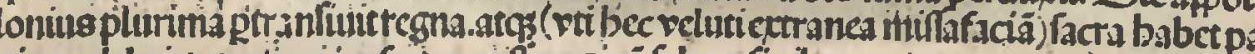

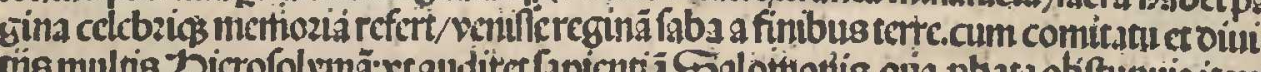

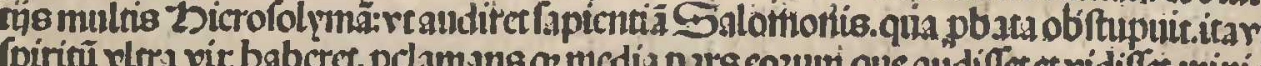

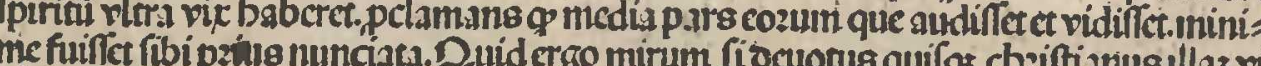

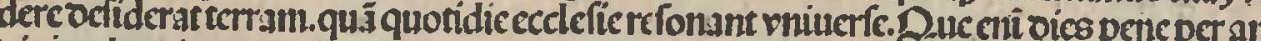

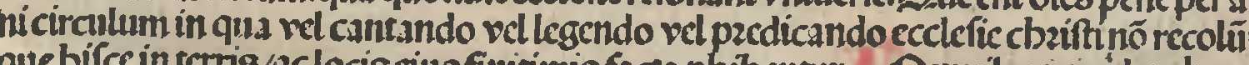
aikus be te

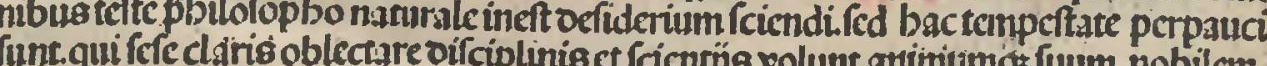

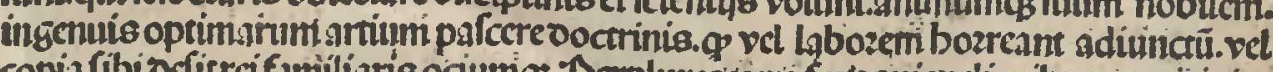

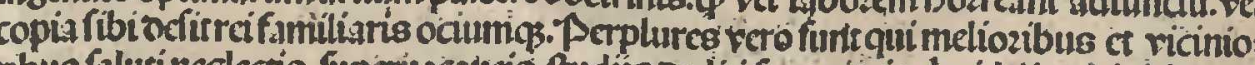

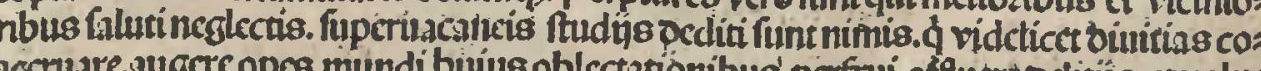

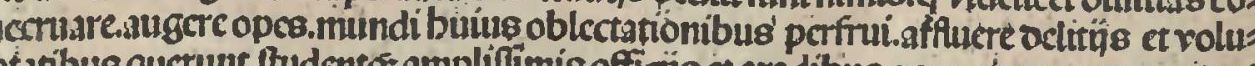

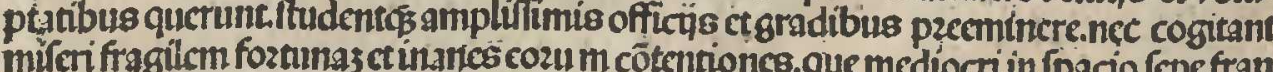
güntir ct cozruunt.relante in

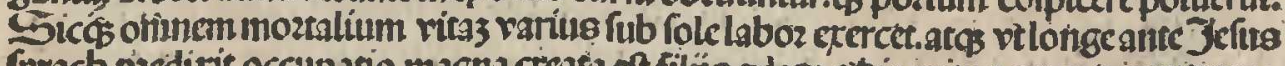

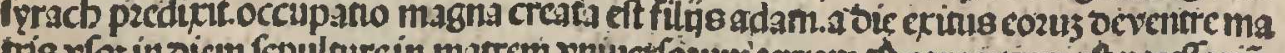

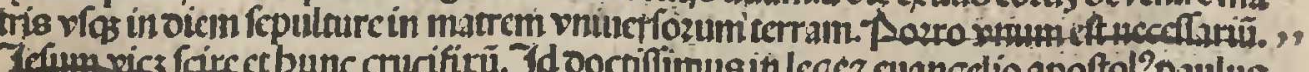

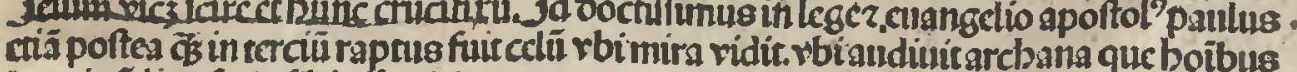
pzof flor Coditing

onisom penome

Magma Conorio Cogimsutas

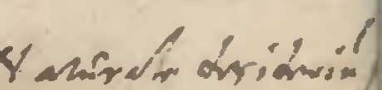




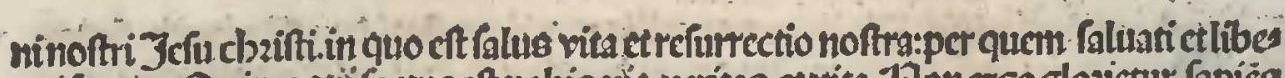
ratifumis. Duipequiifactuseft nobis via. veritas.ct trita. Fon ergoglozictur fapiés

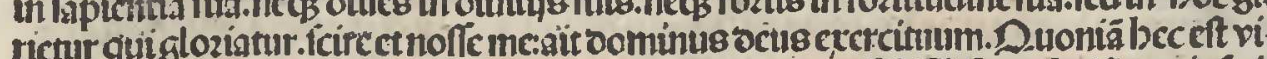

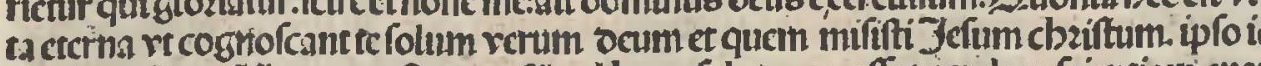

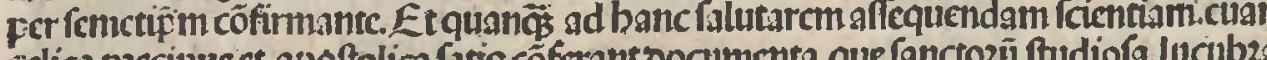

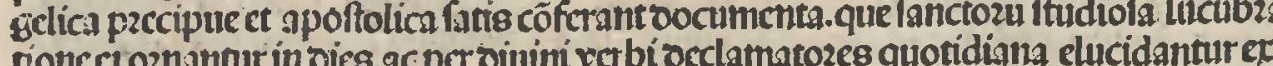
planurione

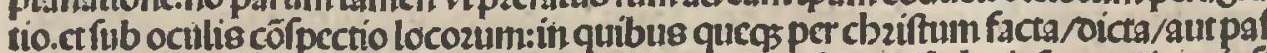

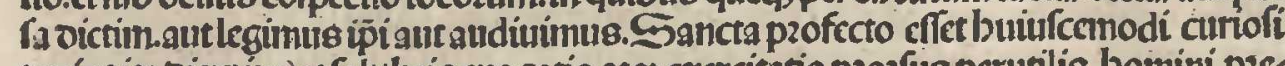

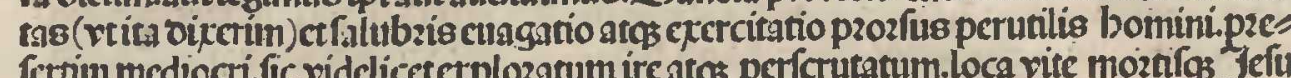

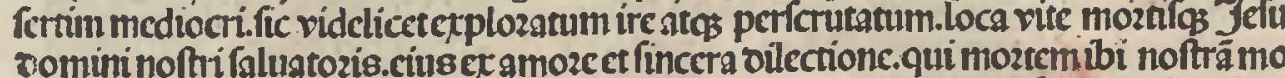

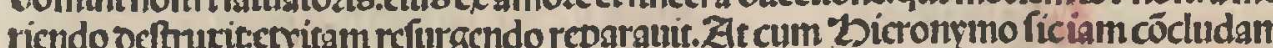

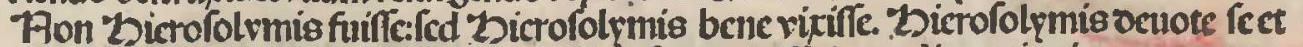

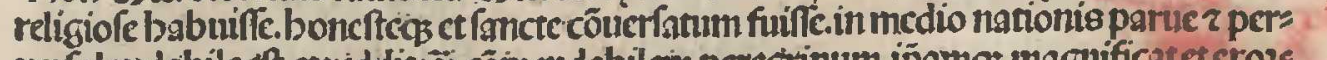

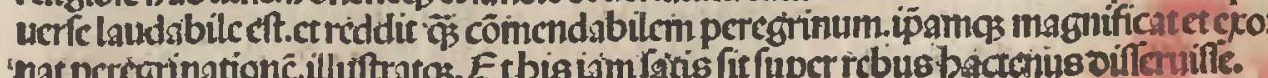
Intentionisexplicario.

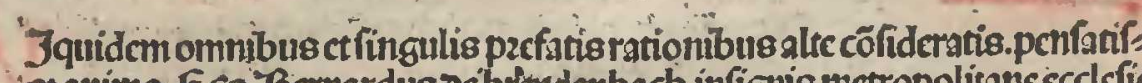

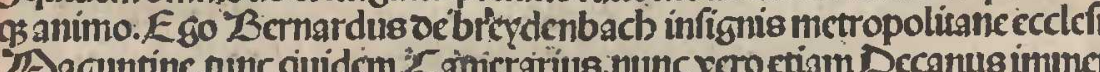

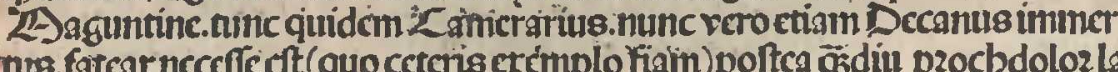

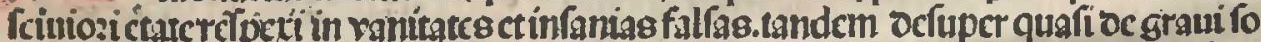

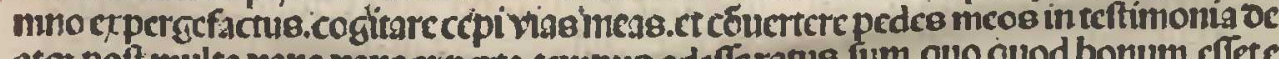

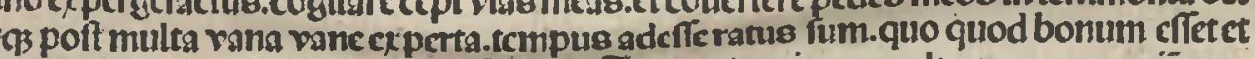
puts.

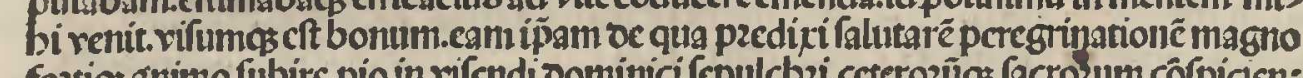

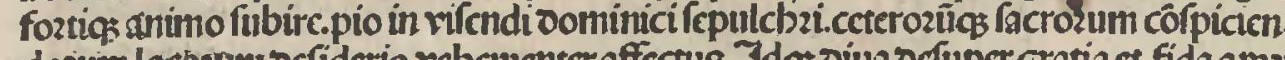

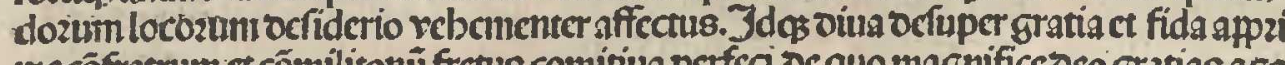

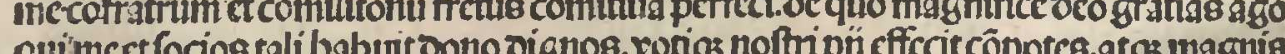
peanguiftis f

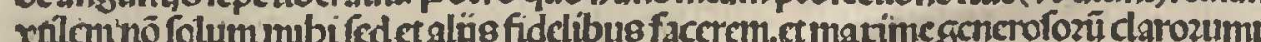
boiminum animos in eam ipam magis magilos cömoucrem. opcram nauaui ä́ctact rt interperegrinandü me oc ommibus que cituneccilaria oigna

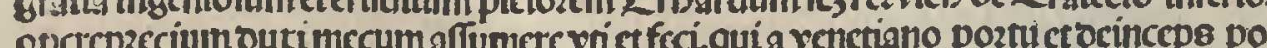

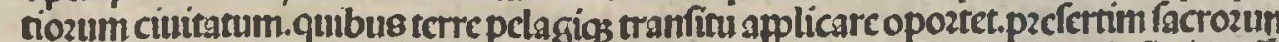

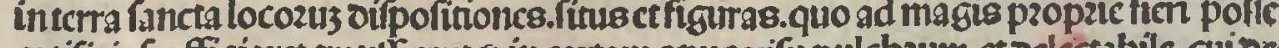
ad rou lo cunctis babererolcnnbus comunicandum rcinam atos vtinam. intento optato

Pperisouitio. ren certiosen

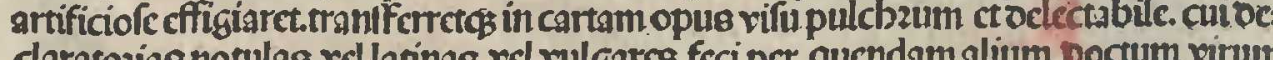
baud racuum fructu cuadar.

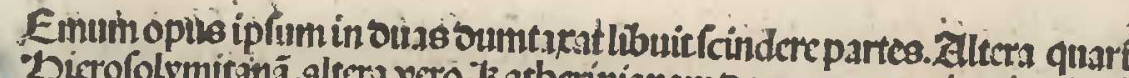
(1)

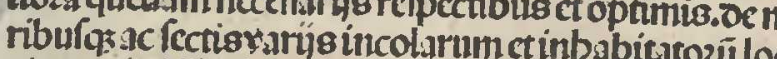

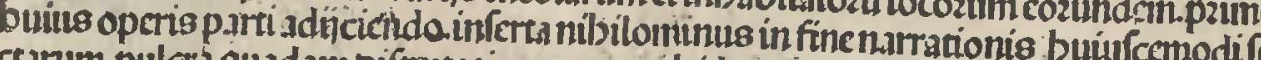

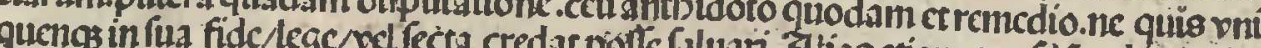

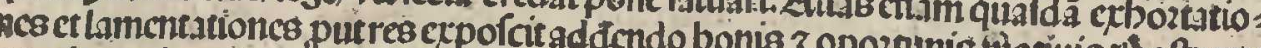

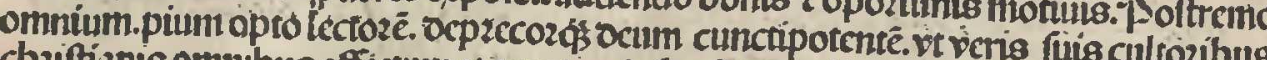

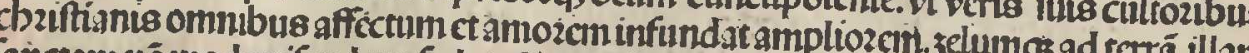
ancipublice

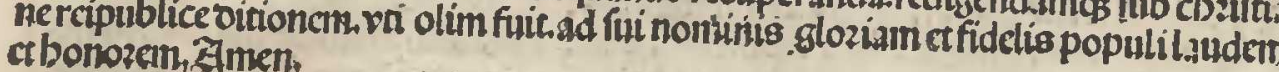

(Sequirur intirium butue peregrinationis a folò natali vfos nerictizo.

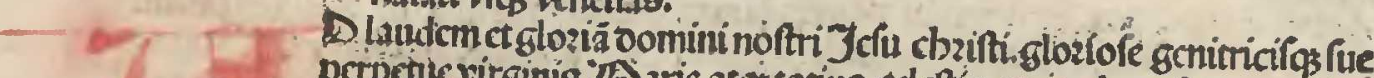

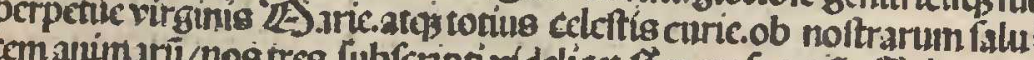

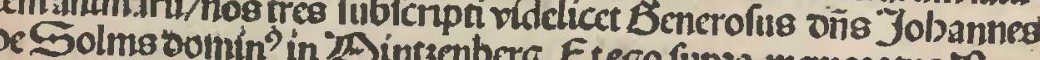

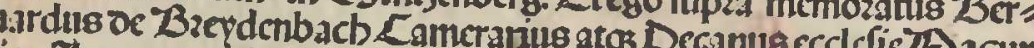

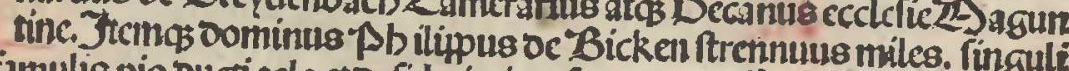
作

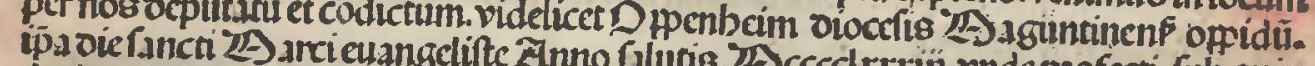
decini oicrum interuallo. renetias oivo niunere fanict incolutives a pzofecti. fub quin agendum ixt.

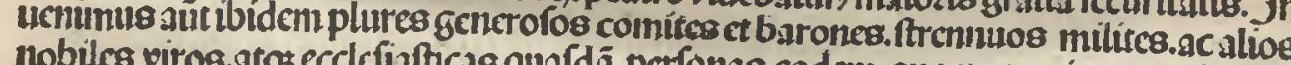
cbziftianis

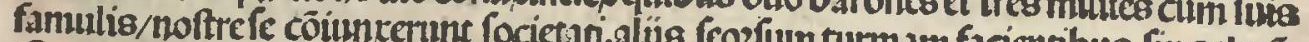
Erant autem nomins costum quiin vna nobifcum galea fierunset focions fingularc.

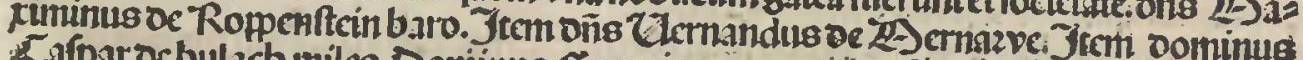

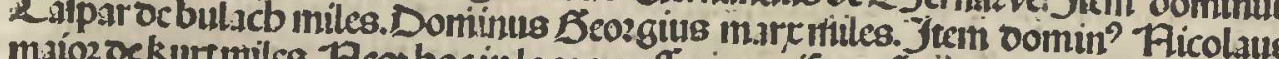

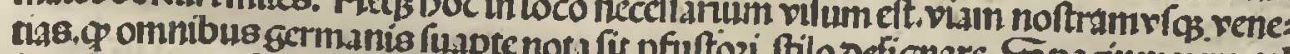

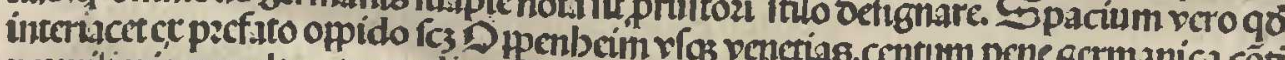

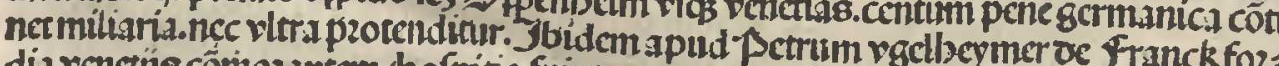

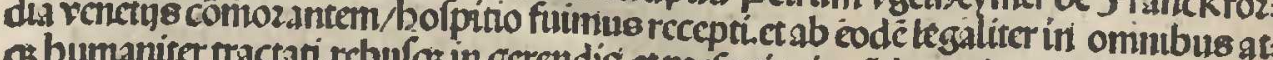
Qurilio fidclior adiuti patrono galce. vnde baud ab reoucieas bic clufurat

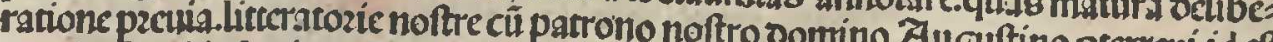

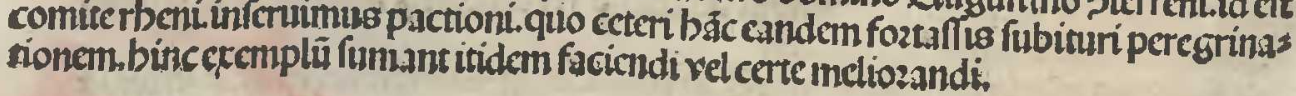

Deformucónactuscuini patrono galé

enum in pucntionc articuli tales inferti.quos quafiad alios factos cur

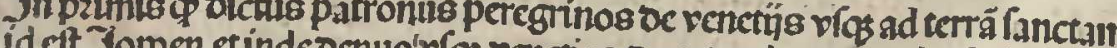

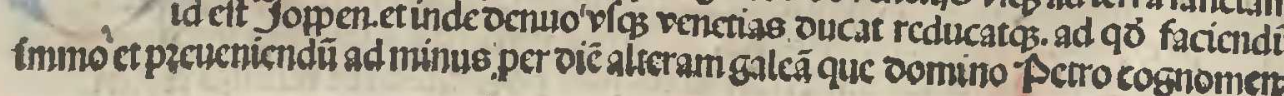

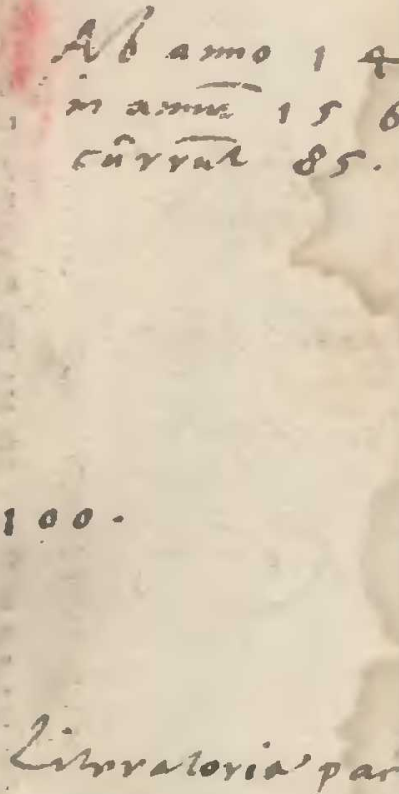$$
\text { of }
$$ 
Indavesttinebat/in qua ali peregrinioe fecunda focietate vebebantur. fub pens peregrnozum. tuctoeinceps in omnibus locis quibus foret splicandü/p2ofpiciantet inquirantan

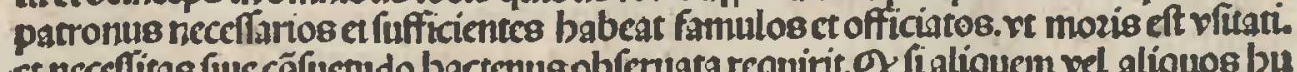

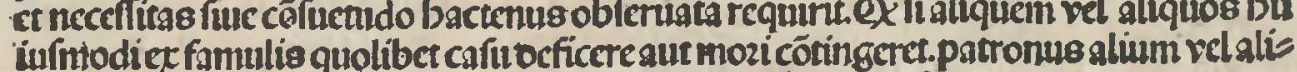

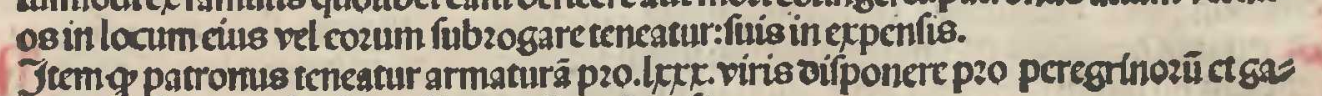
lee offeritome.aut boftium fiopus fieret inuafione. Jtem $q$ patronus ad fingula loca et poztus ap licare teneatur.quibusabolim confue tumeft applicare.neqs tamen vitra bidui triduiue ppacium in aliquo loco immozectur.

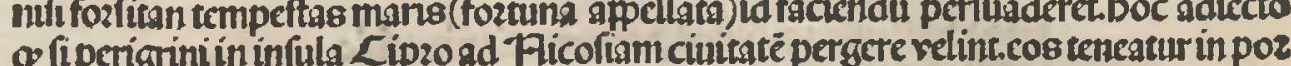
th Lipzinoexpectare. Atun reditu octetra fancta biduo vel triduo in quolibct poztll. mertium vel vendendarü vel cóparandaru gratia. porcent immozarietno vitra. Jta tas men $q$ loca peregrinis oeputata. in Galea libera mancant.necincrcibus occupenturas

Jtem q patronus peregrinis fingulis vicbus bis ad comedendumet bibendü bet ve oect pio viris boneftis. Q) fiex qualibet caufa aliquis peregrinoziñ ad menfampatro nimank aut $v e$ efpere renire nequiret.aur folus foztaffe vel cum fua focietate feozfumbel aursblop córtadictione omnt.

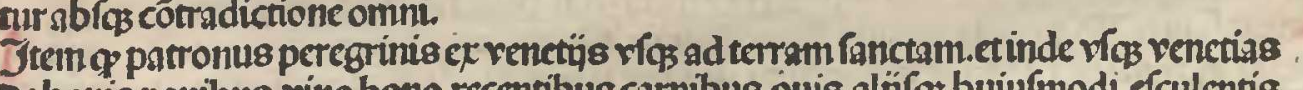

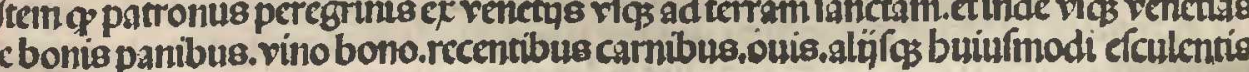
copetcnter pzouldereteneatur.

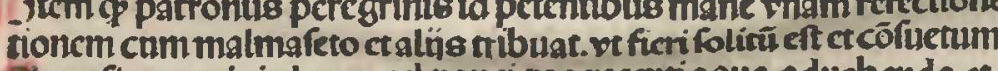

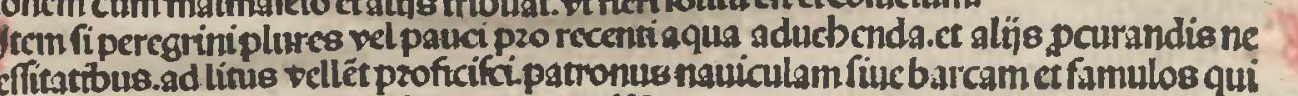

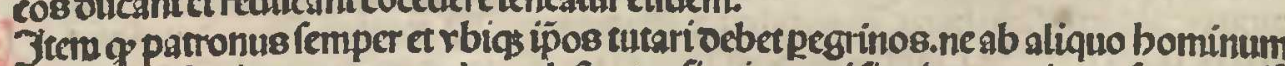
vectis gutfacris quoquo modo moletentur fivein mani fiuc in terta. iupta fuum poffe et noffeablip fara.

Jecm $q$ patronus ípos peregrinos ocbito permittat tempozc in terra fancta mozarict

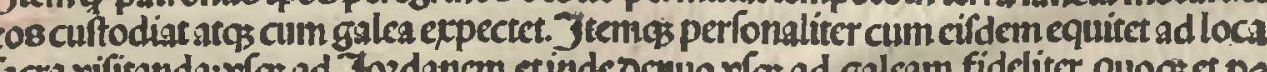

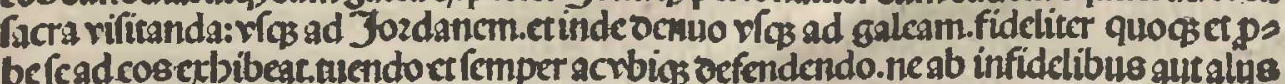
rlto pacto circumticniantur. fiuc feducantur ooloct fraude femotis

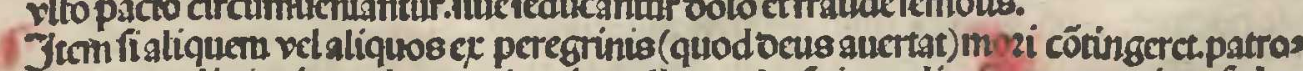
nus oe ocrelichos vel legauerincomunino relinquatintacta.

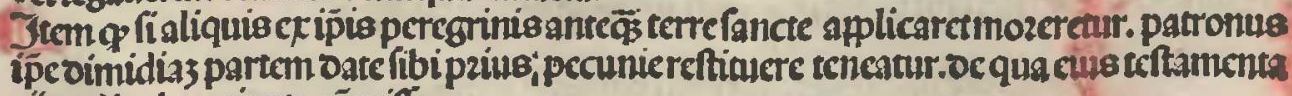

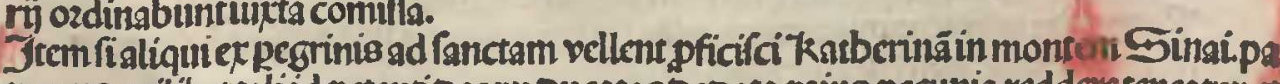

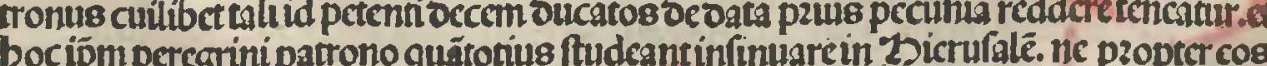

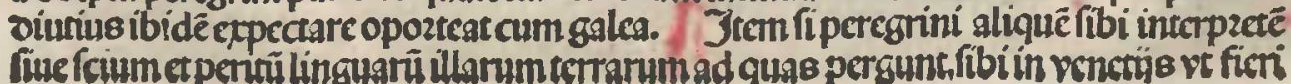

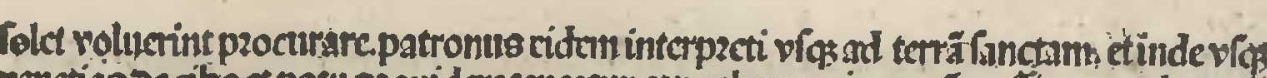
coder

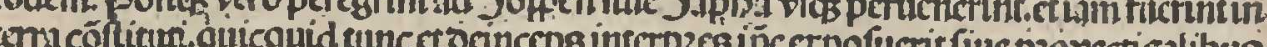

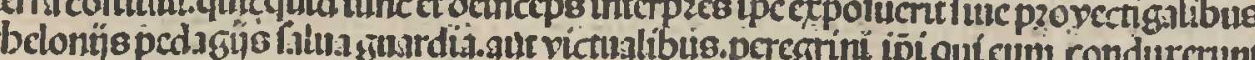

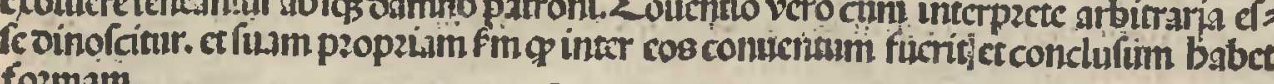
formam.

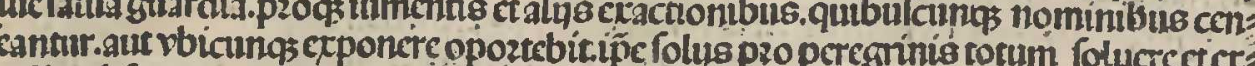
pediresblosecoum tumpta rel oamno teneatar.

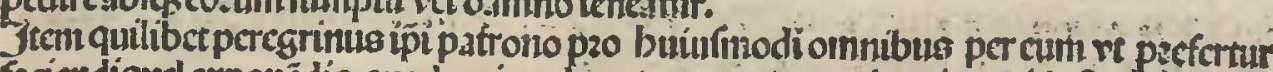

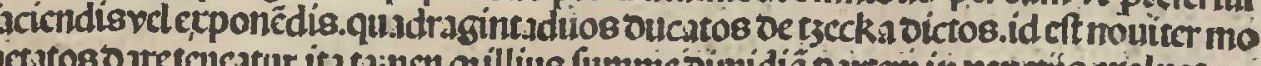
levuane

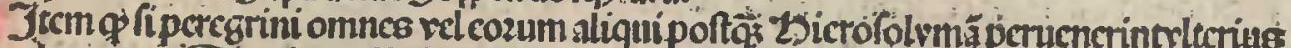

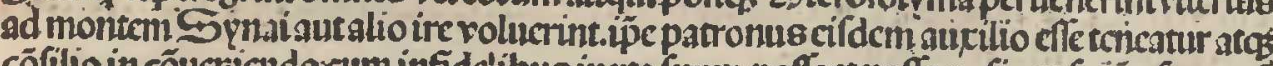

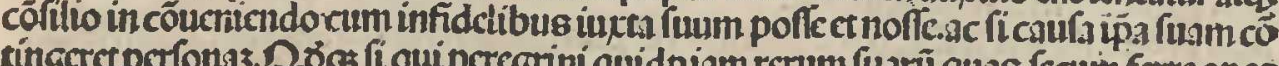

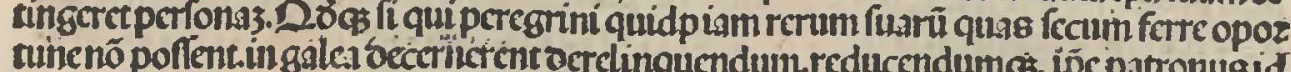

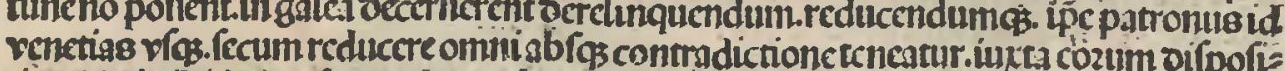
ronem cis ibiden referuandum et bocitcrum propzińs fub explenfís.

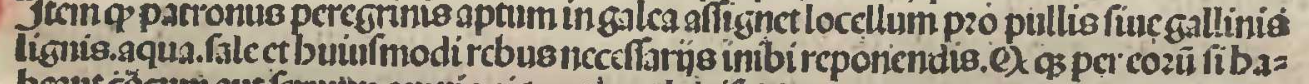

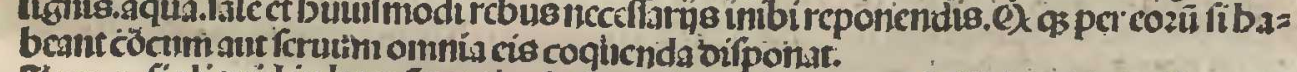

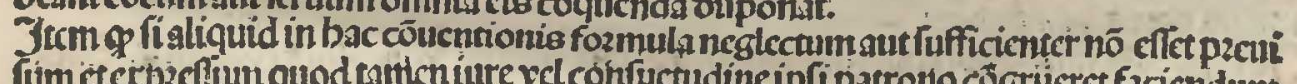

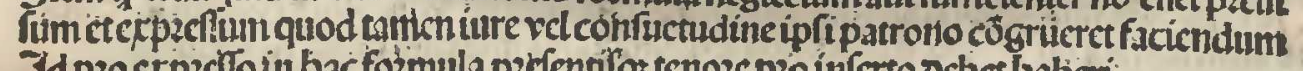

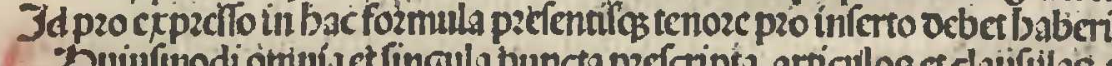

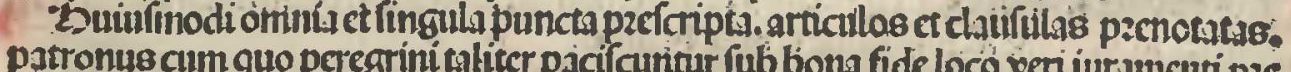
pastronus cum quo peregrini taliter pacicunitur fub bona fide loco veri iuramenti pece

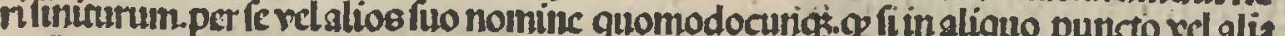
quibus ipfe patronus cötraucnire pzefuncret.quicquid inde oumni allasü fuertí peres Gerints

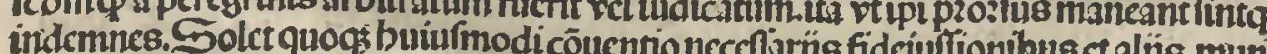
mentis robozariquo firtiioz fitce piligentius ebferuetur, Dereliquás pteciofiset venctandis que bit
bentur venerins.

Onfequenter becuotis peregrinis vent confidotandum oq in cintute ip ra renctianim plurime pacciole reliquic conferuantur. queab cif

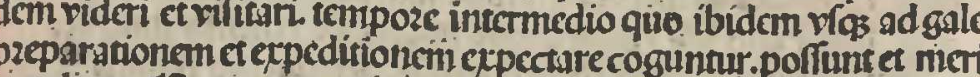

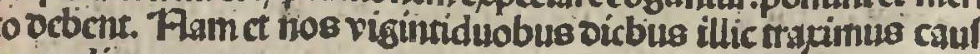

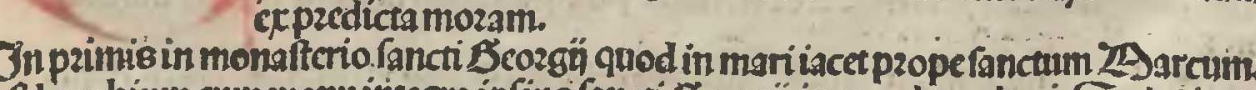

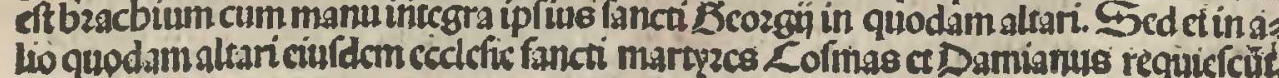




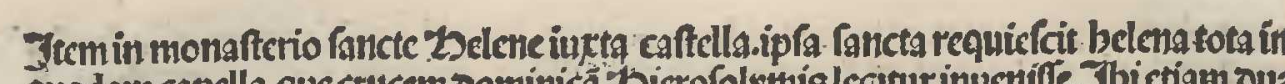

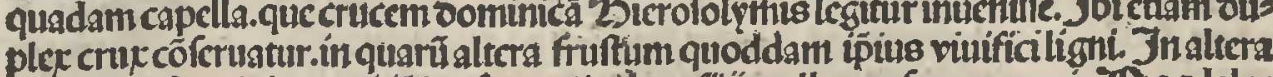

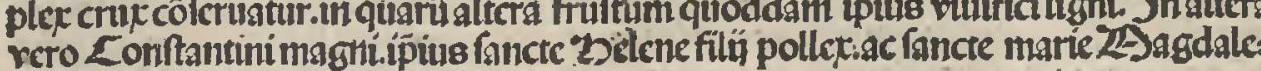
nc os quoddā be pectozcótinetur.

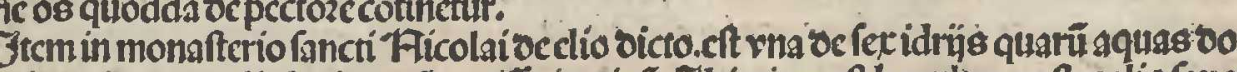

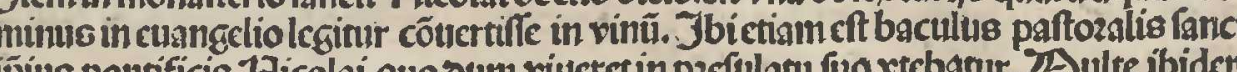

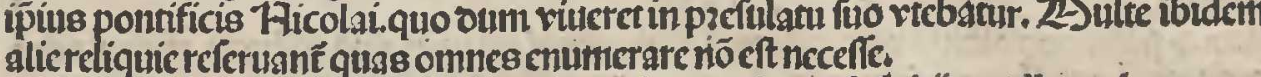

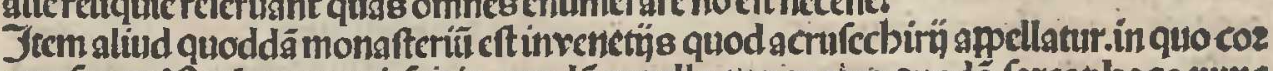

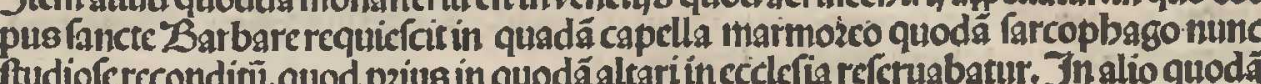

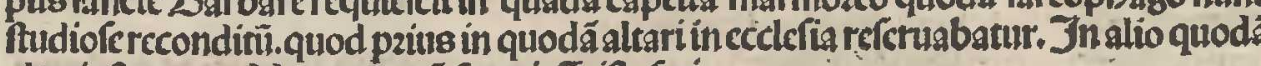
altaricft os quoddam magnil fancti Eriftofer

Tccin in alia quadectich

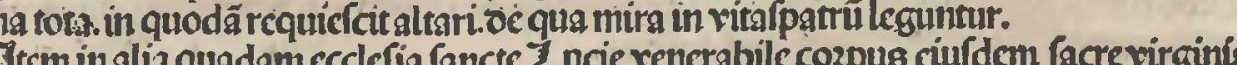
acmarteris 2 ucierequicfaitadeo integrum et illefium. vt maknmilleciuls viderietreco

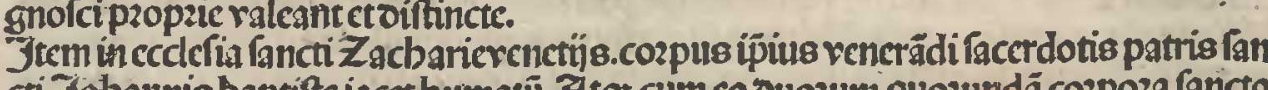
(n) Gbteriam pac fozibuts eccléficc curfdem.eft fepulcrumbomini inftar cius quod eft bie rofolpmis formatumn.

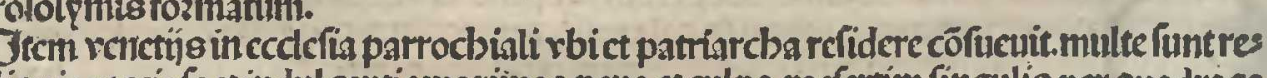
liquic pzeciofect indul gentic maxtine a pena etculpa.pzefertim fingullis per quadrage mam oominicis dicbuls.

作 bidem tbciaurue renctozĩ in teftudine quadam. fummma cum oiligctia reclulus.

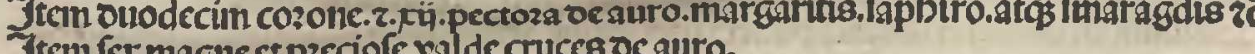
Jtcm pilcusoucisquo quilibet ouk venctozü. fingularipzinilegio fiute pactogatiuace ecij incetumabilis.

Jecm ovo candelabaa aurea et magna.occem lapides pacciofos babentis.

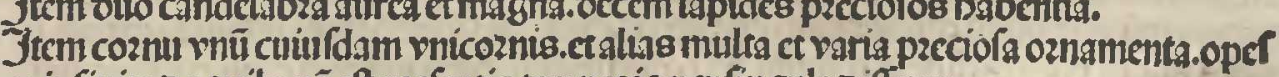

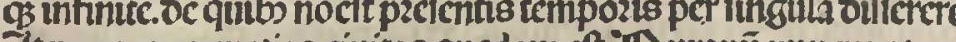

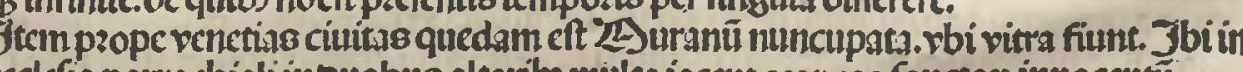
Tcletia parrocbill in

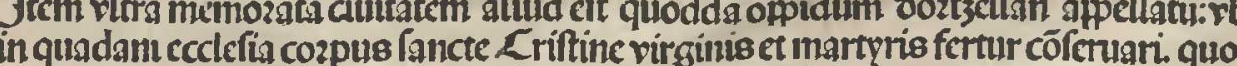
multo pzins tempoze in caftro bulleno nō longea vitcrbio quicuit, rbictipfa martỵn um fulcepit.

\section{Dereliquịs in padua.} (Inilluculas piatrarectitc (a)

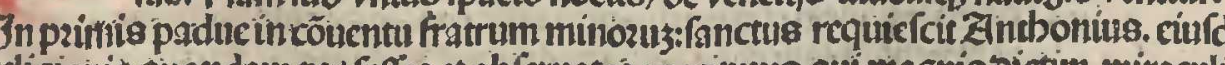

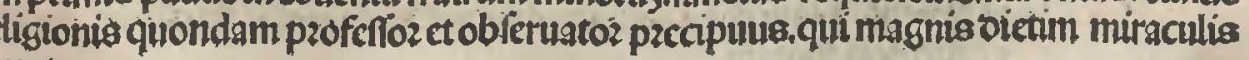
claret.

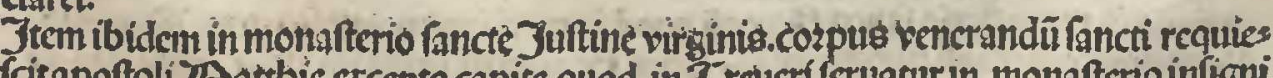

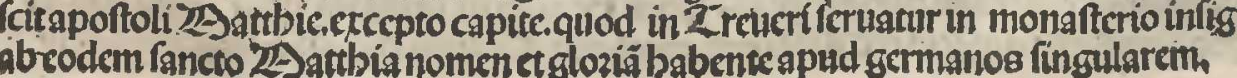

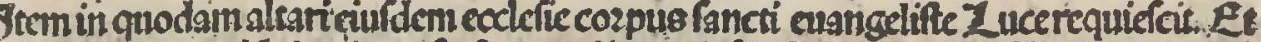
bide in (1)

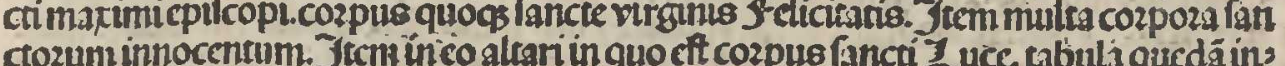
作

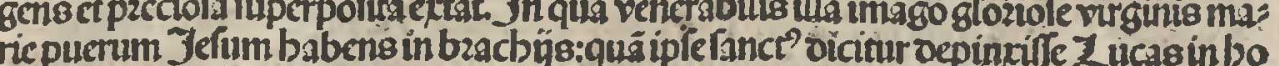

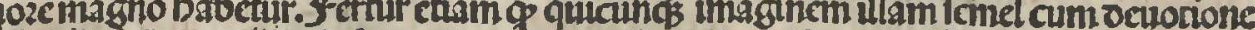
excosdis copunctione inf pexerit.nun

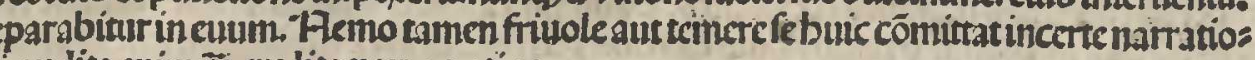
Jem in eademecelefia natto potive

Doza in p2cfatum monafteriü oeportanic fuis accepta per mare renctias tranffictret.rogaus a collega fro. fancto quiodam viro.

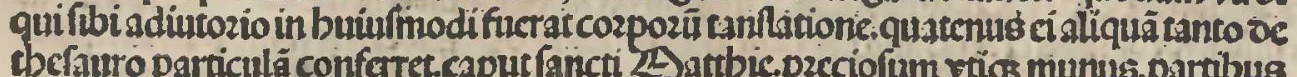

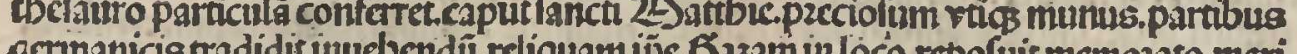
to proundemulta babendusveneratione apud paduanos atos ocuotione opibuslocuplctarit.

$$
\text { Sequỉurozatio cómiendanida ciultatis ctoomi= }
$$

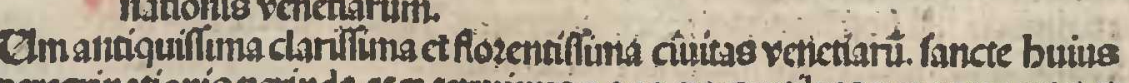
peregrinationis perinde as@ retrminus a quo pergenceibus per mirecetcetet

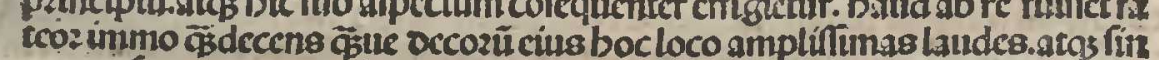

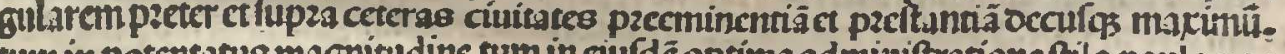

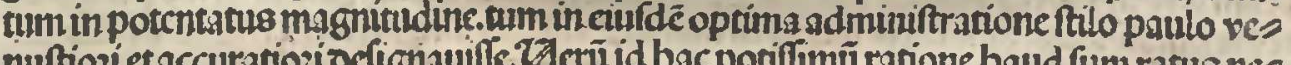
cheriction

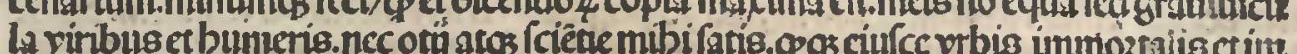

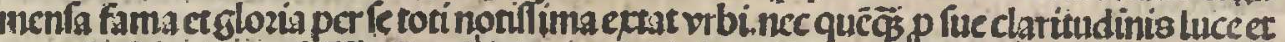

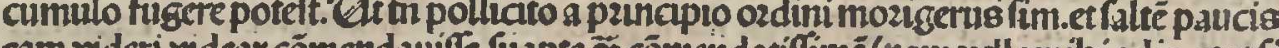

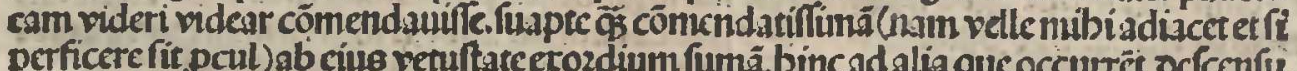

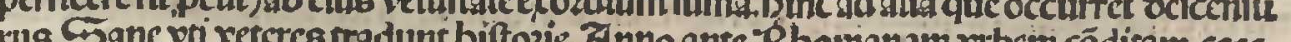

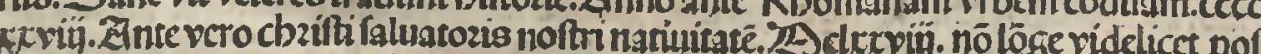

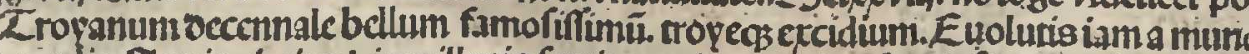

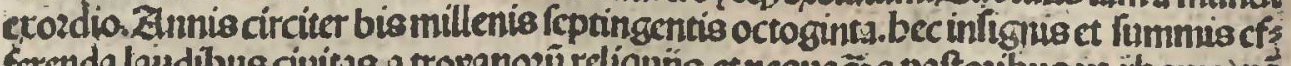

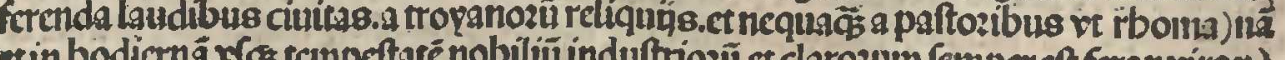

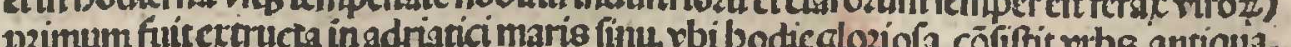

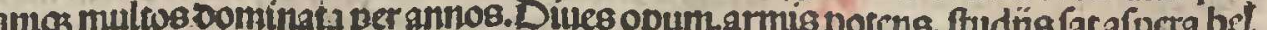

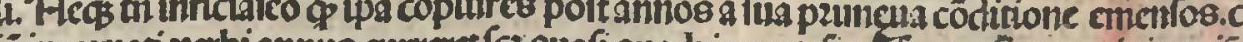

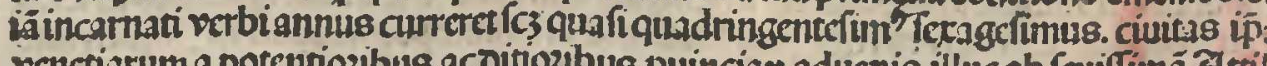
(anchir

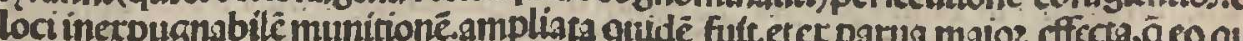
oictum eft tempoze. bumili faris intino fuerat fundata. Ex pfecto sicu mirabilc of ert

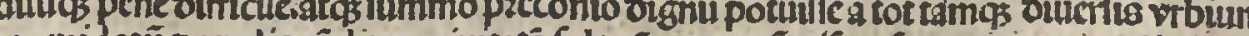

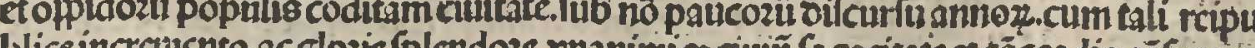
ficeraugaificctornariogio rrbé 


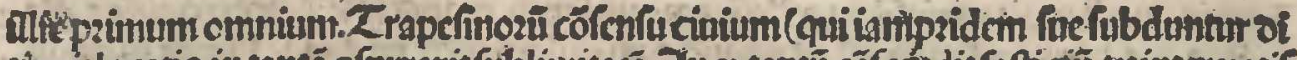

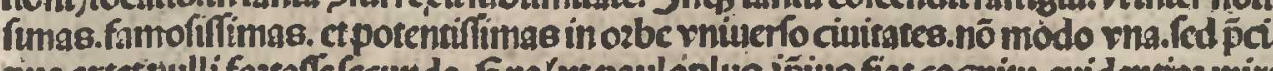

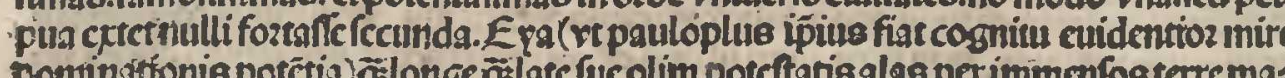
rifos tracuis

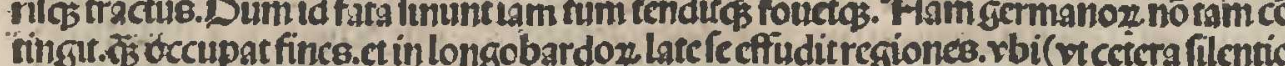

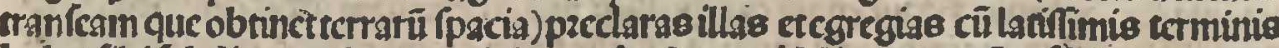

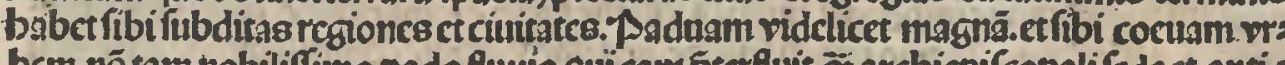

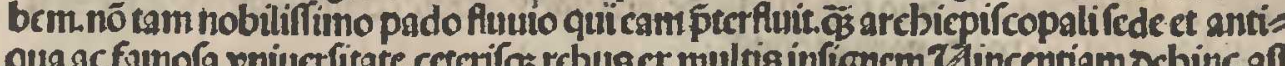
Ztrona

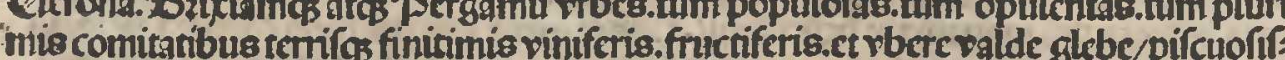

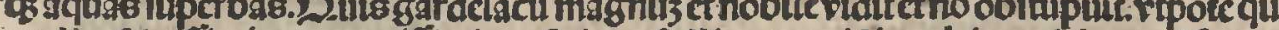

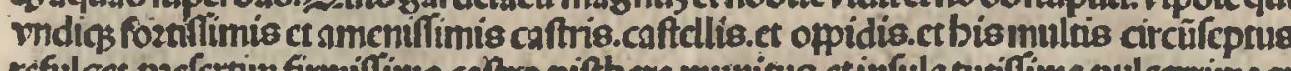

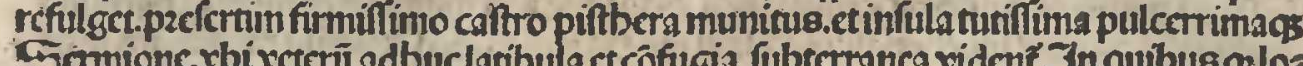

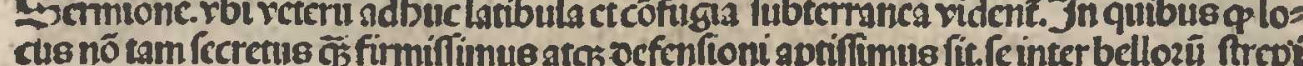

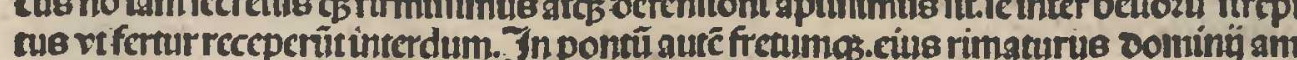

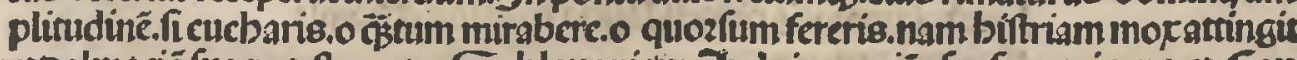

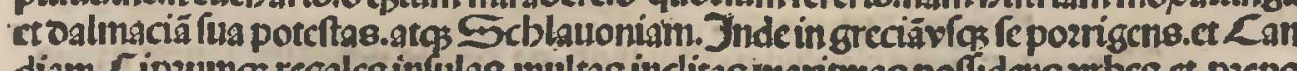
diam $\angle$ ipzungs regalcs infulas. Imultras inclitas inarimas pofledens yrbes.et prepo

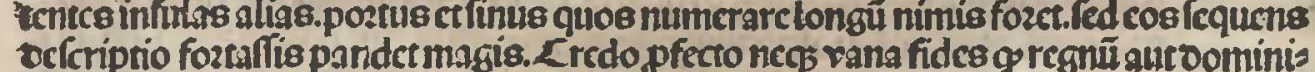

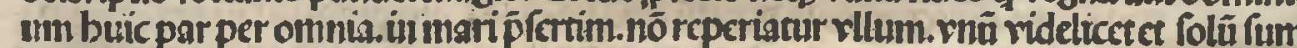

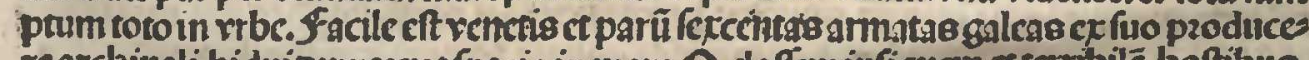

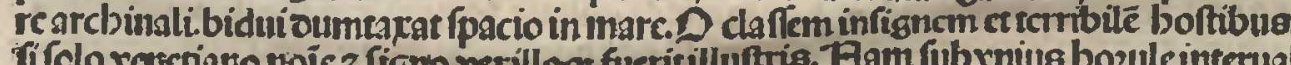

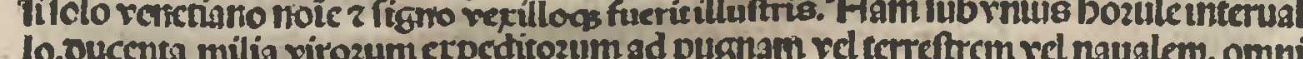

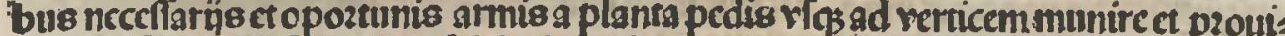
derepoffunt:ad inflicndum fubito in boftes.ct congrediendum animofé fineceffe five vitcum eiddcm. Er quod magnum eft fue argumentu puidentic.pzeter alias copiofín mas in victual

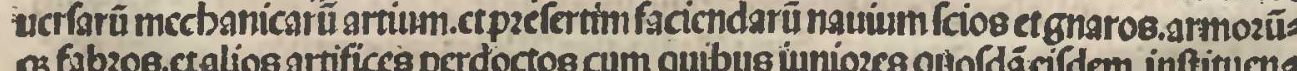

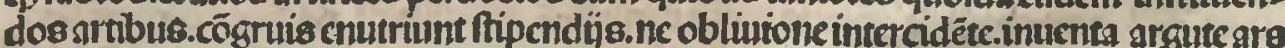
ocperire poffit. SS ullictes vero quinquagints omni babentecmpoze p polis velis fas acndis.orbito prectio in codem loco cóductas. D mnia vero beci pergo meis perf́pexi

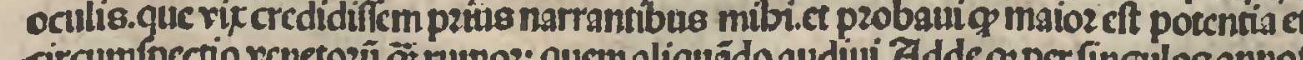

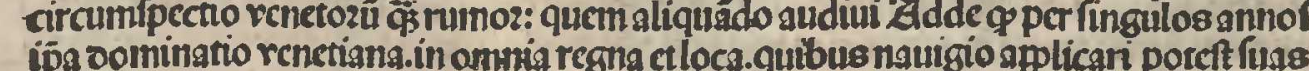

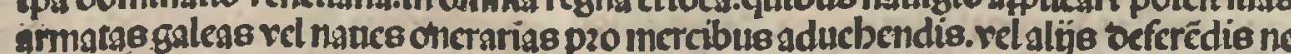

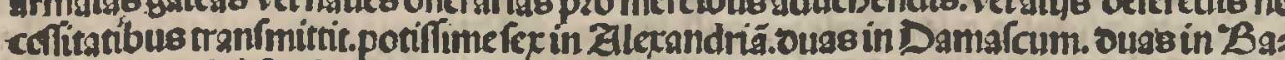
ruttum.ousa in Barbarozû régionce. Duas in Loftantinopolim. Ouse in Jopen fiue

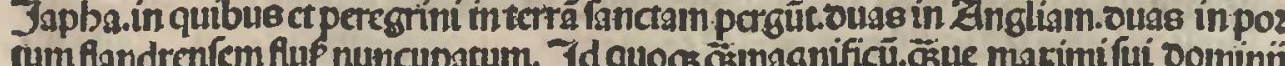

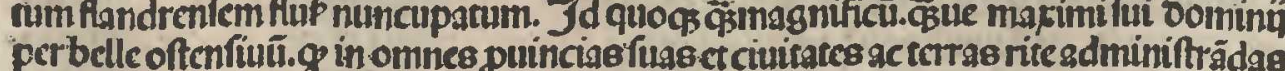
p2obers Gubernandas sinn

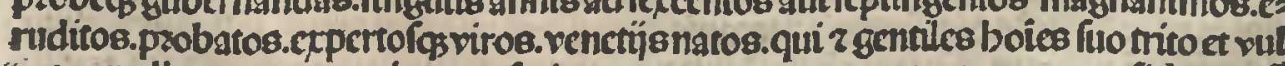

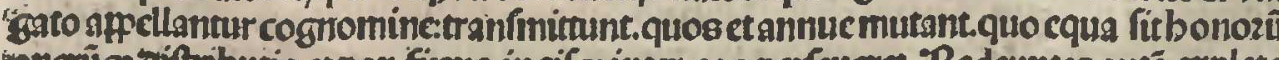

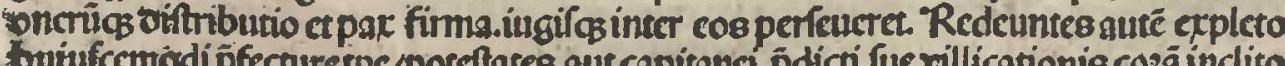

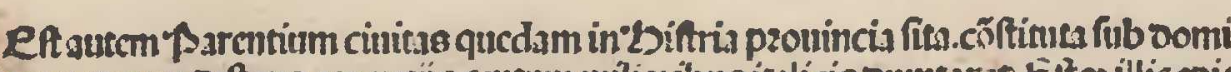

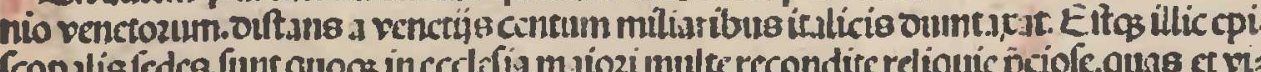

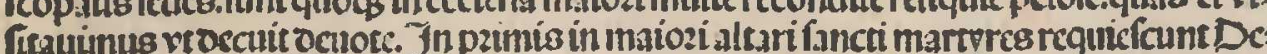

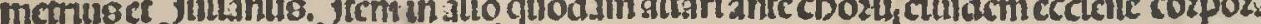

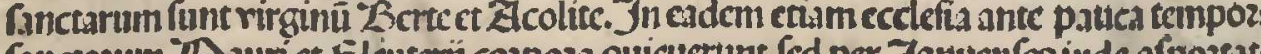

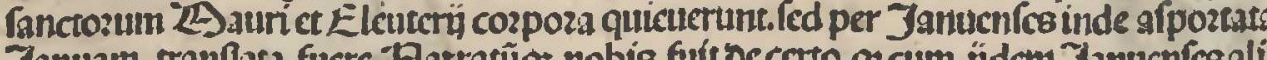
Januam tranhata fuerc. Flarratün nobis

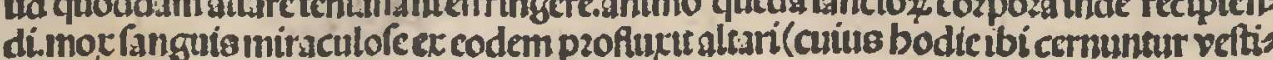
giaderclicta) vnde territi a cepto defierunt.

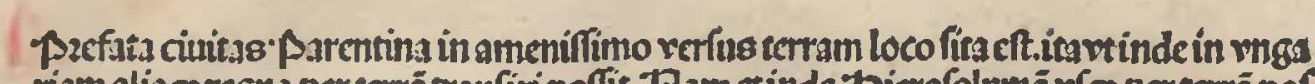

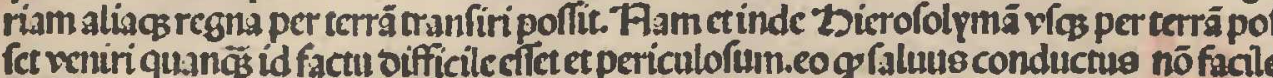

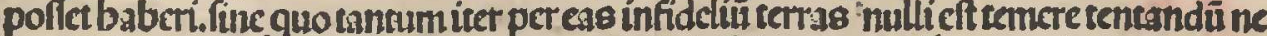

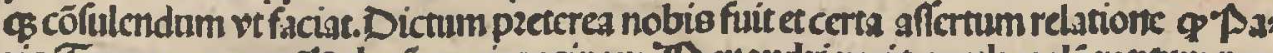

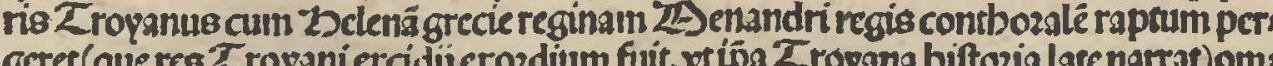

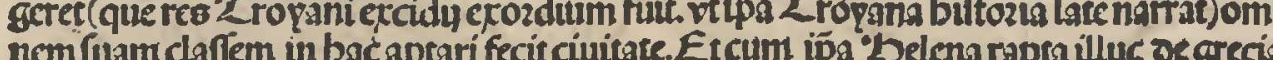

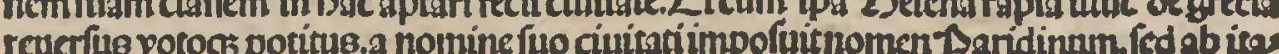
lis bodic Parentium appellatur.

(Scquitur vrbe parentina bicfubfequentereffigiate. 


\section{(5)anit?}

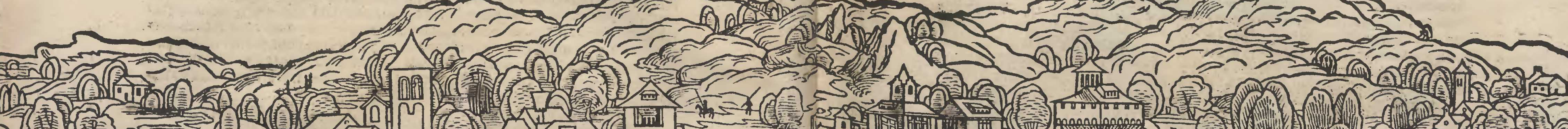
m) 然 $-$

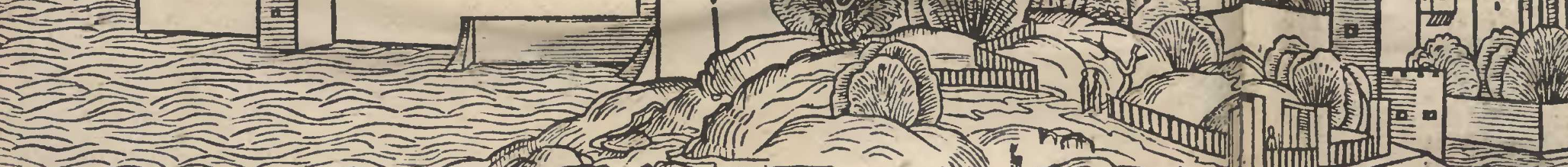

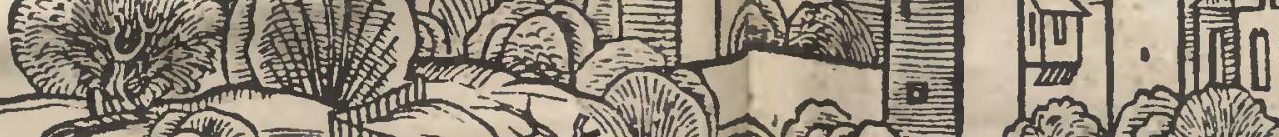

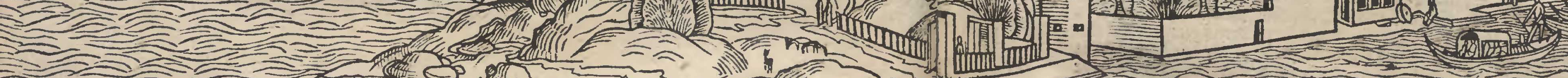

(5) -

(1) $10.1-7$.

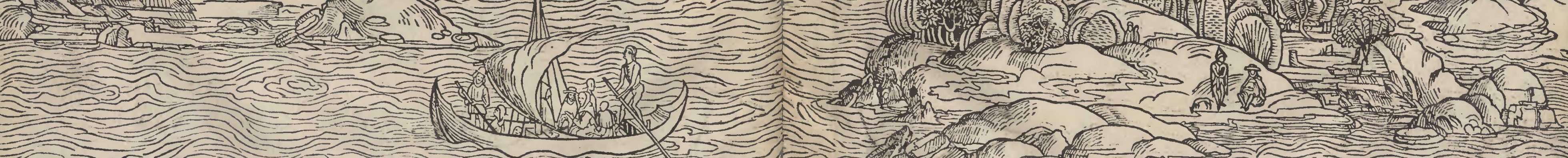
(1) 


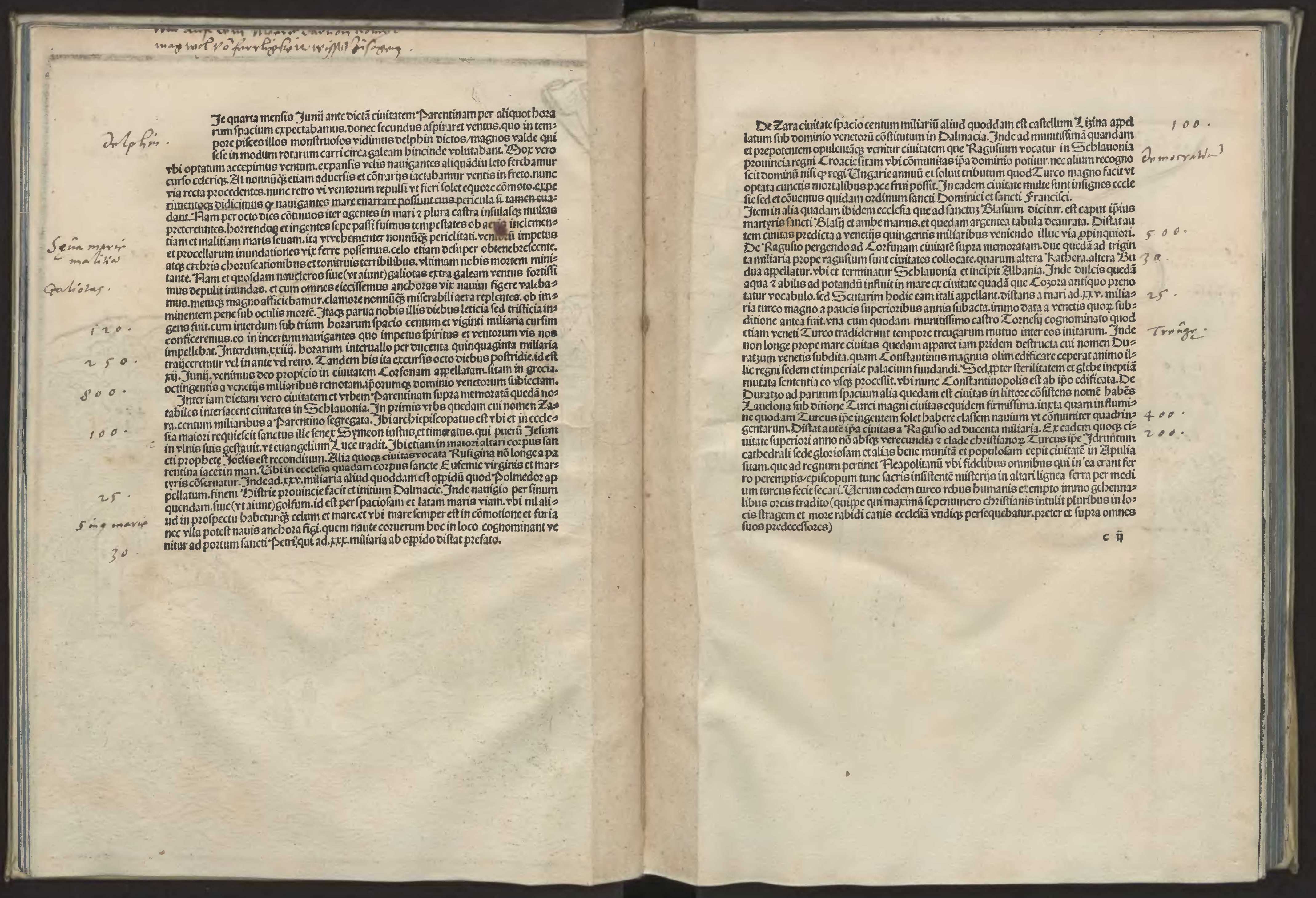




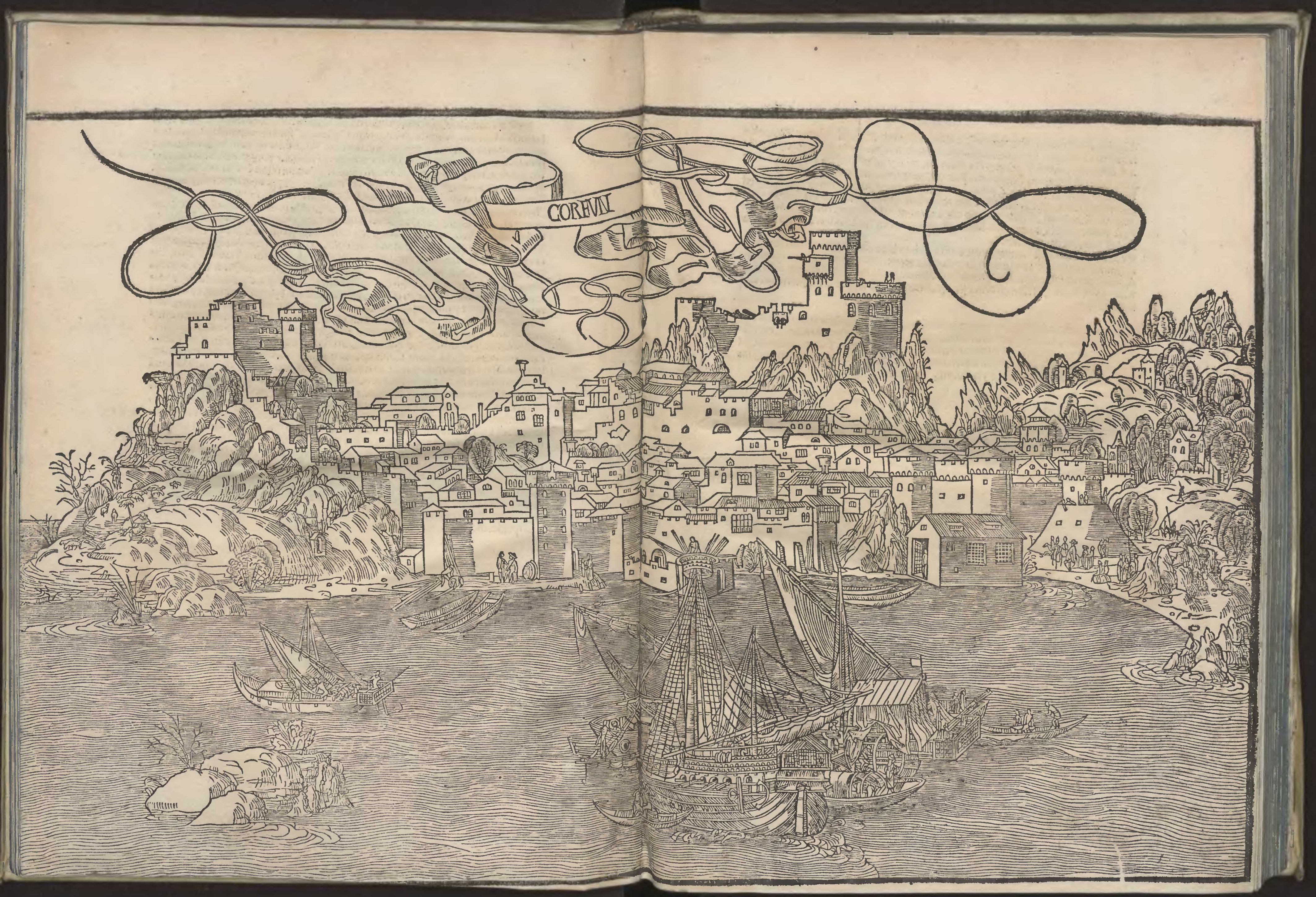


Zanden filiug futs qui bactempeftate cills loco oomintutur regi reddidit Feapolis tano tasm padatam

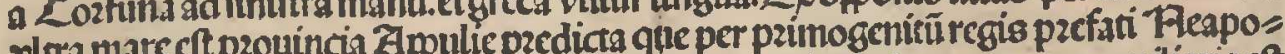

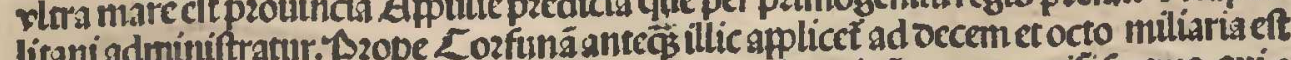
portus quidani ct opoidum oef tructum rbinullus bominü mozatur nilif fratres quis

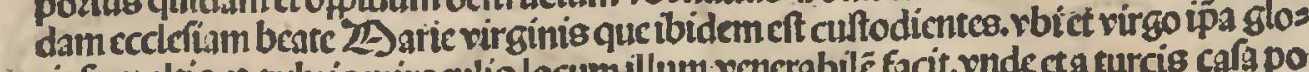

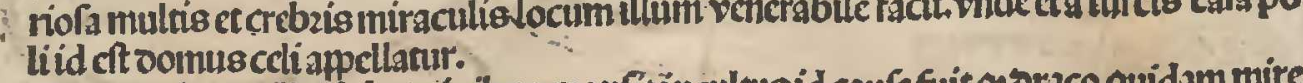
Qx gntem locus ille ab bominibus manfit incultus id caufe fuit toraco quidam mire magnitudinis er marino quodam fcopulo illuc oictim natando venitet plurinos bos

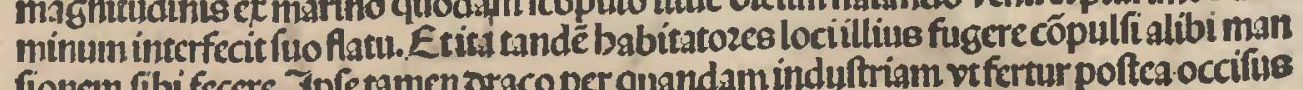
fionem fibi fecere. Jpperamen oraco per quandam induftriam vefertur poftea occilts
fuit ab eifdem.

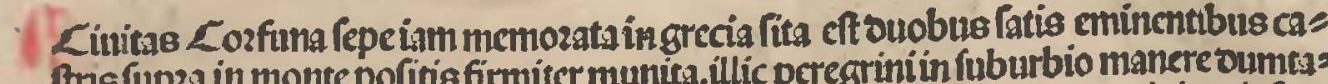
rat finuntur vel apud fratres minozes qui ibicōuentum babent:'porro cinitas ipfacs infulasdiaccns quesb ea nomentralit centum occoginta miliaria occupat in longum et latum.effog tota fub oominio venctortm.

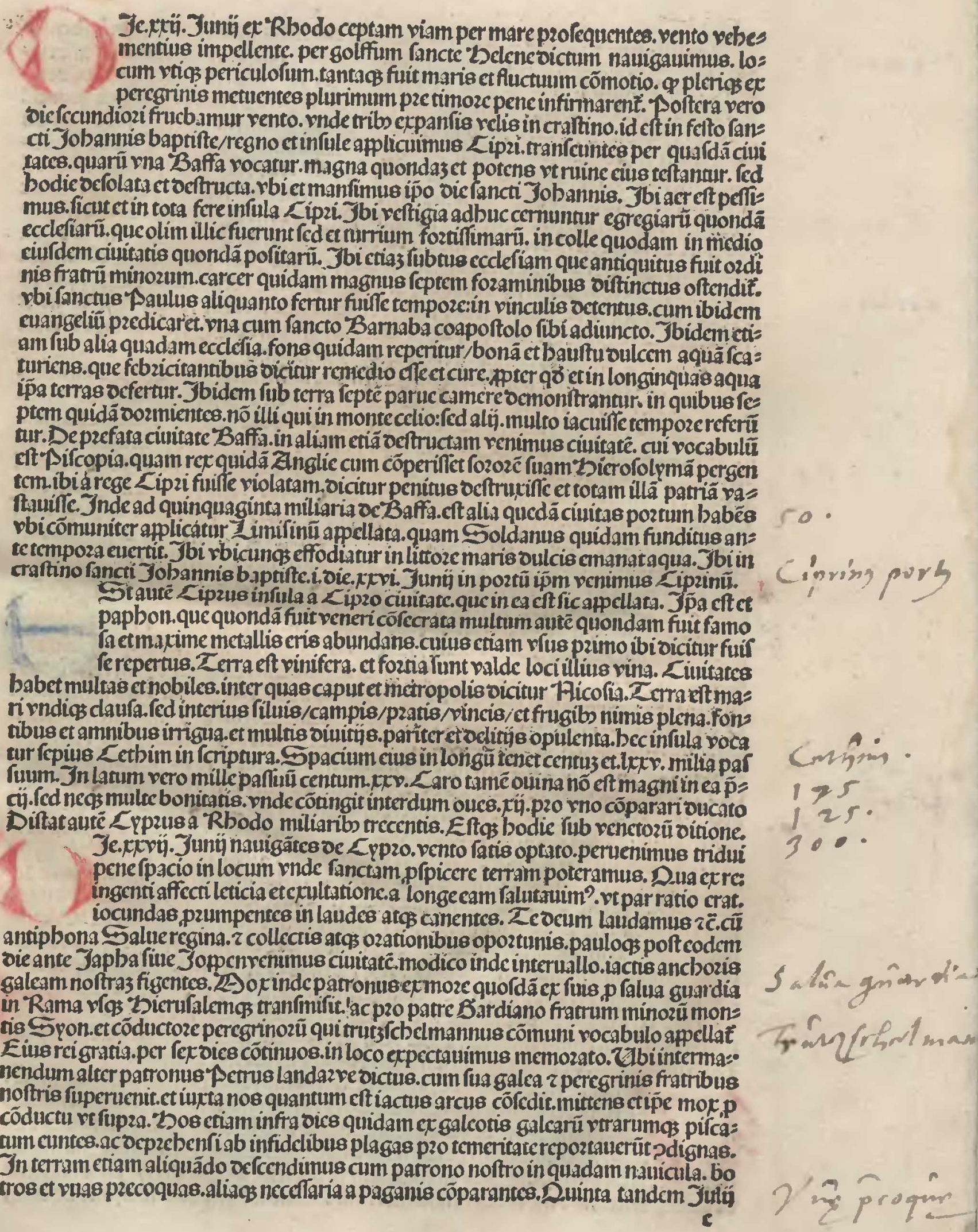




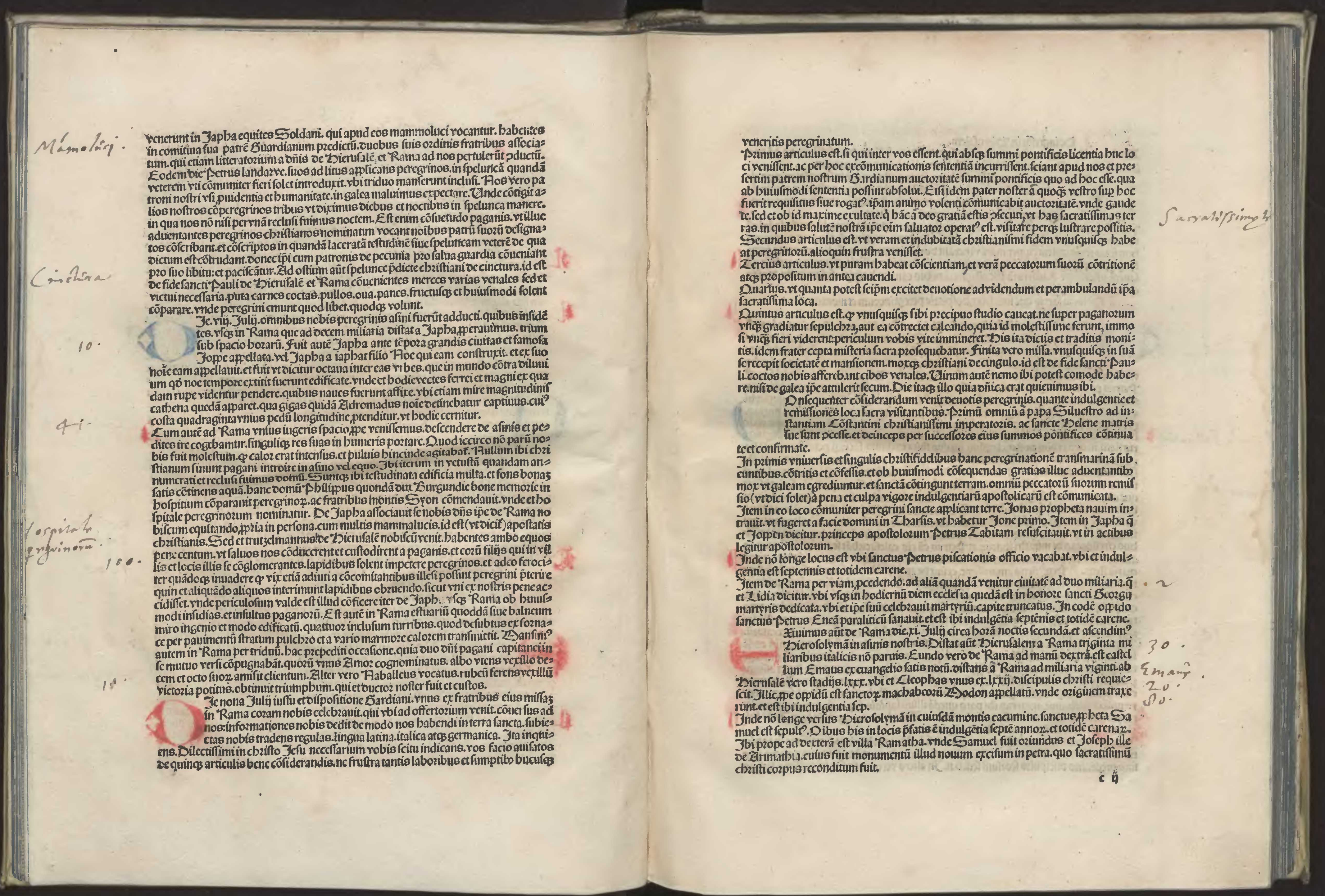


andis Saliater $3 m^{\prime}$
Deingterfuin bienufalem

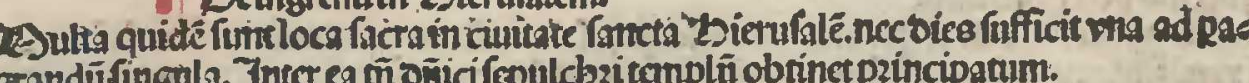

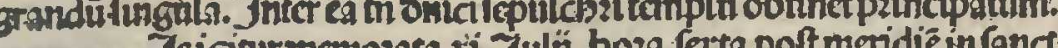

(a) Gecaliquis pereginorillic permitrinurequitare

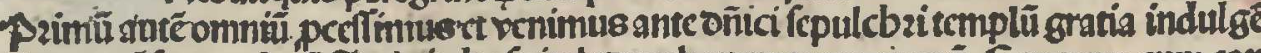
aarum cô cquendaru. Inde in zorpital couccbantur peregrinozu. E go vero cum con fratrbus melis qui mecii per marc renerant bomi intraumus Lalini minozis.iuts

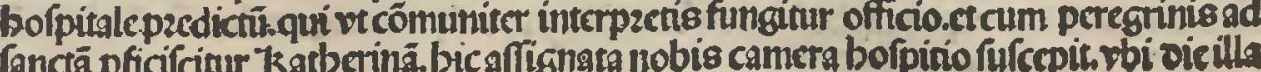
quicuimus longi írincris fatigaticôfectione.

Delocis facris quefuntinter bofpitale peregrinoum et montem

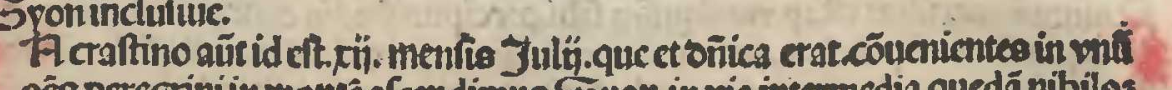

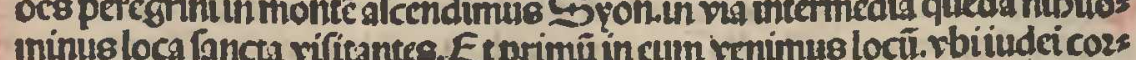

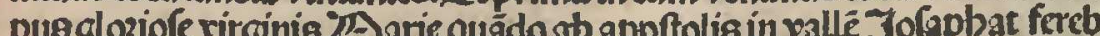

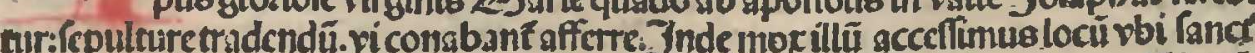

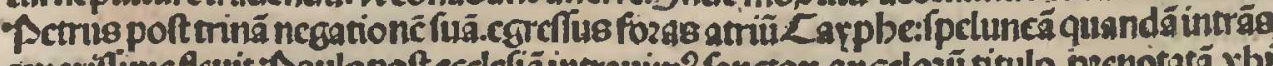

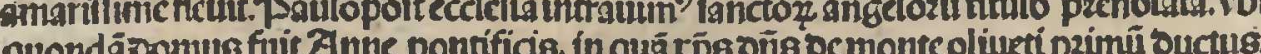
quie

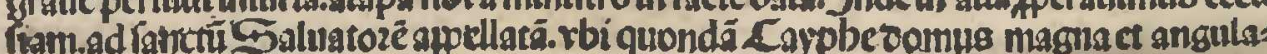

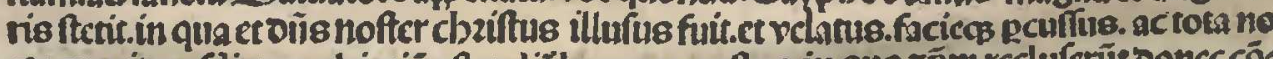

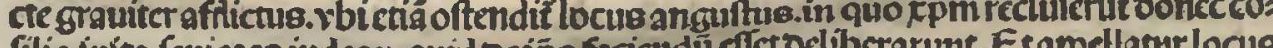
illecarcer

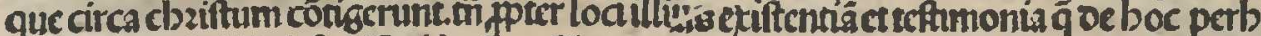

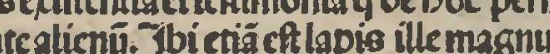

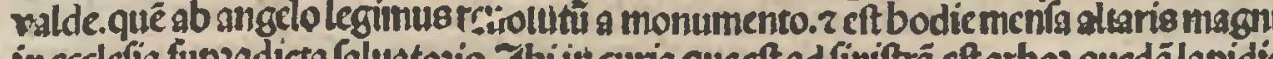
incccelia

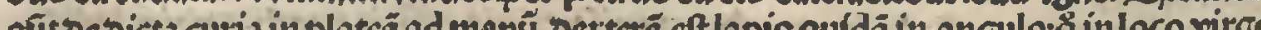

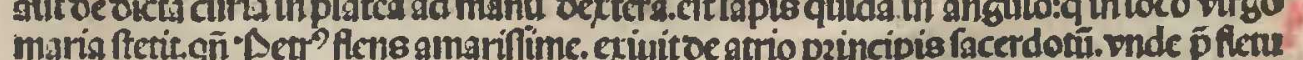

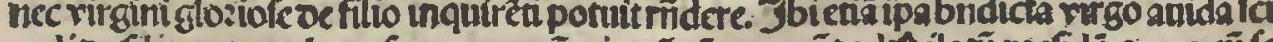

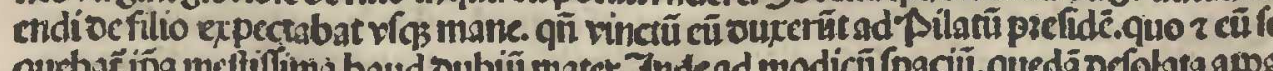

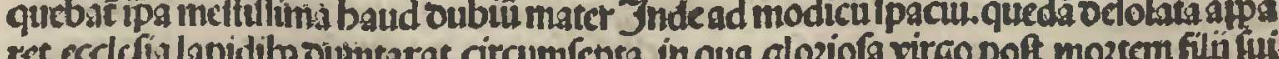
ret ecclchal lapidits ouxthtarat circumfcpra. in qua gloziofa virgo poft moztem filt fui

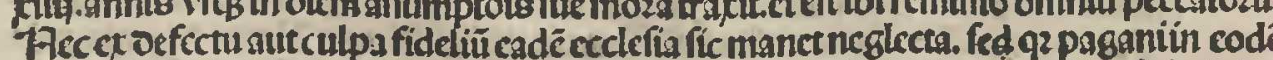

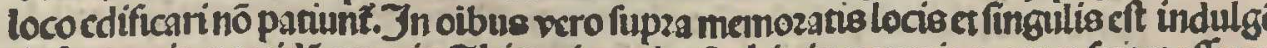

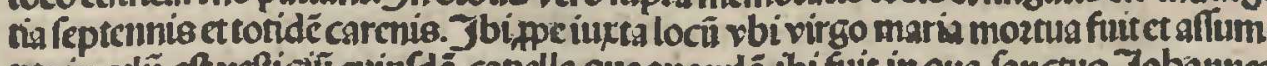

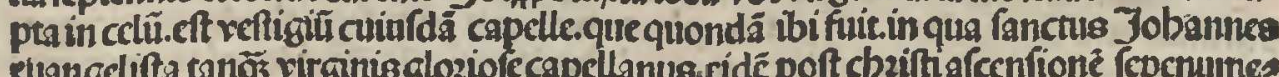
romites

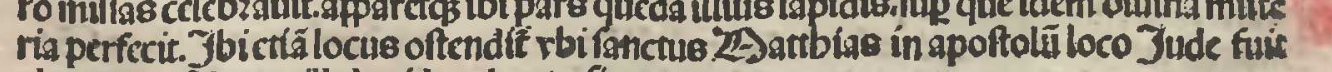

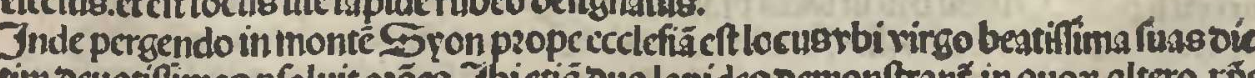

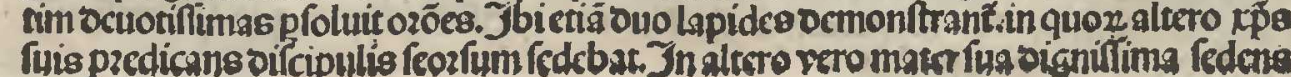

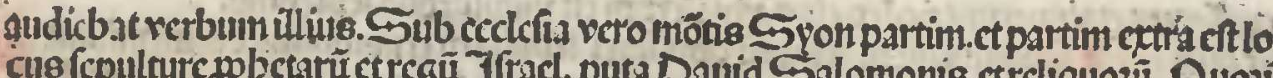

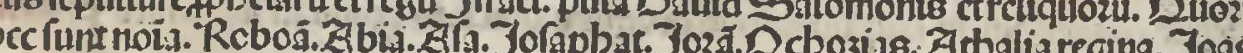

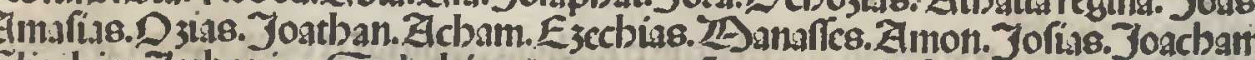

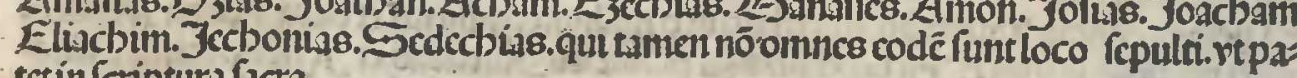

Itctinlecrptura facra.

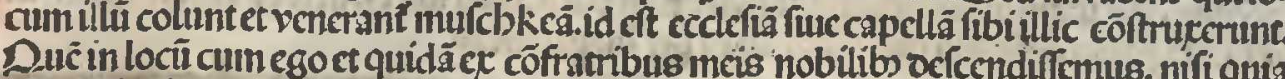
On8 aduut no8 paulominus

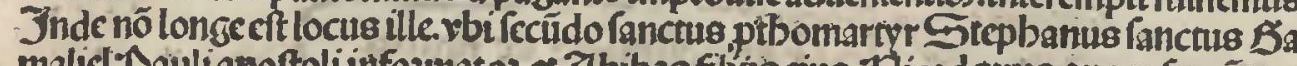

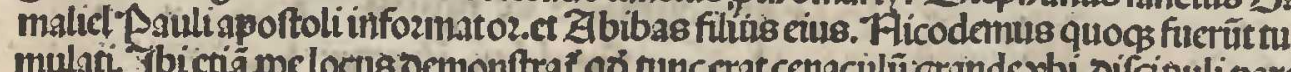

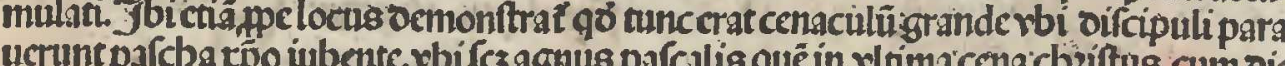

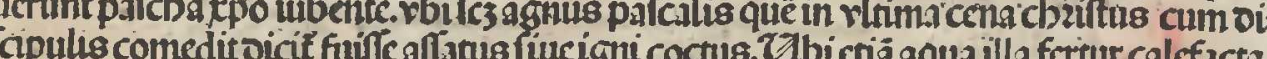

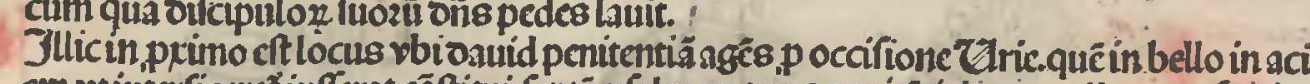

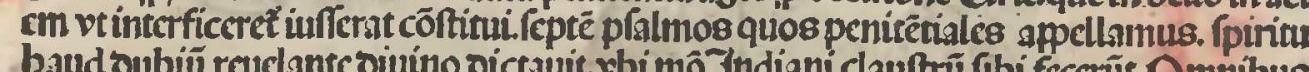
bis in locis fupa oficme

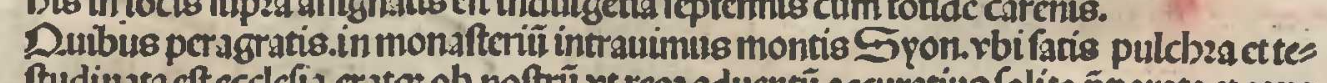

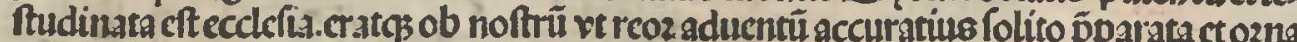

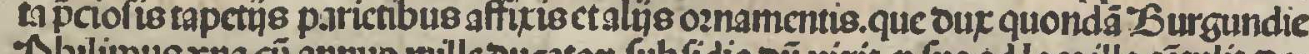
potion acf fuit in bumanis ctia fecir. Fam ct fruccefforciven

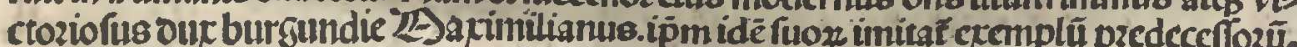

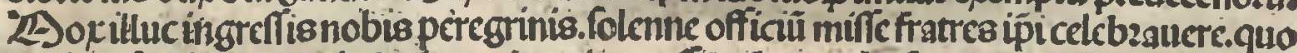

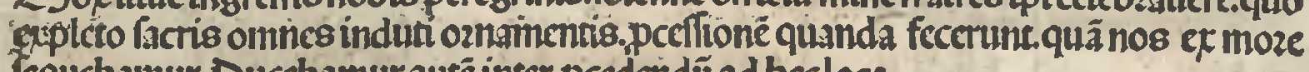

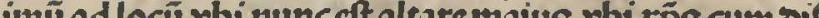

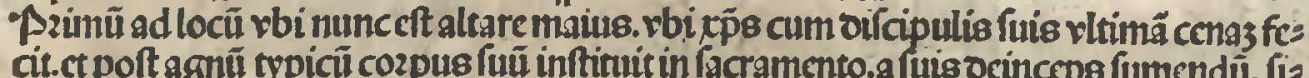

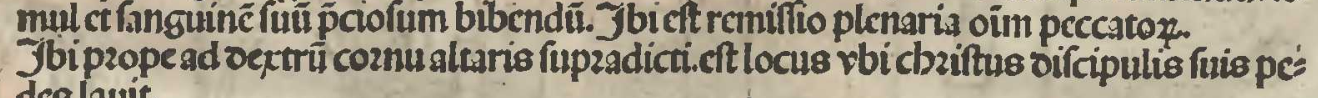

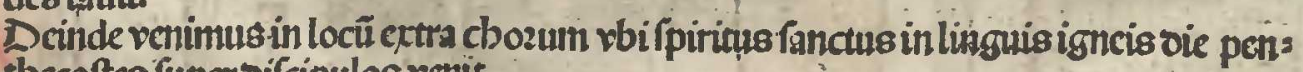
Joi fubtus cit capcla illa oe qua ftiano pbibencibs paganis. Onipo loco isctu plenana cit remintio.

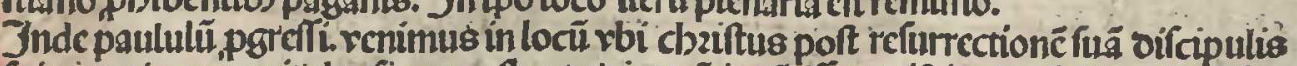
The

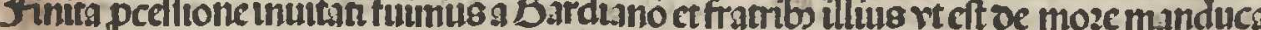

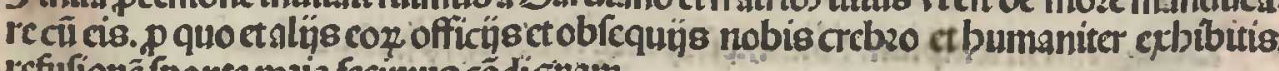

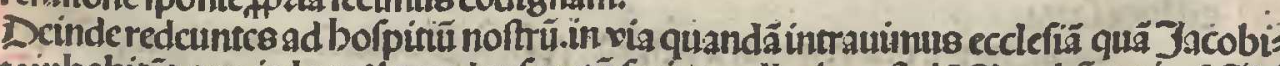

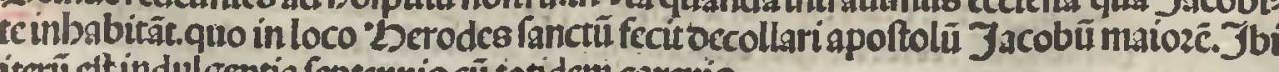
(a)

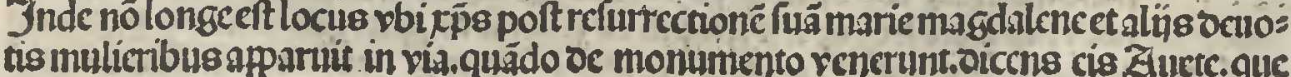




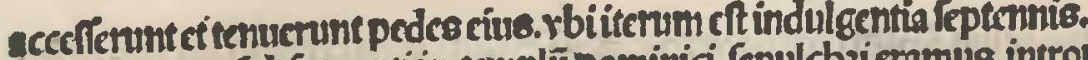

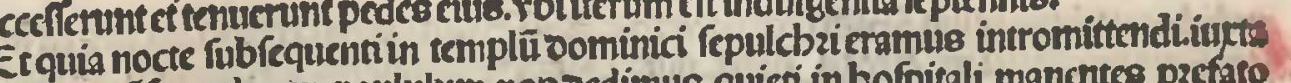
etuduncm paululum nos ocdimus quier.in botpitali manentes pacturo

detpaucsis bozas. biset mons Zabarim er Fcbo.etpbafga planicies Jozdanis et Jericbo ctmare moz

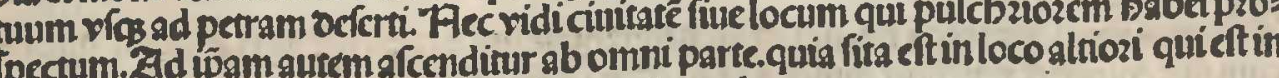

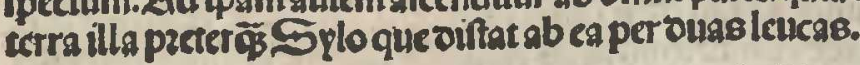

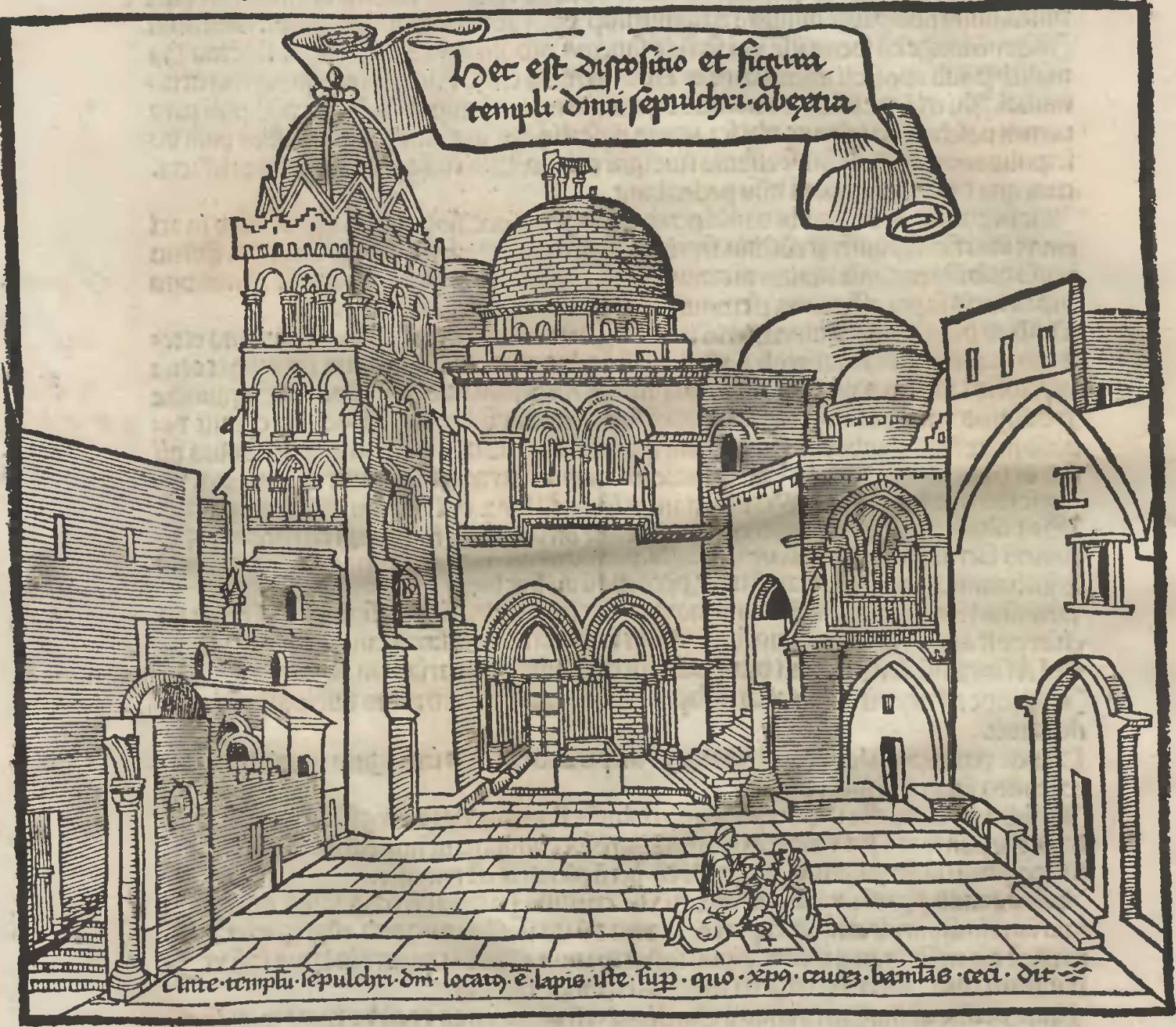

Deingrefiu in templumsominicilepulchri, ct pceflione inibifacto

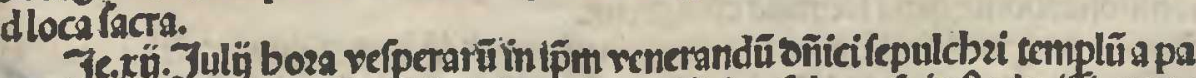
ganisid eft rectosibs ipt" cillitatis lancte zierofolyme tuim" admilti etnus merationthy

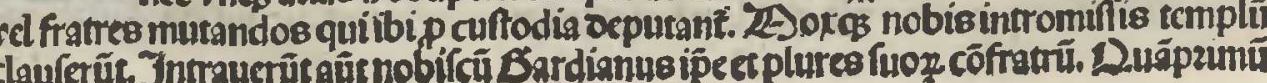

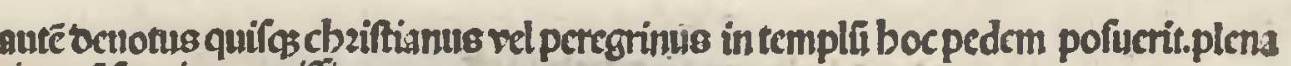
riamcốrequiturrcmiffioncm.

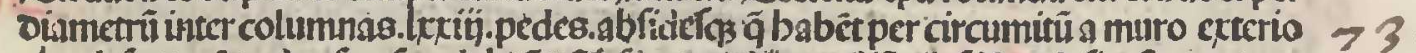

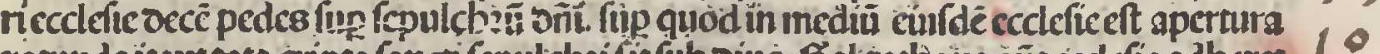

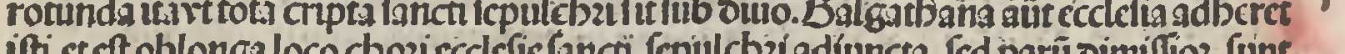

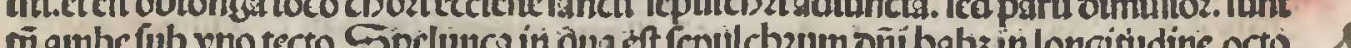

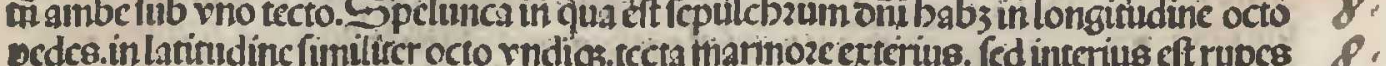

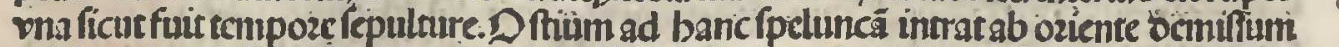

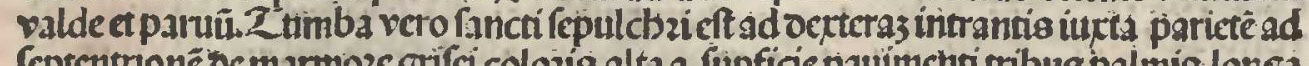

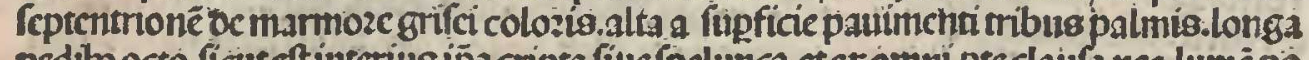

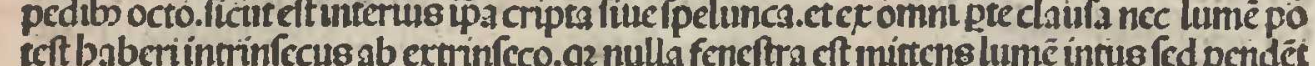

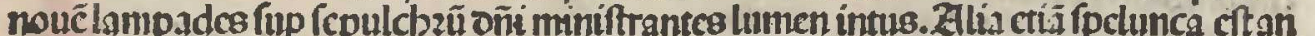

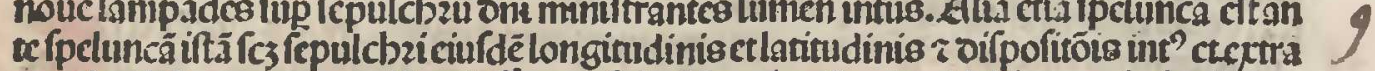

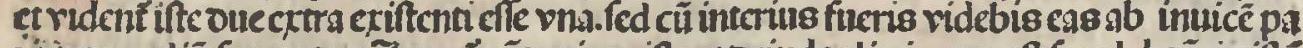

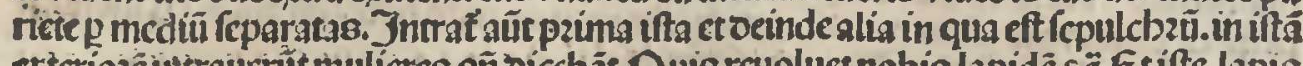

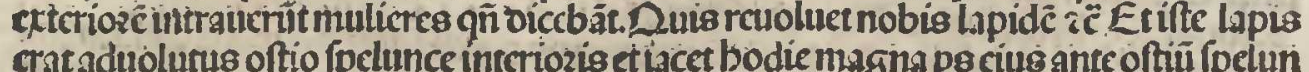

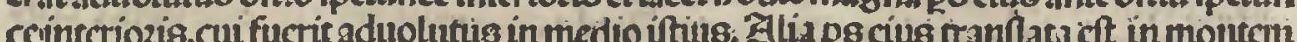

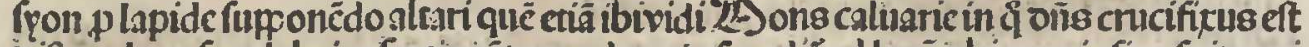

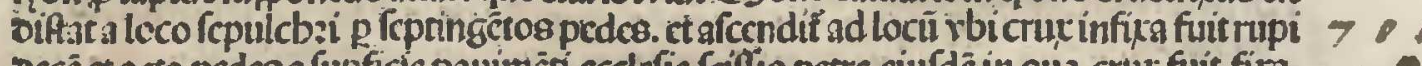

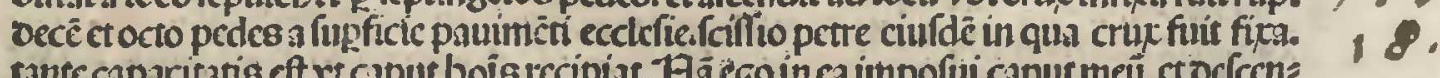

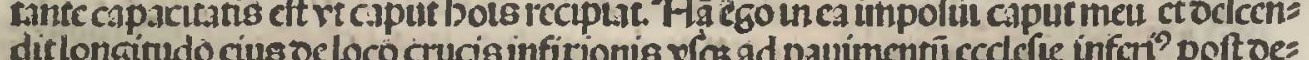

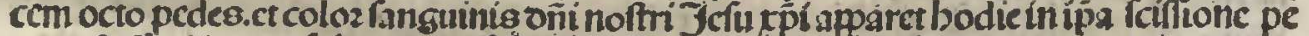

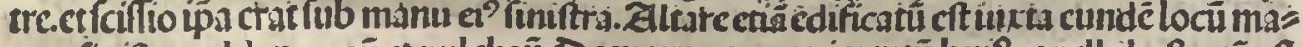

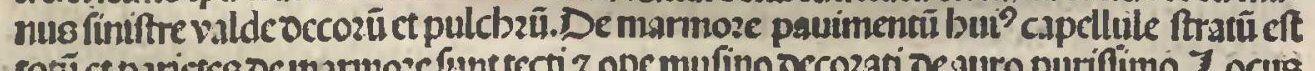
coctin

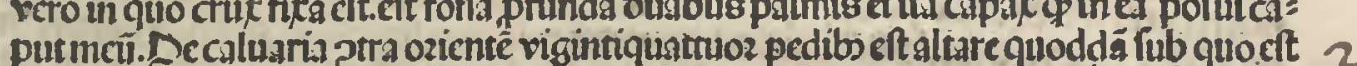
pars cotumme. fun quã oñes fiut flagellatus illuctranflata oe oomo pylati.et fub lapide

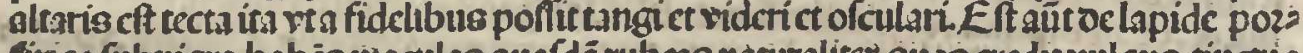

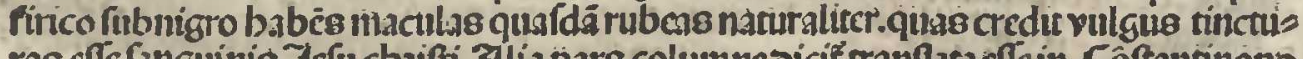

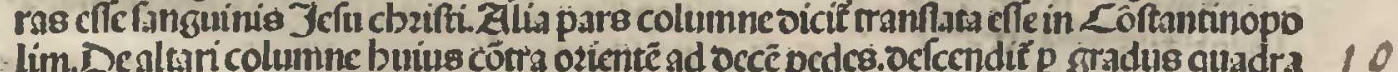

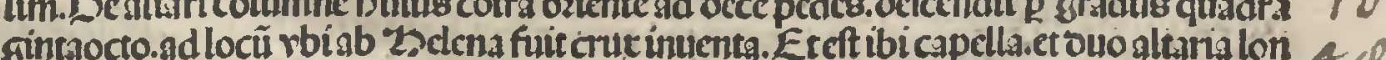

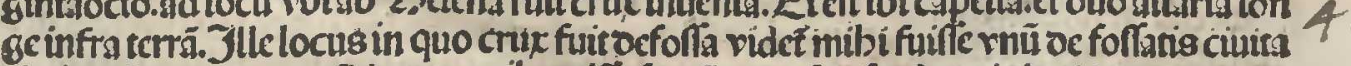

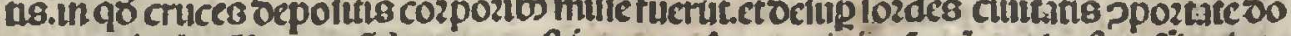
necper Escena loco múdato crucent inuchta.' (nit)

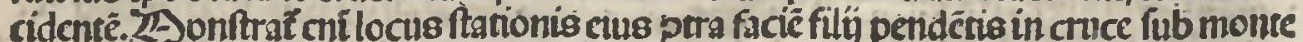

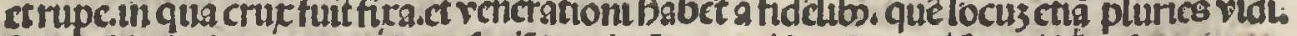

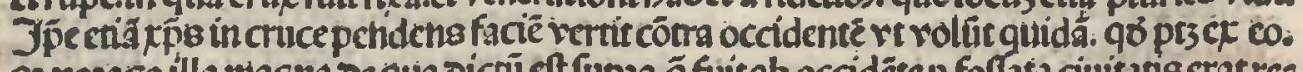

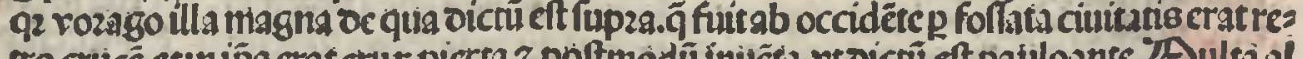

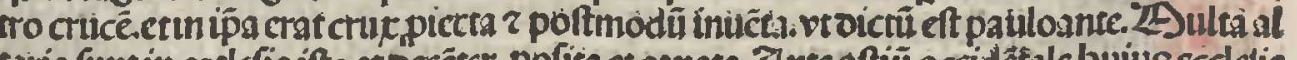

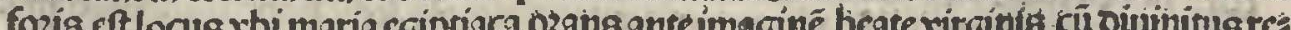

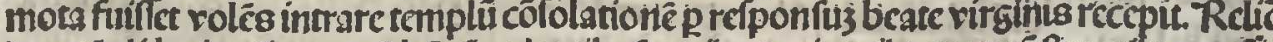

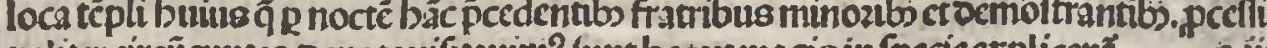




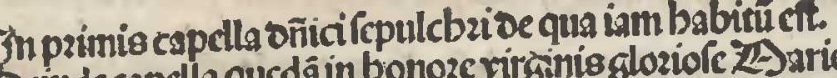

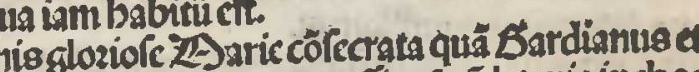

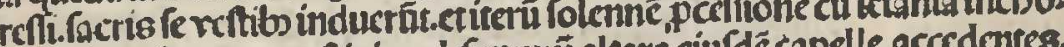

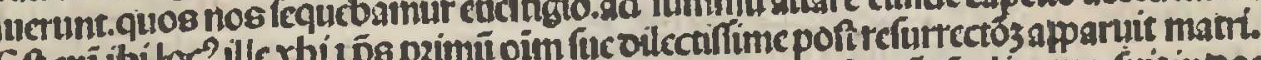

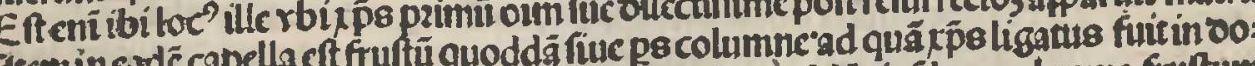

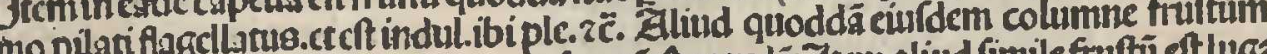

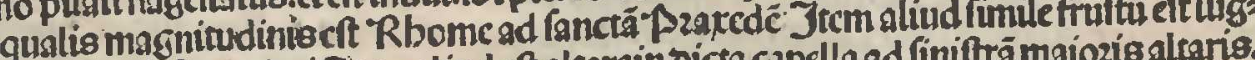

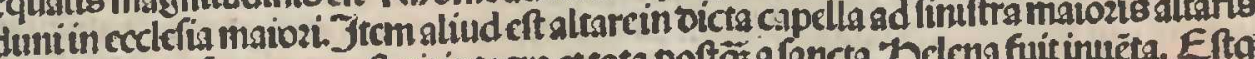

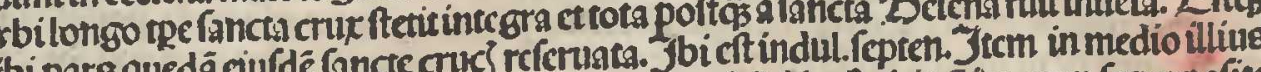
bi pars queda ciuterid

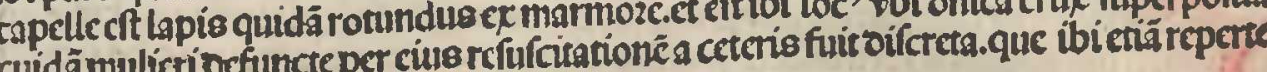
fucrunteteft tbindulgcntia feptennis.

1 Jremípamegrefficapelläouos al ios é marmoze reperimus lapides albos etrotun

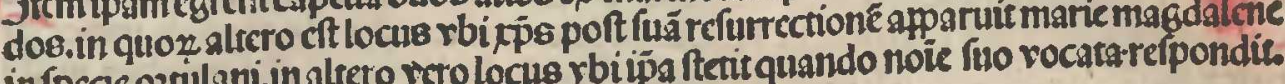
inf́pscce ortulani.in altero recto locus rbi ipa

6. Tha Jnde prectendo in remplo.ala quanda

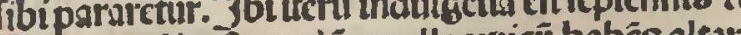

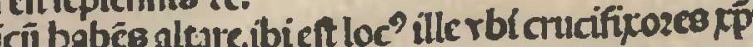

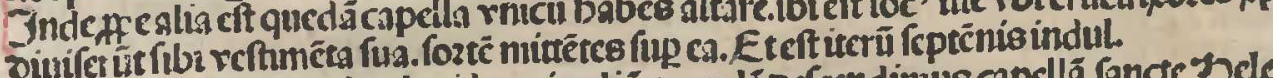

30

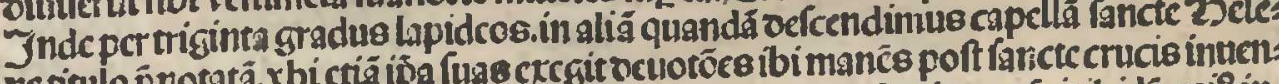

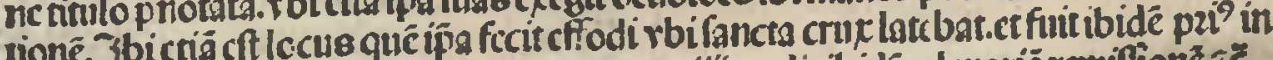

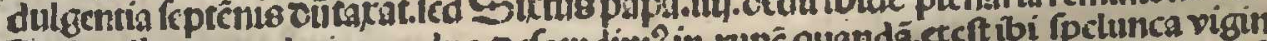

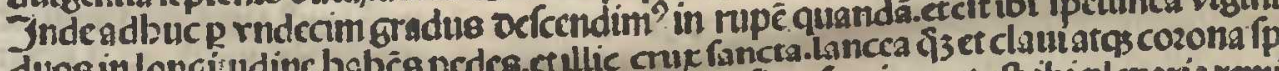

2.

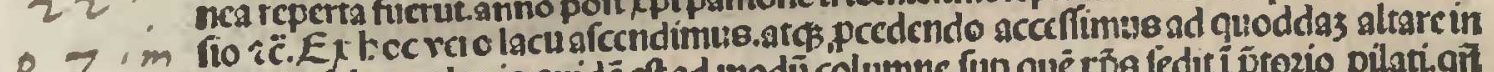

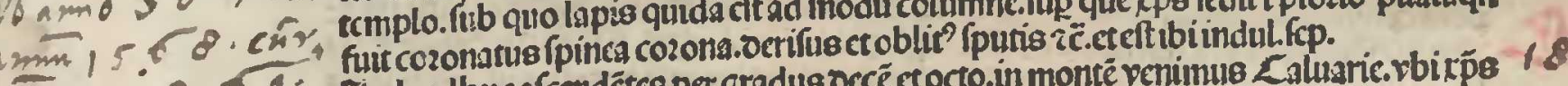

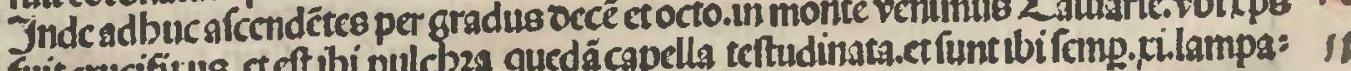

dcardentes. co o locus ralde fit facer et renerabilis.

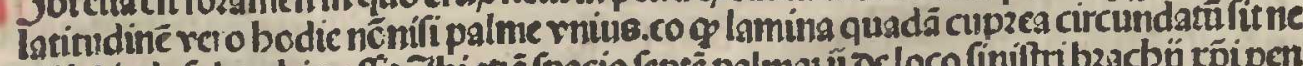

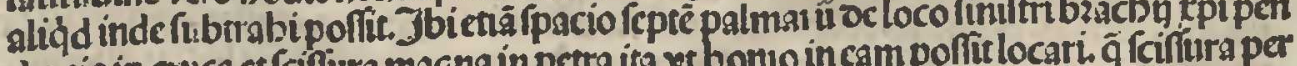
dentis A

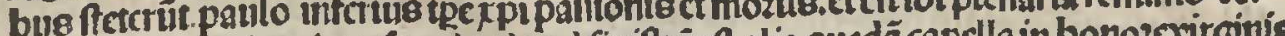

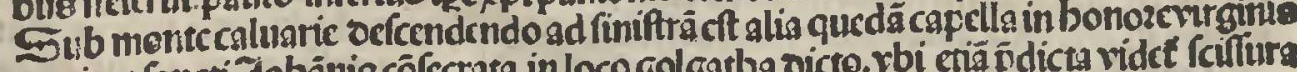
maric ct fanct J b

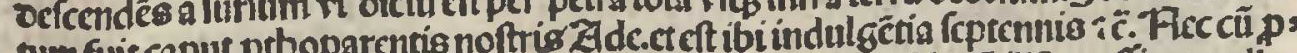

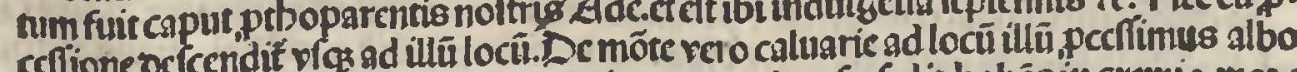

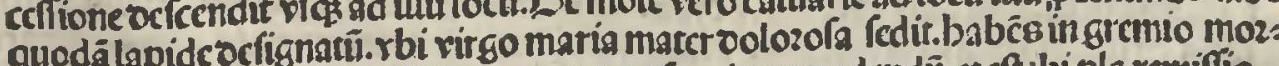

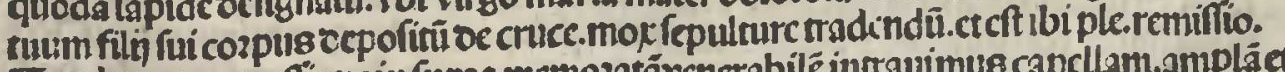
Zandem cum pecifione in fupza memozatarcherabile rotundam.in cuitus medio glozion

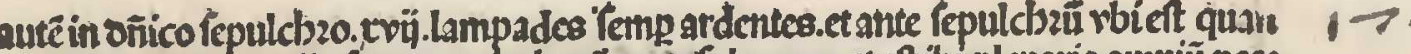

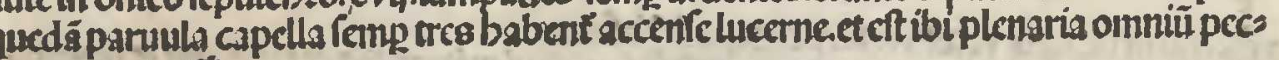

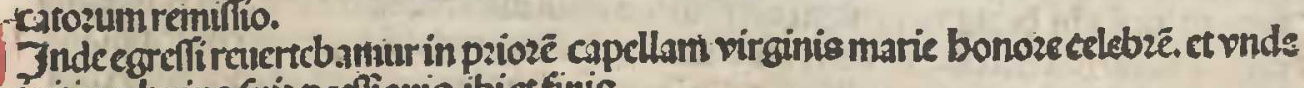

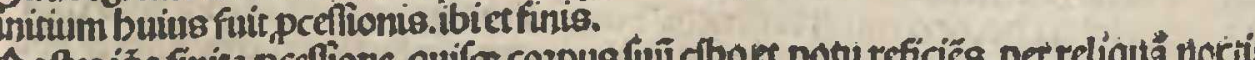

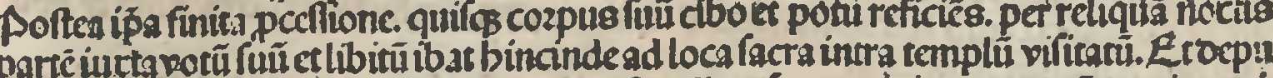

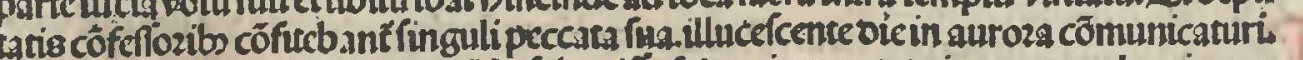

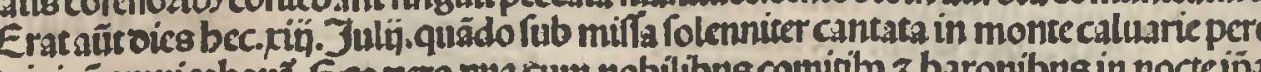

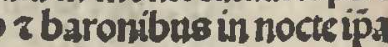

Dergreffuertemplo oñicífepulcbzictlocis alĭs factis

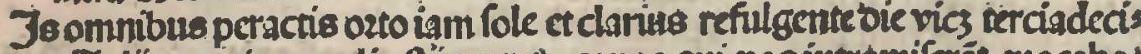
ma July.apertis templioitus per paganos qui nos intormiterar chocaba

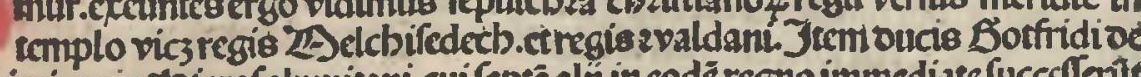

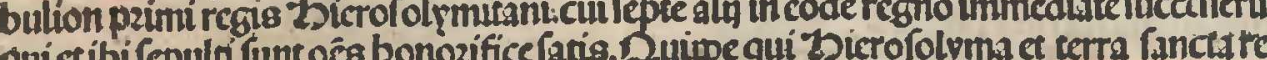

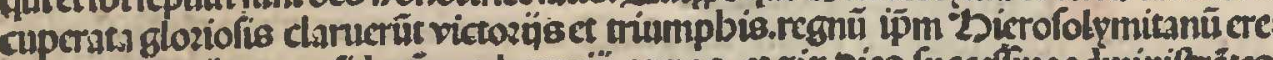

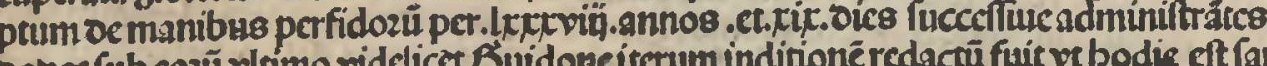

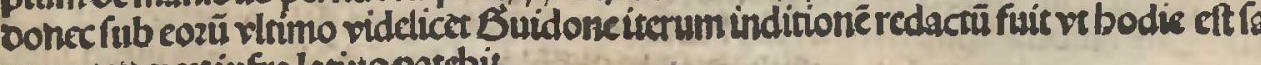

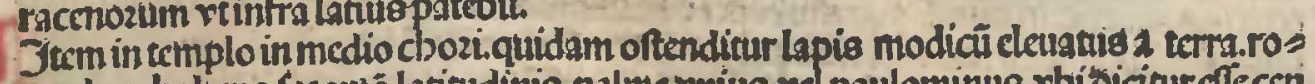
nundum babens fozamé. latitudinio palme vnius. rel paulominus. vbí́bicinur cflecen (rumerabisteme

(a)

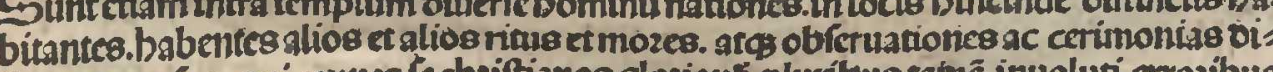
(a)

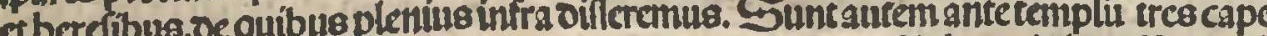

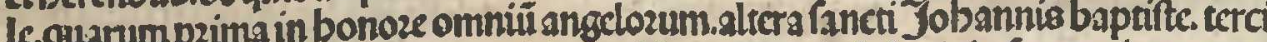
mariemagdalene cff cosfecraca.eteft in qualibet earum indul gentia feptennis cum to ridem carenis.

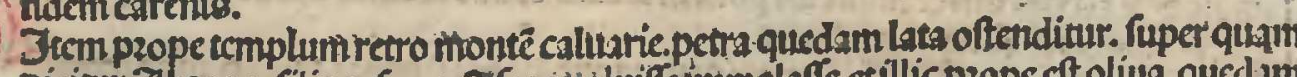

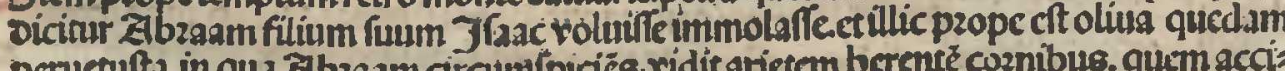

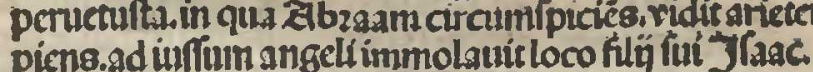

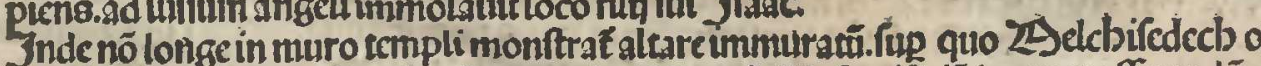

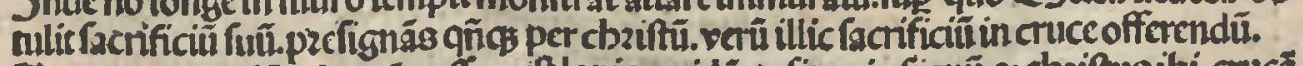

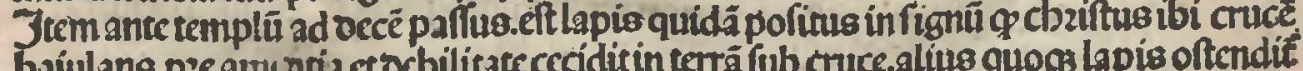

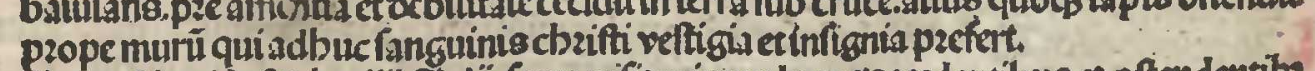
Sn craftino id cet oie riii - Jnlï.facra vifitauimus loca.pzecedentibuset oftendentibs nobis Bardiano ce gliouibus fratribus ciuseandica pzius mifra in bofpitali fanct 30

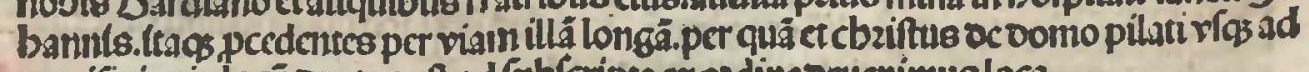

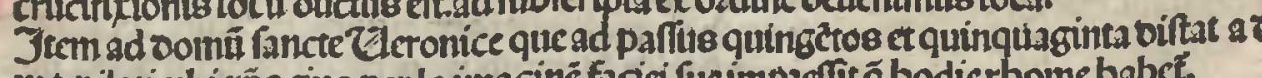

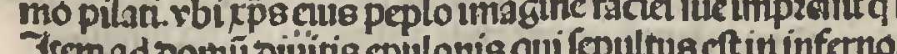

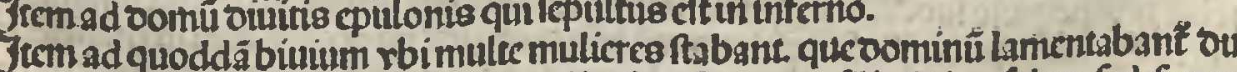

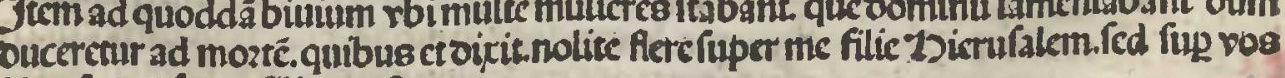

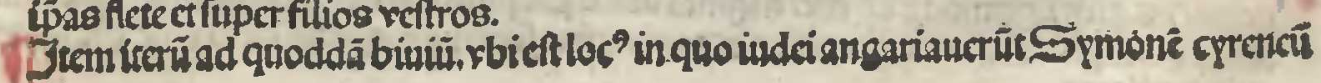

B

\section{ithe}

88.

Crrovénations 


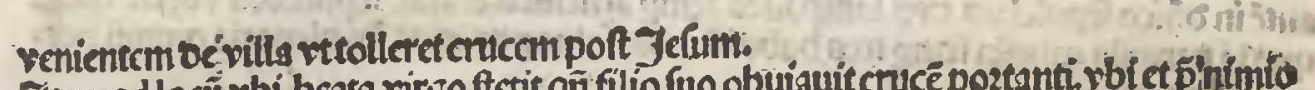

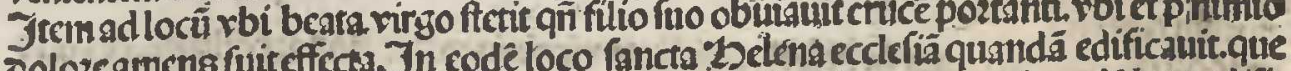

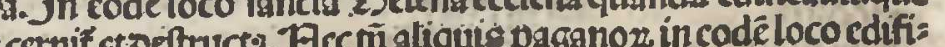

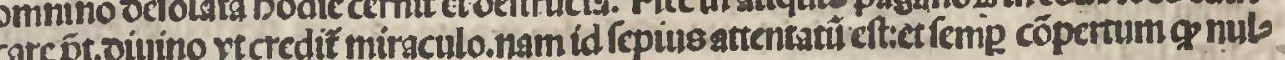

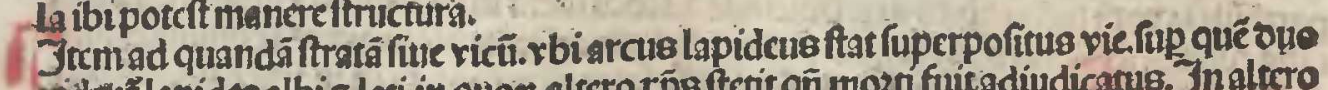

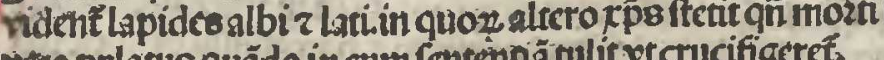

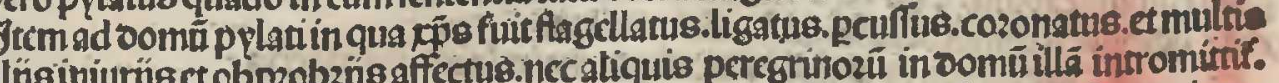

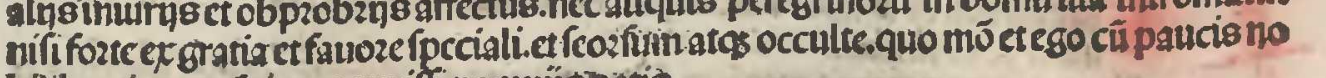

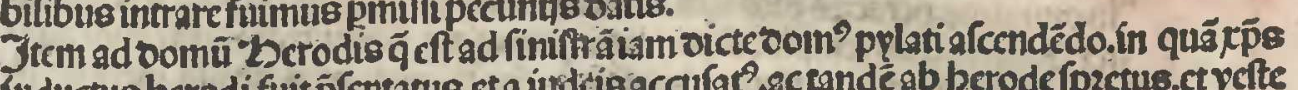

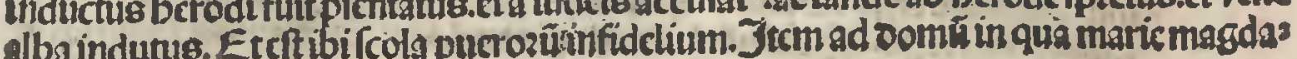
tencoiniffa fueruntpecests

- Jtcmad locti vertemplo Salomonis.

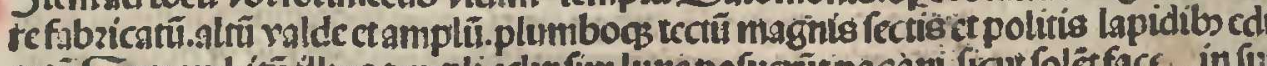

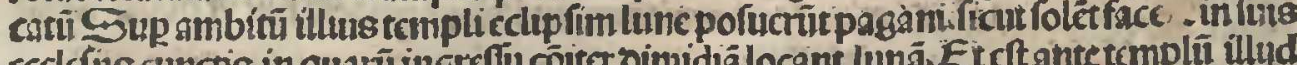

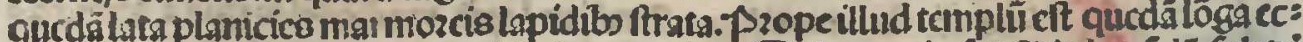
clctia.plumbo bectup tecta.quonda olcta poztcus Salomonis. 3 oun bientale fíb oi

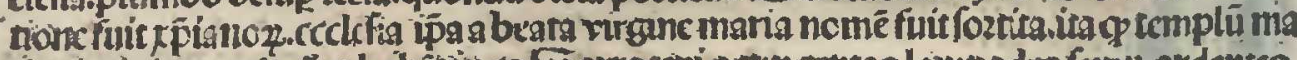
ric virgints rocabat.ct babeting In templo recro Salom

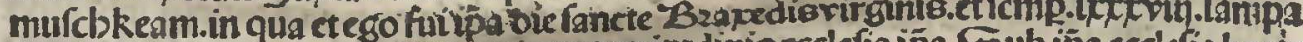
desintrea babent accenfe. Eftag miremagn

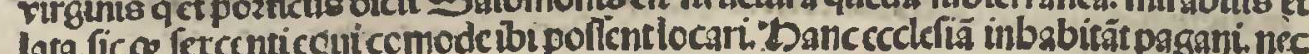
facile perminturtebziffianosintroire. Jtem Sarraccni tomplũ Salomonis in magno babét bonozc.nullas inco inmmuns

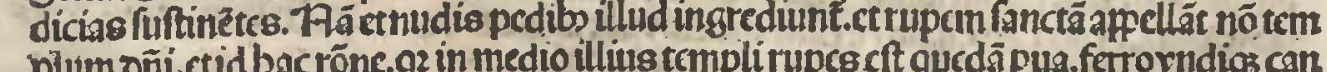

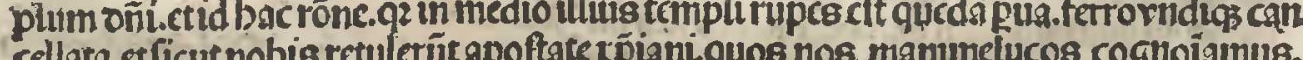

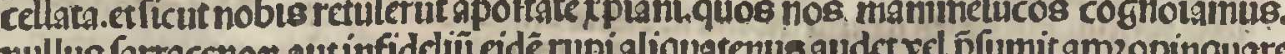

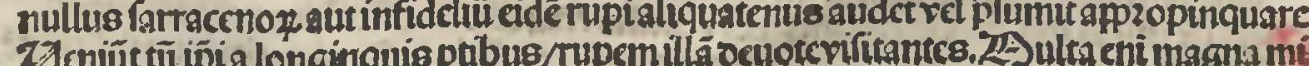
rabiliain nupcilla legunt facts.

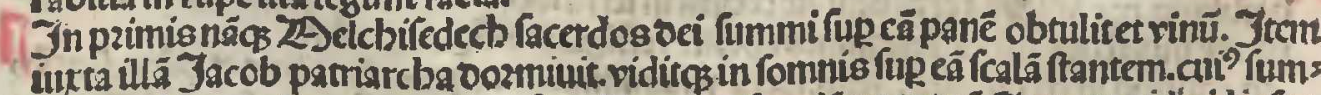

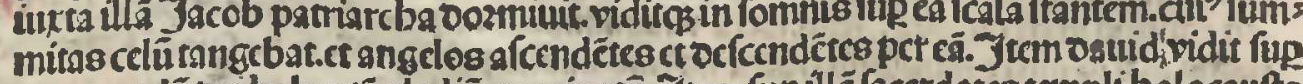

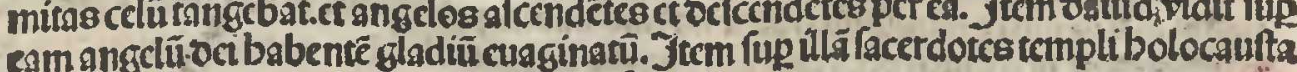
ponc bant.queignis oiumuse tocaliter abfiumplifi.

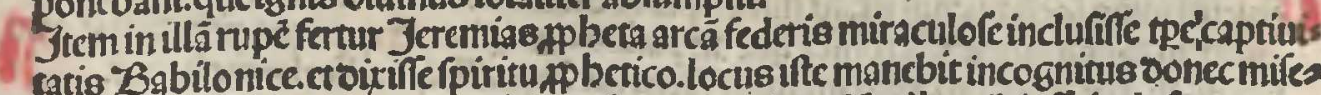

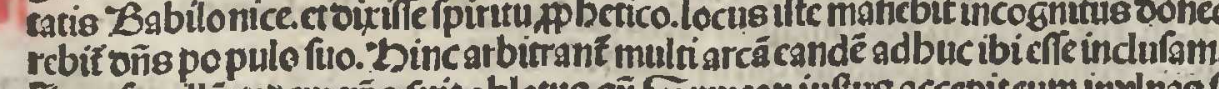

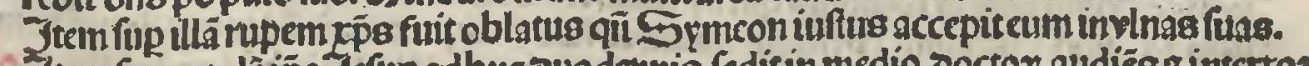

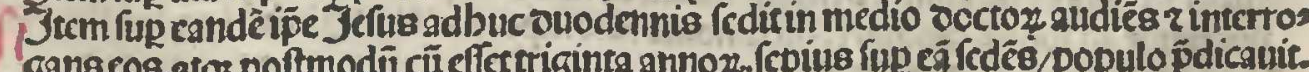

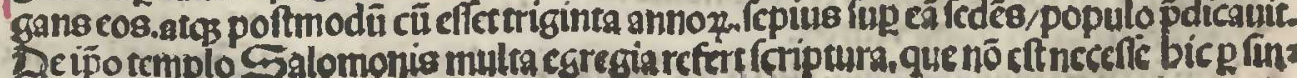
Gallactplicare.
Dcinde venimuse ad poztam fancti Stcpbani per quă ípiceductus nö longeab ea .us

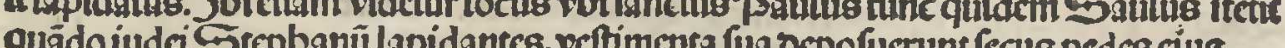

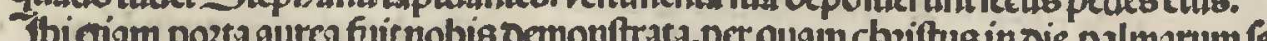

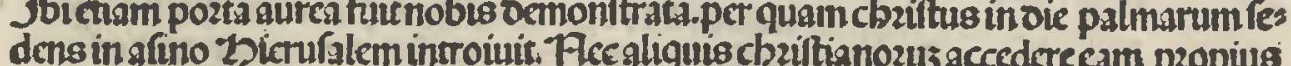

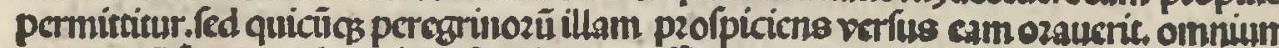
peccasozũ fuozum plenariácófequinur remififionetr.

Derefcenfu in vallem Jof́apbar. (n)

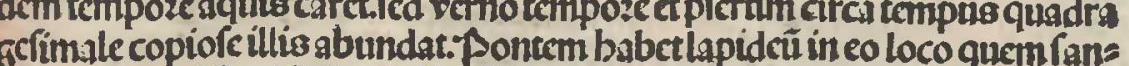

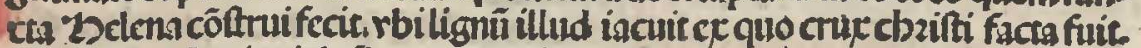

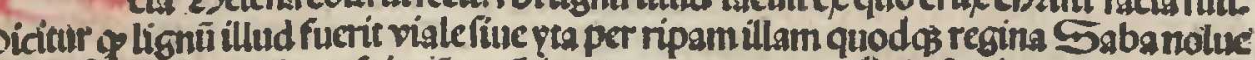

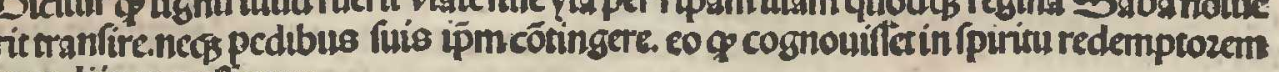

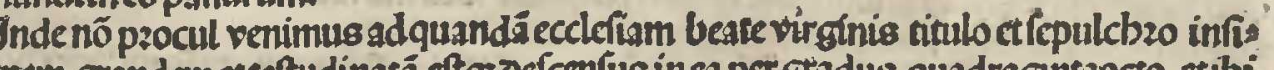

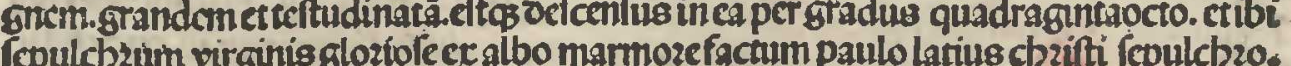
potef to ibicelebzarificulct fuper fepulch zo cbzifti $E_{t}$ babet ouss ianuas p quas ins

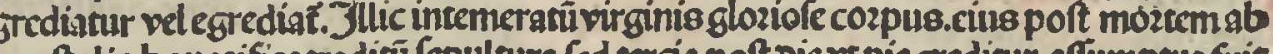
poftolis bonozifice tratica

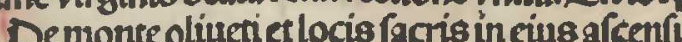

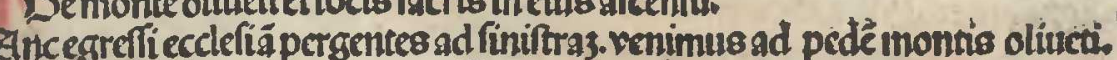

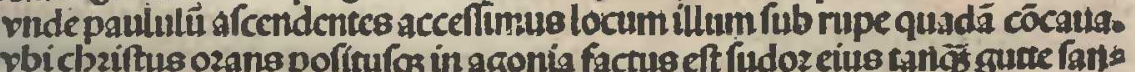

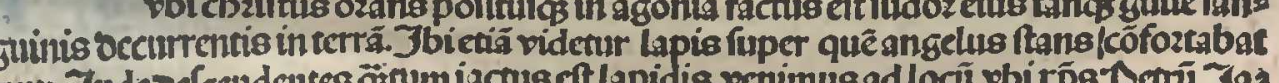
cobum et Jobannë retiquit oicés cis. fédete bic oonece vadă illucet orem. Indeafcendimnsoenuo in locí illum rbifanctus R bomas apoftolus fertur cingus lum virginis gloziofe accipifíe oum ipa affimeretur incelum. J Inde pcedentes intraut

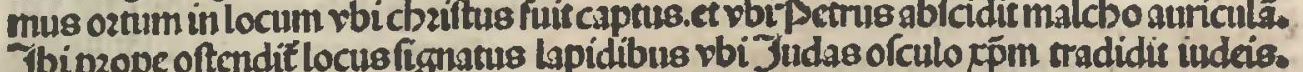

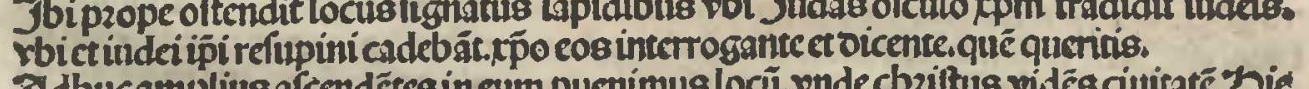

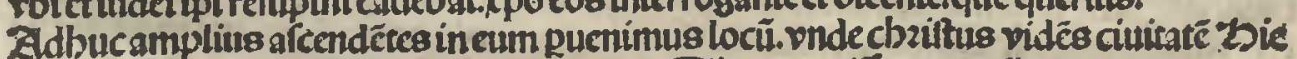

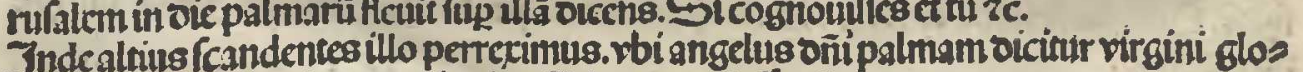
Inde althing (candentes illo petrecimus.robi angelus onimpalmam oichur vighin glos

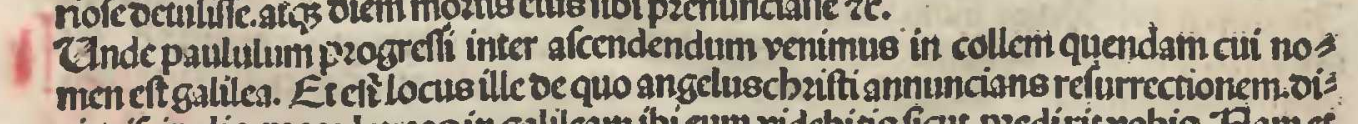
pir oiffipulis.precedet vos in gallicam ibieum videbitis ficut predítit vobis. Fam

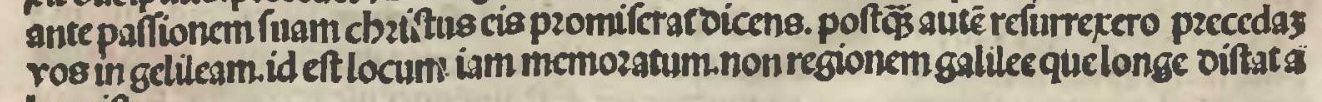

1) Randem in cacumen montis venimus. vbieftectelia quedamineo'fabricatalloco in

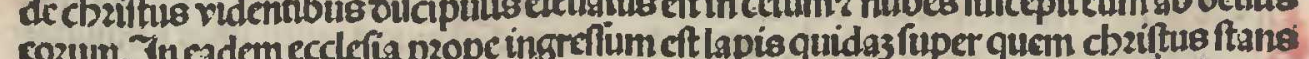
alcendít in celum. in quo adbucpedum cius relicta veffiga cernuntur et pefertim pes

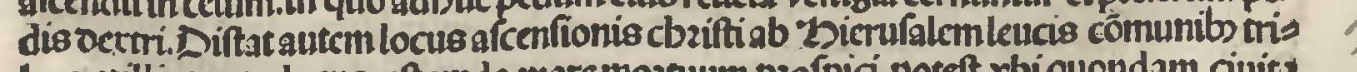

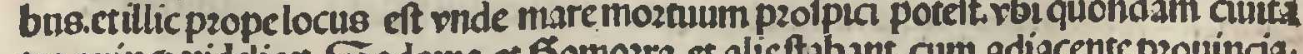

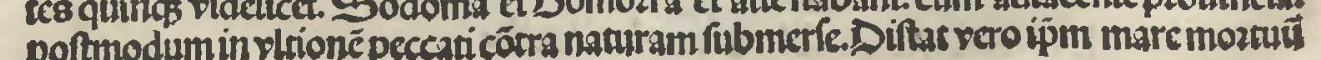

Porta aning

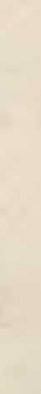


7

ab hitoloco miliaribus feptem.cetamen vicadaimidium biftare videtur. Delocis in ocfcciflu montis bliuet.

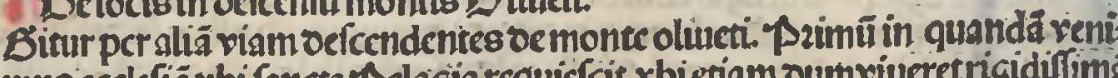

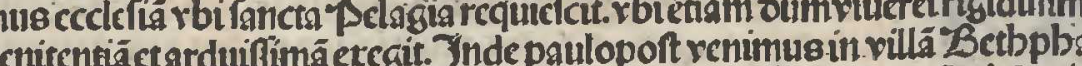

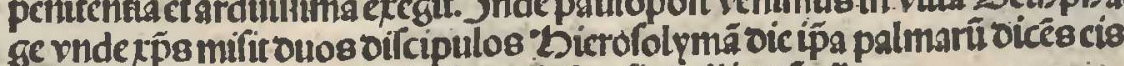

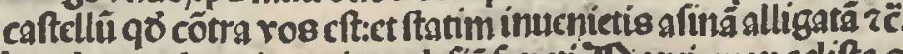

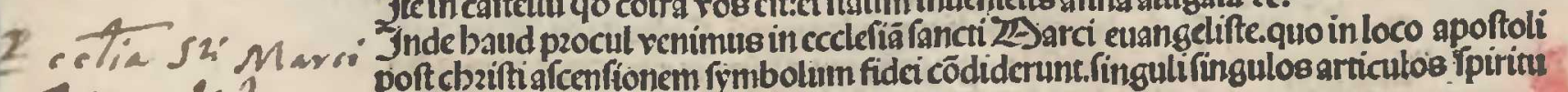
Ziangulirim

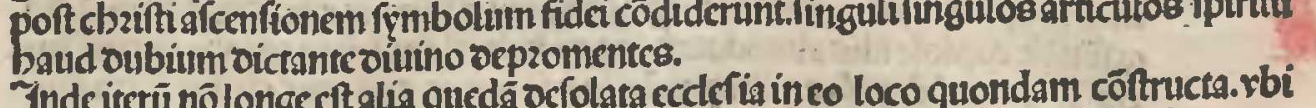

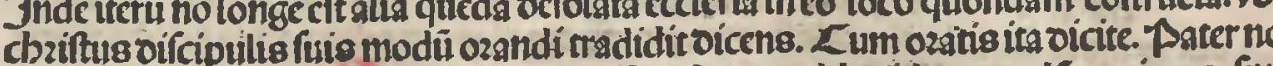

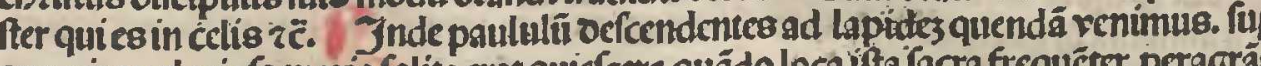

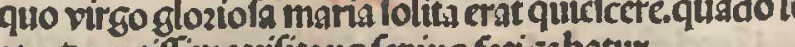
arto epilcopiocicend

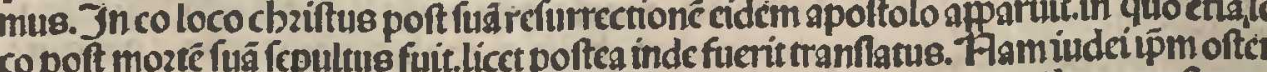

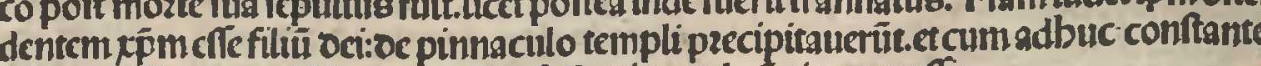
LF m predicarct.quidấ cumpartica fullonis cerrbzun ciuls crcuffit.

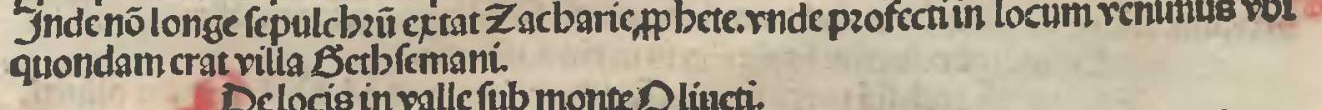

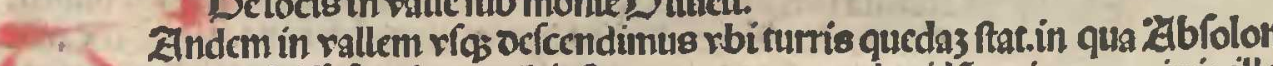

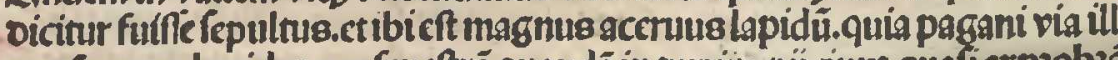

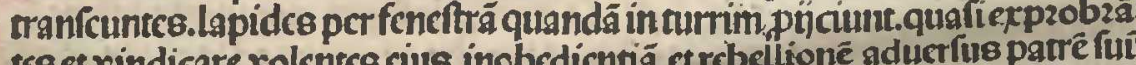

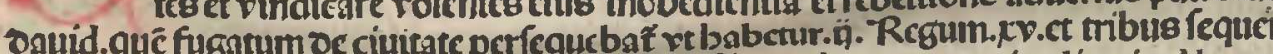

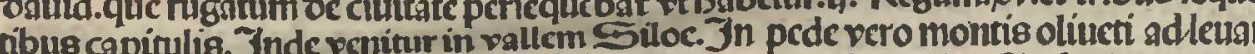

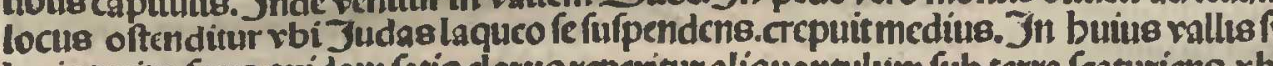
locintroitu. fons quidam faris clar us reperitur aliquantulum fub terra featuricns. rb virgogloziofa pannos illos Icpenumero hatuic.quibus filium fuum adbucinfantulum

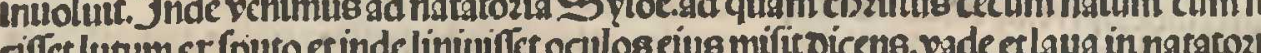

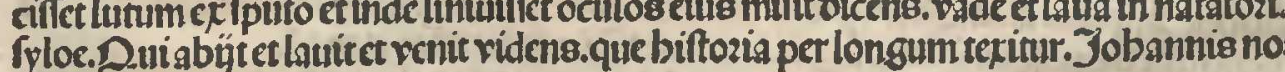
no capinulo.

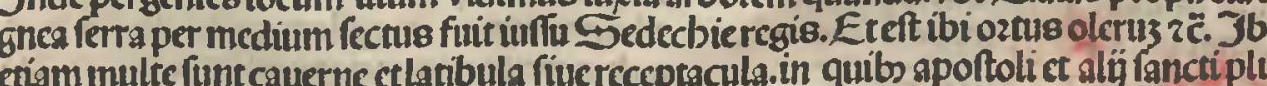
resob metum iudcosum latuerunt

reg ob metum iudcosum latuerunt.

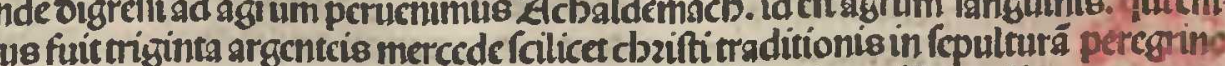

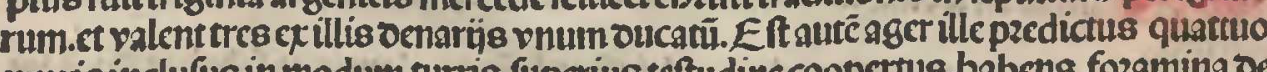

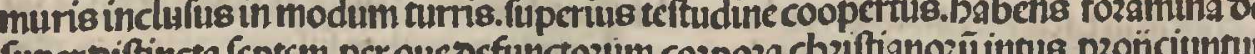

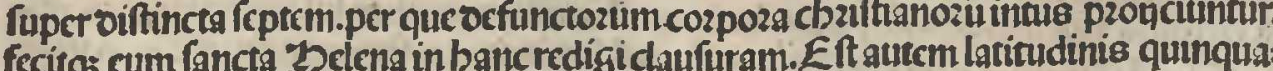
ginta pedum. longitudinis vero reptuagintaduozum.

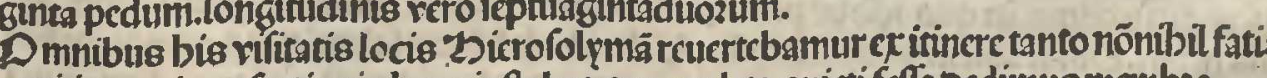
gatiliam enim reffectionis bo:a inftabat.qua expleta quicti feffa oedimus membza. 1) Detranfituin
centibus,

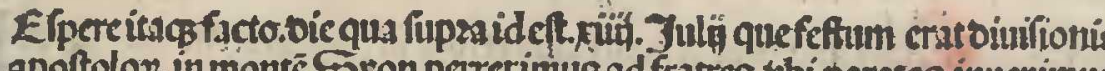
A

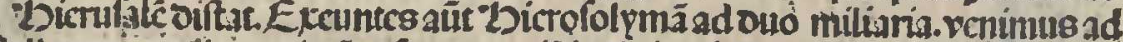

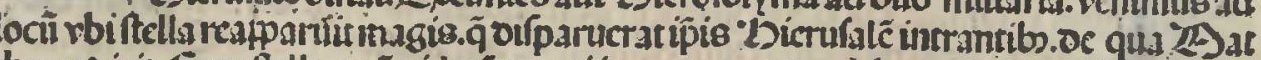

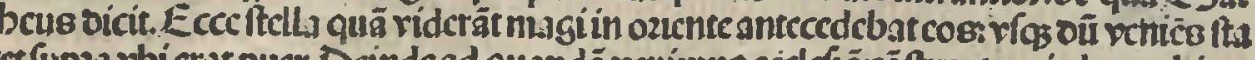

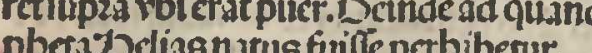

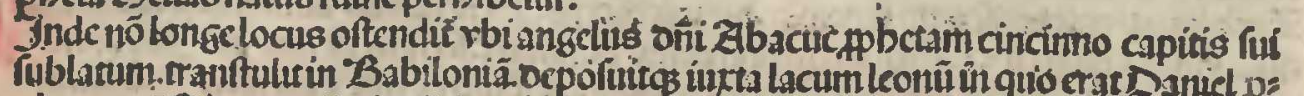

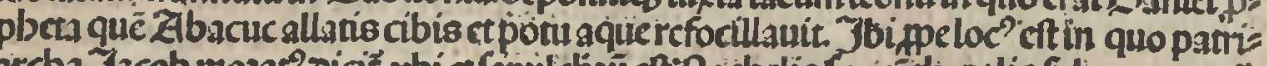
ycba Yacob mozat" oicit. .

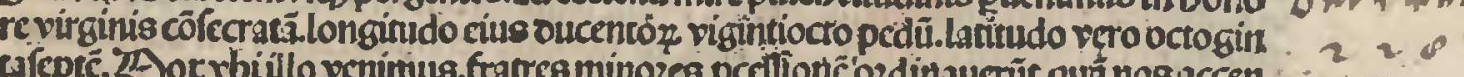

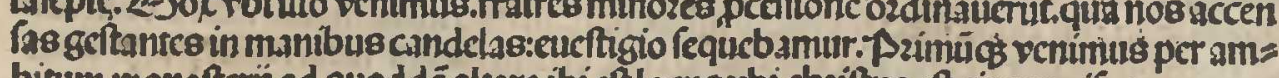

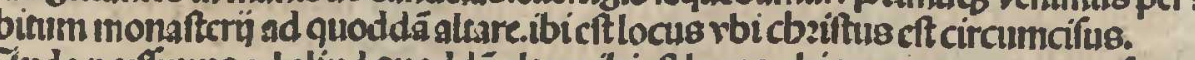

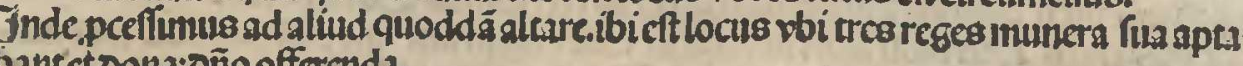
Tndepergiadus quofdälapideo

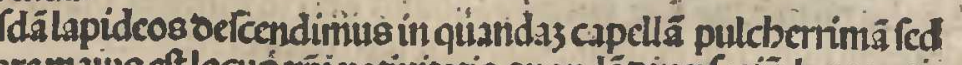

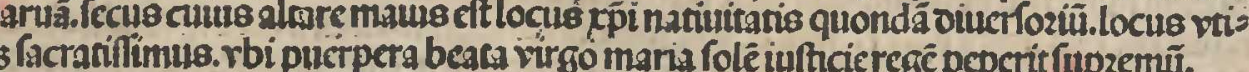

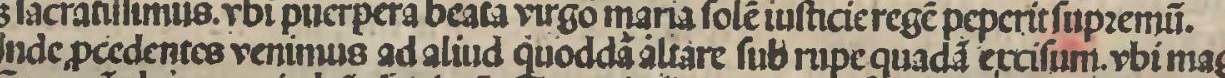

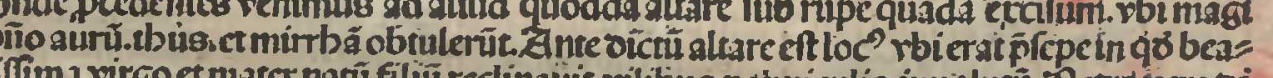

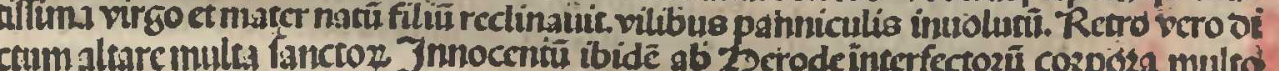

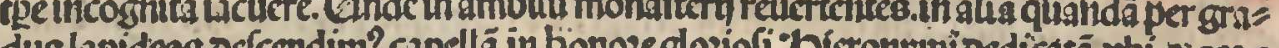

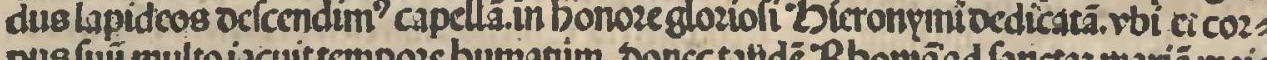

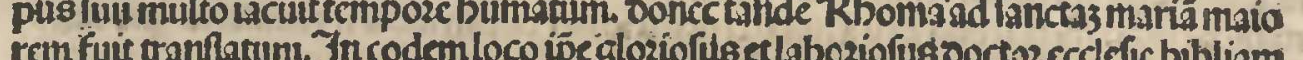

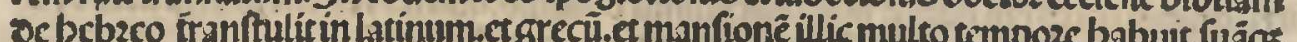

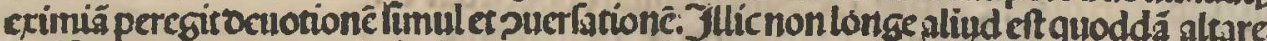

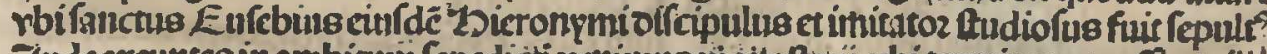

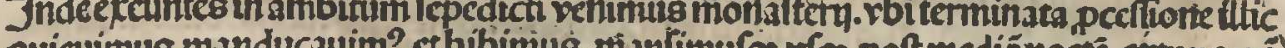

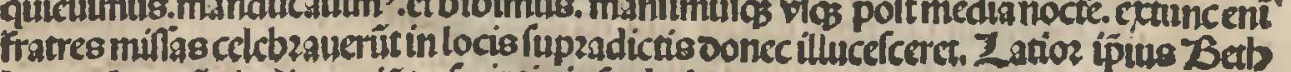
Lecm et loco3ñ ciadiacentiü oefcriptio infra babetur.

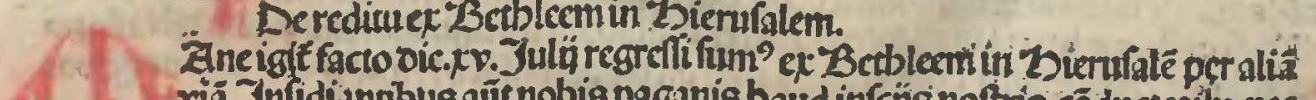

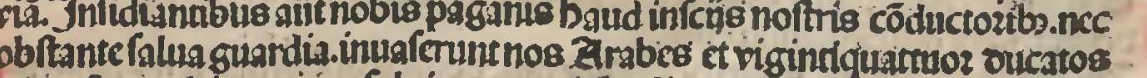

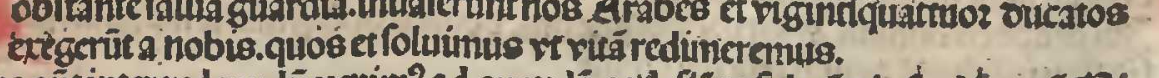

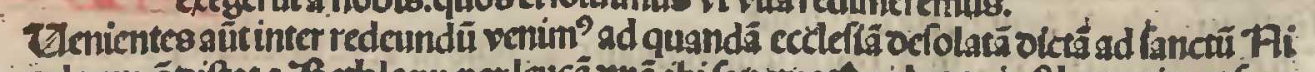

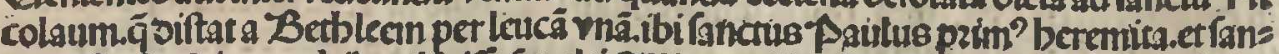

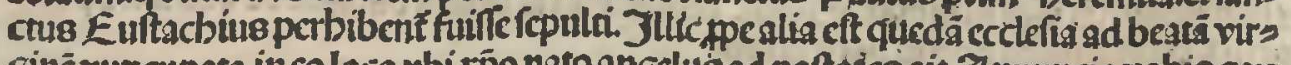
Eunc nuncupata.iv coloco obi tppo nato angelus ad partoces ait Annuncio robis gau

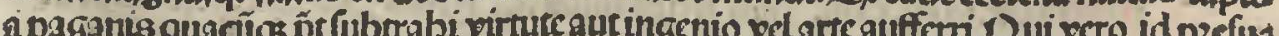

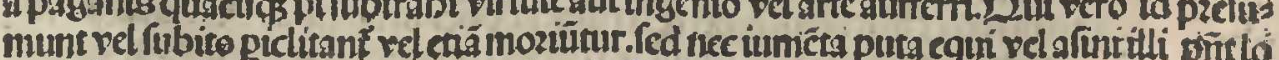

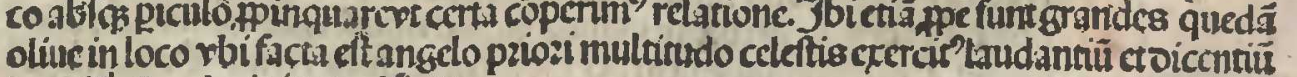

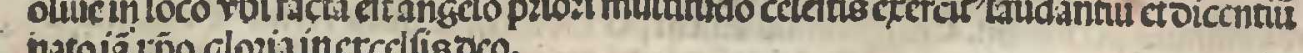

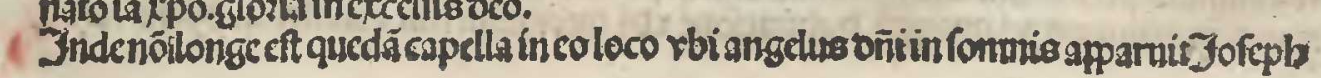

\section{5 .}

a

$\rightarrow$

2.ritralionilats 


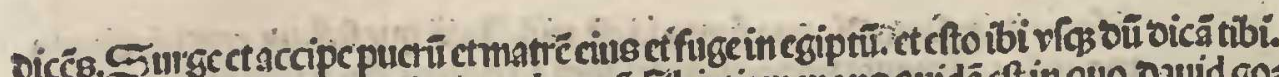

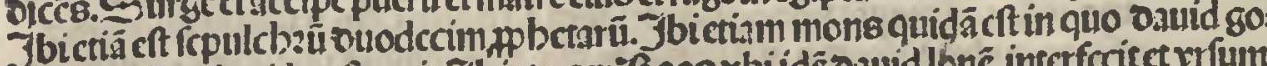
liam funta ct lapidc, prtrant. Jndesd o ont ptronetn virgosbinn

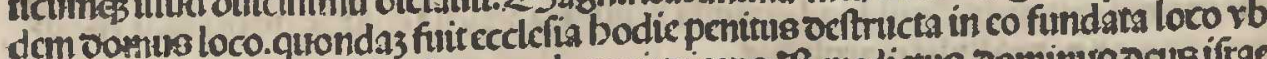

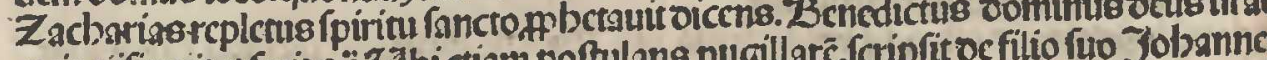

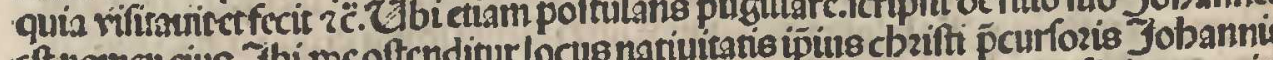

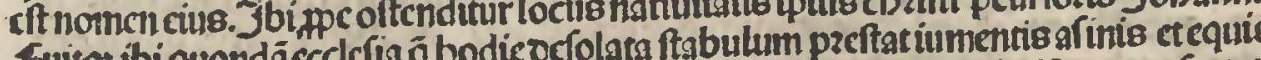

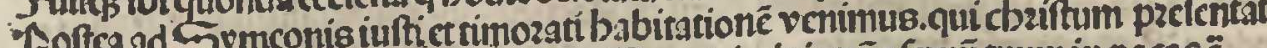

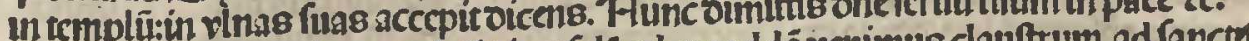

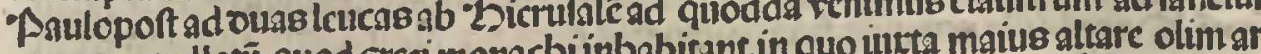

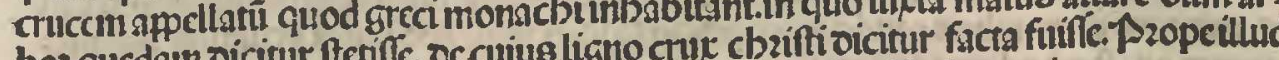

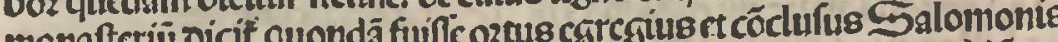

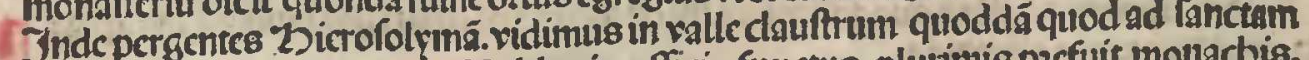

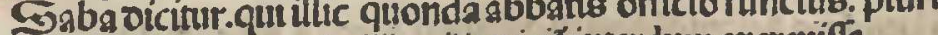

nimus circs bozam pzaindù.facta Jister

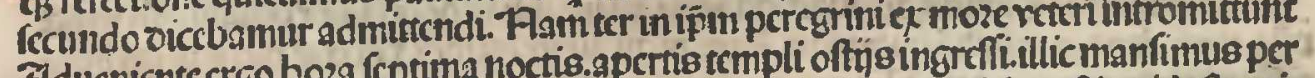

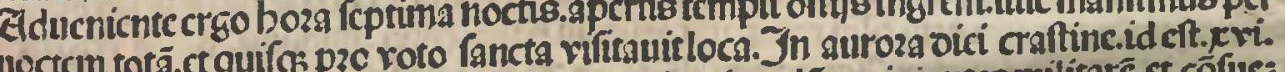

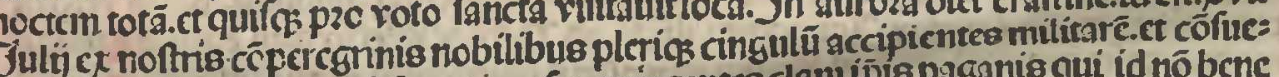

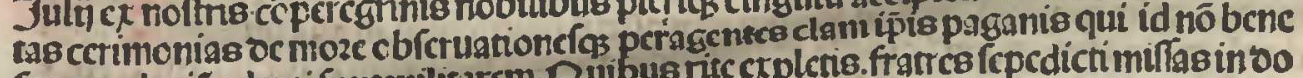

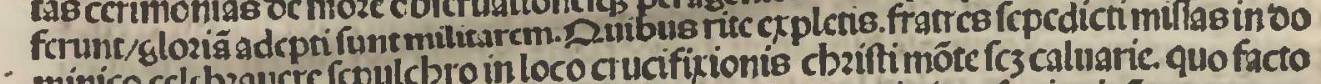

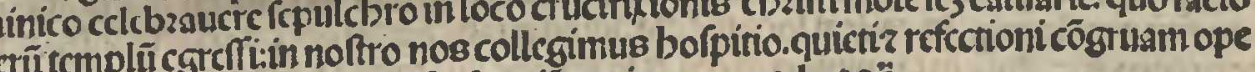
ram oantes.co gy codé fero in Bethania equitarc opoztebat ?

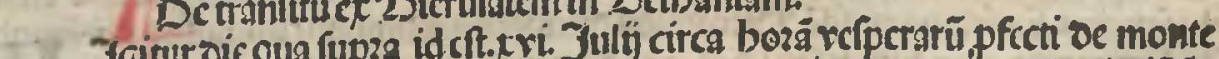
(a)

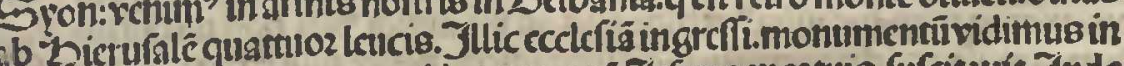

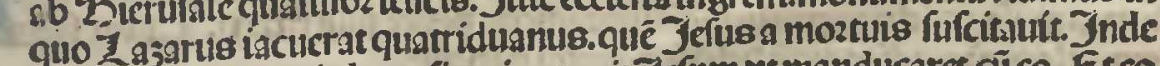

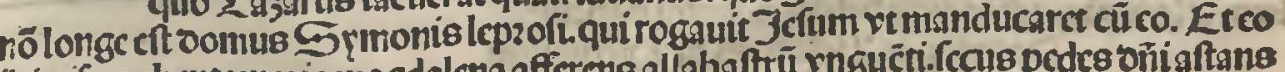

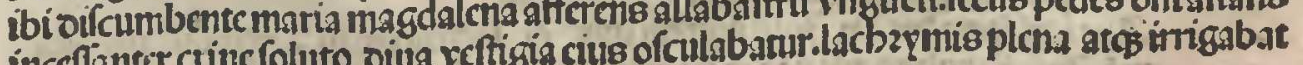

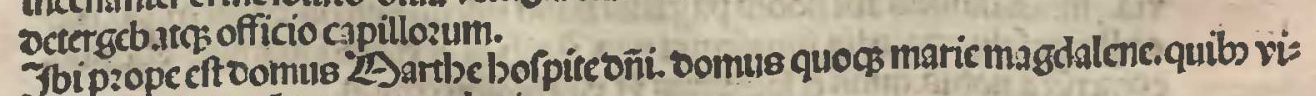
Tis bierofoltymä ocnuo repcdanimus. In crattino id citr. etralua guardí

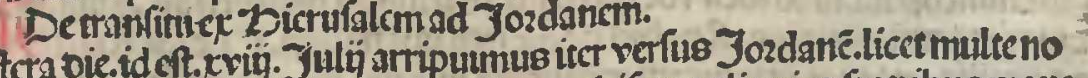

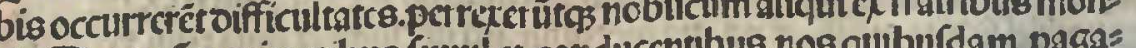

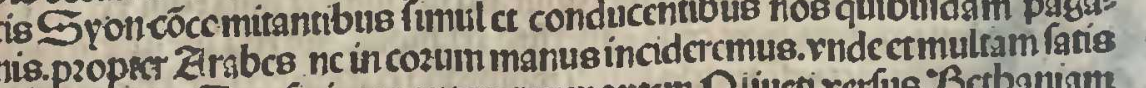

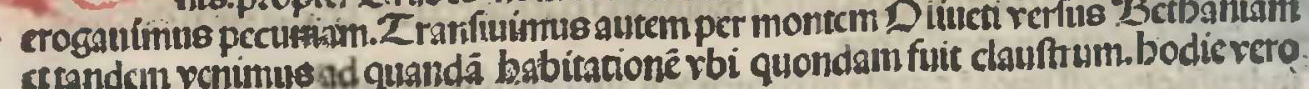

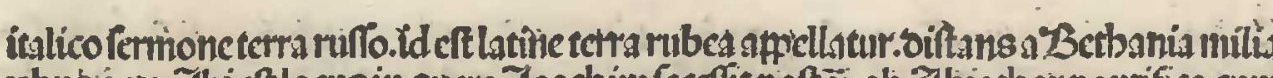

\&. Tubis octo. Jbicftlocus in quem Joacbim fecellit poft tö ab abiatbar pontifice ctin to

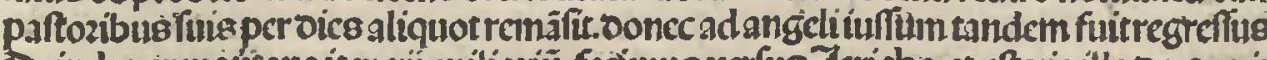

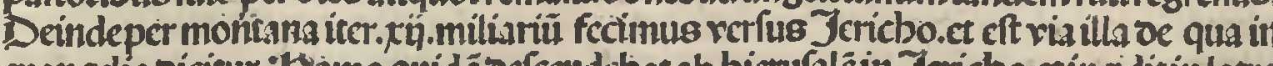
suangello oicitur. E omo quida.

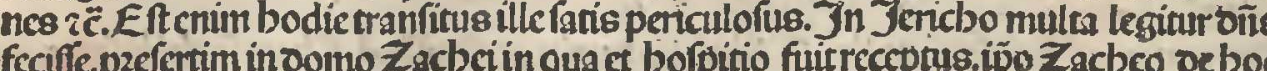

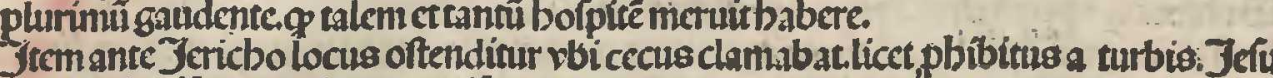
filiosuid mifereremei.et mor vifumi recepic

6. nicricbo nocte vna pnoctantres manefacto.ad loodanê perrectnuilis qui eft in fpacio

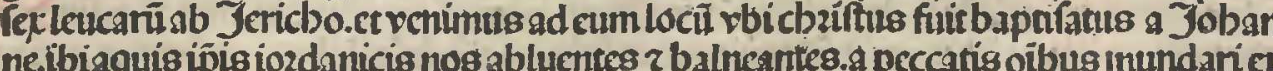
oprabamus.näctibieft plenaria remiffio onmnia peccarco?í.

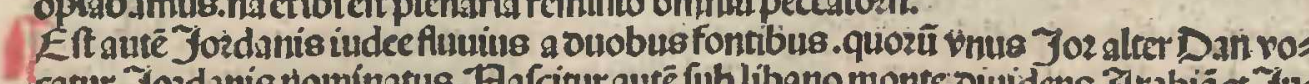
catur. Josdanis thomínatus. Fafciur auté fub líbano monte:ouildens Arabiäct Jut deam qui per mulios circumitus inkta Jericbo in marc moztūi influit.quod iṕm abs

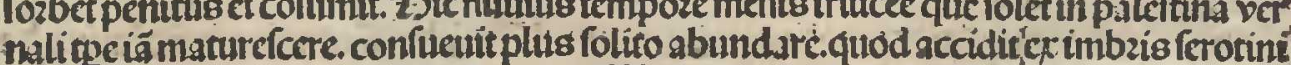

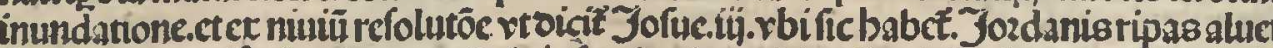

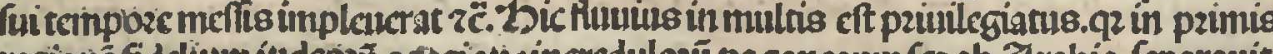

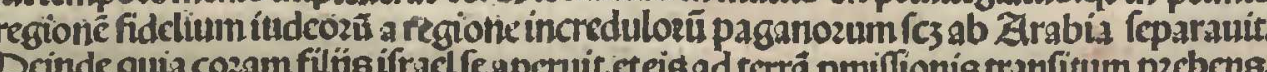

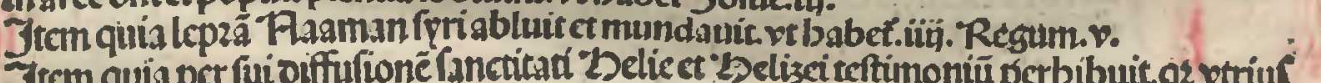

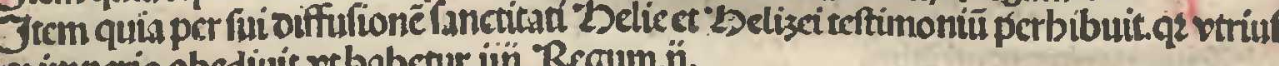
gs impetio obedunit.rebabetur.uq. Regum.

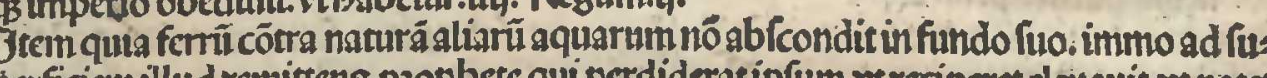
a qui perdideratipfum vtreciperet cleuauit.vt pate

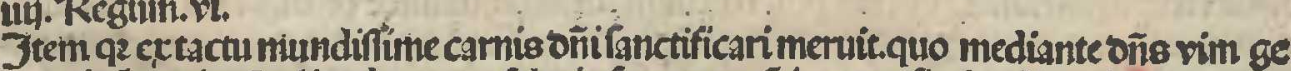

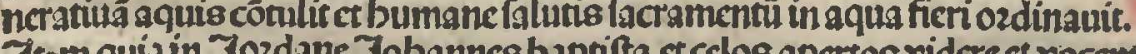

3 tem quia in Jozdane Jobannce baptifta.cectos apertos viderect rocem patris au

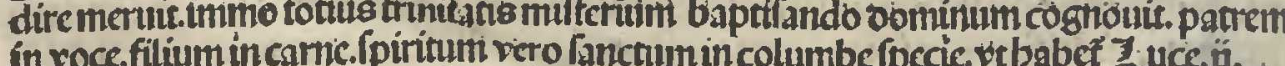

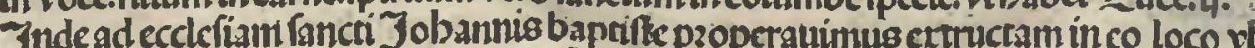

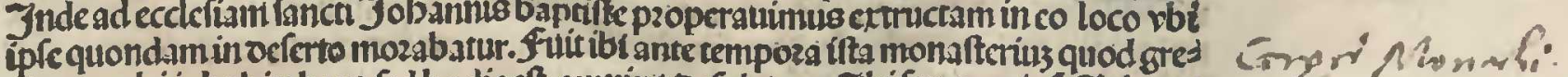

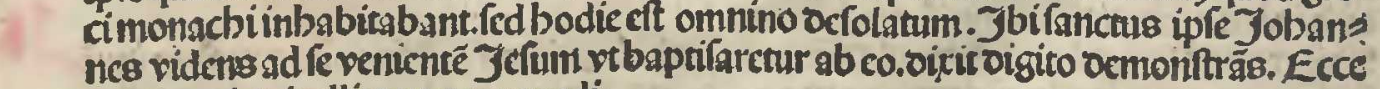
agnus ociquitollís peccata mund

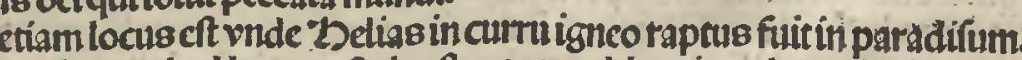
Tigdenö pzocul ad leuame eft clauftrum quoddam in co loco rbi fanctus Jetontymus maremozthum.patus tamen rallem llam inquirumume ct intraumus $r$ bi quondan

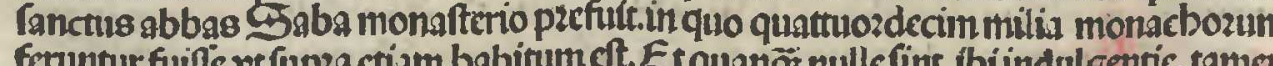
feruntur r finte verapaz

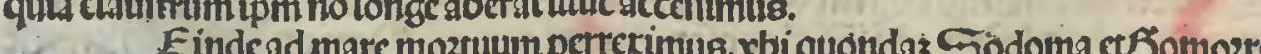
1. aliçs fuerunt cinitares.neceft ibialiqua indulgentia. Sed oe bocmarietce icris locis fupza memozatio infra laguse fro loco oicerur:

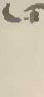




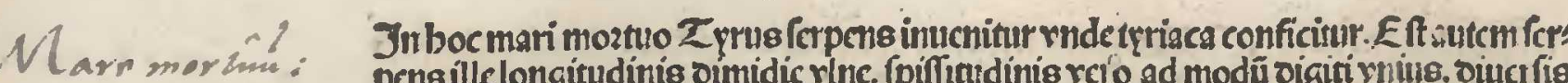

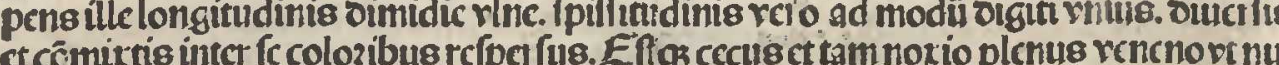

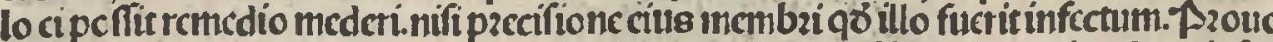

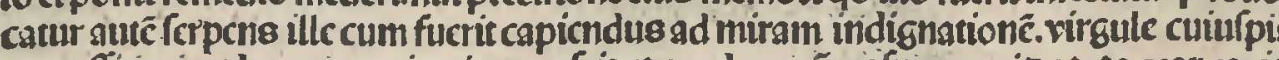

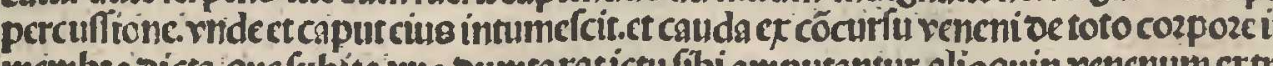

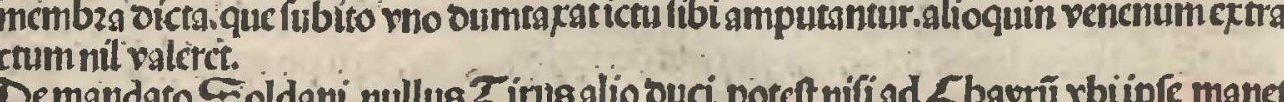
Soldanus rbiet renglis reperitur tirus oumtarse

Merr inining

Christ.

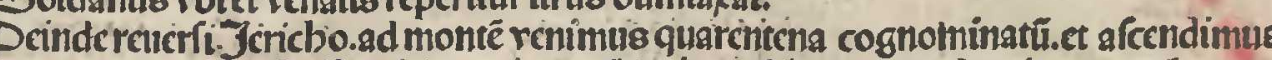
incim locum rbichziffus iciunauit quadraginta oicbus ct quadraginta noctibus.

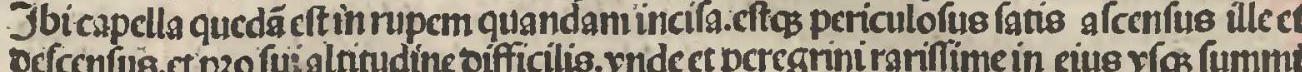

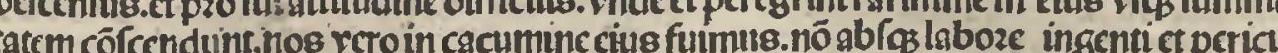

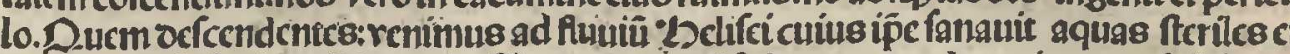

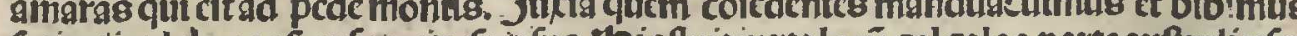

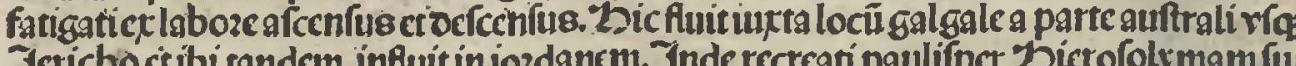

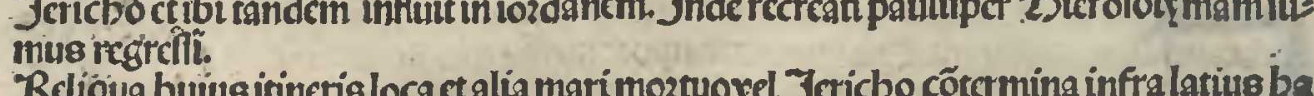
bentur efertipta

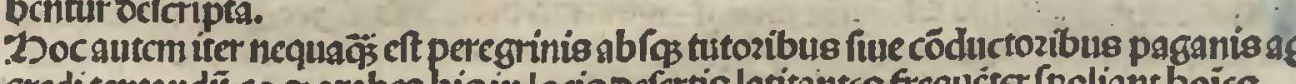

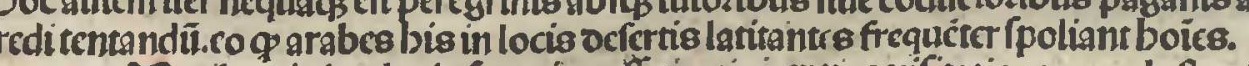
Simous igitur locis fupcringalignanio per

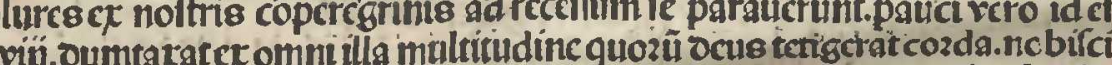

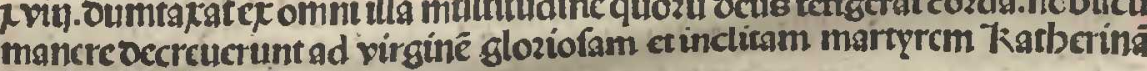
nobicumi parisct pzofccturi. orcteimentis 3ulty.quecrat folcmniras fancte mariemagdale ne.folead occafum rectgente.alq in Rama rig redictut ctinde Japba rbi quifas inf fus

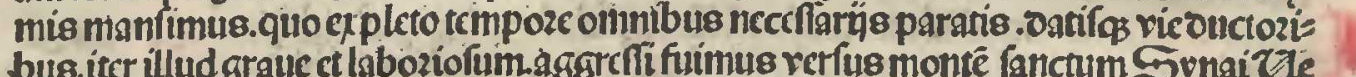

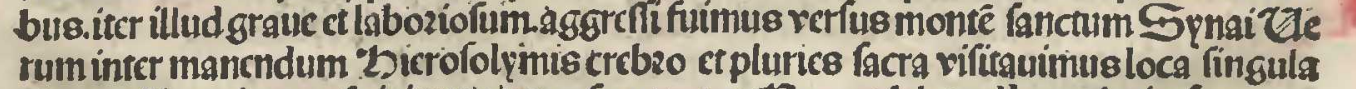

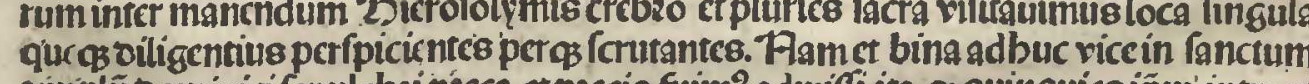

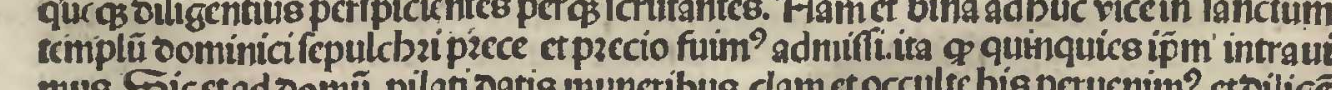

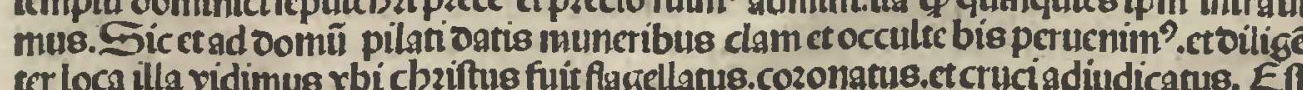

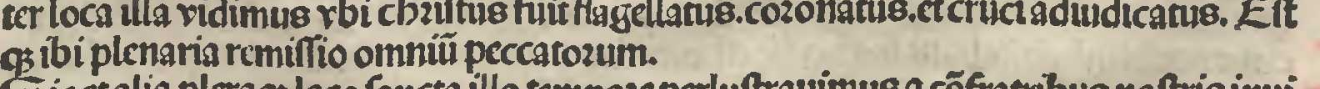

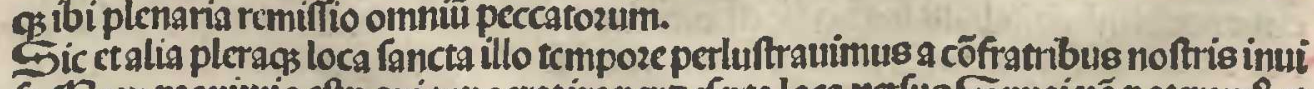
Fa. Pam prenimio cftt qui tunceratire per ocferta loca reffus Symaino porcram? b

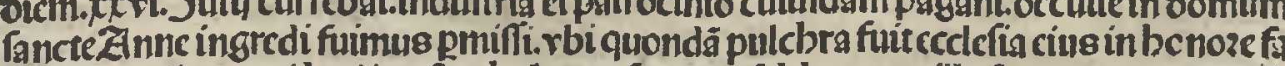
bicara.quä pagani bodic p pruo babent rfu.ct mufcbkeamex eilla fecerunt. nec permit cuntur peregrimtillam introire nifi fozte valde occulte. Erillaccelefia renimus in ams

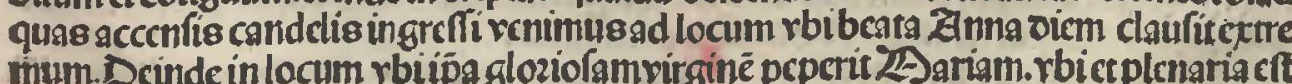

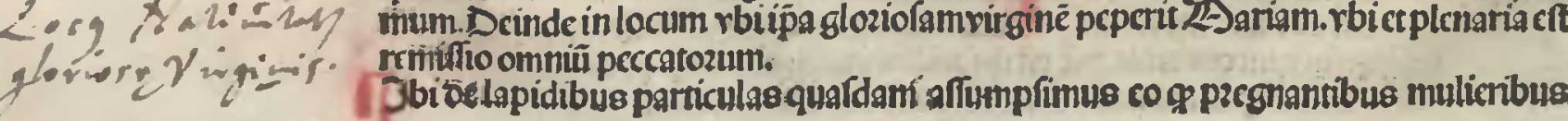

confolationictmedele forcticantur

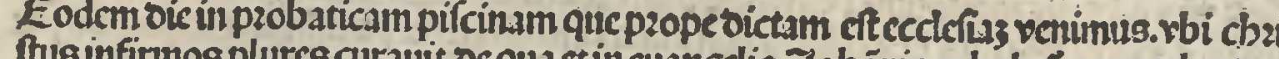

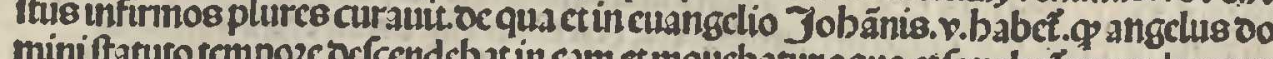

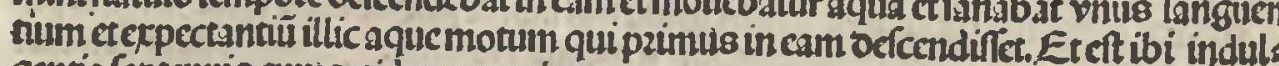
gentia feptennis cum rotidem carctio

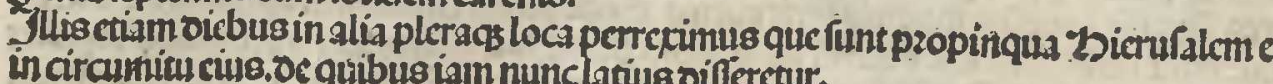

Sequitur cōpendiofa tetrefancte ocfariptio.etfingulozum locozũ quozum ba

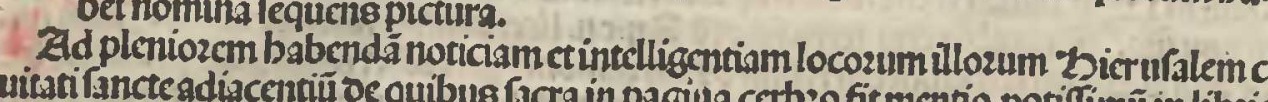

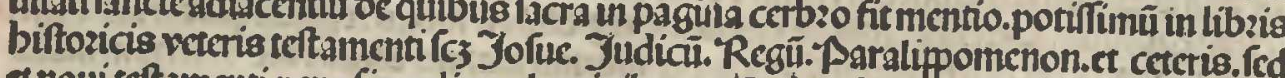

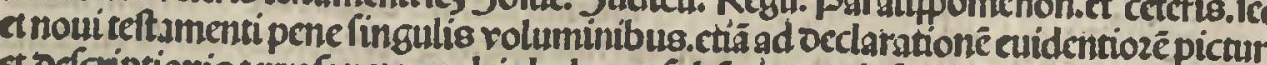
gre

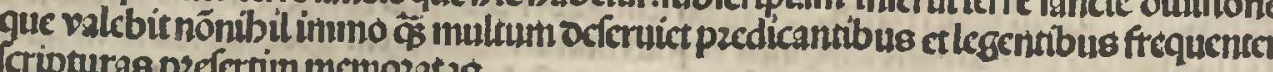

Cicndum autem in primis q terra ifts quam fanctä oicimus.que cccidi dec.que tratouarum tribum ifrace pzo partegliqua oiccbatur regnum in cebatur regnum Samaris cintitatis f́c. quenüc Sabettecoicinur. Eterat

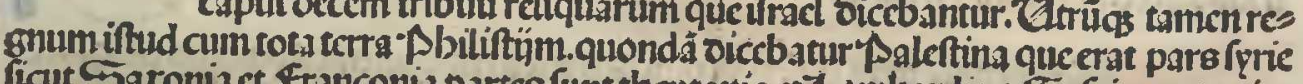

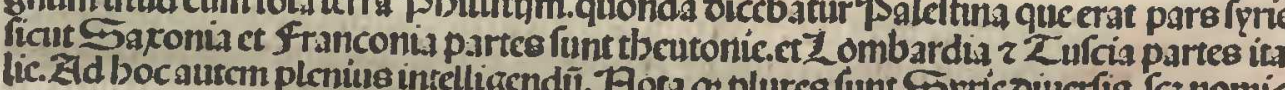

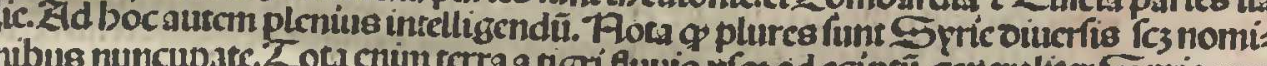

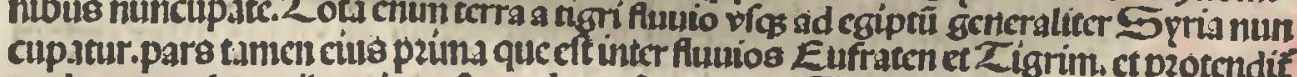

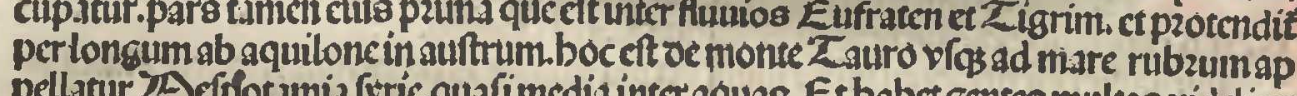

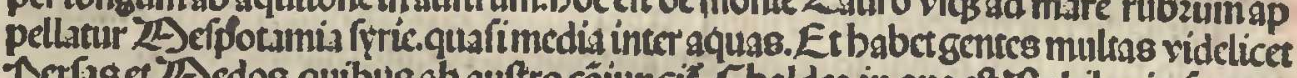

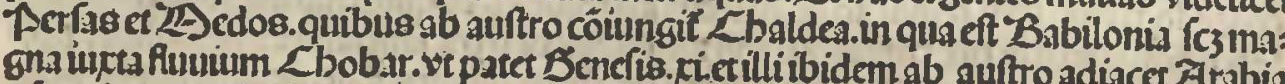

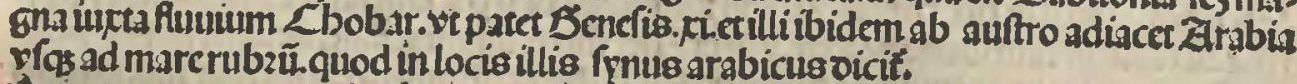

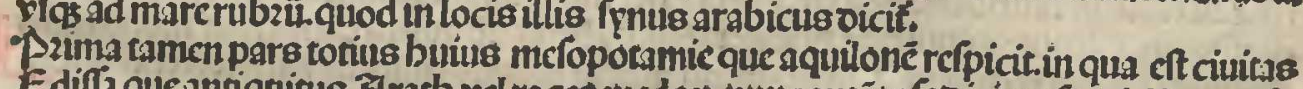

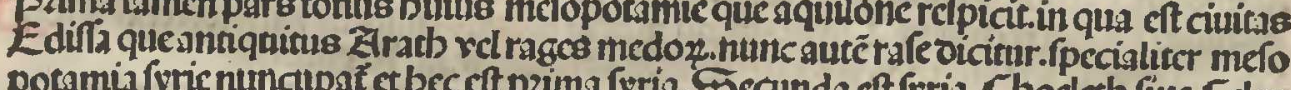

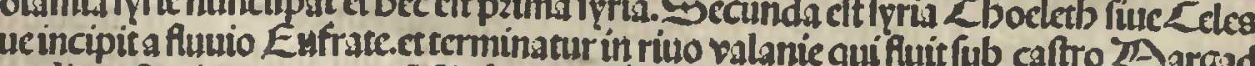

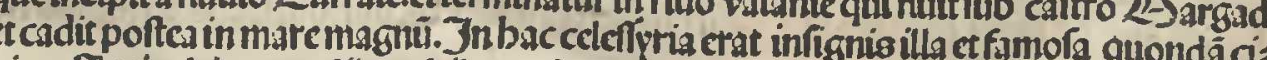

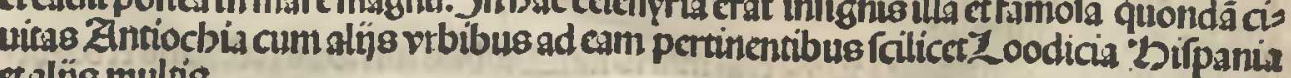

Zercia eft fyria pbenicis.que incipit a pecedicto flutio yaloniesb aquilone.ctextendit

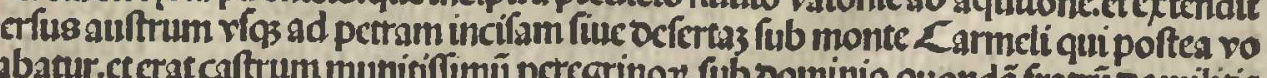
mpli cớtitutum -

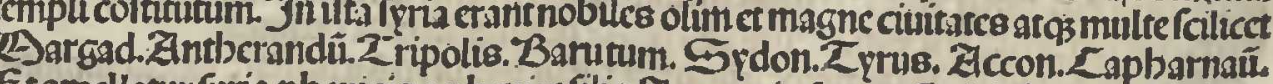

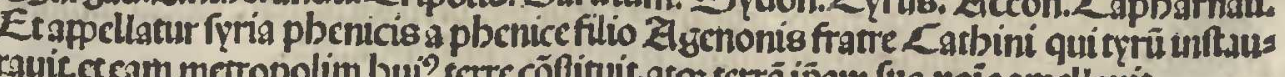
Duarta cf furia oamafci in qua ct cinitas $\mathrm{D}$ am

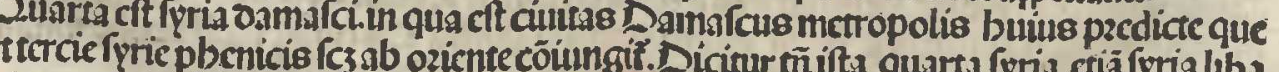

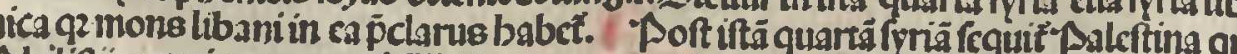

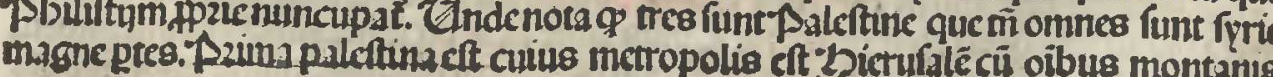

pria Damariogmón 


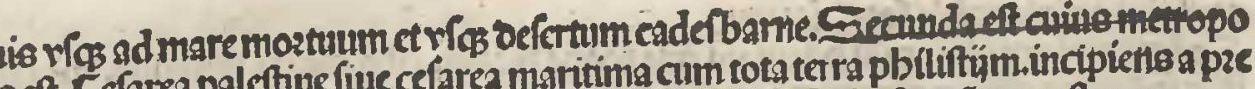

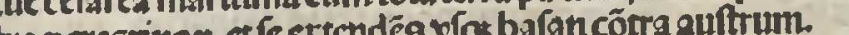
dicta perra in

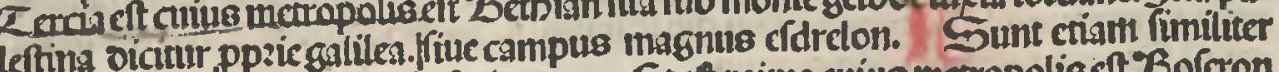

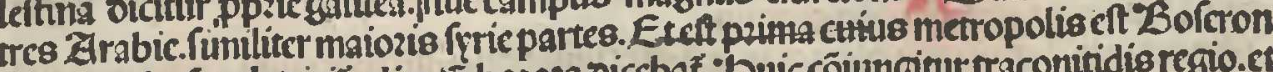

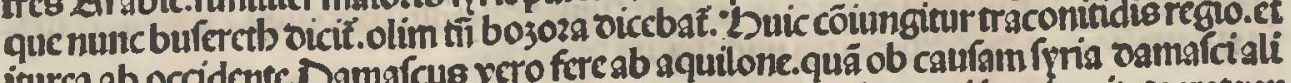

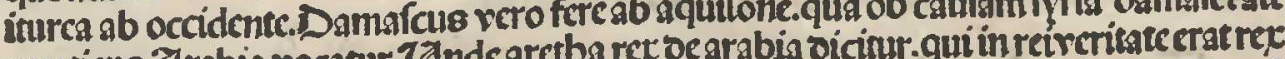

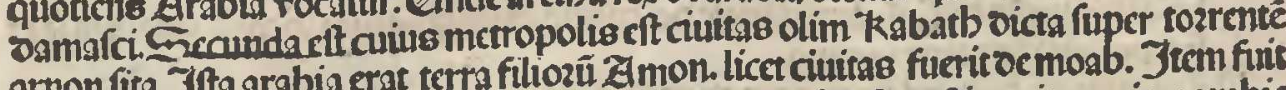
Deregno

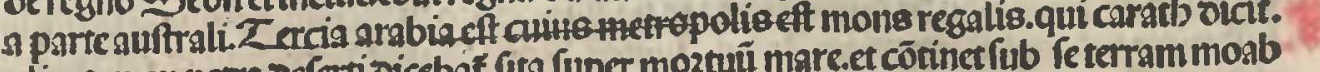

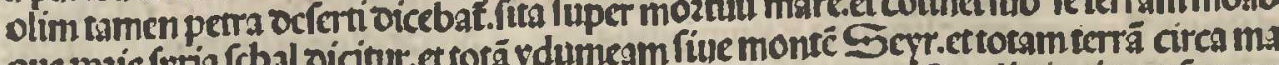
que,pzic Yyria cas rerubsum per larifitimas folitudines vfosad fumen Eufraten. "Decelt arabia magna

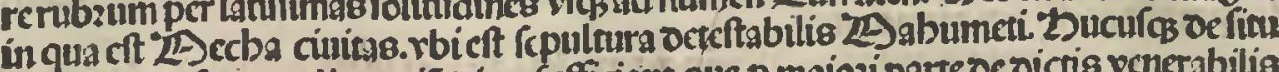

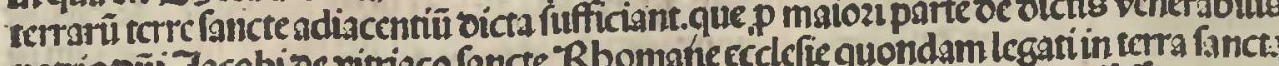

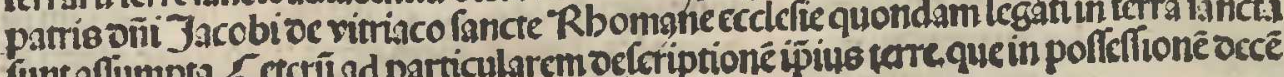
tribuunc cofitiftilum retramus.

P.tomaida

Tuidinur aurétetra fancta in plures partes refpondentce oiucreie plagisces

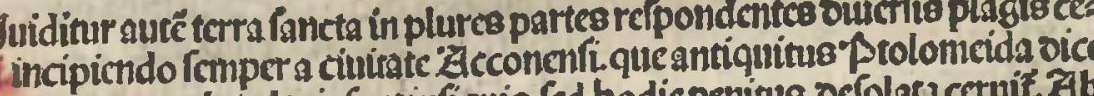
atur.cterat vbo gloziofa ctinfignis. Fed bodie pentitus ociolata cernit. Ab

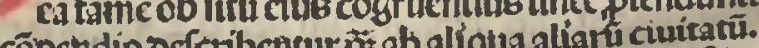

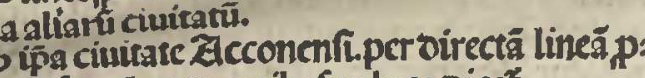

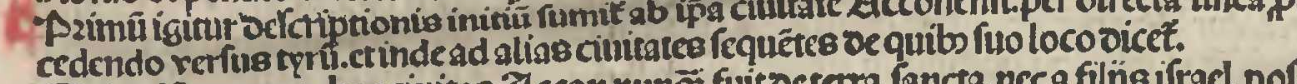

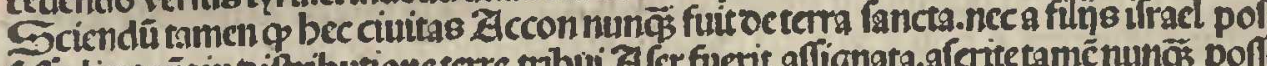
Iefla.licetalt $E$ ft

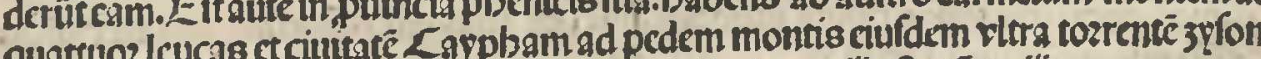

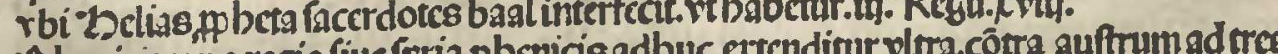

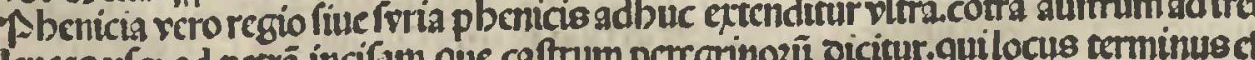

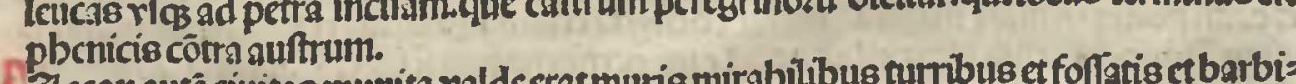
Accon autc cuitas munita valdecratmuris mirabilibus turribus et fonlatis ce barbi

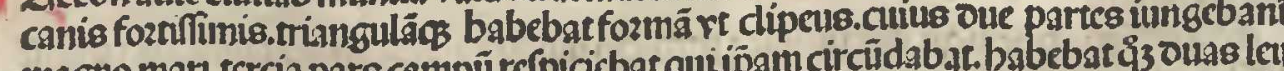

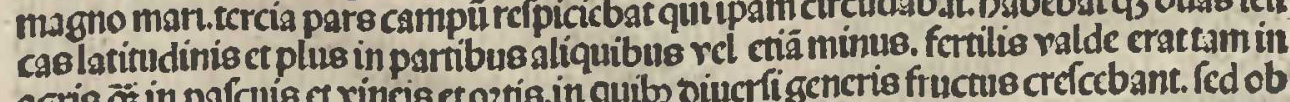

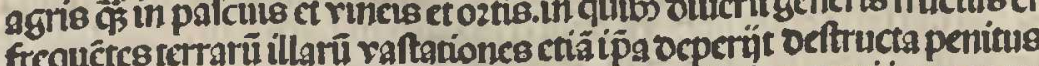

Torrie squis fummibur

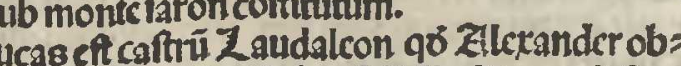

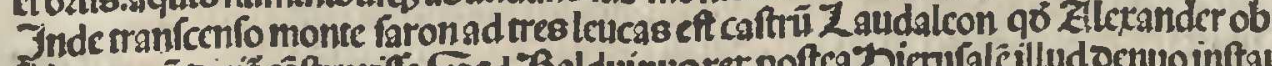

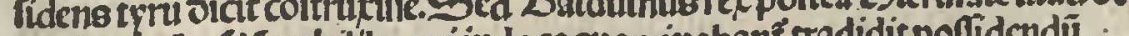

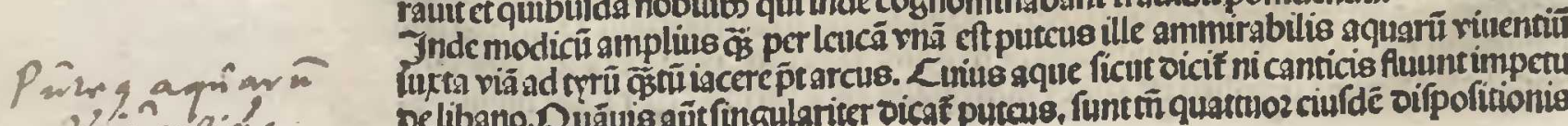

- Ped oíparis quantifatis. Cnusecnim qui precipuus eft babet quadraginta cubiros Las

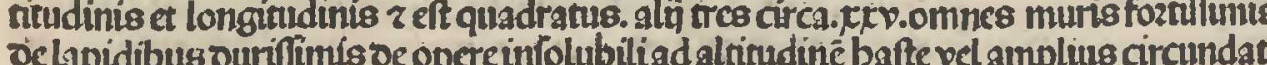

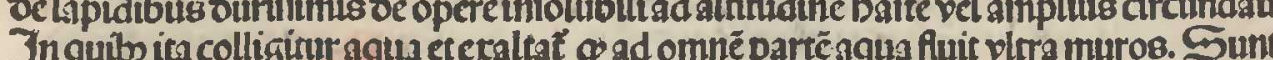

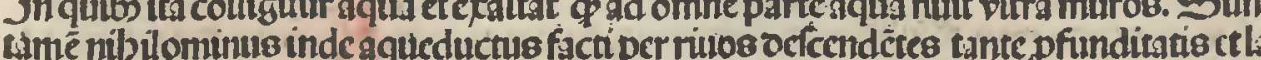

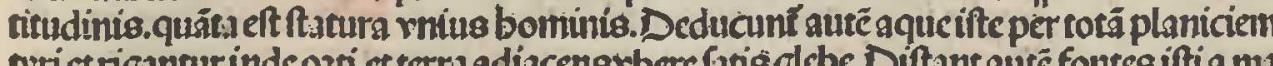

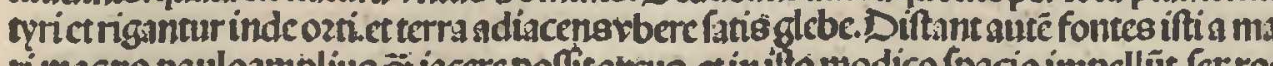

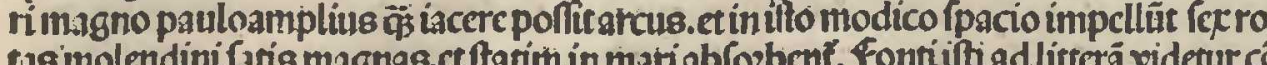

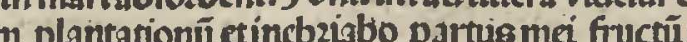

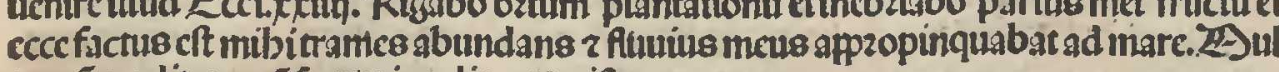

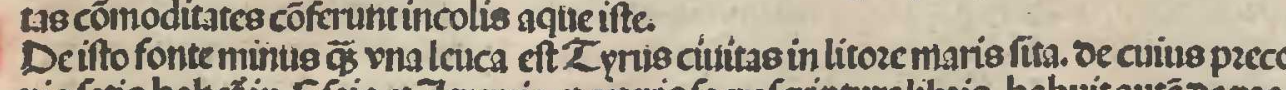

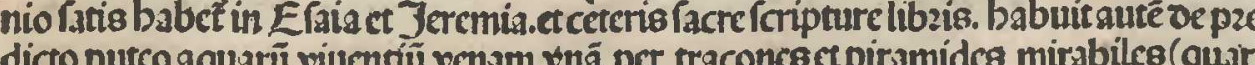
dicto putco aquarü viucntä venam vina per traconce ce piramidce mirabilcs (qua)

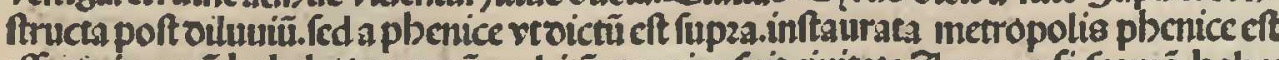

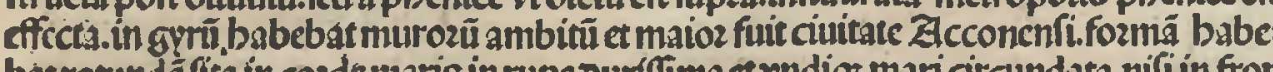

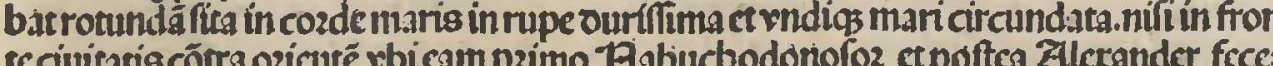
puncén des fpifo. quictiun

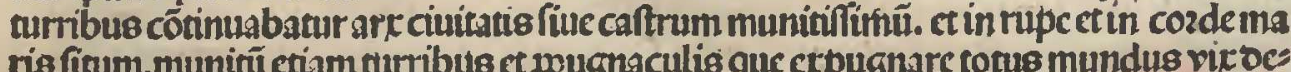

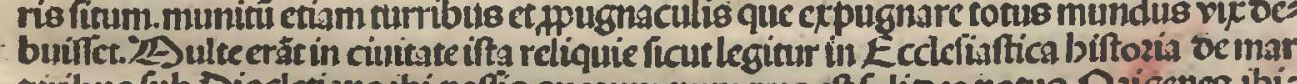

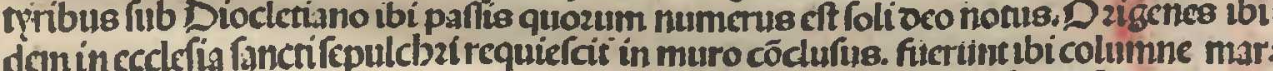
morcc ce alion lapidum tam magneg ftupozerat videre. Sedes arcbiepiccopalis erat

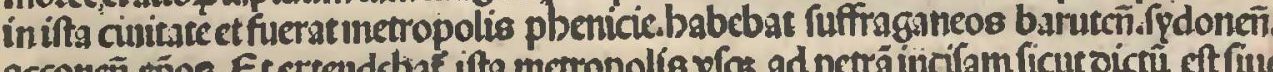

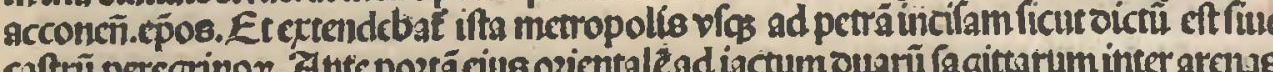

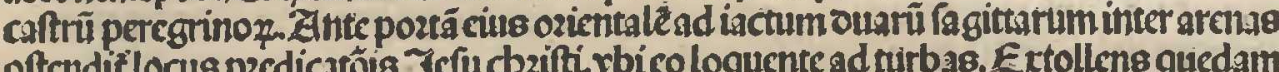

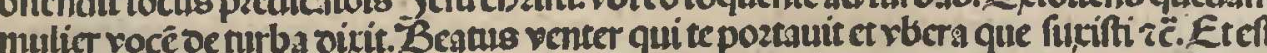

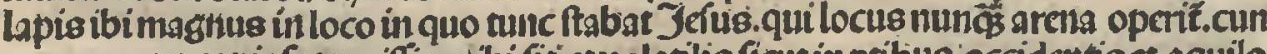

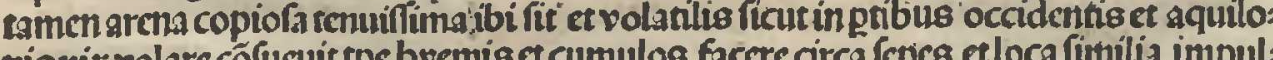

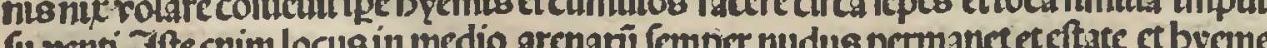

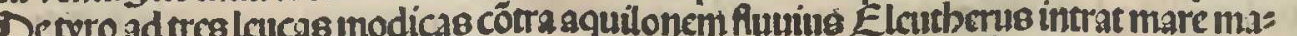

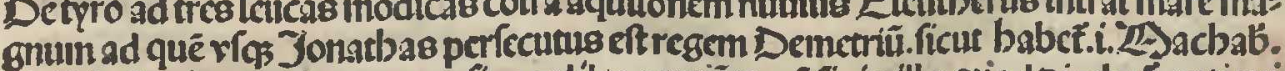

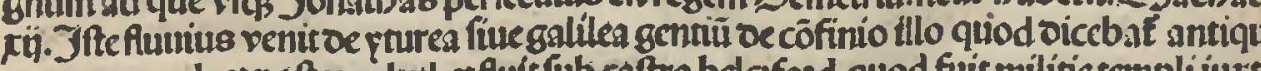
sus ctra roob etpottea zabull.st fillt borma riqucque J ad

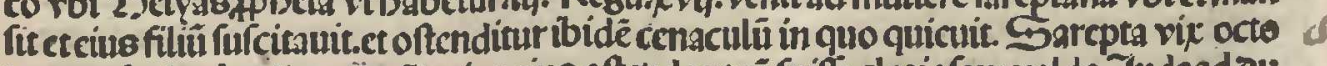

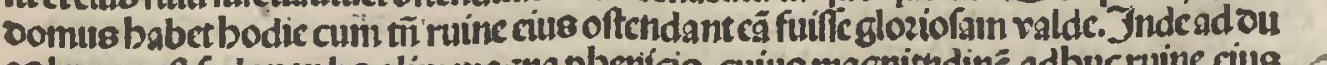
as lencas cft fídon yrbs olimm magna pbentcr. cullis magnitudine adbuc ruine cius tettantur quectia vitecrederent. Fuin in

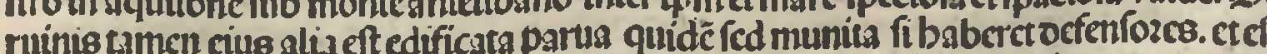
quiloncin rupequadam in cosde maris fitum quod ibidem edificauseñt peregrini qu
Anicinis. Maris magam Tryos.
Lore Prydionision atis $\sim$ 


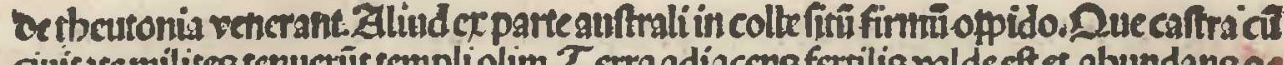

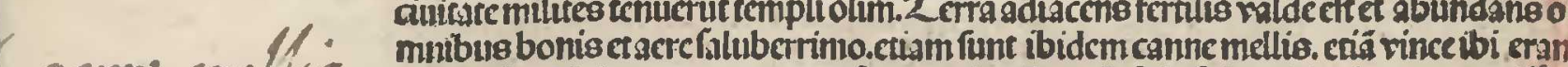

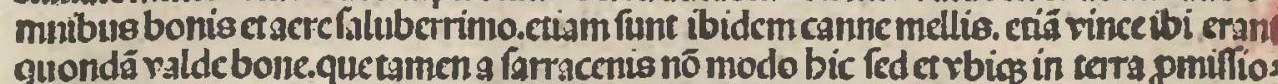

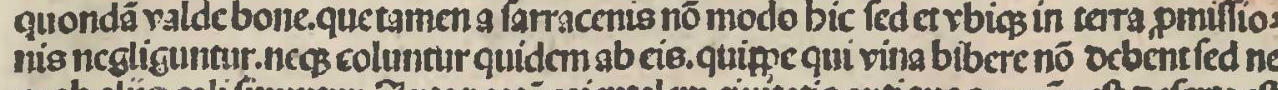

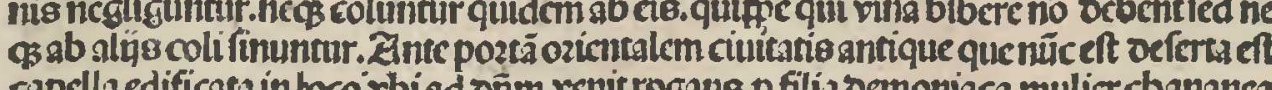
capella edificast in boco, ibbiad oñm venitroganes p filia ocmoniaca mullict chananca

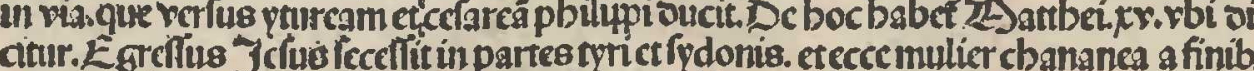

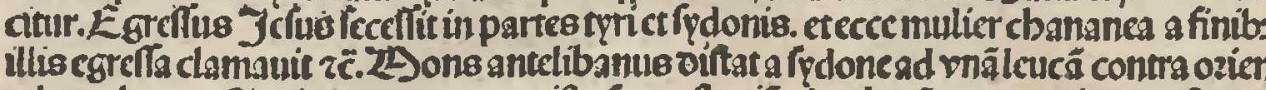

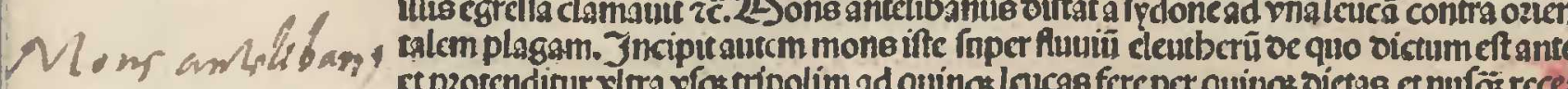

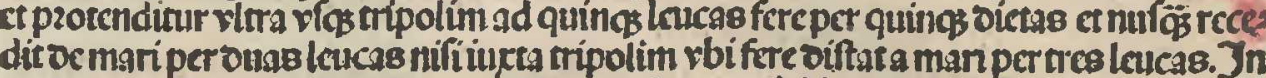
tcrdum tamé ira acceditad mare go locus eft inmeabilis omnino.abundat autem vi:

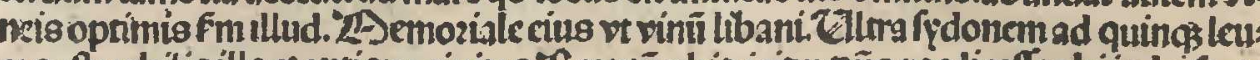

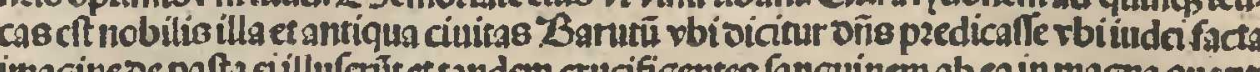

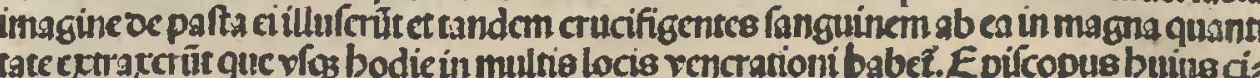

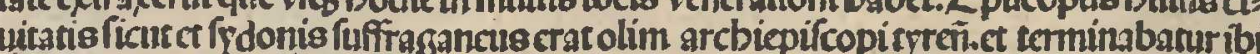
metropolistyrencnfi. Etoltra ad tres leucas in flutio qui paftus canis oicitur qui bi dcm magni mare ingrcditur. cerminabat fimilicer patriarchatus "Dicrofolymitanus.

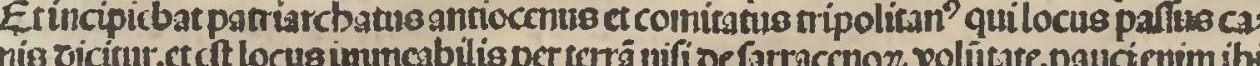

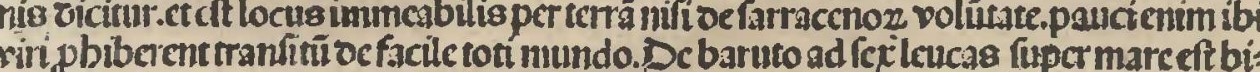

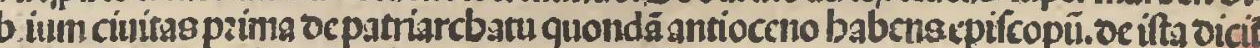

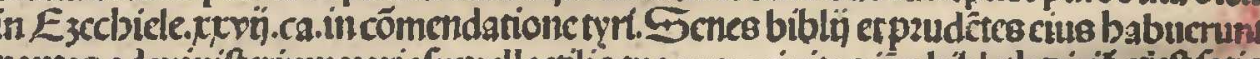

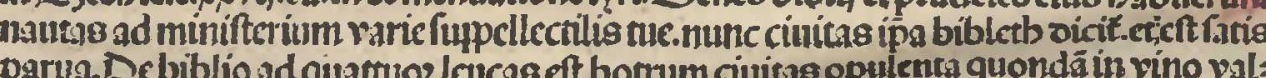

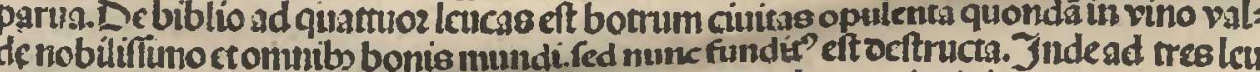

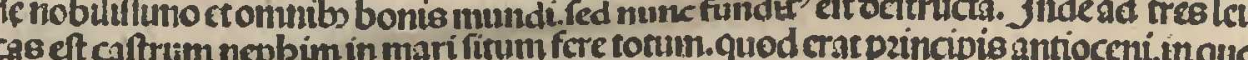

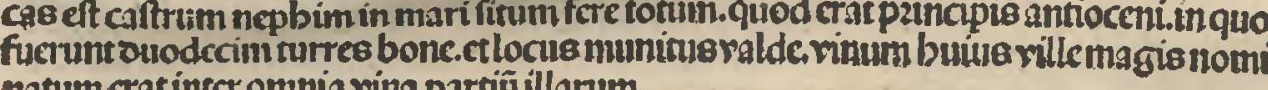
natum crat inter omnia vina partiii illanum.

B. $10 . \sin m$

botrin

12

Tripsin.

Savrá Povadip

refonominata

3. . .

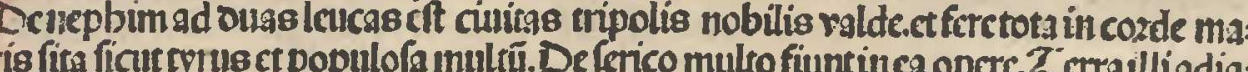

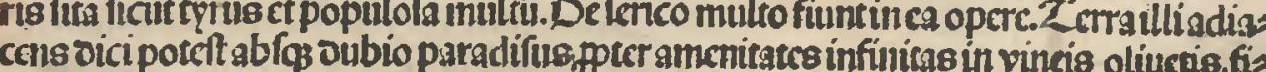

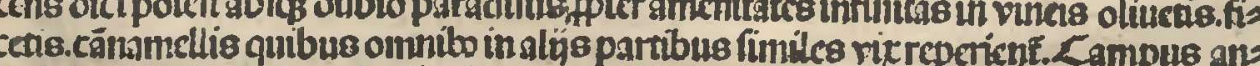

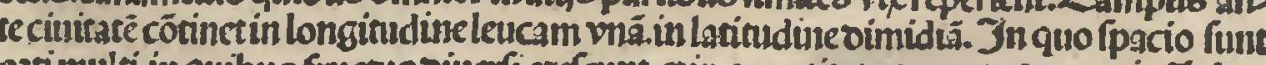

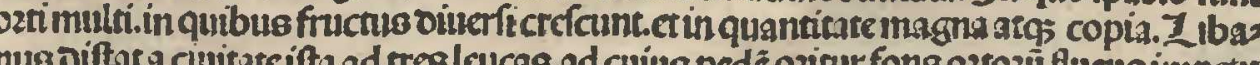

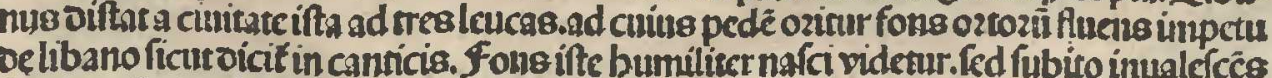

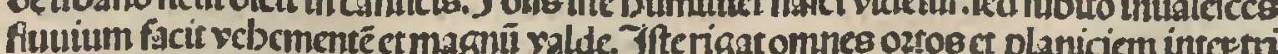
polimet tibanti.etcōmendat mirabiliter regionce. Aque cius optime fiunt frigide et ouls

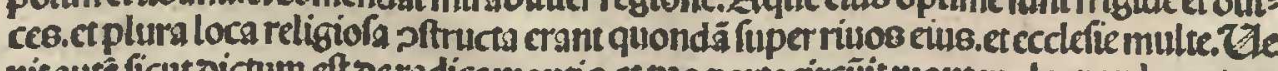

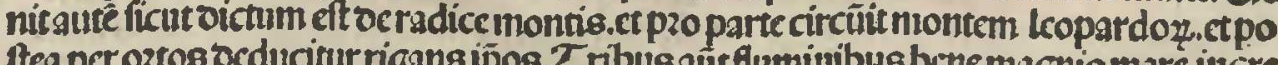

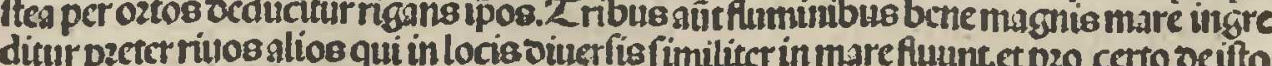

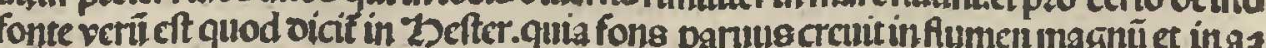
quas plurinas redundauti. De tripoliad ouas leucaset mons leopard oz. rotundue in afpectuct ratis altus. oiftane a libano ad vnam lencam. In buine pedea quilonan

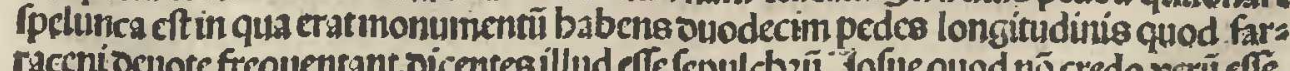

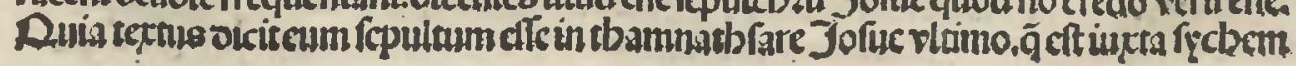

rratroru a noln'firmón.

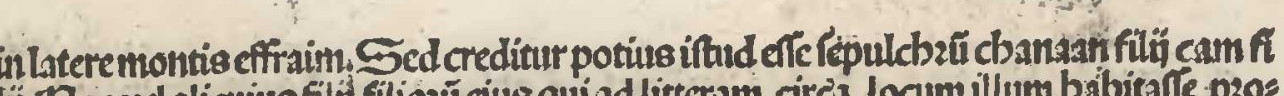
if Foe.vel aliculus filit filiozú cius quiad littcram cires locum illum babiterfe p:o

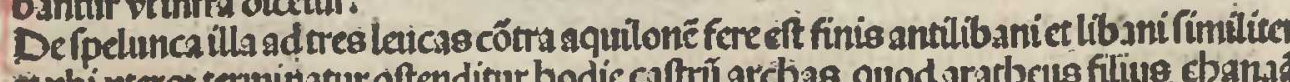

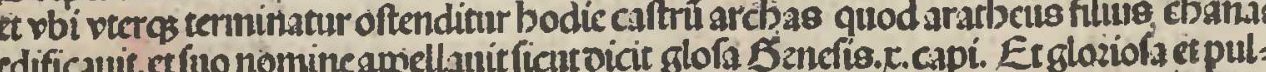

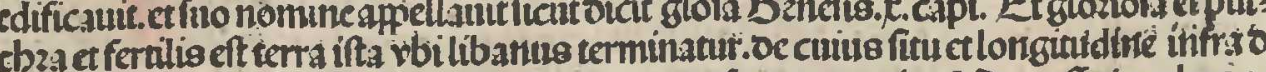

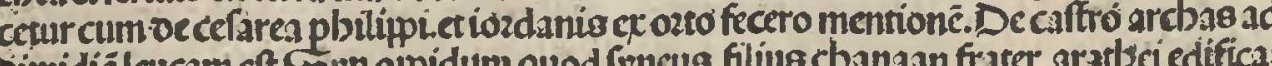

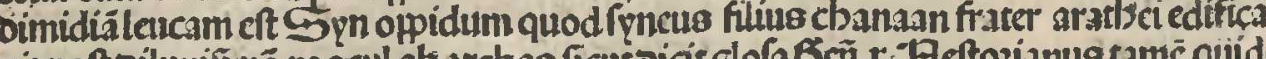

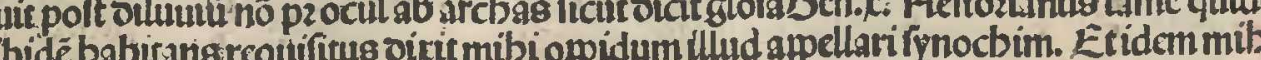
oiritquidāfarracenus.

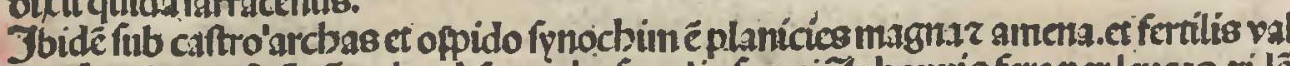

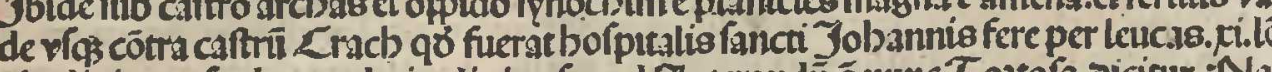
gíndinis ctp per leucas latitudinis of

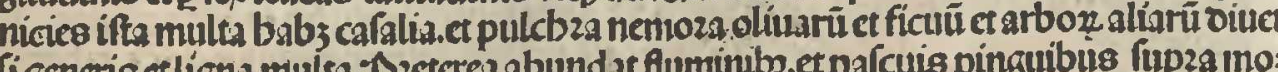

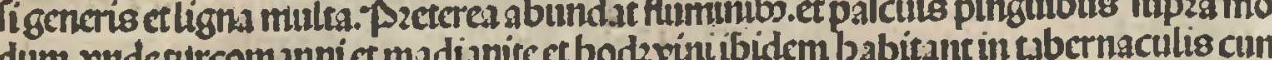

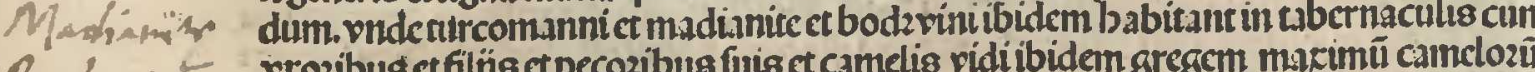

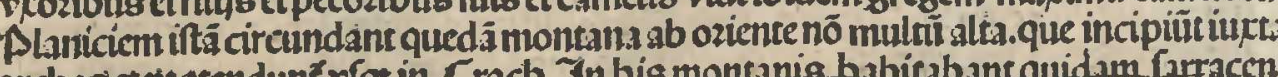

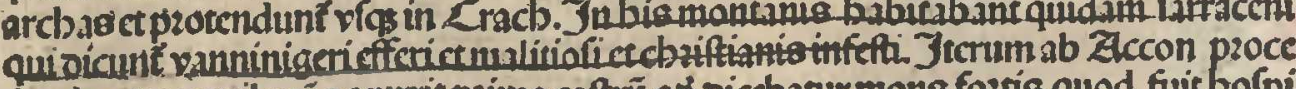

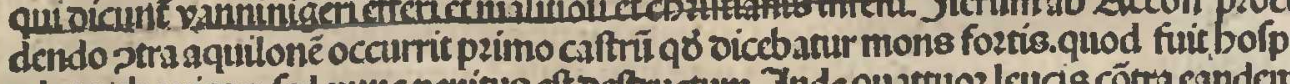

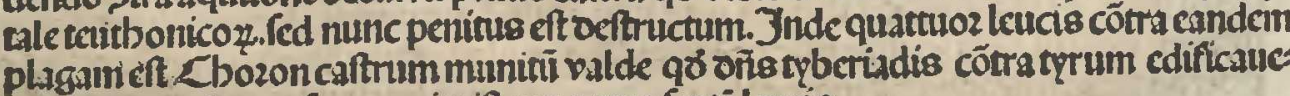

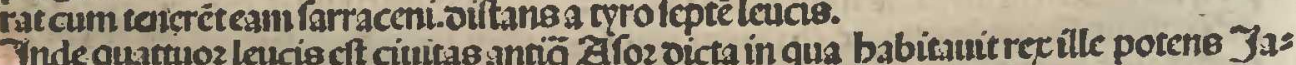

$24 \cdot R \cdot$ bin quic cunt vigintiquartuoz regibus gingnauit cörre Jofucet ifracl ad aquas muron

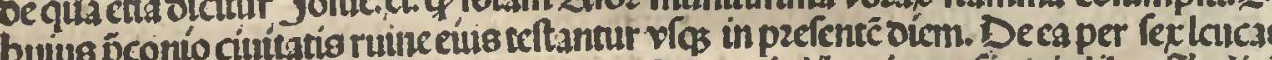

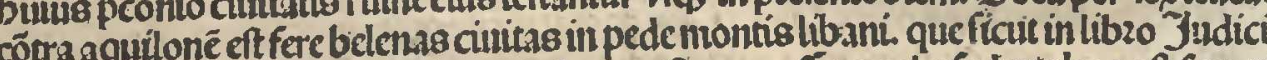

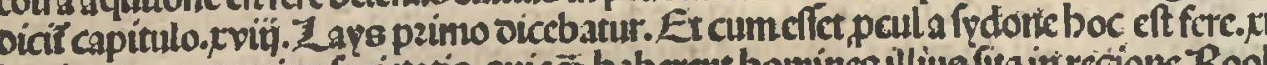

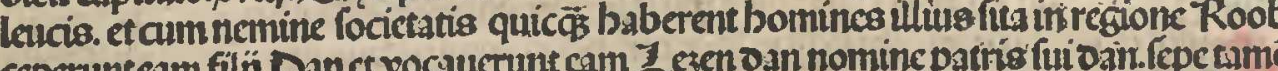

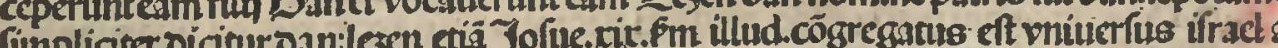

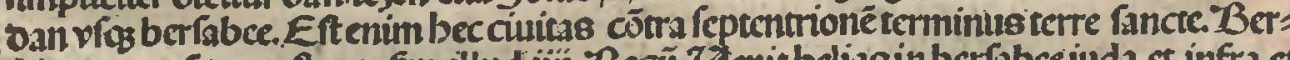

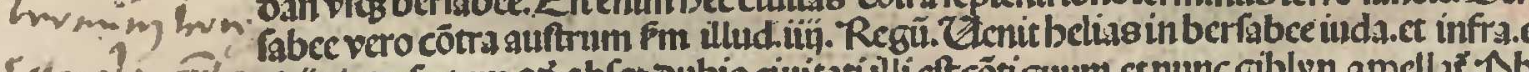

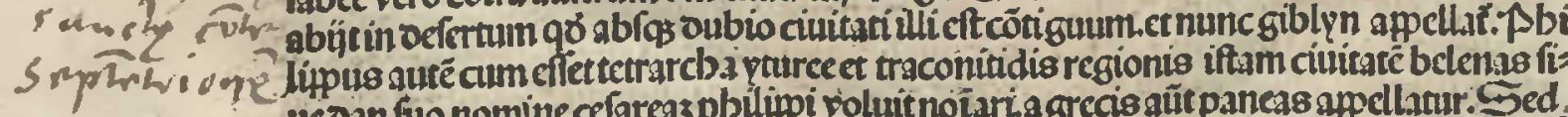
ucoan fio nomine cefareas p pulippi voluitnoíariagrecis aût paneas apellatur. Sed

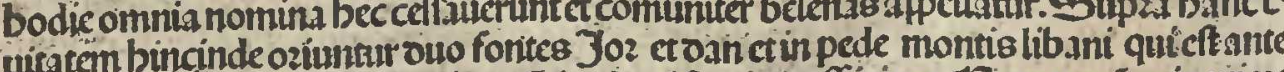

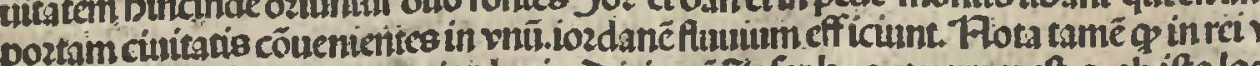

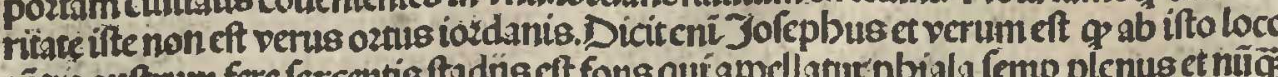

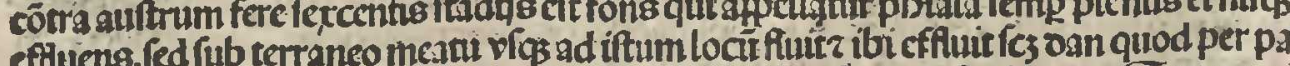
efreg in phialam fonnomiffas et in fonte oan receptas f́cpurs ett pbarum. Garracen

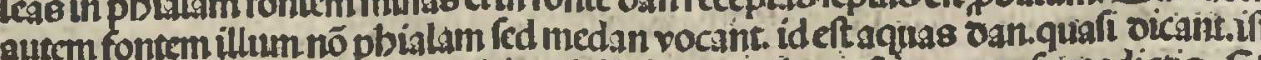

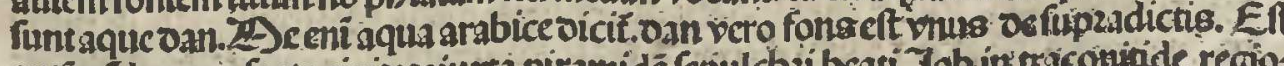

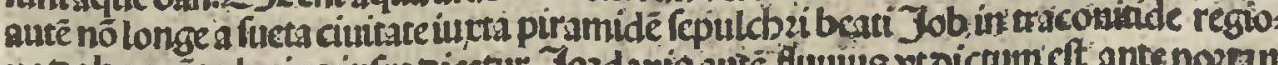

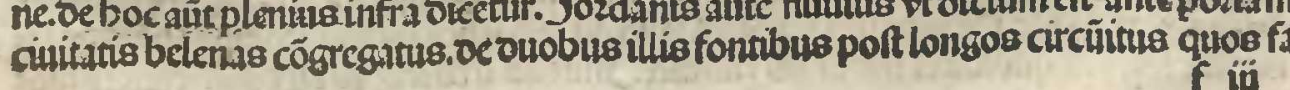

Grm maxim

now fort
Panvias. Srpantrés 


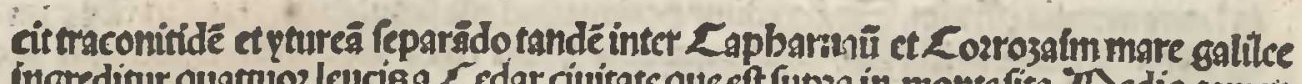

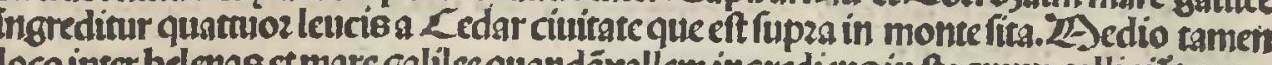
rerefolutionis nuiuium oc libano. oicituros bodic gd gquas in tiagnum colligit tempo

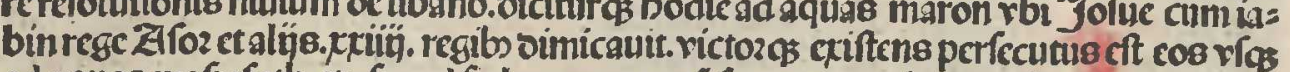

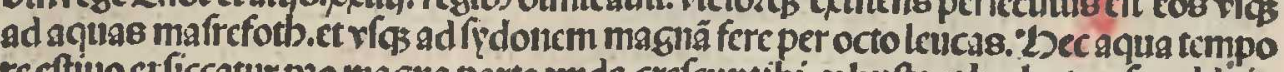

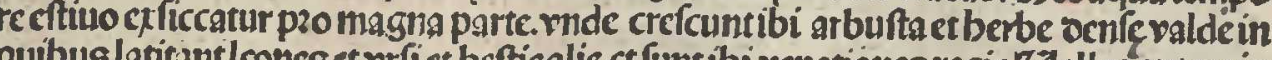
Wolibano vincis ortisct

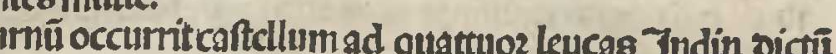

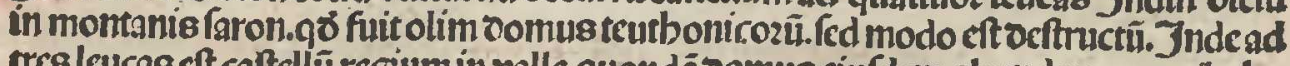
nomin Cong Tinst.

Tant.

Crphen is et fructibus quictiä in terra illa rari funt nifi ibi. In de lcucis qundans omnibs bo

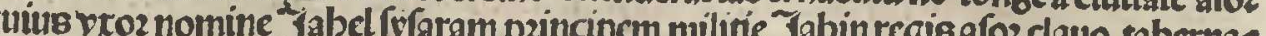

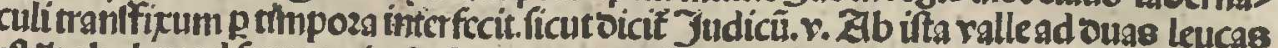

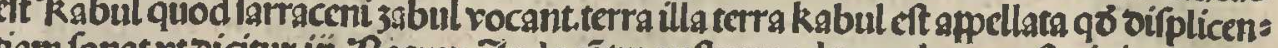

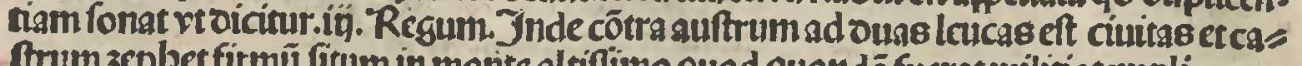
3ndec côtra aquiloné quatruoz lcucis iutra rallcm fenym eft cedcs neptalim. oe bacfu

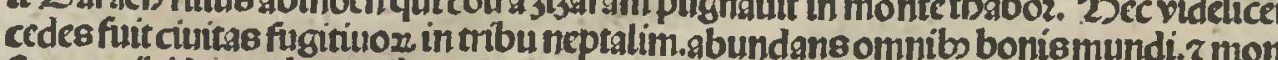
(r)

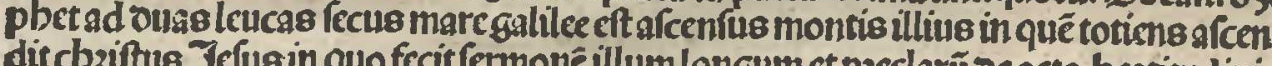
bus ctive rituris

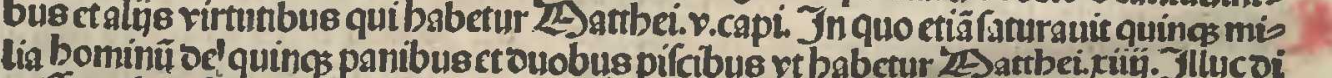

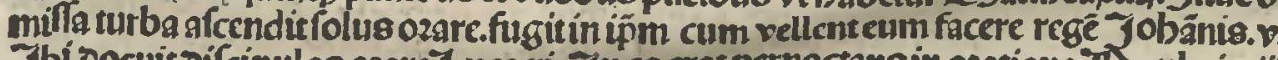

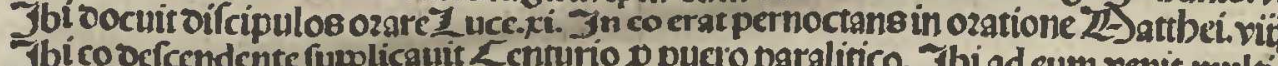

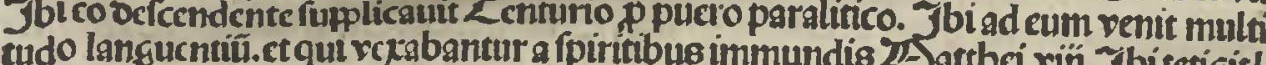
profum et fanauitcum. Jbiftetitin loco campeftri et turbs bif cipuloring. J J bitetigitle monte videtur rotum mare galilecet ynurea etraconitidis regio vfos ad libanui monte Jnfuper lanir etbermon.terra 3abulon et neptalim vís Ledar etralia loca multra.

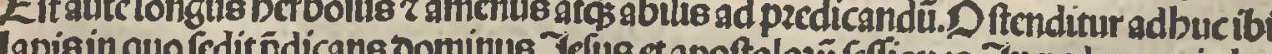

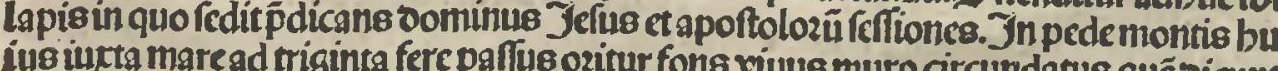

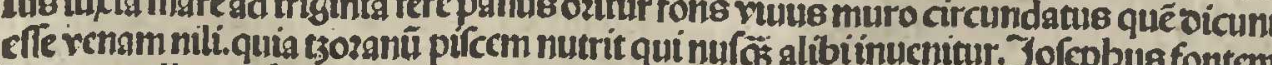
bunc app ellatcapbarnaum.quia tothe campus qui eft ab ifto fonte vias ad iordanem per ouas lcucas capharnaum appellatur. Defontciffo advigintipaffue fupza mare ga likections

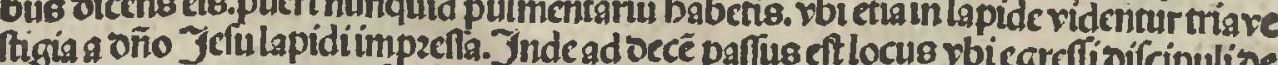

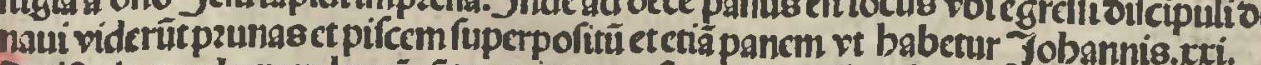

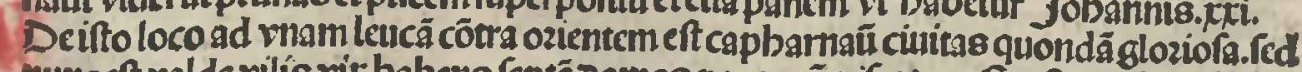
nunceft ralde rille wir babens repte oomos pauperü pifcatoz. Eteftrecrein ea imple num oetrabcris. DS- Tat xi.De grmedictrab chin.

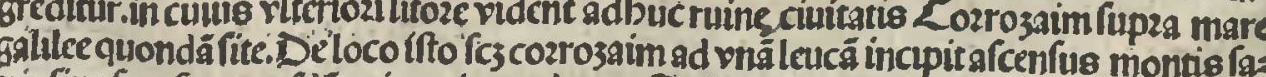
nir fiuc fęr fm quofdäet introitus y̧dumec, Jndead tres leucase eft cedar ciuitras glos

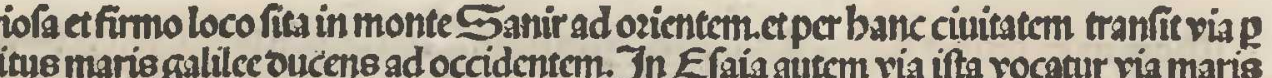
litus manisgallkce outcens ad occidentem. In Eraia gutcm via ifta rocatur ria maris

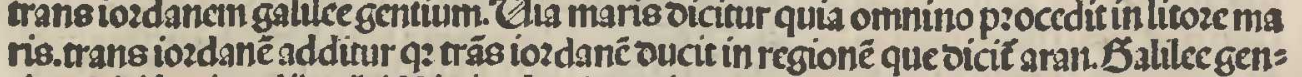
nuum oicit quiagalilea ibidé ín iozdanč terminatur. qui io: danis per mediü buiug rall

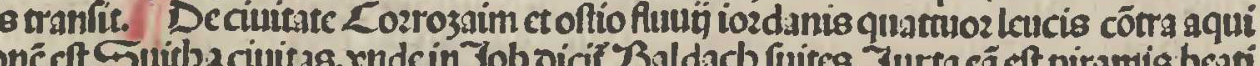

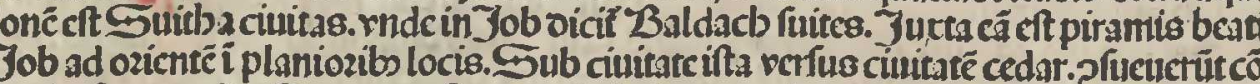
uenire farracenioe Aran et mefopotamia frrie de mosb amon et róta terta ozientali cir

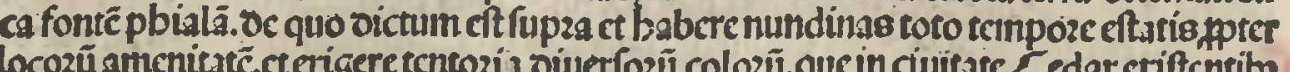

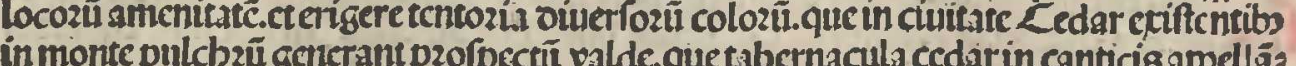
urr. Deciuitare $\mathcal{L}$ O2trozaim quattuoz leucis contra ozienté éf cinitas Ledar in monte

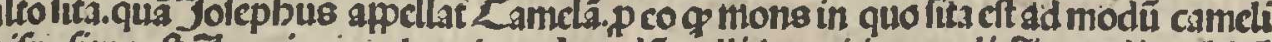
oitpolittus eft. In pzincipio longioz ad modũ collietc capitis cameli. Jn medio gibbü

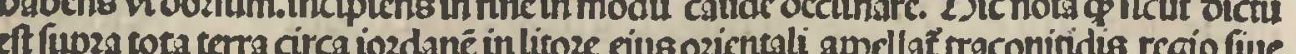

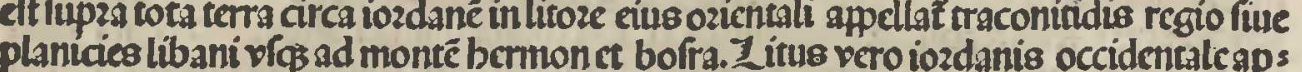

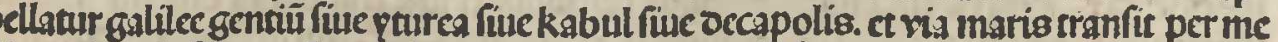

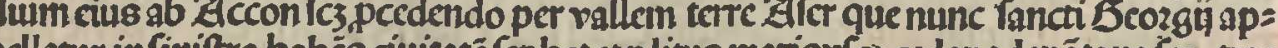

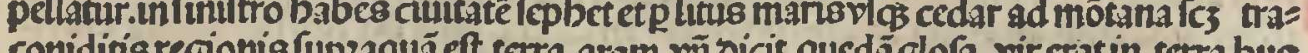

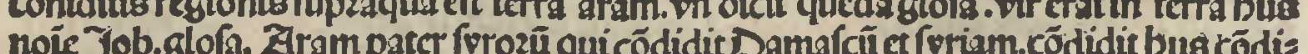
toremos babuit'pbilippus tetrarcba ficut et puream que eftcirca regionéiozdanis ad occiden

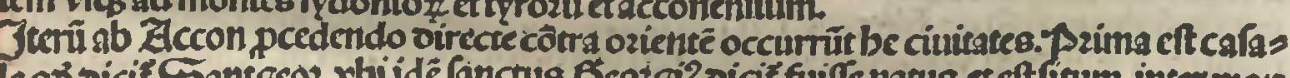
(a)

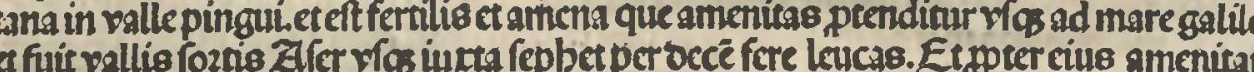

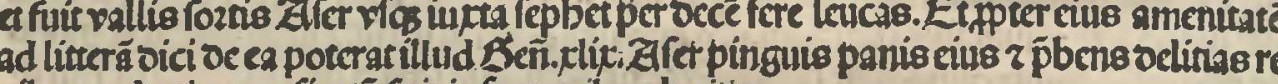
Gibus.qo vtigs verfficaû́ fuitin forte tribus buitis.

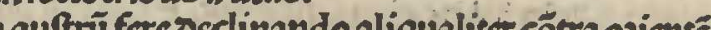

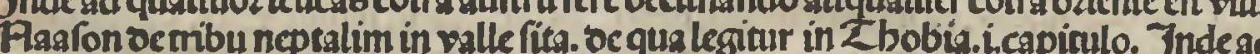

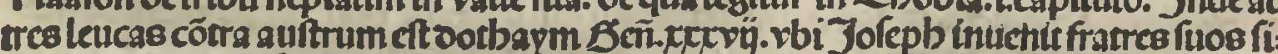
sum fub monte beth buliead vnä leucam oppidu valde amenut in vinctis olluctioet fice

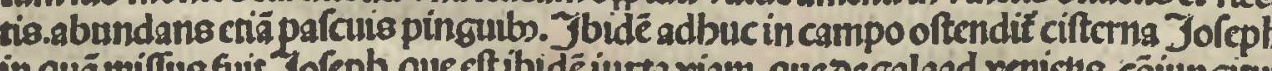
in qua in las fuir Jolepb.quent betbulicetinde percamp ü cefdrelon pcedene fúb monte $\mathcal{Z}$ babozad finiftrâ per cam pum mageddo alcendit monse effrayme c penit in Ramatbaym rop oucir in egiptu. Per banc viamvenerunt by rimabelise qui cmerunt Joreph. De it a vi

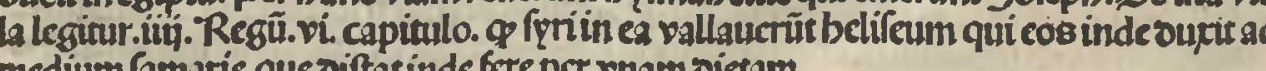

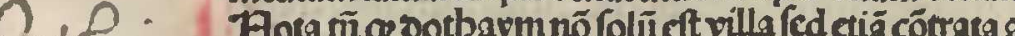

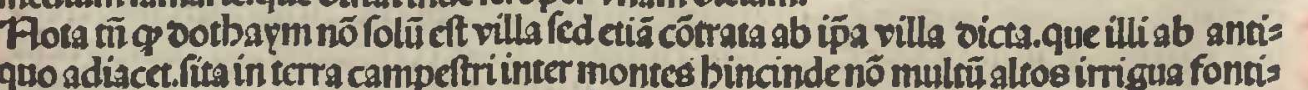
bus et ideo parctiofa atç alendis pecozibus apts.

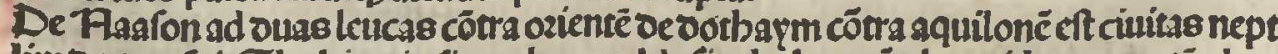

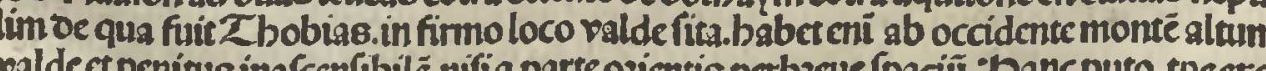

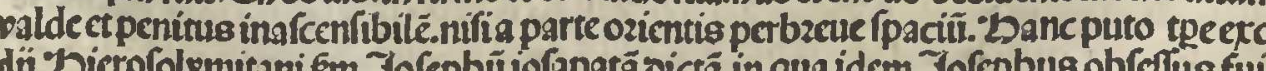

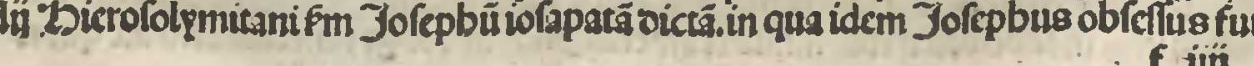

Mons

Yis Mavi

$\sin f a$

Tamnar

Cromar

Tracomitá

Juinas

Him

cants 
Syrm

Bmsainan

Parming foroing

Caprowasion;

Burfitia

Smasa

Croing barm

Tammi.

Cripe

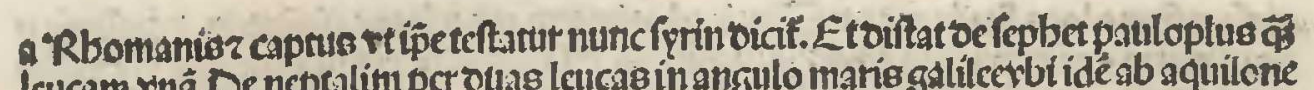

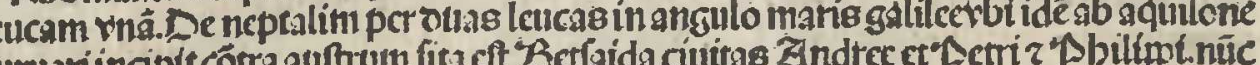

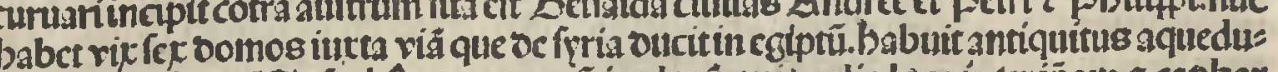
ctus se cfunio qué Jof naumingredit mare galilec buins reftigia adbuc apparctr. Indep ouzel cucas cötra

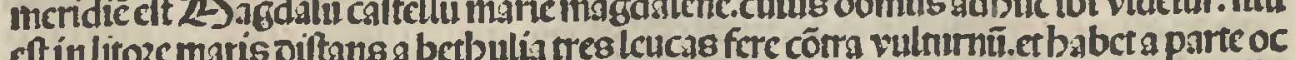

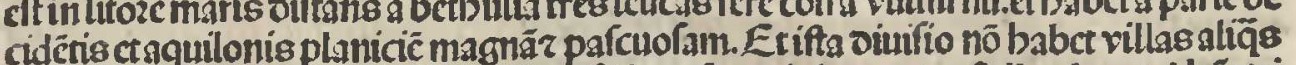

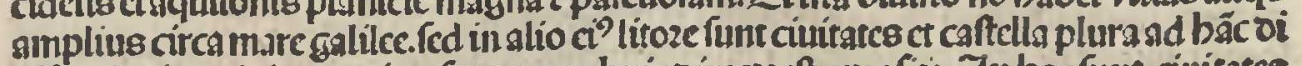

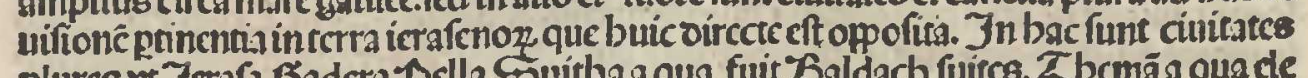

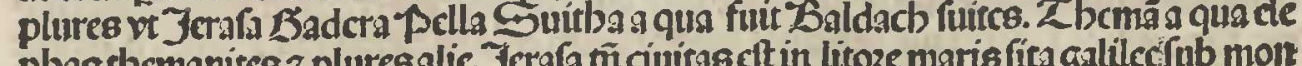

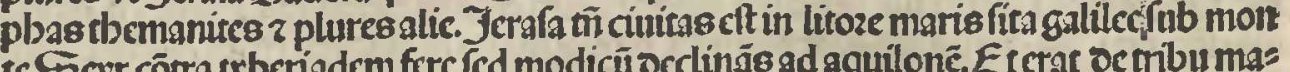
mafecoimidia

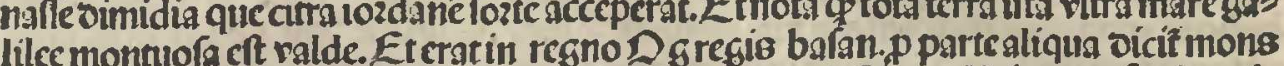
fevr.quia E fau ibi babitanit ref ftatim Dicet. Diccbatur infuper alibi mons fanir quia

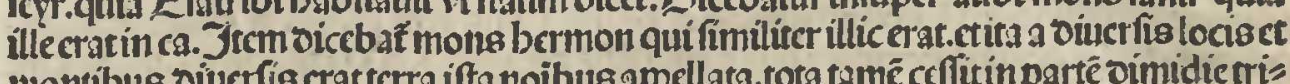

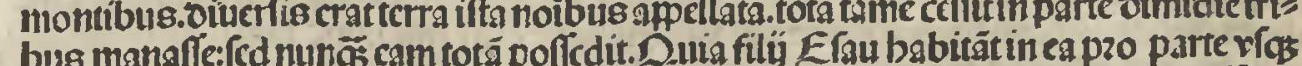

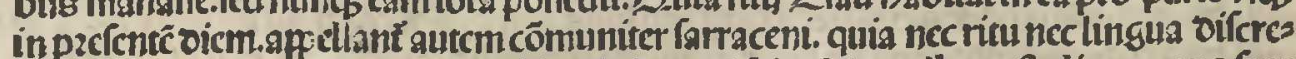

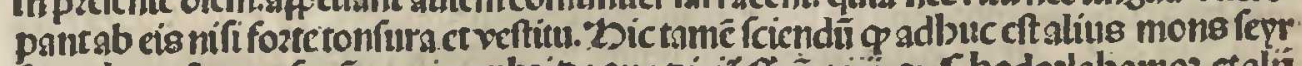

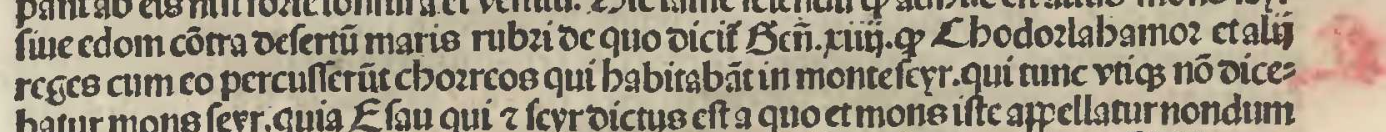
batur mons minos fratrum veftron filiozü $E$ fau qui babitantin ferr ct timebunt ros. boc pzimo

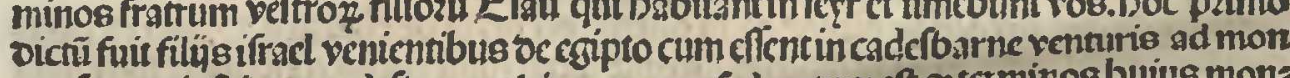

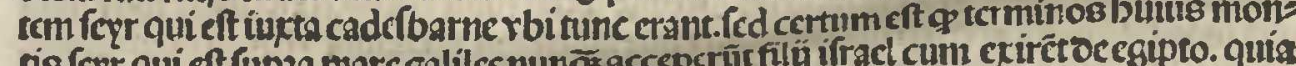

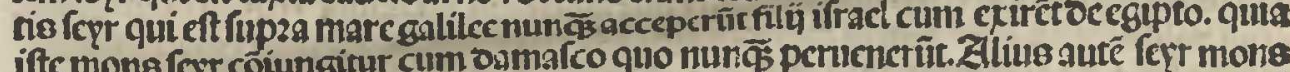
oc quoibi loquitur cóiungitur cumbeférto pharan. qué longo tre circumicrut filì if

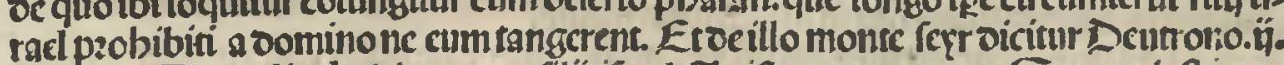

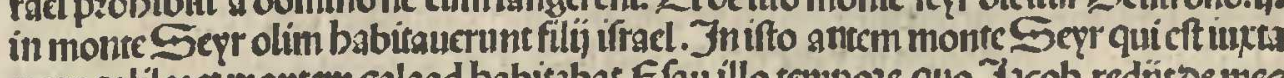
marc galikecect monten galaad babitabar Efau illo tempoze quo Jacob redít oe mes ropotamia fyric cui occulnt iutta vadum Iabotb vitoicitur Bencfis.xprip. Eftads

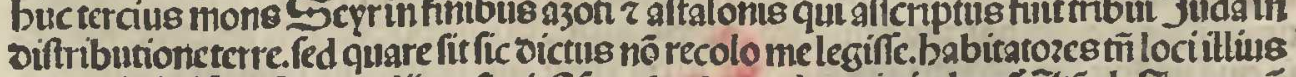

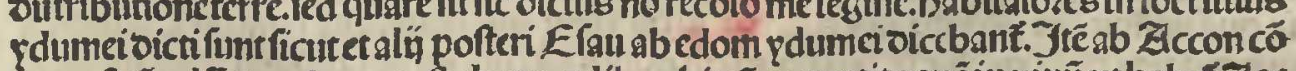

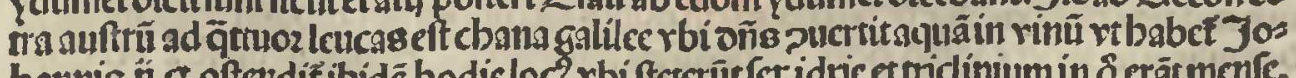
bannis.î́. Eunten beclocalifom

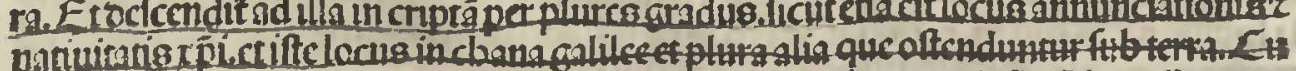

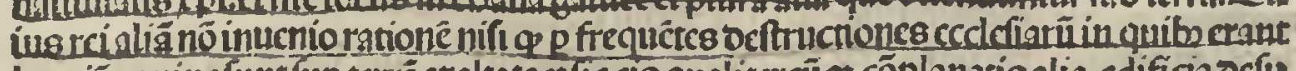

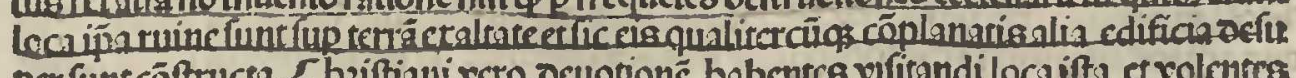

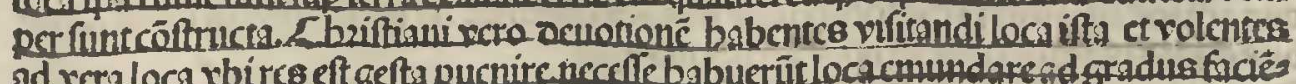

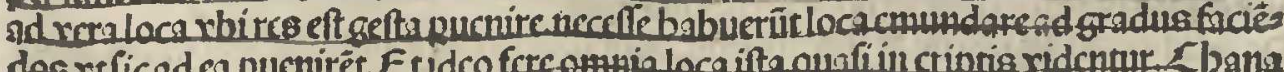

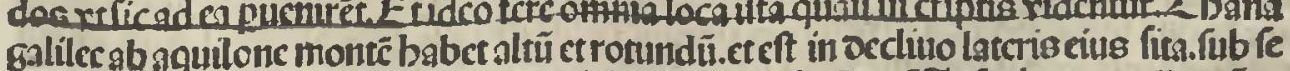

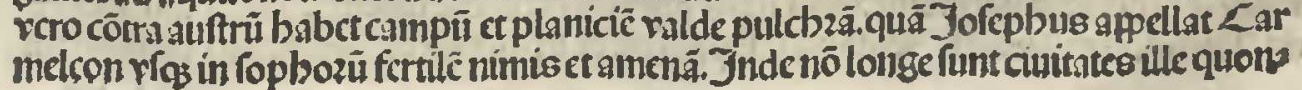

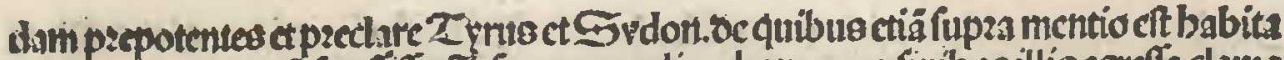

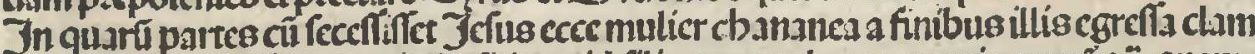

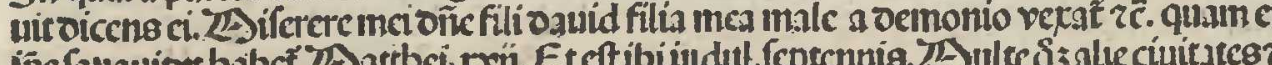

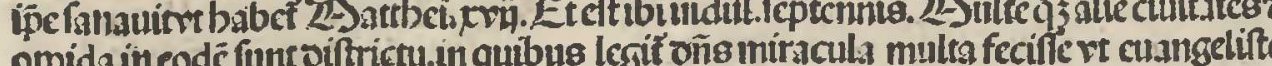
oppda in cod

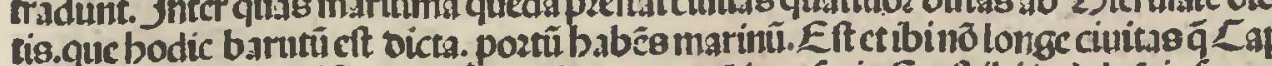

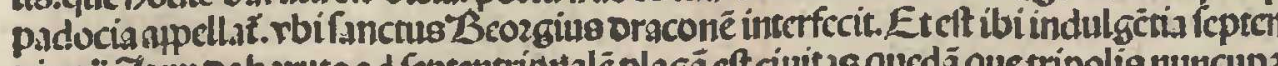

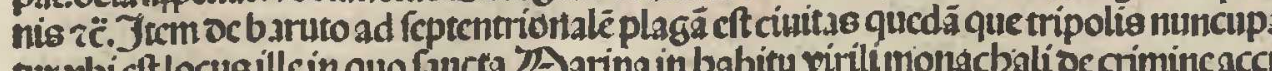
arr vbictt locusille in quo ancta 2 artha

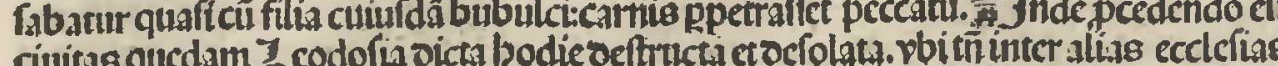

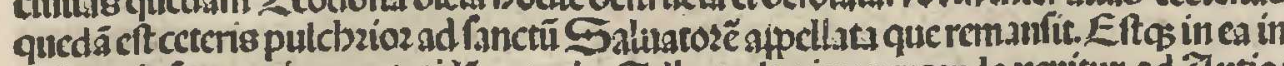
dul gentua leprennis cuin totide carentia, Adhuc viterius pergendo rentur ad Antio

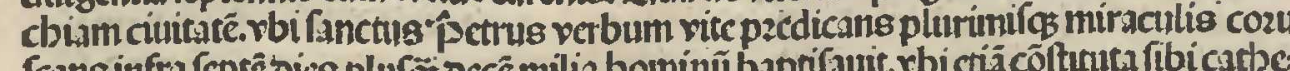

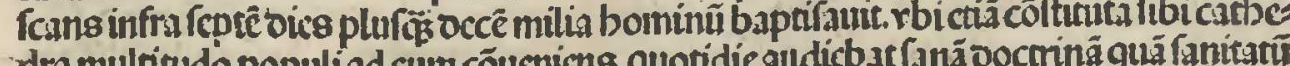

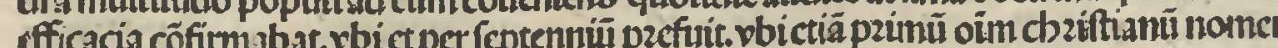

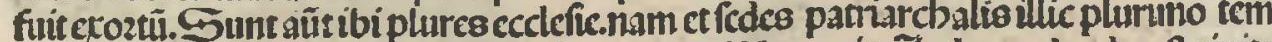

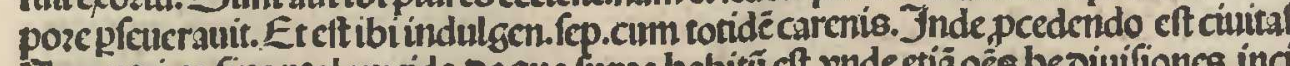

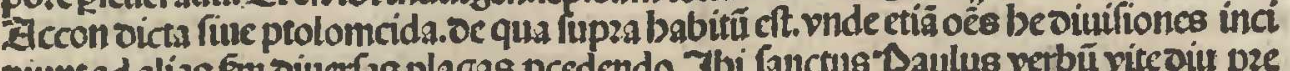

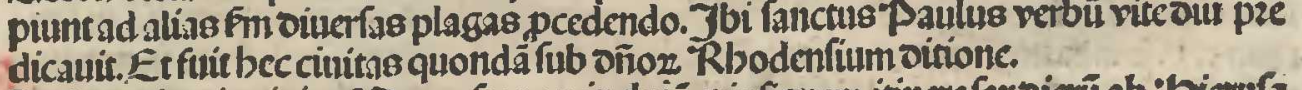

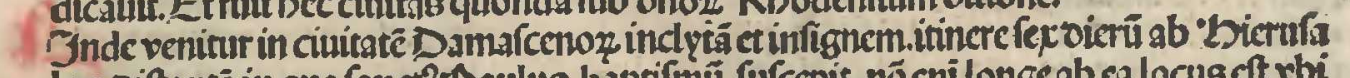

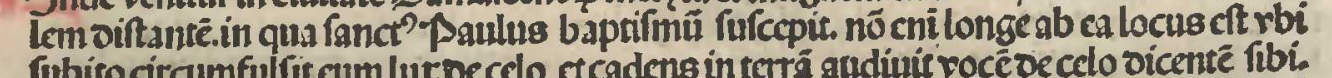

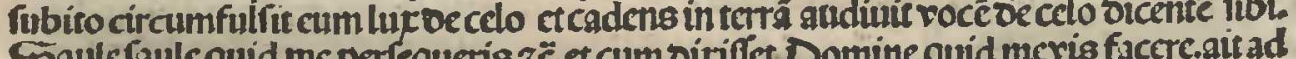

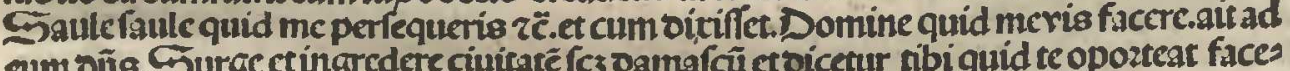
a 7 . manduciuitncos bibit Tandem poft baprifinü acceptocibo côfoztaruseft et manfit

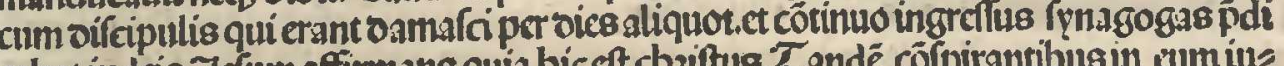

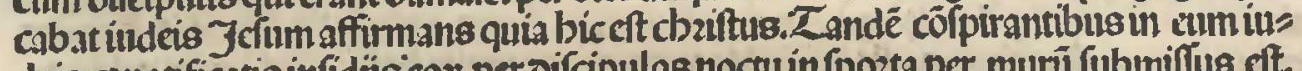

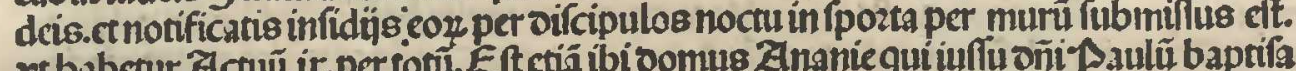
uit ct in bodienni v ros ojem locns oftenditur in muro ciuitatis. vbia fratribus in 1 po2 a fuirtoimifius querentibs cum uldeis interficerc. Et eft ibi indulgentia leptennis $\tau \varepsilon_{\text {. }}$ ficurct in loco fue conucrionis oe quo fup2a.quiad oimidiáleucam oiftara oamafco.

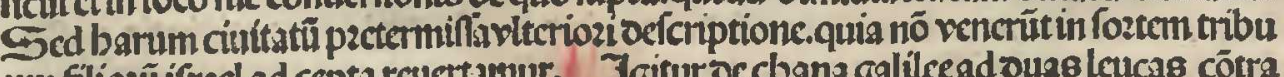

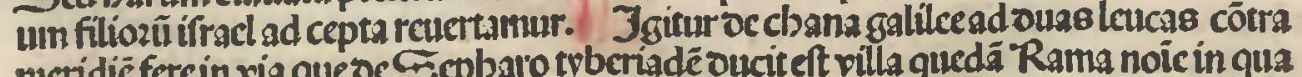
meridic fercin via que oc

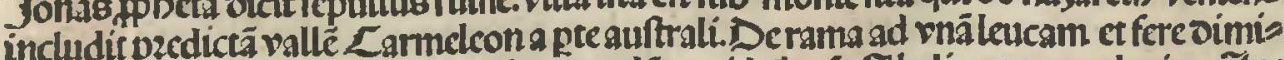

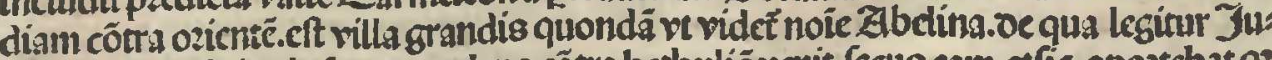

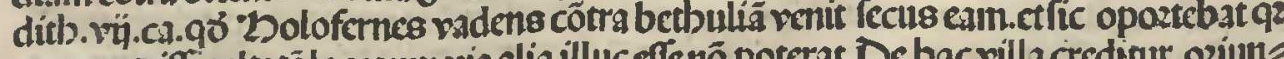

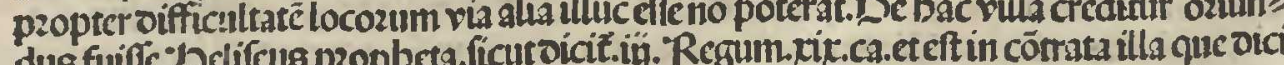

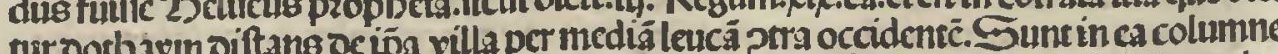
multemarmozecetruine magne que oia oftendürcea fuifite gloziofam.eteft in loco alto

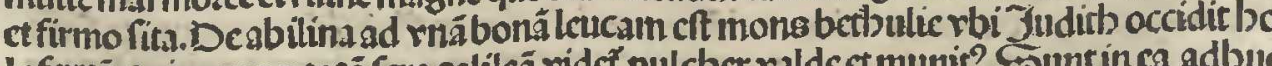

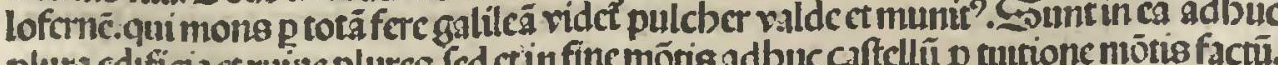

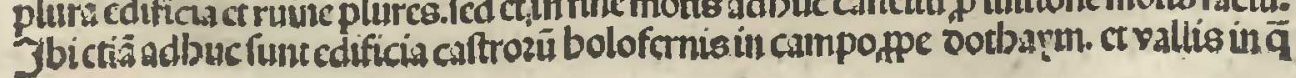

Capposionir

Tripeis:

Aimentim

1000

Phlommián

Damares

am

Alusisin 


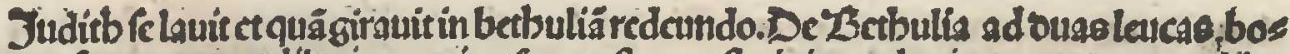
mare țbectiadisoictil aliquoticns inuenitur vit babet Jobannis.vibec antiquirtus oi

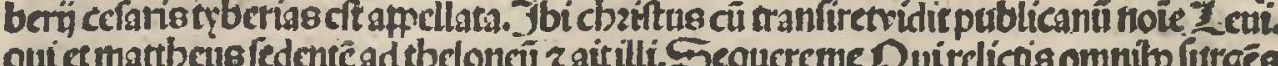

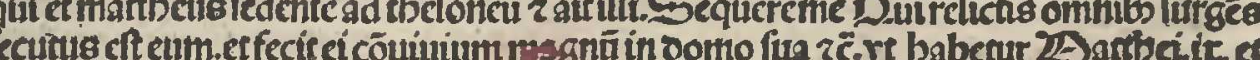

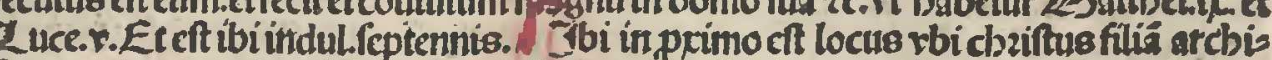
qnagogia moznhi

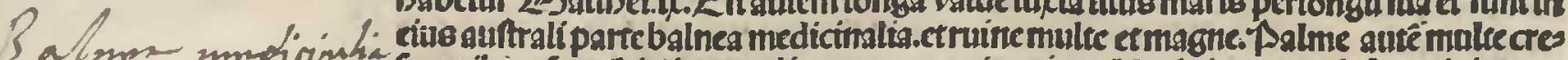

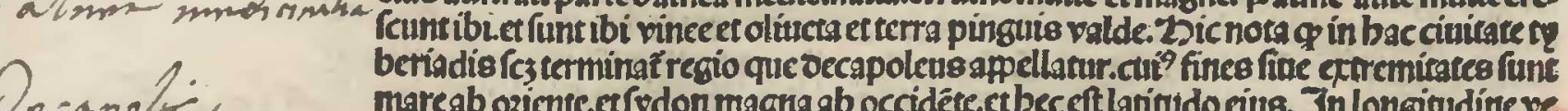

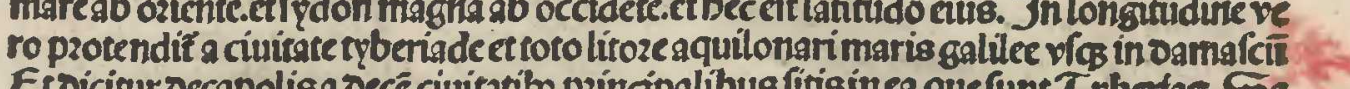

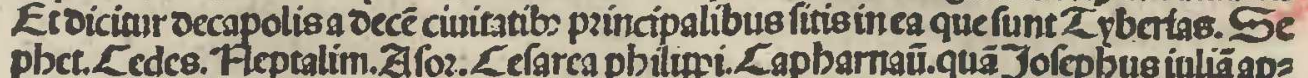

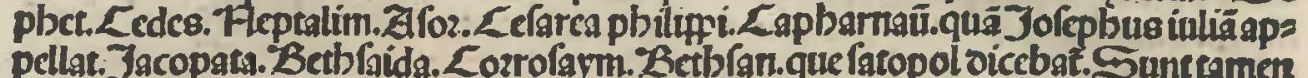

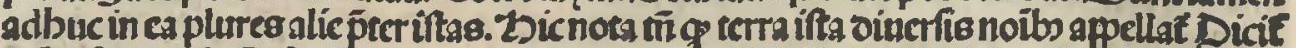

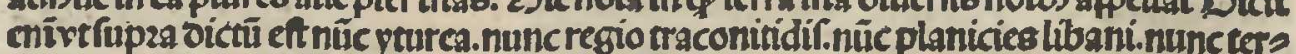
ra roob. ntrinc kabuln nunc galilea gentiü.,nunc galitlea fuperioz.eteff femper eddé regio

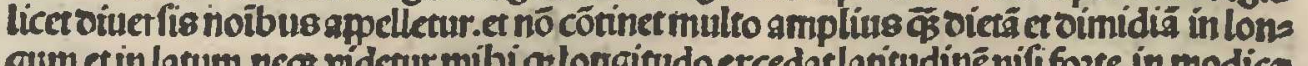

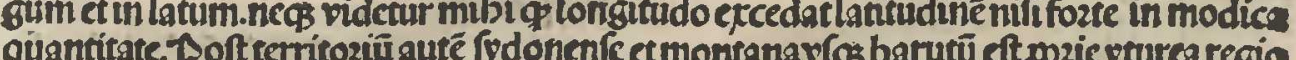

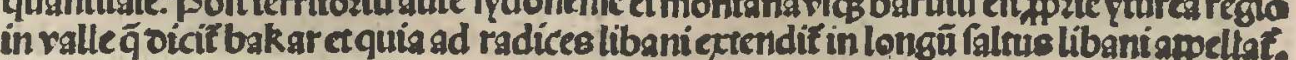

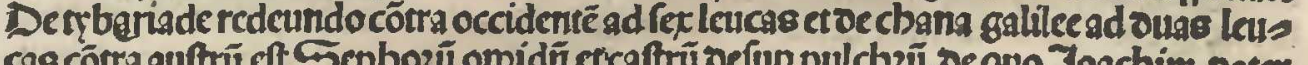

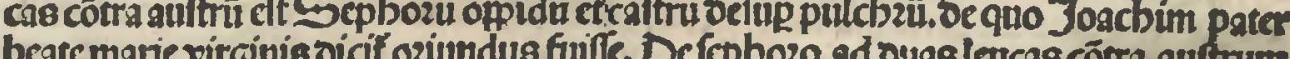

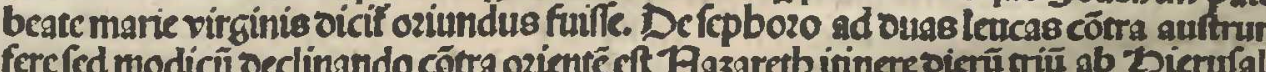

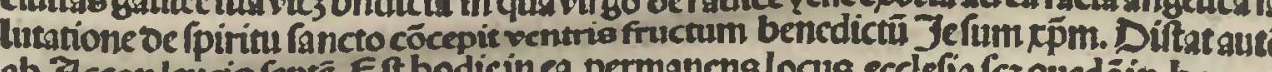

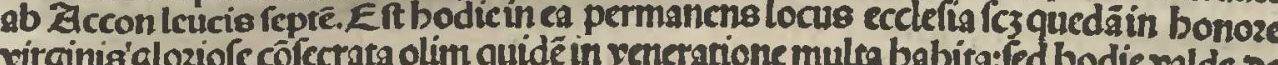

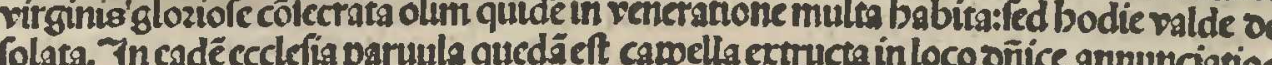
Oñs trecum benedicta tu in mulieribus.

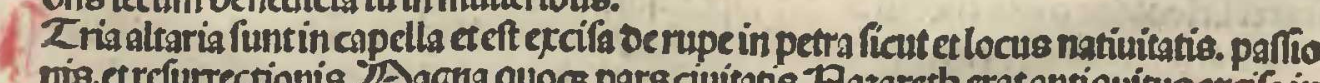
mis

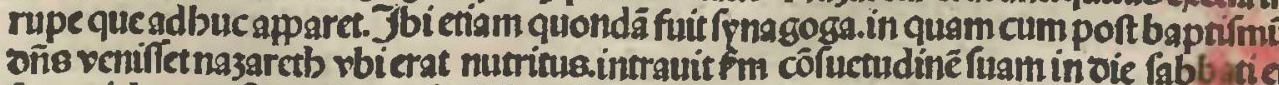

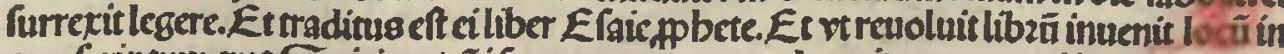

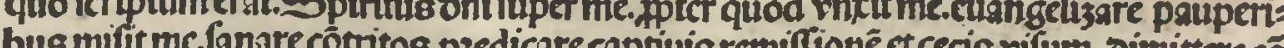

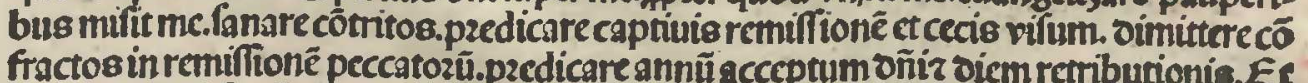
fractos in remifitione peccatozu.pzedicareannin acceptumm oñiz oicm retributionis. $E_{t}$

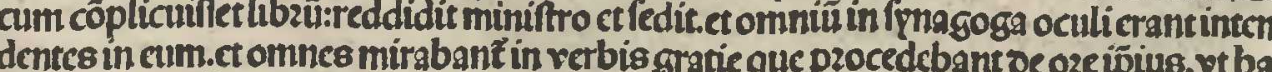
betur Y Lce.ïin.capinulo.

Eft infuper in finc cututatis vbi quondä erat ecclefia fancti Babrielisnuncupata fone

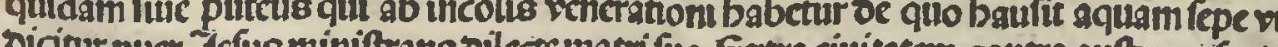
quantü quarec poreft arcus iaccere cff locus qui vocet faltus oomini in monte. in cuive

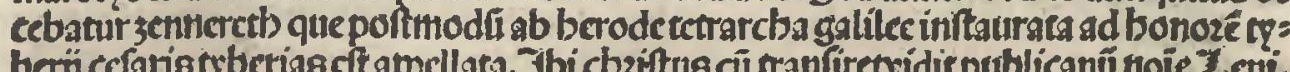
nis.rbictiä bodie marnmozea quedă manet columna cui virgo î̃a fuits apodianta qü doangelus gabziel miffive a oce fíbiattulitnuncium falutare oicens. Eure gratia plem

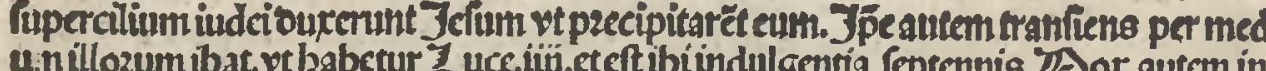

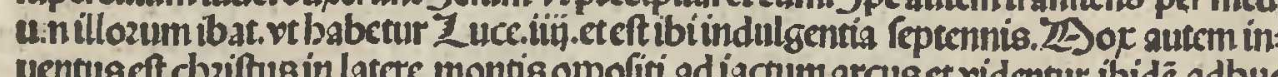
uentuseff cbziftus in latcere montis oppofiti ad Laccumt arcusest videntur ibidé adbuc

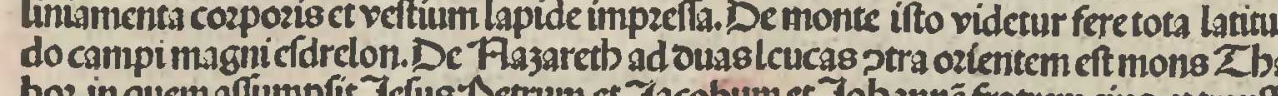

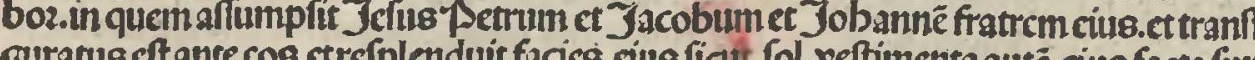

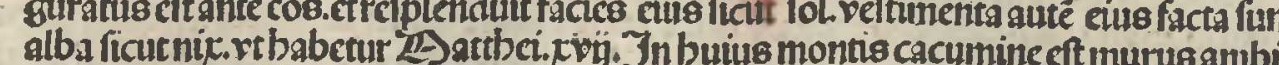
cns locum trantfigurationis dominice. Intra quem eft oztus arbozibue côfitus et fon

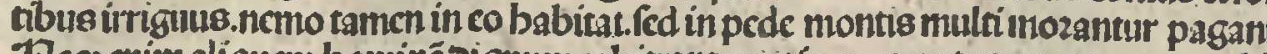
Heqs chimt aliquem bointine oignum arbitrantur:ert in eo monte trancat quem vald peccatozum remifio.
. Jbiectium bodic oftenduntur ruinetrium tabethaculozü fine clauftrozum fm ocfideri

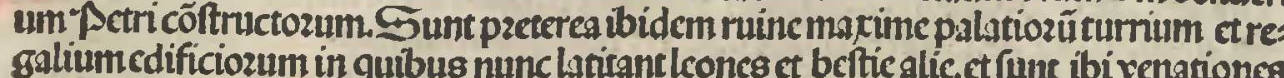

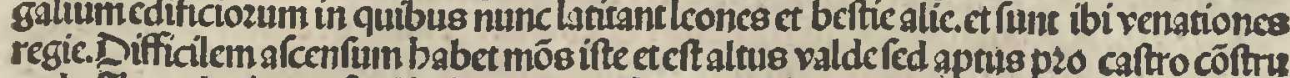

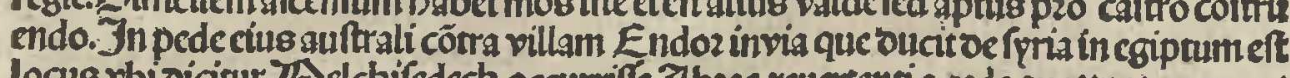

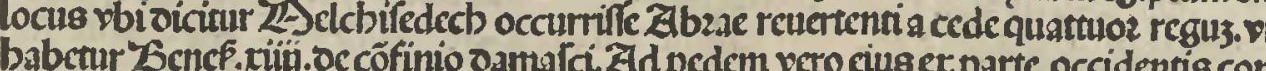

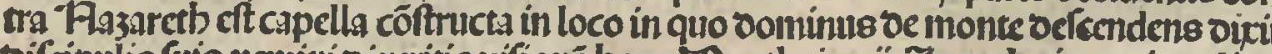

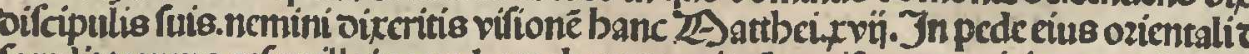

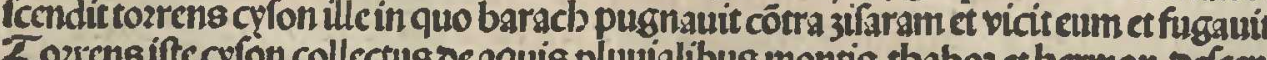

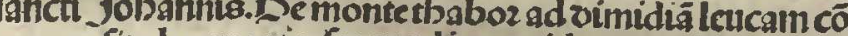
monte fít bermonio $\mathrm{f}_{3}$ modico.qui bermoniü non eft mons per fe. fed quafi tunnor

Etinco eff villa Endor fita.oe qua oicitur in p8.Díperieruntinendor. Jn bac villa

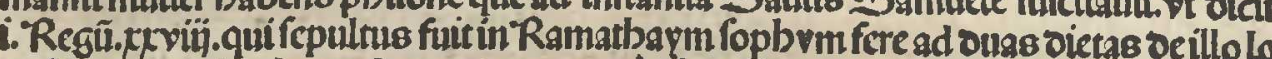
co. De nazareth ad outas leucs se monte thaboz plus vna.cötra auftrum eft bermon

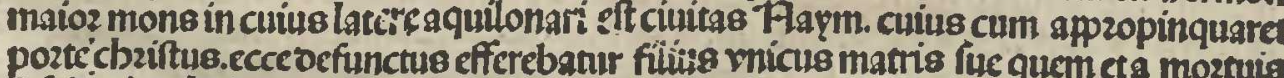

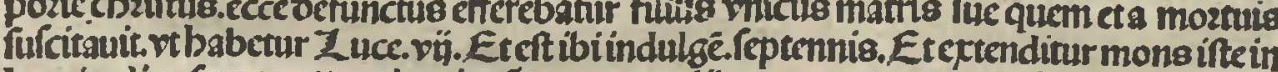
longitudine fere quattuoz leucis cörra mare galilce.et terminatur non longea loco ifto vbiiozdanis flunius cgreditur oc marigalitec.

1. Zerumab Áccon cōtra notumn occurret pzima pars mötis carmeli ad quat tuoz leucas oc accon bbilocus clt in quo belias ppecta occidit faccrdotes

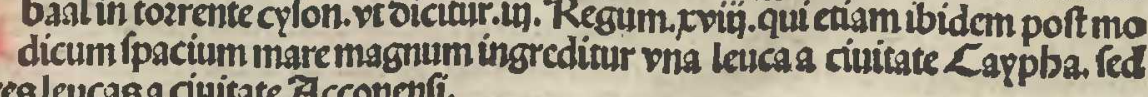
Seread tres lencasa cinitite A Acconenfi.

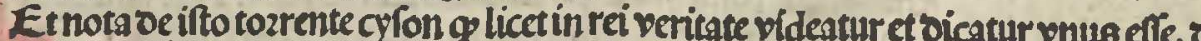

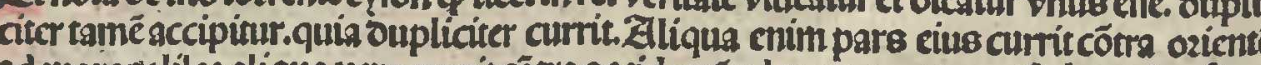

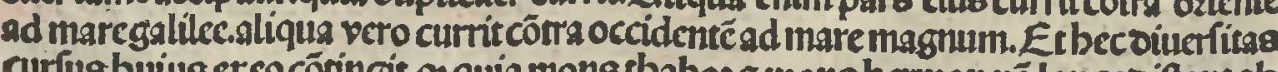

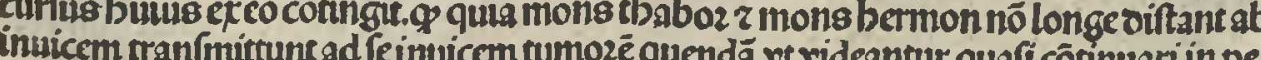
demontis vmifor

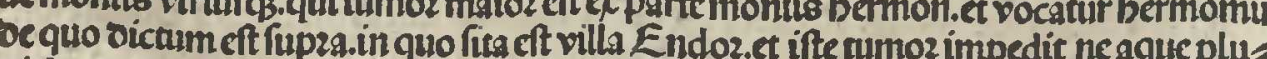

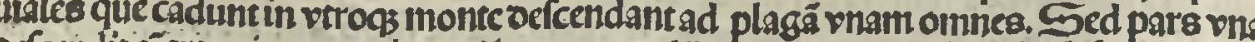

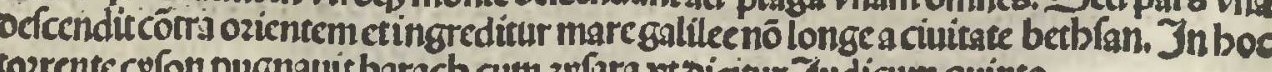

Thabok

Nous $2 m$

Rngan Truation

Enaín
Y arm

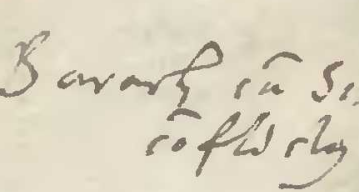




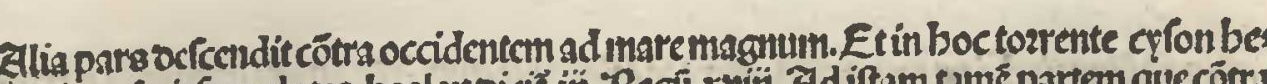

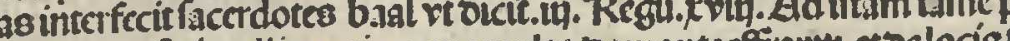

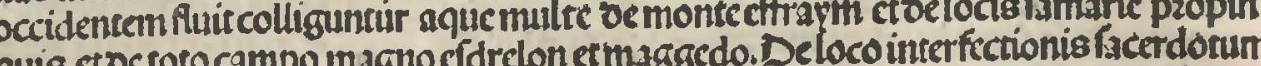
action

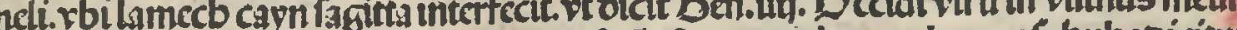

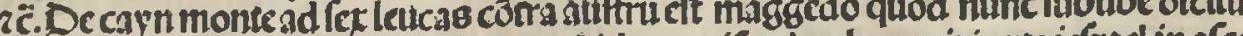

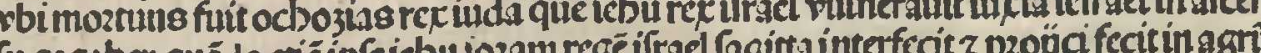

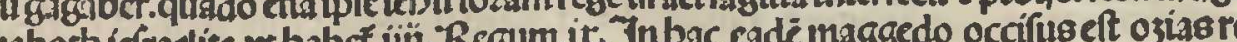
nabo

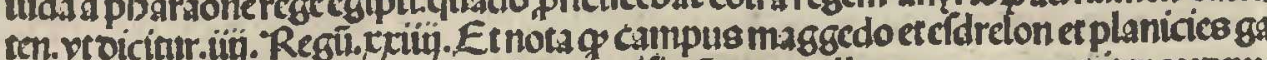

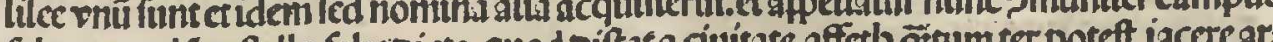

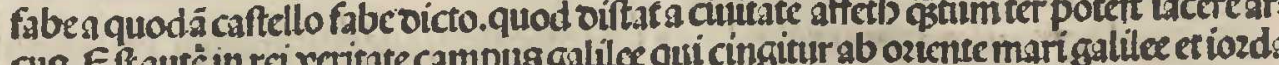

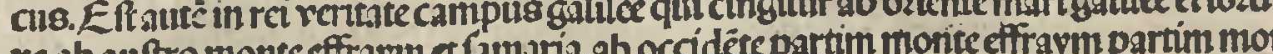
nc.sb auth to rudine oecé leucus ferc in Latitudinc fer vel amplius. in partibus aliquibus fertilis fur pza modú. in fruméto vino ct oleo.ct abundans ommibus bontis mundi. ita $q$ videtur

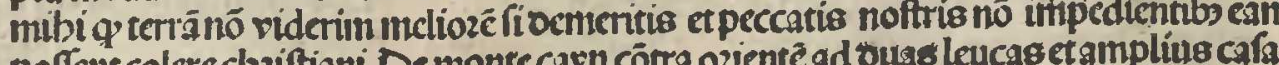

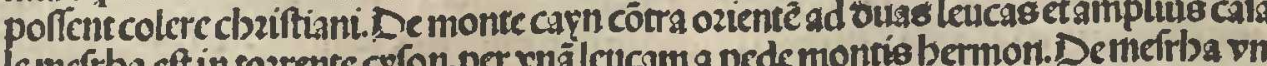

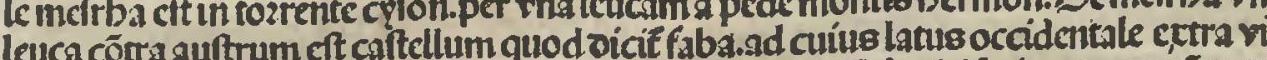

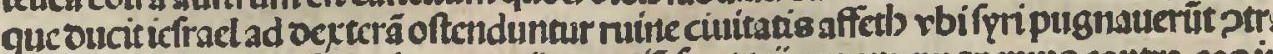

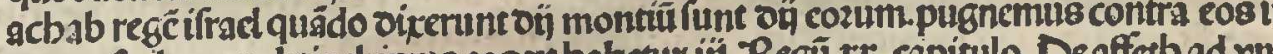

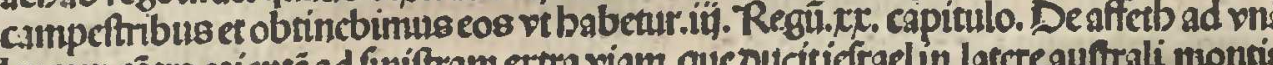

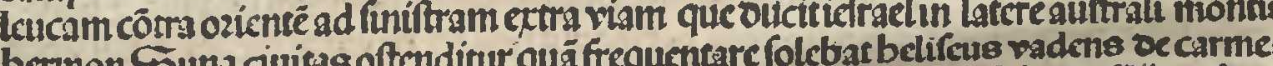

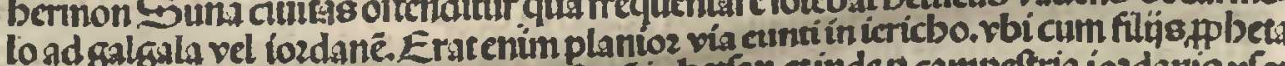
runmozabat vadens vecarmelo per finna in betran. opoztcbateum tran

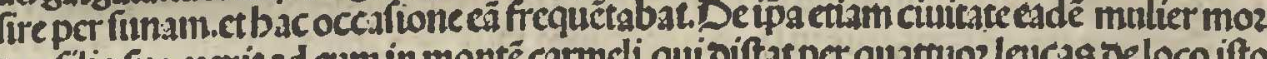

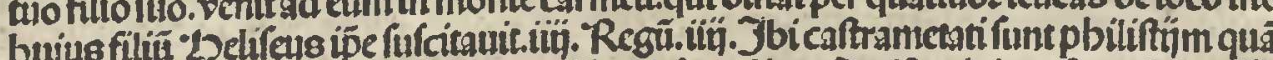

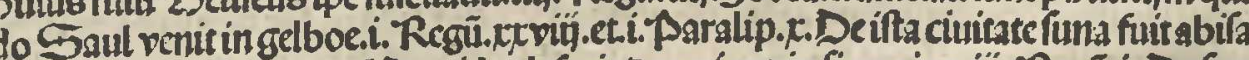

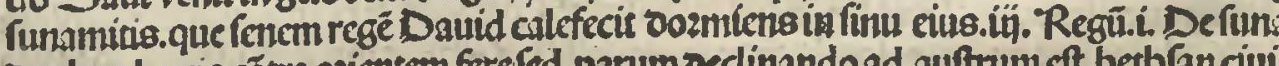

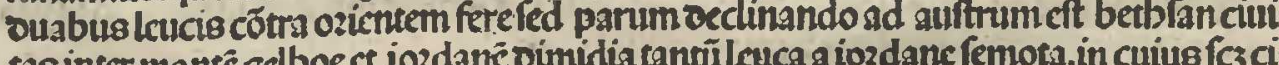

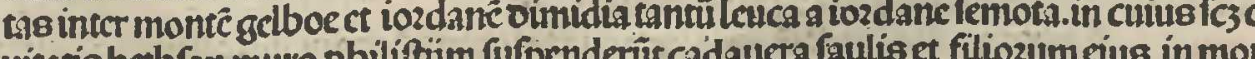

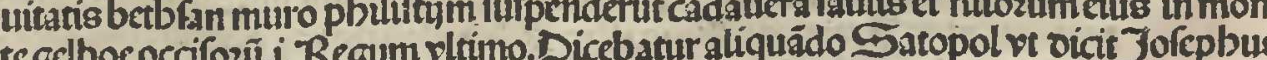
red nunc sb omnibus betbfan appellasur.ct eff locus oelicanus valde. Supza bancc

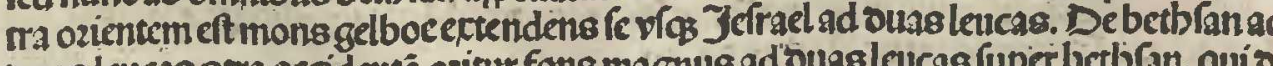
oulas leucas ofra occidente. ozitur fons magnus ad ouas lencas fuper betbifan. quio citur fons

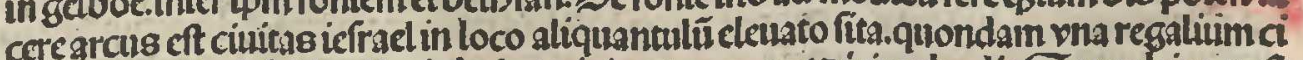

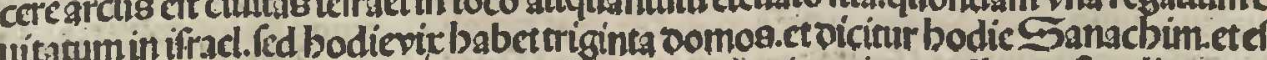
in pede montis getboe ad occidentem iua.antectulus introtum adbuc oftenditur ager .

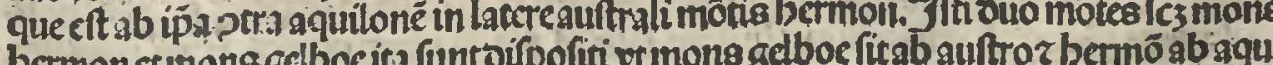

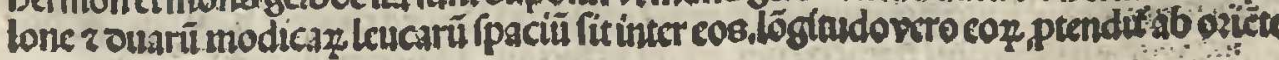

in occidentem.eterminantur amboin iordunelongiouabus leucis vel plus. In plant

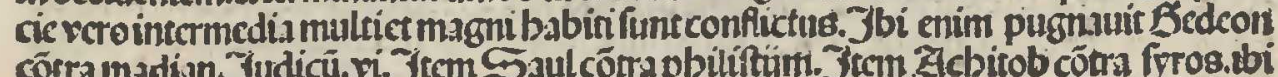

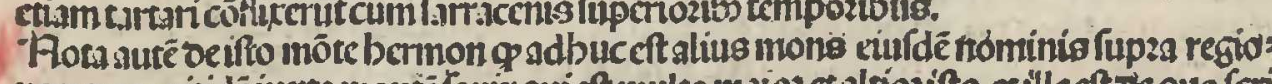

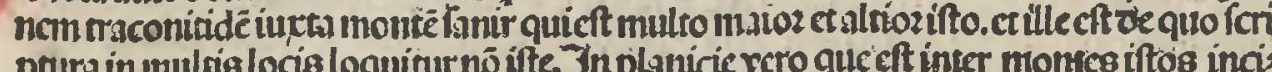

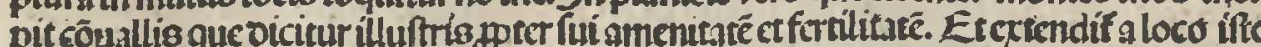

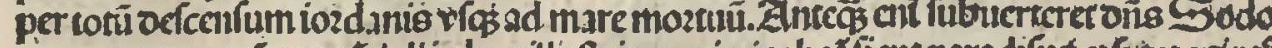

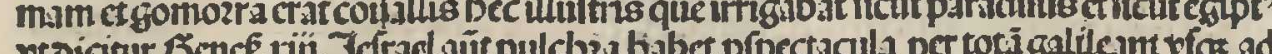

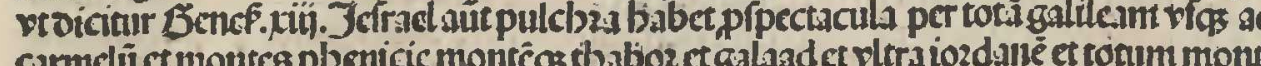

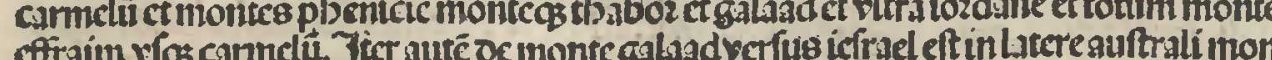

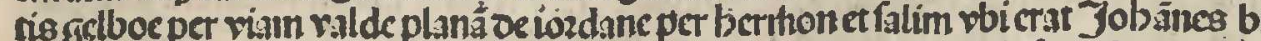

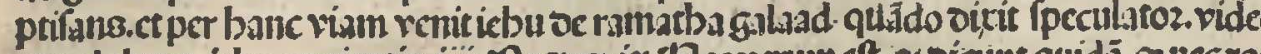

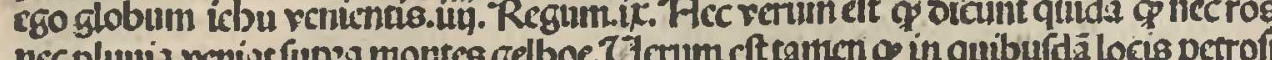

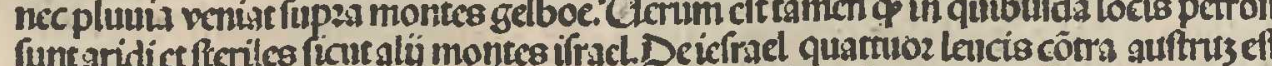
(1)

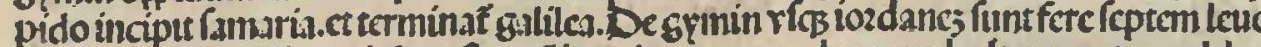

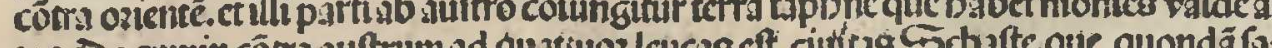

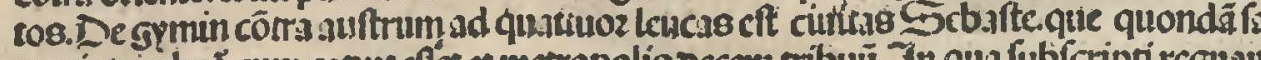

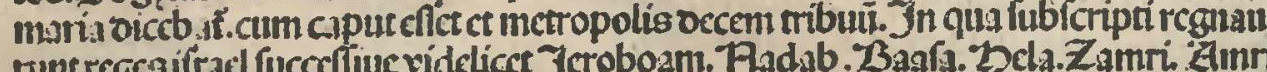

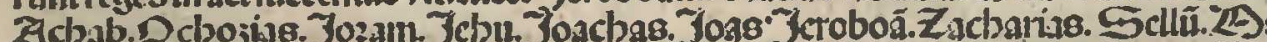

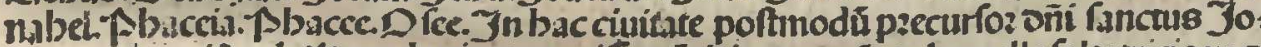

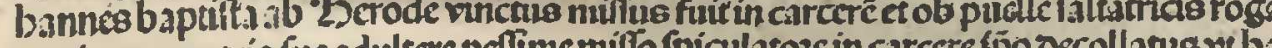

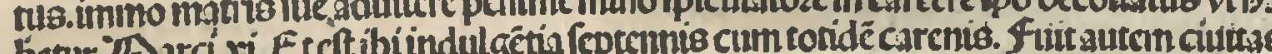
betur

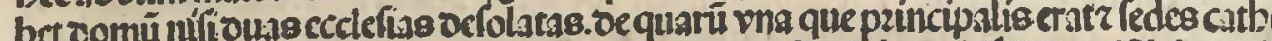

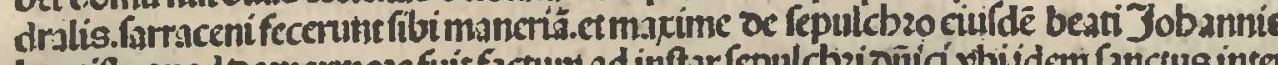

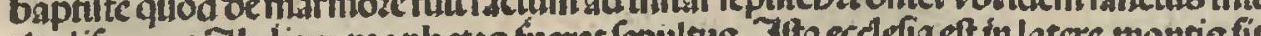

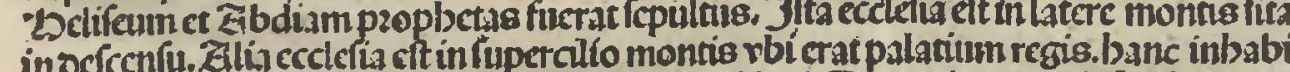
inocccru.

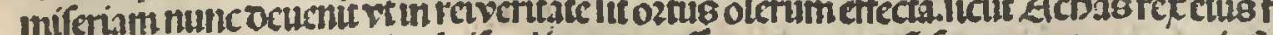

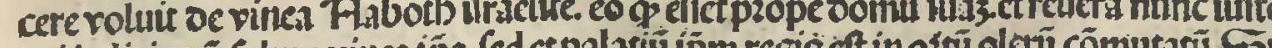
acitidictio no folum

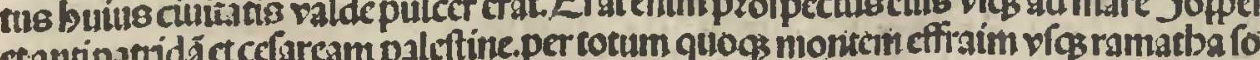

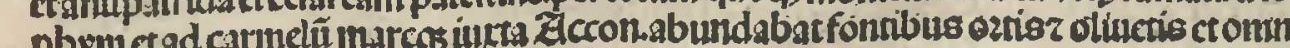
busbonismundifftius.

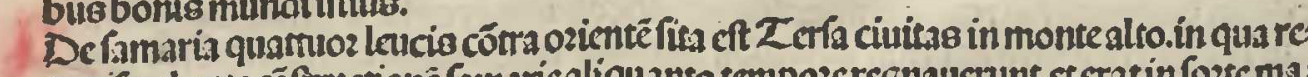

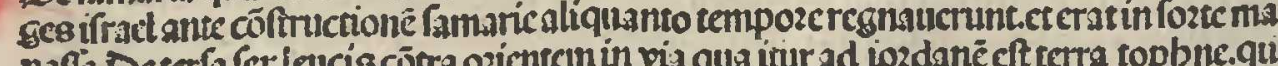
ind

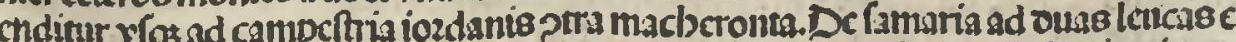

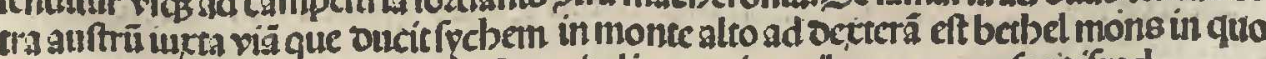

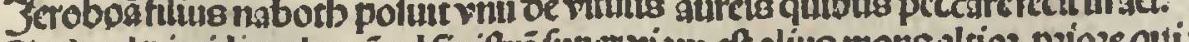

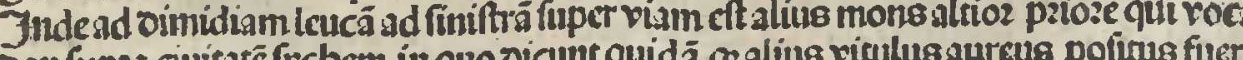

Salim

Crymin

$5 x^{2}+a^{2} y^{2}$ crabibir

Aremo. 
C

Piliopi:

X ypolore

Fonstare.

Trarism

Lmon

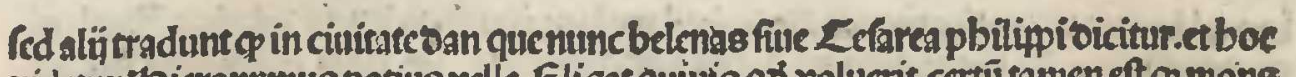

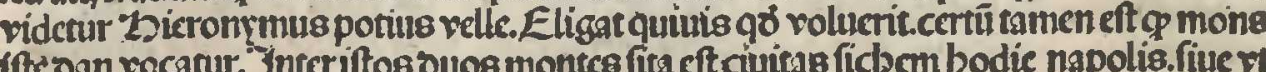

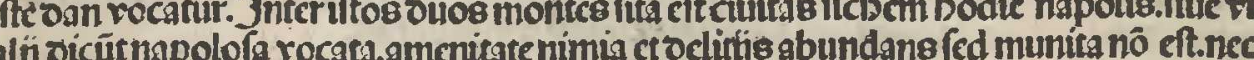

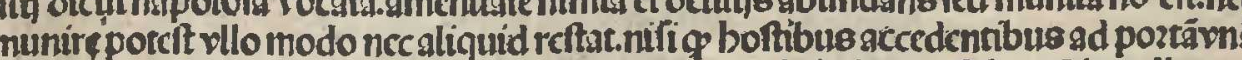
babitanozcs fugiant per gliâfif fuctint cis pauciozes ct inferiozce. Eft auré in valle me

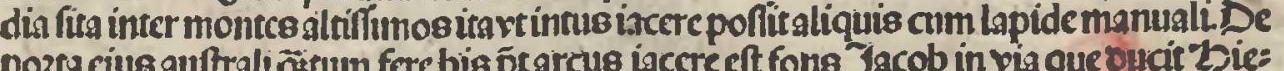

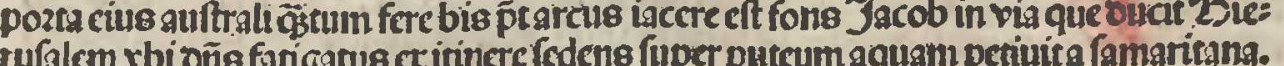
(a) Garifim.aliud cbal apellstur. In monte garifime dificato alterri Jofuc ferip pit Deutro

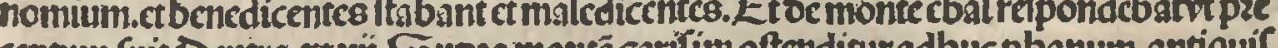

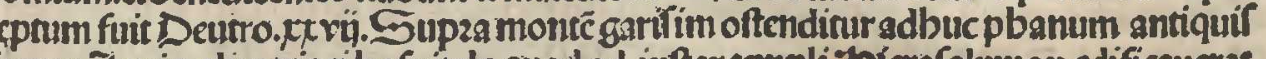

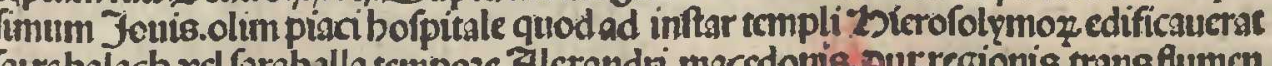

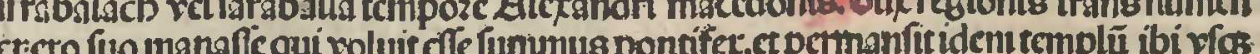

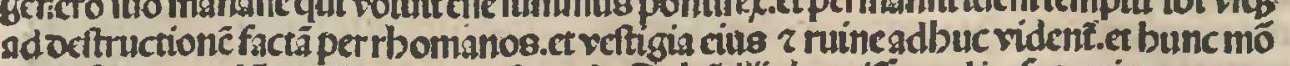

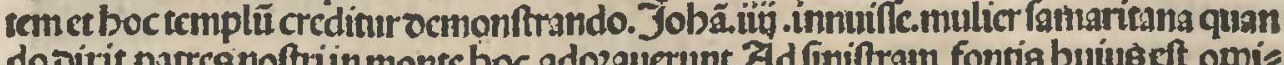
do oixtit.partresnoltrinmonte boc adozauctunt. Ad fimitham fontis biulustt oppis

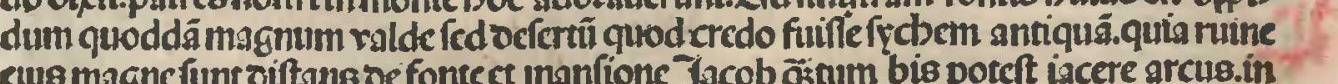

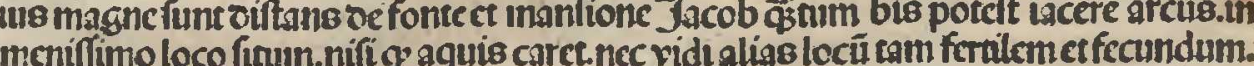

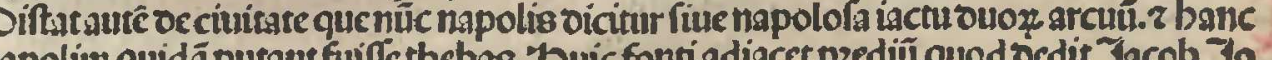

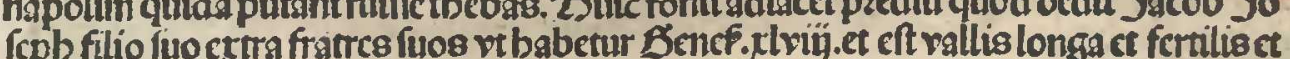

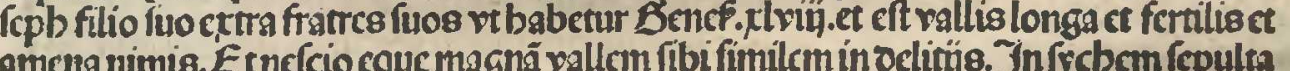
fimt ofla Jolcpb allara illncoecgipro.

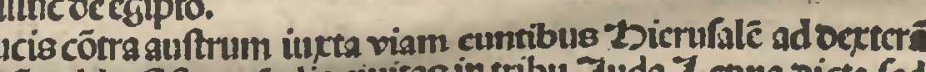

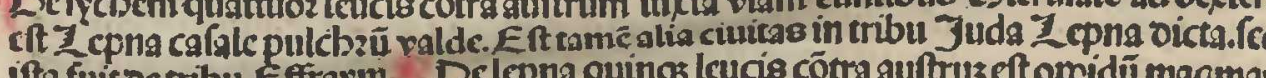

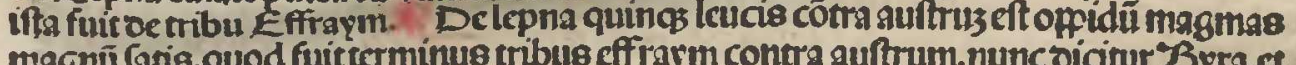

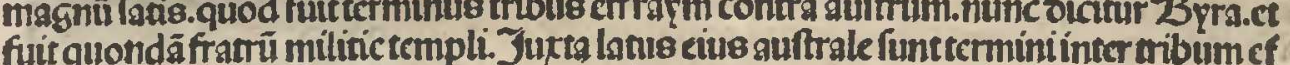
fracm et beniamm. Demagmas cotra suttrumad via leucamet gabag Saulis.rb

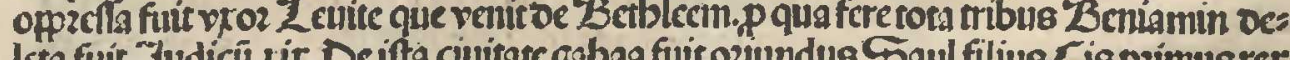

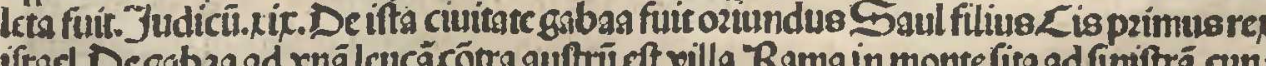

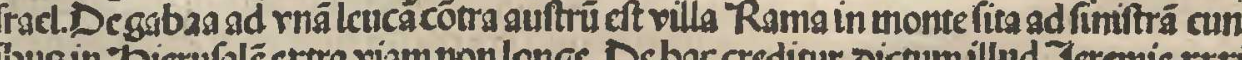

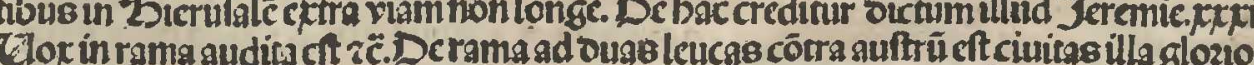

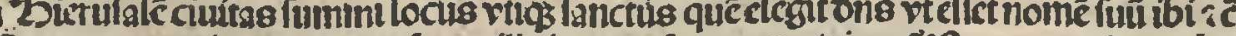

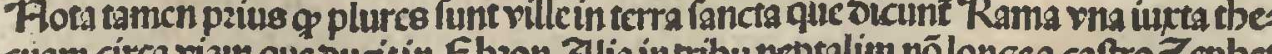
cuam circar ram que ouctin Eb20n. Alla in trbu neptalin no longea caftro Zepbet

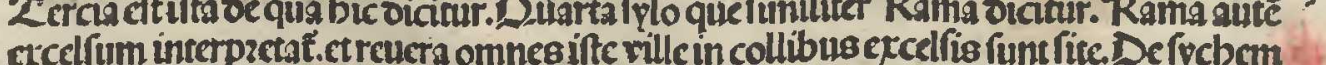
igitur pzocedendo 2 tra auftrum ad ioz dancm pzimo ad quattuoz leucas eft Emon op pidun raldebonum in ameno loco firtum sabundans quondá omnibus bonis. Et fu

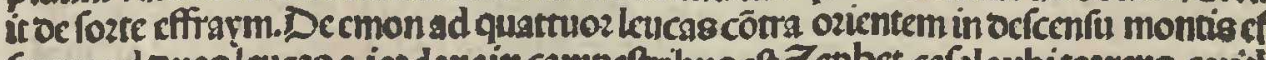
fraymad ouas leucasa iordancincampetribuseft Zepbet cafale rbitorrena canth cibummane cr vefpere. Dezephet ad vnălcucäadfinifträrectus terä topbne écaftrũ Docb in quo ptolome?

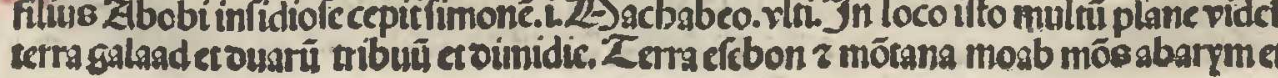

alga etnebo.ctocfenditur bicin campeftria Jozdanio.ct funtlocaplanaw fos in $3 e$

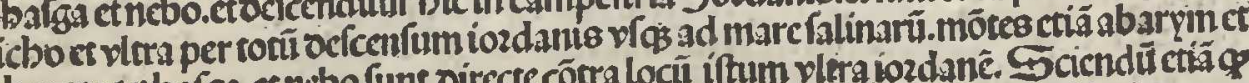

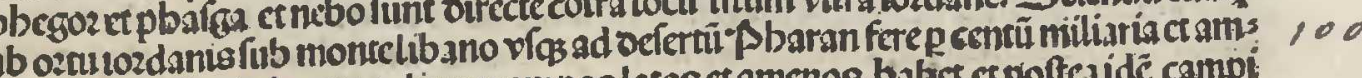

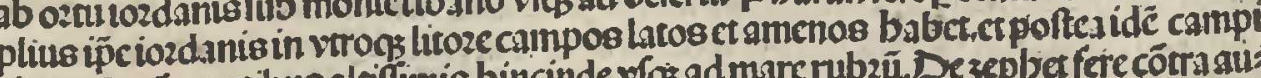

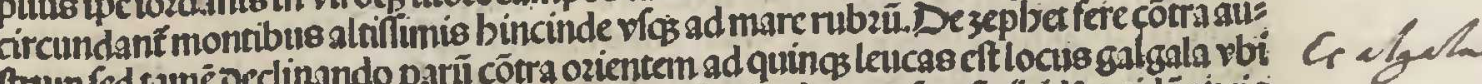

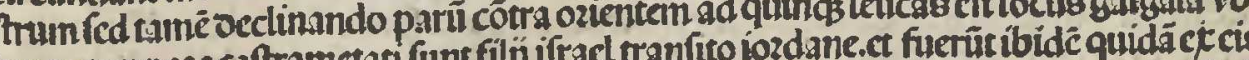
corgo tempozecter

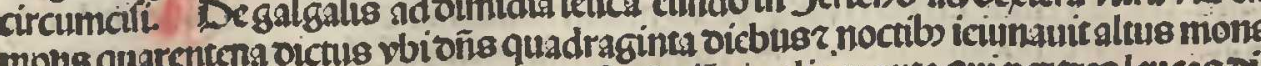
etoifficilis ad alcendendü. Fed tentatus eft on s ipe in alio monte.qui per tres leucas o

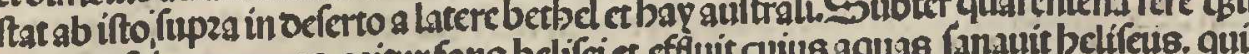
bis potect incerearcus. ozihf amarecrant

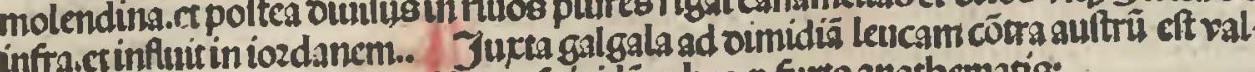

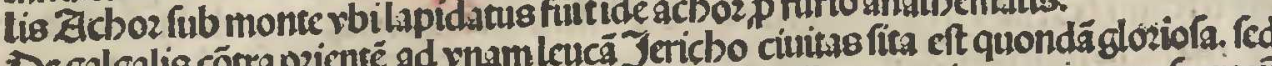

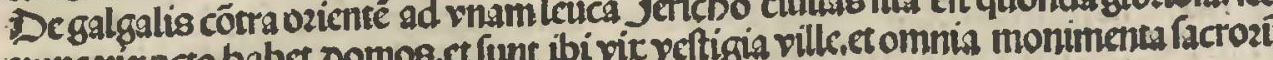

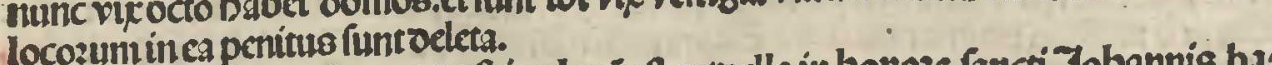

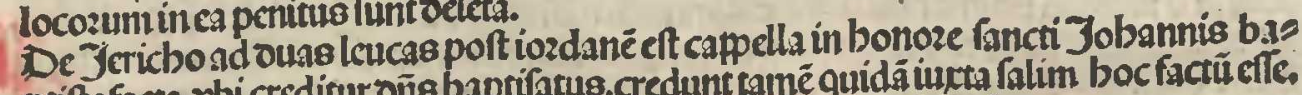

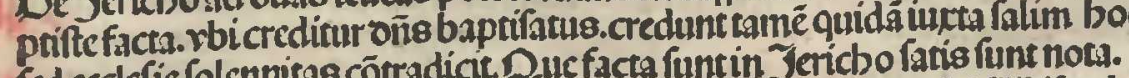

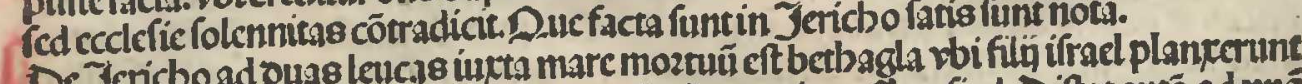
De Jericboad ouas lenc.1s iutta marc moztuin cet betbagla rbi filn itrael plankertun patré fuum hacob moztull quado cunt malerut oe gipto Denctis.

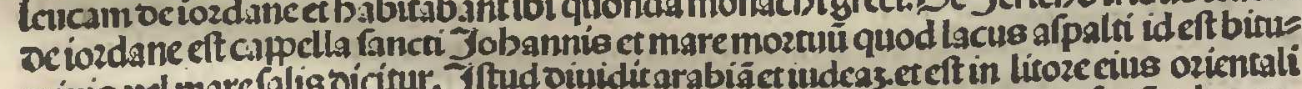

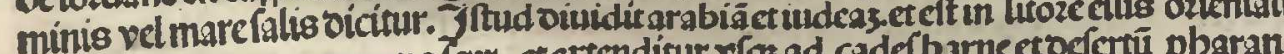

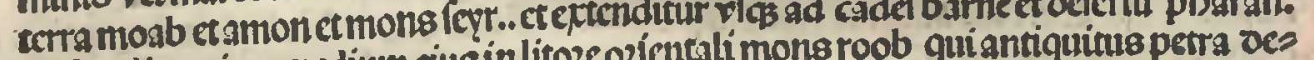
Dftendirur circa medium cuss in litoze os

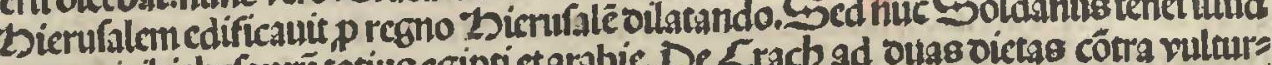
etreponit bithefaurnt

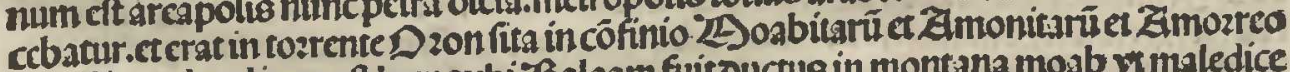
rum. In codcin litozcenton

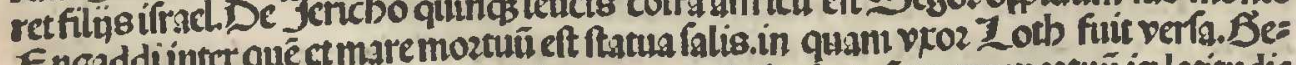

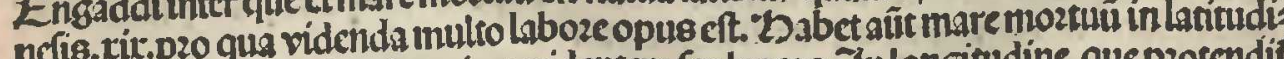

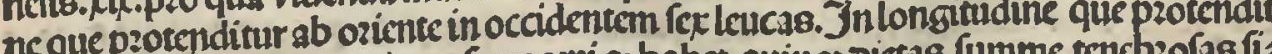

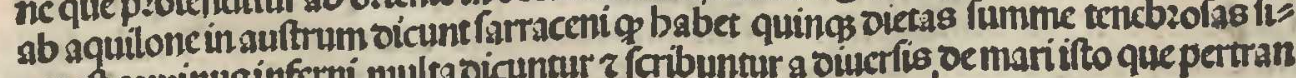
curcit caminusinfermi multas oicun (co tanös multris nota.

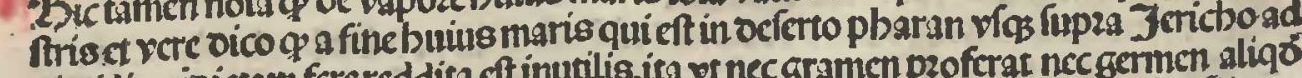
oimidiam; oictam ferereddita eft tinuthlis.ita ve nec gramen proferat nec germen aliqo

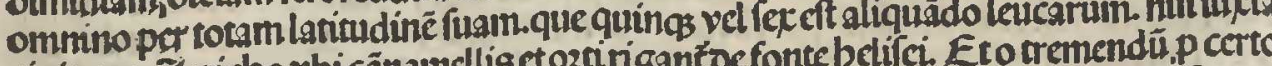

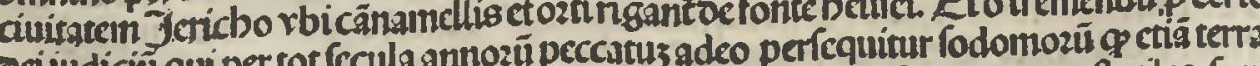

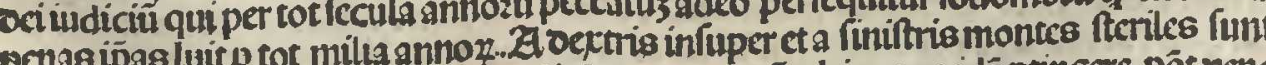

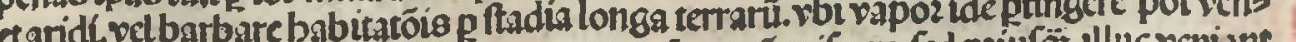

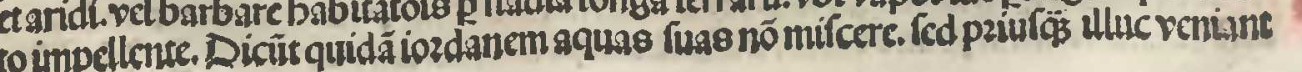

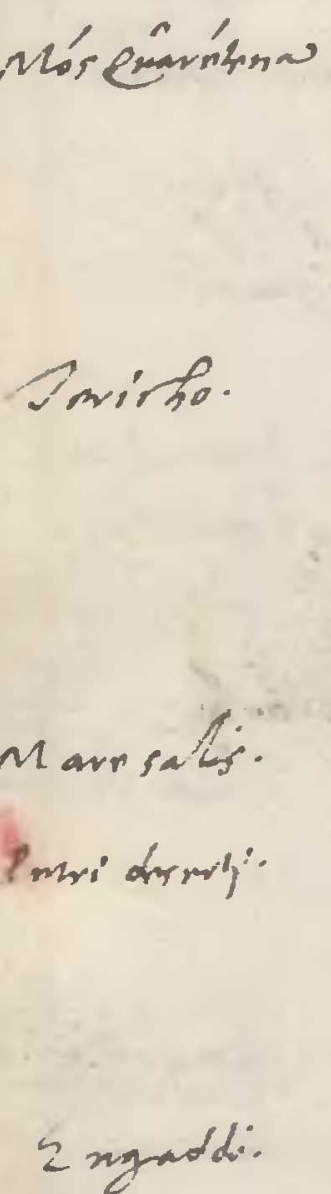




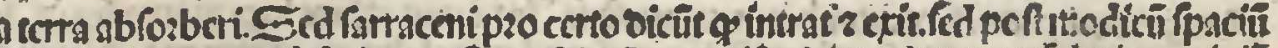

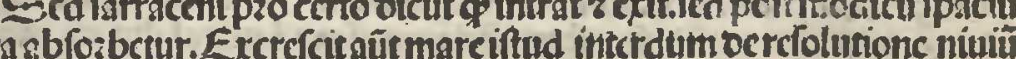

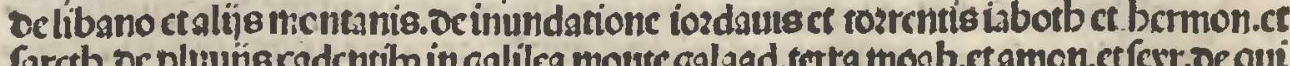

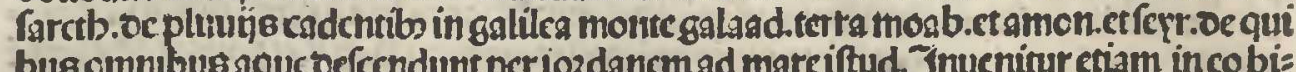

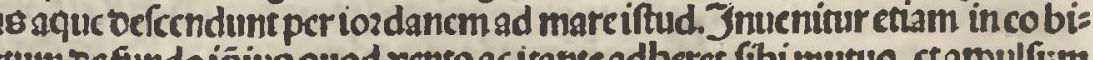

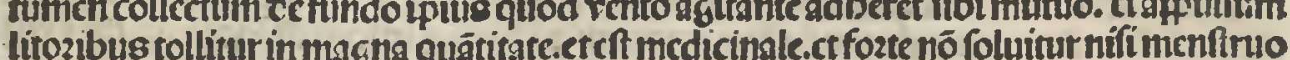

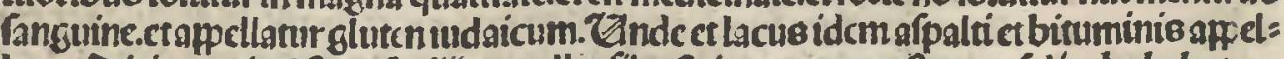

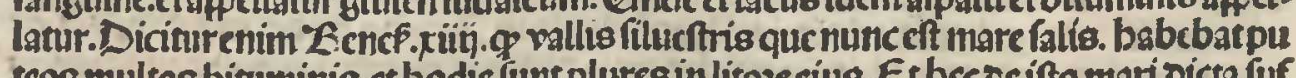

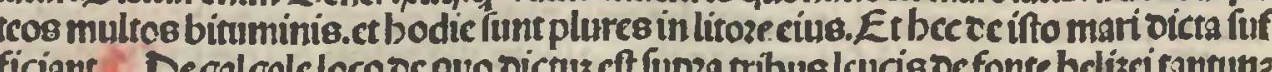

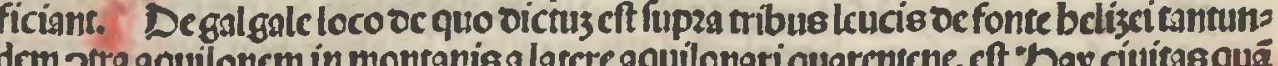

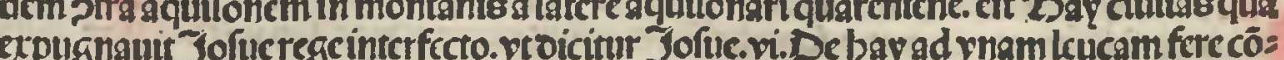

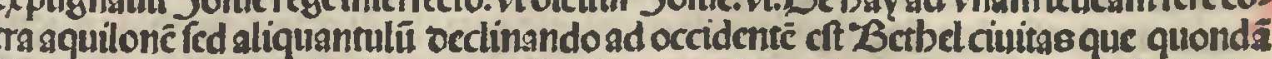

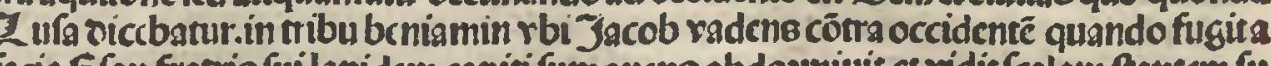

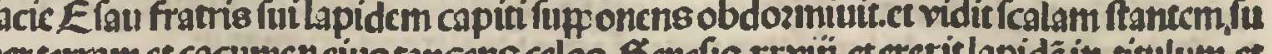

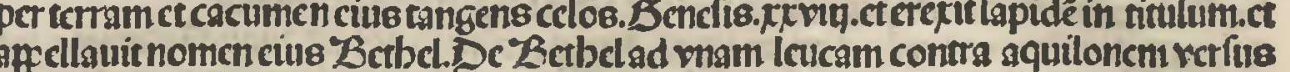

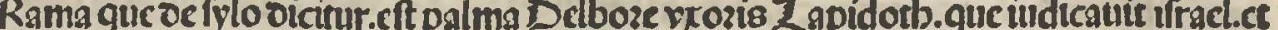
Earacb mifít å pugnandî́ côtra Z izaram in monte $Z$ babor. Judicî.iiì. De betbel

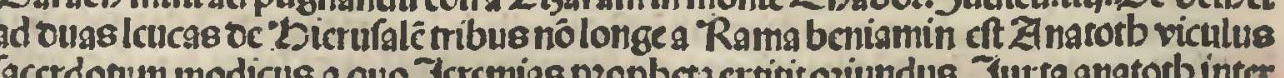

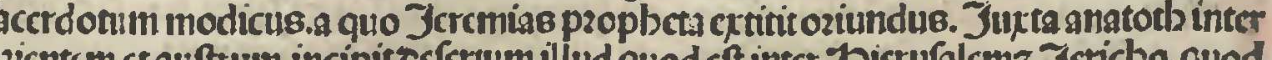

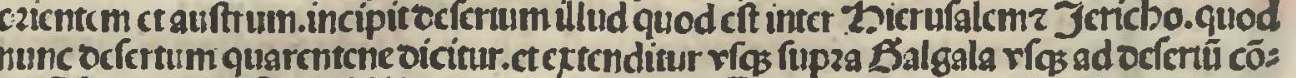

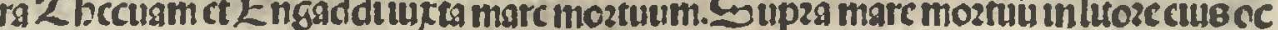
idcntali ioc Srgoz ad rnam leucam of afcenfus montis Engaddi in quo aliquando inurnitur Dautd laturfecum Saul pertequerct cum. On circumtu montis illinget in

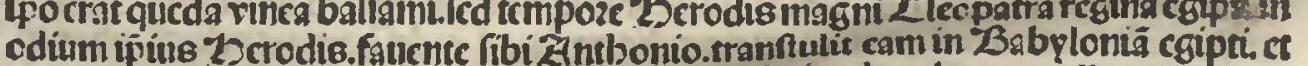
culrozes balfami Dicunt a meridic fabbari vigs ad Dicm lune boucs nullo mö trabere

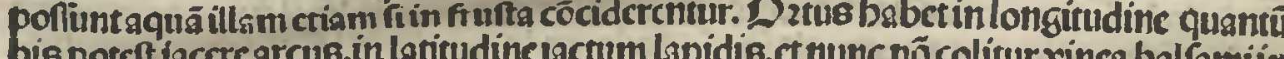

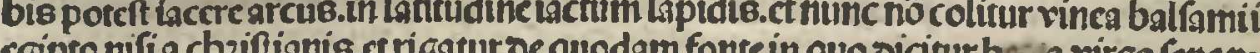

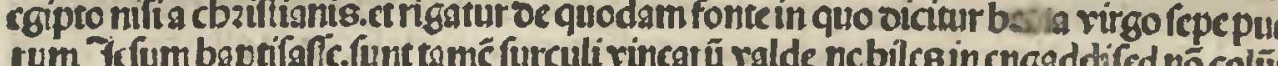

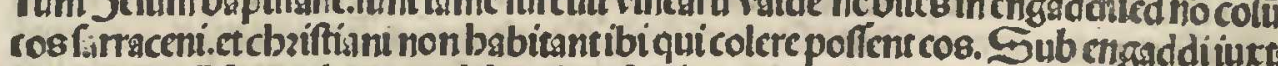

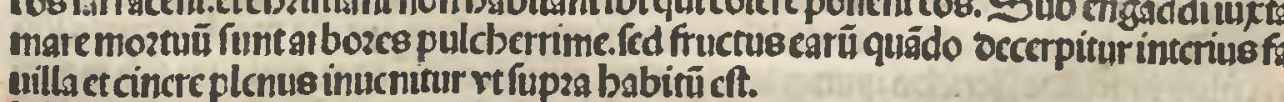
nillact cincreplonus inucntur rtfup 2 a babirū ct.

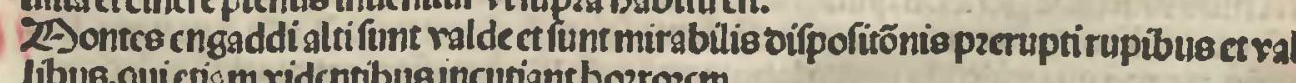
libus.qui cris midcidcntibus incutiant borroscm.

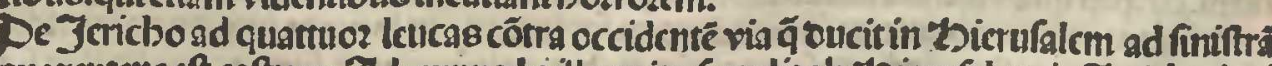
quarentene eft caftrum Zadominn rbi ille quiockendit ab 2Dicrufalcm in Jericbo inc

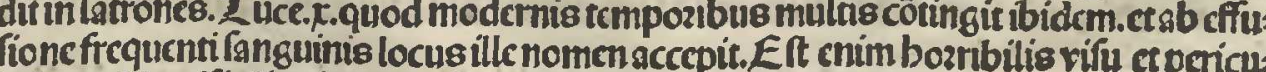
lofiz palde.nifíaliquis padar fub cóductu.

Aóomyn

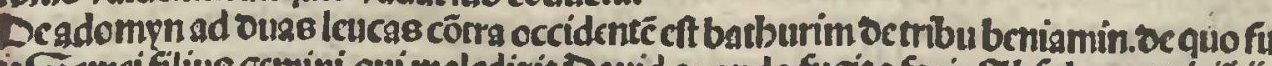

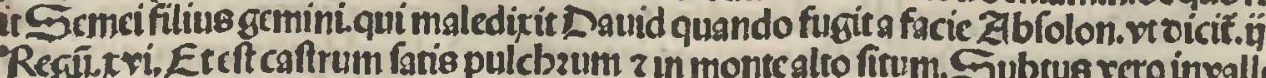

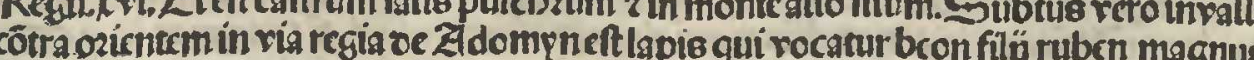
in modí clibanict videter ce man moz.De bathun

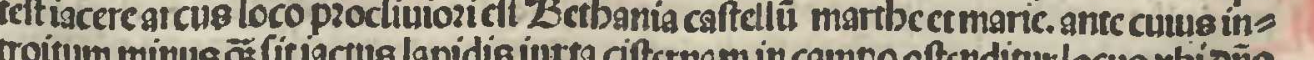

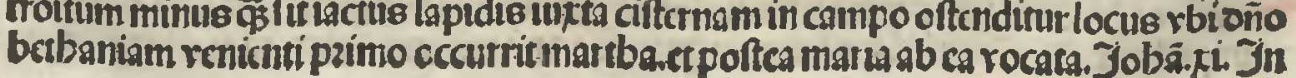

betbania adbuc oftédifur bomns Sq̣monis lepzofi.in qua recubuit bominus Ycfus quädo maria magdaleng venitad cum affercng alabaftrum vinguenticet ftans retro le: cus pedcs cius lacbrymis cepitrigare pedcecius.z capullis captis fuitergebatct orcu

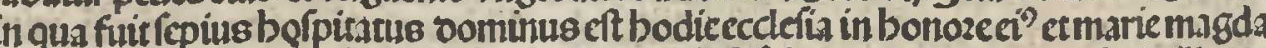

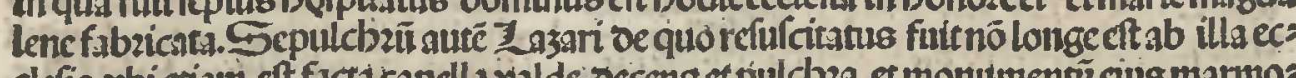

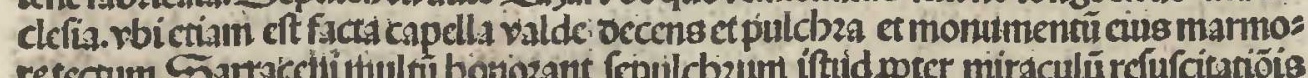

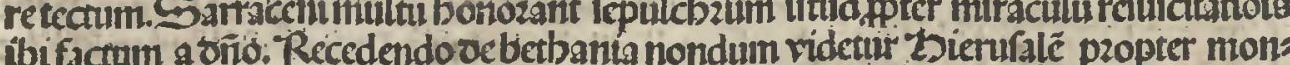

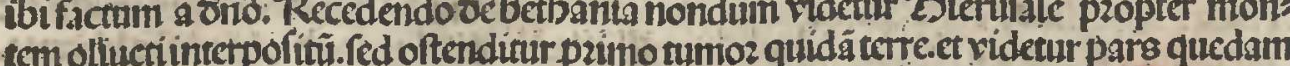

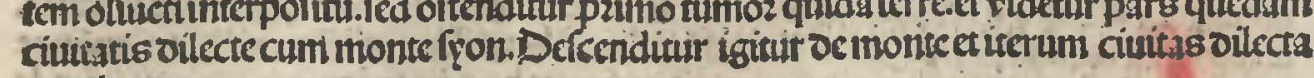

Itcen fub latere ozientalimontis olineteeiutta betbphagequead iactum lapidis oimit ticur ad finiftram fub monte offenfíonis in valle. vicalus palde paruus a afectiditur per

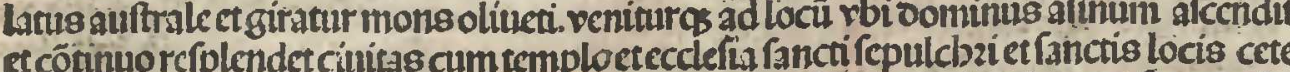
ris. Zlenitur crgo gd orfenfium monnis olineti rbi turbis pzecedentibus et tequennbs

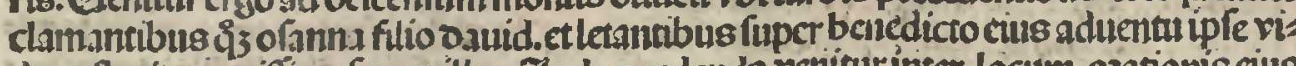

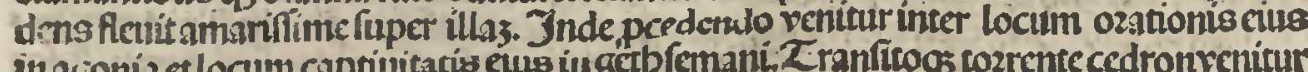
insition

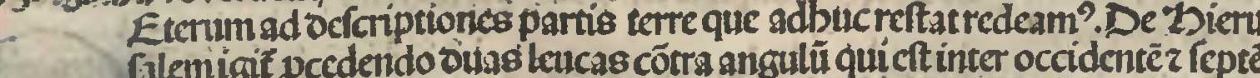

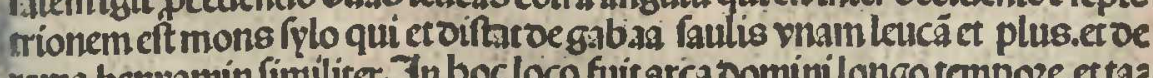

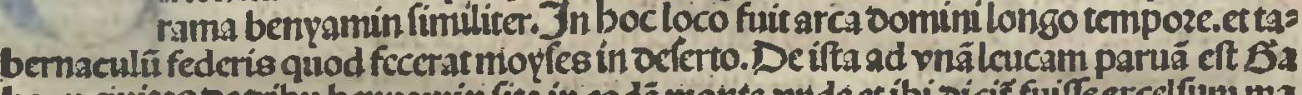

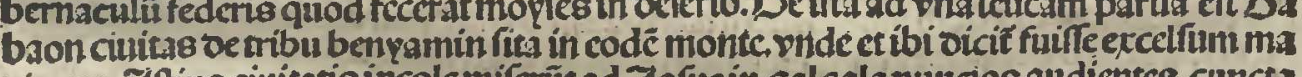

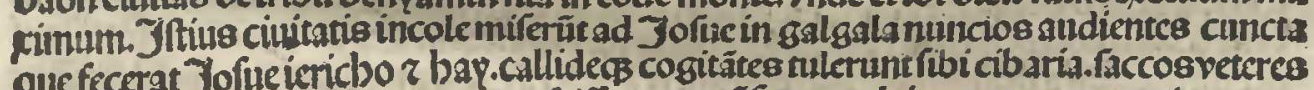

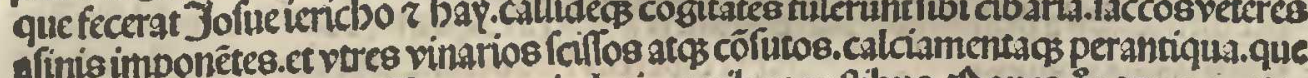

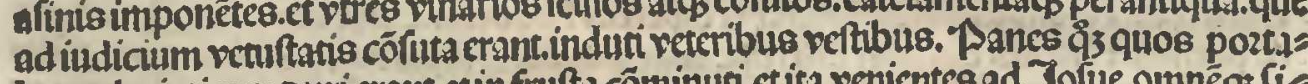

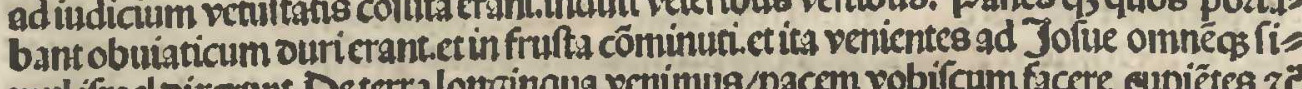
mul iffael oirerant De terral longinqua veninus/pacem vobilcum facere elpietes ze

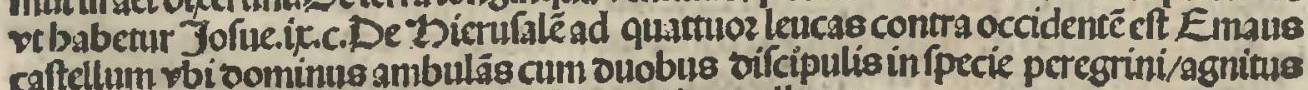

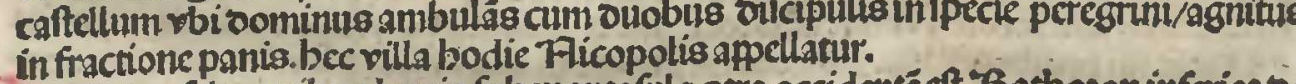
in fractionc panis.

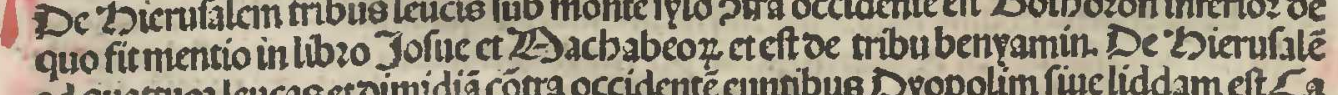

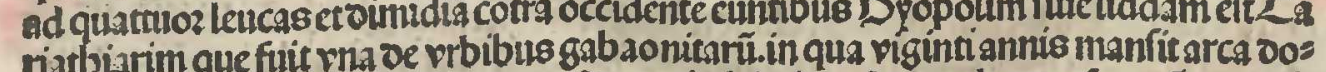

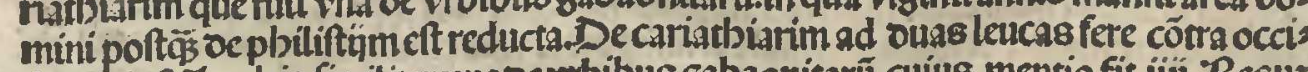

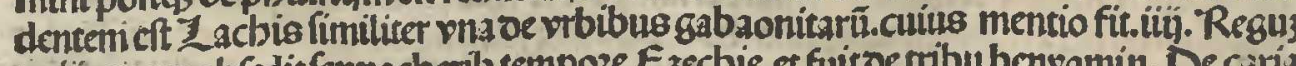
zriit.quam ob feditfennacberib tempoze Ejechie.et fuiroetribu benziamin. De ciria viam alrerius beth fames que in neptalim eft oicinur betbfames Jude. ENramen in rei veritatecrar de tribu Dan que fuitcarca forté Jude untra mare. Jucampo buins ville

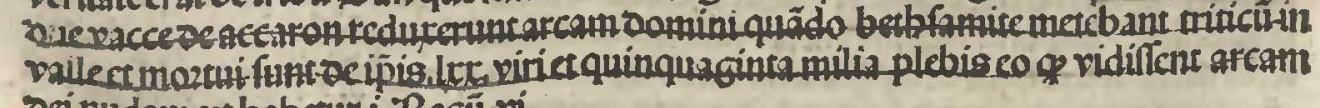
Deinudam rtbabeturi. Reguivi

De Dicrulalem ad ougs leucas cótra occidenteme ft Ramatafopbym partim oetribu

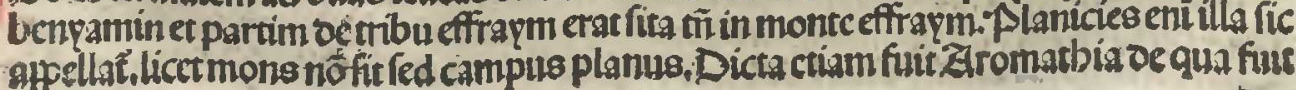




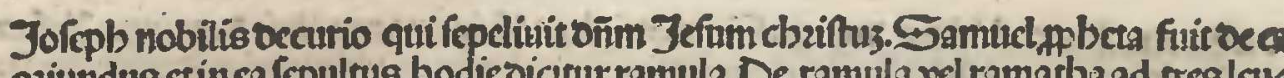
cascōtra occidcntê cef lens fugerea facieoñi in tbarfis. boceftad infulas marisvt babet Jone. i. Jn ei quon

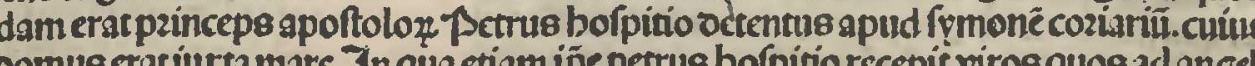

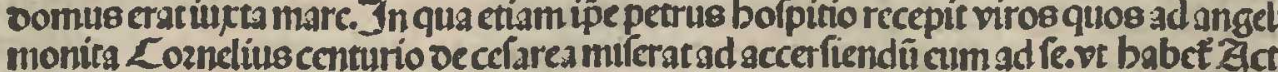

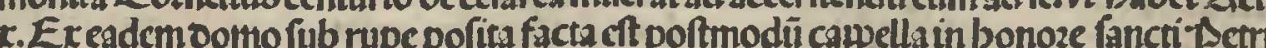
cốfcrata bodicocfolata. Sunt etiam ibi copuli in mariad quos fim fabullas andre moda filis 2 bepbci religata fuitocuozanda a belua marina. fed a rege perfeo liberata Sum ea

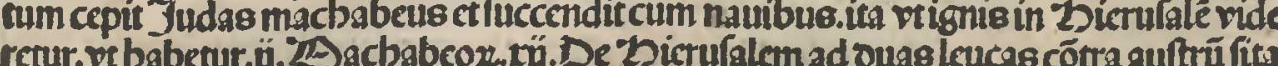

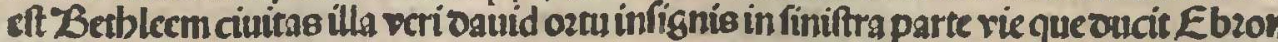

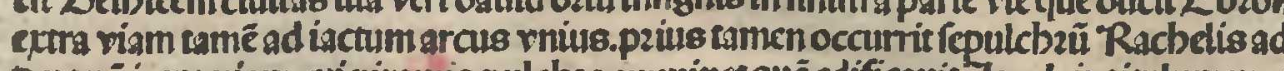

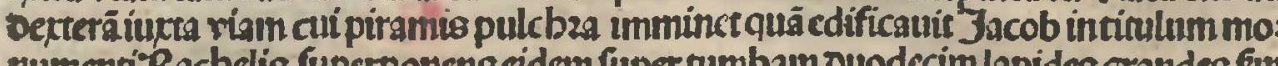

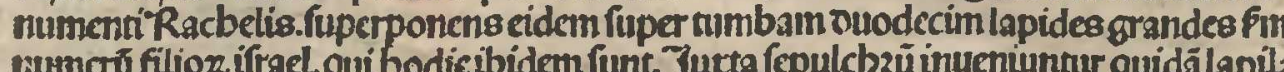

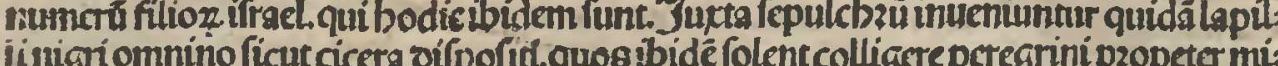

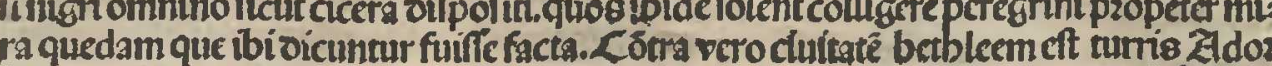

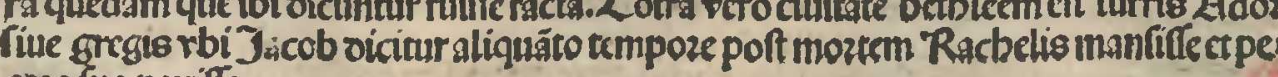

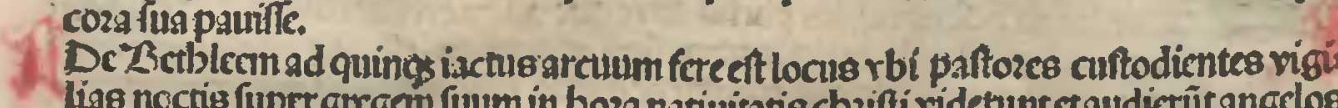

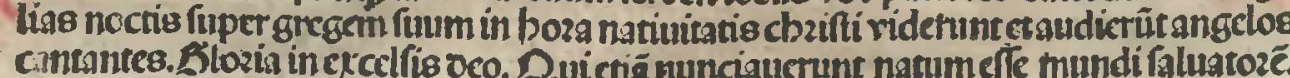

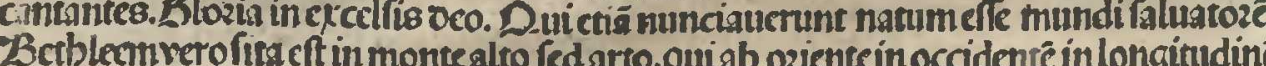
fectendit Z b occidente baberintroitum et ibicft cifterna intra poztam oe qua oauid

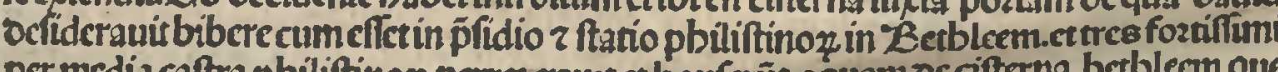

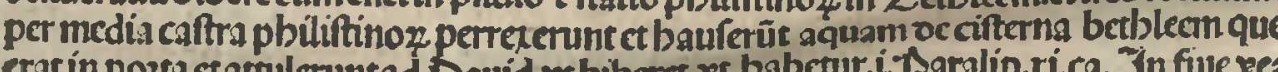

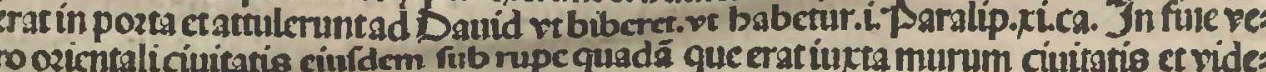

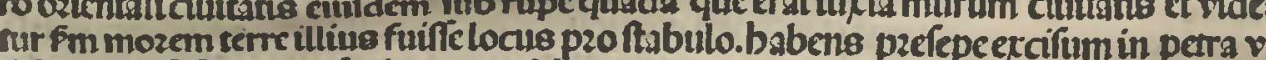

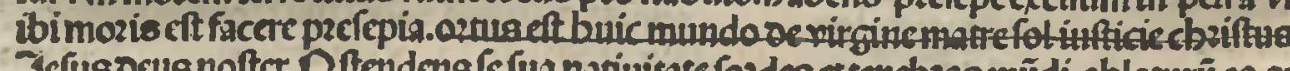

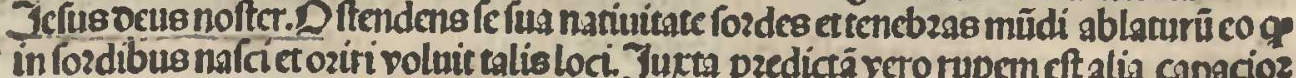

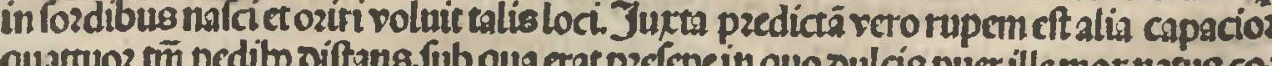

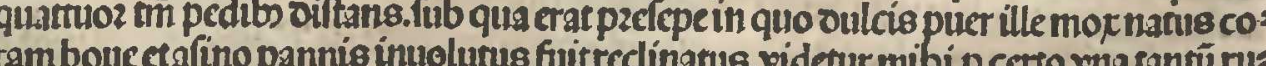

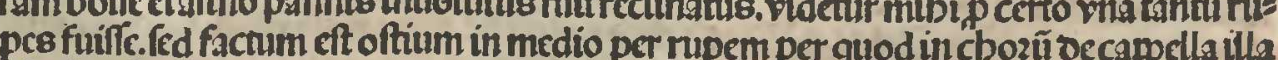

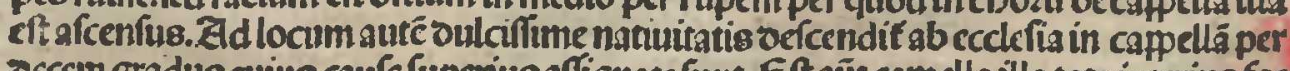
occem gradus cutus caule lupenius affignate funt. Eft añt cappella illa tora interius fas

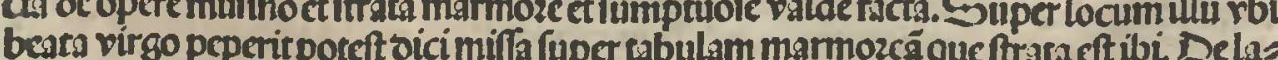

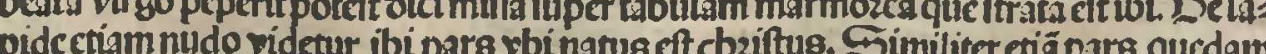
pecepis in quo iscuit cbziffus relicta eft nuda. que loca fiddes ocuotifime of culant.

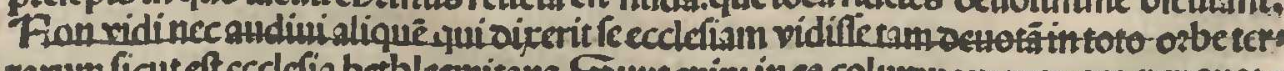
ranum ficutertecedria betblectitrana. Suntenint inea columne marmoze per quats terea

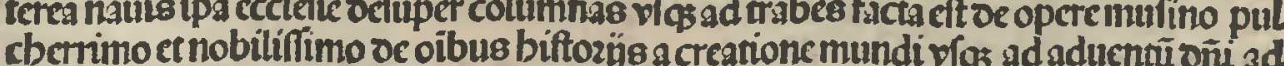

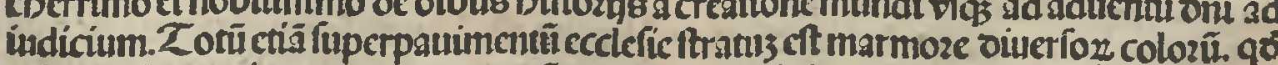

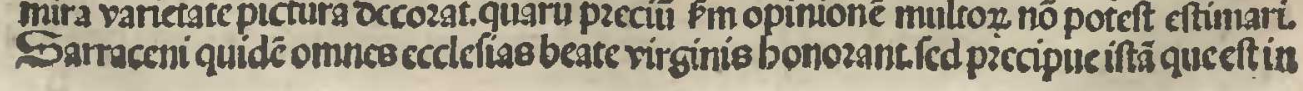

betbleem. Soldanus vero vidès in ecclefia itts oznatũ et tabulas ct columnas ormnee

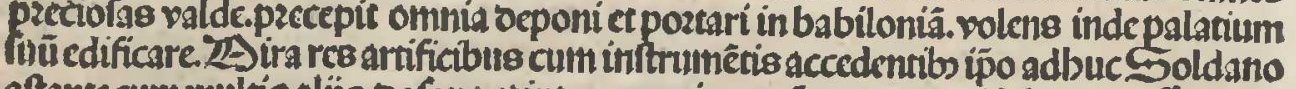
aftantecum multis alij8.se fano et tintegro paricte que nee acus videbatur poffe pene:

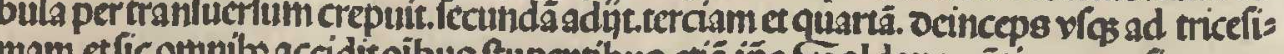

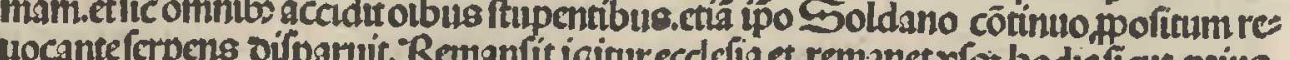

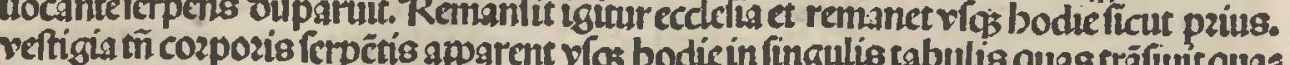
cöbuftio quedă igne facts. Et tuper omnis mirabile videctur

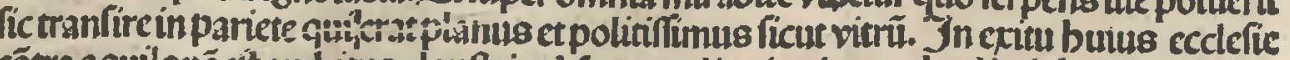
cotra aquilone eit ambitus clauftri qo frattres aliqui minozcs bodic inbabitant. 102 Dececléin ift a Prochium filice eins. vbifuntectiö

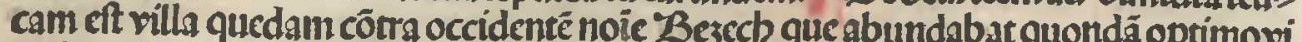

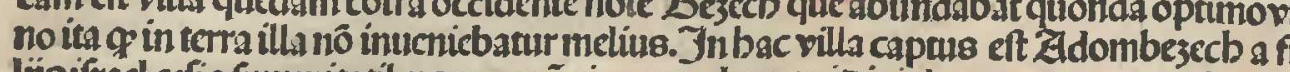

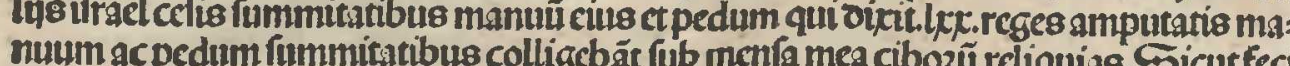
num acpedum

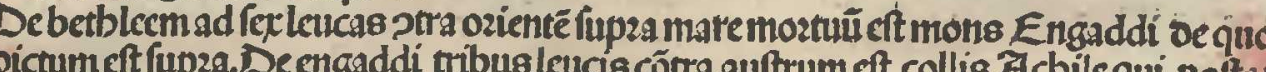
chificato ibidem ab engaddi trbus lencis cótra auftrum eft collis Acbile qui poltes co Dauid faulem fugiens oicitur aliquotiens latuife. vi babez.

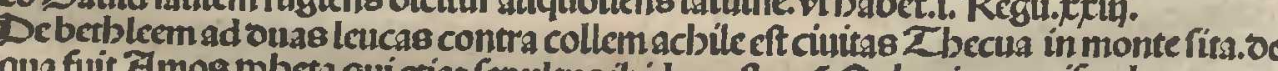

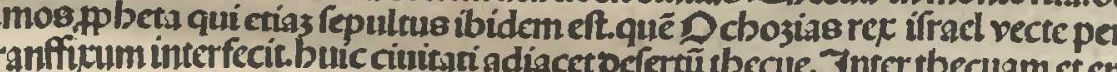

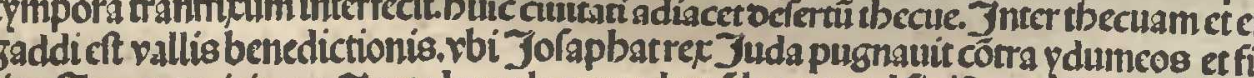
los Amonct ricitcos. Jurta bunclocumad vinälencam ad finiftram coutra defertum

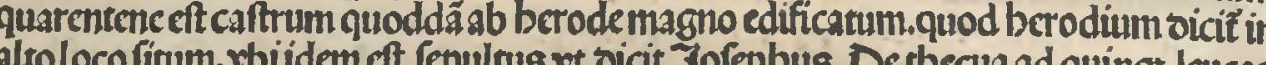

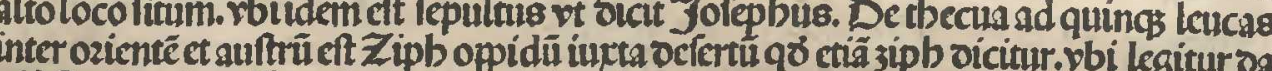

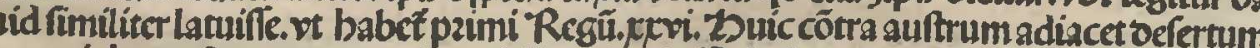

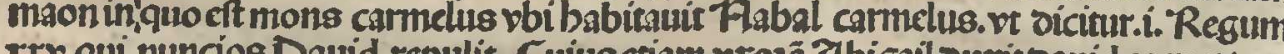

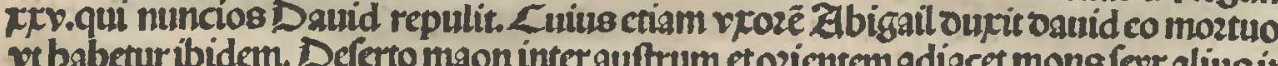

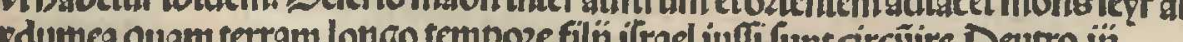

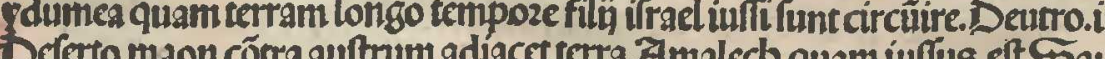
rei. Regum, cy.interfectis babitatozibus eius, Zunteterram tuilinguameft cadébarne. oe quo loco \$S oiles mif́t ouodecime explozatozes ad côfí

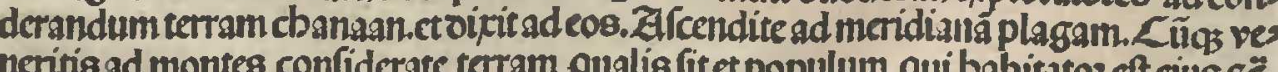
nentis ad montes confiderate tertam qualis fitet populum qui babitator eft eins $z$ z

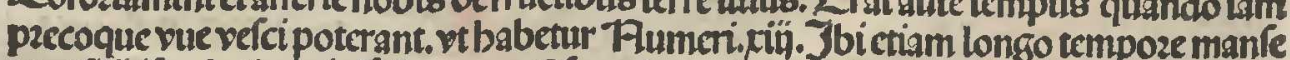

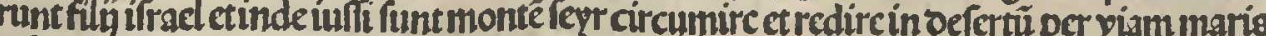
rib:L Debetbleem ad tres leucascotra autrum in ria que oucitcbzon eft betb3acba

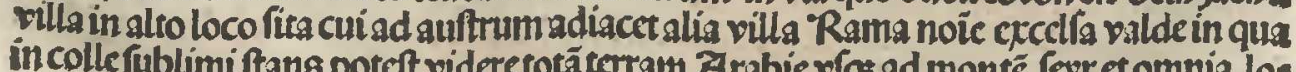
cacirca mare mozruivet potet videre to

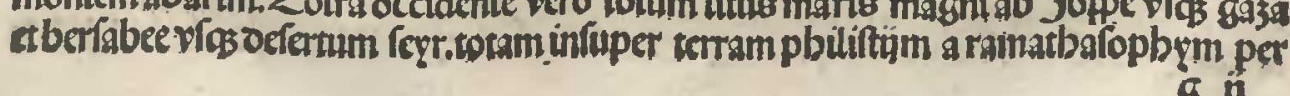

Pratri imisoris Adom anciers. 
Bctb ct Zcbaronet Azotumet Jamniam et Zfrealone cum omni planiciefub monte

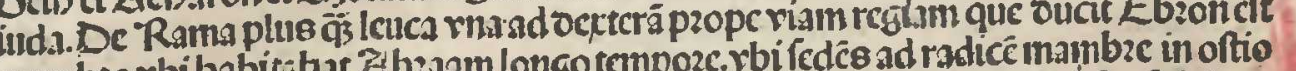
mambzer.rbi

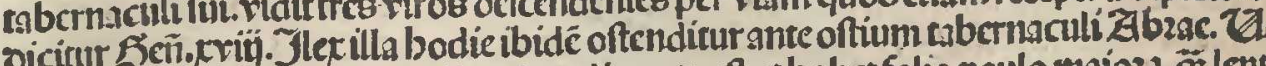

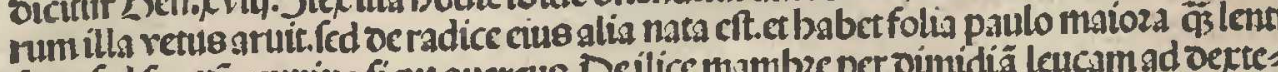

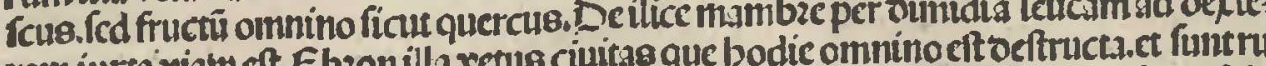
ram acris

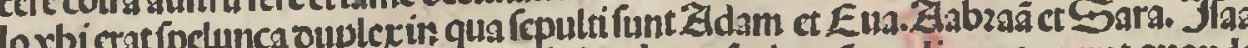

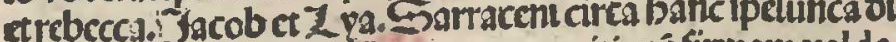

gor Dampinng. bonuset ager Danarcenus

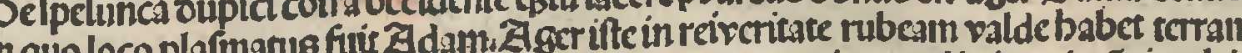

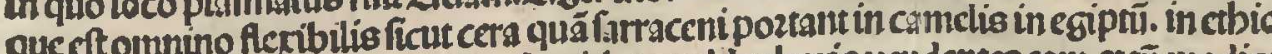

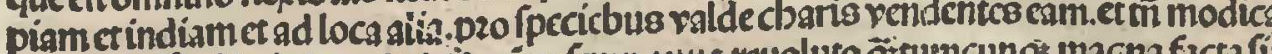

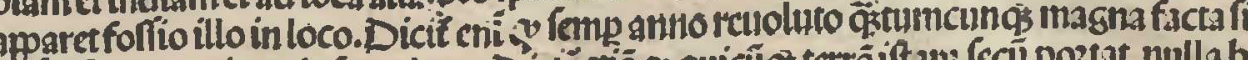

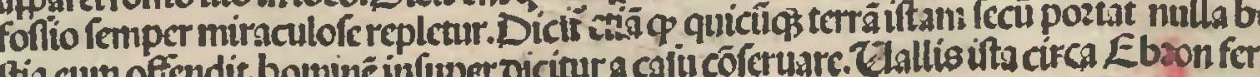

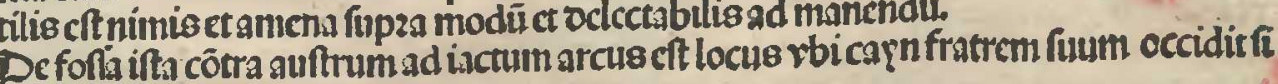
cut oicitur Renefis.iii.

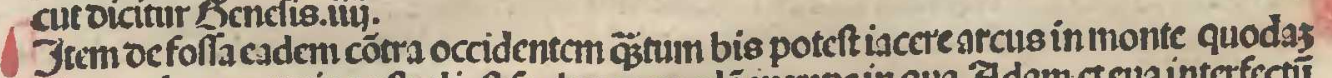

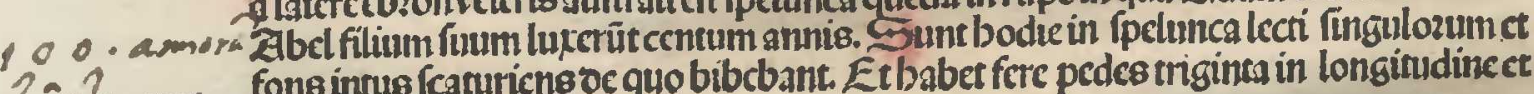

$2 a, y$. pro latimdinc fpeluncaifta.

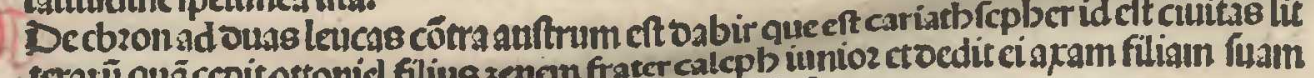

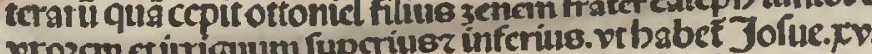

Decer

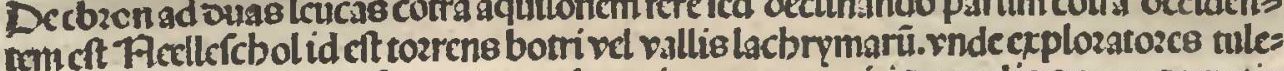
runtpalmitem cum rua fua que poztabant in vecte out oviri. De malis quogs granatis etoc ficis locillius rt babctur Futmetr.xiy.

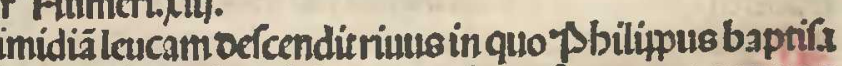
uit virum ctbiopen eunucbũ candacis regincectbiopum quícrat fupcromunceg gazas Dencellicfchol quartion

政

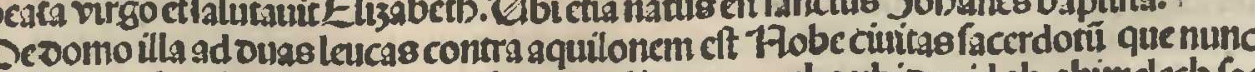

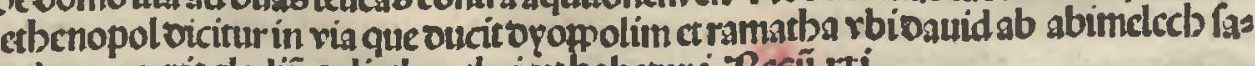
(a)

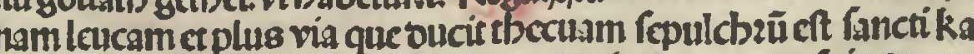
riorb sbbatis cum fuis monacbis qui omnes co mígrante migraucruntecf fuit quons

dam cócnrfirs magnusad locum iftum. b pedemontis carmeliaquilonari.

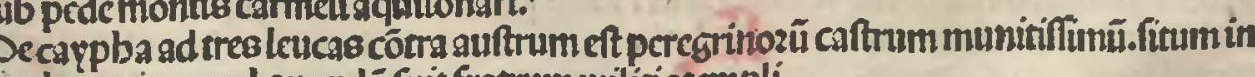

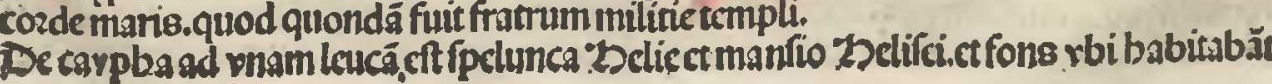

Filï propbetarĩ in carmelo.

Decaftro peregrinoztum ad quattuoz lencje eft ECfarea paleftine metropoliz/que

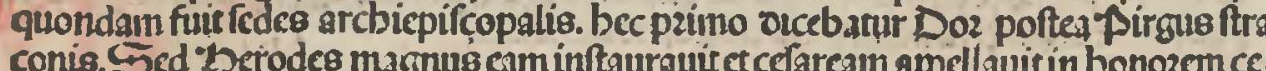
faris.occuius ftructura ct munitione Tofepbus multa feribit. Lingitur go cccidente mani magno.ab ozicite palide quadgoulict pfunda.in qua eit multitudo cocodrillo

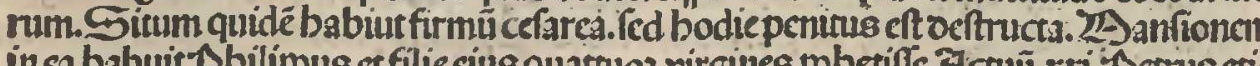
urcer bab

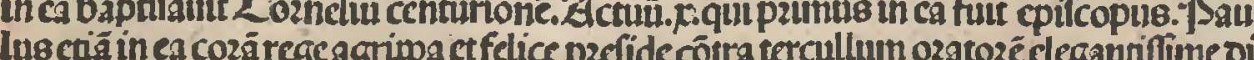

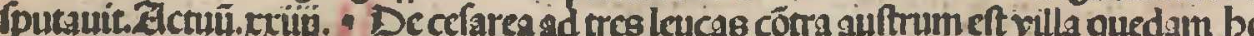
die affur oicta.quonda tamen anthpatrida oicebarur. ab antipatrepatre berodio mas

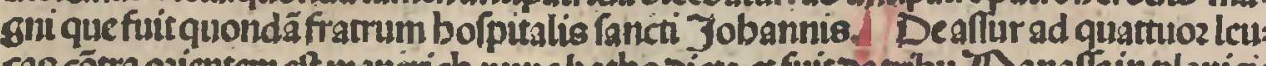
caub monte Effrim fit nó lonocamente Dekatbo quattuoz leucis cötra auftrum eft mons Saron etoppidú farona.cuius mê

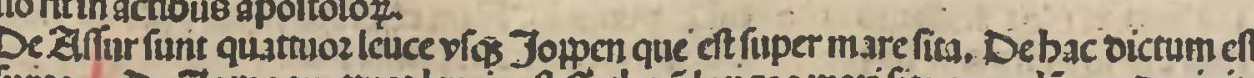

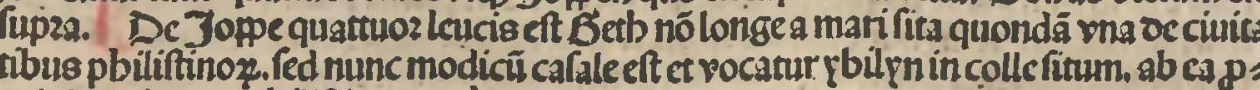
Degetb ad ouas leucas cốtra auftrū eft betbfames inda. oe qua oictū cft fupea.

Debetblamesad ouas leucas córtra auftrü in montc inda vidét mons modynoe quo

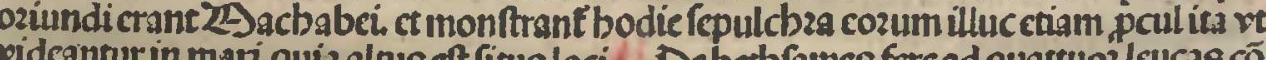

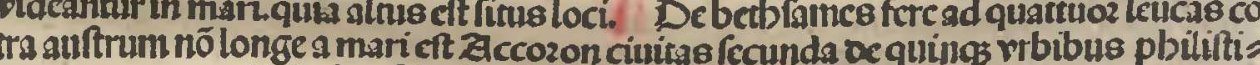
$102 u m$ tunn modicu ct cafale th adbucgntiqui nomen retinet.

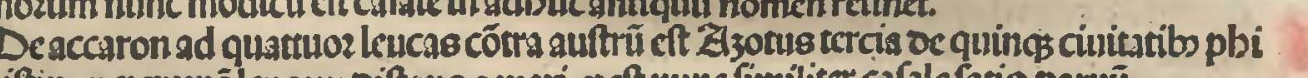

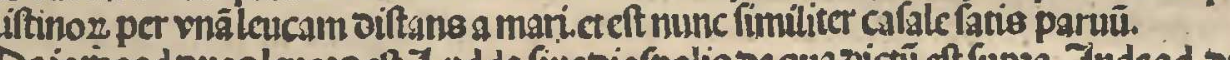

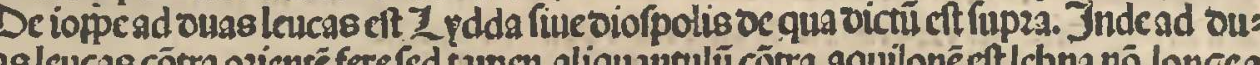

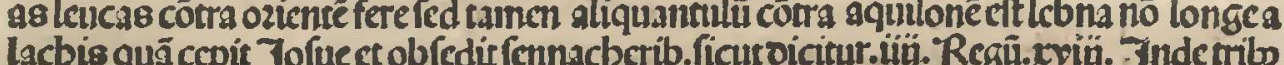

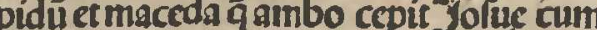

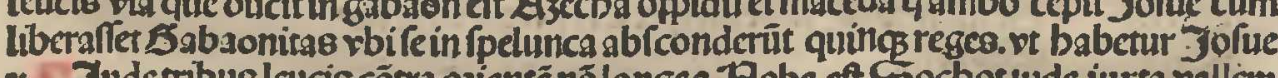

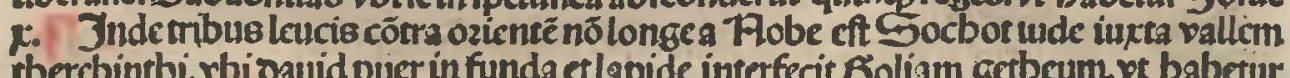
i. Regum

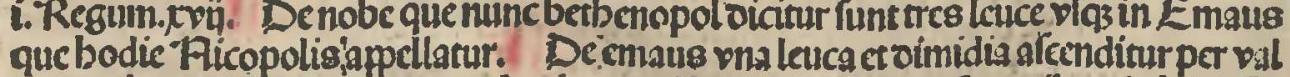
lem rapbaym a latere oomus 3achane que relinquitur ad oetctera cuntibus in bicrufas lsm. Et oecadcm bouno per rnamlencam et Dimidiä eft caftrü valde munitü Betbfus

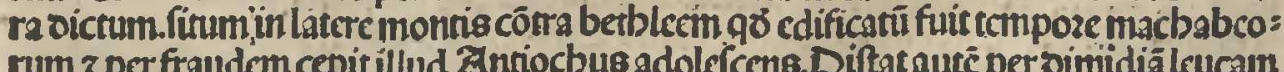

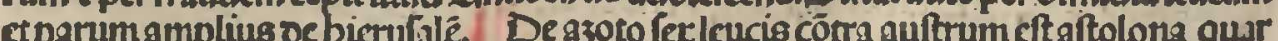

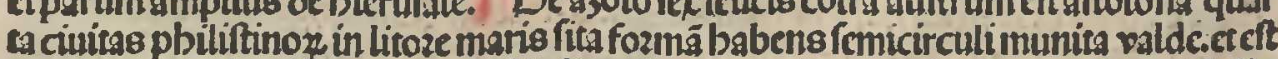
quodammo robur arracenoz in ila. Deantolona quings leucis cotra autrumet ba

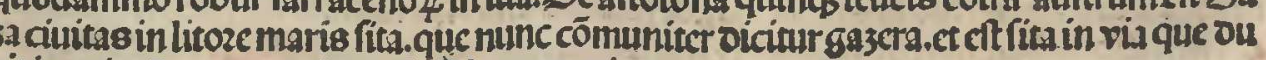
Desipti. ocqua infra in fecuda parte oicctur.

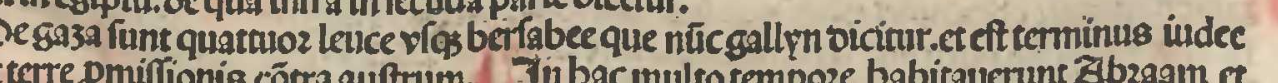

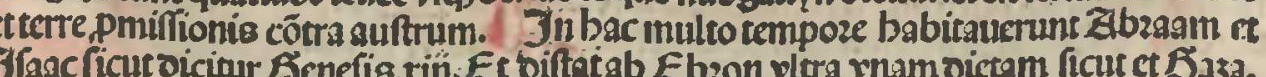

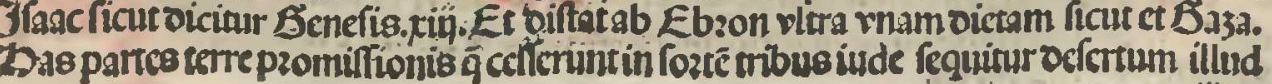




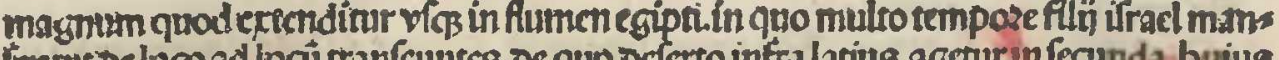

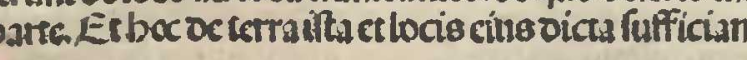

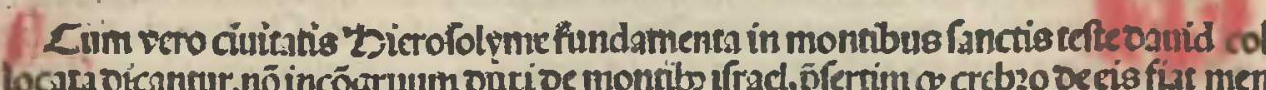
to in facra icriptura paulo amplius oiflerere. Erpaimum quidemin gencre. ocinde in Ipcricocaliquibus.

(1)

Demontibus Jiracl in genere.

(t)

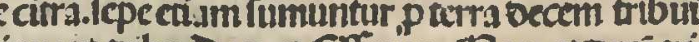

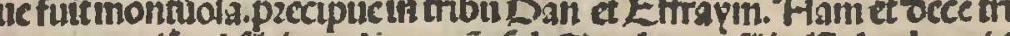
Gection

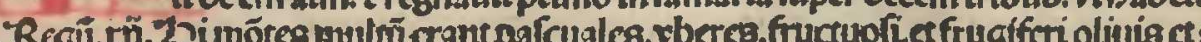

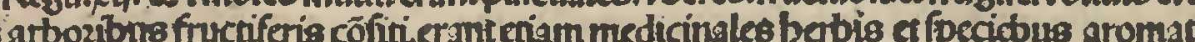

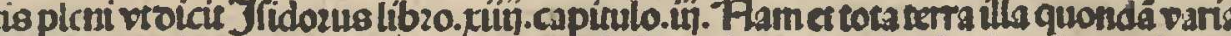

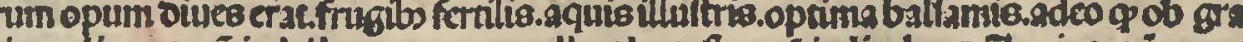

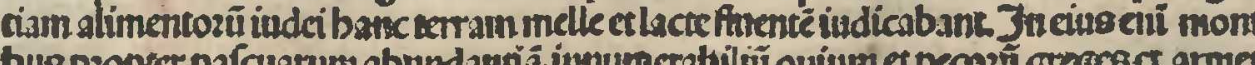

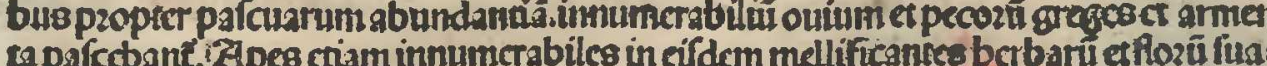

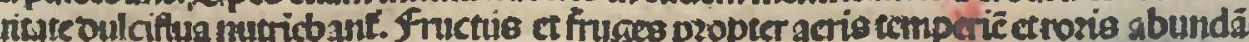

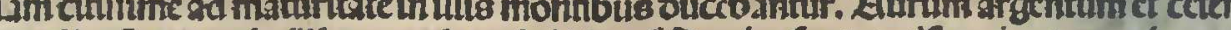

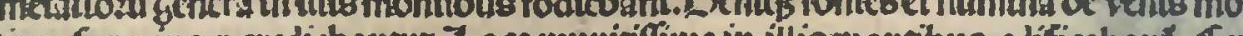

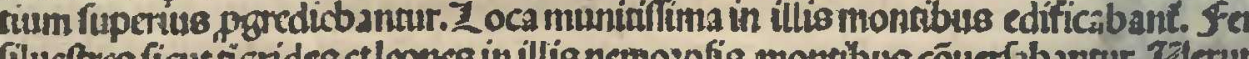

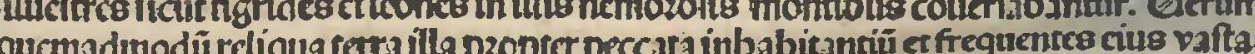

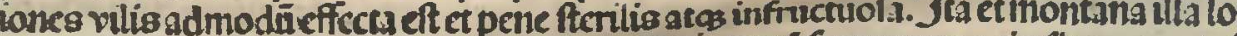

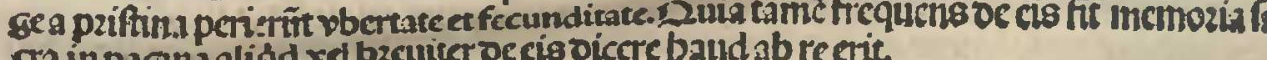
cra in pagura

$$
\text { Tequiturnune semontibus Jifracl in fpecie. }
$$

$$
\text { Demont }
$$

Yon mons crat in bjierufalem. fruper cufus verticem erat arxfiueturis os

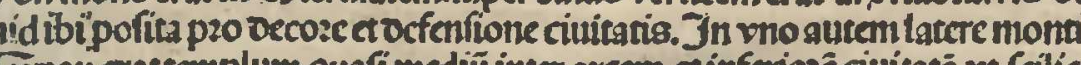
Fyiph ratrimplam qus

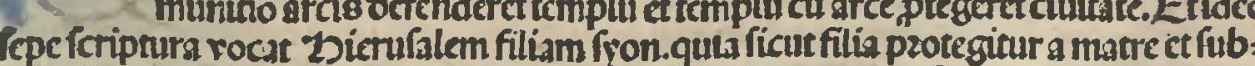

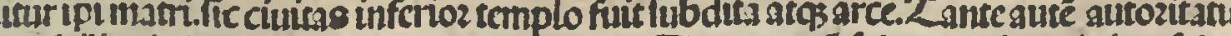

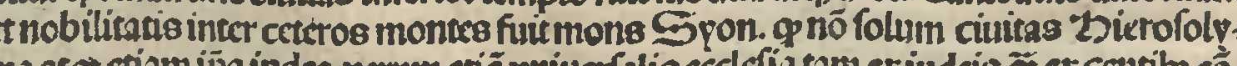

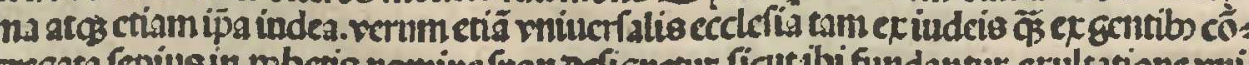

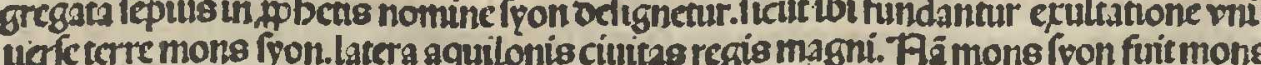
magnealtitudiniset fublimitutis, magne fortindinis et firmitatis.magne plenind dini

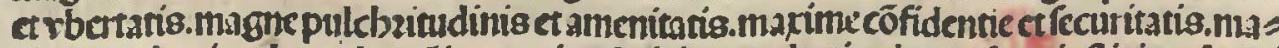

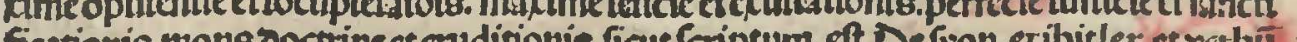

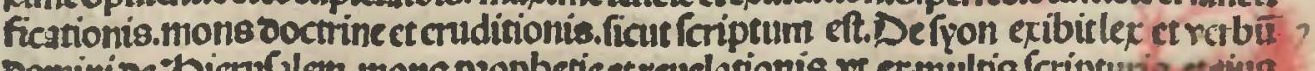

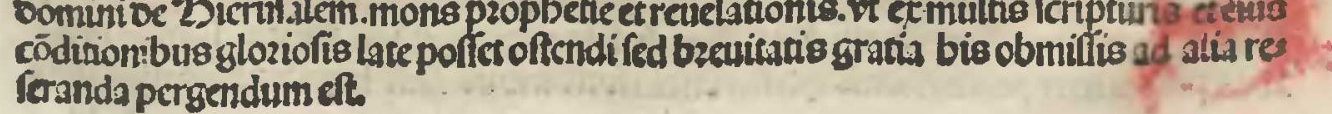

Demonte 2Aozia

múale in quo edificatum ent templum Salomonie

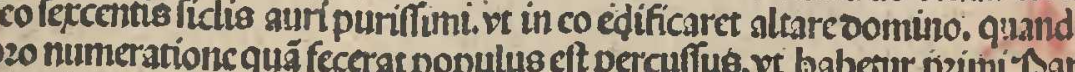
pomenon $x+i$

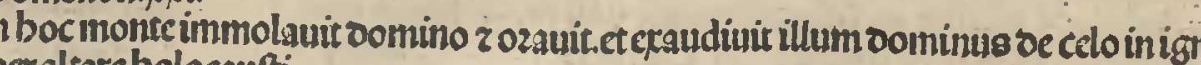
arcbolocaufti

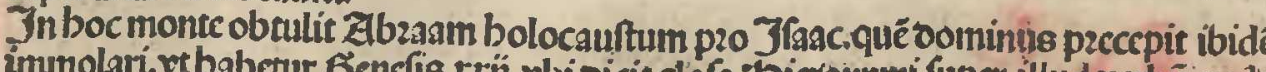

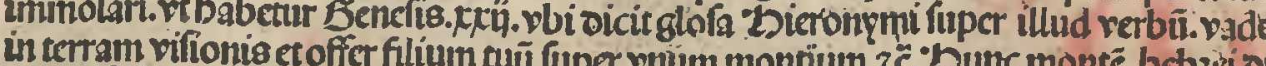

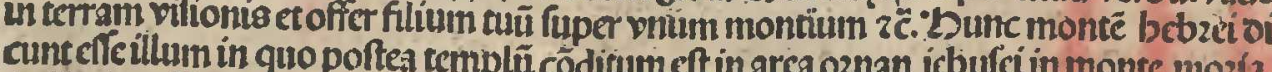
quiticcirco illuminuans et litcens interpzectaf. quia bieft toabir boc of ozacultum ozi.c. in codem loco crediur 7 mob oo er

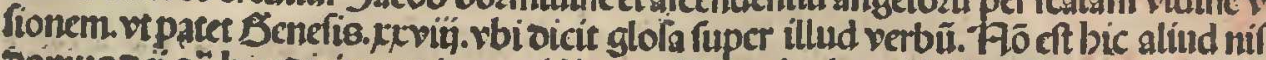

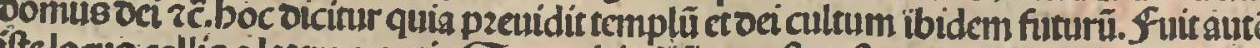

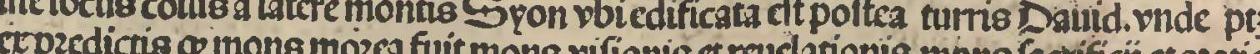

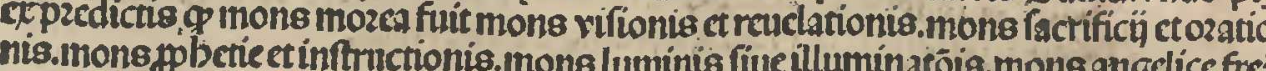
Deimonte Dliveti.

Dns oliuctieft monsin iudea iutta Zicnufalé fic dictus.pzopter copiá oli

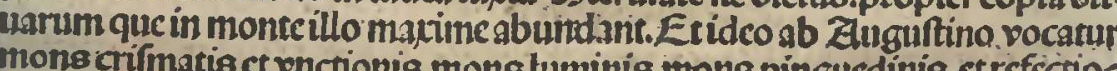

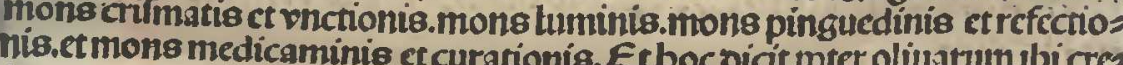

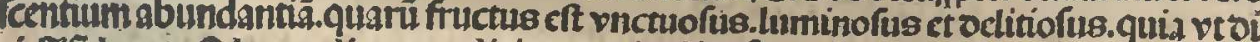
cir 3ridozus. D leum oliuc exradicis amaritudine furgit in pabultú luminis.in medica

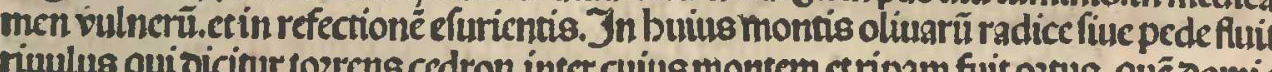

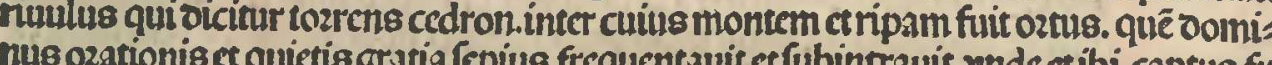

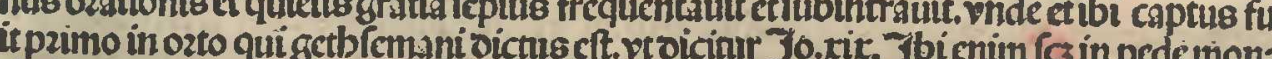

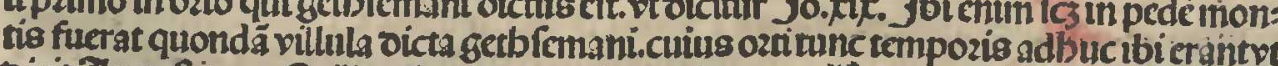

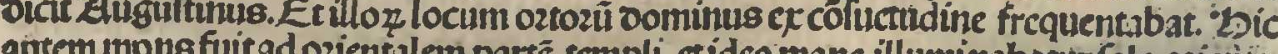
ocvépereveroctocnocteillum parre rempli et ideo mane illuminabatur fole ozierite. Etideo merito mons luminis oicebsar fum

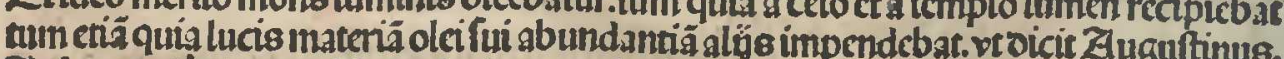
In boc moniccrat quidam viculus nomine beth phage.qui faccrdoctum crat. in cuius

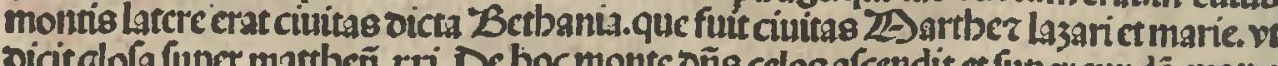

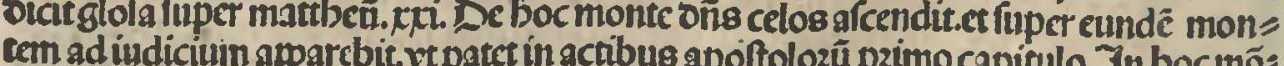

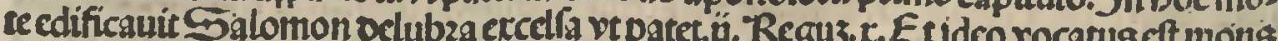

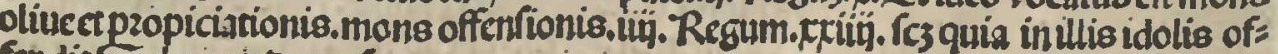
Fendir Salomonocum fuum.

\section{Demonte Ebzor.}

B2on mo valle oicit TSambzc wurta 2on recus rpe

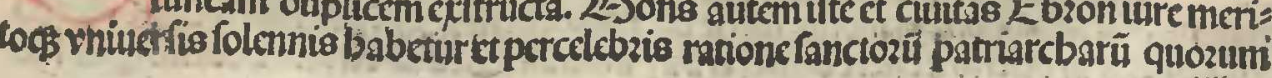


Carrintherom

Yorags

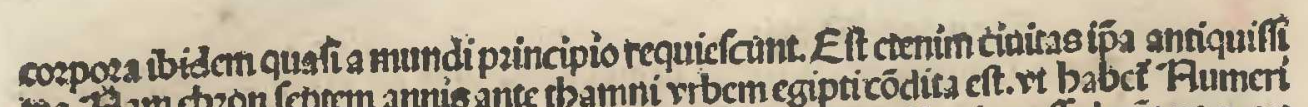

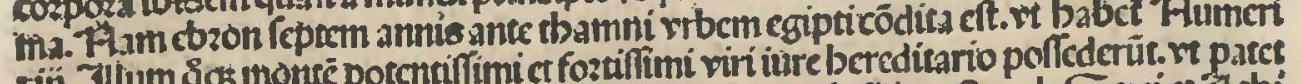

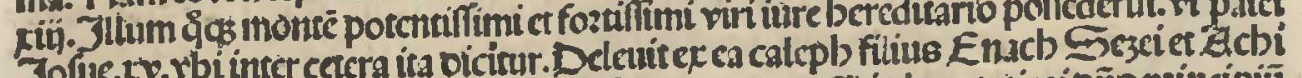

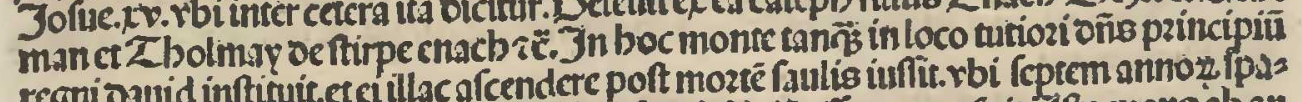
goregna tiquis 2 riatbarbe futrpzimus nuncupante.

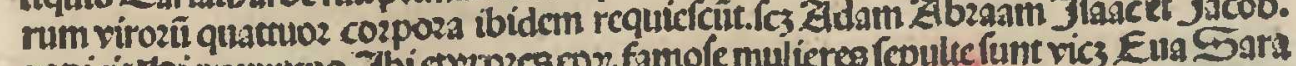

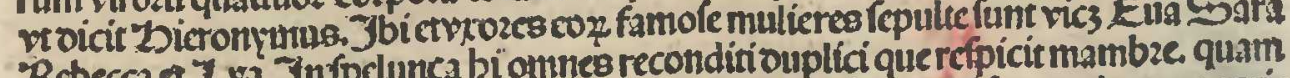
Rebeccal a Xa. Jn pelu Heiudeum ingre

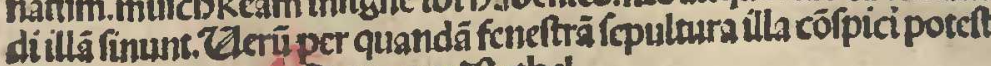

Drices bethel funte in iudca vicini bientalé rbioom? oni fuit fub Salos frundi graminibus etberbis aromaticis pleni.etideo cenvicapzecet bỵn" nolifreguétrare illoz montiu cactumina funt côfueth

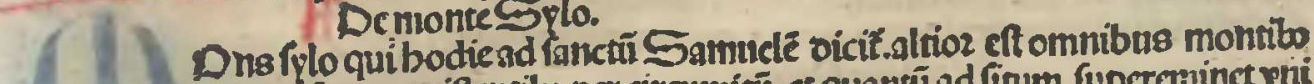
.

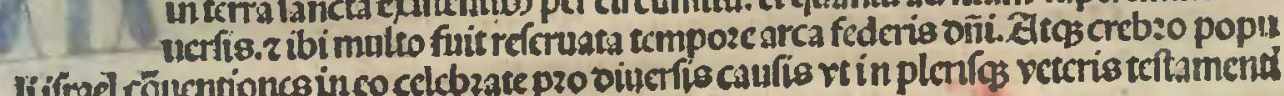

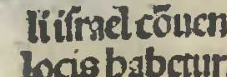

$$
\text { Demonte Barilitit. }
$$

cuivicin? of mons

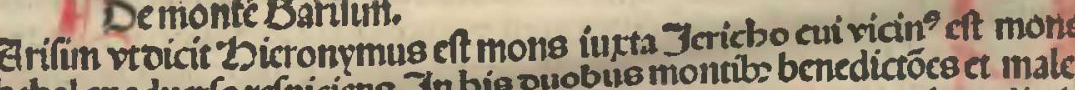
(1)

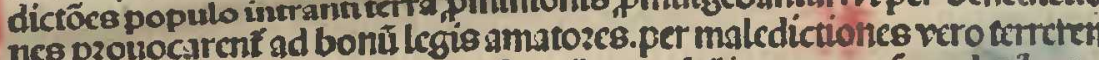

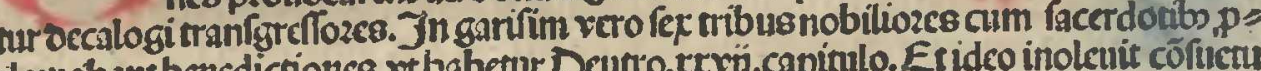
clamab.snt bencdictiones. vibabctur

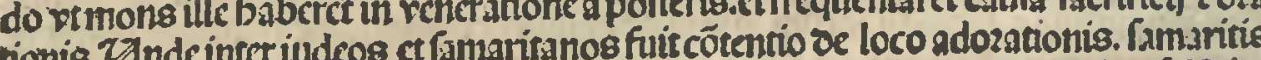
pferenobus

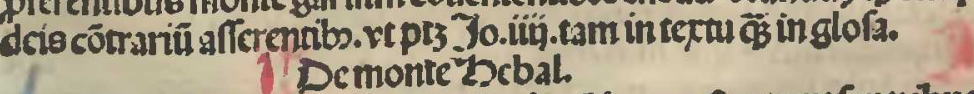

Ebal eft mons trane iordane in quo feccrunt fectribus poftiozdanis tram isumad maledicendü illis qui pzecepta occaloginō feruarent.ret patet etri

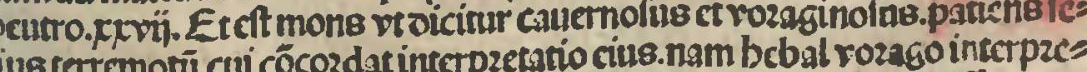

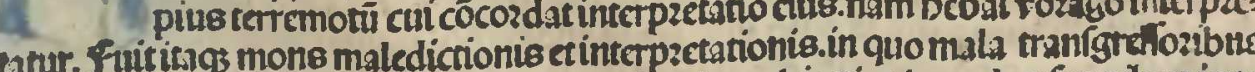

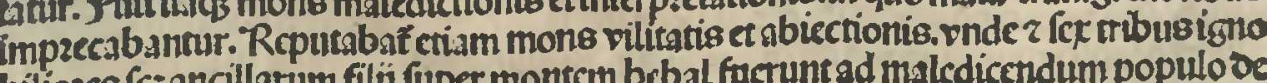
biliozescs

arencenis eft mons eccelfius valde iuxta Jericbo.ad vimidiam leucie euns

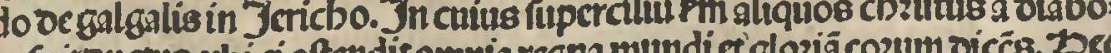

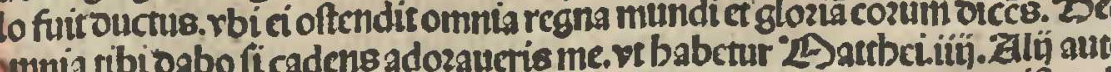
adunt in slio quodam monted furrit cbziftus tentants qui pertres leucas oiftatab monte fuitc cbziftus quando icimauit quadragints dicbus ct quadragintanoctibs.on

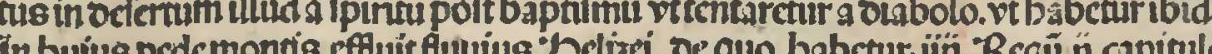

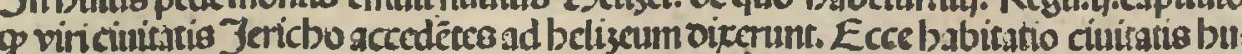

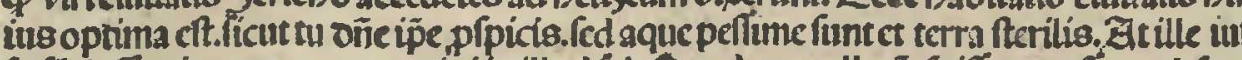

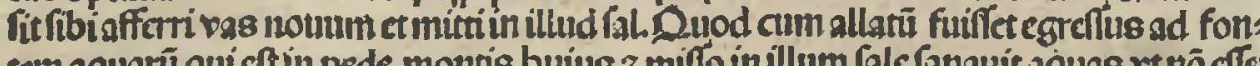

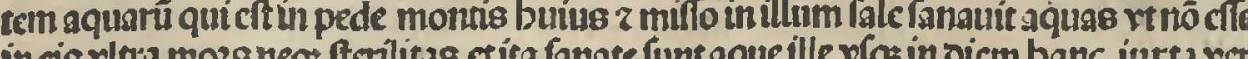

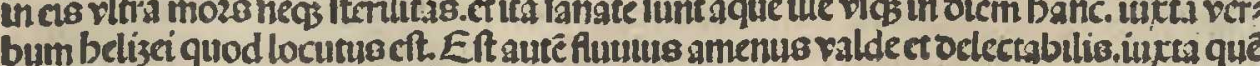
peregrini illo venicntes coquiécereet paufare folcht.

$$
\text { Démonte zsermon }
$$

Enmoncft mons modicus fuperiozdané fime:quiberbisct palcuis eff f undus. 'Plam circa radices cus func cres pedem fluentris iozdanis imbui reet rozisetaque influentia occozaruret infignitur.,pecr quodet ibi nutries Gattur peco2s que incemplo ocbuerunt immolarionio ct offerti. Et quia antimalia e rozefruminis ct berbis impingnara in monte front id eft in atrí templi offercbantur.

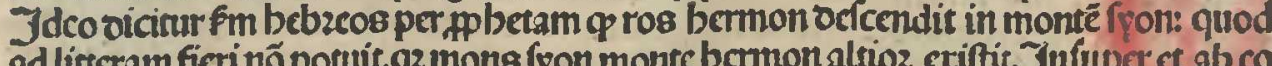

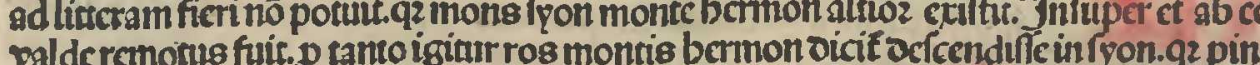
gucdines er gdipes animaliu que in illo monte pafccbantur ad paftuni ignis altranis in

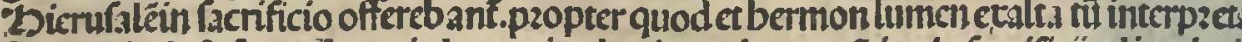

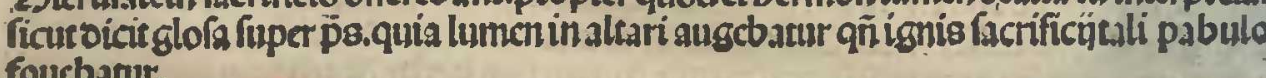

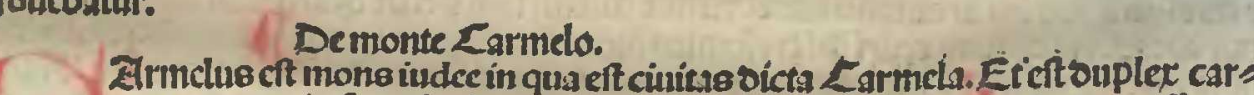

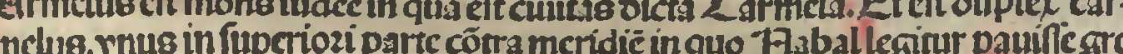

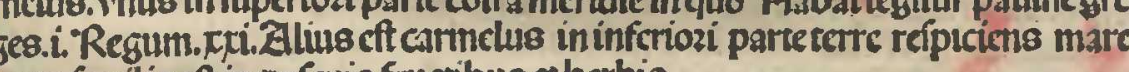

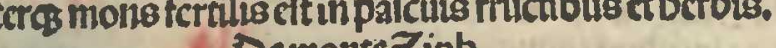

$$
\text { Demonte Zipb. }
$$

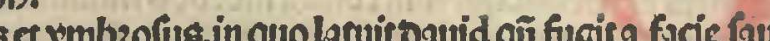
The

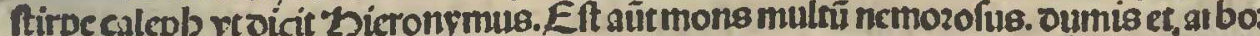

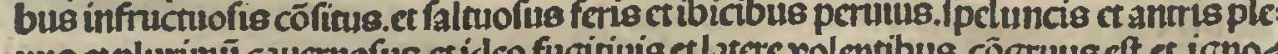
nusctplurimú caut tis tranfeunsibus periculofus.

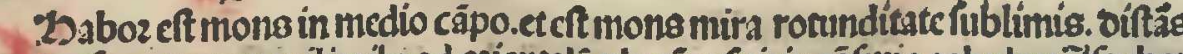

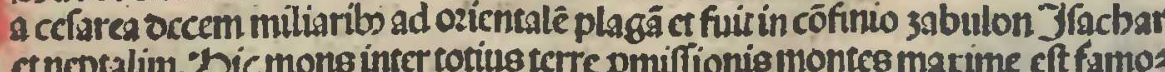
etncptalim. "Dicmons inter totius terre pmiffionis monteg marime eft famos

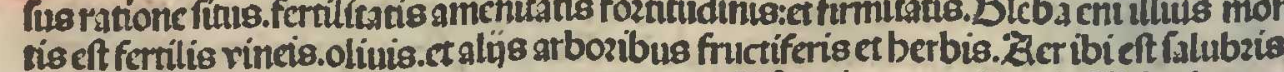
ros frequenoctoulcis imbor tam temperancus ö Terotinus mediocris 3 bi arborum

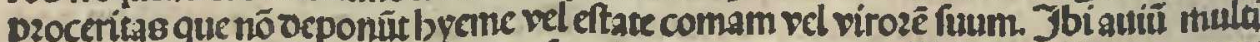

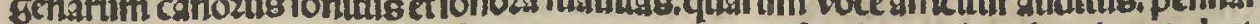

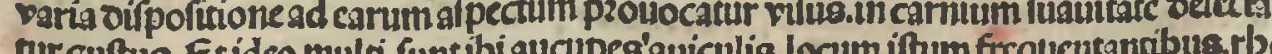

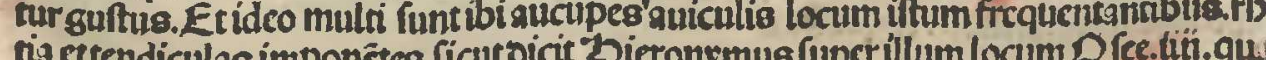

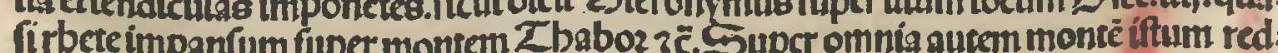

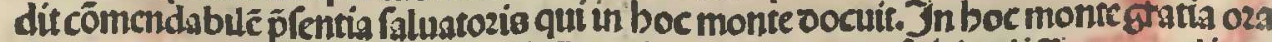

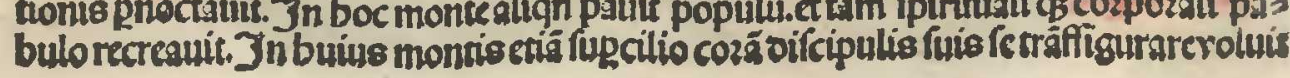




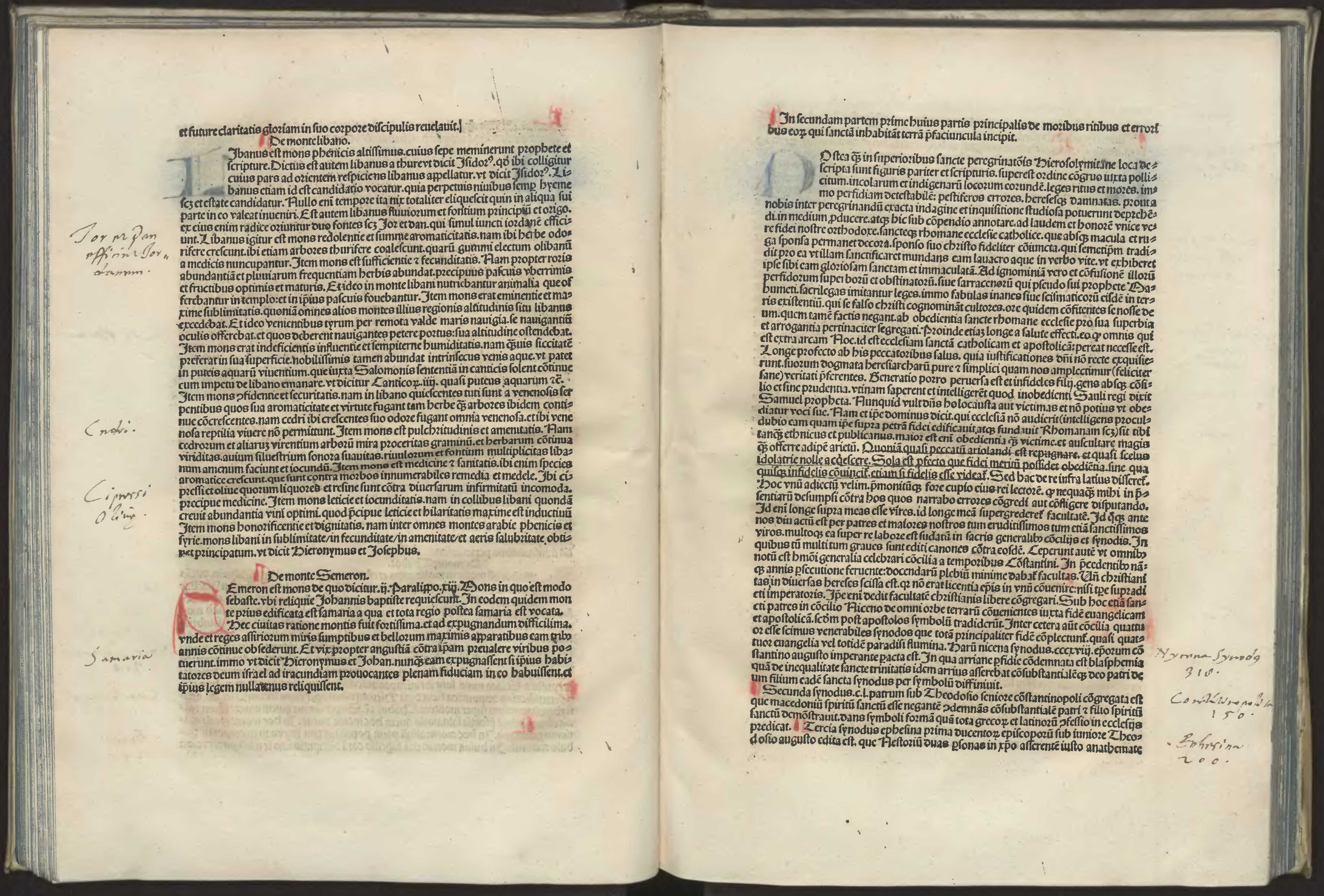


- hosommsis

30

22arnilatoriso Sarvorat.

65

5.6

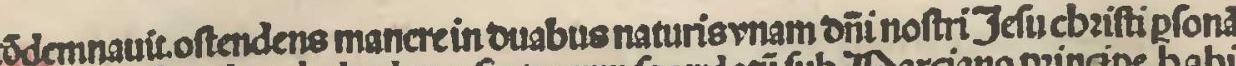
Duarta nod aeft.th qua Ẽ

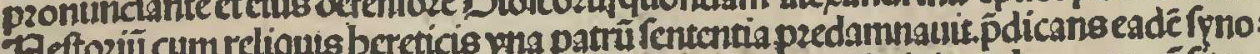

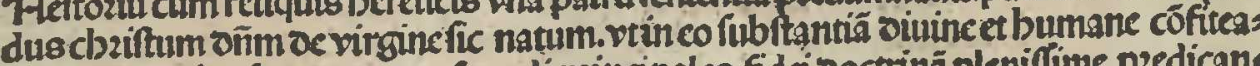

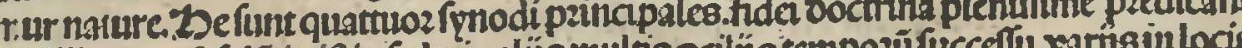

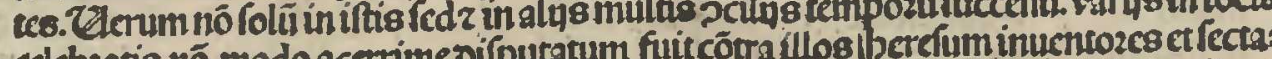

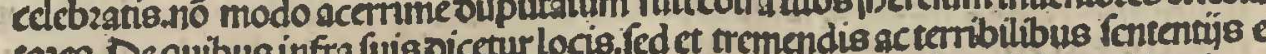

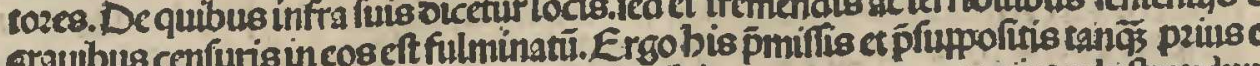
bolum fudiofiffume ventilatio animuse ft mibioumtacat cos quos inter tuftrandun

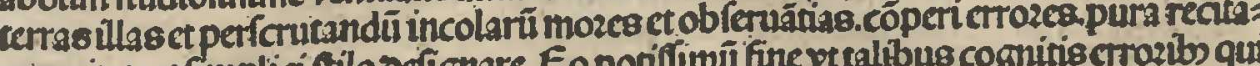
revertaatse et numplicit thlo odignare. bub ancea lla

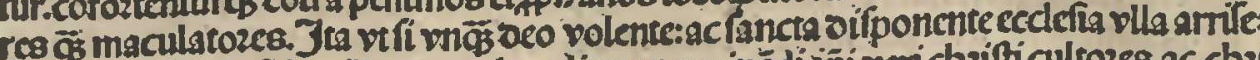

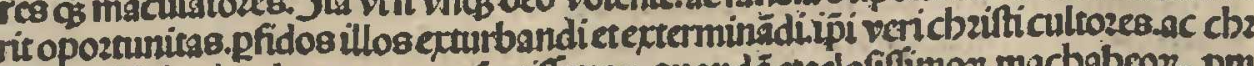

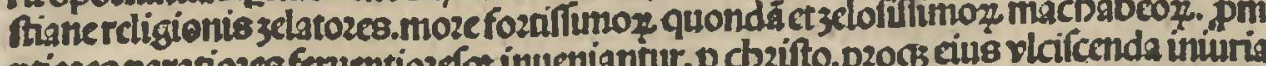
prios 20 ficiectiligst

Tecerñ vt ordinatius inrem veniam.pzimũ omnium se farracenis ec coaum legibo

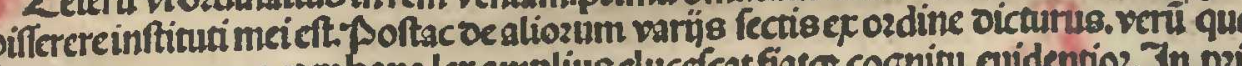
artacenozumetroz et,phana lec amplius elucefcat fiatg cognitu cuidentioz. In p2 mis ociponfar Sramo Scruio

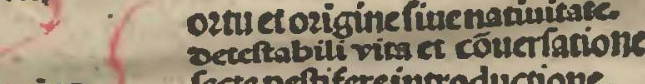

crcio oecius 2 rectepeftifercintroductione Do

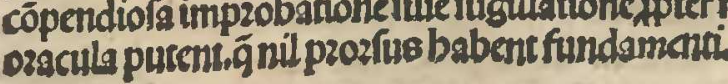

$$
\text { Deormetozigine 2Sabuneti. }
$$

- O ranpozequo 'Bomifacius papa quartus ecclefieribomane pzefidebar

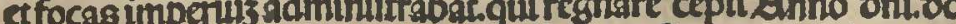

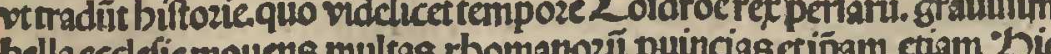

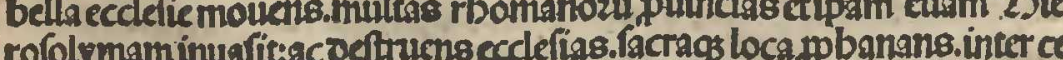

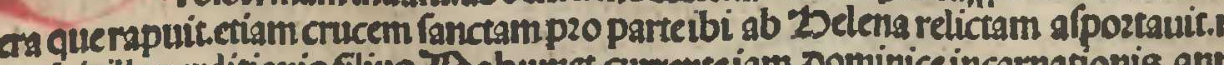
as fuitille perdítionis filius 20 abumetcaurenteiam Dominice incarnationis anno

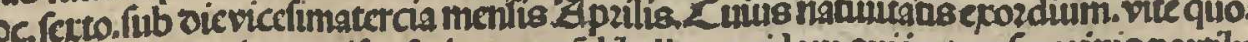

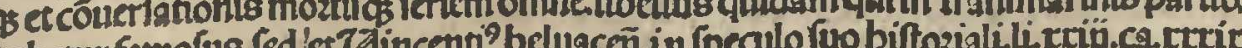
sabecurfamolns. blequentibus millib.oif

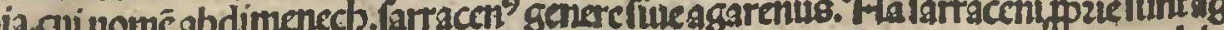

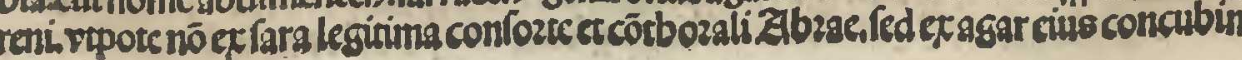

oxkendentrs.exprogeniefc3 J Fmaelis.vndect ifmaeliteetiam dicuntur.cuúus munus

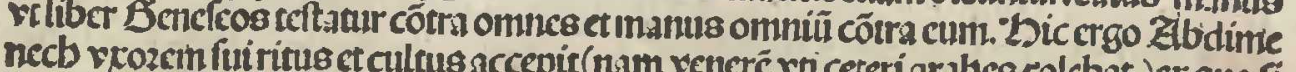

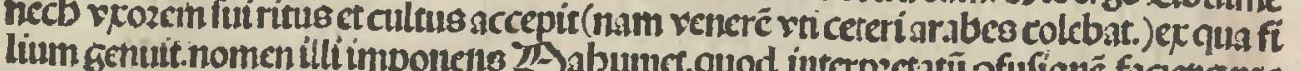

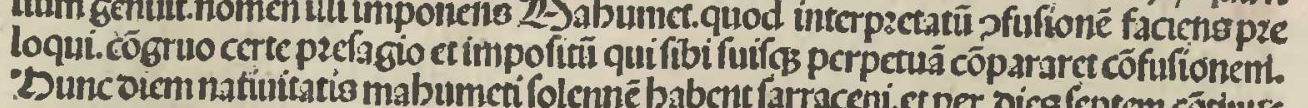

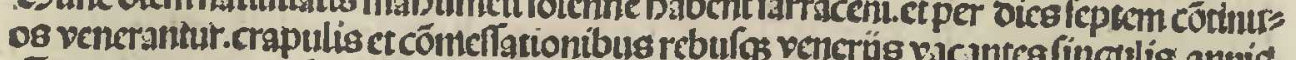

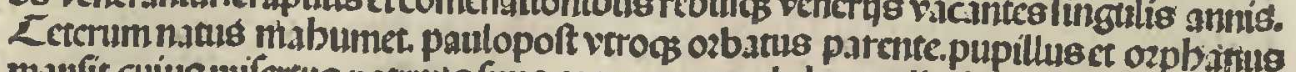

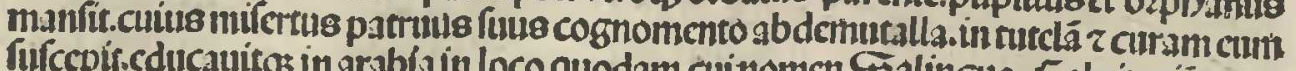
bumetidols cum our a

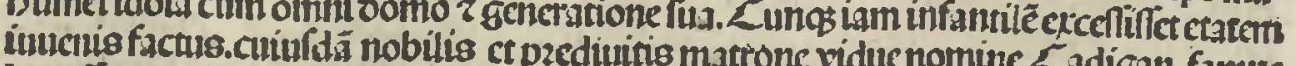

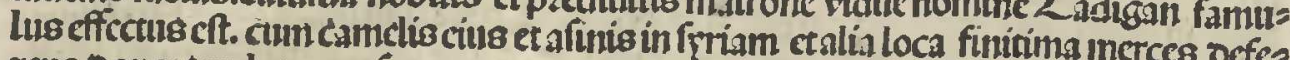

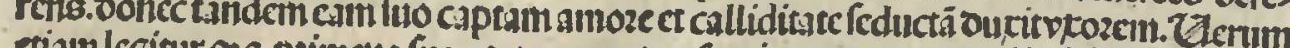
pact tinä cum

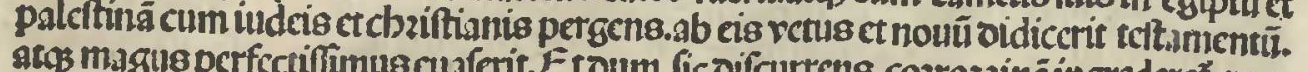

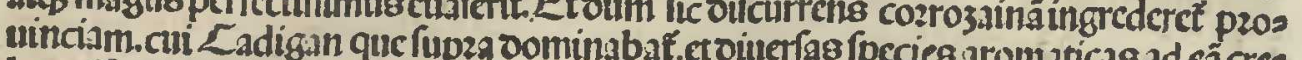

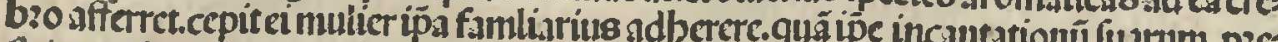

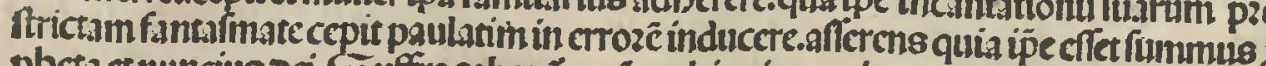

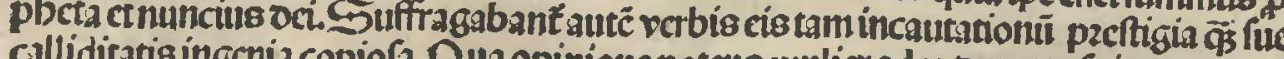

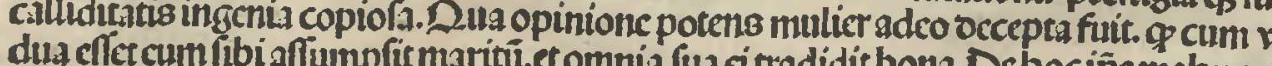
inglkozmo fuo teft

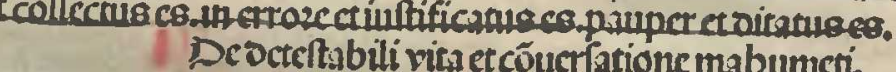

Drovif

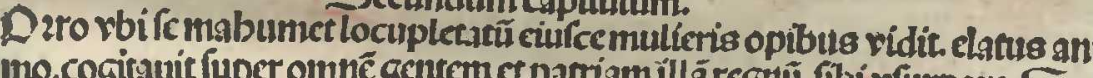
oum boceffectui oaref facile nō poffet pzéfertim cam paucos ou parc. Se ret fautozes, arte et ingenio vt poterat vecbatur. funulans $f c$ oci mbetimet

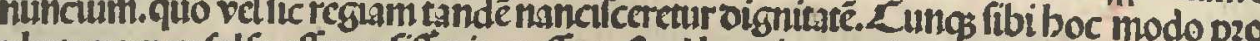

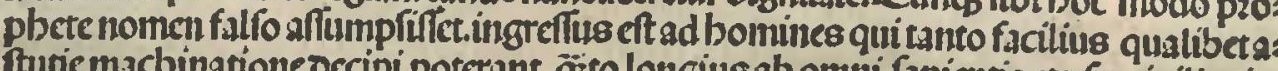

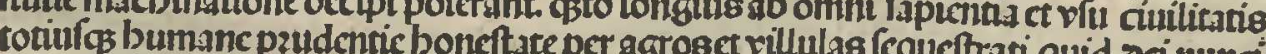

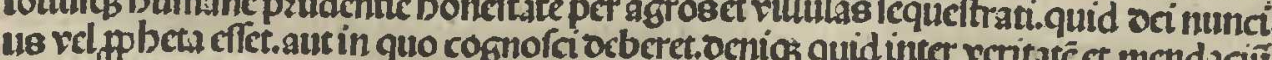

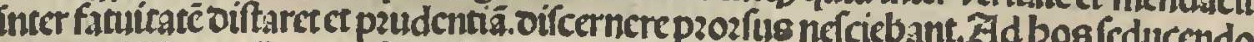

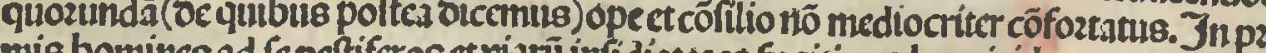
ror

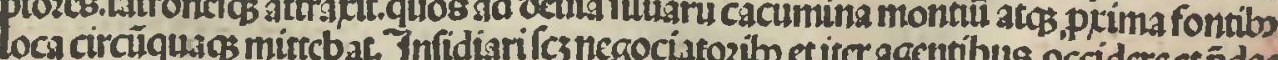

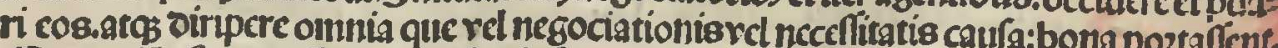

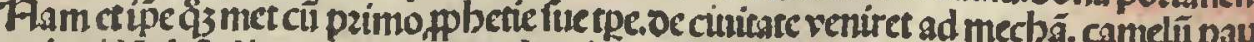

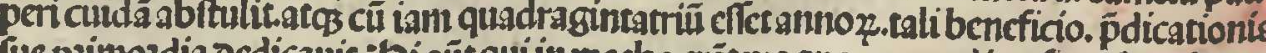

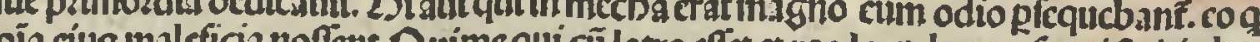

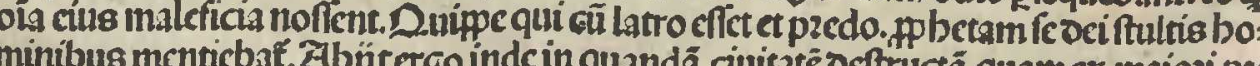

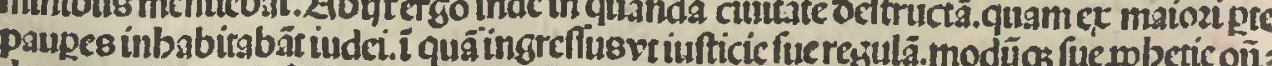

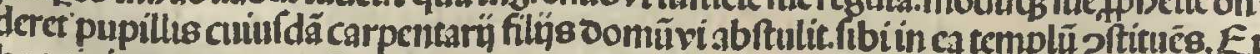

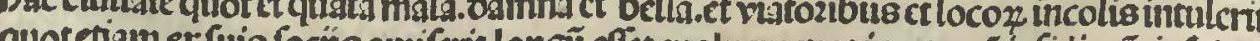

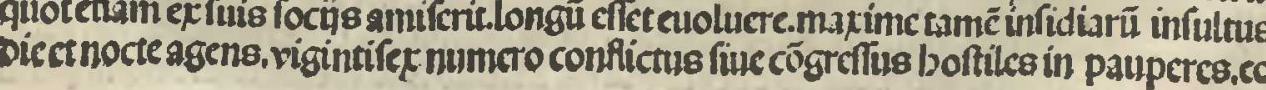


Sisper

Aditionitu

40

pores

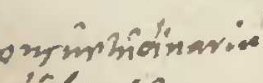

óithorisim

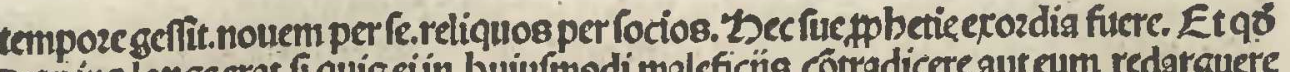
Gfumpfiffer mor cum rel per ferel per finos pzoditozie ingullabat. ficuti (quod certitith eft) fenemn quendáuindeum in lecto oommienté ideo pzeter ceteros fecitiugulari. quia fe

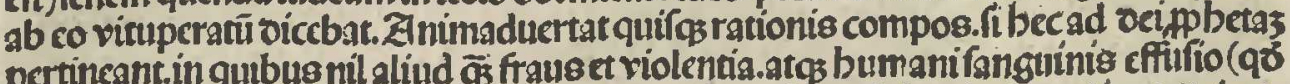

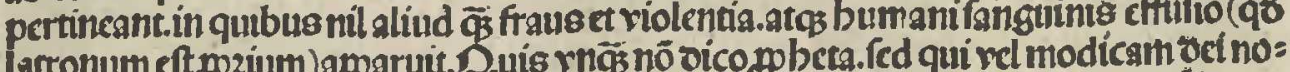

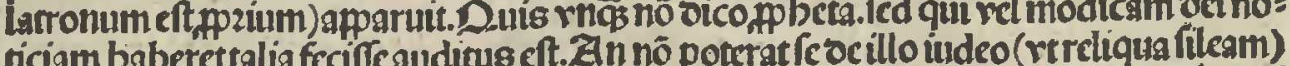

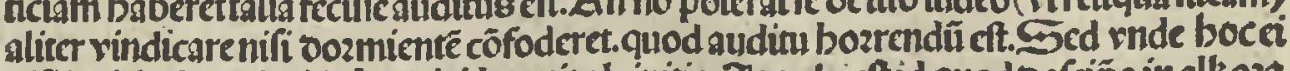

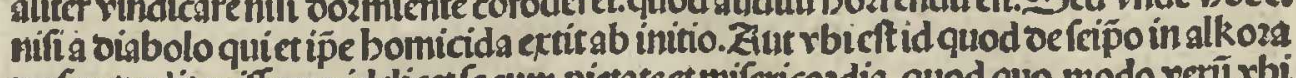
no fino traditit.miffrum ridelicet fecum pietatcet mifercicozdia. quod quo modo verü rbi

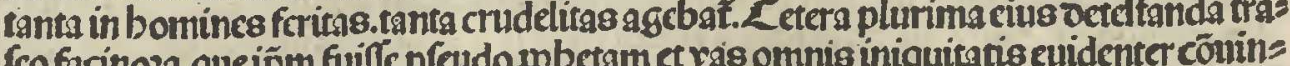
cerenc fi pre ocio in medium adducereliceret. fed rationé babendam pio lectozi cenfui magis. bo oc rnum cx multis relim adicctũ. ríf pzofecto oignum. ox cum ipe quadam

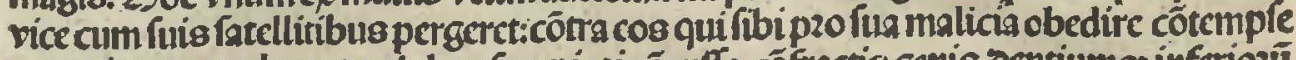

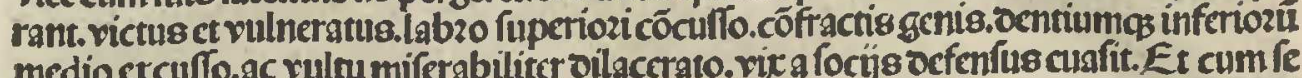
(a)

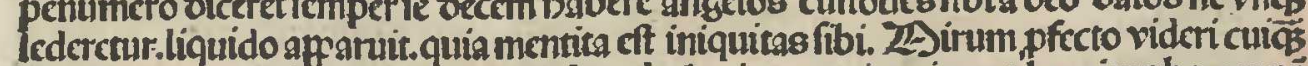
poteft.quo pacto is sci nunciusetpphera baberi potuerit.cuitus th bzeui rerbo omme

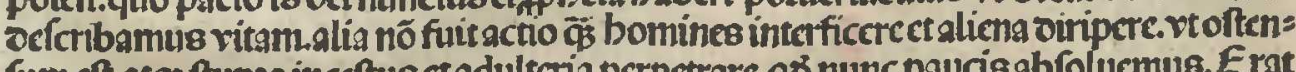
Inmeft.stg ftupza inceftus et adultecta perpetrare.qo nunc paucis ab folucmus. Erat

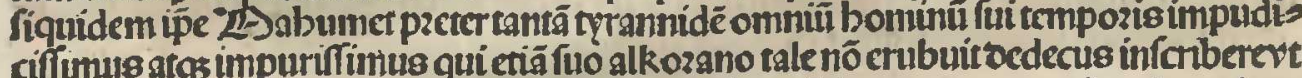
Diceret (ficut patectegenti) talc o onum a oeo renibus fuis oatum rt quadraginta viros in coitum quantumlibetpotentifimos pof fet adequarc. rol quogs inter cetera rrbus fe

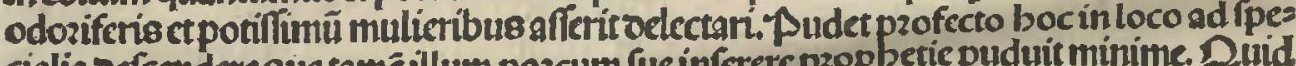
chalia oefente cher

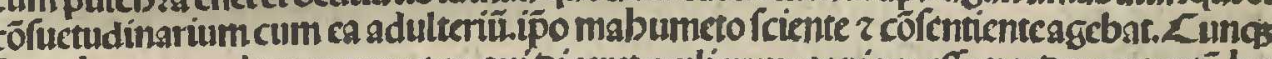
fuper boca quodam argueretur.qui Diceret mulicrumn copiam effe.nec decere tantü bo

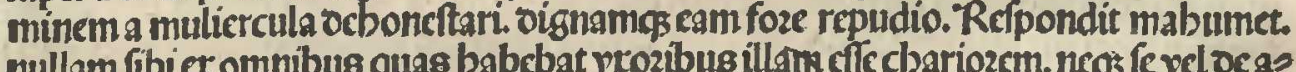
liazum in alkozano fuo cam affirmat innocentem fuiffect boc fibi Diuinitus reuelatum. $D$ e bu

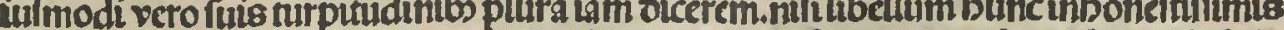
recbis fozdidari formidare.per ormma fimilis factuseft equo et mulo quibus nô eft in

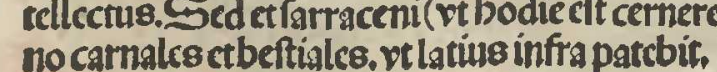

$$
\begin{aligned}
& \text { Detempozestmodo quo mabumetfectam fuam } \\
& \text { incepit. } \\
& \text { Zerciumm capintulum. }
\end{aligned}
$$

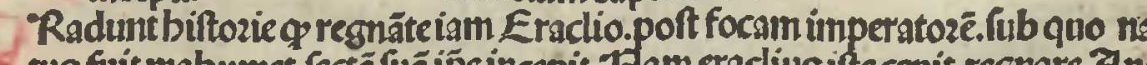

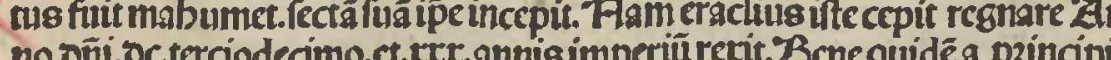
ho

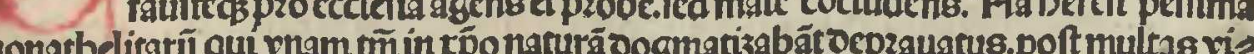
ctozlas z gloziofos triumphos (oe quibus ctiă partim in biftozia (anciecrucis babet)

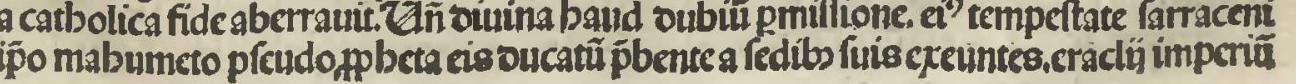

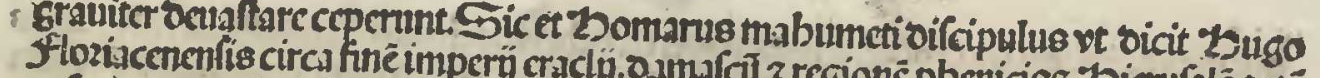

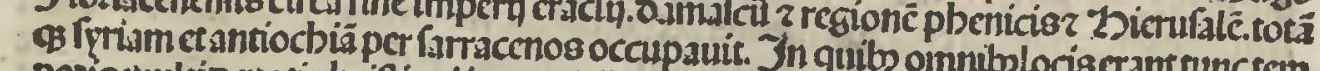

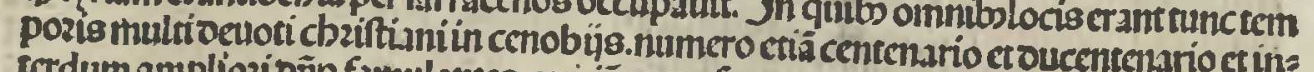

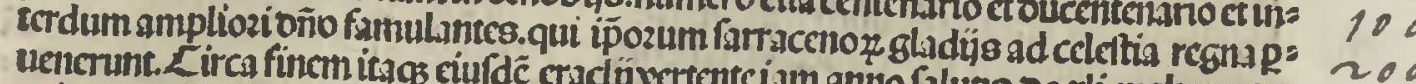

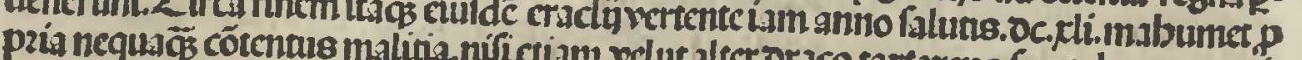

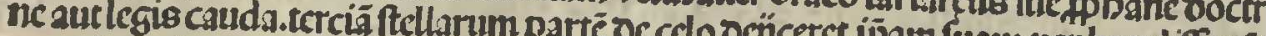
ctam inccpitatc auctozc obubolo perfecit bocmodo. 7 .

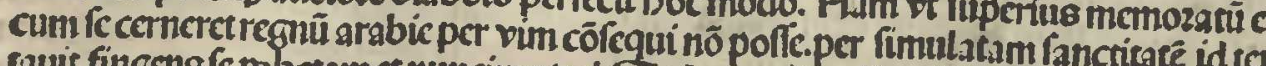
ioi

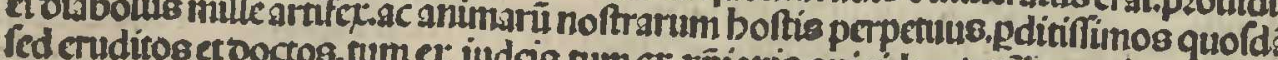

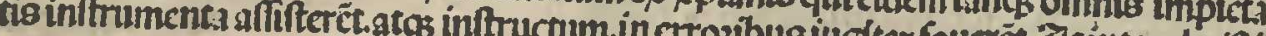

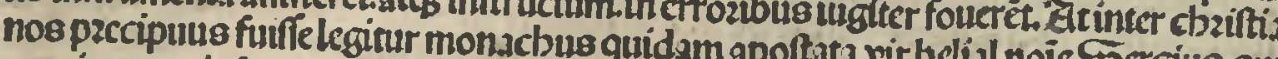

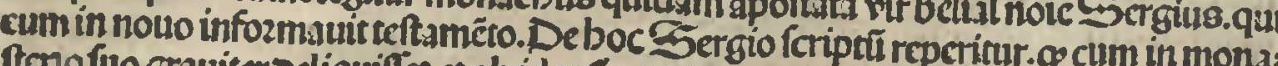

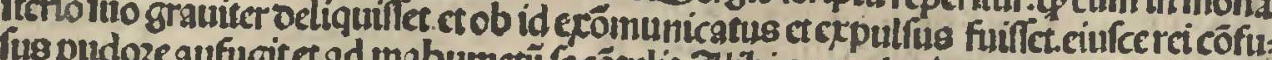

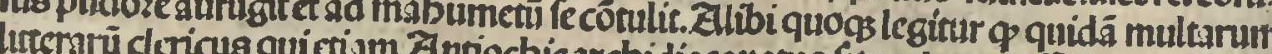

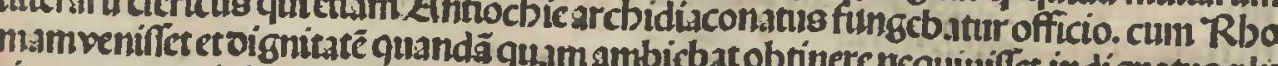

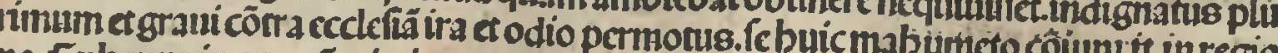

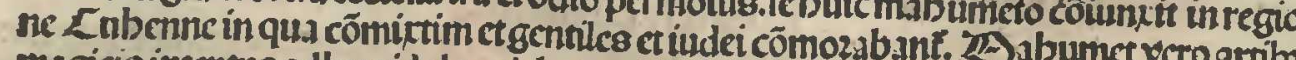

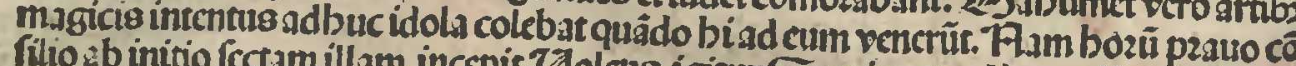

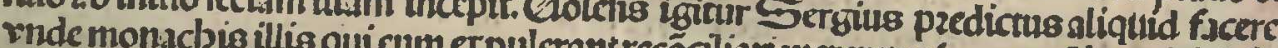

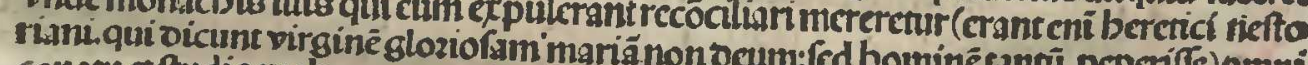

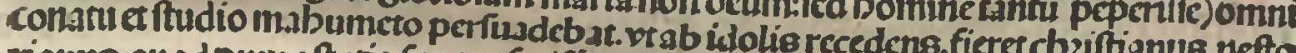

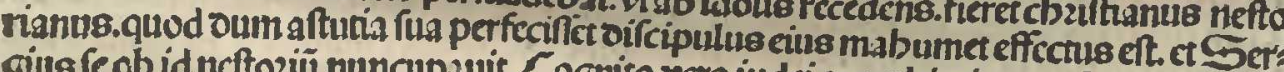
gatls lecbidn fea per mabumetũ effectü eft per í̄m neflorium

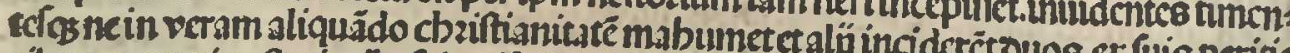

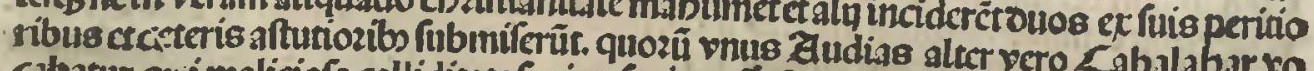

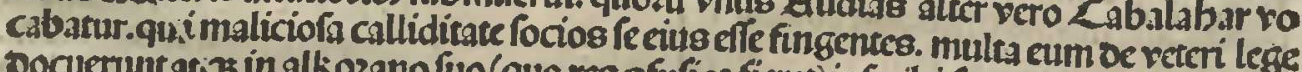

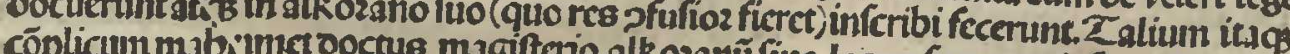

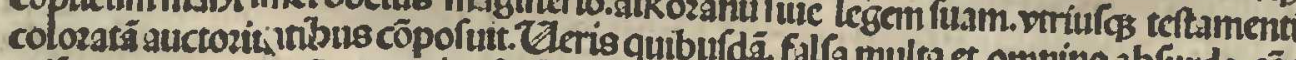

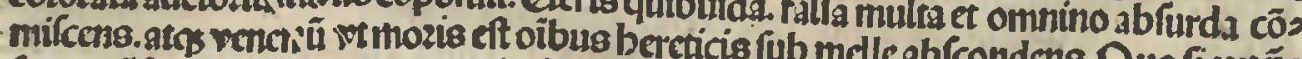

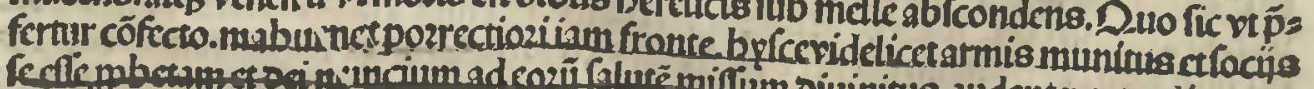

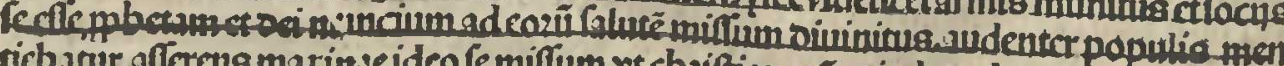

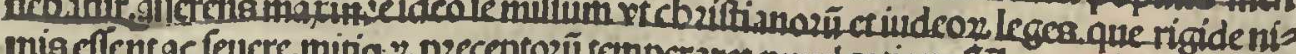

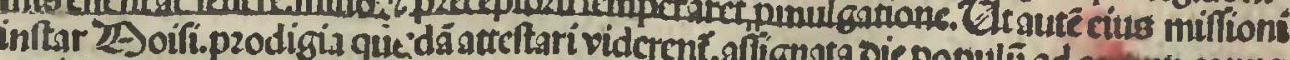

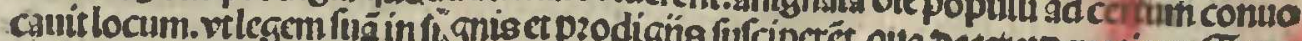

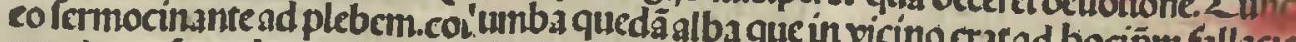

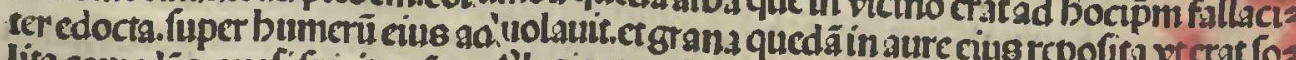
rus \& 3 ferur fimulinducrue

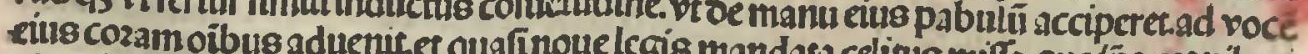

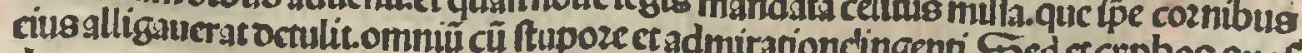

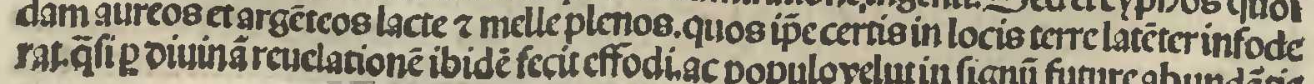

rare Tarkorno,

Sunging 2ior Bro

Anstiofimong Aso

Donara Crrifíam

Alcovang

2Chriphisonar

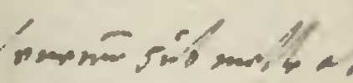

Prochigin

Unfoummet 


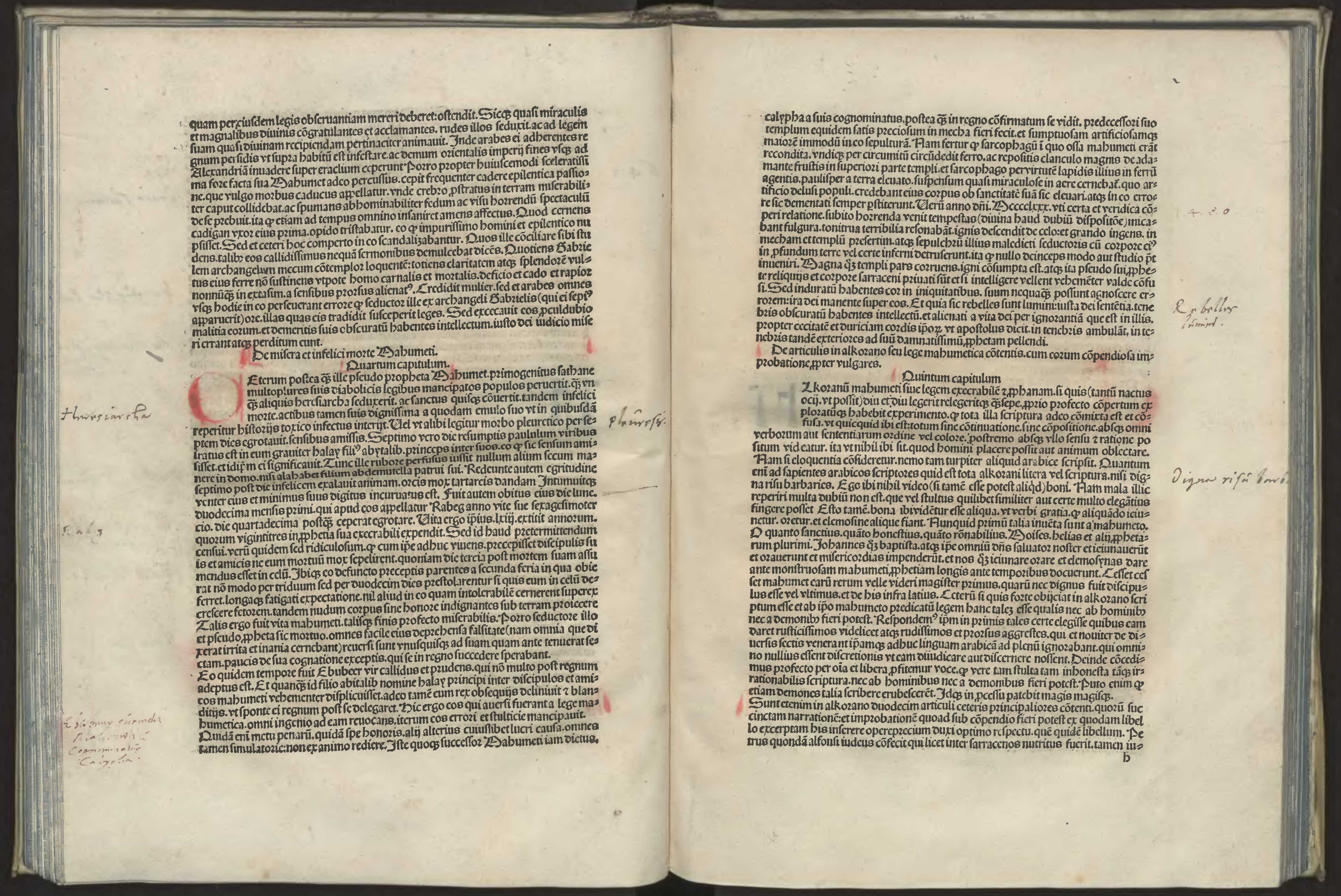




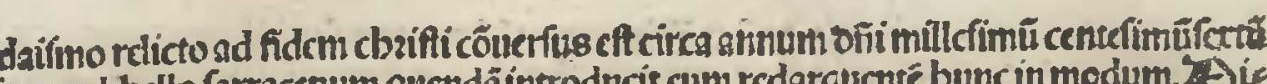

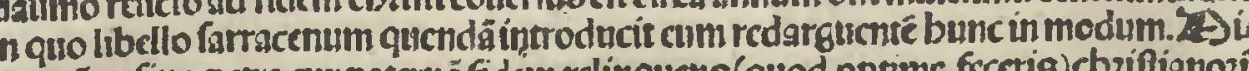

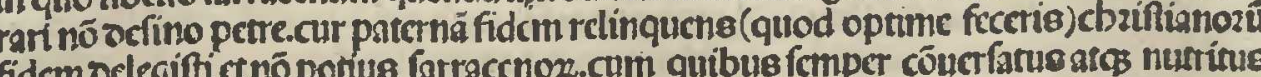

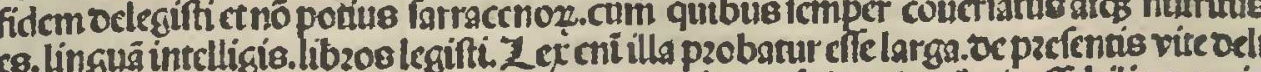

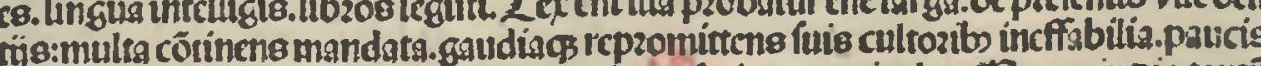

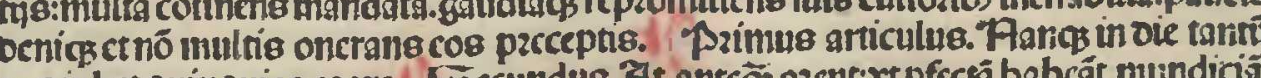

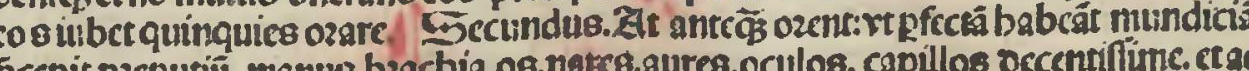

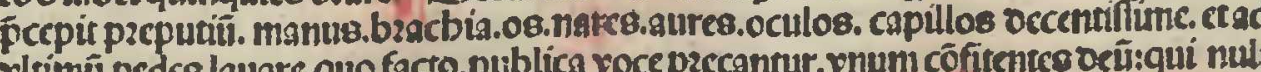

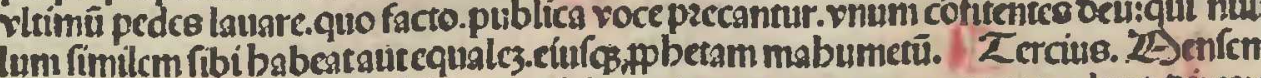
pzcterea integrum in anno cicunantet iciunantce nocturno tempore comedunt oiumo sbflinent. Duartus. Scmcl surper fingulos annos pzopter folam recognitioncm

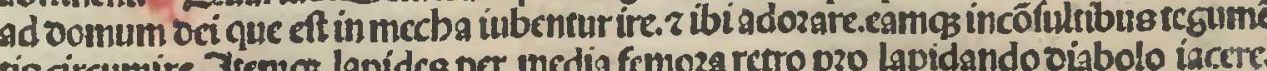
niscircumire.

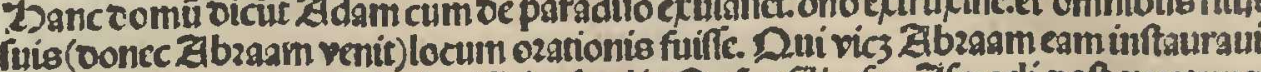

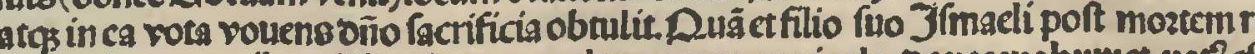

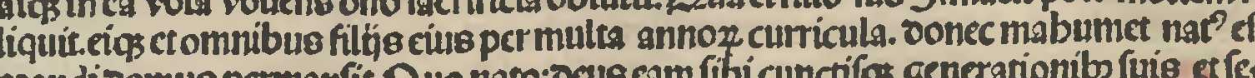

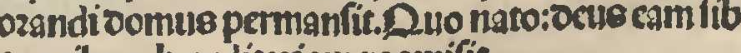
Q ilacibus. betreditariam p2omifití.

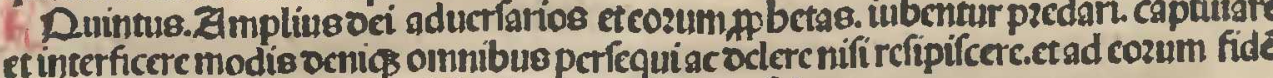

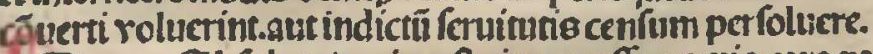

Setris. E. broluta di

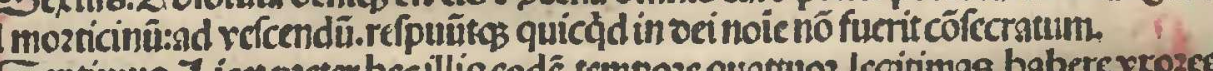

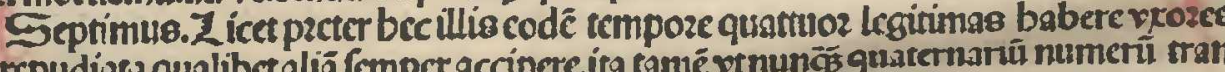

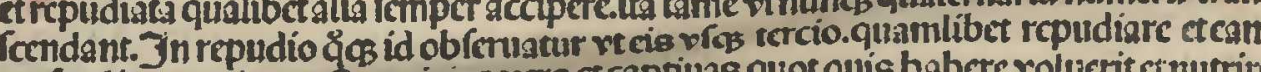

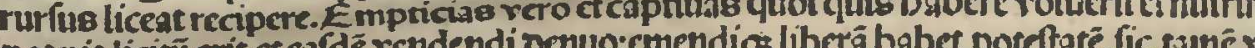

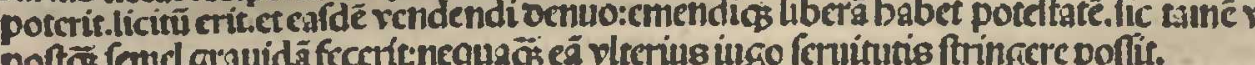

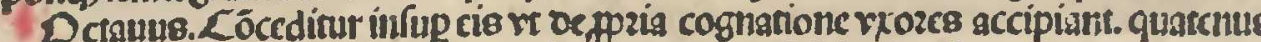
fanguinis fui pzoles accrefeat.ct foztius inter cos amicicie vinculum rigexh.

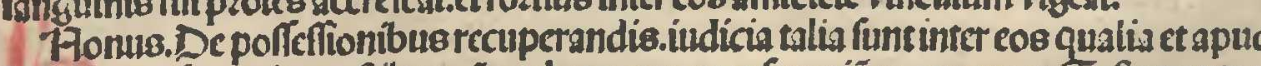

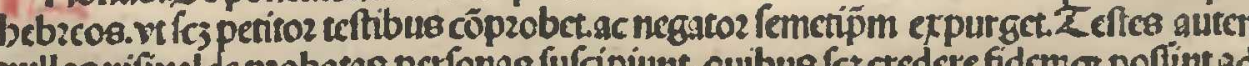

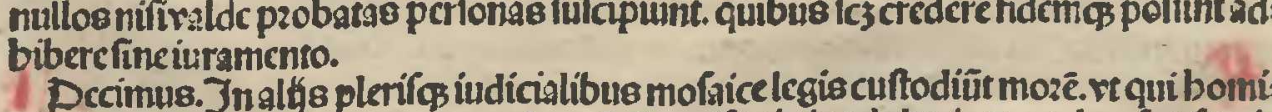
nis languiné fuderiteadem pena plectat. El quirquis inadultertio ocp:cbenlus fueri

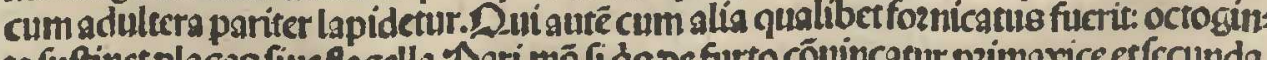

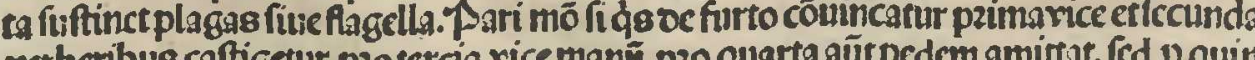
retbertbus caftigetur.potctela ricemant.p2o quarta

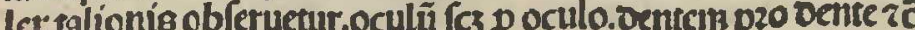

andecimu8.a vino femper abftincreiubentur.quontam feminariú etfomcs omnis

peccaticlic probartur.

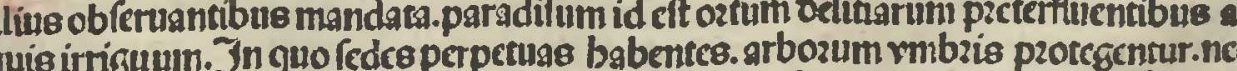
aloze affiligentur nec frigoze. Ied omnium cibozum et fructuum getheribus refcentur-

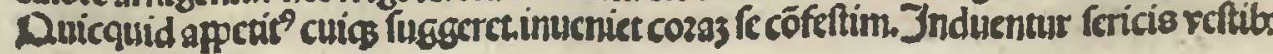

Ommi colozibus.in oclitīg accubabüt.current angelipincemarũ minifterio inter

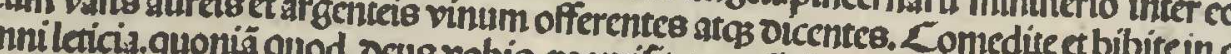
Pccionthis quas nec bum

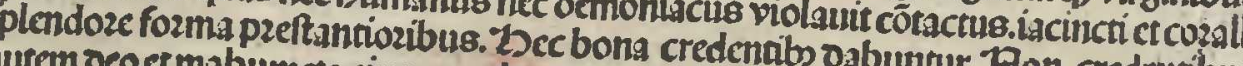
a secredideris ípo inter

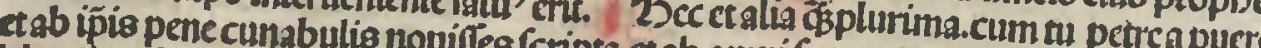

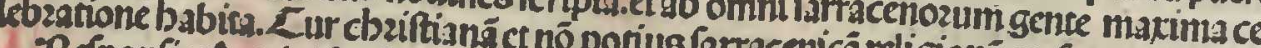

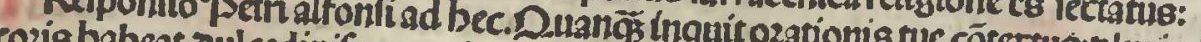

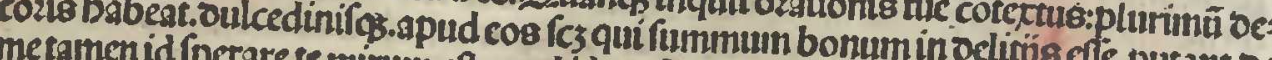

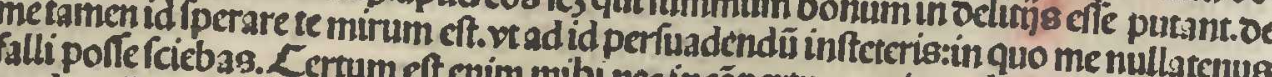
modo callida fe fimulationctum

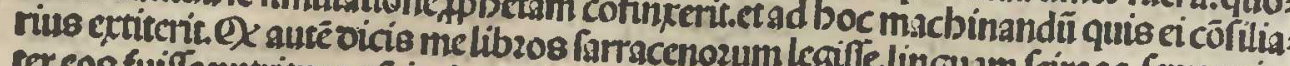

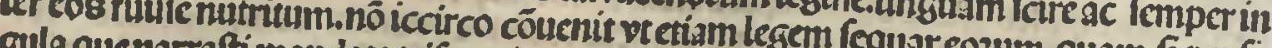

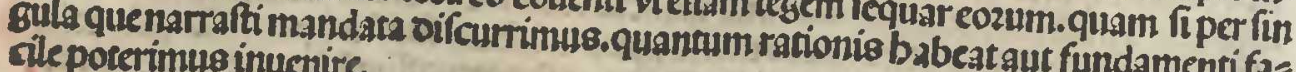

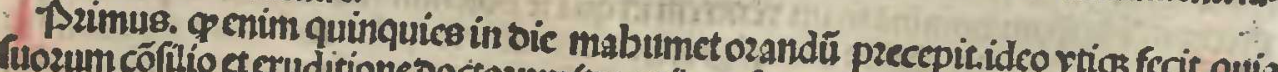
inter iudcozum et cbziftianozoülozem (oe quibus fupza imentio facta eff) mediancricem

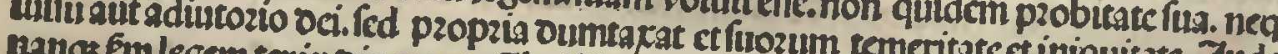

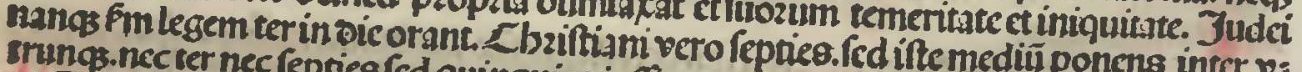

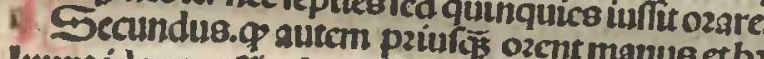

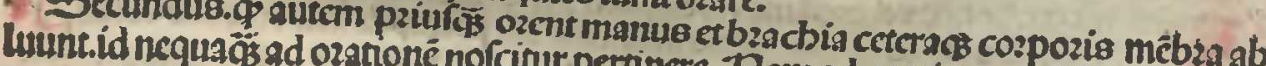

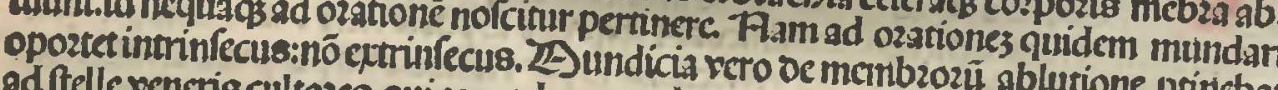

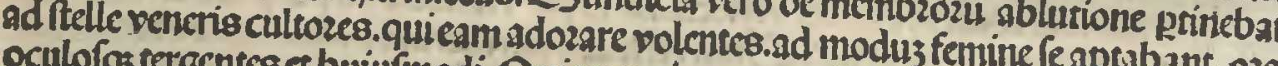

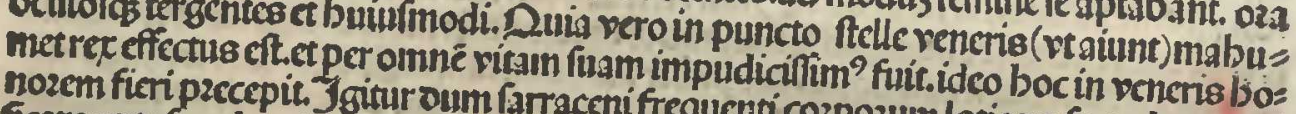
ficarcapte ranc bocillisoici poteft quodet iudcis fimulia facientibue fecredunt pur

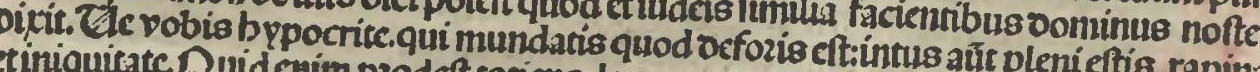

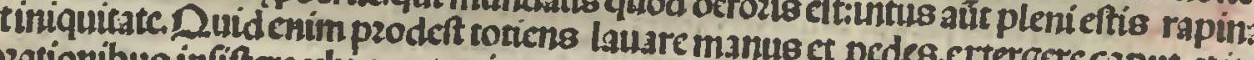

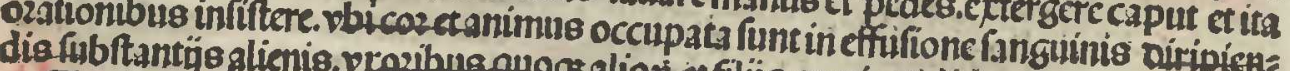

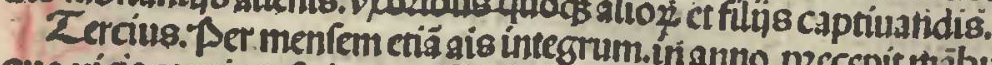
quo vicia carnis reftringant. Sed oic quefo nibiouid pecpit thäbumet fitos iciunarc:

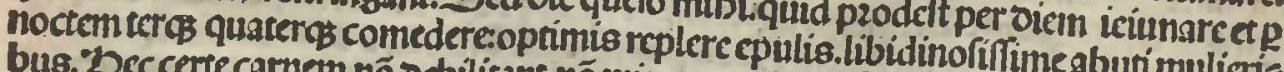

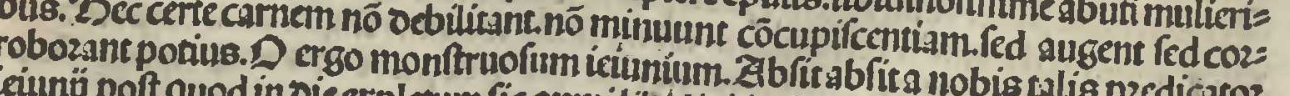

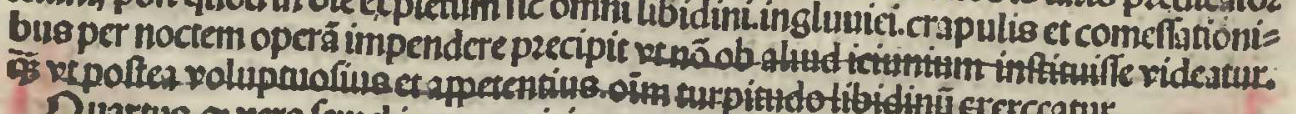

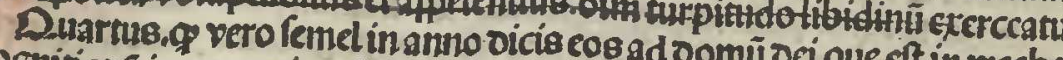

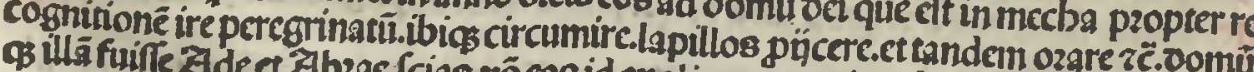

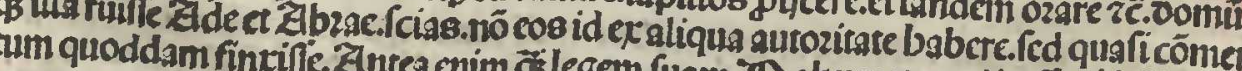

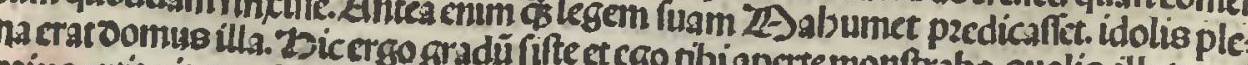

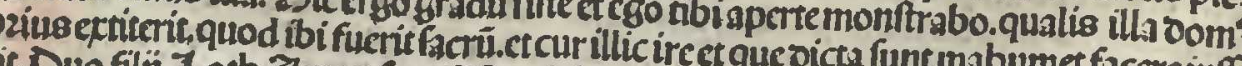

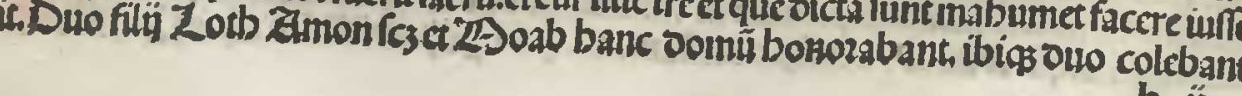

Mafomin 


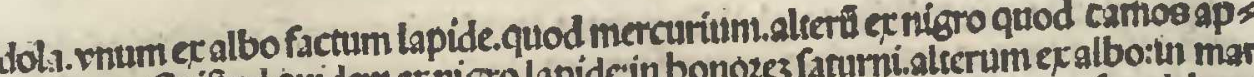

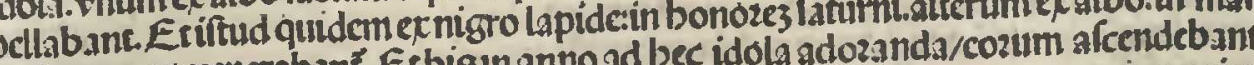
tis bonos

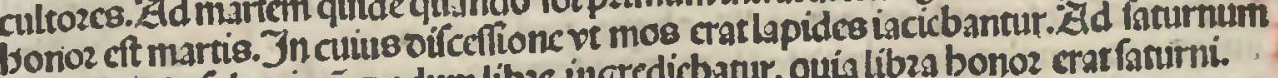

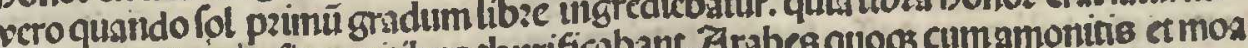

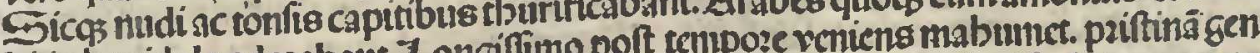
bitti sec idola ado tiscorectude

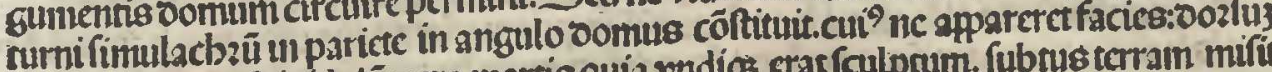

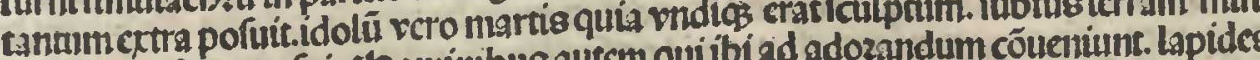

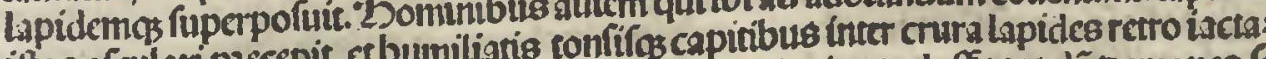
it os ofculari przecpic. ch re.quiter

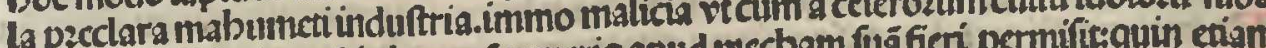

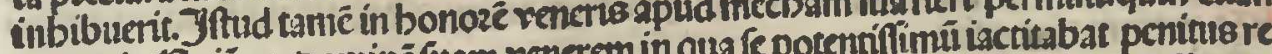

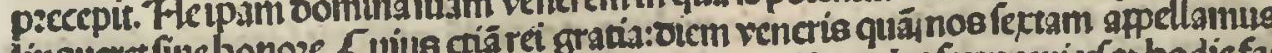
linqueretfin

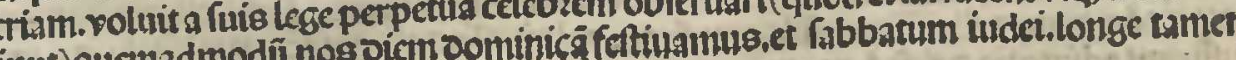

alia cealia ratione.

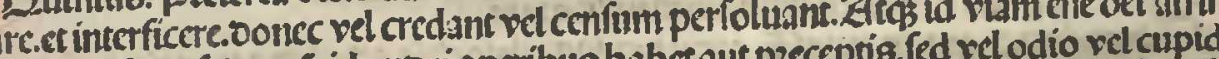

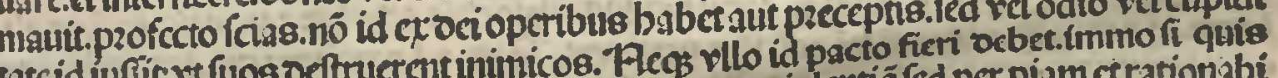

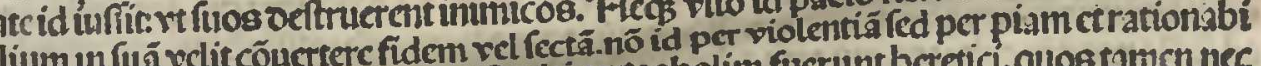
em informationem facere ocber.

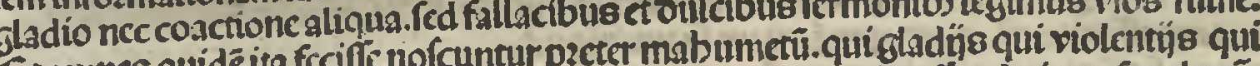

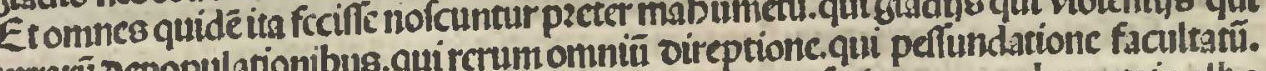

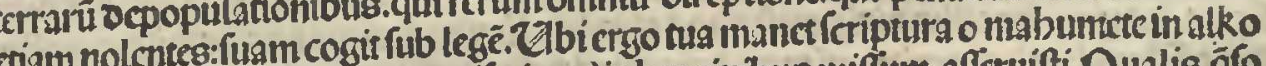

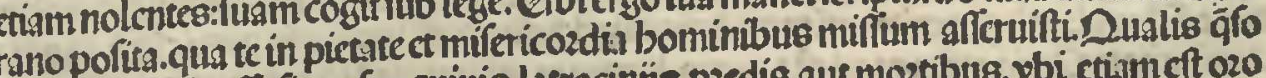

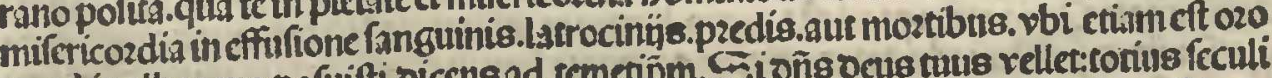

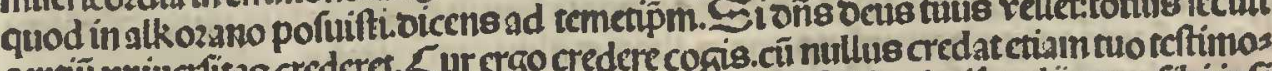

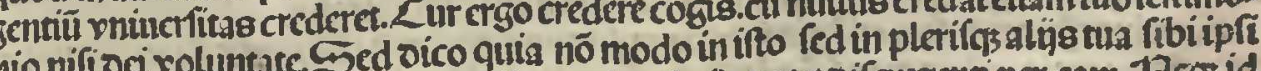

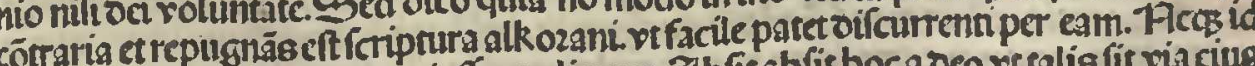

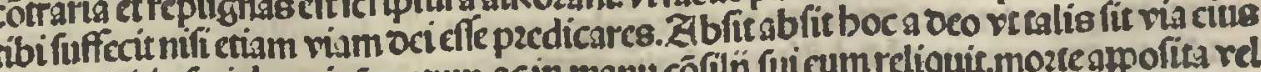

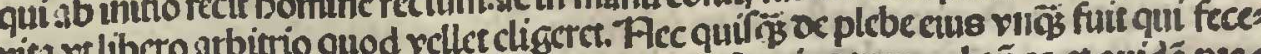

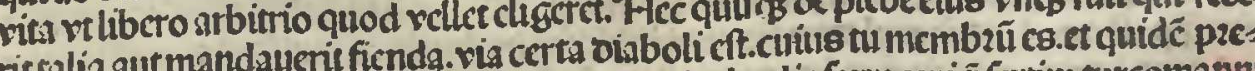
(a)

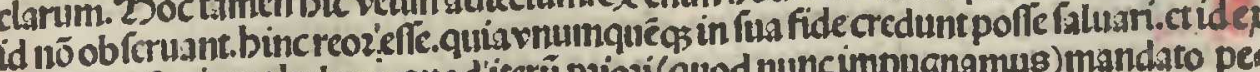
nirusett aducriun

政

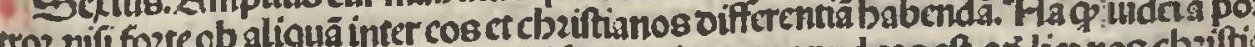

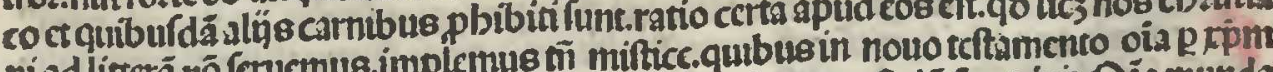

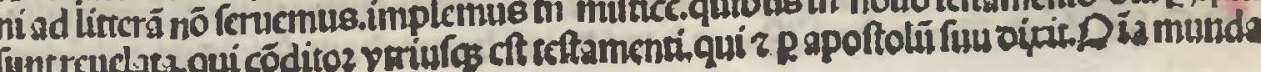

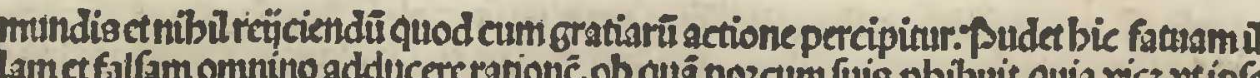
ind

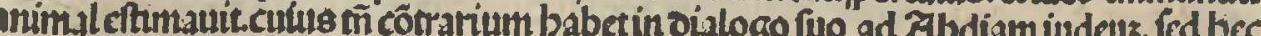
quonia fabulohapzoz (us funttrantico

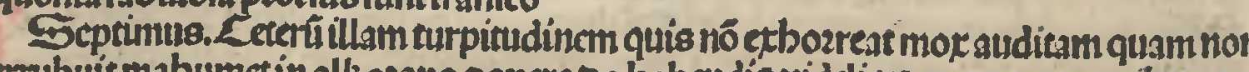
moetcodétrepudiarsozano ponere.oe babendis videlicet quastuoz veozibus tq

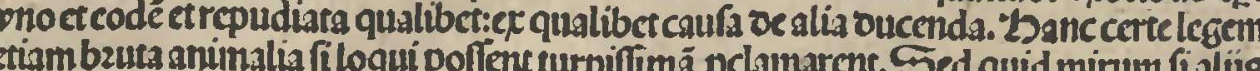

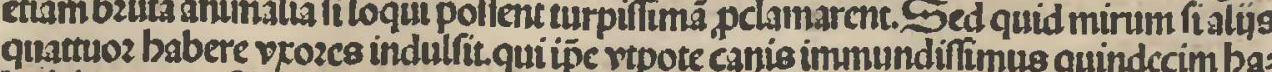
buitingenuas. Ancilhs vero etcócubinas fine numero. quas omnces selotipiesmants

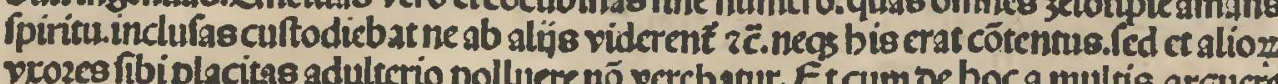

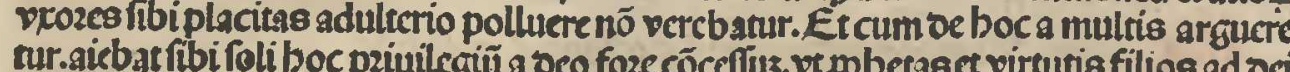
cultum kozano legenti. Ox ctiam empticias et captiuas quafibet et quotcūos babcrcemerc oै

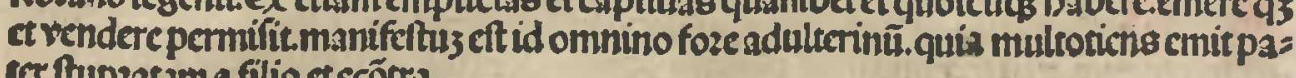

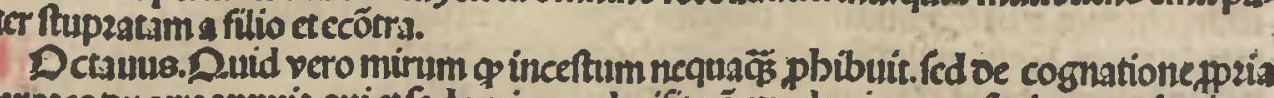

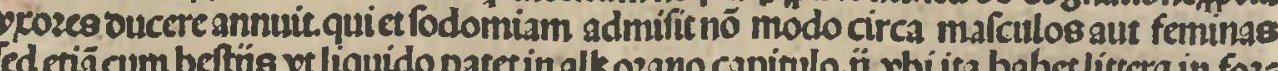
ma. Lótineasis vos a muliertbus in mentrutis et nösccedatis gd cas oonec mundent.

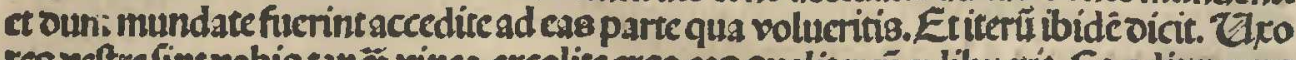

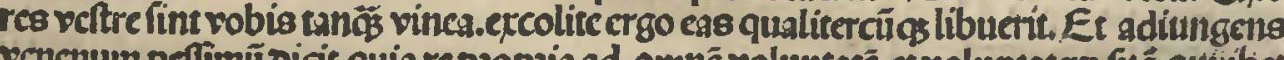

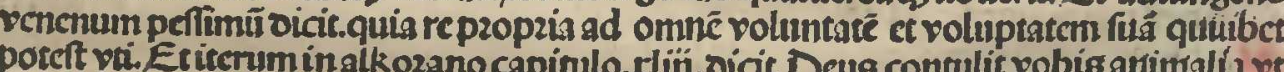

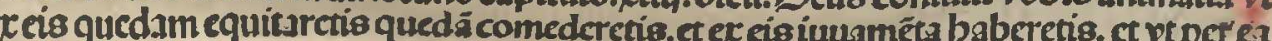
etequereminies que in co2dibus veltris ponerctis. Extlicet vos farraceni tectus iffos

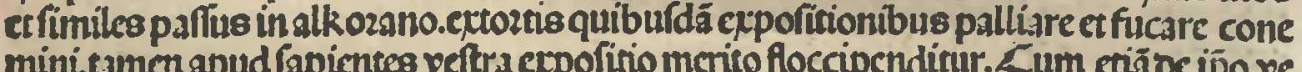
(itre feduccoose legatur

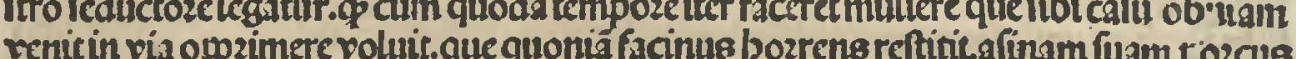

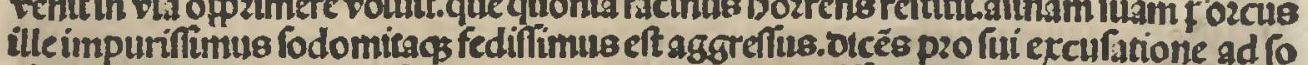

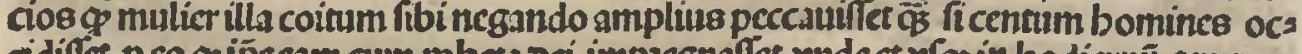

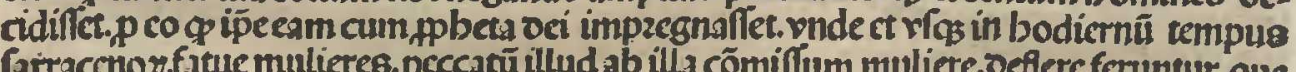

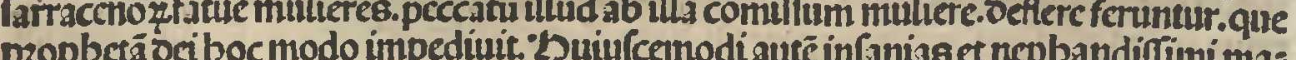
bumeti veftrif lagicia fatcoz ob boneftatem et fitli verccundiam filentio tranfiffem nins

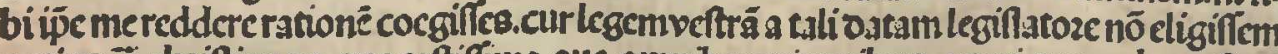
potius ö cbziftianop.que caftifitima.que ommibus virtutibus. omni quogs boneftare refcriftima.

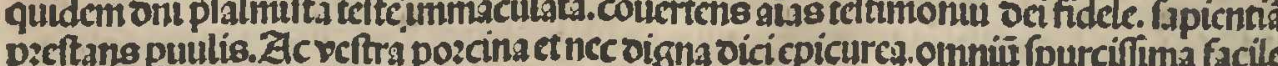
coprobarur dinofcitures. Jamg viltra perg.m.

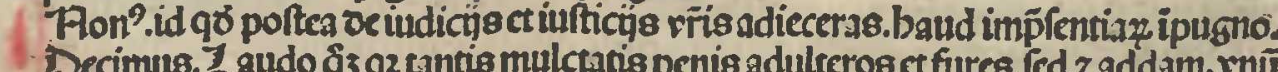
Decimus. Zaudo â3 q2 tantis mulctatis penis adulteros et fures. Fed zaddam. rni

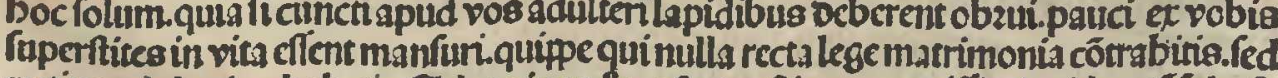

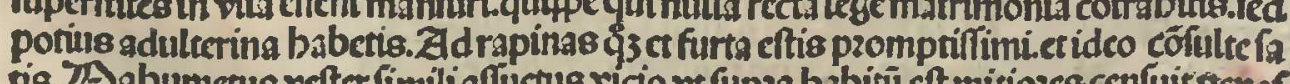

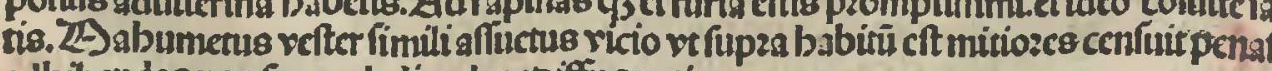

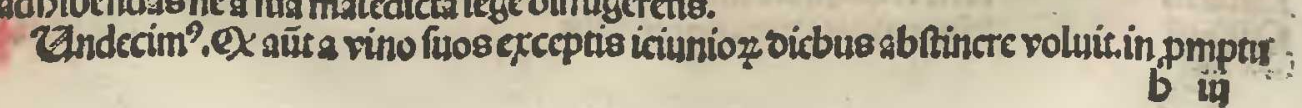


caufa eft.qu etregio ipfa veftra calidifing omnino fit et ob id frigida appetibilioza $\mathrm{ma}$

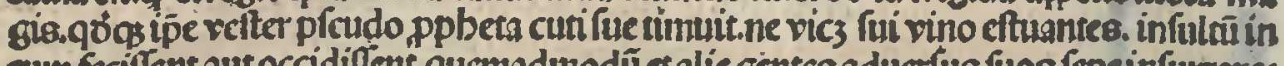

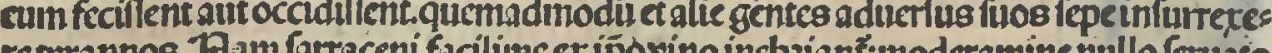
retrrannos. Halch

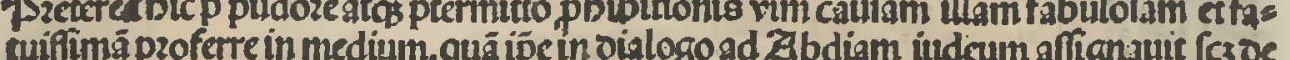

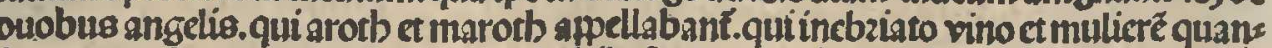

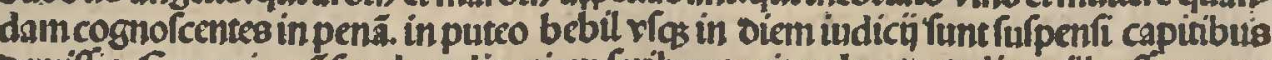
ocmifis. Egoenim cófundoz tslis etiam feribererecitando.que oclinusulleafferereve

Duodecimms.ita ef que oe paradifo pzedicauit vana omnino funtnegs vlla poffuns barivt infra oiffuritus pascbit.

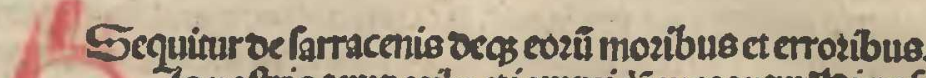

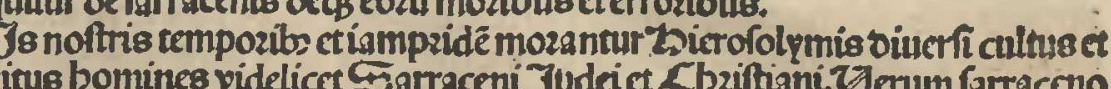
ritus bomines videlicet. Sarraceni Judecet Cbziftiani.ZZerum farraccno

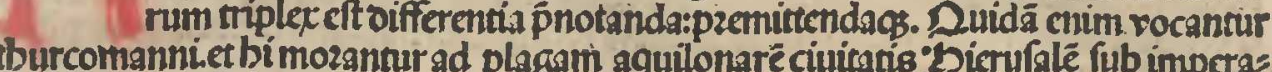

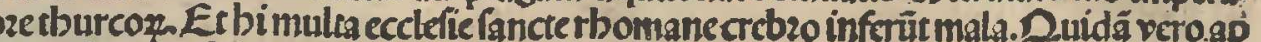

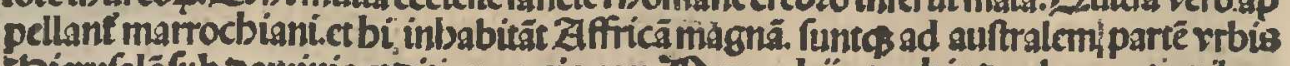

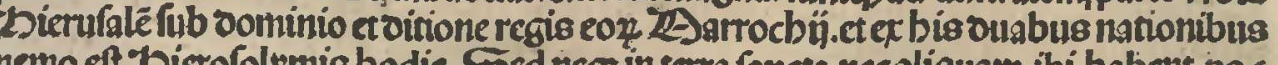
able

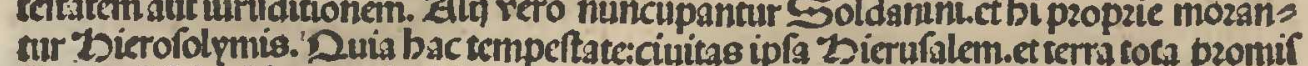
fionis eft fub oitionc regis foldani qui communice refidet in magno $\angle a g r o .20$ mncs

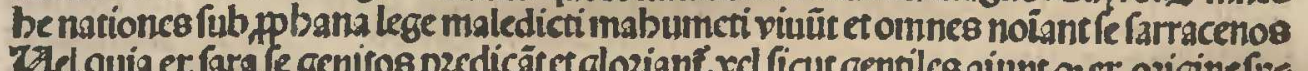
mun fint etagarenicenfendiponing

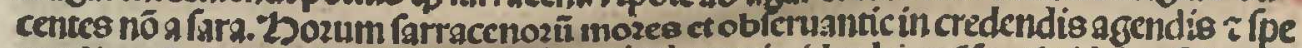
randis infertus Lare fatis babcunar in articulo tercio.ideo bictraffeo. bi bicrofolymis

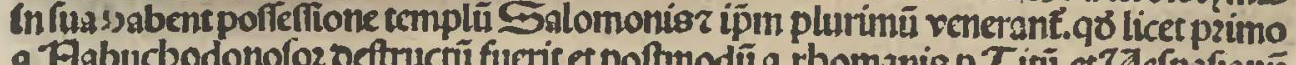

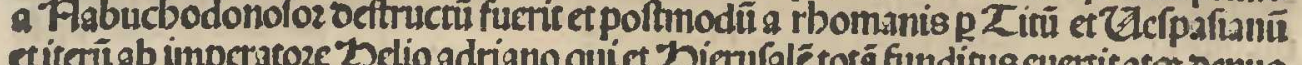

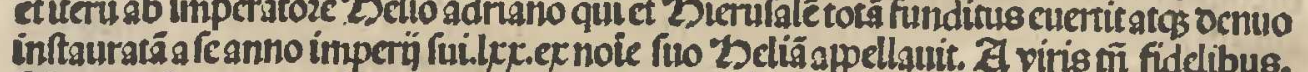

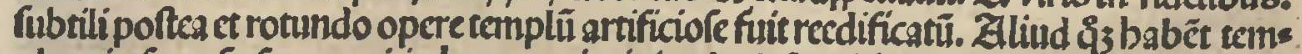
plumn in fito ylu arracenti in bonoze virginis gloziofe edificatĩ. in co loco, ppe templī Diu seuot fime

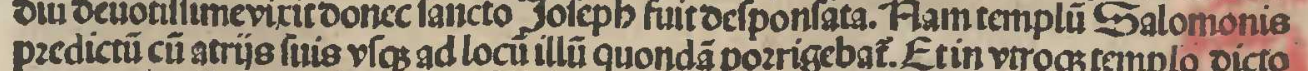

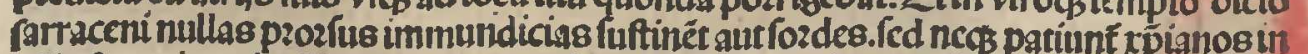

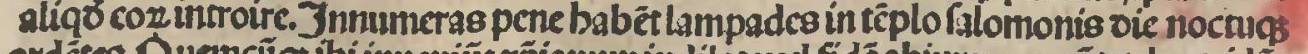

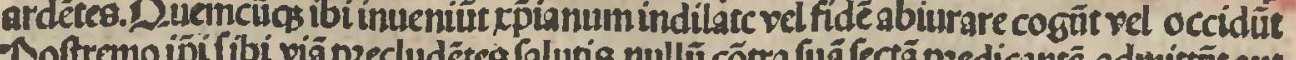

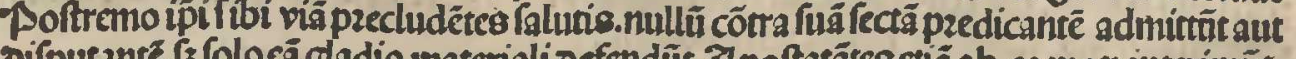

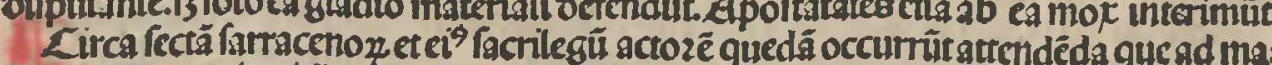
cainum | cius fecteinitio feu inclboatione.

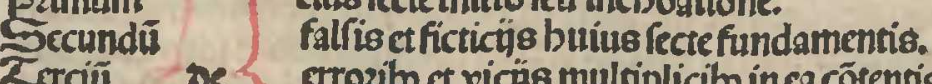

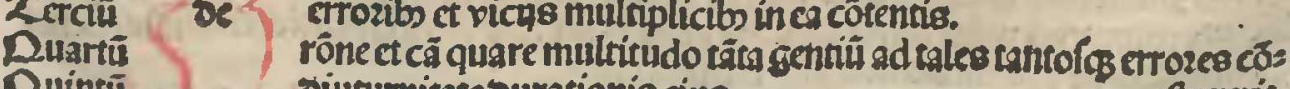

Jrea paimü fciendum $q$ licet omnes biftoziograpbi cöueniät in boc ptem porecraclin imperatozis circa finē vitefute.bec fecta nepbanda babuit initiü

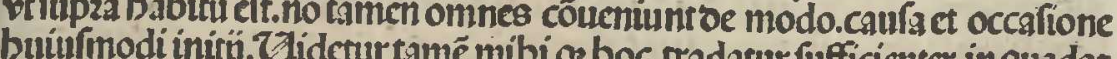
biforia ini mulucollegit ad boc pinentig. In

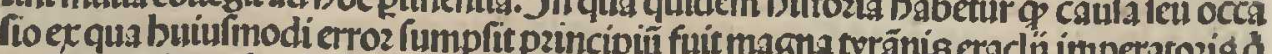

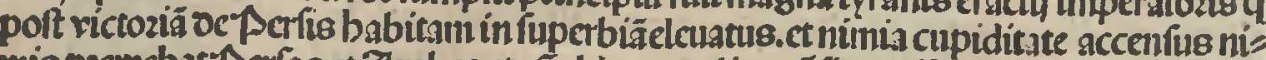

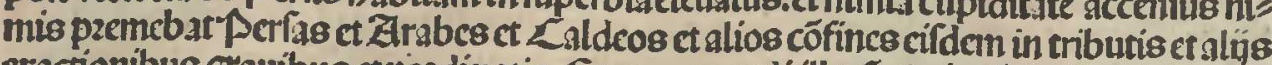
adrefiftendüe Gyrannidem et magnäpotcf tre etetiâmagicis arthous cruditus fe cis adiunris qui inuenicns oi pof

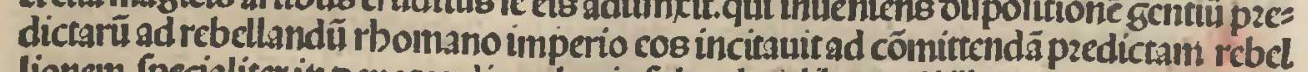

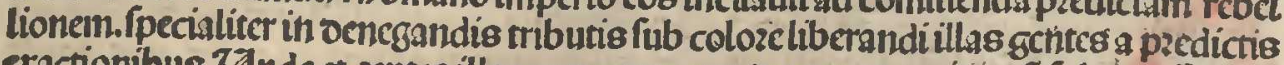

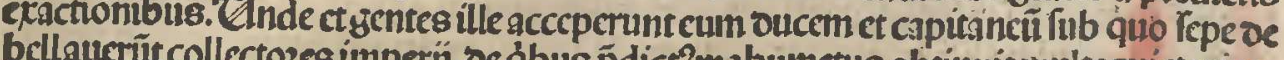

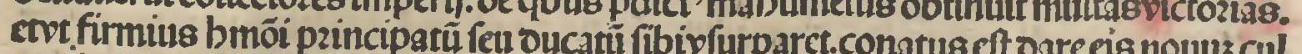

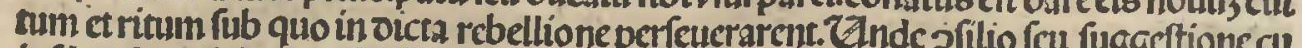
unidam beretici monacbi Sergü nomineaccepit quedam quafi pzima facramenta le: glo mofaicect queda cuangety cbzith be lupza patet we fic crederctur a rudibus popus

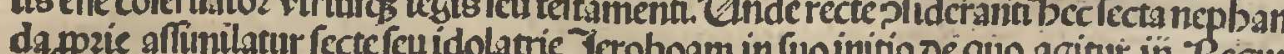

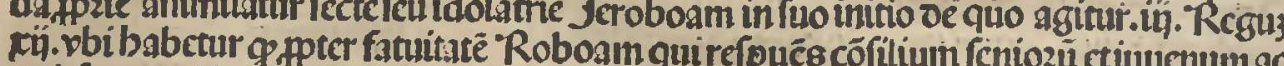
quiefcens ocliramentis minarus eft popthum aggraugre in fuis croctionibs - Jerobo

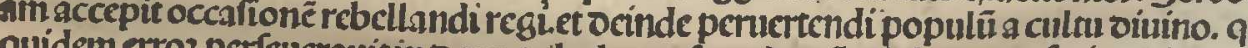

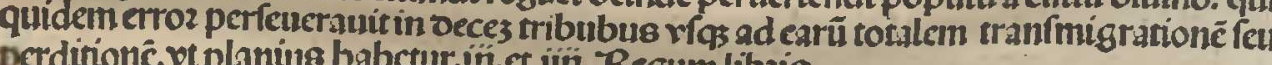

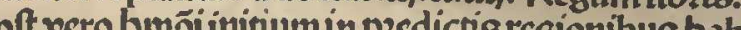
mote ad adberendi predictis fub coloze predicre libertarti metrealie gentes funt con ris caucafi egrefia cet gens pertida stra craclium q que quidem gens nobotbeswocitur

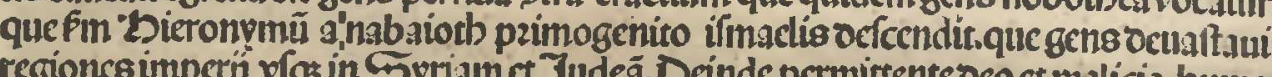

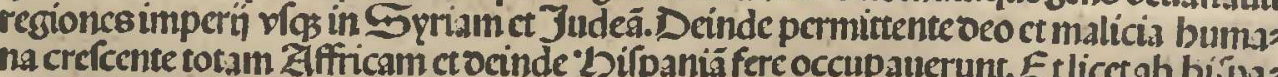

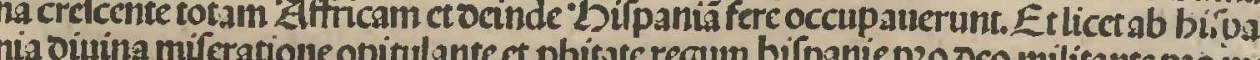
iozi partefint eiectiadbuc camen bodic ad excrciriīi bonozuni sliquar radie germinä

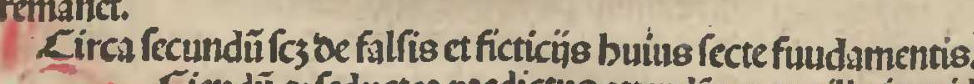

- Liendú $q$ feductoz predictus attendés $q$ tpeillo iam idolatria fuerat ab ozs

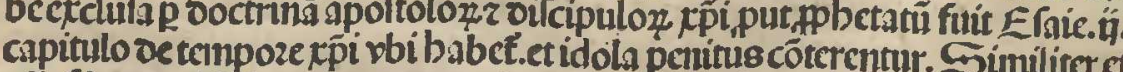

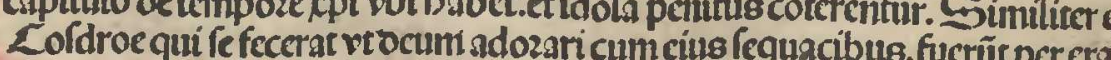

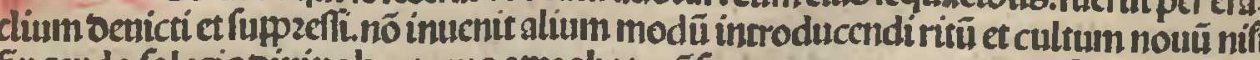
fingendo elc cels

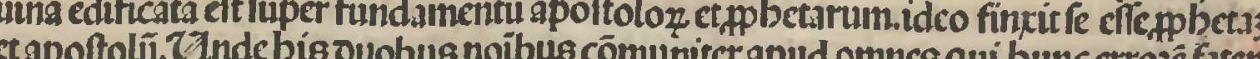

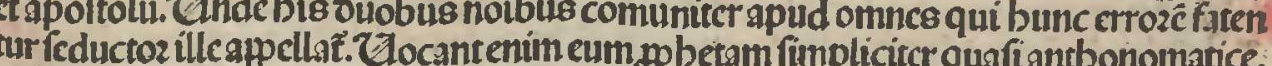

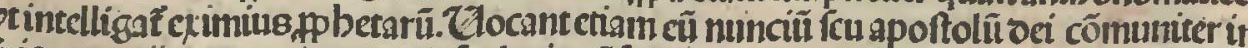

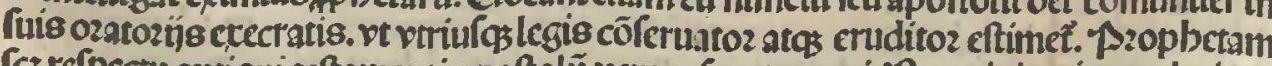
ç3 recpectu antiqui re q2 beftialiter viués oe gentibs et วgnationibs terrenis ad fublintem potentiä mundanä 


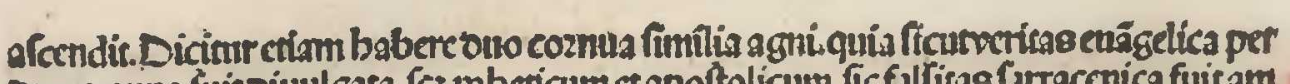

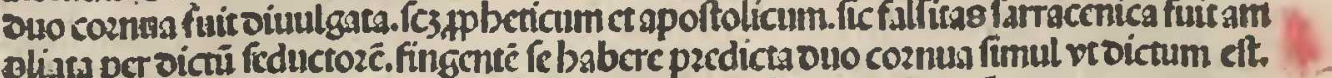

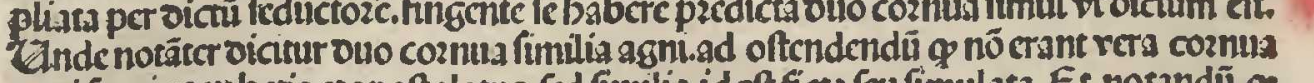

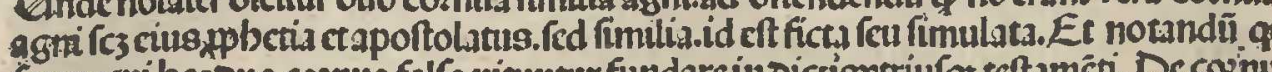

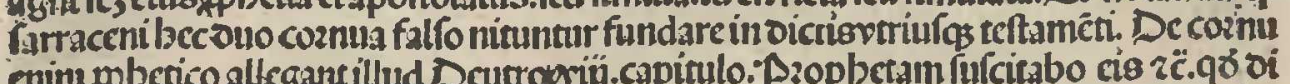

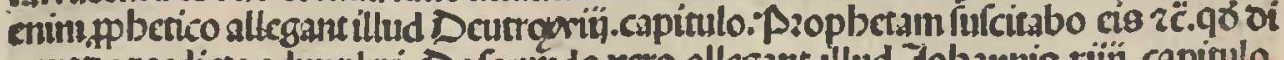

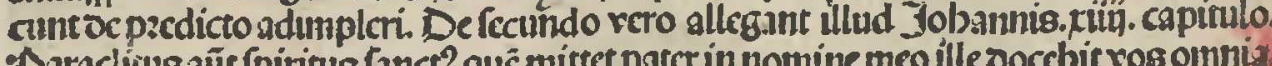

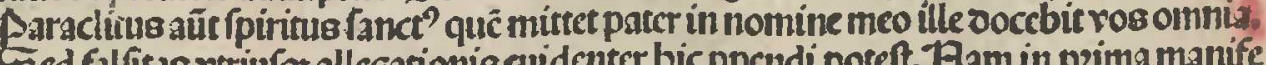

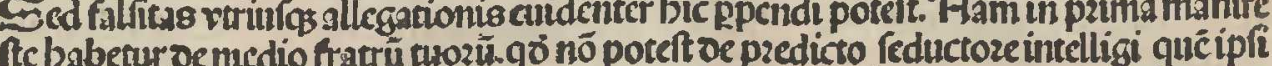

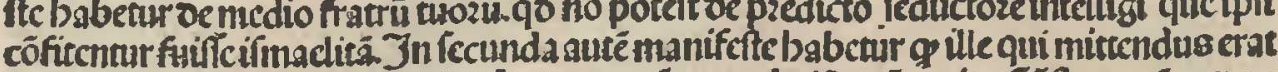

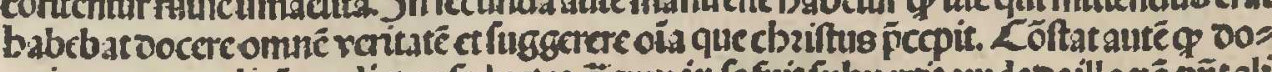

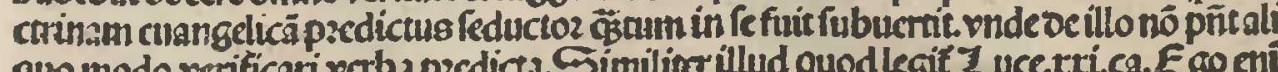

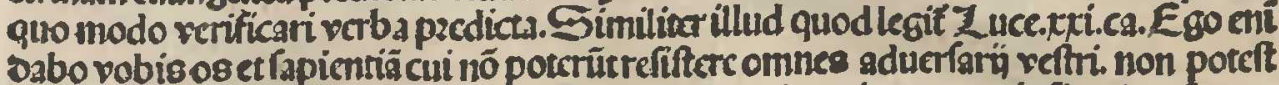

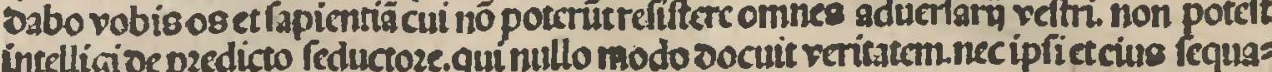

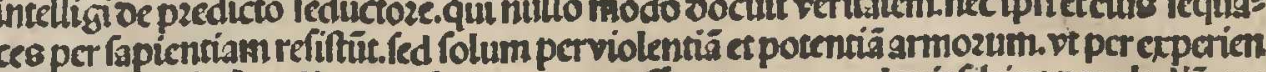

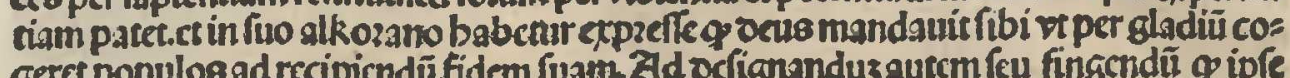

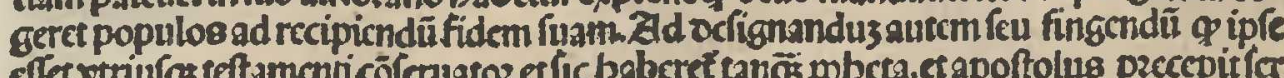

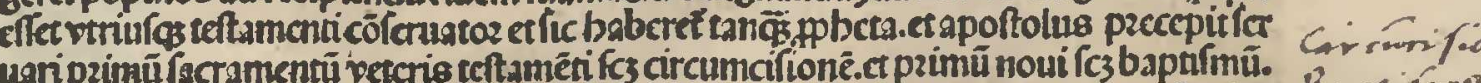

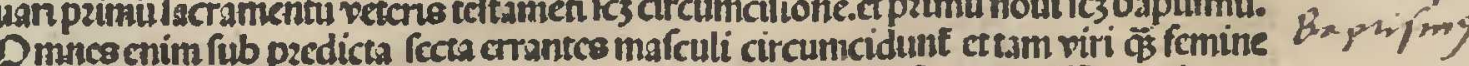

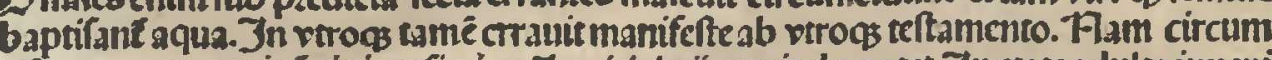

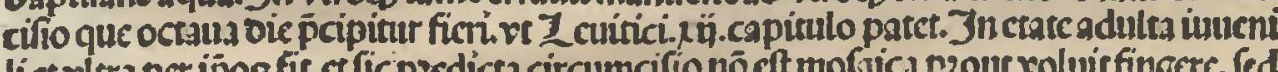

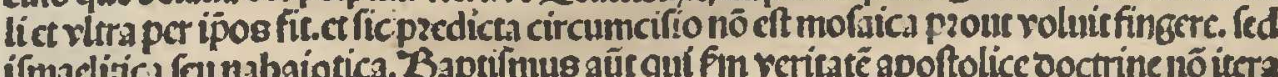

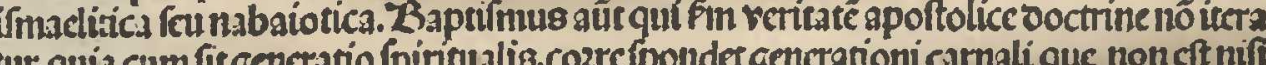

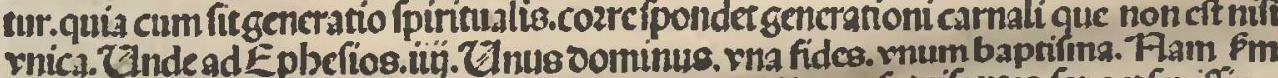

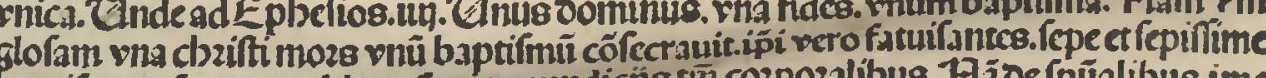

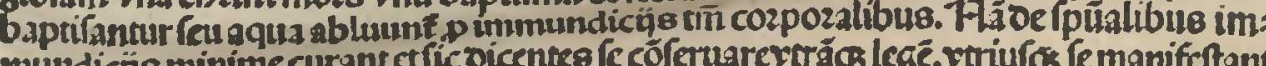

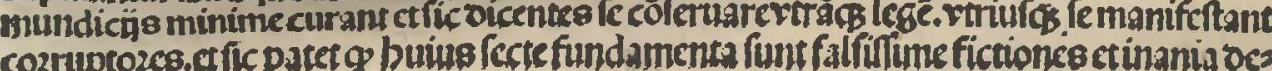
lirsmenta.

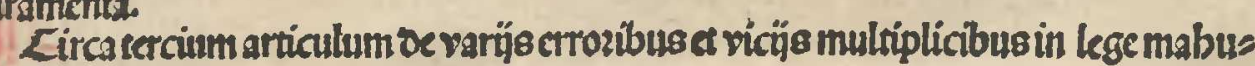
metica contentis.

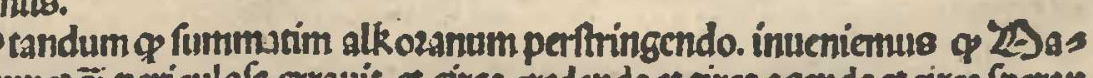

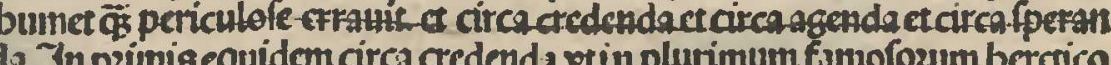
da. In paimis equidem circa credenda vi in plunimum fanto forum berecico

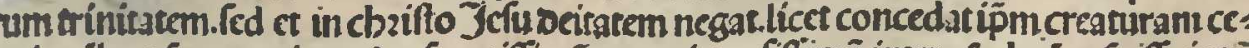

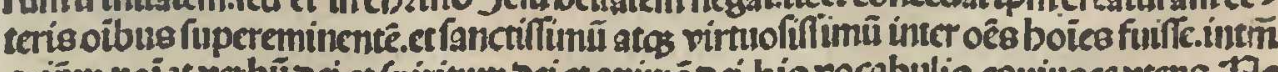

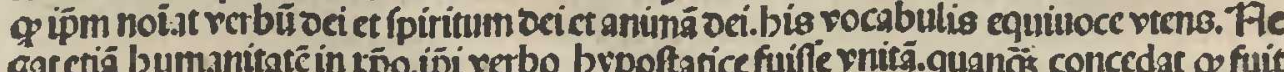

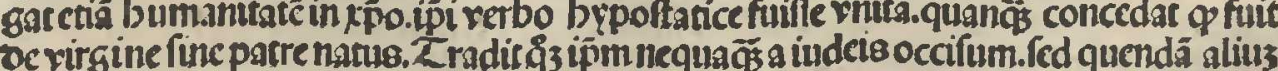

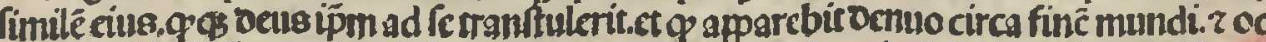

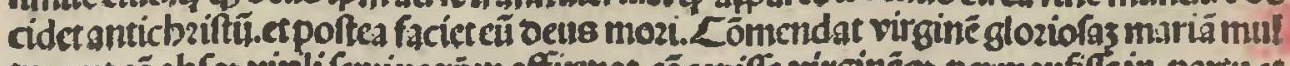

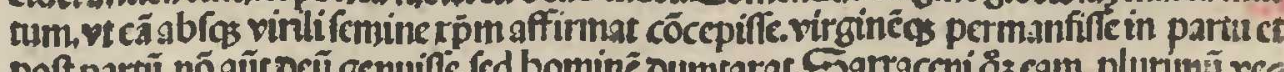

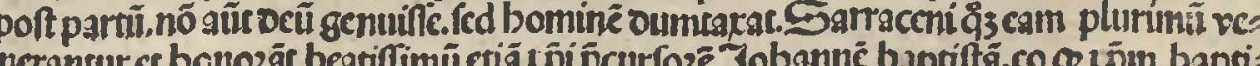

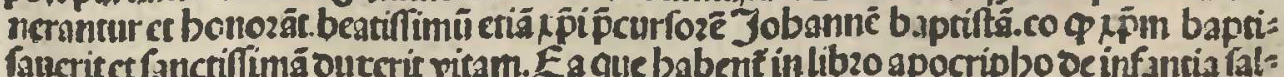

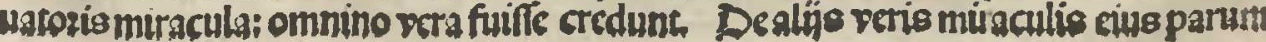

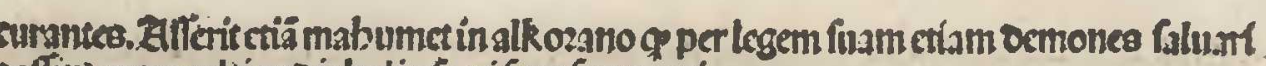

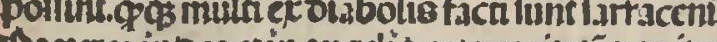

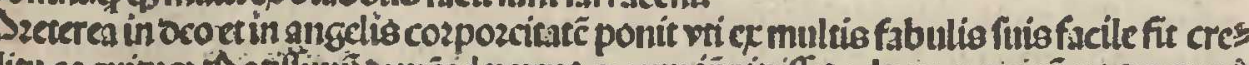

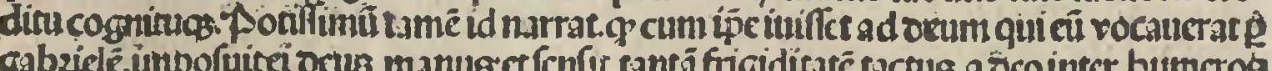

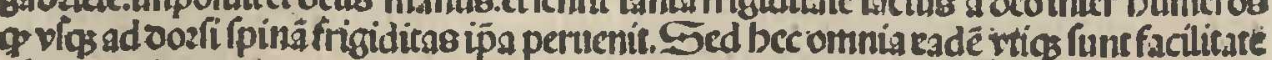
cortemnenda qua pontuntur.negs cnum aliquid babent ratomis aut fund.smentivep ore

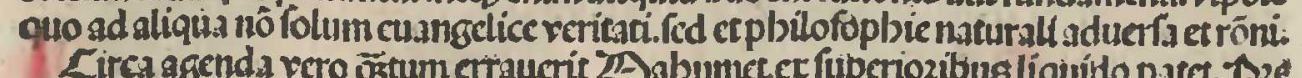

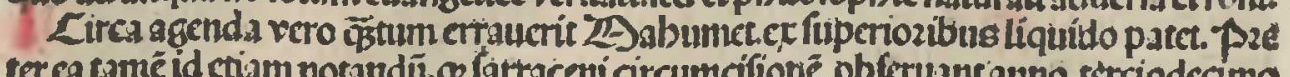

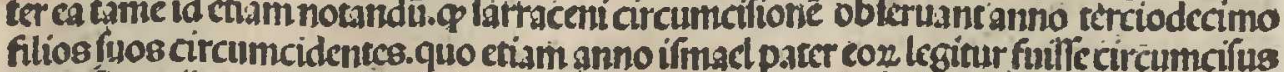

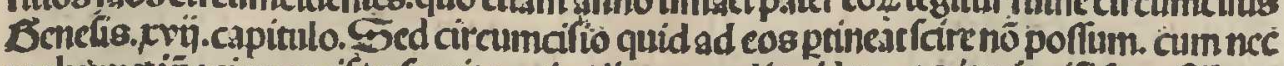

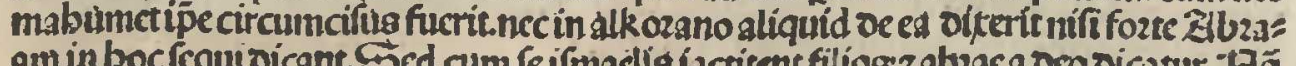

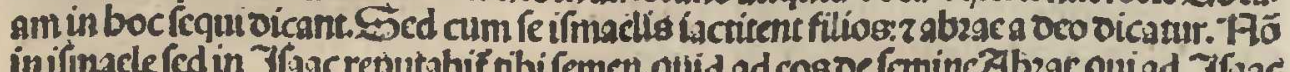

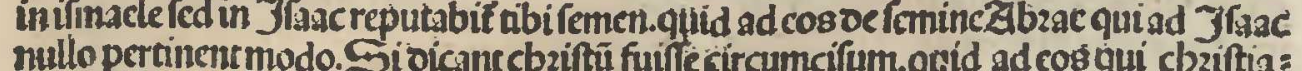

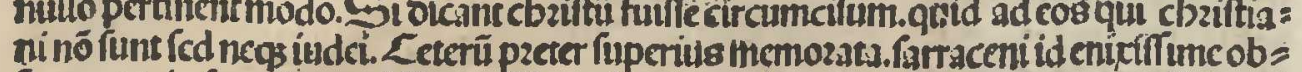
Ceruant

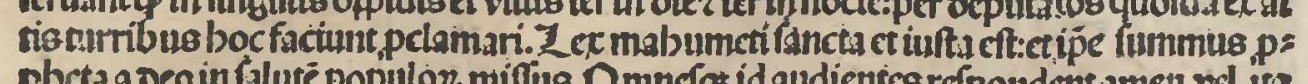

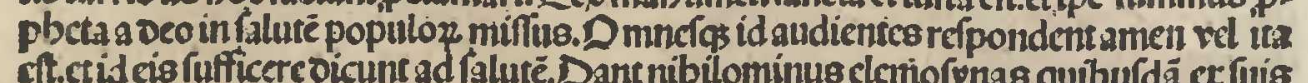
quos repurture fanctos $\mathrm{E}$ ad

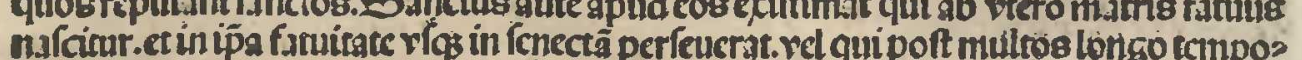

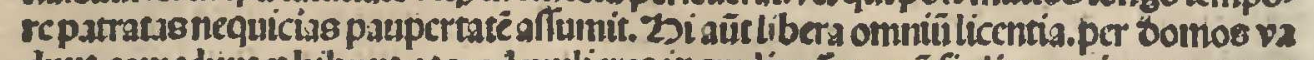

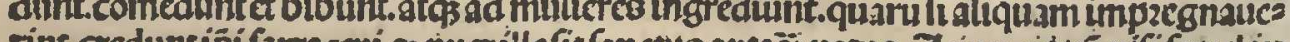

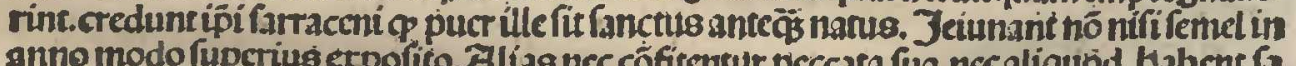
cramentum.ncc occimas oant.nec aliquas faciunt oblationce.nce aliquod oiuinü of:

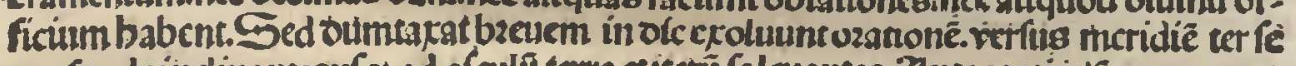

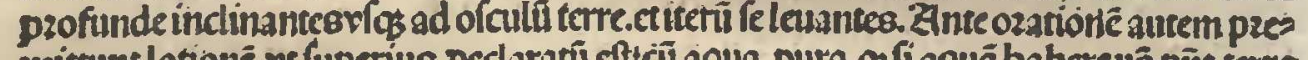

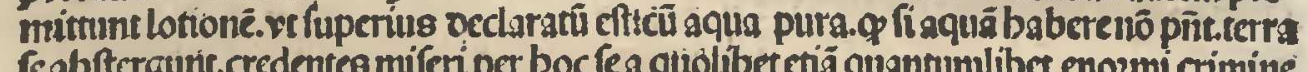
reabrest

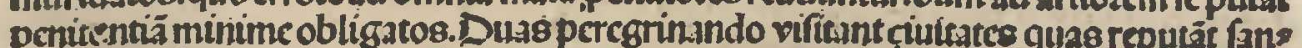

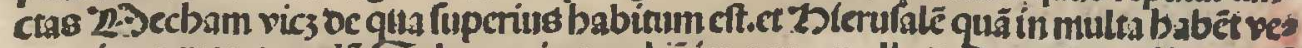

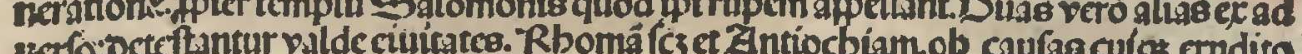
35rio.oct

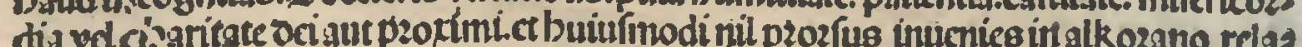

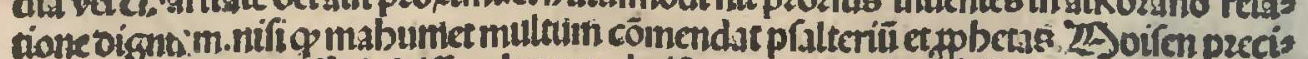

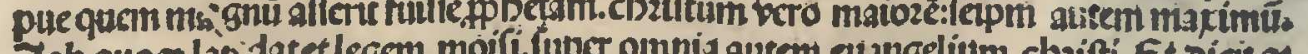

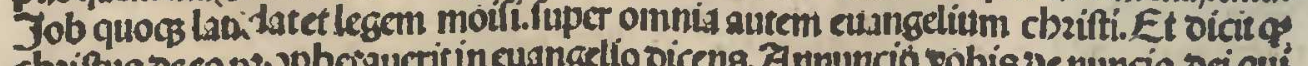

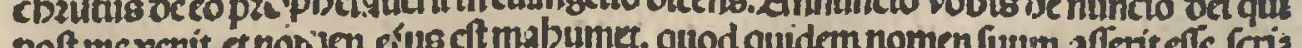

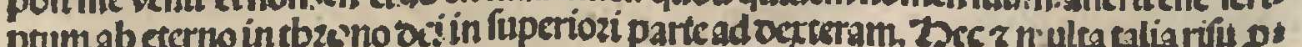
Firctu digna in alkozano cötncestur.

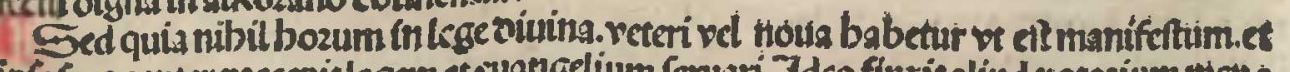

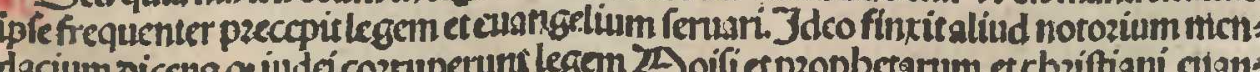
Gelium

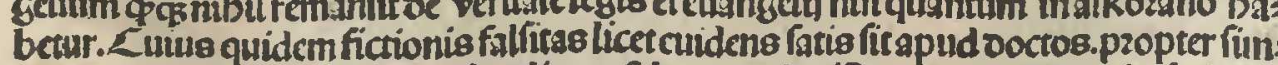
plicioses tamen quof́s clare oftendi poreft bos modo. Fam, talis corruptio icripth: 


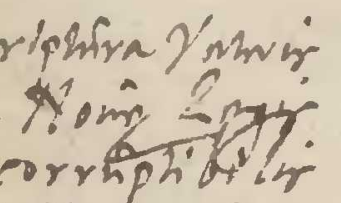

$2.9 n+f 5 n$

hifsion latrals

in oomica

$\sin 00$

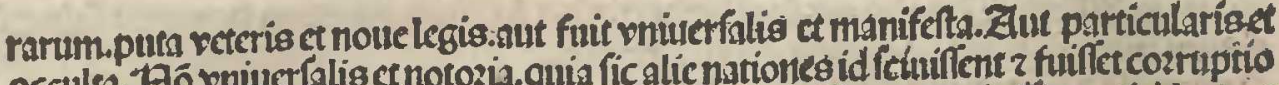

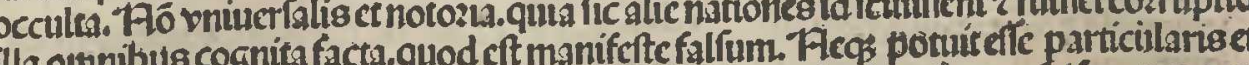

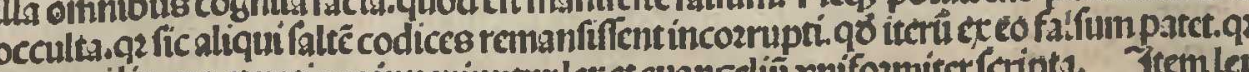
in omnilingua quntione hit

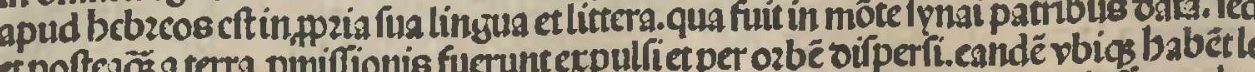

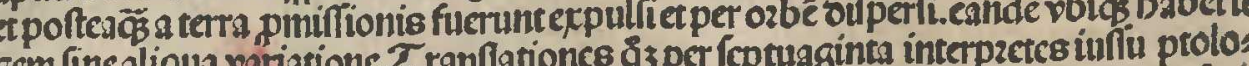
meirecis ptefuifient.quod efficacifitimú eft argumentum incozruptionis veteris legis. DS ulto autem minus bmoi cozruptio potcti nonele giaplicart qo binc partet. Faan quatruoz acta cuangeclia que per oz be pront

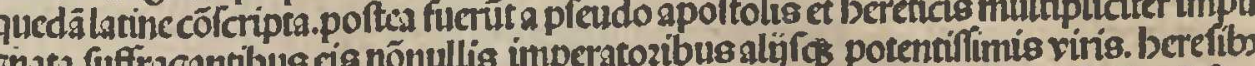

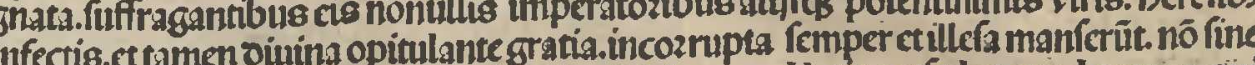

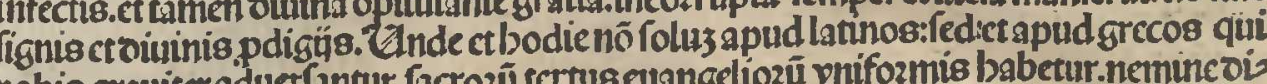

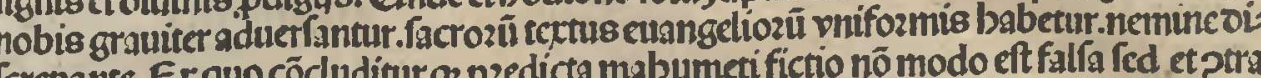

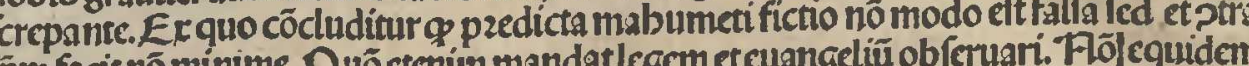
eq

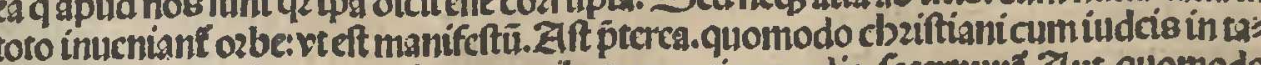

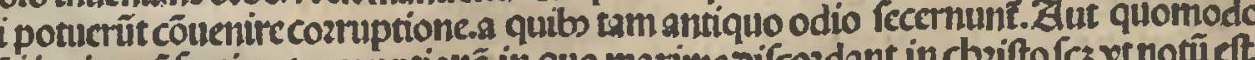

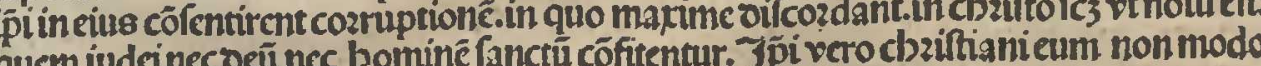

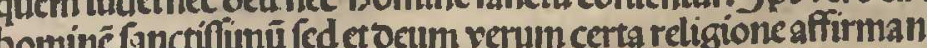

Etquanö p̈ plures ad bocipofitum adduci rônes facile poffent. be tamen fufficiăt cau

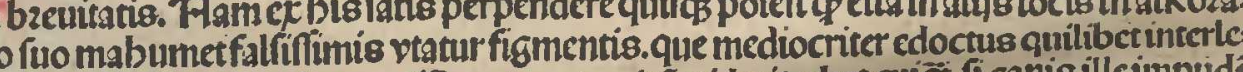

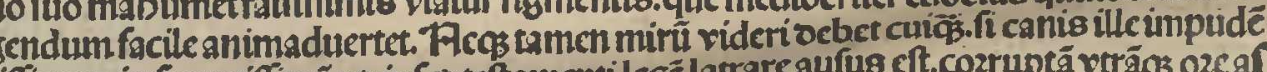

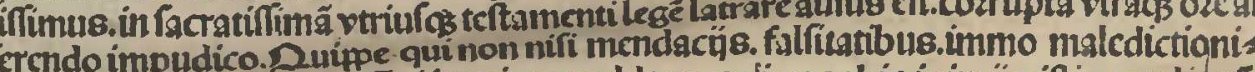

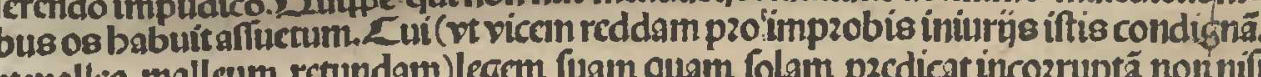
etmalleo malleum rectundam) legem fuam quam folam p2edicat incozruptã nor nif

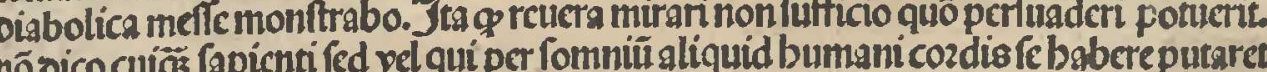

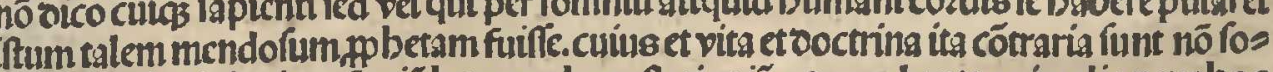

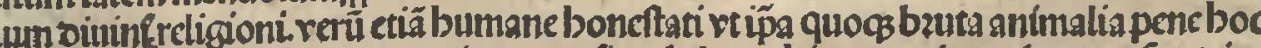

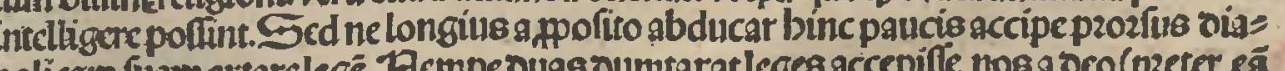

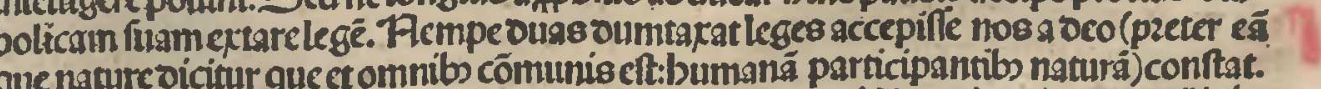

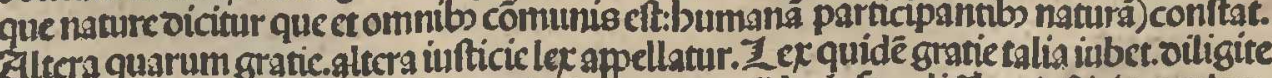

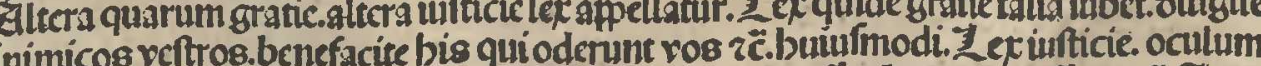
pzo oculo.ocntem pzo oente. vfturam pzoviftura.percuffurā pzo percuffura z $z$. Ittem quead talionem pertinêt reddi imperath barum ouarünullam mabumeticf́e manifes ftum cft. Lumergo itte ouc tantummmodo leges bominibus oate fint. quarnum altera oi nima.altera bumana ina gis efie viderur.

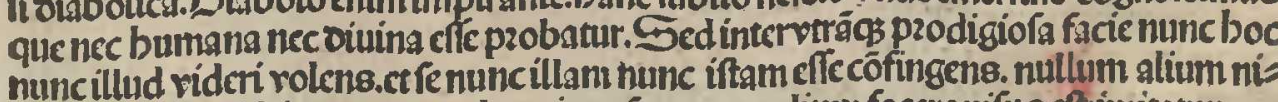

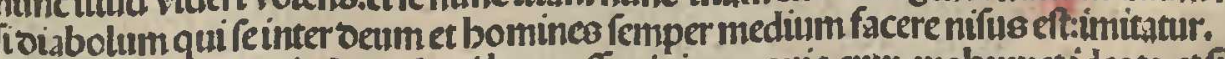

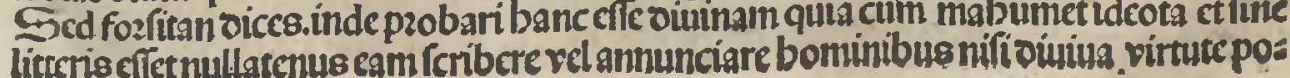

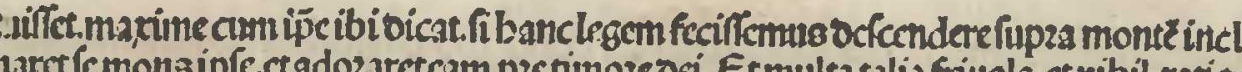

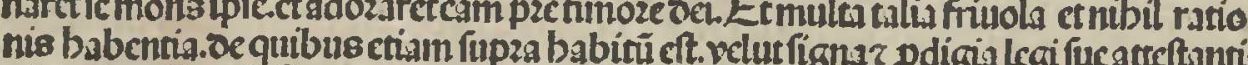
adducipofient. $D$ figna e mirzcula in iftis ait mabumer ip fe. D figna côparâda man

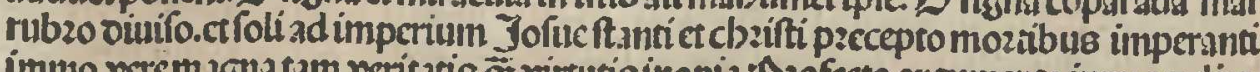

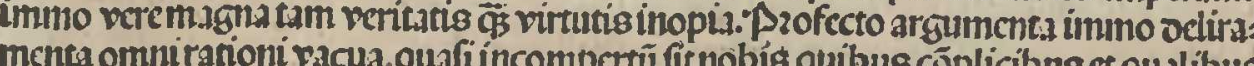

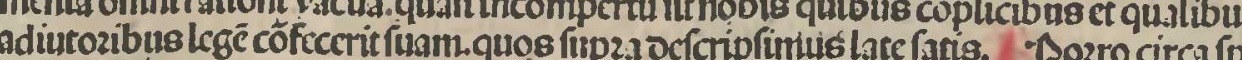

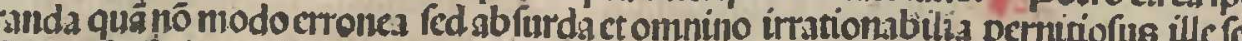

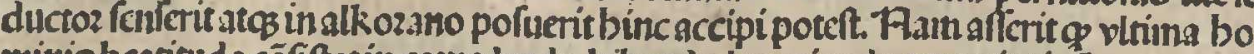

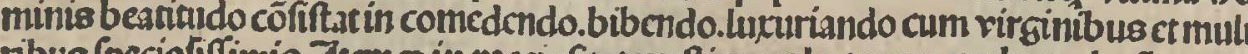

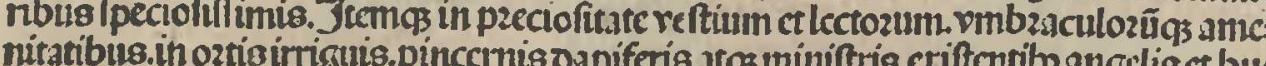

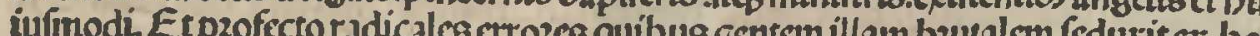
paoftumt fontc. imuno bac Fentina prodeunt. vnde paulo bic aiutius fubfiftendum bitratus fum ne enfuales ecarnales binc baurant venenui. Quozum fenfus et cogits tio proma funtad malumab adolefecentia fud. Yacleautec mea quidé cenfura. becfall tectrocte pereparbericon princelo

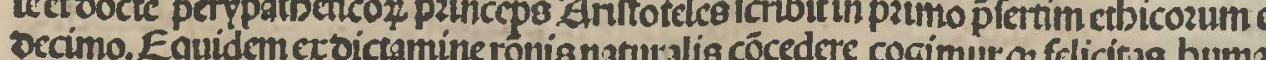

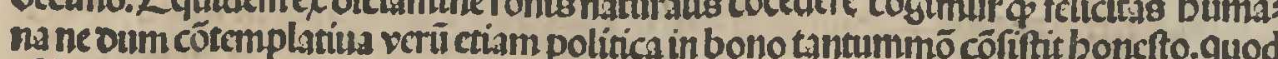

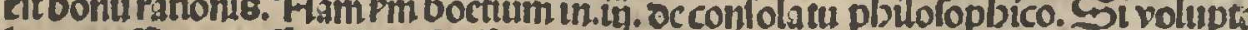

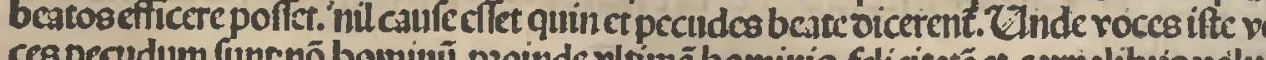

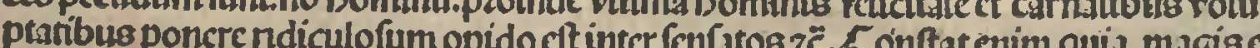
minusno diverfificant fpeciom

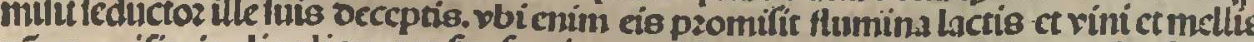

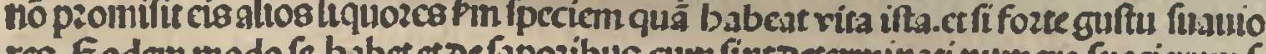
res. modictpoturcozpor

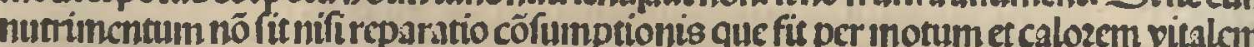

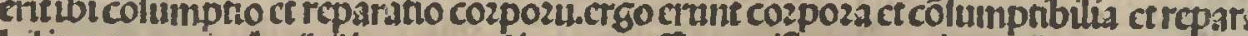

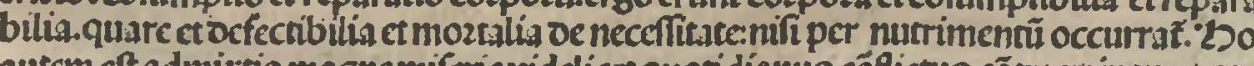
allo

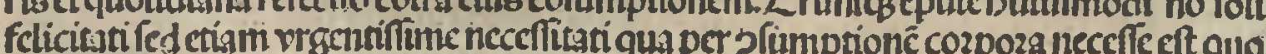

Zmpliins queremusab cis vertum fint ibi digeftionce cibozuz et potutum. quas ibi effe neceffe eft. Lun enim cibict portus multas partes babeant que no cötcniunt nutrime

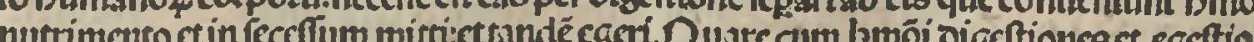

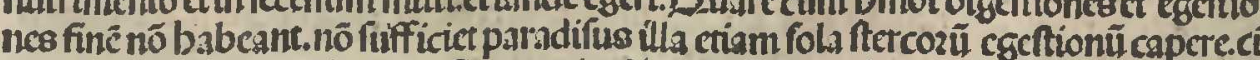

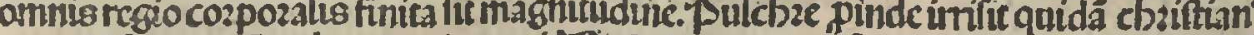

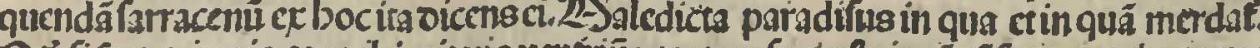

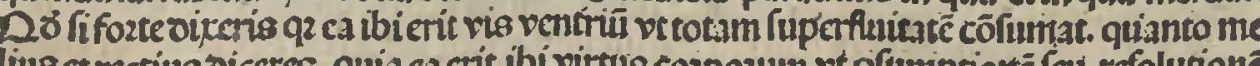
nõ

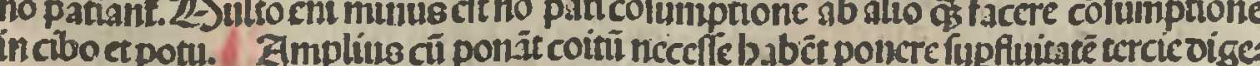

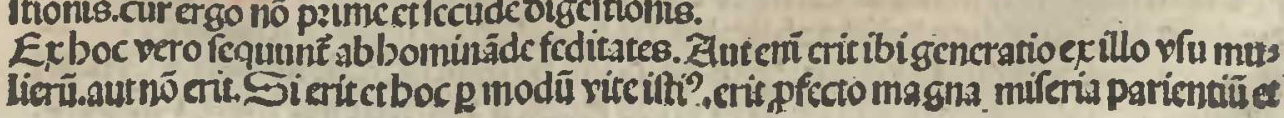

Ontiviman

Ariflololor

Prinatinuiver rinnips 
nunrientium et tanto maioz quanto cedzioz eritbi committio mantum ef feminarum Dnlocun forter

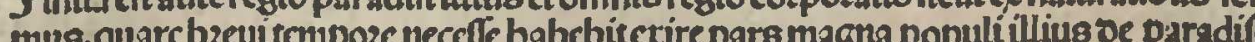
tali.ctopoztcbitcis creari iregionem aliäad babitationé cozum. Etnố vnam folam fé

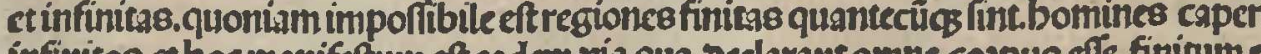

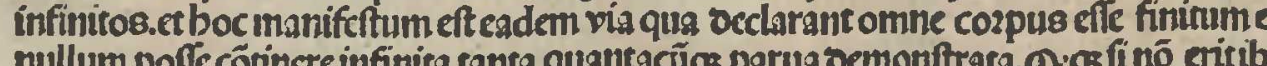

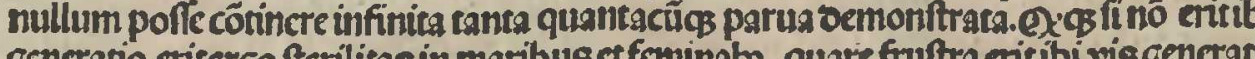
Ben in prifo virozil vel mulierum. pzoptr quod remanet opus generationis incopletum. Jbl aute

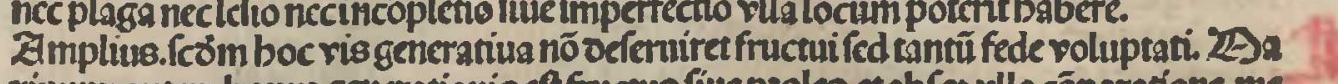

B. nim grewration

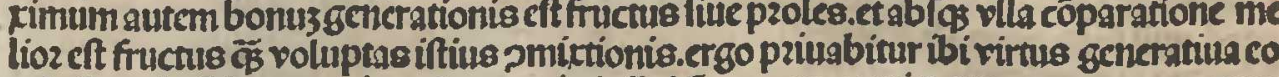

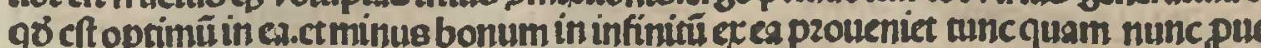
nit in vita ifta. ctgo ris generatiua nö beatificaretur ibidem.quod eft gbfurdum oicere

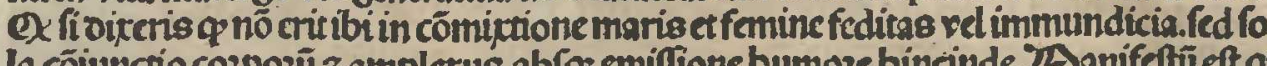

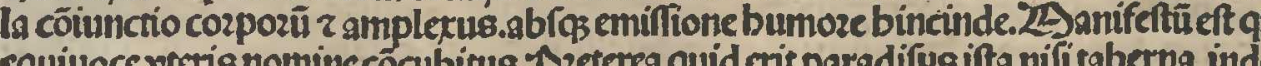
cquleingurgis

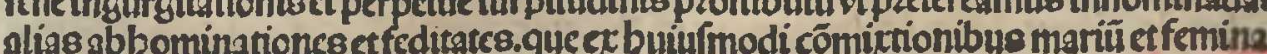

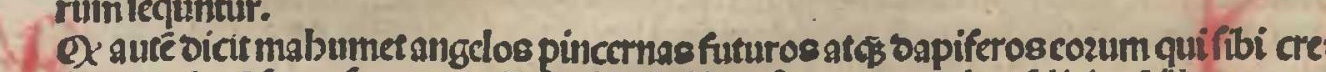
derentct legē fuam fertuarentzę. Quis nō rideat.fanctos angelos felicitaté illam quam

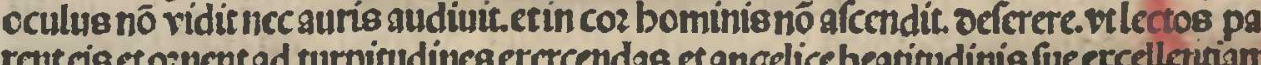

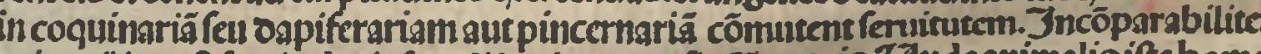

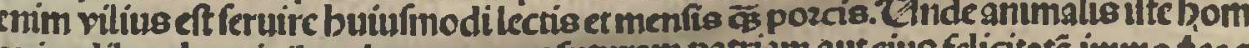

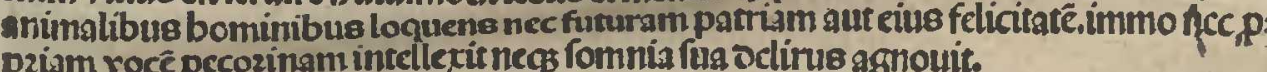
(a)

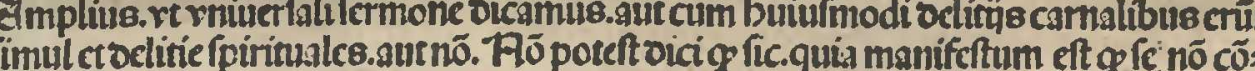

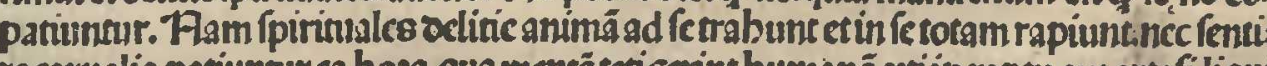

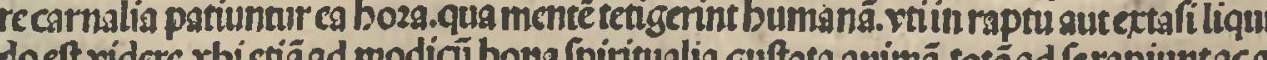

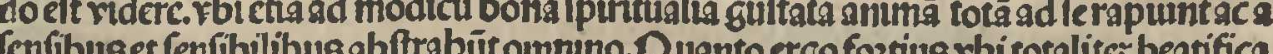
binur carc.

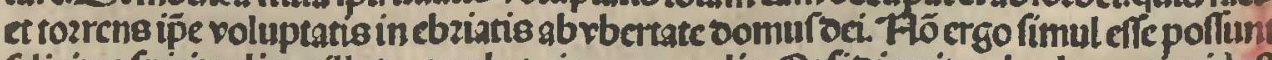
flicicas r piritualis et illa oc qua loquimur carnalis. Ox fi oixerit quia altermanmid on

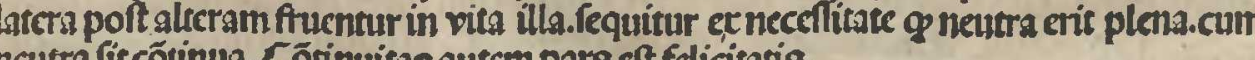

diut

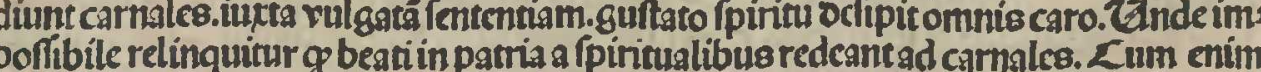

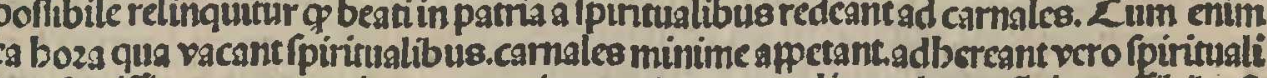
bus foztífimo amoze quinec memoziam pariatur carnalius voluptarũ. impolfíbile êt

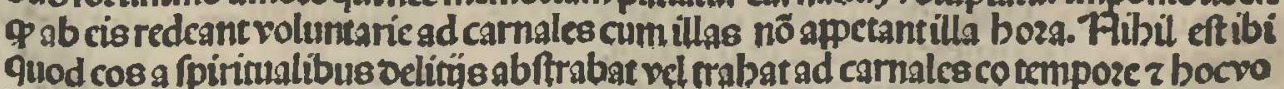

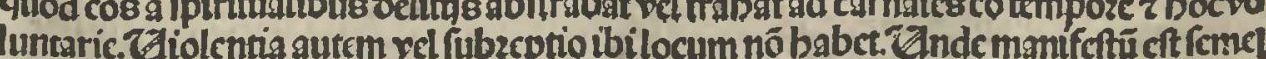

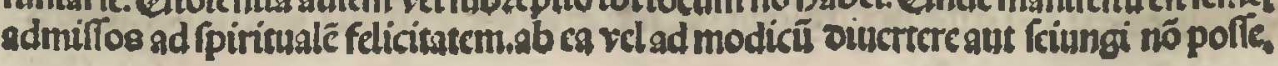

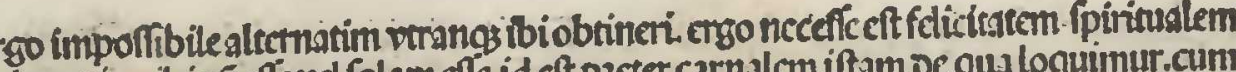
mecle.id eft pzeter carnalcm iftam De qualoquimur.cun

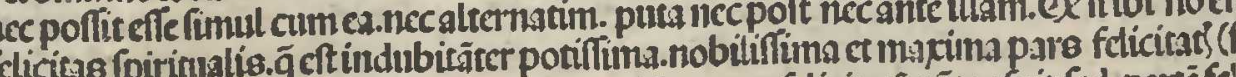

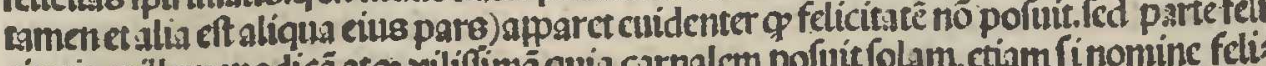

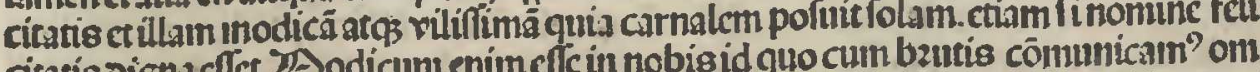

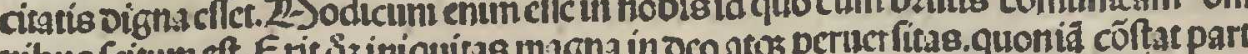

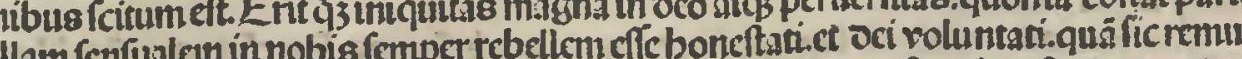

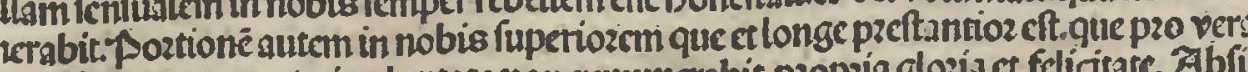

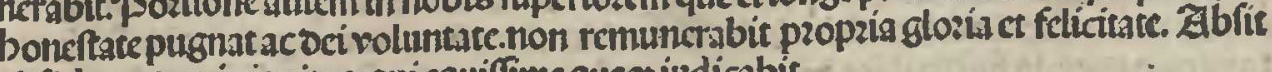

abfitbeca oco iniquitzeqqui cquiftme quecs ind

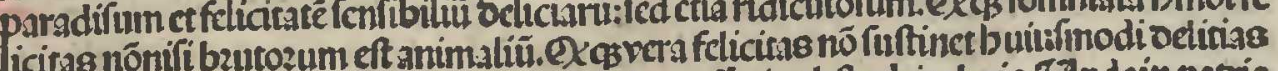

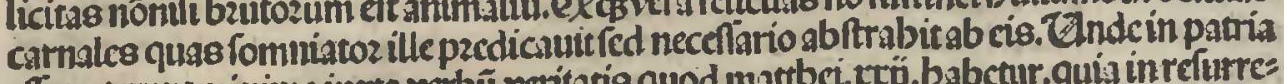

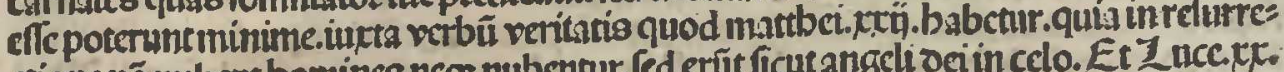

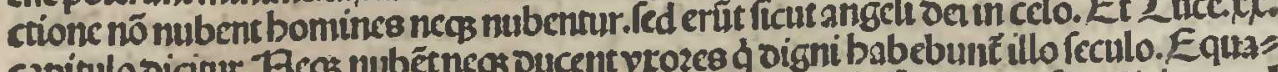
capintulo diciur. - .

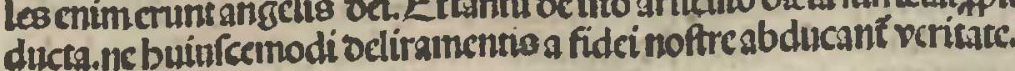

(1) Jrca quartumarticulit oecaufa fezcur ad eam fectam quetof ptinet errozes et falitialcs

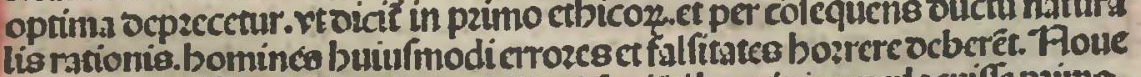

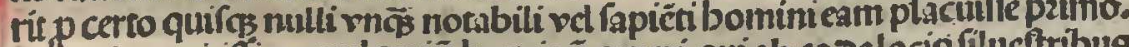

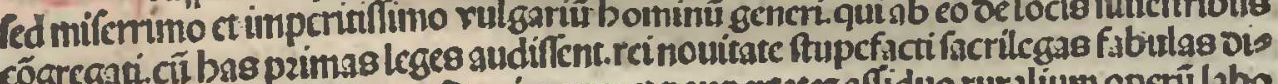

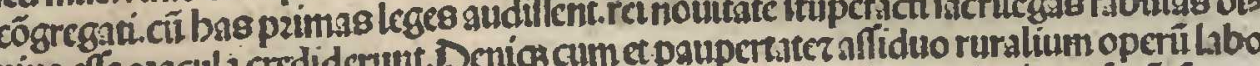

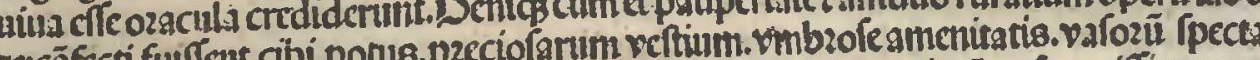

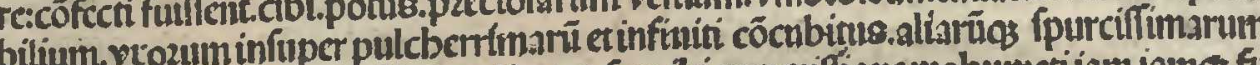

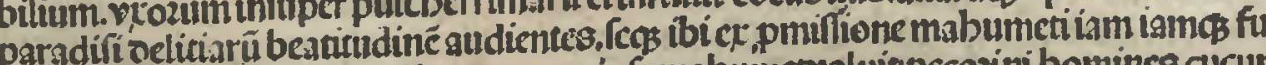

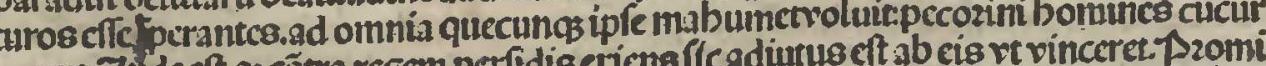

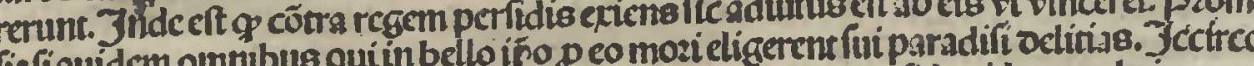
the

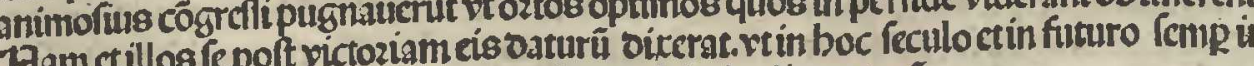

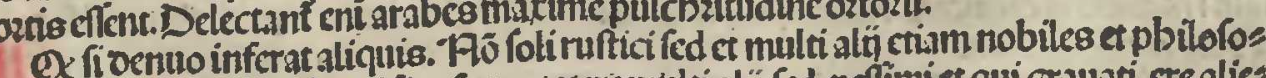

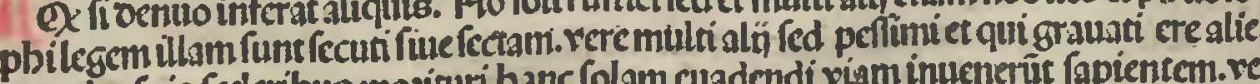

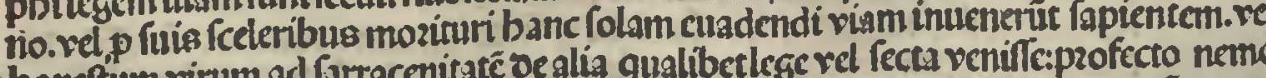

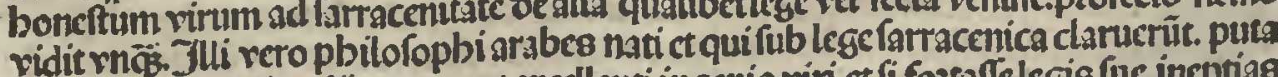

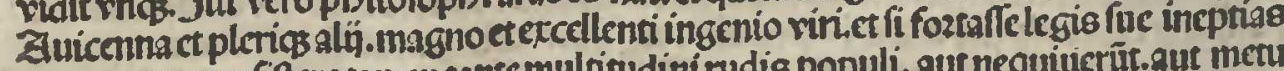

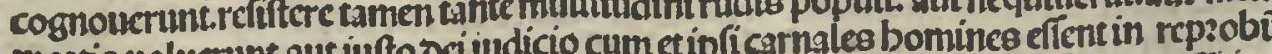

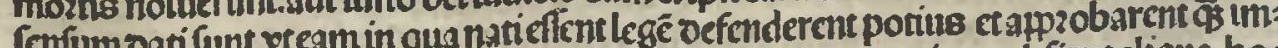
punarente pugnarent. po:

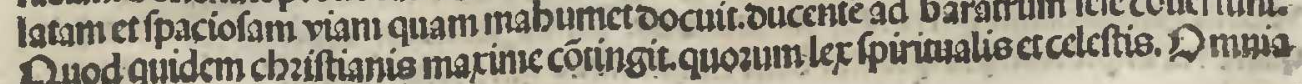

Rilio simat Survitity foreyin Soing ósorikid. 


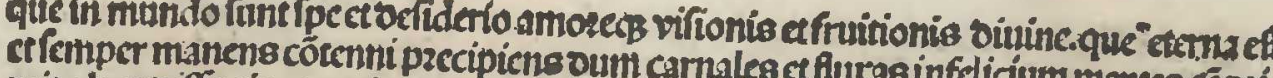

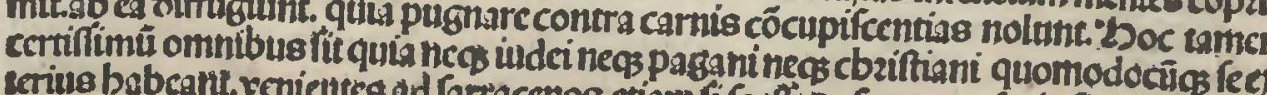

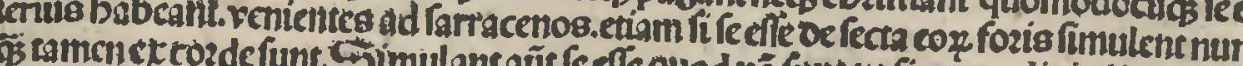

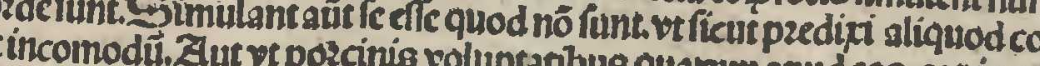
centia eft perffruanrur eterumpinum

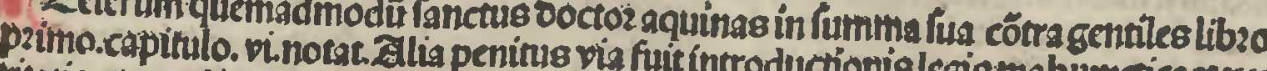

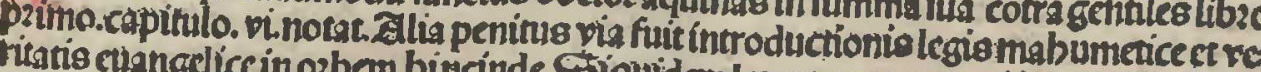

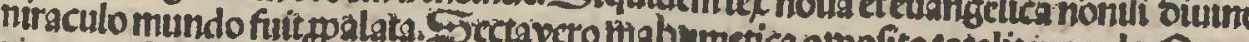

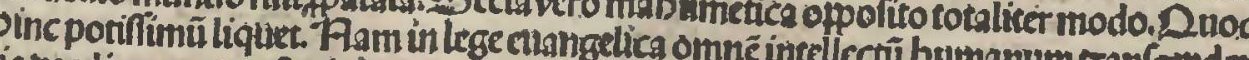

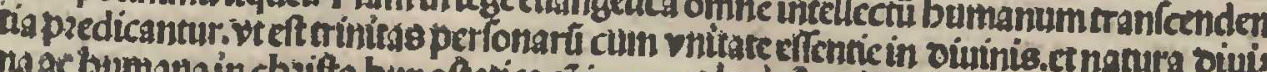

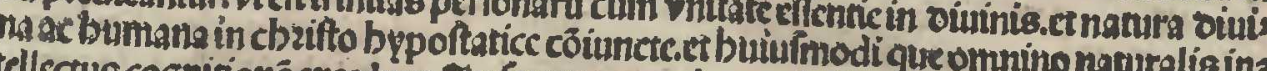

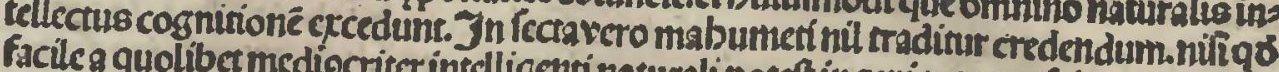

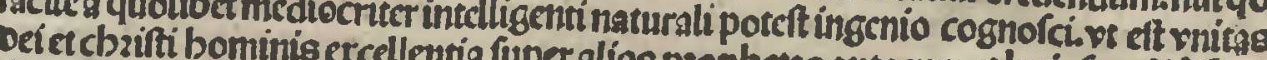

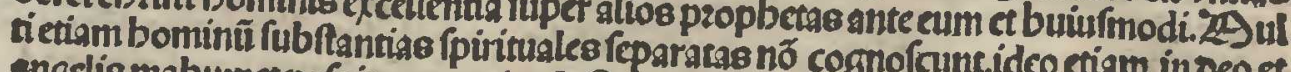

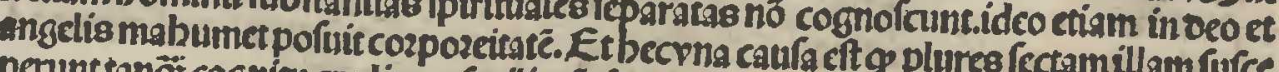

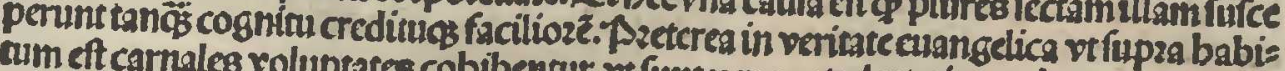

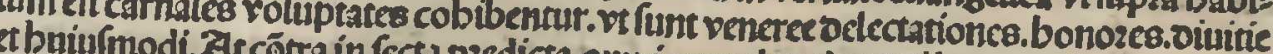

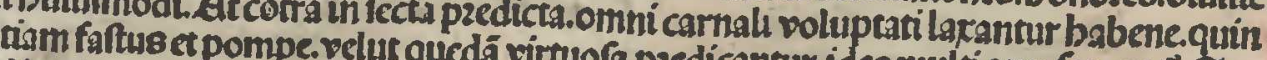
(1)

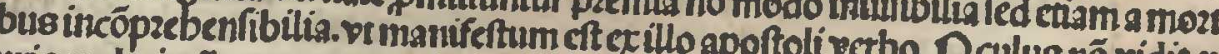

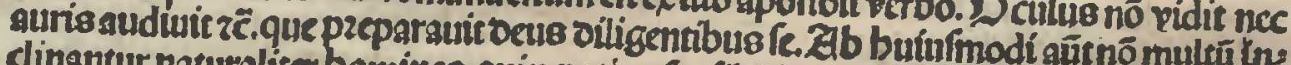
metica mercedis loco pzomimumer 3 inde eff

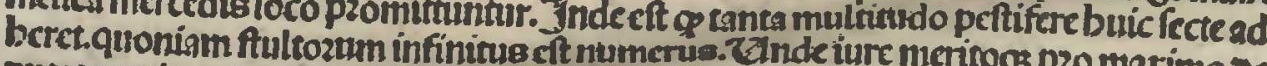

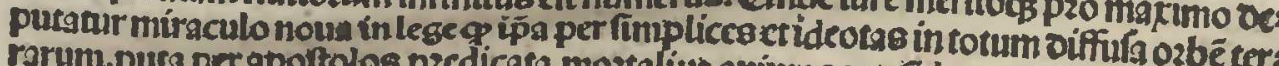
rollit.vin viribilibus ris

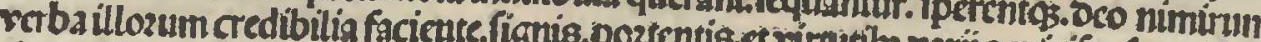

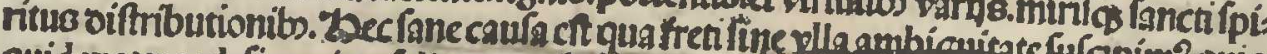

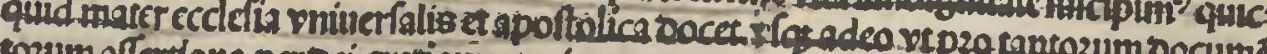
procing (n) a betredictus z men

Trentio de ounturnitatce burationis illins fecte.

mprovising

ir revä.

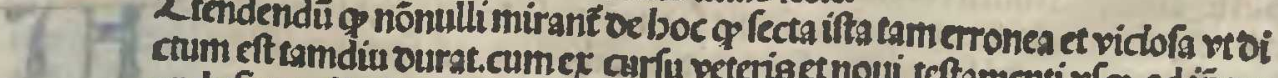

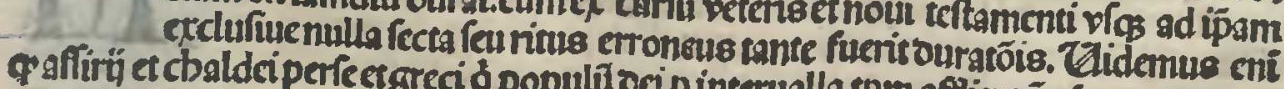

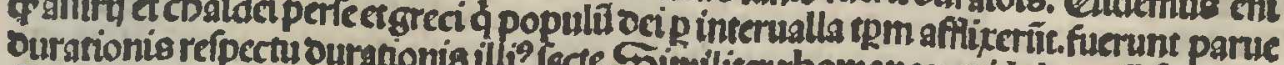

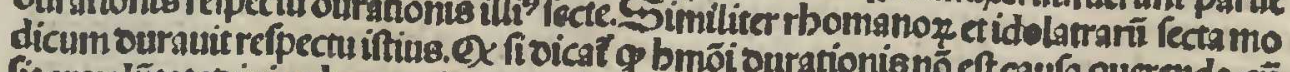

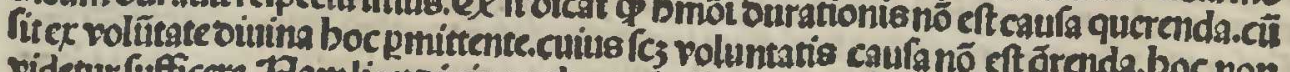

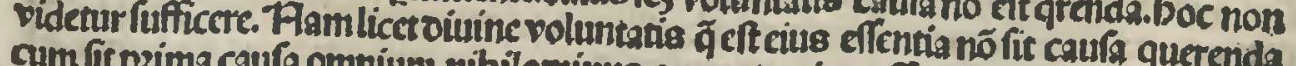

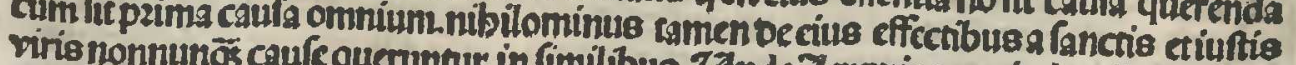

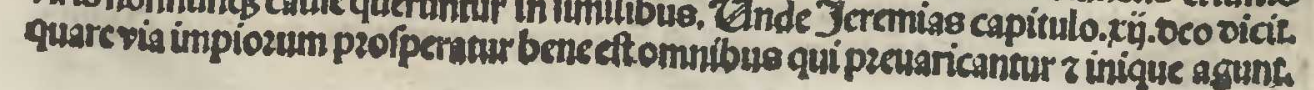

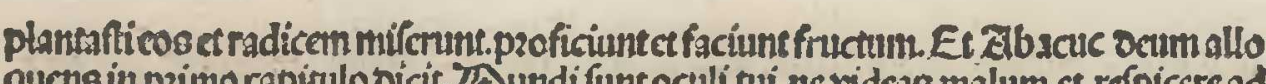
quens in primo capirulo oicit. TSundi funt oculli tui ne videas malum.ct répiccre sd

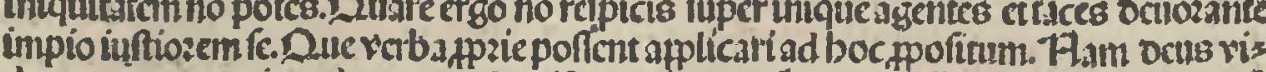

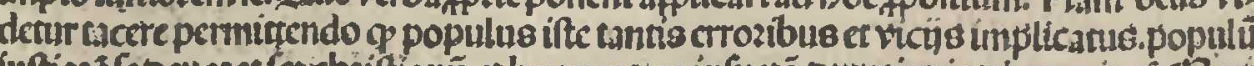

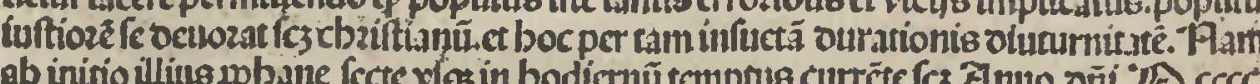

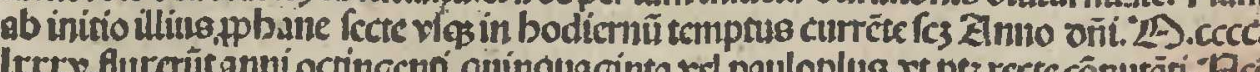

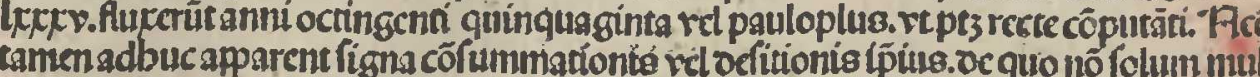

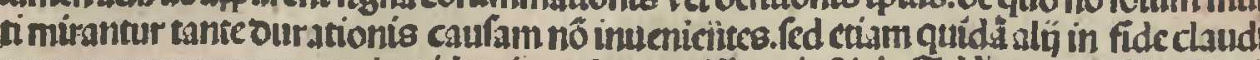

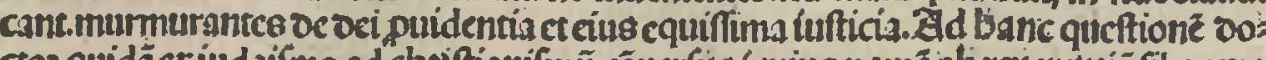

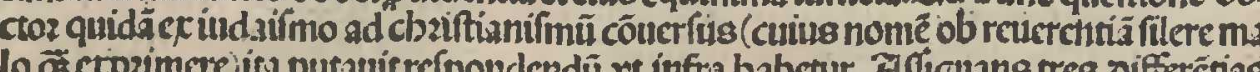

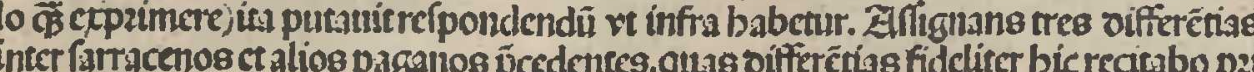

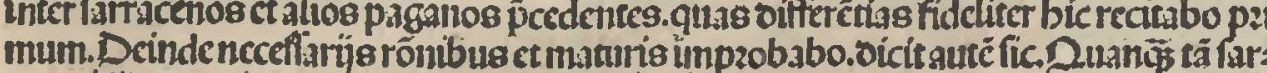
illicarentfide vera, Differunt tamé in maltis et fpcci litect in tribus que ad pofirum fa

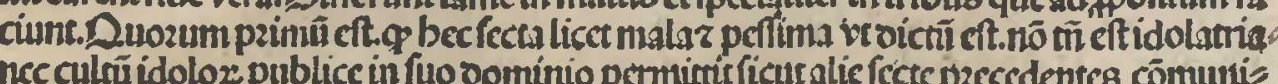

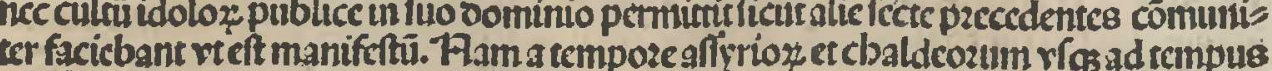

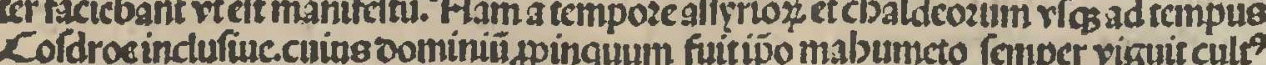

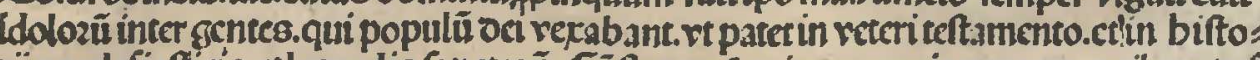

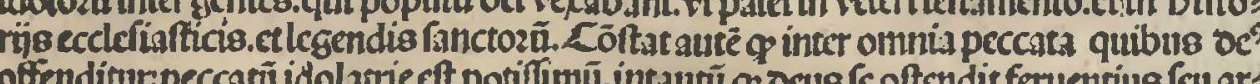

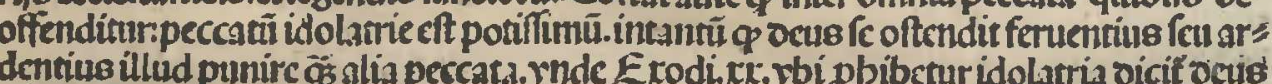

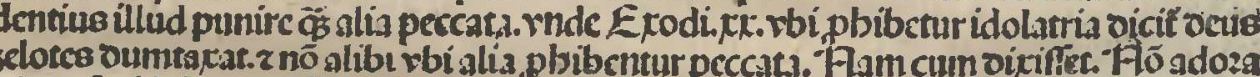

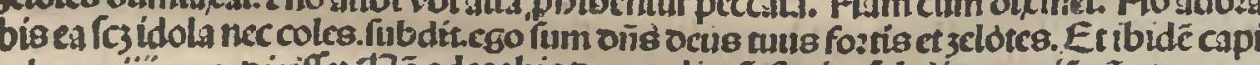

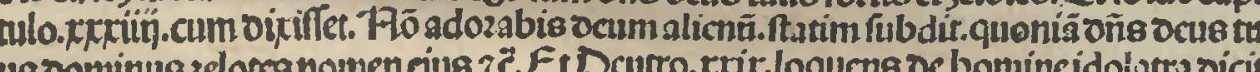

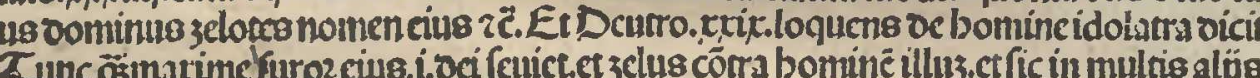

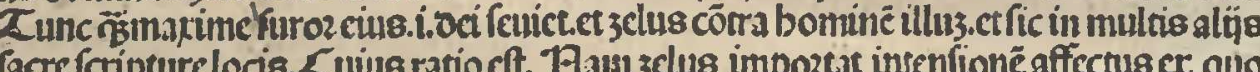

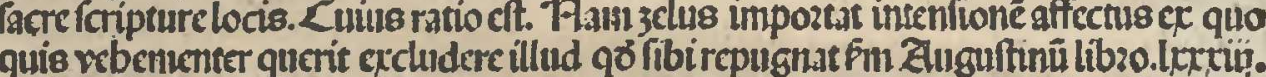

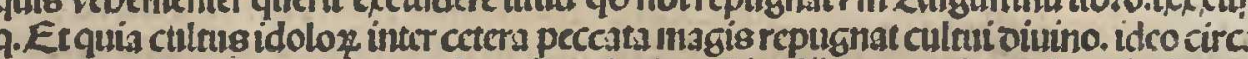

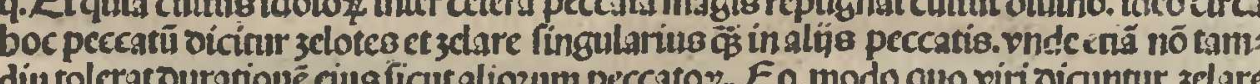
diut tolcrat Durationte cillo ficut aliozum peccatoz. E0, modo quo viri oicuntur zelare

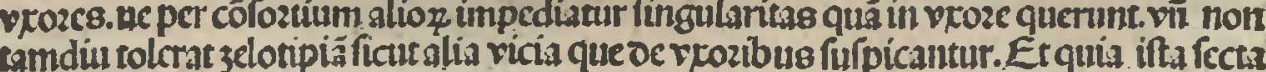

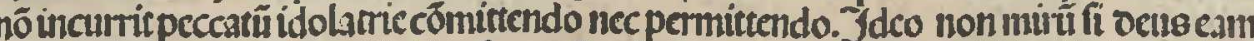

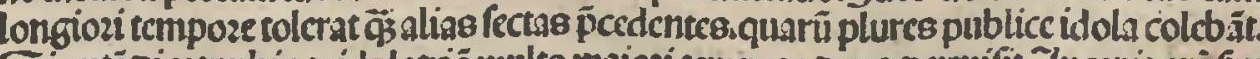

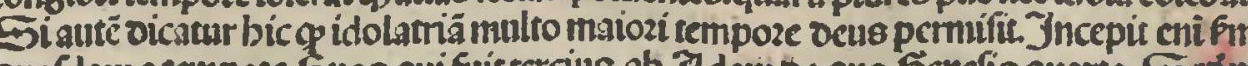
quoldamatem porc

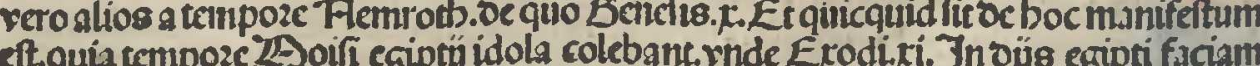

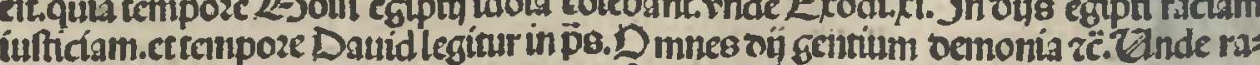

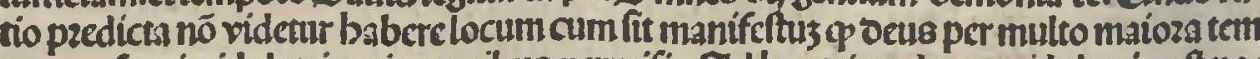

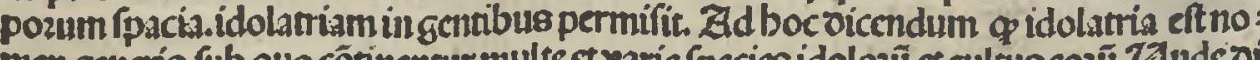
pere

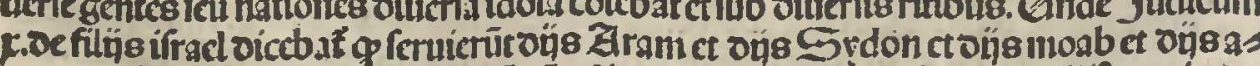

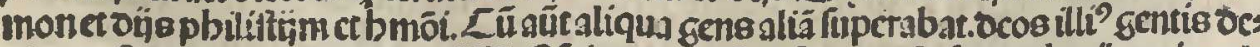

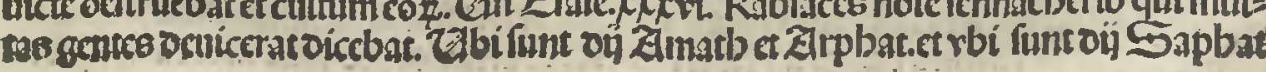




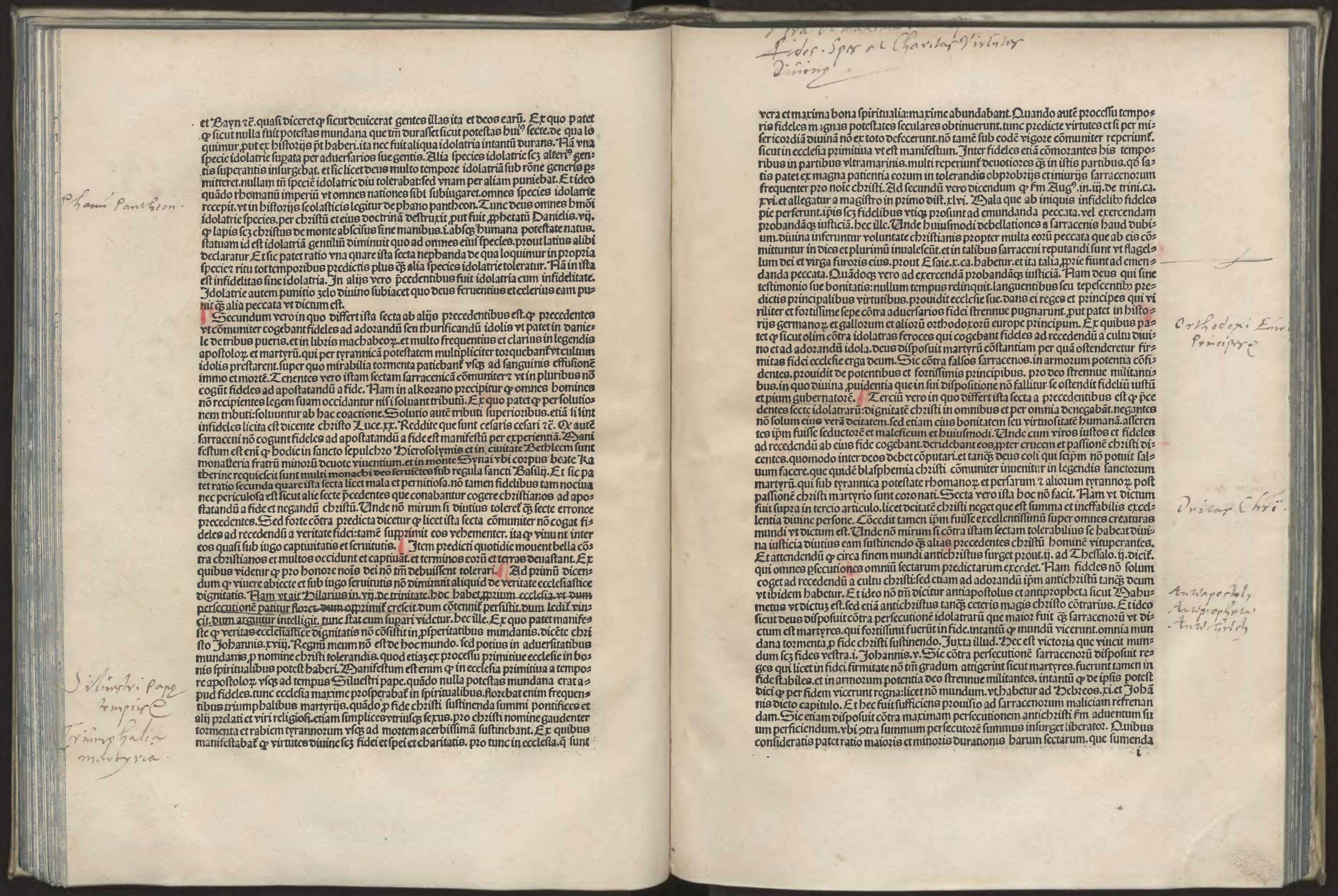




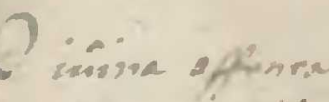
peima procits

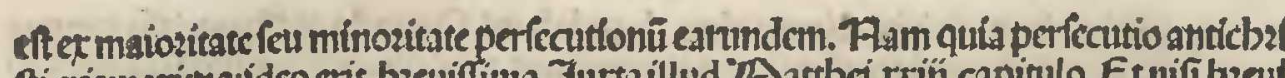
therinn

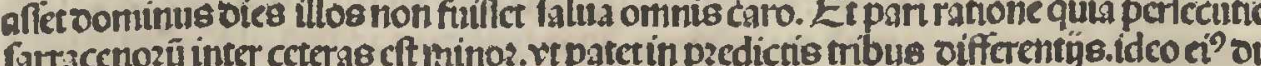
ratio cit valde longio: ceteris. Et $t$ 2 perectutio idolartay fuit ăfi media inter cas. ideo en ouratioeft quast medta inter buranionce pdictas.ert boc loquedo oe ouratione in com

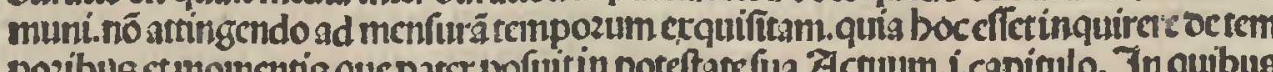

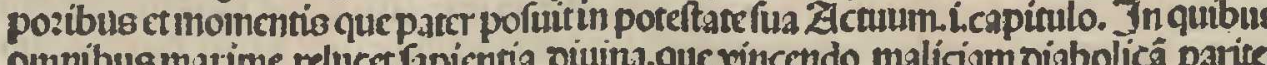

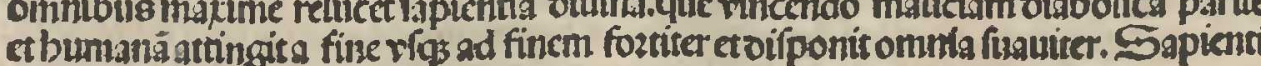

Octauo capitulo.

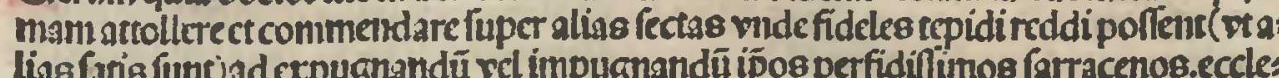

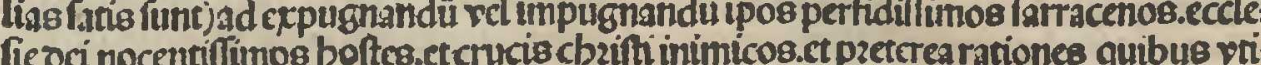

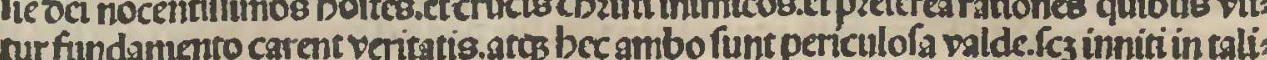
bus nō veritati quo gd fundamentu occtozum $z$ retrabere fideles per buturfmodi Dict

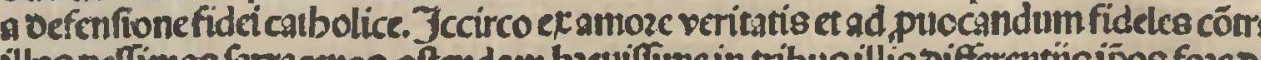

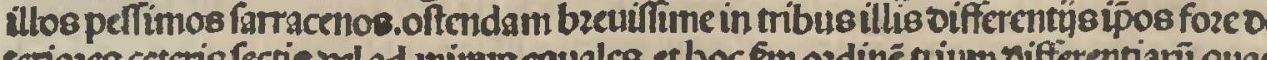
(1)

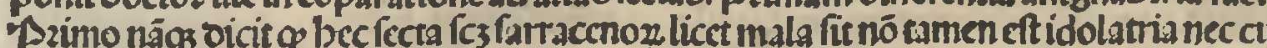

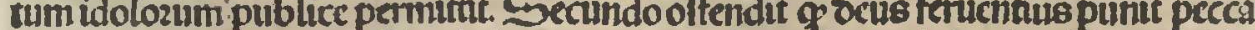

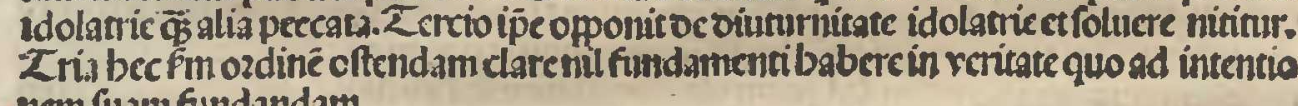
Men fuam fundandam

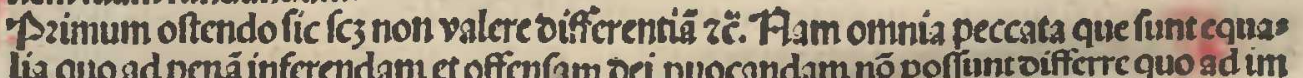

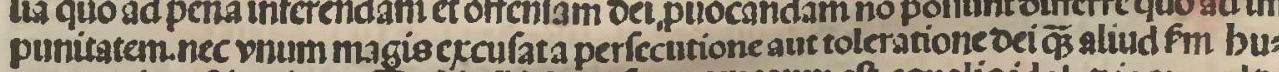

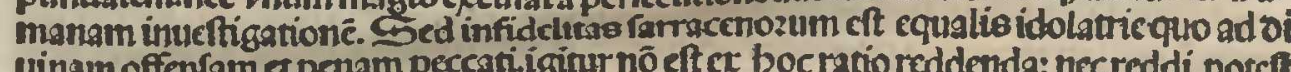

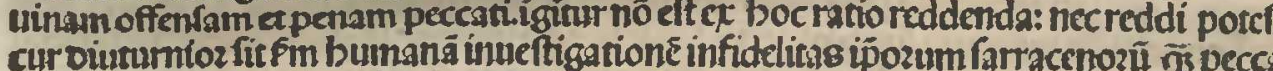

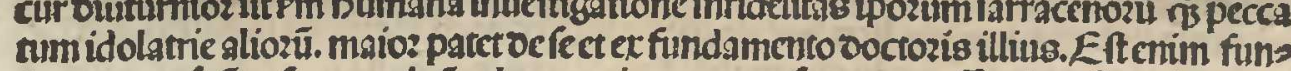

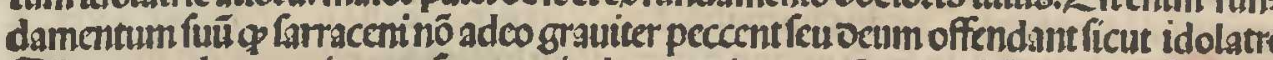

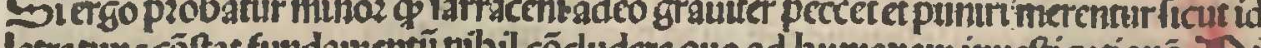

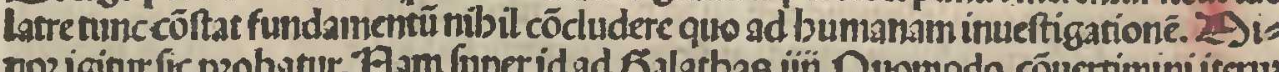
noz iginur fic pzobatur. F. am fuper id ad 5alatbas.ü̈. Duomodo contertimini itenus ris cui tunc oediti erant erarpeccatū pene par feruituri idolozum. Etalia gloga ibidem.

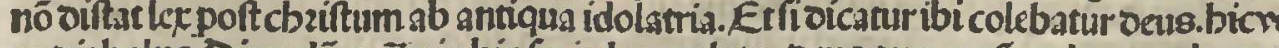

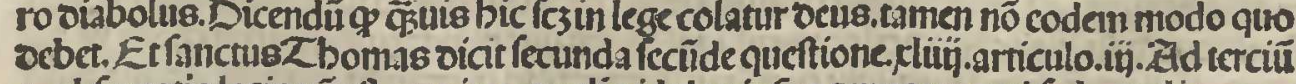
o obferuatio legis nô ctt omnino cqualis idolamie Fm genus peccati:fed cqualis pene quia vatios cet fipecies peftiferefinpertitionis. Ex quibus fic p2ocedo. Si iudeo 2 pec carum quo obfertuant vecterem legecen tempoze gratieeft par et equalis pene peccato id

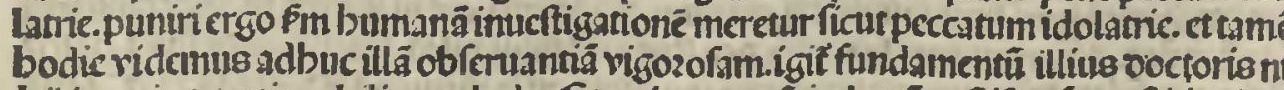

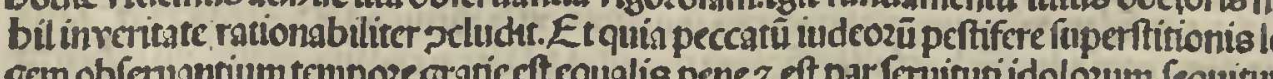
gem obferuantium temporegraniceft equalis pene $z$ eff par fernituti idolozum. fequitur

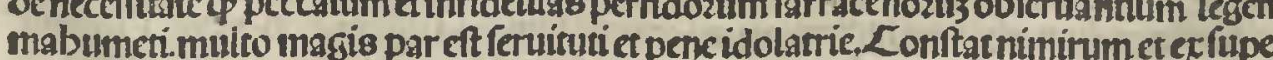

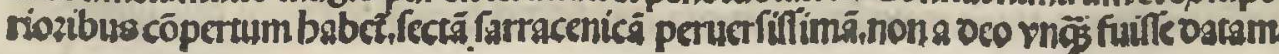

necproaliquo tempoze bonam:fed fuperftitiofam ef fallam femper. Zegi quoos tam omnial longectcedunt obferuantiaz legie

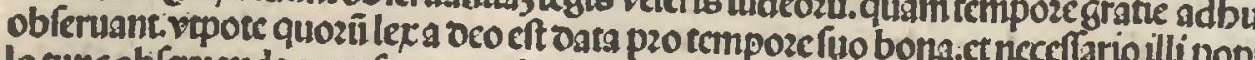
Led fier.

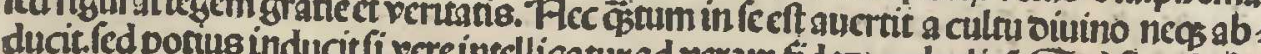
tur lex mabumeti in alkorano

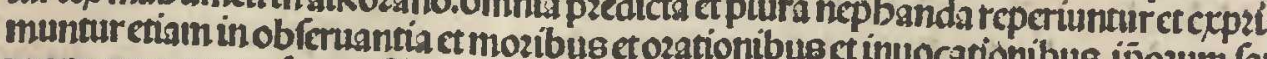
racenozum perterfozum. Jterum fiattendaur o ventrantur folenniffime illium fodu

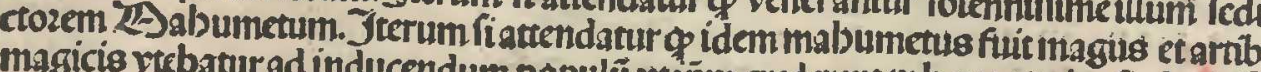

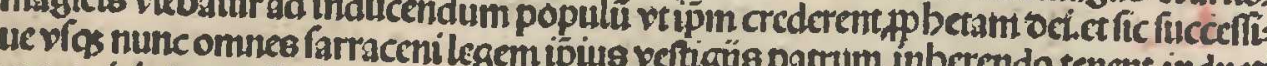

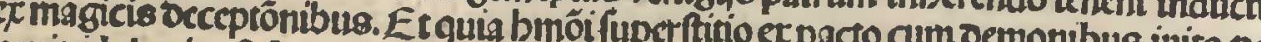
uenicidolatris clf fm ranctum 2 bomäf

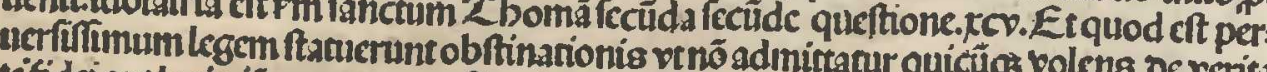
bie

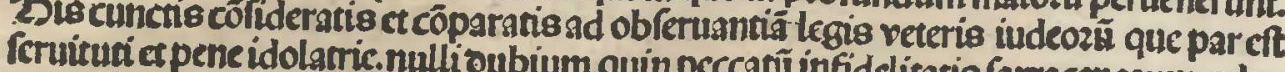

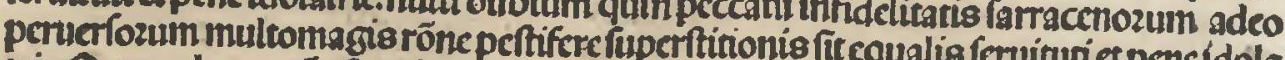

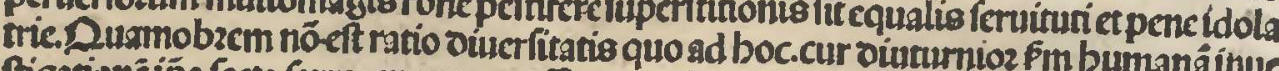

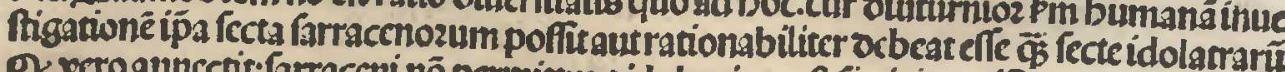

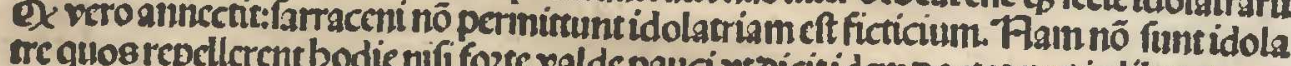

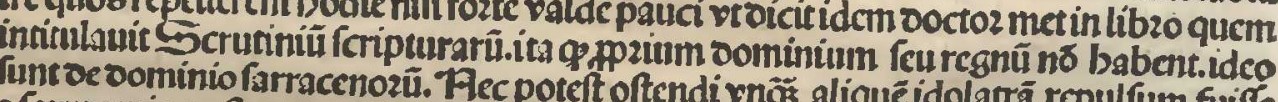
a Durracenis poft motcm impij mabumeti.

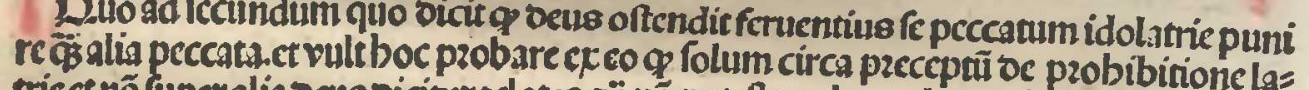

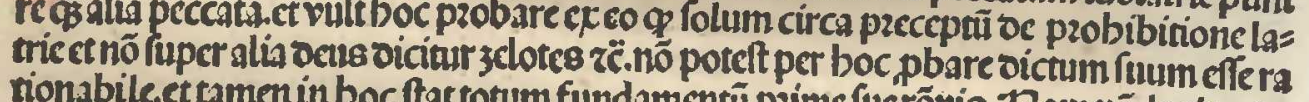
tionabile.ect tamen in boc ftartrotum fundamentü paime fuccrōnis, Fam nō tegitur oe

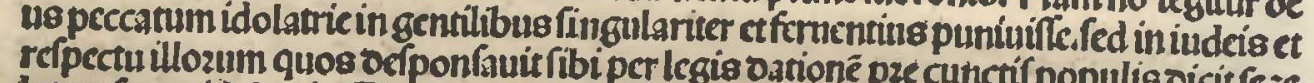

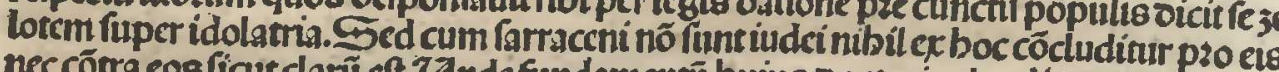
oxtercio om

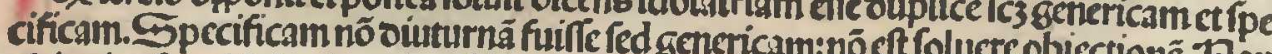

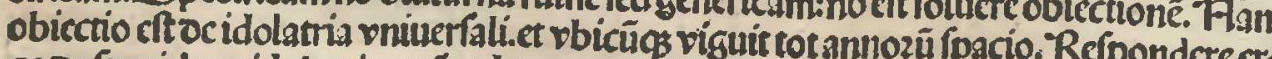

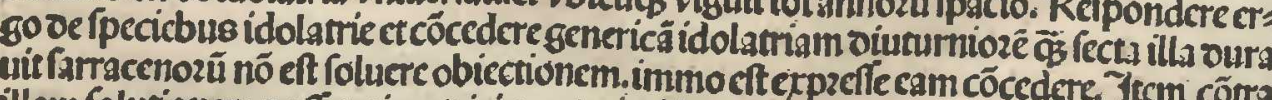
illam folution

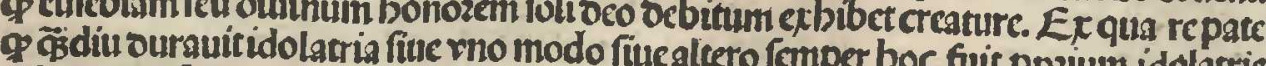

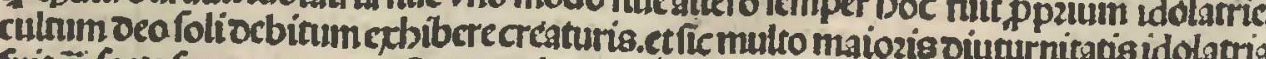

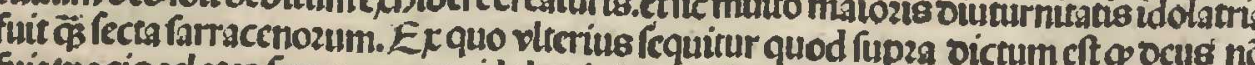

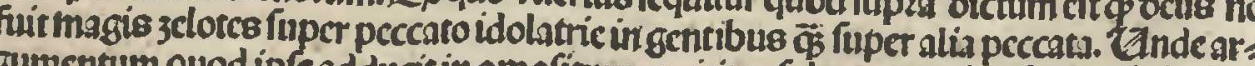

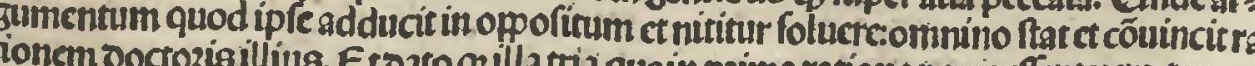
nō oeberctaliquiser boc rationabiliticr arguere

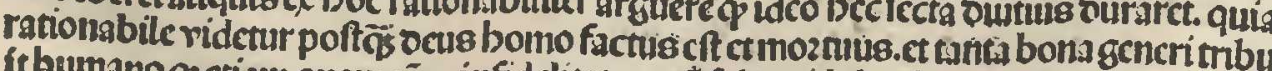

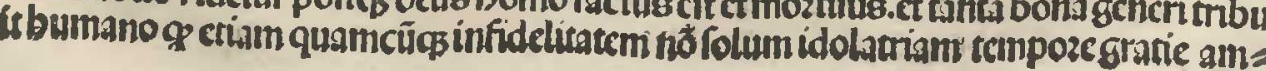

Sorotiond crepinovern 


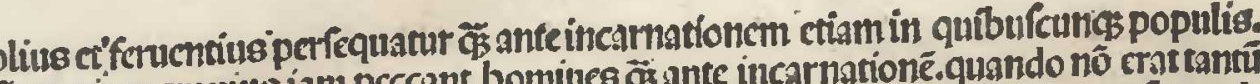

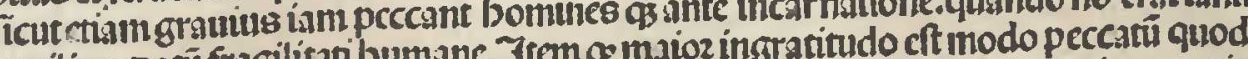

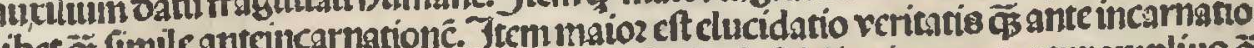

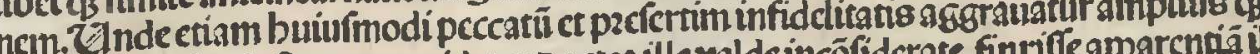
amet oifferentium.

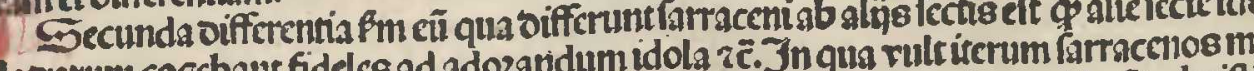

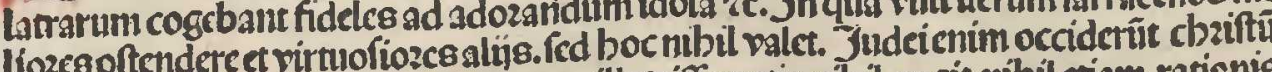

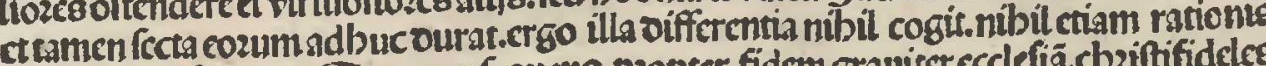
babet.quod fic parct. Secta periequerte propter fided gratho captiuando.incarctrando.occidendo. cocalleando.omneconate munt

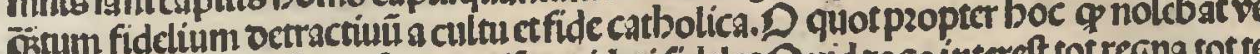

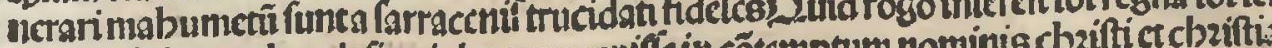

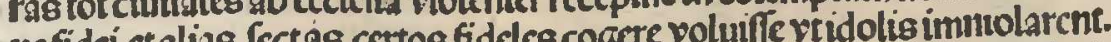

erteliet shis (q)

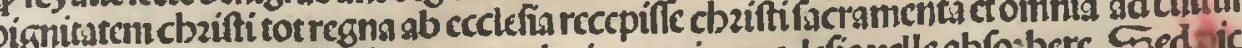

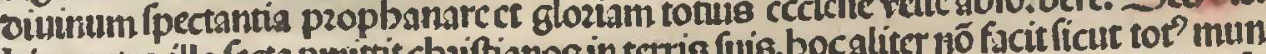
bic Doctoz.

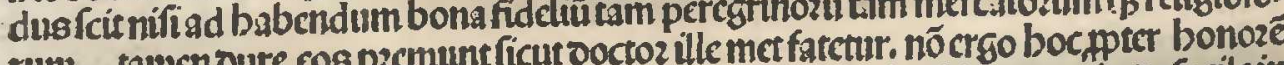

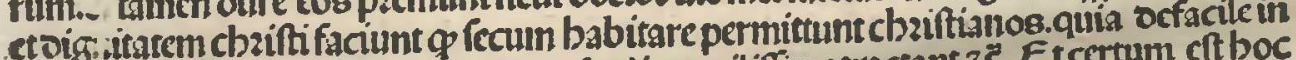

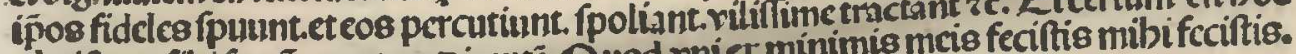

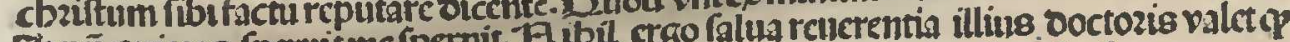
3rertiqui

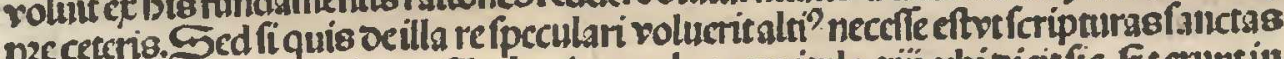

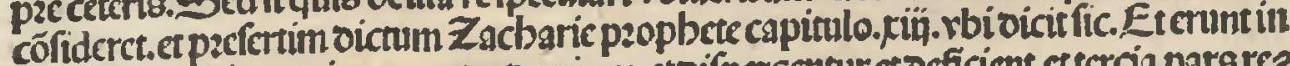

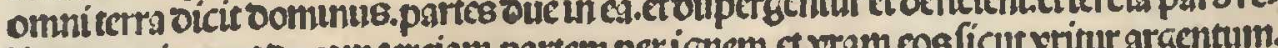

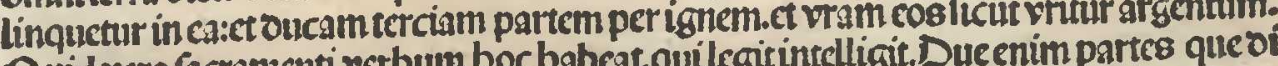

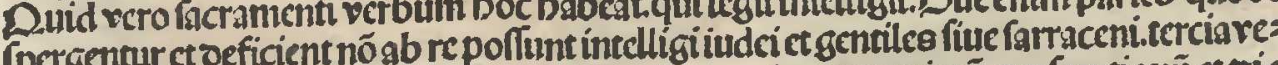

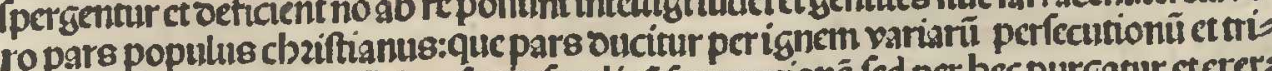

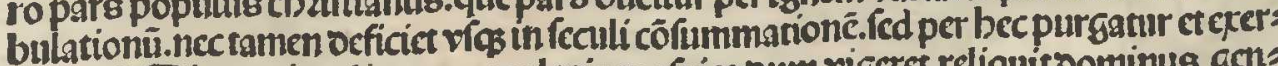

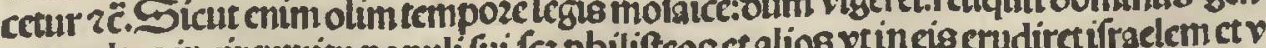
tes multase incircumiln popull thit 3 ph côfuctuding

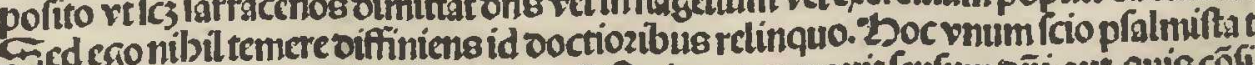

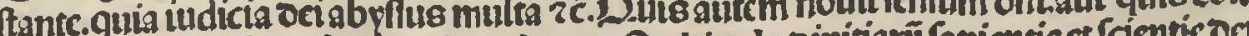

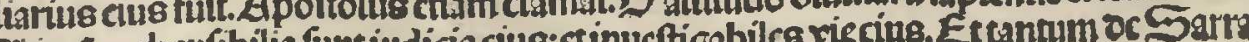
cenis

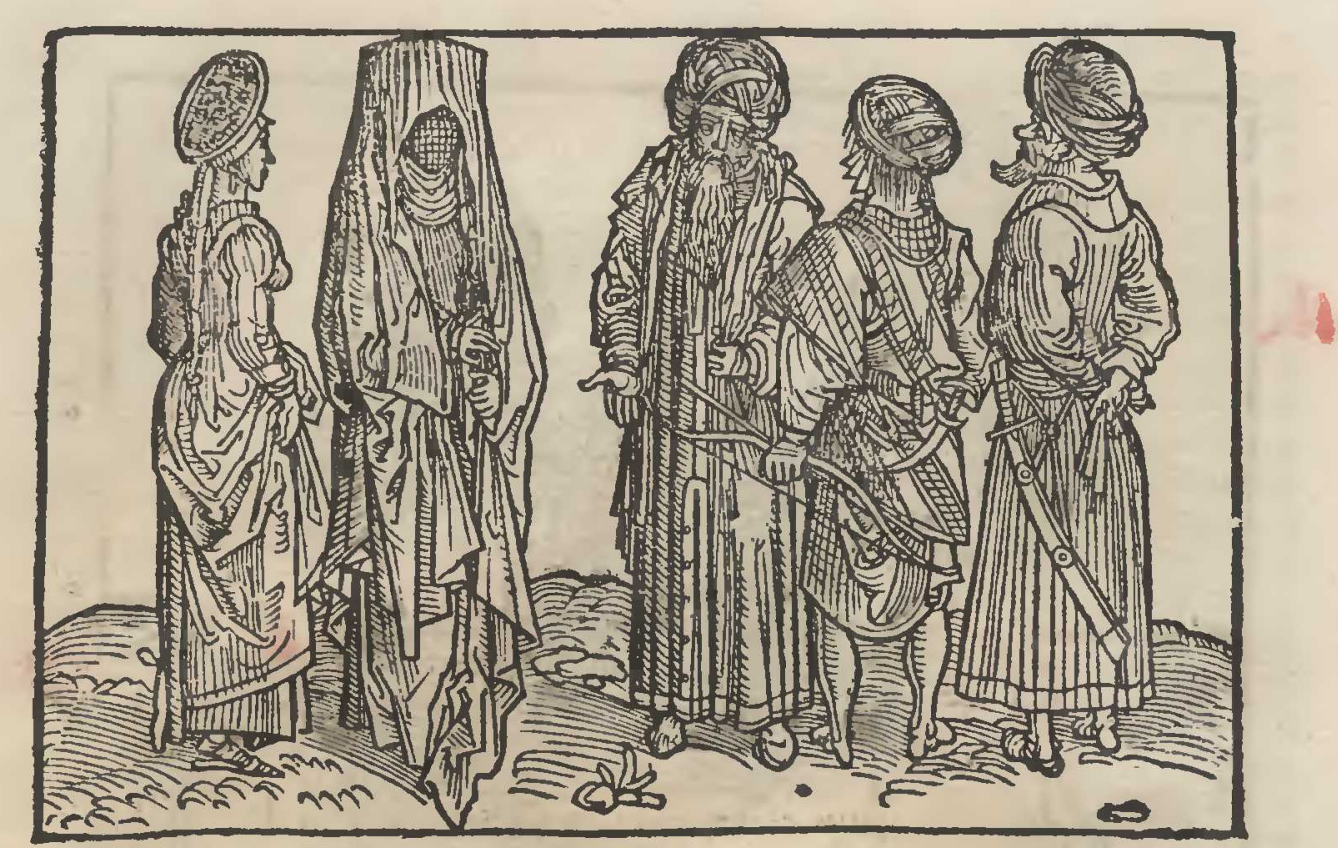

( Sarraceni linguactlittera rtuntur Arabicabicinferiue iubimpzeffa. - -

\begin{tabular}{|c|c|c|c|c|c|c|c|c|}
\hline$\Delta a t$ & $\Delta a t$ & Exy & báf & Bsym & te. & $\tau_{e}$ & B & allepto \\
\hline$>$ & 1 & 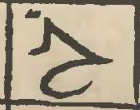 & 2 & $z$ & $\dot{4}$ & $<3$ & 2 & $J$ \\
\hline$\alpha_{y 1}^{\prime \prime 2}$ & Sans & $\tau_{2}$ & sadow & ons & OAfín & Gzýn & faym & tw \\
\hline$c$ & 15 & D & $10^{\circ}$ & $c$ & Cm & Giver & $\dot{1}$ & $\tilde{U}$ \\
\hline betbe & nnum & 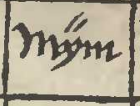 & tant & $I_{n+1}$ & anplo & Endls & ffen & Gougnm \\
\hline 60 & 4 & $e$ & $\mathcal{J}$ & 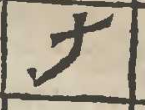 & 9 & $\ddot{g}$ & 9 & $\dot{c}$ \\
\hline 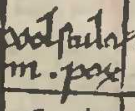 & $y$ & Lomathep & 6) luak & & & & & \\
\hline chind? & $\mid$ & 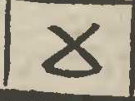 & ق & & & & & \\
\hline
\end{tabular}




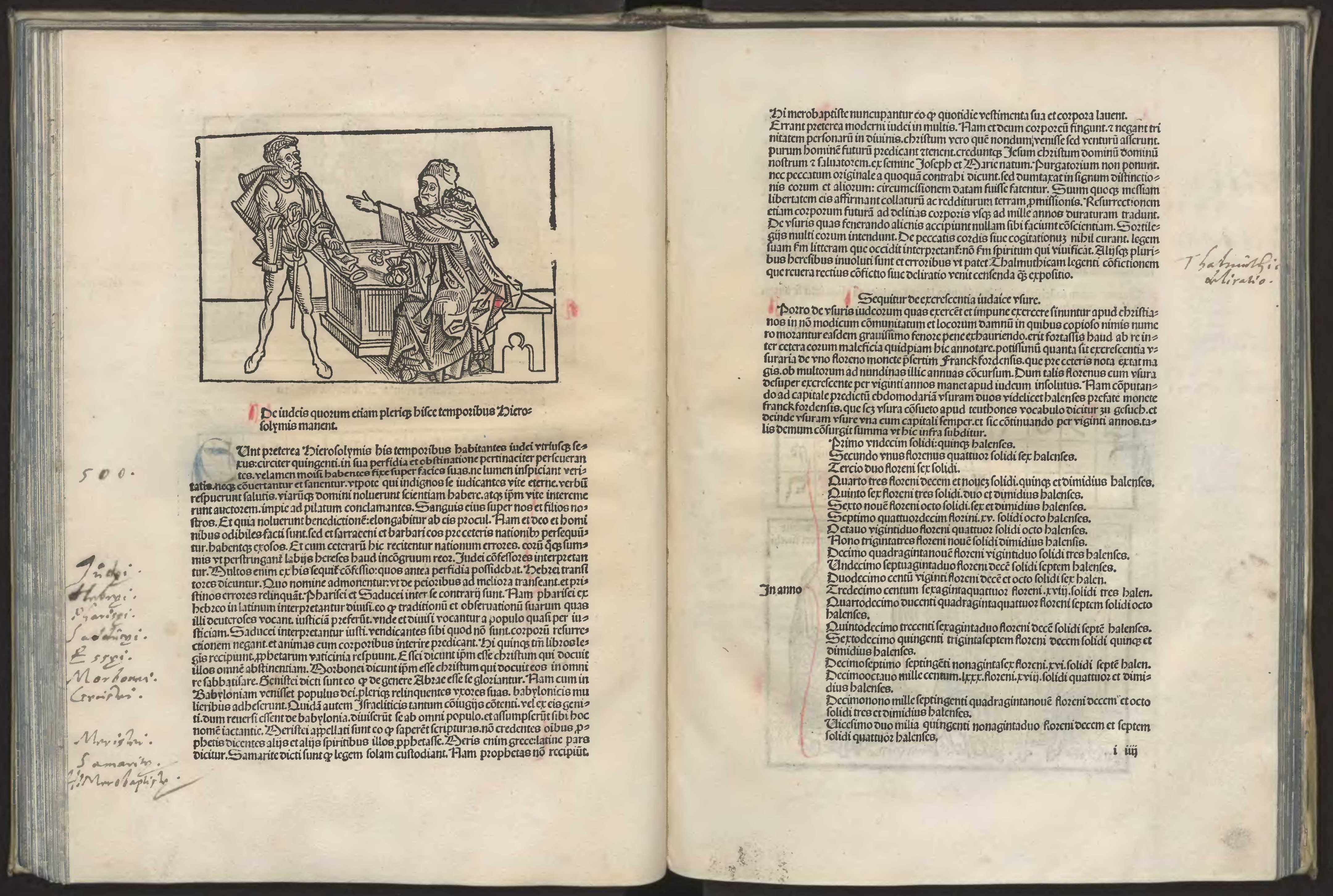


Trem mus flozenus in rigintivno annis bancimpoztat vfuraz. viç mì milia octin

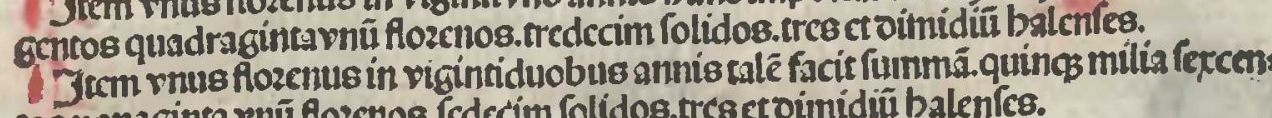

3tem vigintiflozerti in rigintiannis:tantam impostant vfuram videlicetquiquagin tasnum thitia octingchtos quinquagud quädo octo balenfes pro rno albo. ct rigint

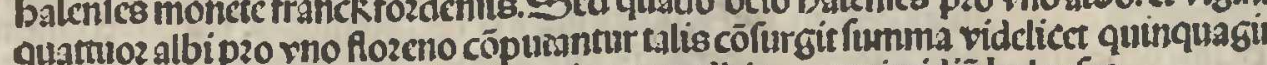

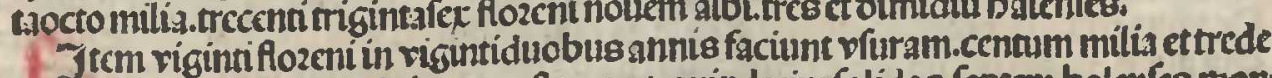

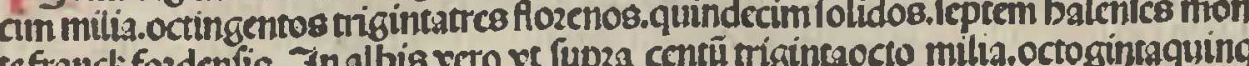

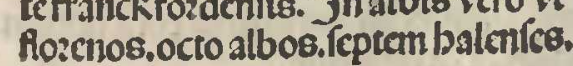

(Eltruntur autem iudé in locis illis extítentes.lingua arabica ad alios.inter fe autern bebzaica cuius biclbabctur alpbabetum bene corrcctum

\begin{tabular}{|c|c|c|c|c|c|c|c|c|c|}
\hline fotla & Therth & brats & Sant & poail & 12a & porlatis & Býmel & Buth & areph \\
\hline 2 & 5 & 7 & 7 & 7 & 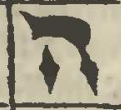 & 7 & $\lambda$ & 2 & \\
\hline pe & $Q^{\prime \prime \prime}$ & Sartran & $h_{1 m}$ & plum & Hem & Mam & Finnos & $\epsilon_{\text {aff }}$ & Euff \\
\hline \pm & $y$ & $D$ & 1 & 3 & 는 & & & 8 & 2 \\
\hline & & & $\operatorname{tanff}$ & 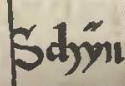 & 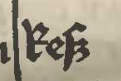 & Euff & sorde & $3 \Delta_{0}+\alpha_{1}$ & ffe \\
\hline & & 1 & 71 & एण & & & & & \\
\hline
\end{tabular}

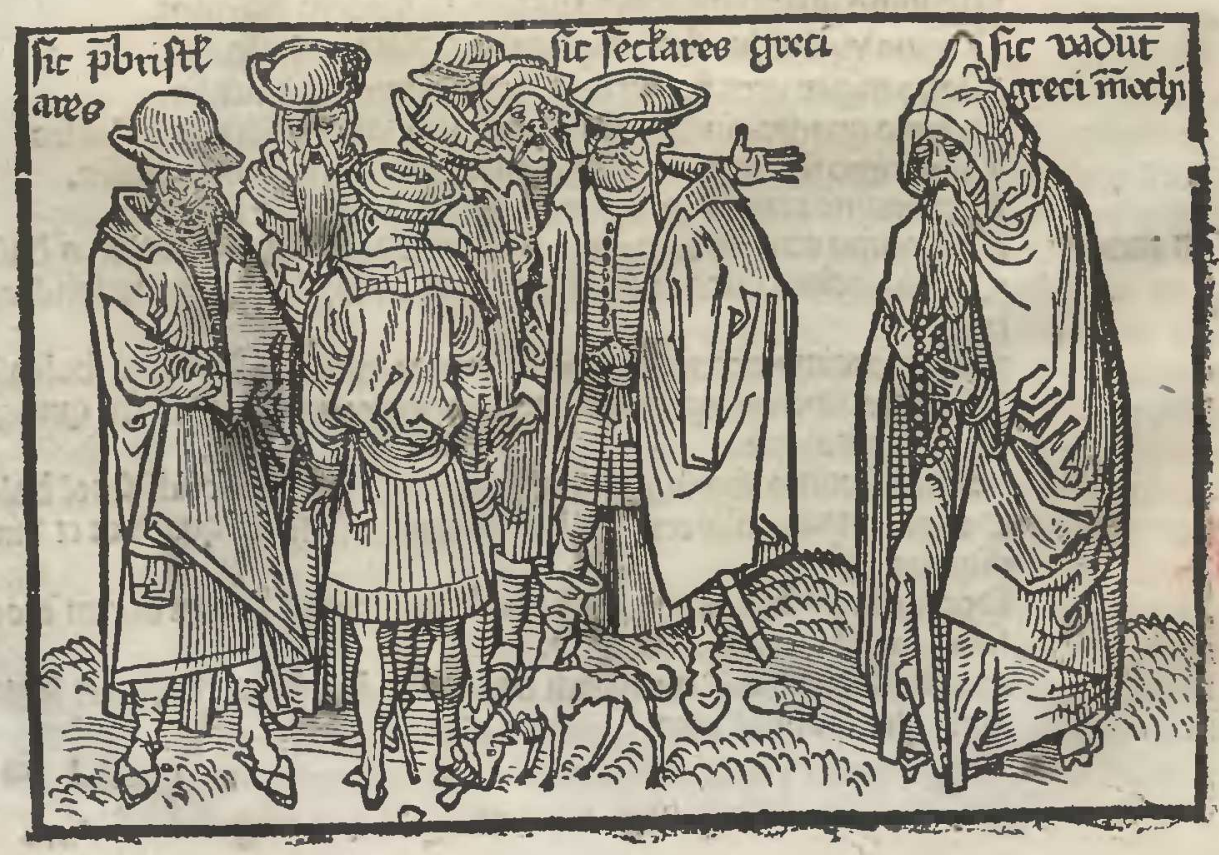

Defrecisquozum etiam plures funtin

7

ciultate bicturalcm incolunt bac tempeftate plurimioucers

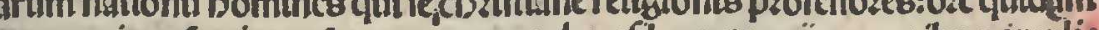

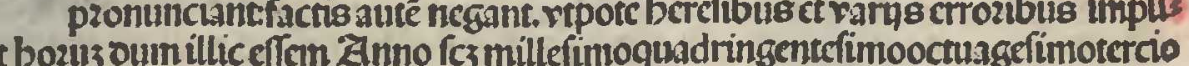
eatict boally

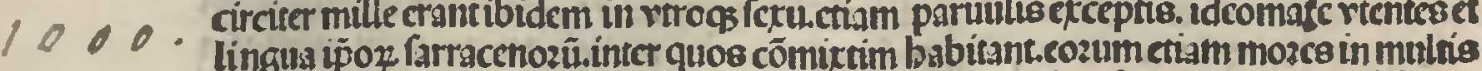

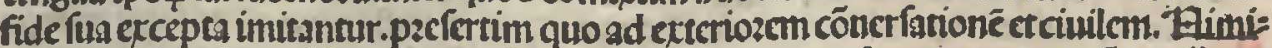
cum quan mimea secece

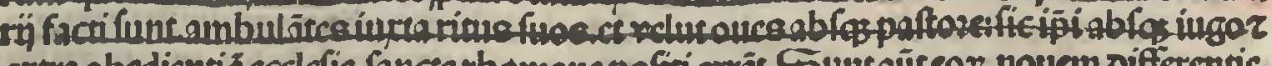
a (uive acobini ali inime

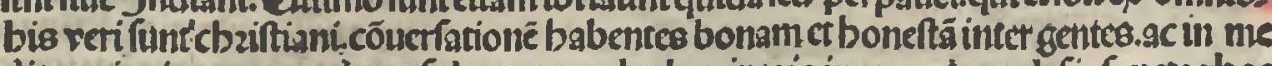

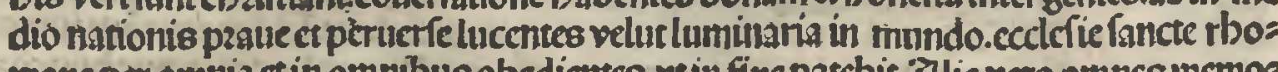
manceris

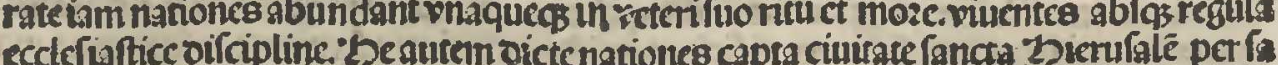

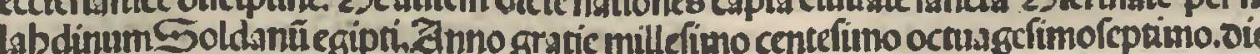

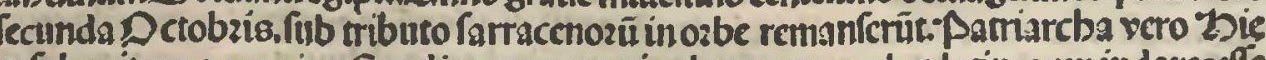
rofolymitanus nomine Eraclins.9tas omnis cenuset populus lannozum indereceffe retcón ros macbinis oebilinafer verentes ciures chziftianicum eq nullo loco aurilium pzefto

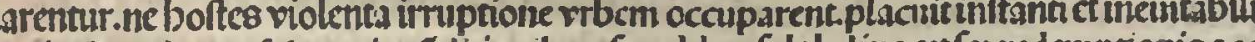

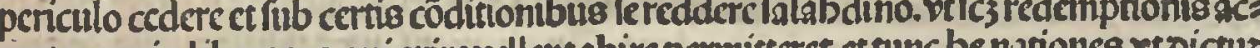

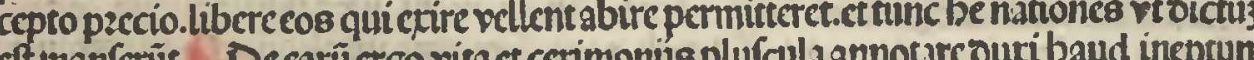
cron

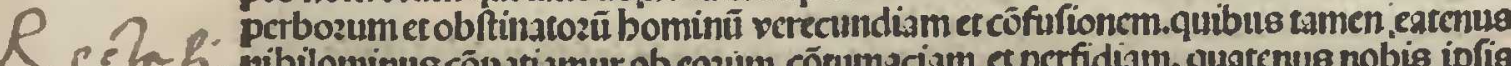

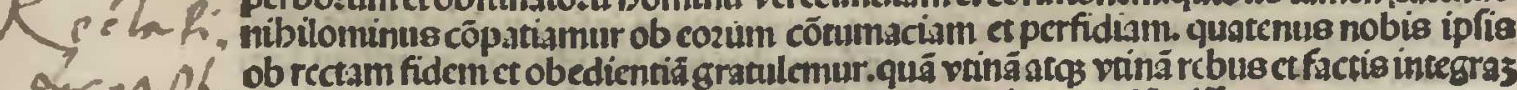

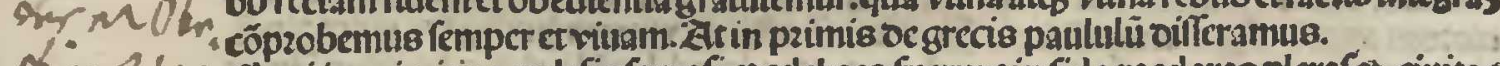

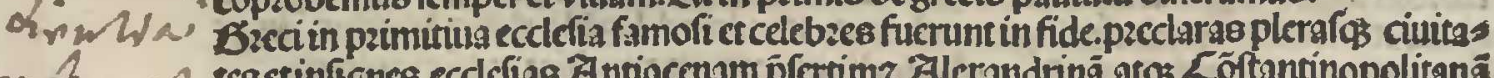

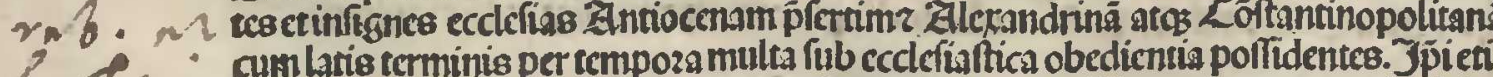

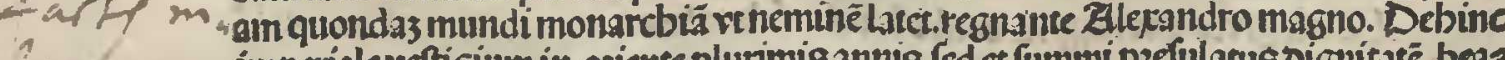

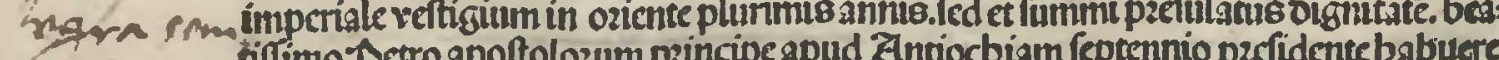

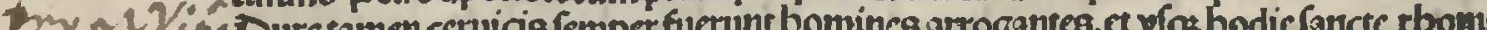

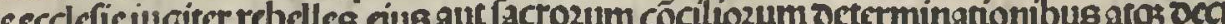

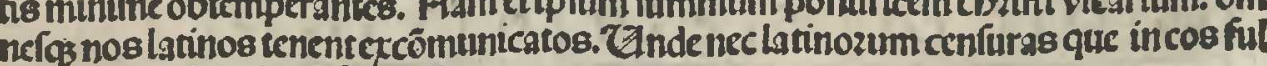
minantur ct fulminatefuntal iquatenus curant.

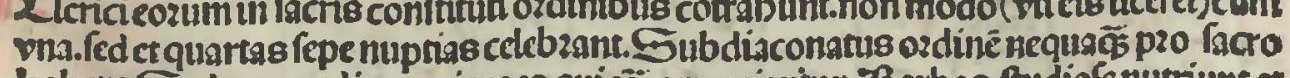

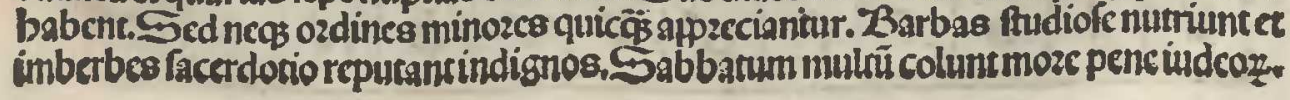

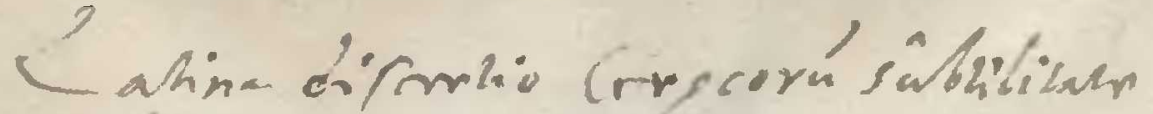
$483 .{ }^{2}$ $560 . \sin ^{2}$ Ex Cosing Cren: Jeriotis $A$ is

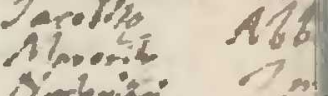
Arovem. Hin: is 7 . ioprens 150.38 Proe Érolationo Tremetion Congrionsens Crión Treprera iniriónfiom inción. mis phinusb. ma crimon CGriblinorn Te imardior 


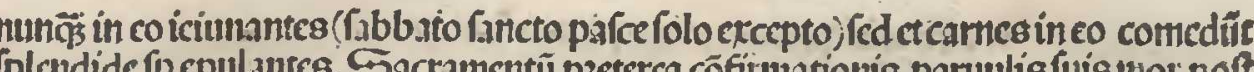

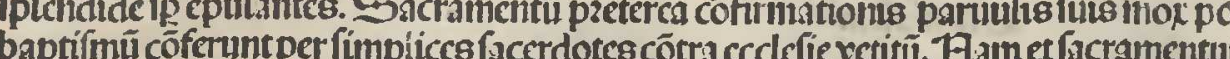
encsaritice

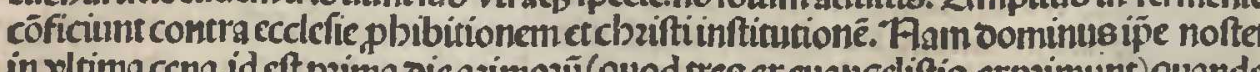

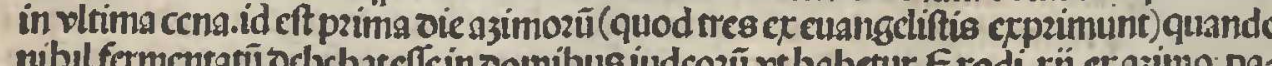

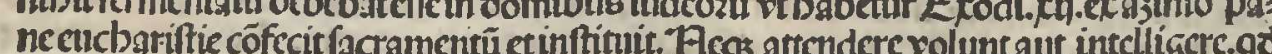
iurta apoftolicam ooctrinä ex purgato fermento vereri malicicetnequicic in azimis iun

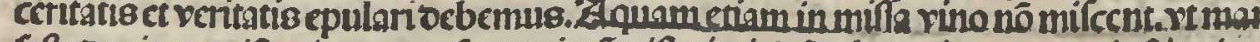

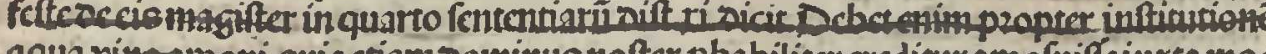
conillivo s.

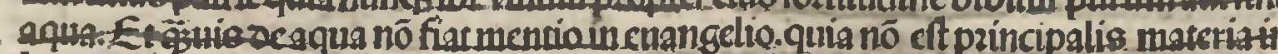

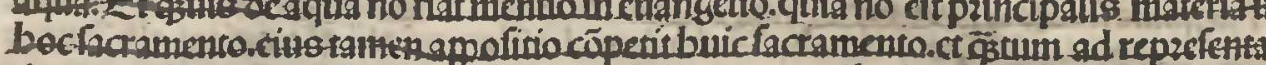

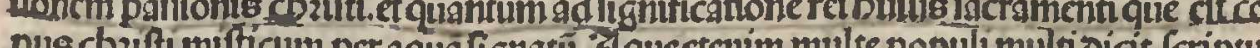
pulscbalti m. cóiuncrionis Etan

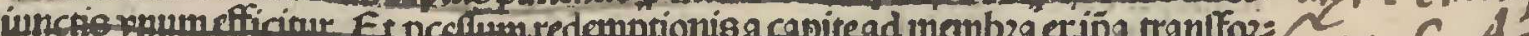

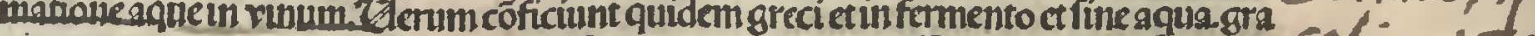

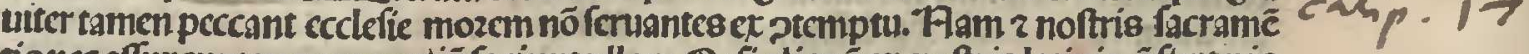

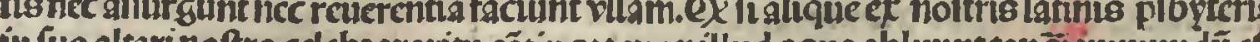

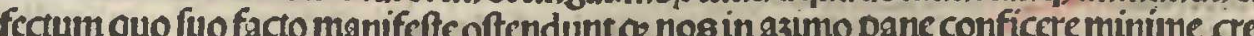

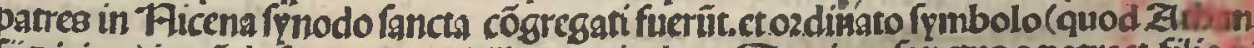
ty ocitur ita cocturctunt

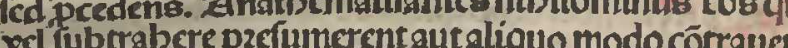

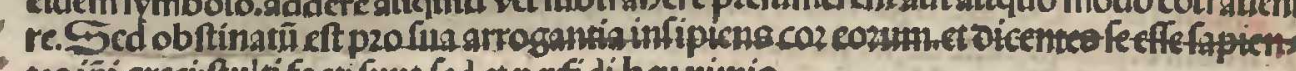

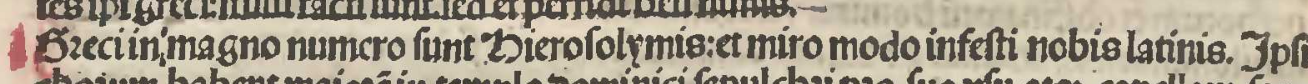

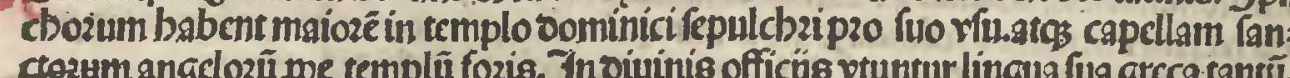

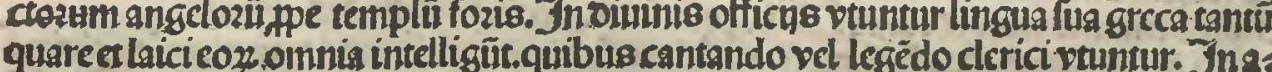
lige vero negocủ s vel cōrractibus cu alğ\&lingua vtuntur arabica autfarracenica.

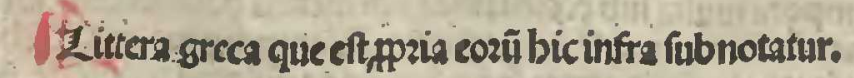

\begin{tabular}{|c|c|c|c|c|c|c|c|c|c|c|c|}
\hline & Bitit & grama & $1>0<t r a$ & .e. & $z^{\text {tita }}$ & int & thitita & 2000 & ory & Llalds & imín \\
\hline $\mathbb{C}$ & $\beta$ & $\Gamma$ & $\Delta$ & $\varepsilon$ & 3 & H & 8 & 1 & $k$ & $\lambda$ & $\mu$ \\
\hline $\mathrm{Mi}$ & $x^{i}$ & $\overbrace{n=0}^{\operatorname{mini}}$ & $p^{i}$ & 30 & rigme & tatur & 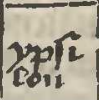 & phen & $d y i$ & $\mathcal{H} \hat{i}$ & omiege \\
\hline$\mu$ & $\xi$ & 0 & $\pi$ & e & $\sigma$ & $\tau$ & $v$ & $\phi$ & $x$ & $\psi$ & $\omega$ \\
\hline
\end{tabular}

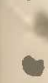

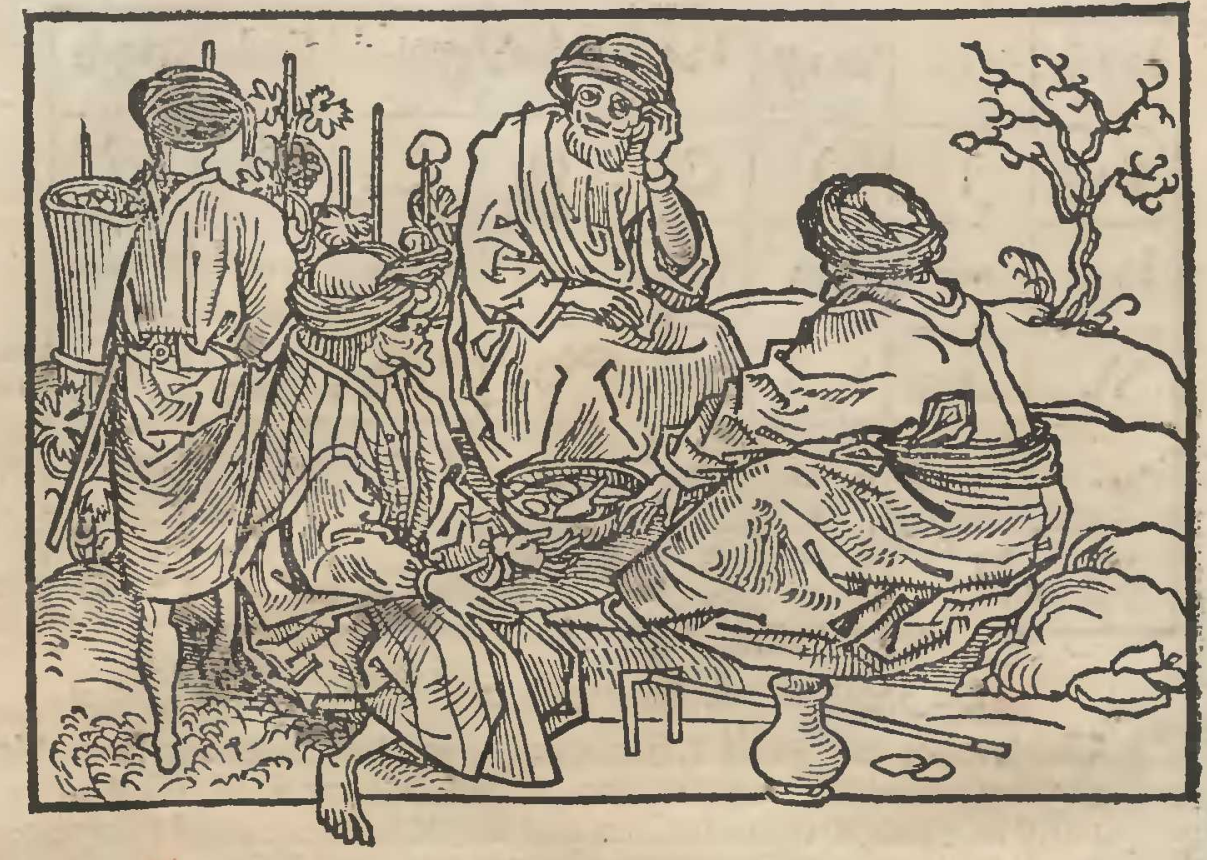

\section{1) Se Surianisqui bicrofolymisetlocis illismanentes}

ciam feaficrunteffec cbziftianos.

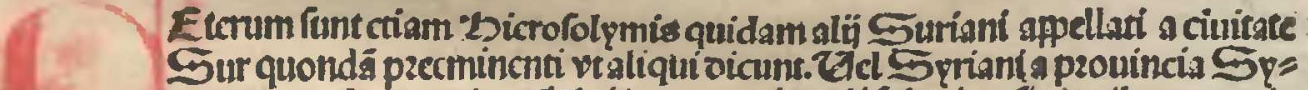

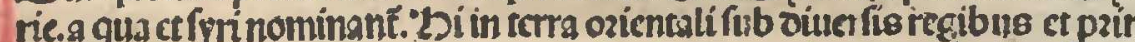
cipibus farracenis atç barbaris iugo fcruíntis opp zununtur abolim. fem

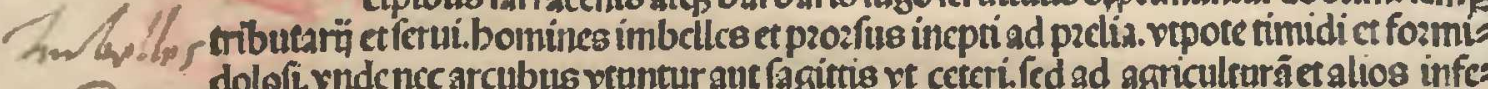

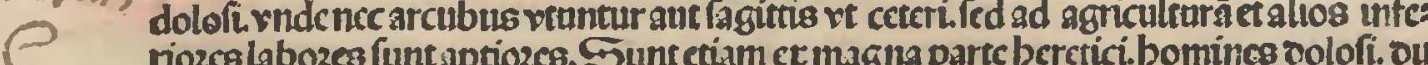
plices animo atto mendaces.antici foztune.ctad muncta sccipiéda pzomptiffini. Fur

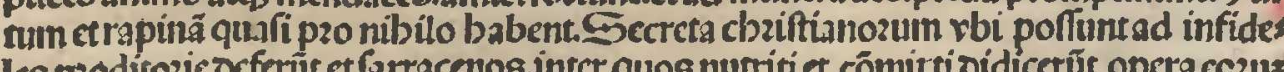

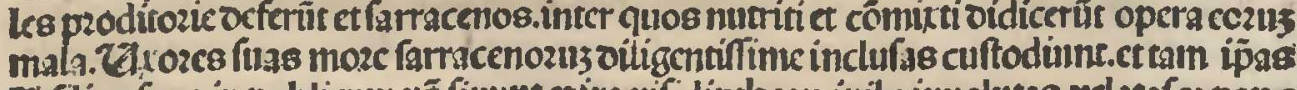

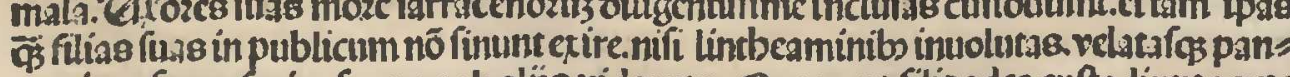

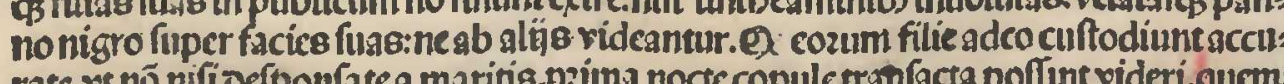
filias fias itidcm foruant Excmplo pariarcbe theob

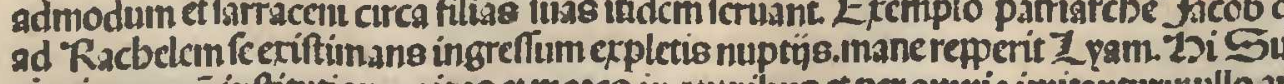
riani.grecozīi inftitutionce rinus et mozes. in omnibus ct per ommia imitrantur:nullo ar

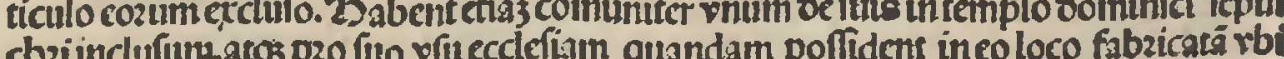

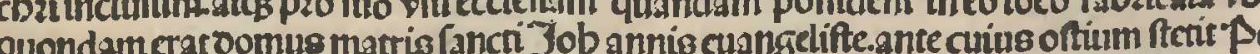

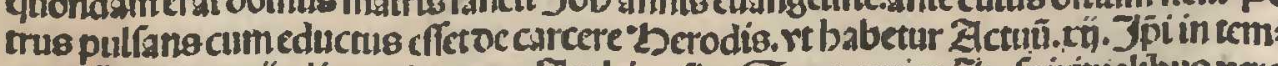

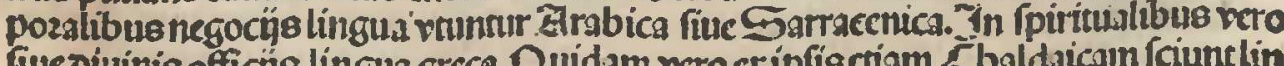

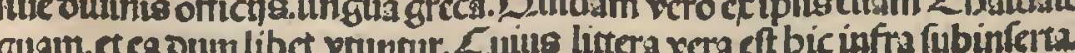

Sirviame $\therefore S_{m}$ Syrianialyr Tolori: $3: 1$ amine 


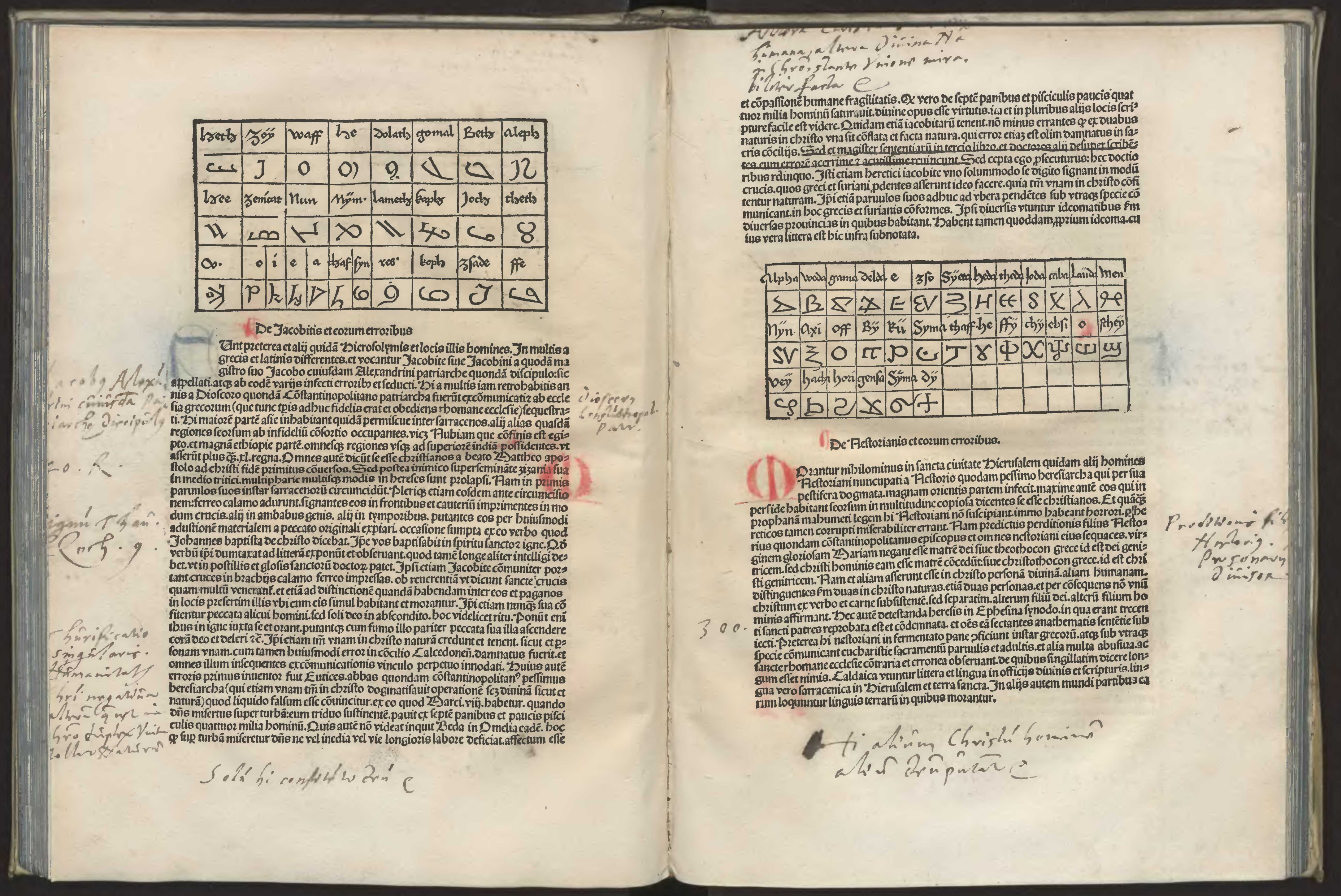




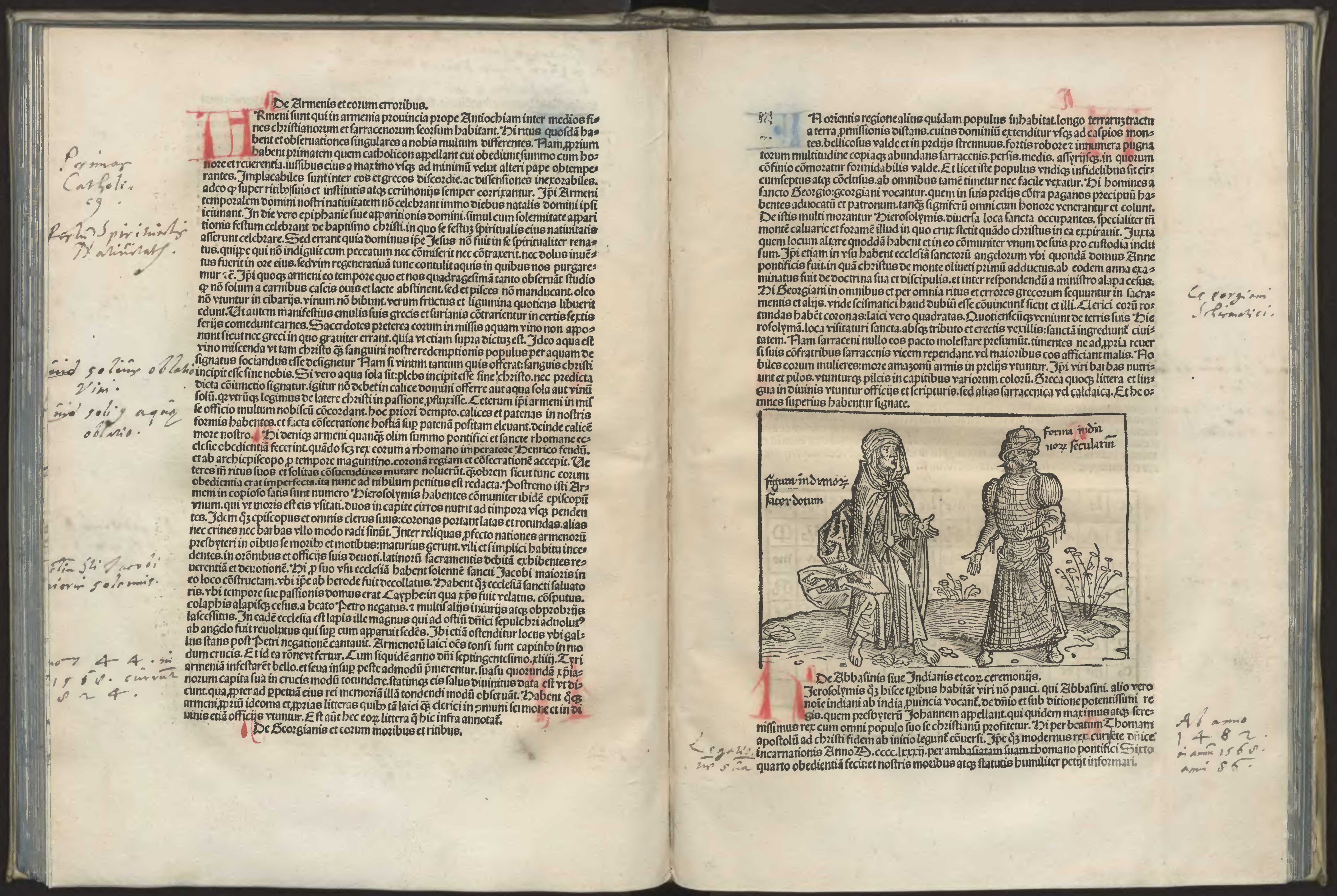




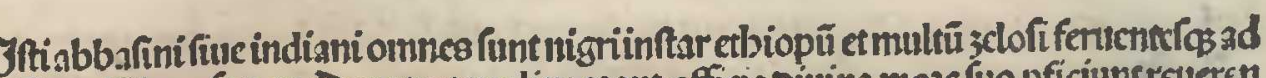

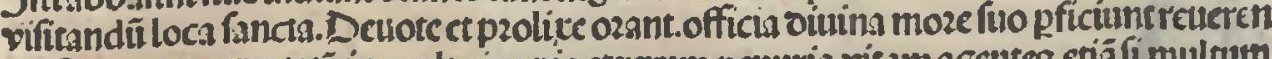
cet. Antusn

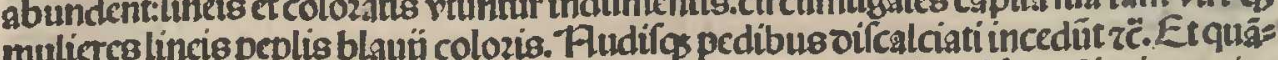

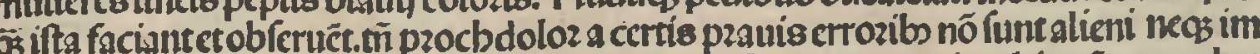

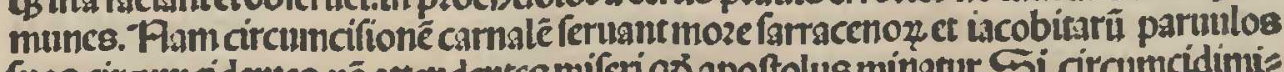

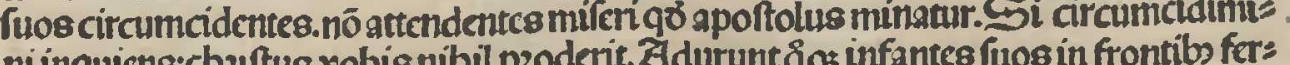
ro locis. credentcses per buiufmodi aduftionem ab oziginalipeccato mundari. $\mathcal{L} u n g s$ ego miferis cópaniens qui alias lanis boni boicevident:p interpaeteinquireré.cur bec faccerêt que omnino rbomane eccléfieffient aducria. cui ienuper.milla obedientria fua

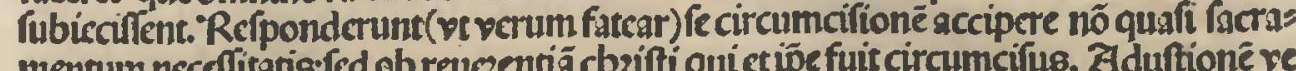
1 to ob

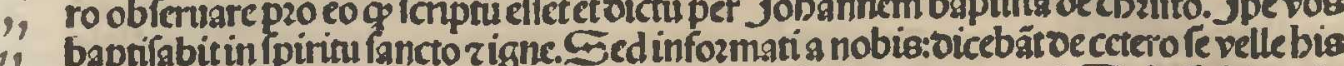
pzete miflis baprifmui in aqua furcepere fmeccletierbomane moze. Sed no folum in

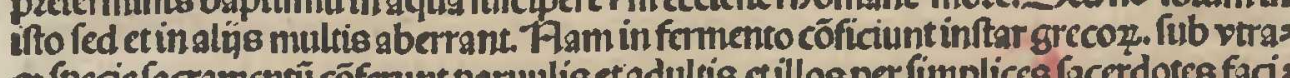

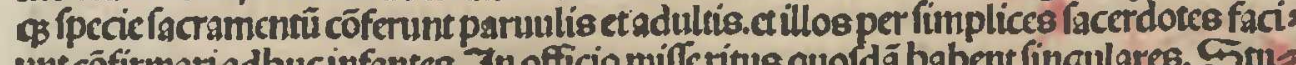

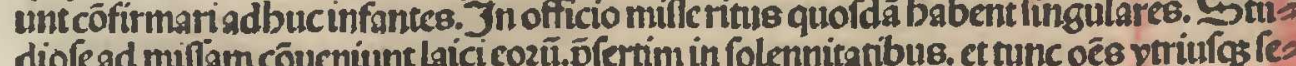
tus incipiunt alris rocibus coctepantes inbilare. flattare pedibus, plandere manibus.

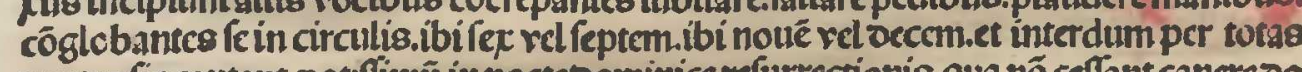
noctce fic cantant. potulfimut in nocte oomunitice reurrectionis, qua no ceflant cantere oo necillucercat.ctnonulli becadeofencenter

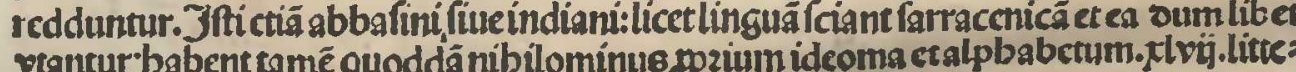
rascōtinens,que bicinfra fuis figuris funt formars.

\begin{tabular}{|c|c|c|c|c|c|c|c|c|c|c|c|c|}
\hline$\alpha$ & 10 & photit & 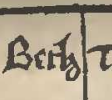 & & & & & & & "yothin & & $500+2$ \\
\hline K & $\Delta$. & $5:$ & $\Omega$ & & 8 & $\mathbb{A}$ & & $x$ & L & 3 & $\longleftarrow$ & $\Phi$ \\
\hline Suf & $3^{a}$ & Jit & ber & $7 B_{3} 4$ & $t b e$ & $a_{34}$ & 10 & Dut & 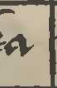 & protit & $2 a$ & tome \\
\hline (D) & म & 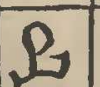 & sh. & & $\mathfrak{L}$ & $F$ & 8 & $8:$ & 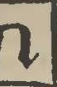 & 40 & $\Lambda$ & $a$ \\
\hline 84 & 20 & $n_{4 \mathrm{~m}}$ & Sard & & & $\Delta \mathfrak{u}$ & met & Alan & 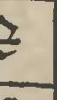 & $\mathrm{ffe}$ & 305 & $s_{0}$ \\
\hline $8:$ & G) & $\xi$ & Љ & $P$ & 5 & 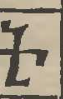 & q & $\infty$ & 8 & $\Delta$ & $\pi$ & $\rho$ \\
\hline$\infty$ & $f_{i 2}$ & $k_{0}$ & 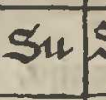 & Sand & & $t_{1}$ & $\sin$ & & & & & \\
\hline$\beta$ & $\mathscr{S}_{0}$ & & & [1 & & & D. & & & & & \\
\hline
\end{tabular}

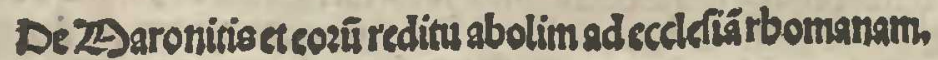

abitatetiam gens quedam frroun fub monte libani in prouincia pbenicis

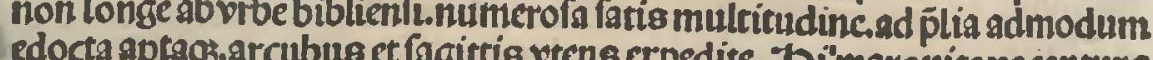

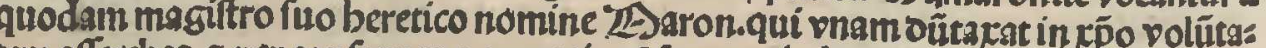
tem afferebat. z per confequens operationê éccutus in boc quendam antiochenum

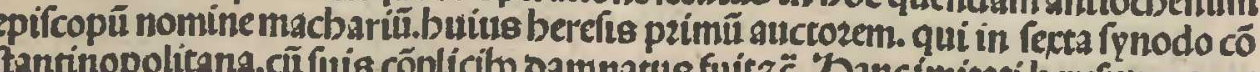
mite

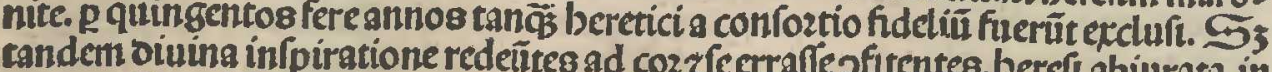
manus L meric cuintdam antbloceni patriarche romanecclefic obedientrata in unt adrumcrati. zad vnitatem cartbolice fideci reuerfi. Zindecĩ omnes alí ozicnta

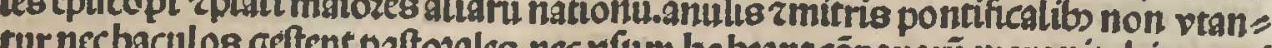
bus pruntur vrinos in

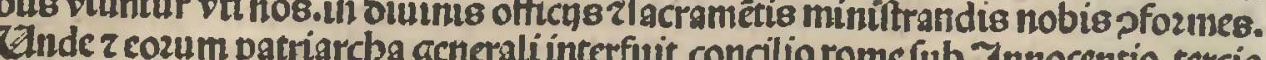
celebzato. 'bi maronitenonmozantur Jerofolimis continue fed fepius illuc ecio uotione ventut peregrinatum ad qualdam folcnnirates. nec pzopziil babcnt idios ma.ed vel caldaica vel farracenical tingua vetuntur.

quolibet mormo quíf piom quas narrautmus fectarum aut legũ. sut alio in fua fide lege vel fecta pofféfaluari. oummö credat came efiécbonum

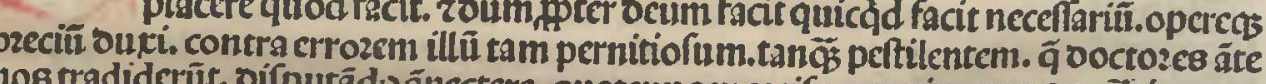

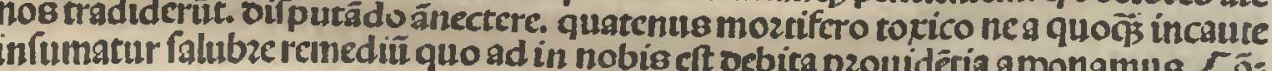

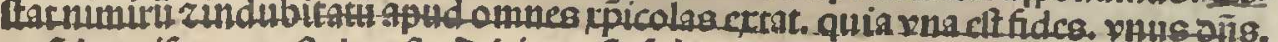

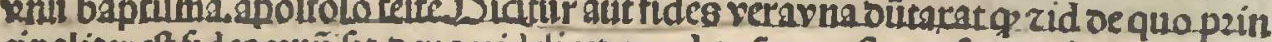

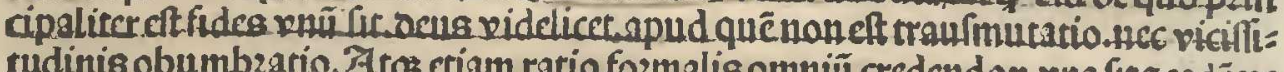

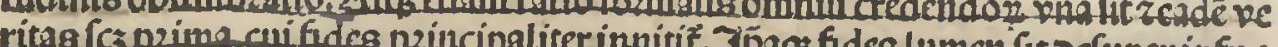

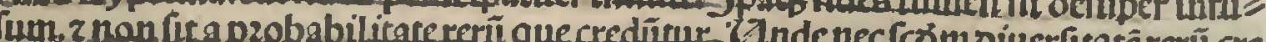

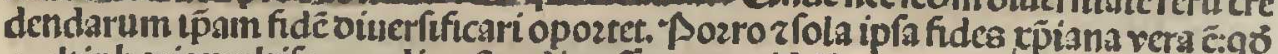

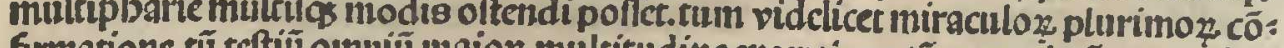
rmationc. tas certiflumas bahe

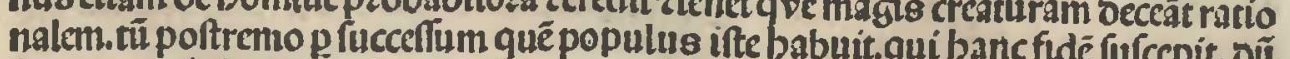

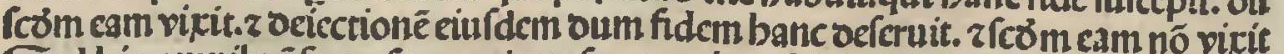

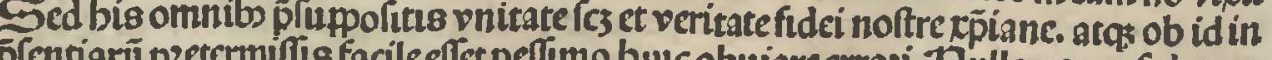
Teditur prer banc znullac aligaigna

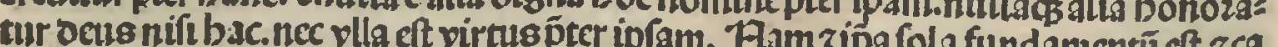
put omnis religionis in vita itta ficurzeius opató pzima eft in ociopibs robfeanin

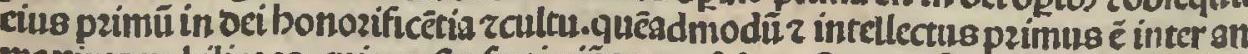

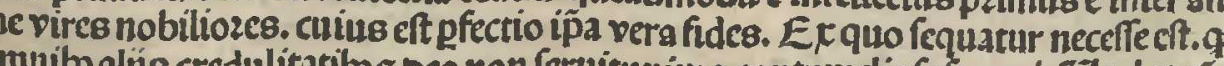
ftatnullam al iam creduliance undamêto siccus erroz pcefferit videam? ve ficip fo clifo fundamerto onunis quo

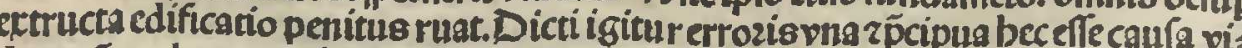
cas faluandosiü. Zünde zmulti inquirür quare tam paucos elegerit oei inifercozdia
Hemtis?

Movafurén iox

Ras. Sirm enconor 


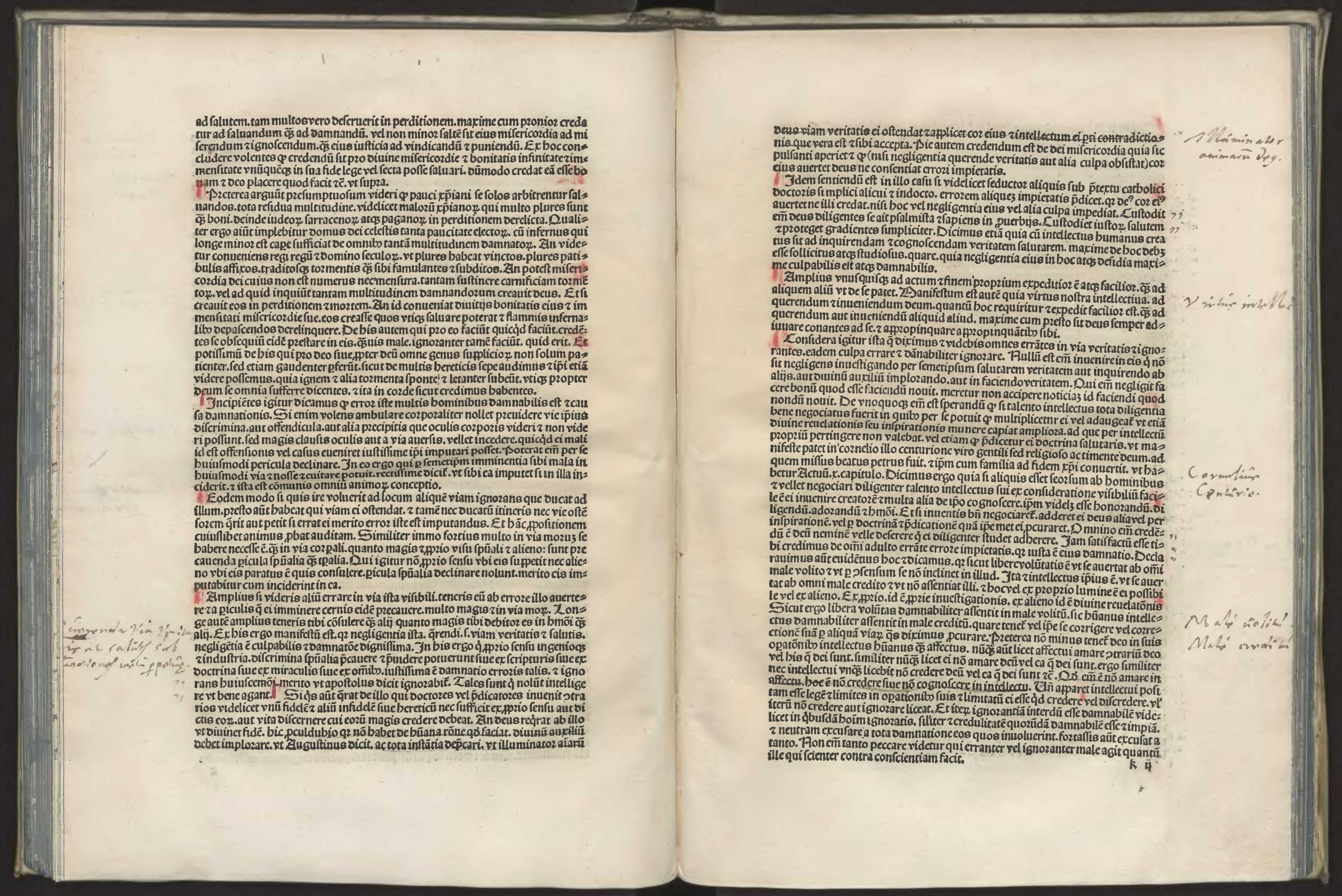


(3girurvt in fumma dicamus, illud nobis potius videtur diune confonü pictas

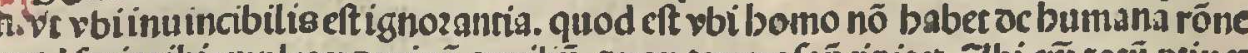

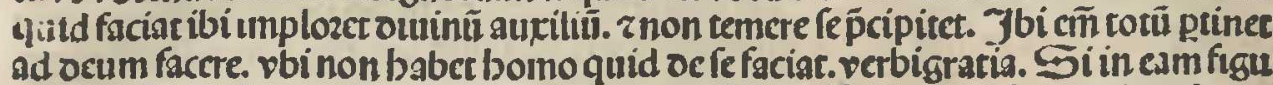

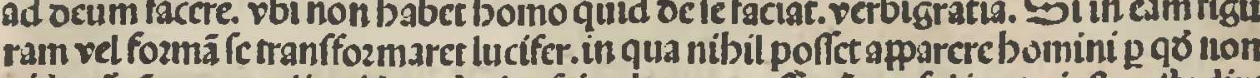

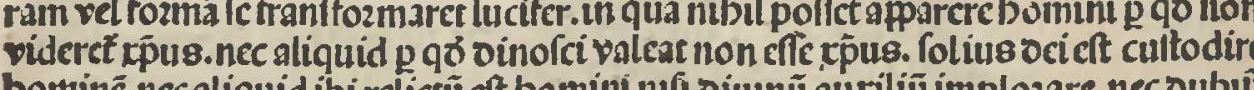
1) quin pzefto fit auxiliaroz ocus inuocantiberi.nificulpa coz obftitcrit.

oz ad quid ocus că creaucrit. centĩ eft quñ

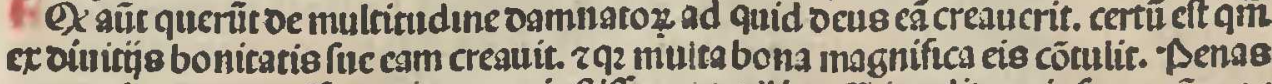

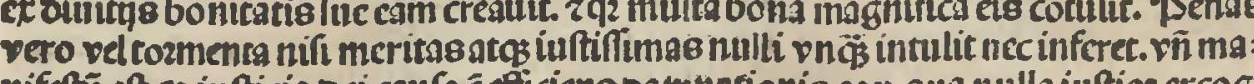

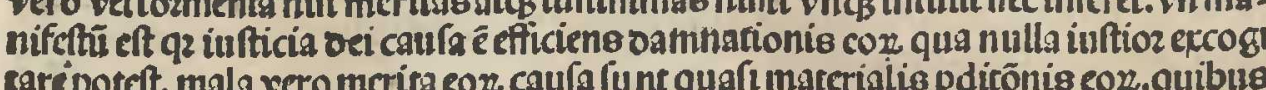
unftifimo iudicio zlaudanda in omnits iuftica. o. amnatio illa cis redditur

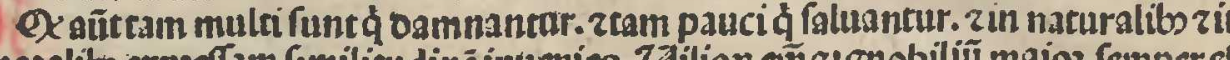

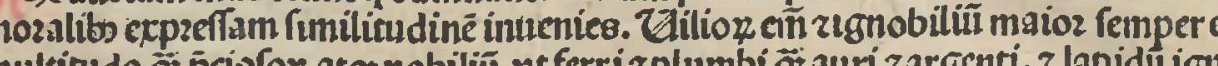

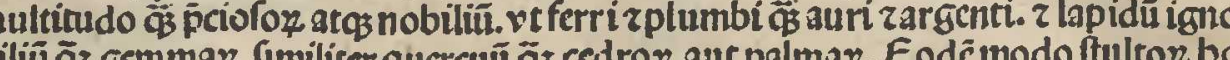

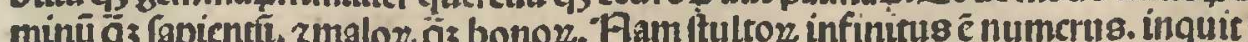

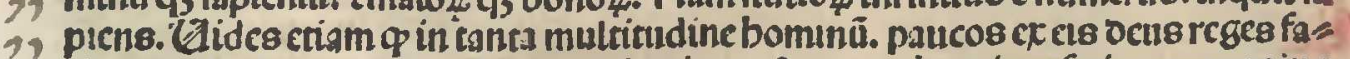

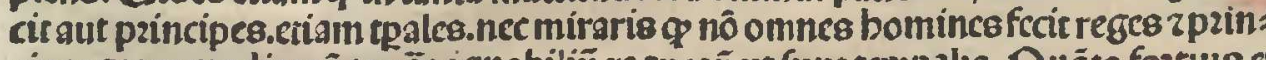
cipes ztam modicozin tantaj ignobilu regnoti ve tunt tempalla. Duato fortlly

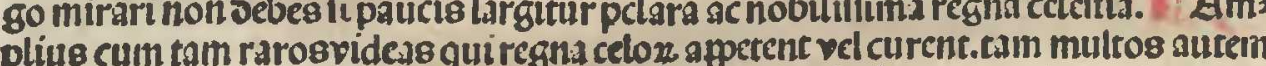

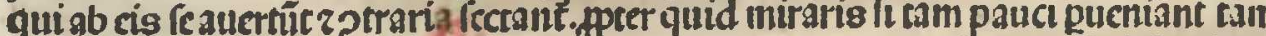

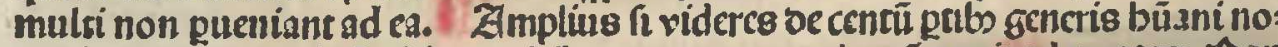

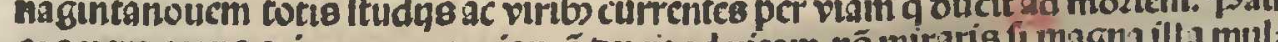
等

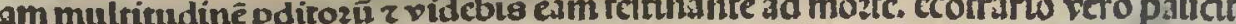

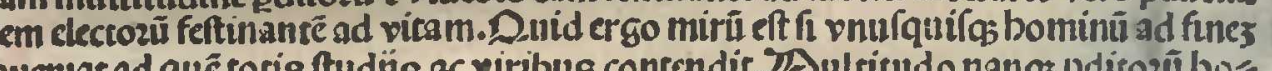
pucrar aduer pula

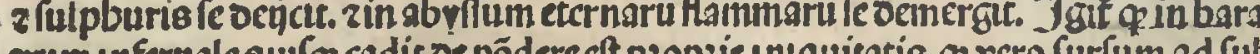

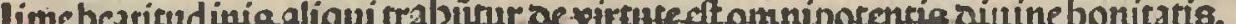

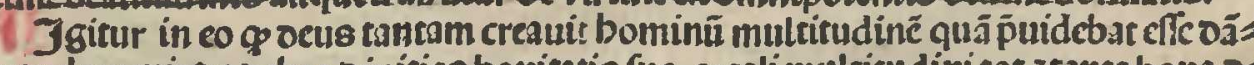

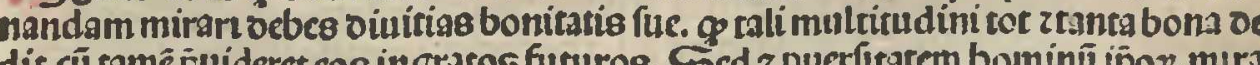

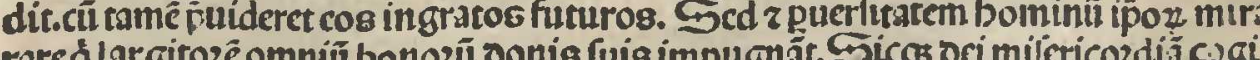
to prziulticiecills memineris,que illos quos milericoz día crat pafcit.regirzcons

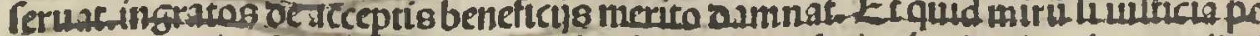

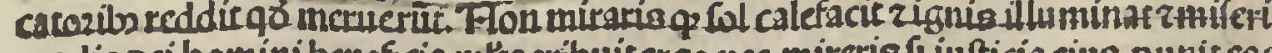

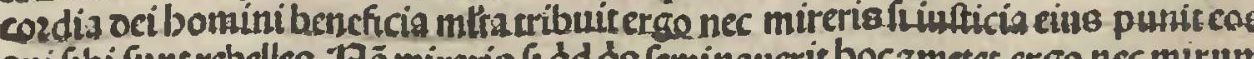
(1)

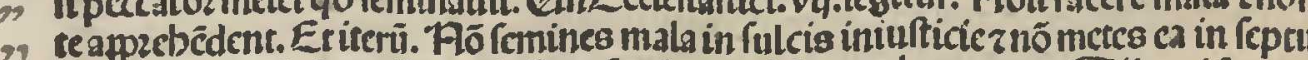
plun. Et apoftolus. Bala.vi. Due feminaucrit bomo bec ametet. Siboni fumn

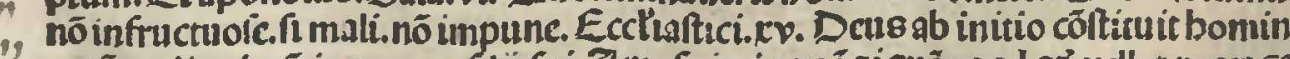

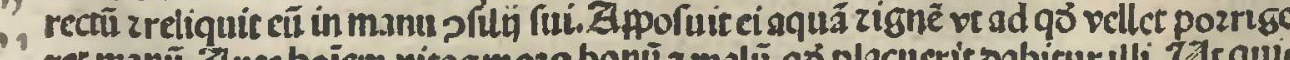
renain

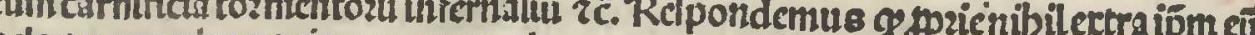

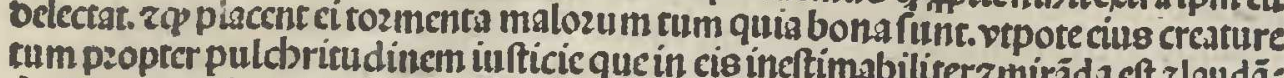

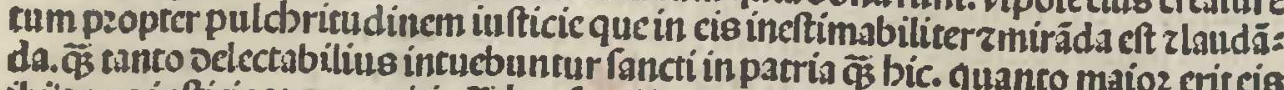

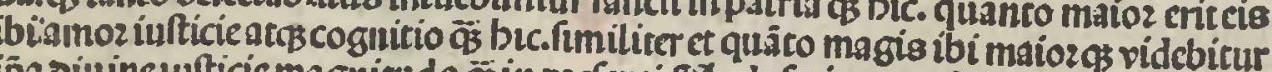

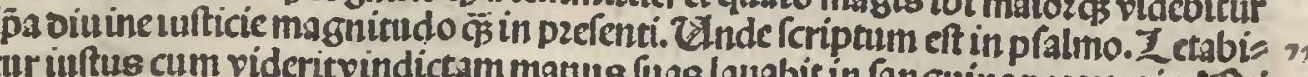
tur iuftus cum videritvindictam manus fuss lauabit in fan guine peccatoris. DS i " "?

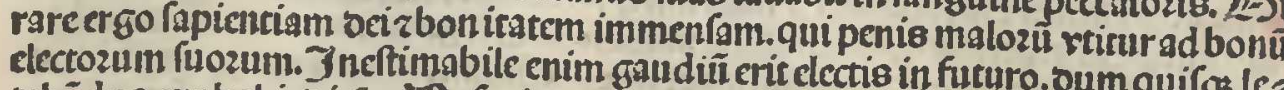

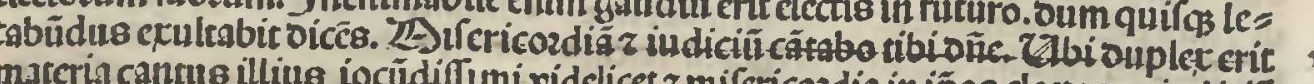

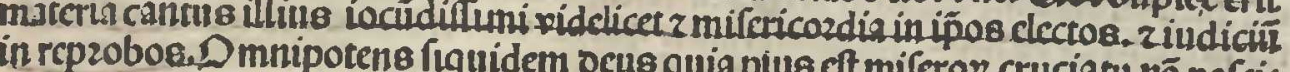

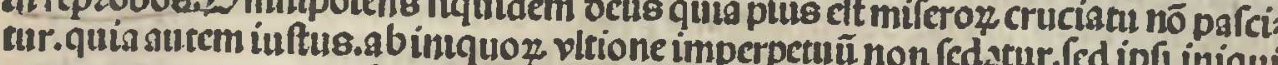
omnes eccrno oeputati fupplicio fua quidé iniquitrare puniūtur z tamen ad aliód ar:

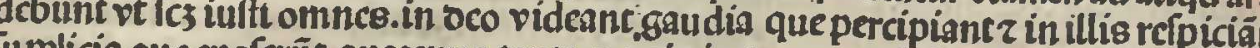
upplicia que cuaferint.quasenus tanto magis inetermum oiuine fe gratic ocbitozes inetcrnũ mala puniri confpiciît. quecius adiutozio vices Ex biscrgo que oiximus manifeftum tibi ocbet effeo bomo. quia laudanda eft

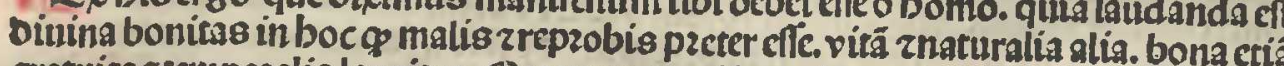
Gratuita zcempozalia largitur. Qx autcem amplioza eis non oat non ideo boc cft. .2 eft vt co minum

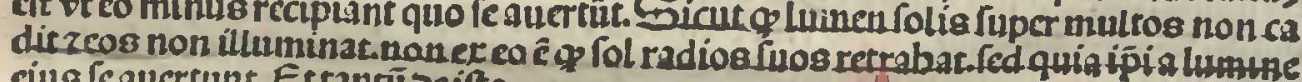
Decaufis varietaris crroz zfectax multiphtciis.

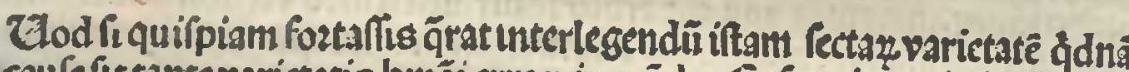

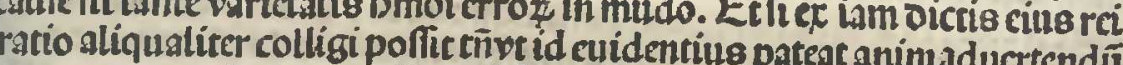

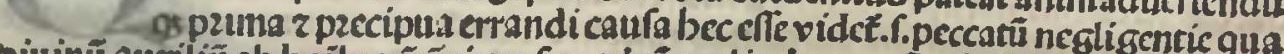

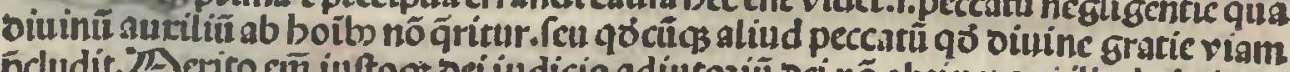
nō arat z ecte

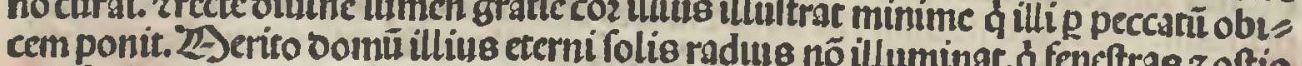

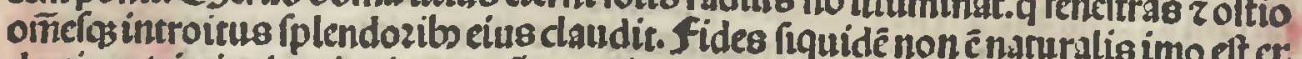

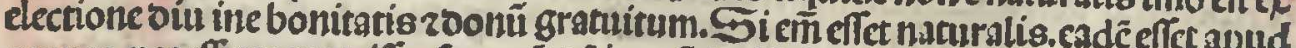
onnce.ncecher Tolus Ephe in Sicur erco fol nō videtur níf lumine fuo fic fol inelligentic

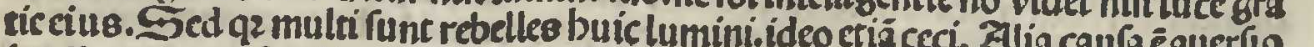
Dectus a cedendia zab bis a pofient induceread credenda z ouerfio ad errores dereñ podef zfectas re 0 cốtarije nec

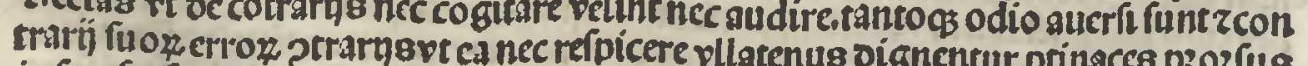

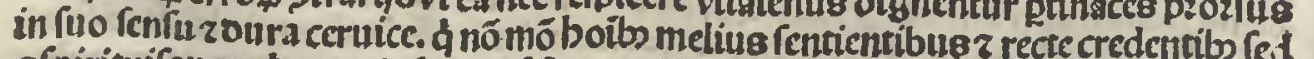

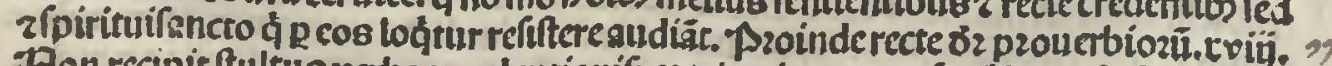
Fañ

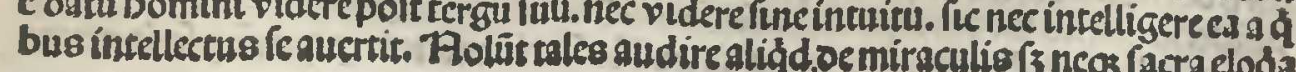

Pnerathéning Precalinólo 
crationes ooctozum quibus poffent ad veram fidem induci. ideo non mirum (a) .

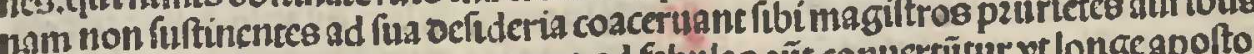

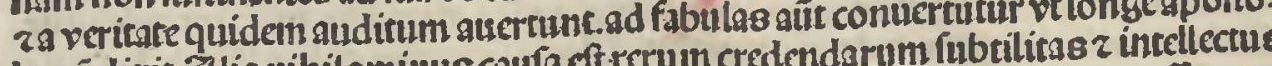

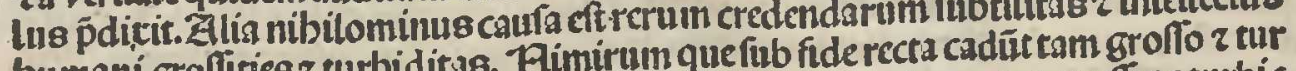

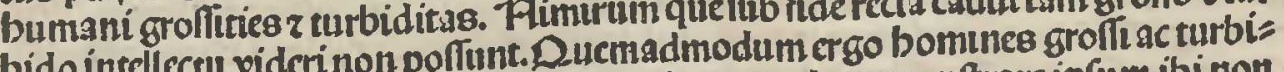
di vifus pilum fubrilem nonvidentes vbteft. Contendunt mont trare iplum ibi nort effe.pzopser cius fubrilitatem vel gracilitatem ipm non videntes, necosiprum vidch

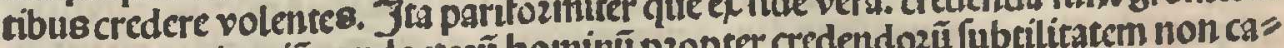
inteltectus vulgariin z undoctozin bom

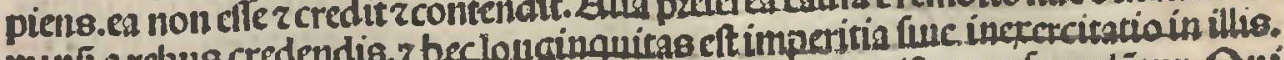
pucing enim in fenfibilibus verfanturz 2 mozantur aflidue que longe funta credendis que inuifíbilia hint.p2ofecto longer

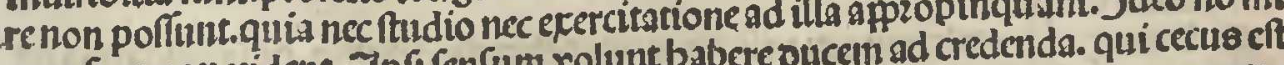

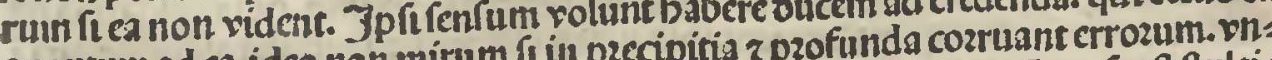
quantum ad ca.

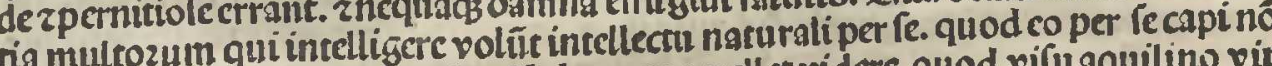
poceft. quemadmodum fi quis vifu bumano vellet ridere quod vilu aquilino vile videri poreft. fic intellectus naturalis ad ea que fidci funt per fe tuultatenus fecenten dit nifi alciozi lumine perfundatur. Bi tunt qui burecifitimantr. zquod illo non ap

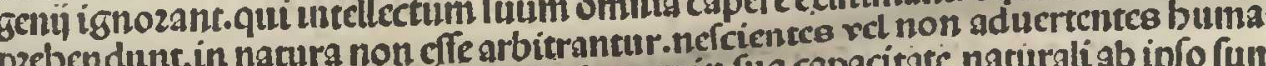

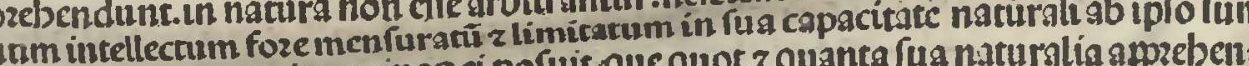
mo crearoze oco qui rerminos ei pof

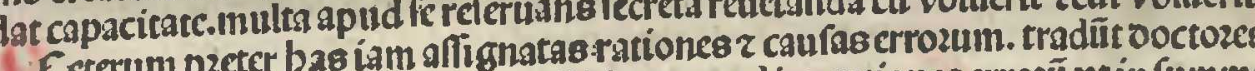
siths Dicentes $q$ omnes falfe fuperftitiones et adinucntiones crrozĩ vt in fumm oicatur ant ex impzobe pbifice inueftigationis aufu procedint. Acut errozes pbilof

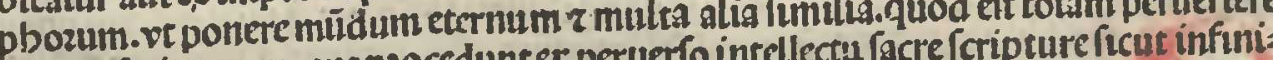

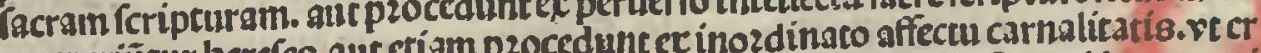

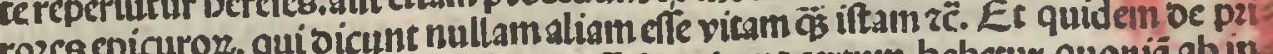
mis puta pbilofopbis qui fé fcientes efre iactabant certum babecur.quonia ab is

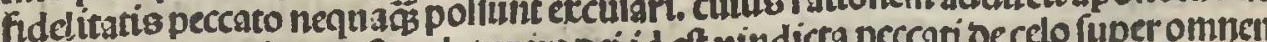
Roma. pzimo oicens. Reuelatur ira oci id eft vindiéa pecectari contra mozes, bo impietaremitdell pecalis mintum boztum qu

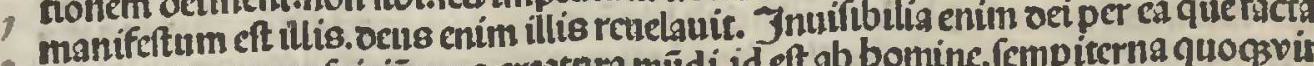

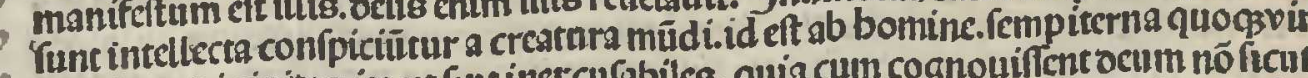

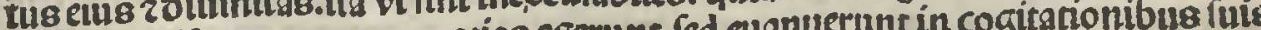
Deum glosificaucrunt aut gratias egerunt. zobraranta

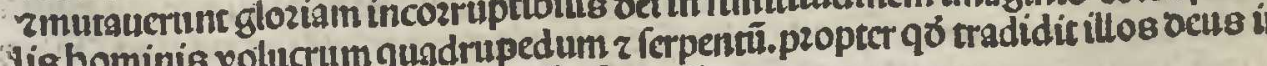

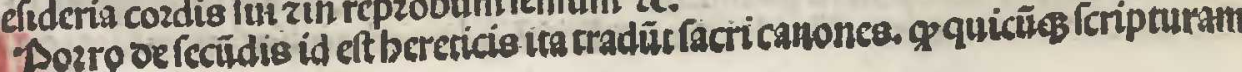

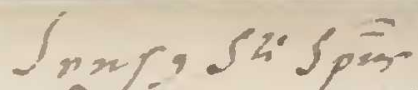

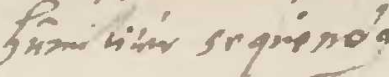

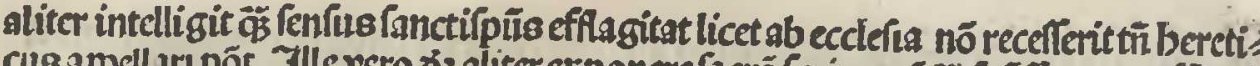

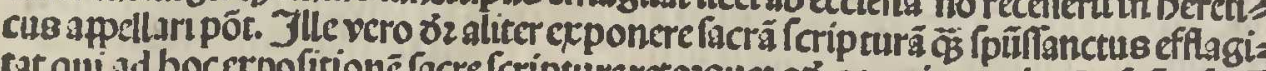

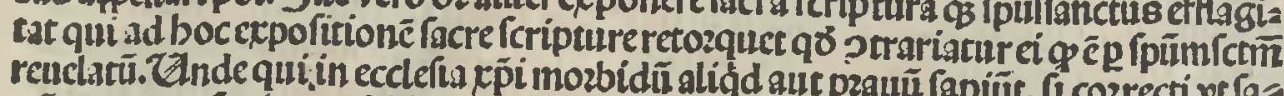

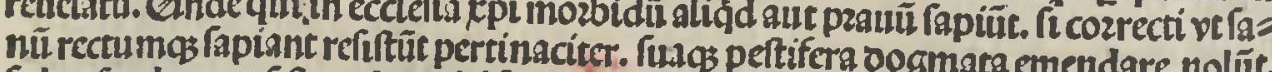

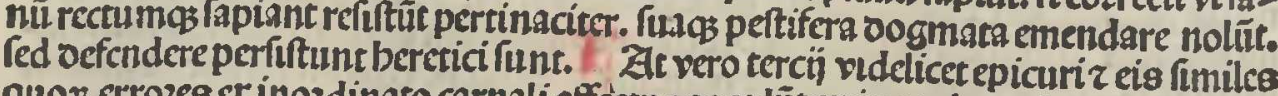
quoy errozes crinozdinato carnaliaffectu procedút ex improbs voluntater electio ne liberi arbitrü peccant malicieadberenteg zita cec certa malicia. Puod quidem $\mathfrak{e}$

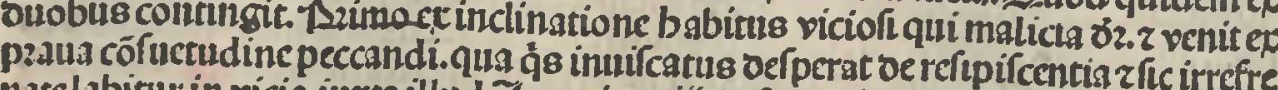

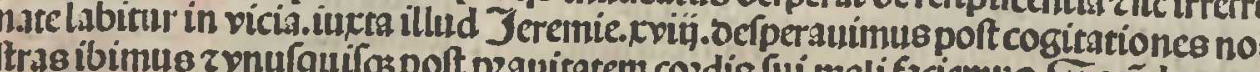

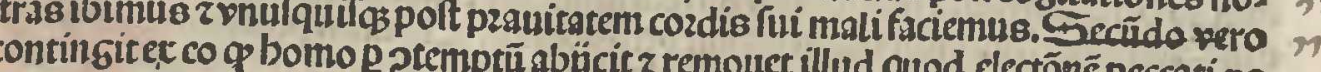

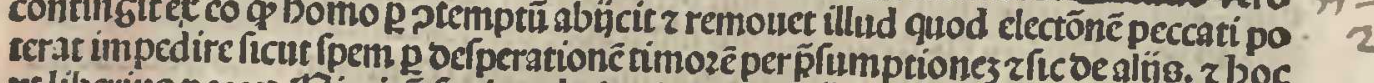

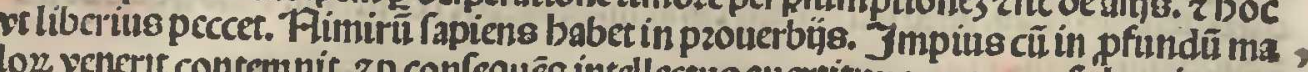

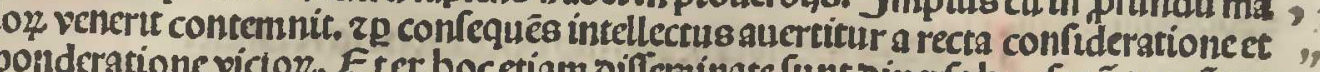

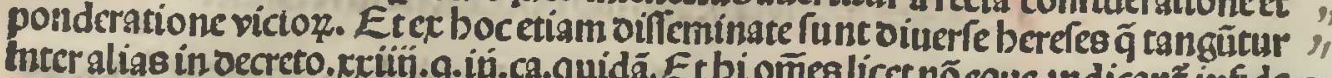

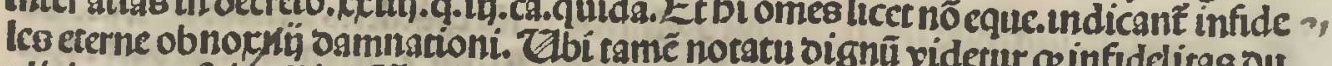

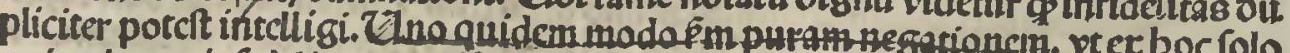
quis bicatur infidelis co $q$ nô babet fidem. vt in ill to à nibil audicrunt oe fide. zvt rü ce peccato pzimi parentis eft fecuta. Dui ergo fic funt infideles oamnäntur ouino pena fenfus pzopter alia peccata fi

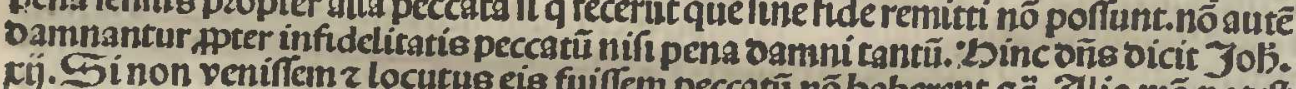

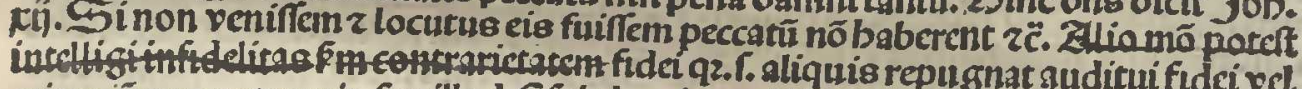
crian ipam contemnit. Fm illud Elaie.

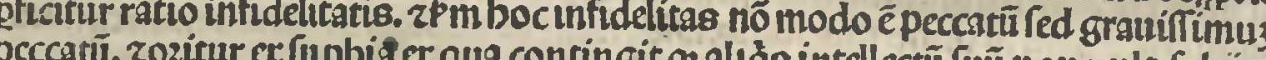
re regulis fideizfano intellectui parrü. rebellis z inobediens ffectur vult fubiłce romane zeills capici fummo antiftiti.cul perfidiezvicio oés fubiacent nationce fits

pza menzozate. De Zatinis quifunt in bierufalcm.

Oft intericctam baud inutilem (pero digreffionem inter ceteras nationce fupza oefignatas. zbanc oe qua nüc oicemus latinozz $z$ catbolicoz ạ etiä nem 6 . cuimus fectarum illarum fiuenationum varietaates.artos congregationum farbane oifferentias multimodas paucis abfoluimus.pzout cracta indagine in locis illis D fcrusantes fingula comperimus. fed pzecter eas. funt etiam poftrema bacnoftra etras rezab aliquot annis fuperiozibs in bierofolimis quidam ortbodore fidec ocuori $;$ retroze. viri rcligiofi. qui a ceteris nationibs ibidem( (ve funt) Latini. a farracenis ve:

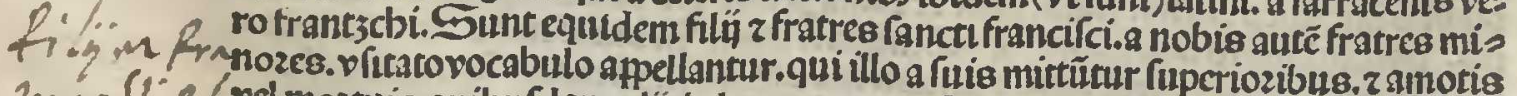

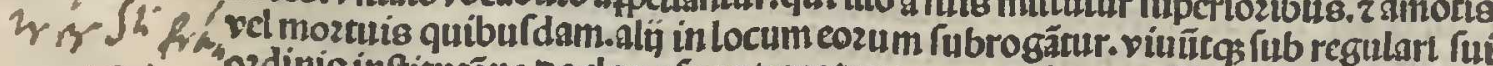

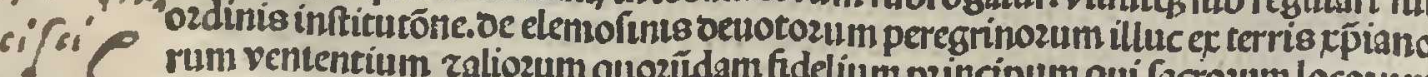

rum vententium zaliozum quozüdam fideliums principum qui facrozum locozun

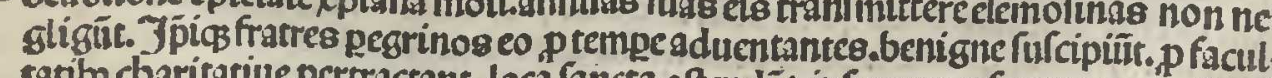

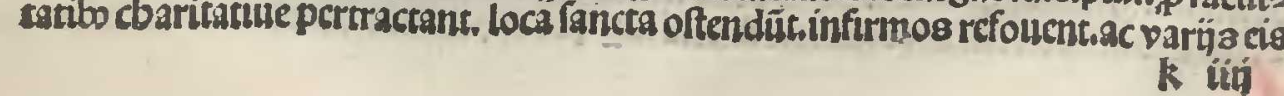

7tempirion apranistio. Epirim .

$$
\text { (1) }
$$




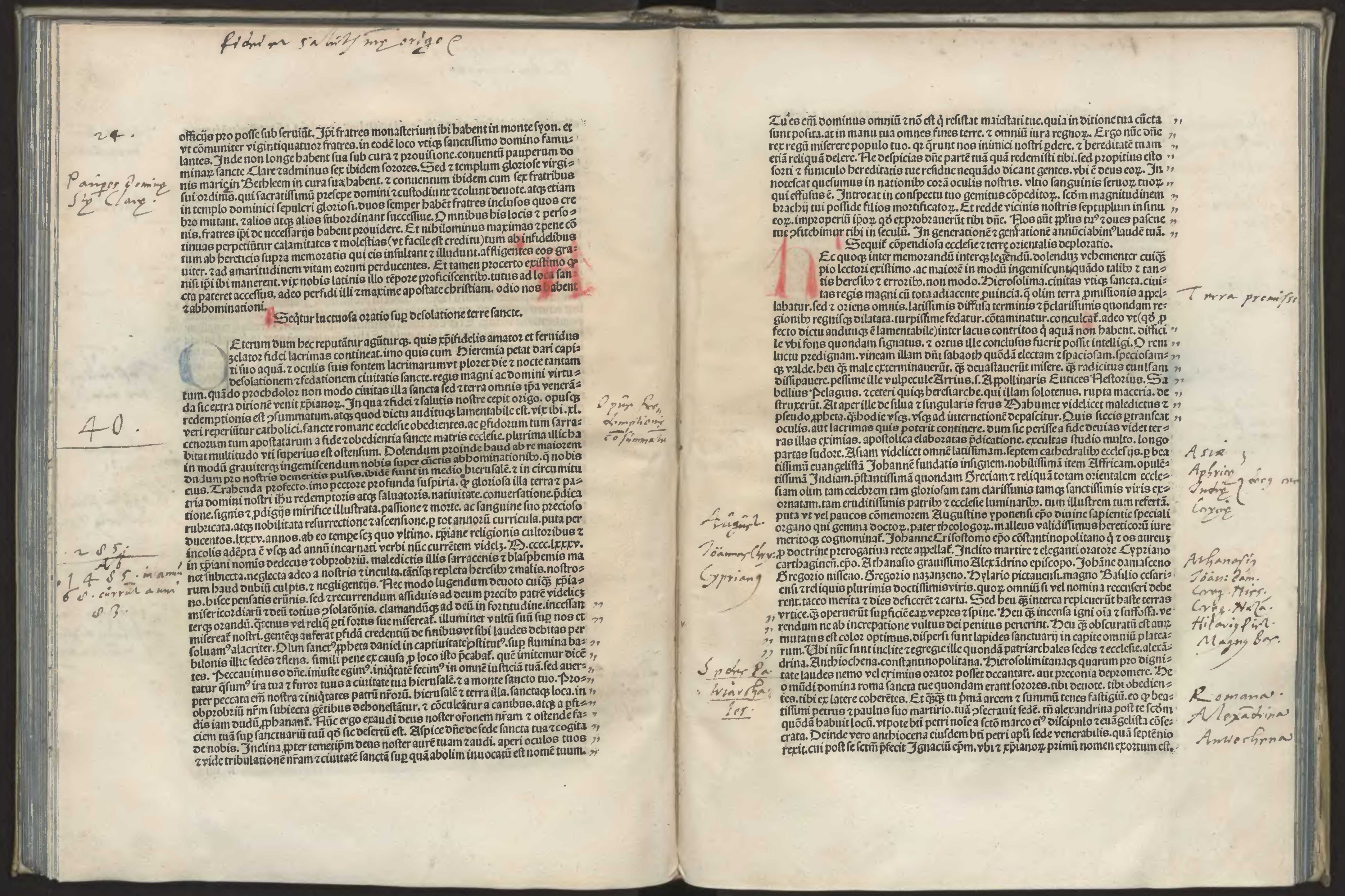




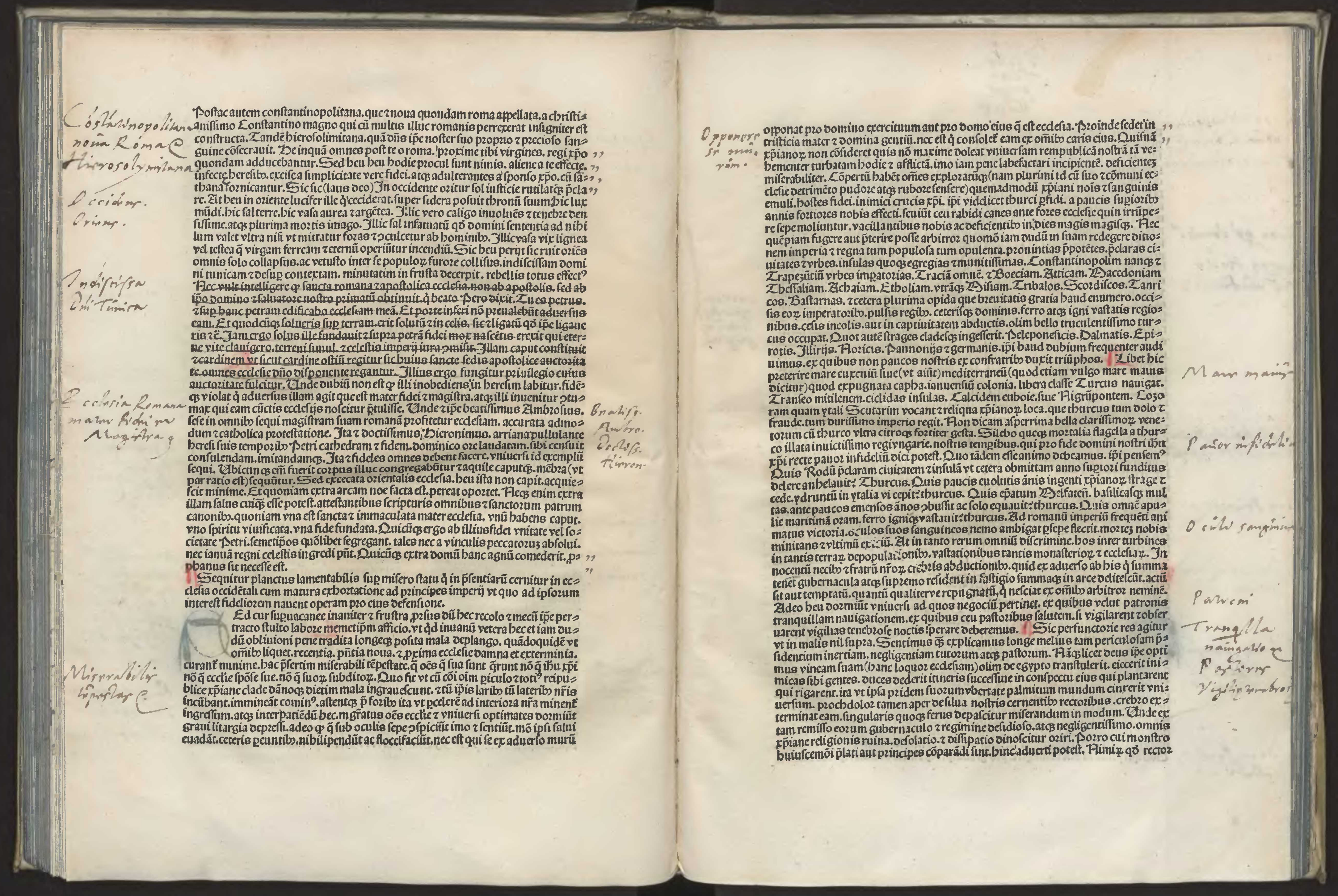




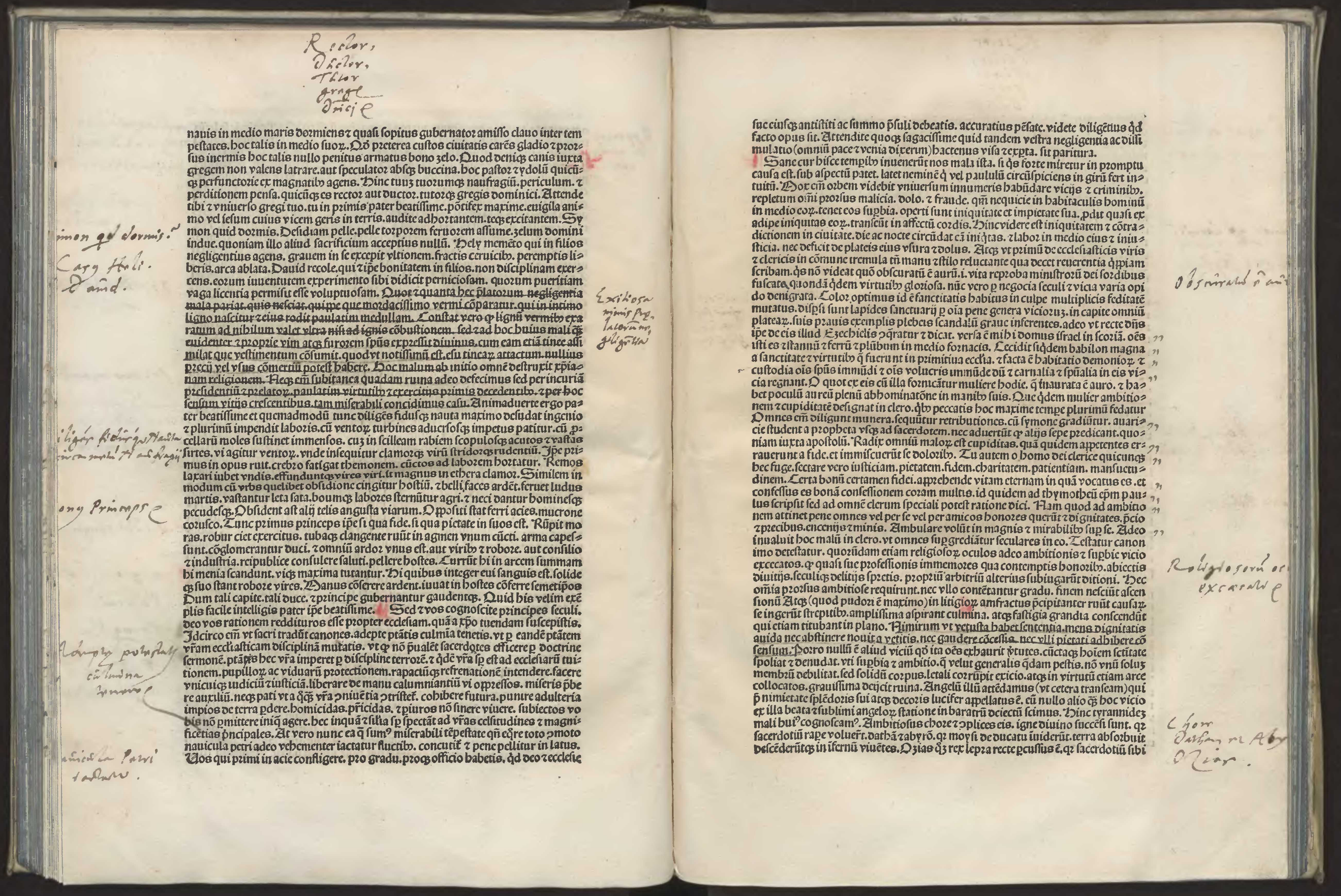


7 agon oxmiln at Fravor 0 min

Traopin siming Parna arolrose morte-

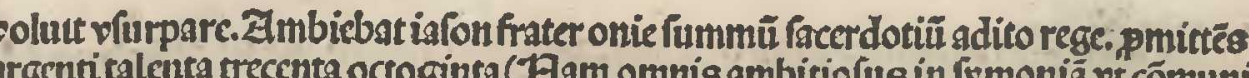
年

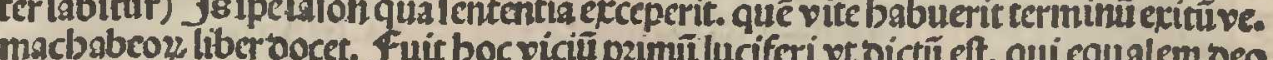

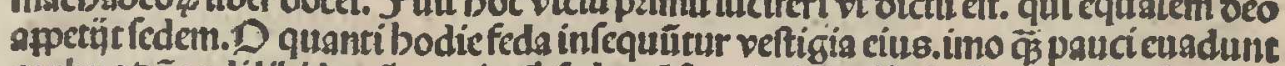

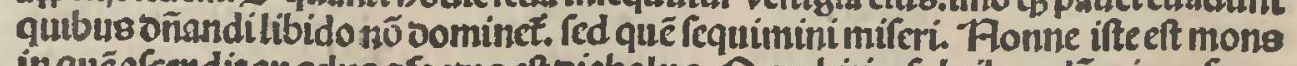
me ne fomes virutün

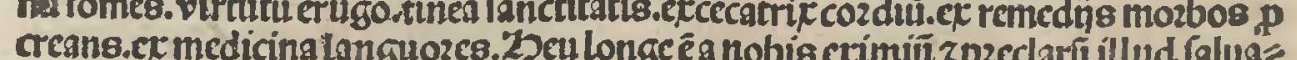
tozis noltri extemplũ qui oblatam gloziam culminis fugit.cû eum vellent facereres

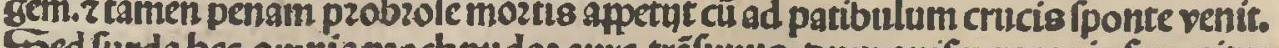

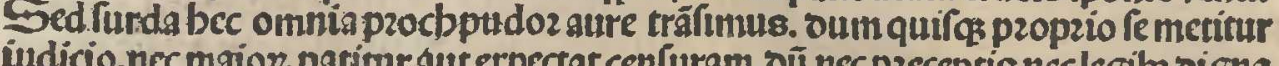
iudicio.nec maioz patitur autt expectat cenfuram. Dü nec pzeceptis neclegibs digna

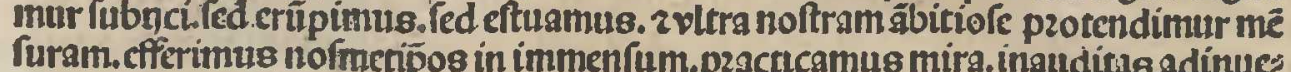
nimus vise. Hecs patimurad nos oeferribonozes. fed pzo bis plequendis putols= mus.contendimus.certamurqs iure nobis oeberi omnia arbitrantes. oũ onnibus

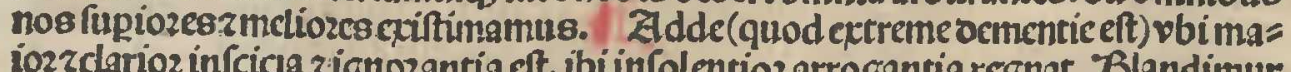

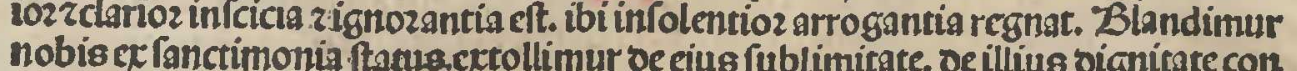
fidimus.neg cogitamus of ficutcenon inquit non locus non oignitgen nö catbes dra.fanctü faciút. Fed vita zmozes. Zinde ex officio z ftarus eminentia non licentiä

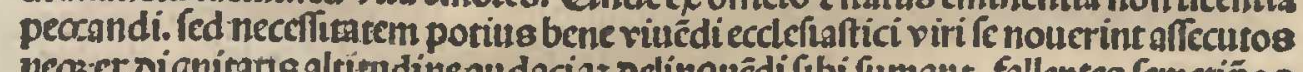
zonos oubicareoser queg petranterignitatis meritum.fufficiut. quea loco z catbedra teftantur. En ricerü.catbedra aut claros ad becfaltigia erigit.aut qui enigutur illuftrat. Ad que oicumus. plurimu fallicos doc

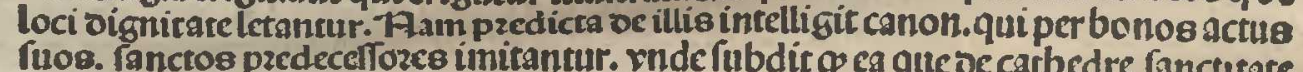
oicra funt. ad illos pertinet.quos pzopziccóner fationis folend oz occorat Gitate tanto Fanctos rocatcanon.quia pzopter locizcatbedrefanctitatem.boni z fanction

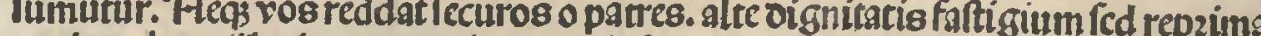
potius z bumillict.ingens periculum zipfis ceruicibs veftris imminens fi vitas obeft

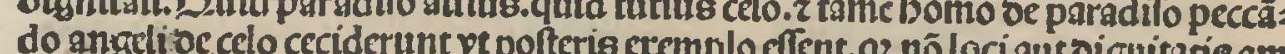
plinudo. fecurin

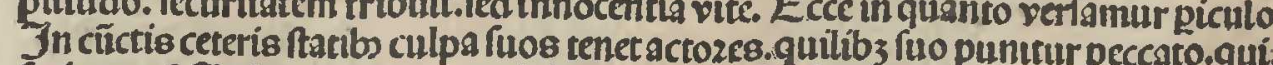
frriprume. e. Anima que peccauerit ipa mozietur. In boc gũt vno viuendi genere no

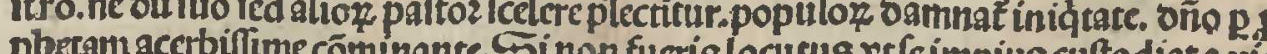

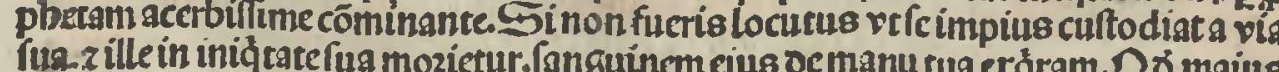

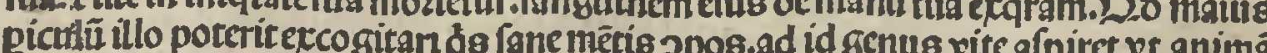

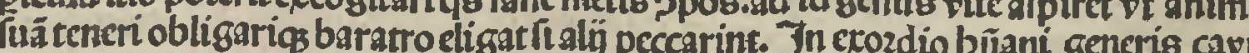
. rius pro fratre teneref

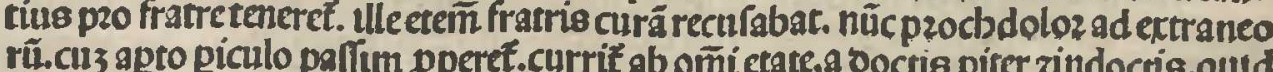

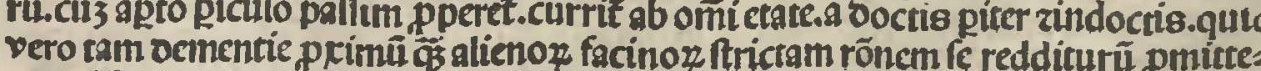
re.qui fua aut nonvidet aut non intelligit. Brandis pzoinde z periculofa farccina ef

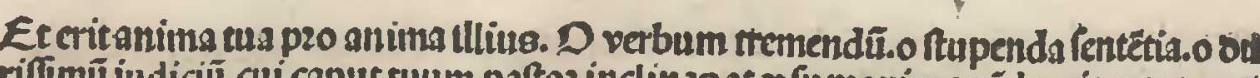
cibus oigen nemoer nobis bec fecü co gitat. omnes felices nos bearof mur curam. fi paltozale cuiméadipícimur. Labozamus p bis nancícendis conter

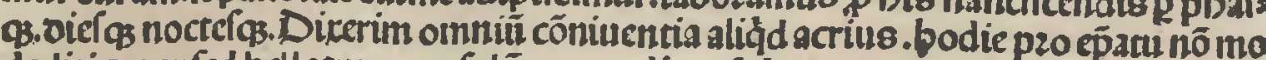
do litigatur fed bellartur. Hon folü contenditur red pugnatur. qo ceteris omnibus lítigarc,

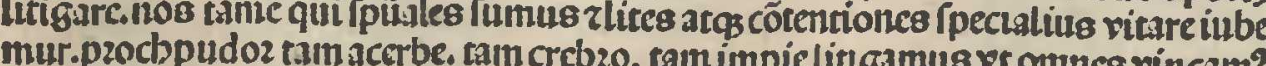
in cö́rendendo Laicos. Et certe pletūos nó fine ozbls f́andalo z piculo. ita fub colo

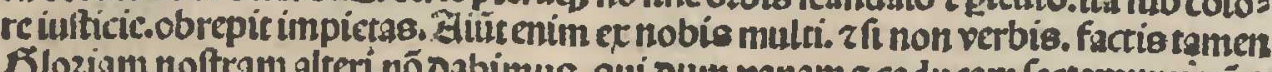
Dloztant hoftram alteri nó oabimus. qui buin vanain z caducam fectamur verä z

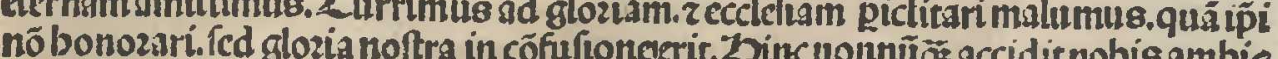

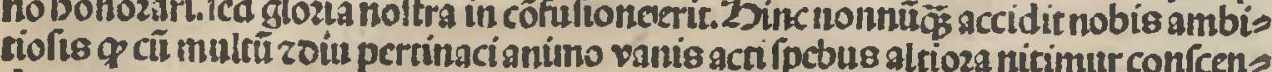

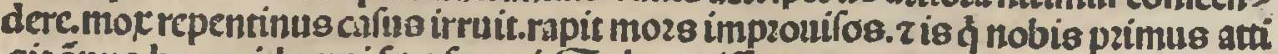

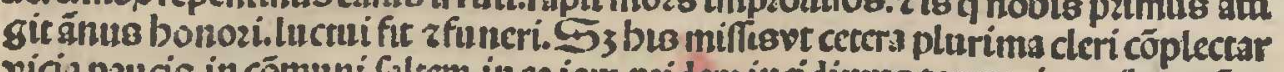

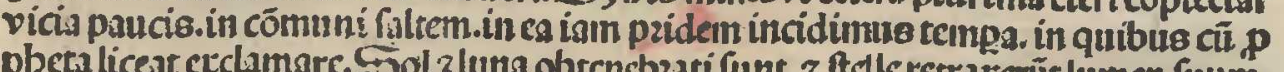

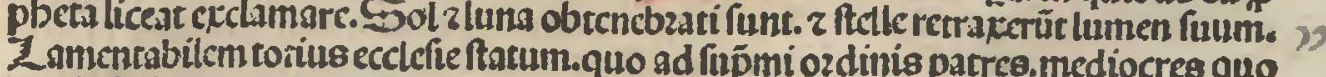

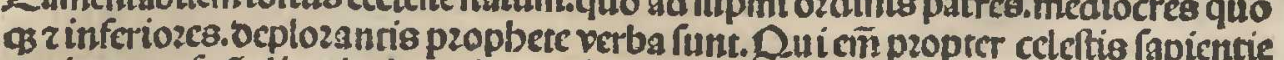

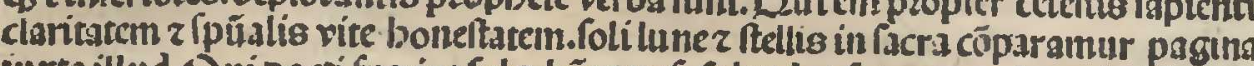

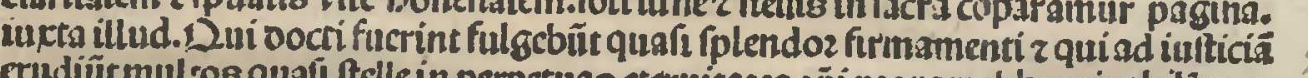
cruduturtmul:sos quafi ftelle in perpettas eternitates.

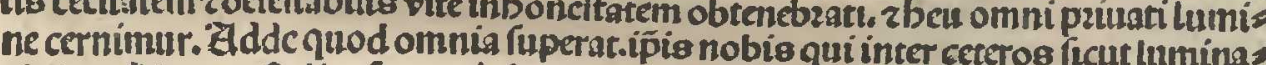

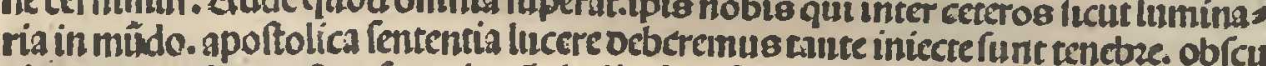

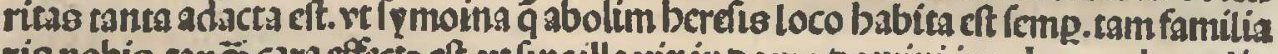

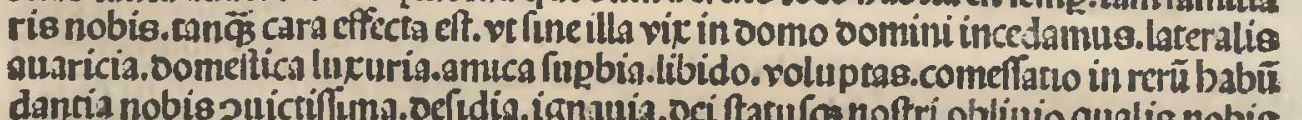

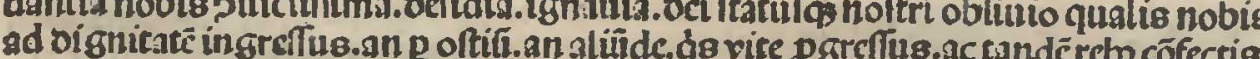

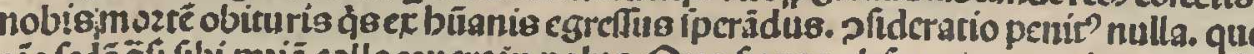

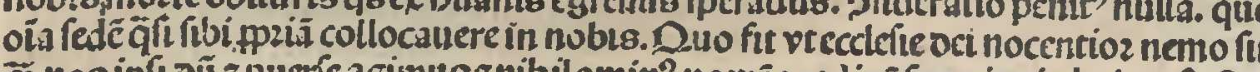

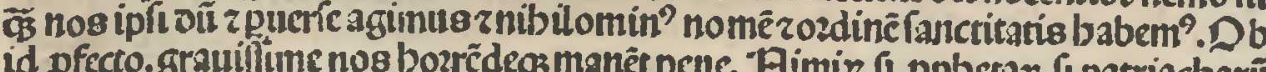

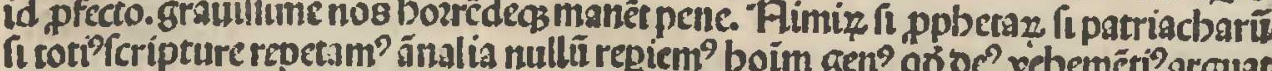

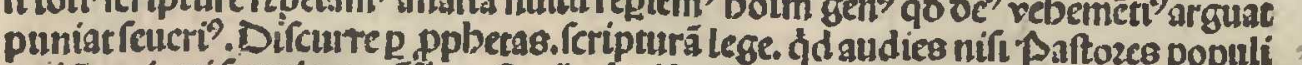

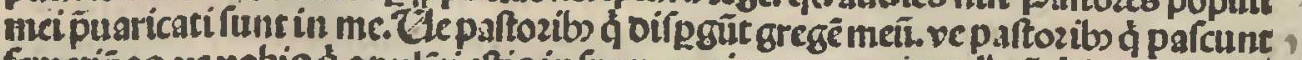

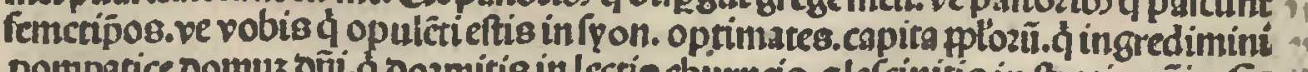

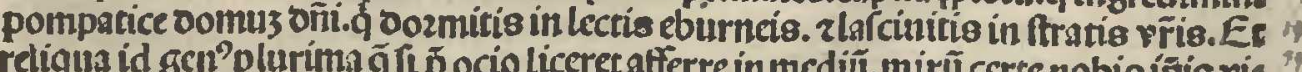

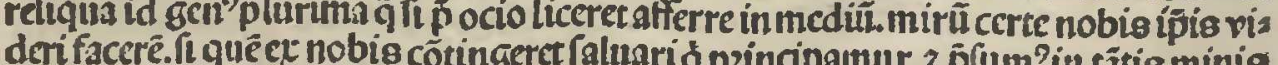

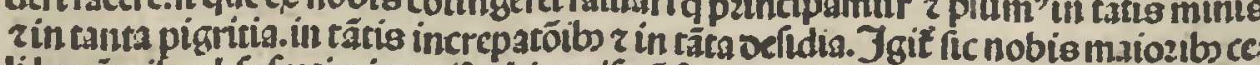

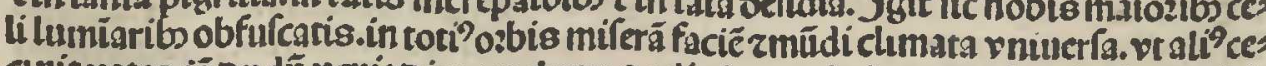

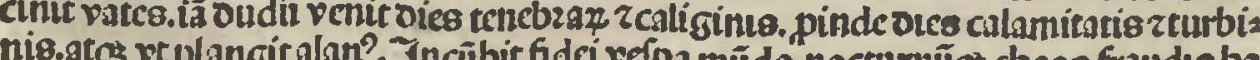

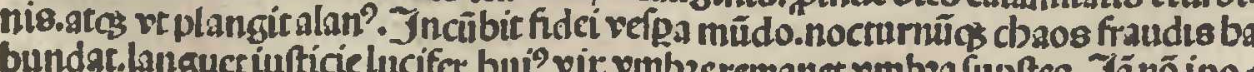

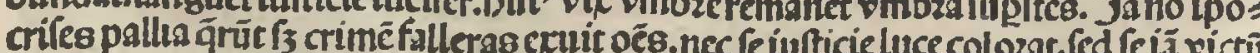

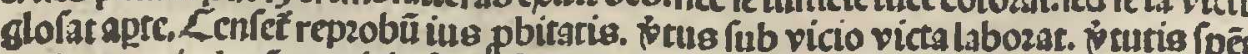

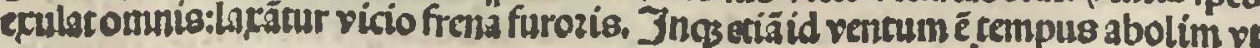




$$
\text { "simg, Liming mping himesce }
$$

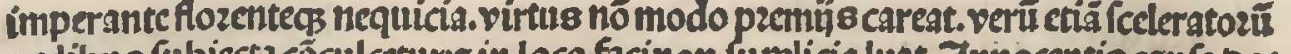

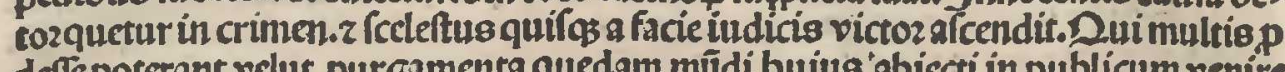
denepor non audent.2 mircer maceros fenctatoz infuniram au get pecuniam. at qui cuncta vellet largiri. tes nuiaffe viuitinglozills. matrone relicta grauitate inluets merctriceos refoluuntur zfanctum Jofepp mulicr emula caftitartisad becperfequitur. Et quid fruftra mul:

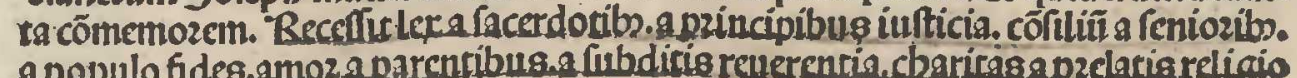

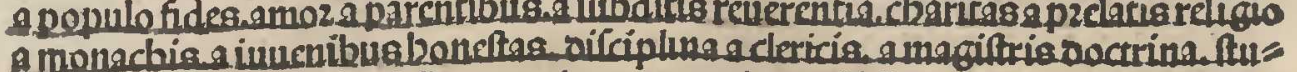
dium a Folaribus. a ind dicibus equiras protectio a milicibus a ciubus concordia timoz g feruirozibus a rufficie compunitas. veritas a mercgrozibus. a nobilibus

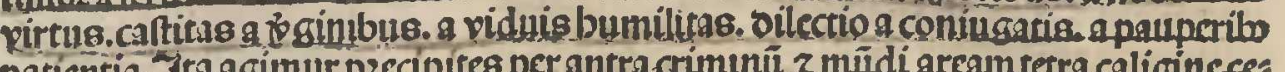

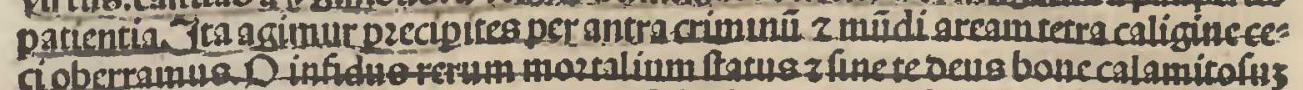
omne quod viuimus. Eccefi virture confpicui z mozibus.fide.fapientia.rcligions. caftitste.ceteríl's anum ootibs clari.quos per paucos bec noftra fert etse. repz obo: rum comparatione, rollanturemedio.quid nific caligo intuoltene.pluritha moztis

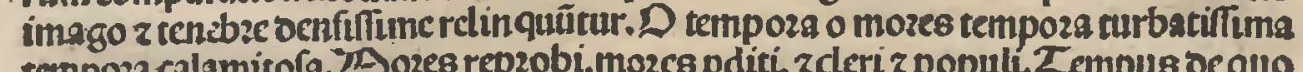
tandem oicetur. Zetenit fumma oies zineluctabile tempus. Zempus in quo iuttave , ruftum pzop bete pariciniū. D mnecaput languidum $z$ omnecoz merens, a planta

2) pedis vlosad verticem.non eft in eo vlla fanitras. 'pz zofecto caput omin.omnilog ver=

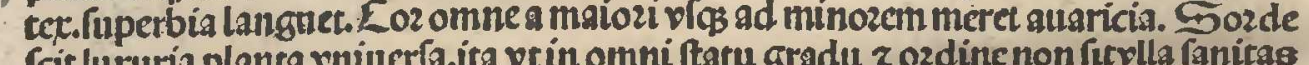

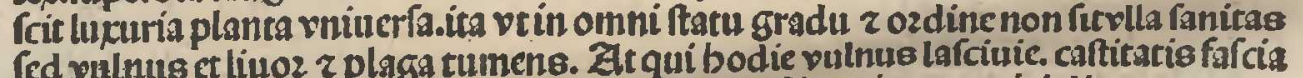
circüliget.eft nullus znemo. Dui paupertatie medicamine, augricie linozem curet. eft nullus $z$ nemo. Dui tumenterm plagam faftus 2 fuperbiefoutat bumilitstis $\mathrm{em}$ plaftro.eft nullus zinctro. En feda laturics quo non penetrauit quo non irrupic regum icandit purpuram.

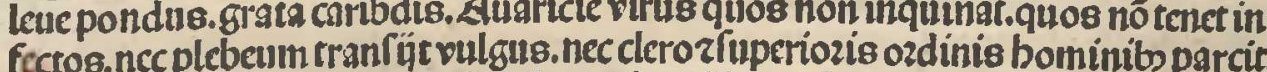

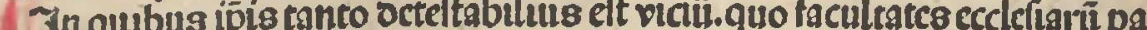
patrimonia certo nofaútur z facril ega cis crudelitate fubtrabitur.quicquid fibi m

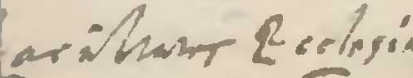

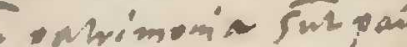
rim

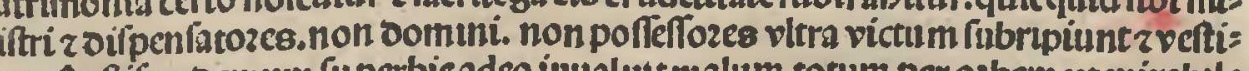
sum. Peftiferce oemum fuperbieadeo inualutt malum rotum per ozbem ve mirabule nofa tranuitus cuins vitam labozióa penalitas concludit.cuius penalisatem pes malioz cücris mozris oemol itur necefitas.cui etiam momentũ eftcarnis gaud dium vita naufragitu.mudus criliti.cul pterea vira aut abert aut fpondetab/entiam.moze

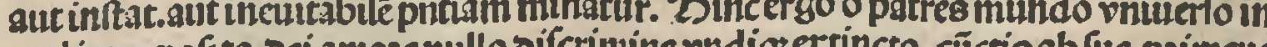

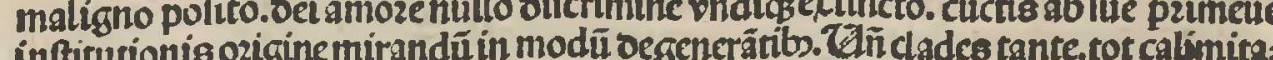

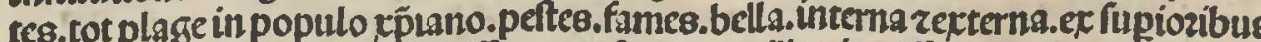

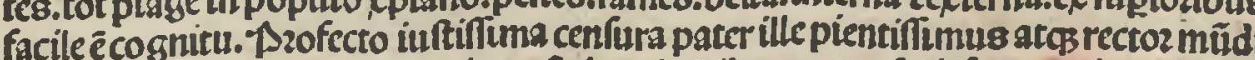

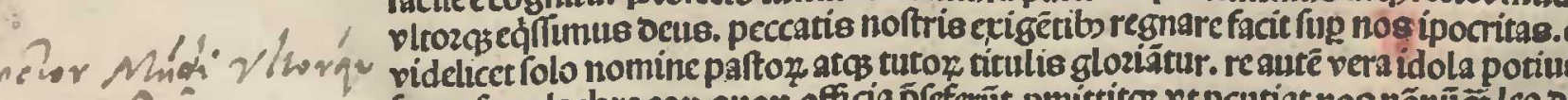
effing anis

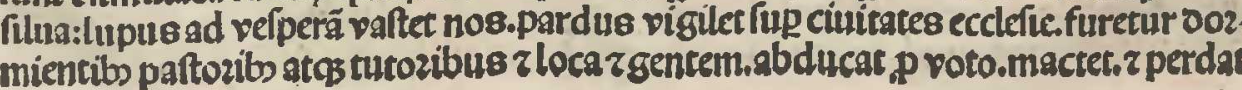

Qnippeneminerefiftente. bifimulantibs vniucris, idcirco etiä digna bei fententio

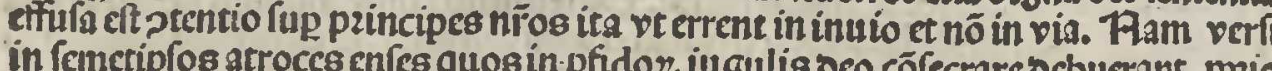
in femetip fos atroccs enfes quos in pfidoz iugulis oec cöfecrare becburant. mprio

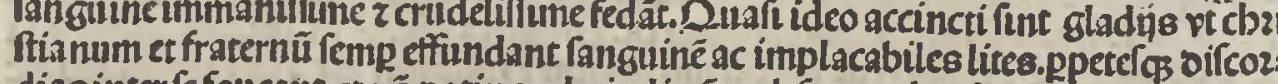
dias inter fé fouteant.et nö potius ad vindictá malefactopz. laudem vero bonoz. ec clefic pacem atos tutelam. Attrendite quefo o reges et principes. 'Aamad vos ocin ceps 1 crmo ifte metus boztasosius. Animaductite tandem quantū potuifet oudín

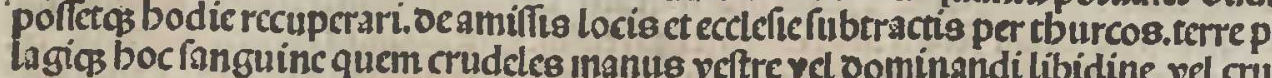

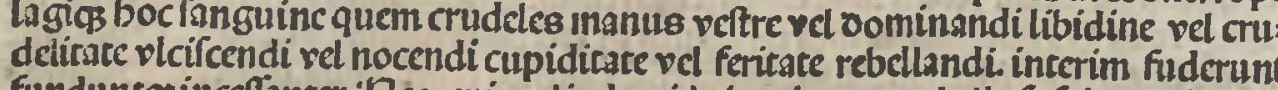
funduntos inceffanter. 'A cos cnimaliud quidpiam inter vos bella fufcitas ve in plu

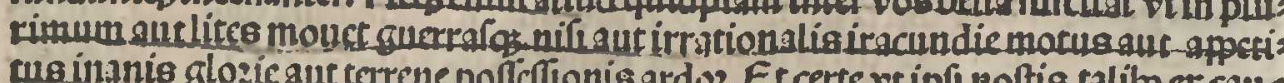

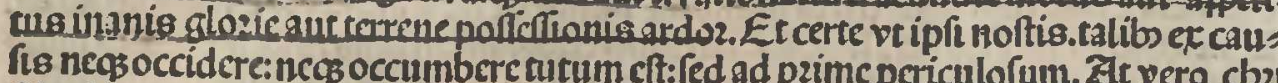

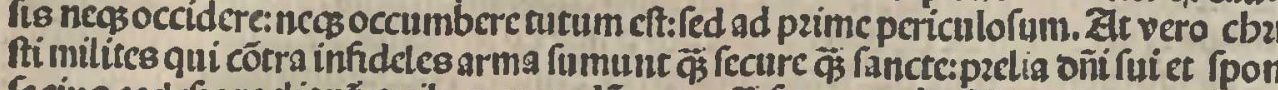

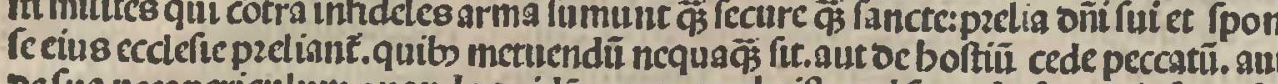
oc fua nece periculum quandoquidé mo:s pzo cbzifto vel ferenda fit vel inferenda 20-ilcs inquam talis et fecurus interimitert interit fecurioz. quia et fíbi pzeftat obfe quinm cum intcritet cbzifto cil interimit, neqs oum occidit mal cfactozé. bo bicida

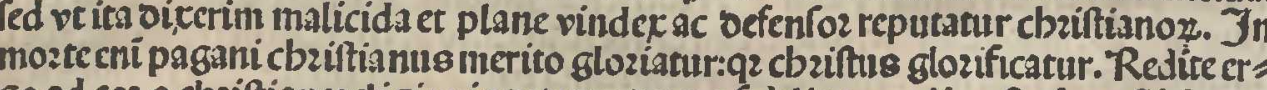
goad coz o cbziftiancreligionis tuto:ce. ouces fidelis populi.cuftodes oñici gres

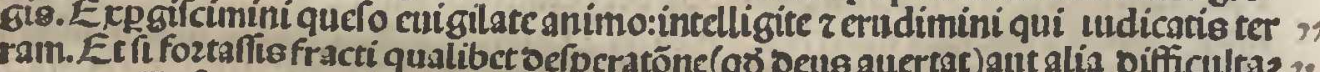
te vectra tha fancte terre bicrofolimiranc. que oeplango osmna. refartire imp offibis"

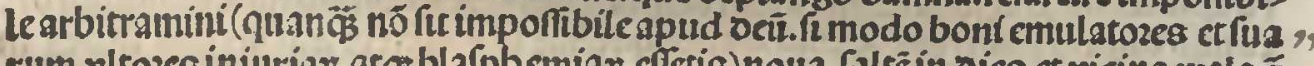

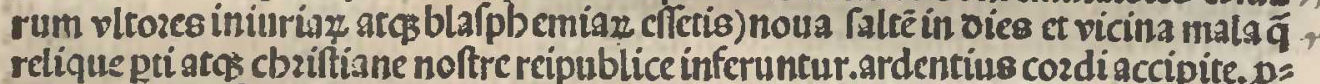

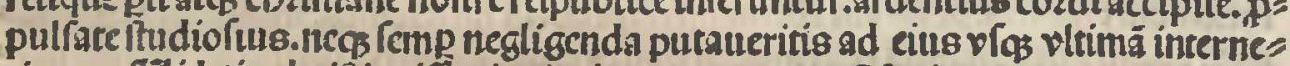

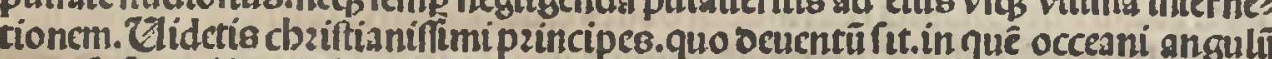

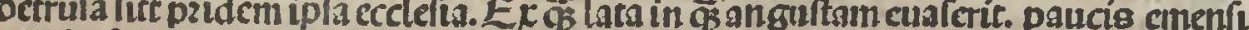
annis. Fitum eft robic. Fleqs ignozstis quo iam in poztll côtitat. Sed quozfium

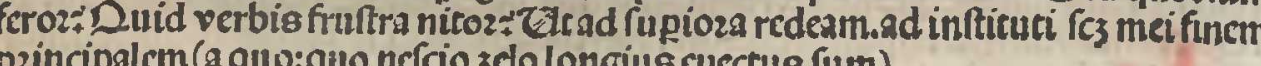
principales cum magnificos adminiftratozcs.bec vna peticiuncula ancer fcularium bignira: pletsd vos deṕpatio.ve maiozî vroy. videre annalia: oignas mennozanu biftozias relegetererenoluere fortia facta partrü. nö grauemini. Karoli videliç quondä magni

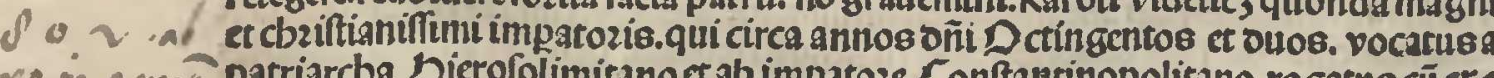
ro in a ma a patriarcba bierofolimitano ct ab impatoze Conftantinopolitano rogatus cū exs if ${ }^{\prime \prime}$ - Ginver et ouces.comites et milites.omnes gloziofis militicic peonüs infignes Jnter quos iñ Rolandus comes Cenomänenfris miles ftrenuiftimus.in rebus bellicis pre cete

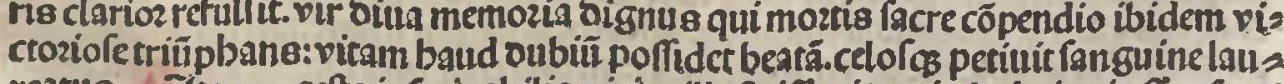

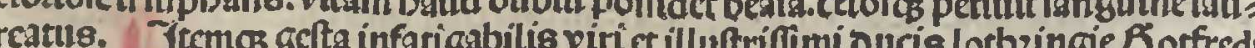
ocbullion cognominatimente recogitate quo ouce irerñ circe annos gratie 2 A

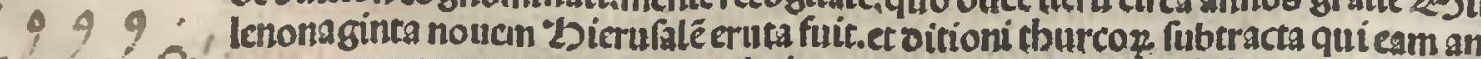

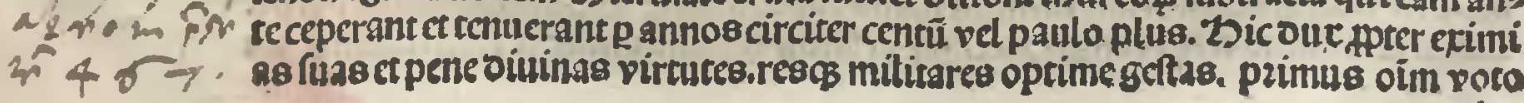

Dizun om' Im

Canpring

bon

Morsecrín

Mentis

avolg Mazng

R.landóncomot

mnombria angon

Sin 2orforiming 


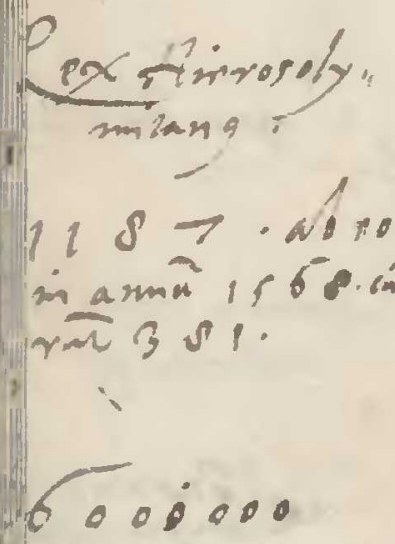

ghilis mintisin

Tosthon et confenfu factus eft rece 2 s:

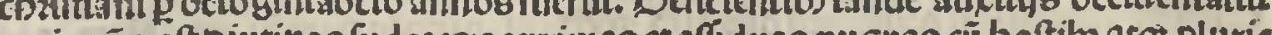
resloning por

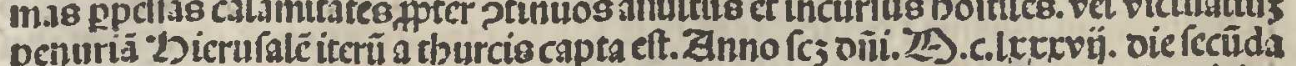
Ocrobzis, Suboctauo rege cbziftinno ibidem 5 undone. Aca Farracenis etrbinc

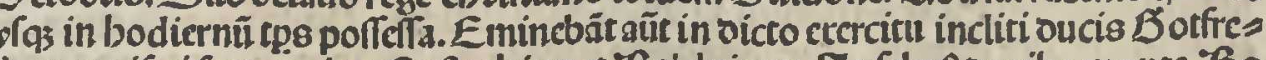
di.mganifici fratres eius Euftachiuset Ba:duinus. Anflim sc ribo monte. Bo

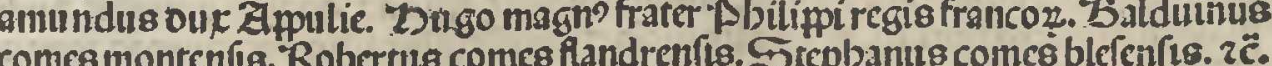

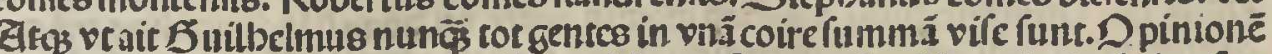

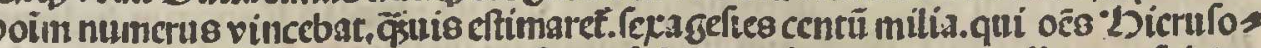

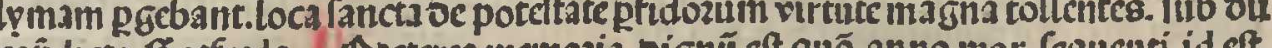
cep̉dicto Sotfredo. Preterea mentozia bignñu eft quō anno mor fequenti.id eff.

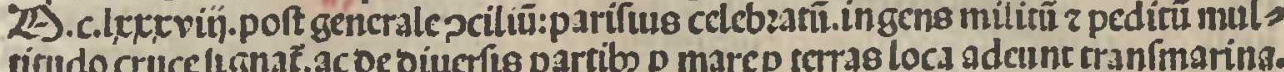

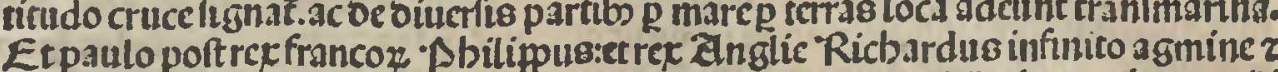

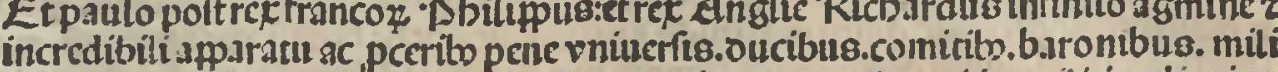

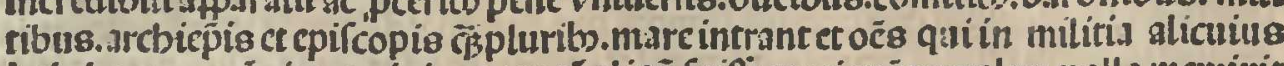

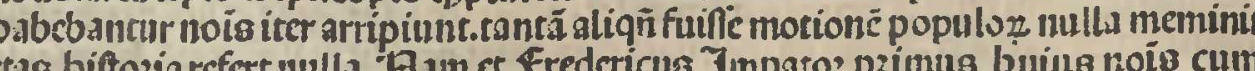
ctas.biftozia refert nulla. Han et Fredericus Ympatoz pzimus buius nois cum

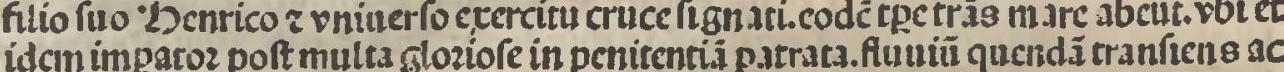

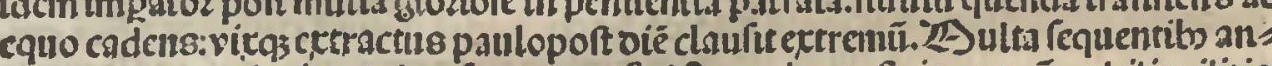

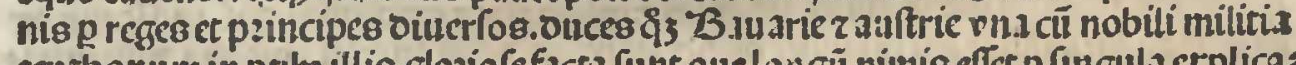

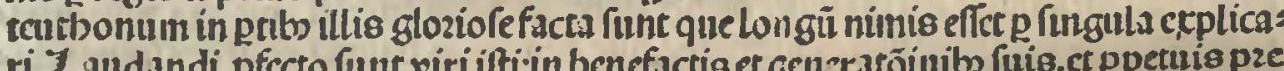
conijs oignia pofteris babendi.quupe qui fide oenotônce et 3 elo magno in ocil in

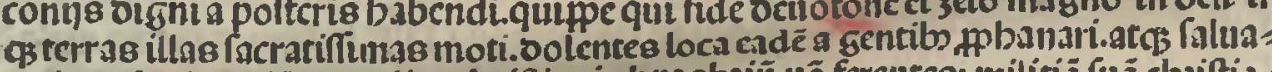

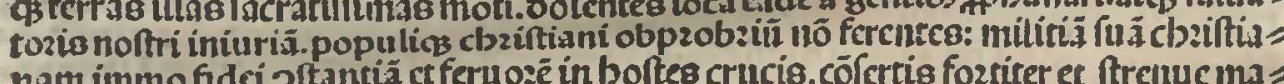

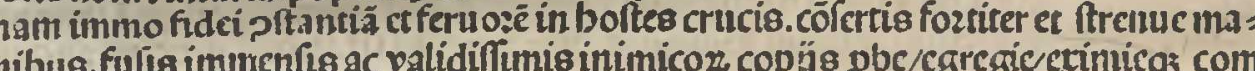
pros

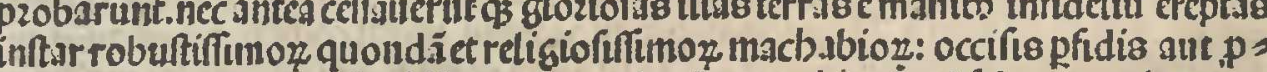
fligatio. in cbziftiane reipublice oenuo:vel redegere oifioné vel faltemiconabantur.

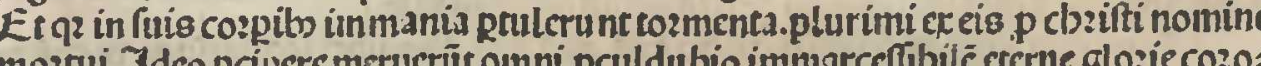

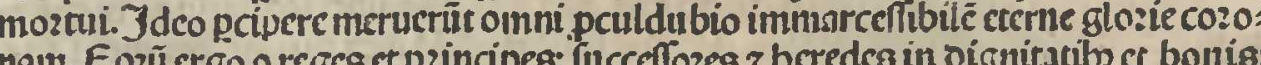

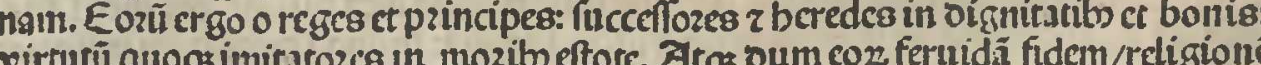

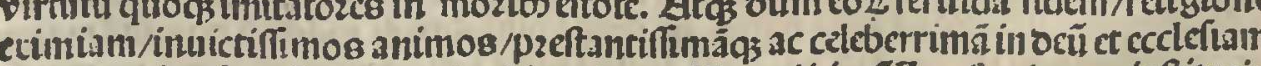

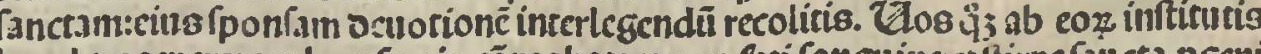

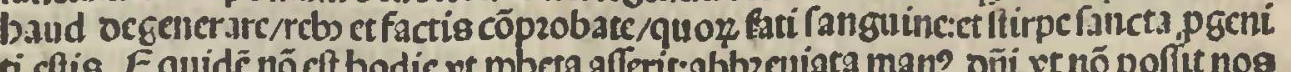

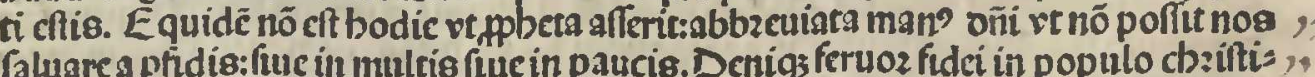

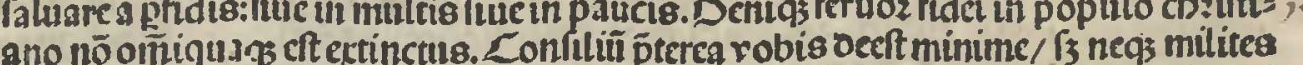

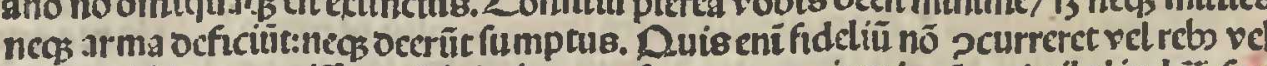

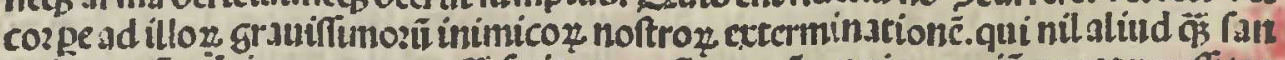

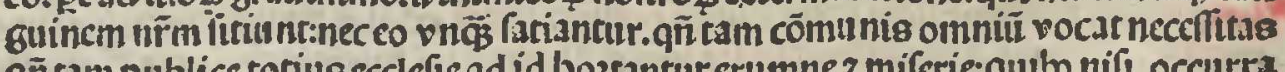
gint am publice tortusecclefir ad id boztantur crumne z milerie:quibs nifi occurra

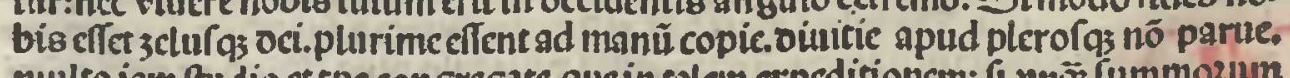

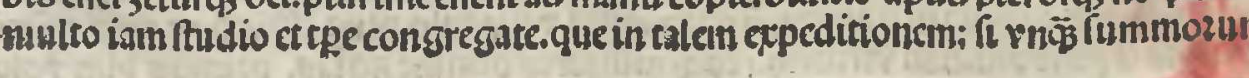

ordinatône capittun indiccretur.baud oubiñ. oco fuoz cozda tangentesio pmpto

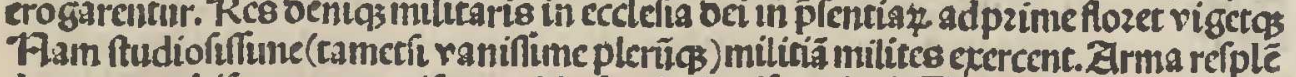
denf cum mírifica tum terrifica valdeficculo inuif́a pziozi. Sed et foztes auriliarú 管 rebulas in ozdinc p cos quoz intereflet dirpofitis.pari voto. côfenfu phanimi cos demos oecreto. ad bumilcs ct fupplicce ac frequentes ecclefiect capituñ: fummoz vi=

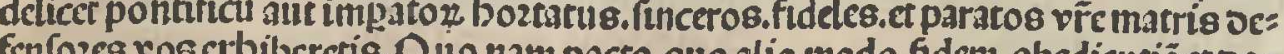

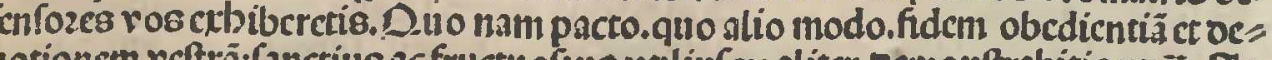

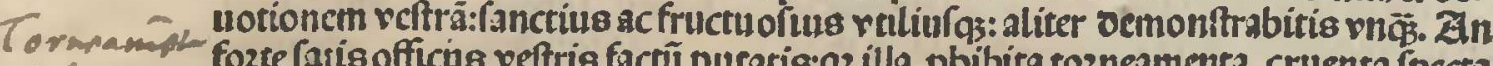

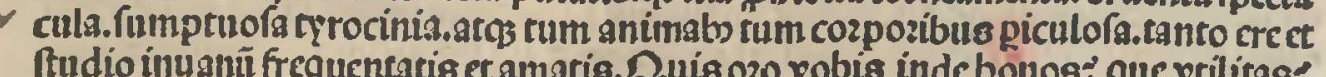
Itudio inuañu frequentatis er amatis. Duís ozo vobis inde bonos: que vrilitas: tantis

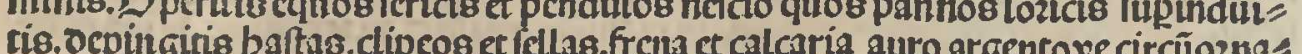

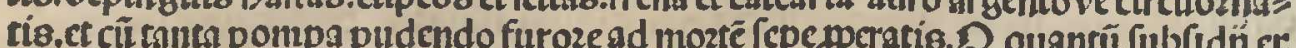
builufcemodi rris fupbis fugftuisç aparatibs quos in vanitates et infanigs fallas

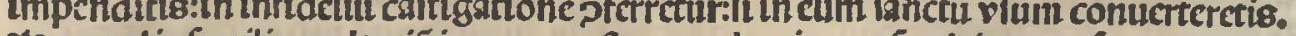

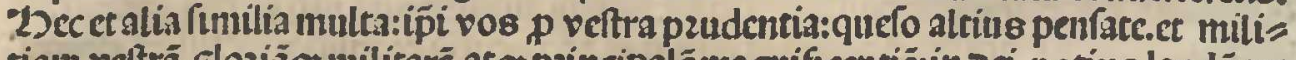
fram falué et

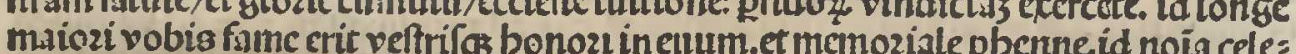

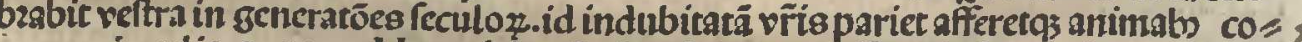
ronam in celis.quam redder robis ons in oic illa nouilima iuftus itdec. pro quo ",

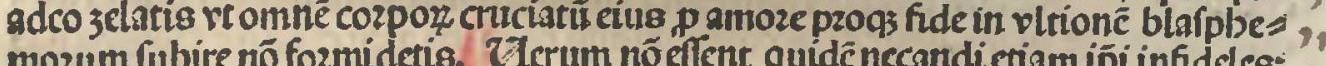
aur occiden di.

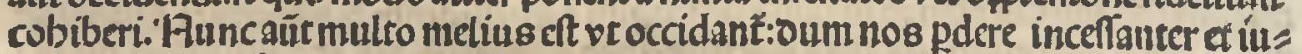

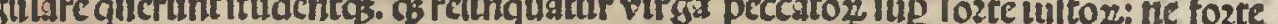
etrendant iufti ad iniquirate manns furs. Difipentur gentes que bella volît. Zabs cedantur qui no lo fi poffent:fup cuncta ocfiderabilia gratifimin baberent. Excrecatur vere oe fub ce

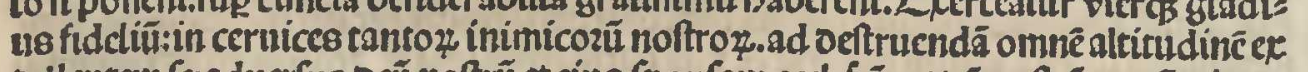

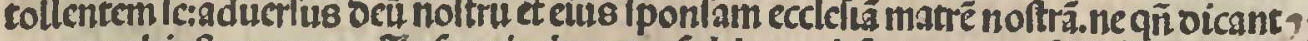

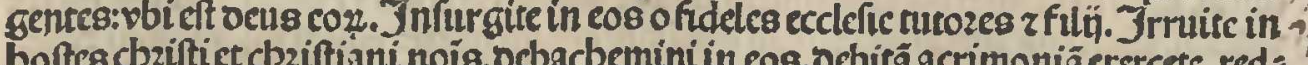

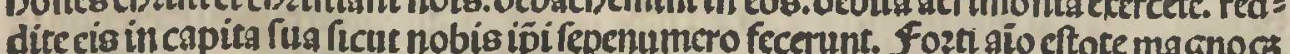

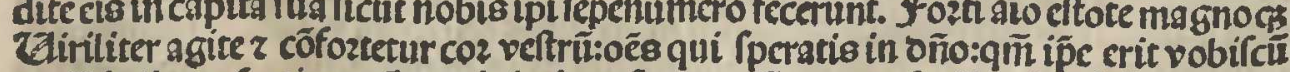

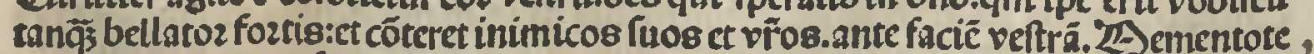

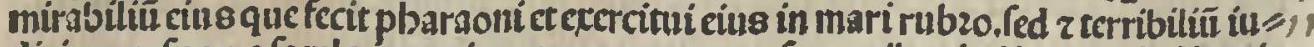

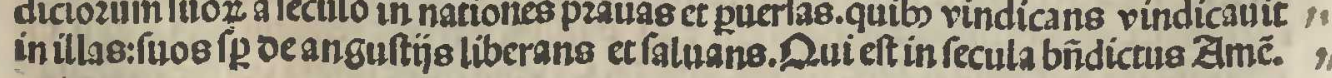

f Sequirur virio quedam borribilis, oftenfa karolo quondä 3 mparciquicals

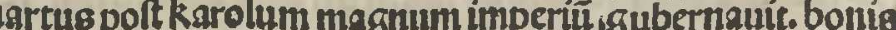
répecribs bicinferta.

1) P1 noic bei fummiregisregũ. Ego karolus impatoz oci bono rex germas 
polt celcbastum nocturnarum bozarum bininum officium: Dum irem repaufarees veltent ntus nis emicceces rir ad me. Accipe fulu glomeris nicanncis ct tiga ec noda furmiter in pollicecue ma

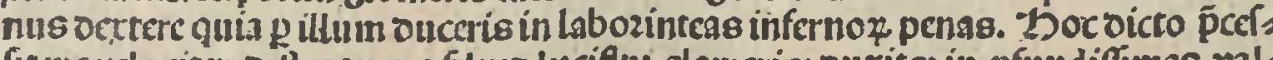
fit me velocicr: ort

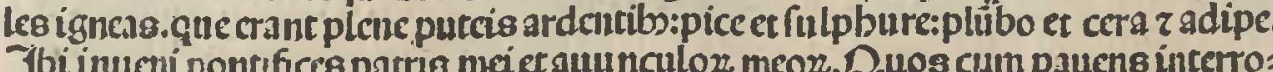
Grem cur pon rui crsuunculoz thoz ct onur ocbutumus illos z populã illoz oe pacect concozdia

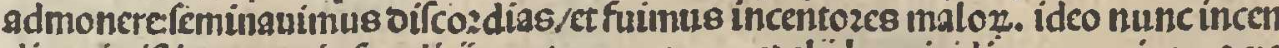

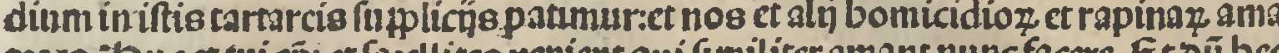
trece Icbant berantibs radis silus glomer is nov um currchtes. volucrut ine aduncare.et in ipos puteos inlpbureos pzecipitare. Fed

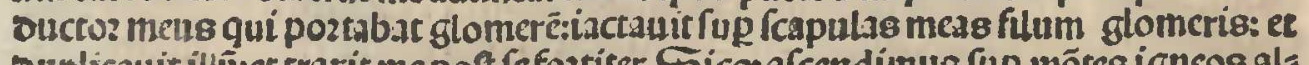

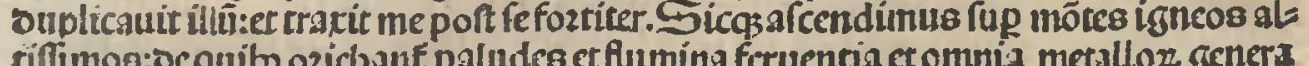

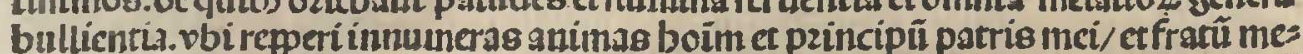

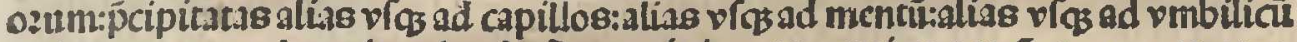

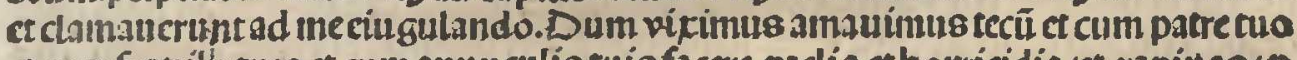
ctcunfratribs ruls et cum anunculis rits

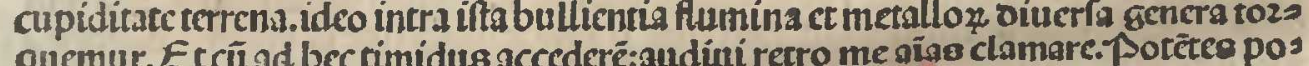
tchter tozmento patientur. Et refperi z vidi fup ripas fluminis bullliencis fornaccs piceas er trat pureas plcnsis magne

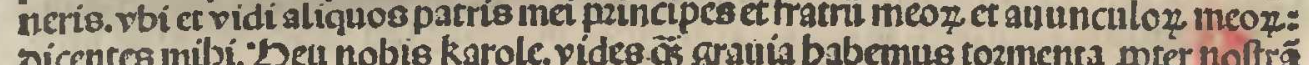

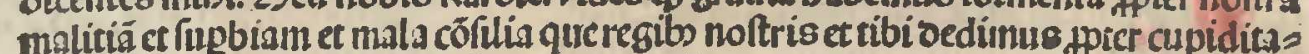
rcm. Lüg:oolendo consemiiceré:accurrerütad me ozacones: apertis fauciboples nis igne ful pbure z picervol entes me in glutrire. \&

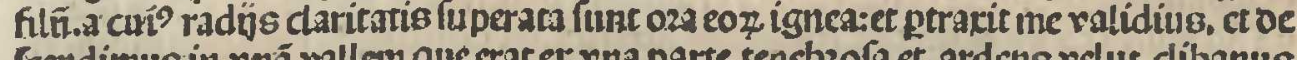
recndinus in vila vallen que carcer rna parte

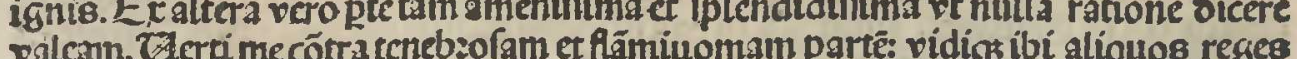
oe inco genere in magnis fupplicje Ex tunc nimis anguftiatus putaui meftatim in illis fupplich) socmergendas gigantibs niserrimis quilipam vallem conflamabant generits igniti.er valde tremene: glomeris filo illuminante ocul os meos: vidie 1 as

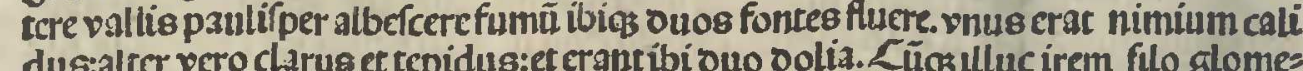

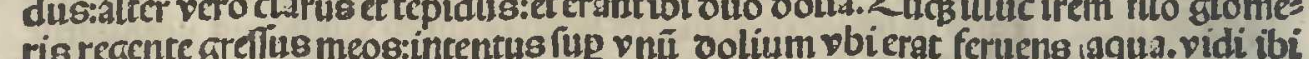

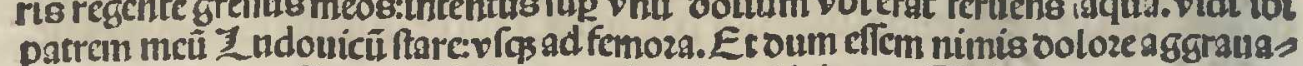
tus:et an gosc pcufius dixit. Dñe mens karolenoli rimere. Socio qz retuertetur rurs

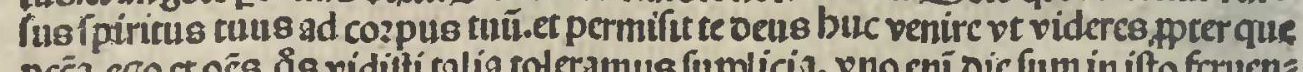

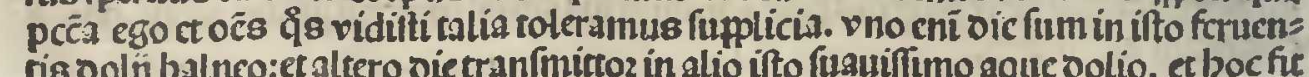

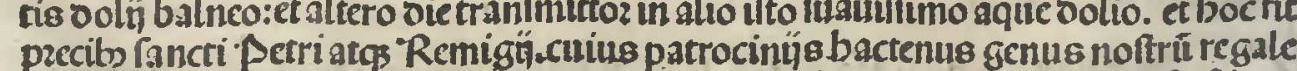
irgnauit. En fi mibi fubueneris citostu et mei cpictabbatce z oís ordo eccléiatticus miffis et oblationibuset ozationibs et pralmodỉs vigilüs ct clemofrnis: velocite

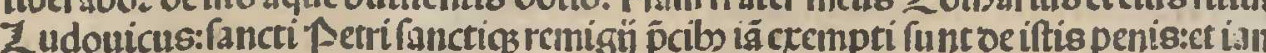

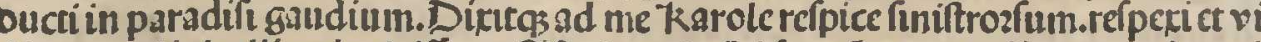

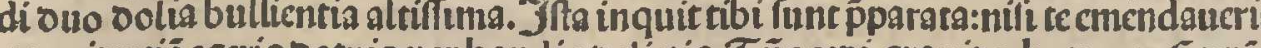

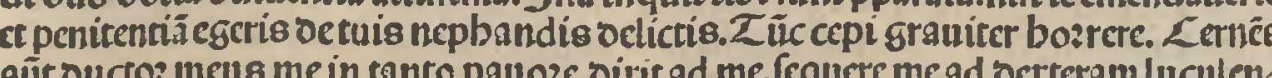
cifimevallis pardif Etcum

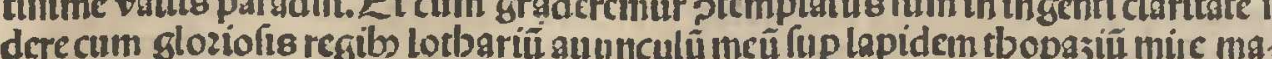

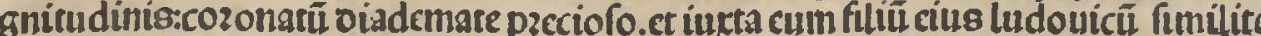
cozonati. Zidmifo mecon us:nunc eercils in imgio romanoz. veni ad me. Scio $q$ g penalem locum venif Mavina sh ocicirinime ociltiopent

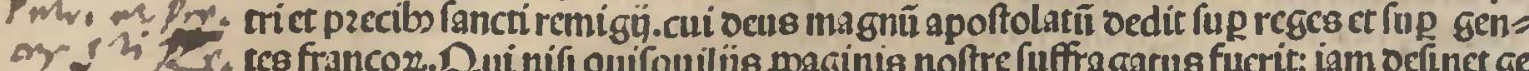

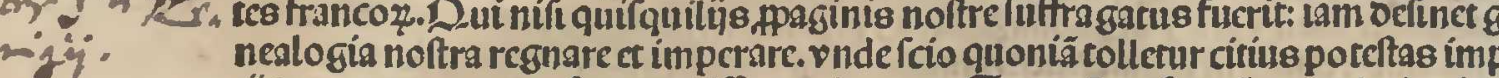

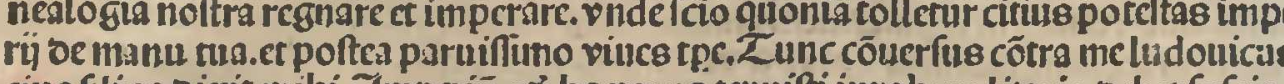

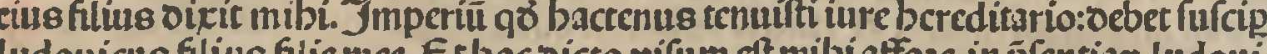

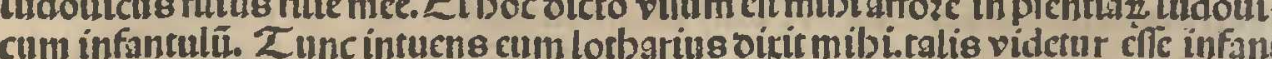
qualis ille puer fuit que ftartuitonis in mediv dícipulozes oirit. Zalium eni eft te

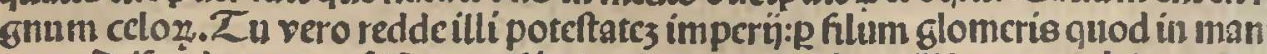
rencs. Difhodans er go filia oe pollice octtre mee oonabam illi monarcbiam oumn imperin per fin us in cozpus meum valde feflite ctcontcrritus.

(1) Aima buiusoperis parsfinito

"Infecundamperegrinationéab hieru falem per folitudiné montem. Synatad fanctam Catberinam ṕ fatio cómendattia incipit feliciter.

Difouis peteris teftamenti fa,

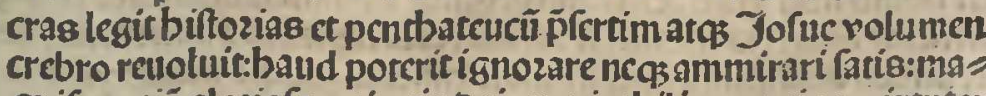
Enificenträ glozicfanctitatis oci. et mirabilia opa cille. virtutez

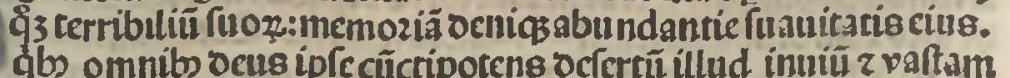

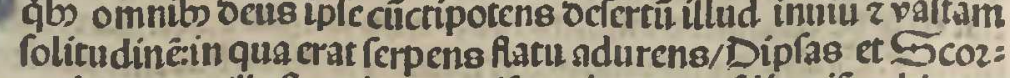
pio:atos plurima moztis umago:illuftrauit.magnificauitq: oum filios ifracl in ter

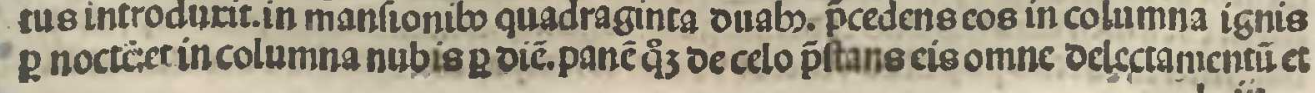




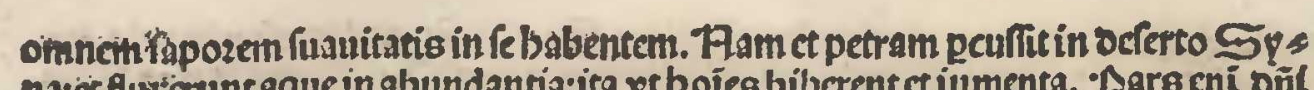

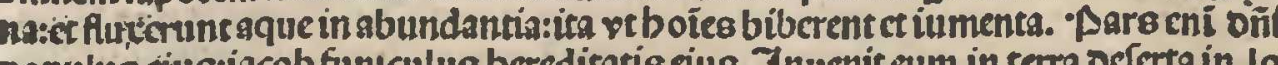
porrozts ce vafte folitudinis. Circúdurit cum et oocuit et cuftodiuit quafi pu villam oculi fui. Sicur aquila puocansad volandum pullos fuos.ct fuper 208 vo litans. Expandit alas fuas ct antump fit com atos poztauir in bumeris fuis. Douius

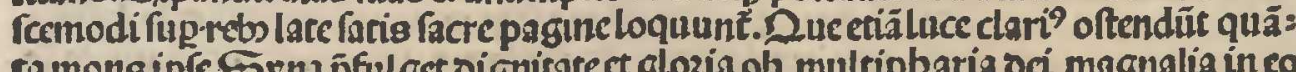

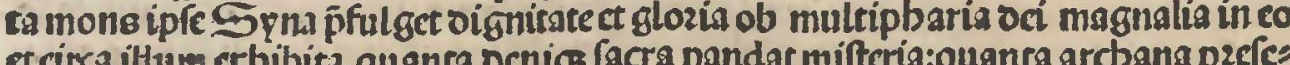
ferat. STiquidem Corm mons eft in Arabia. cuius pars quedam eft ozebiin terrs

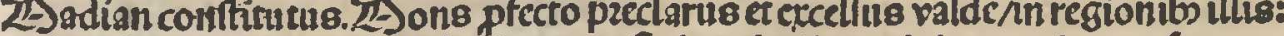
pafcuofus et optimay ferar berbarum. Fuitgs abolimo opinio populoy ac fama p

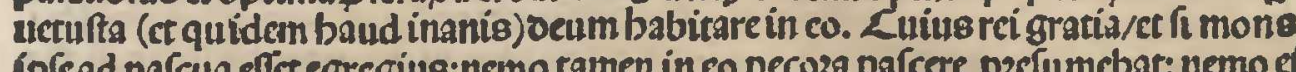

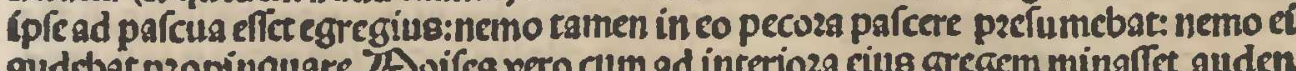
nins:celcbzeiltud vidit pdigium:ignem ícs ingentem rubum cöburentem: virozem tamen cius rel flozem ocpalcentem minime:neqs ocuaftanté ineendio vilatenus ras

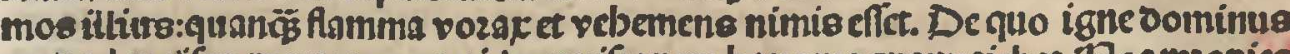
mot ad moilen, perancem ve videret viftonem banc magnam:aicbat. Feappopieg Dro cerce our reflimomio mocpille

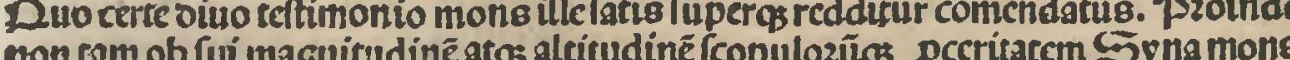

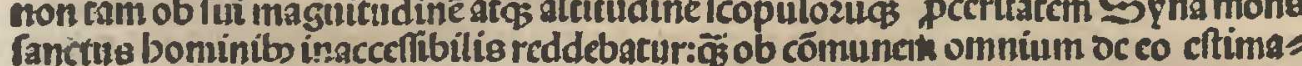
tioncm $q$ locus oci clet tum terribilis tum vencrabilis valde. Cuins et paulo am plitis innotefrat maxima crcelcntia. cimm ouci.quo ea quant babernus in mantily, prectio:ab iplo tanotcrmino ad que

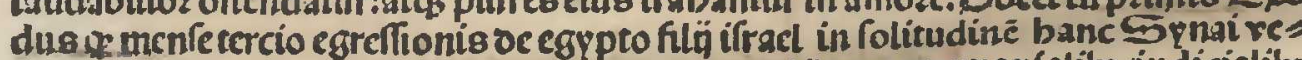
nerunt.et ibi mul to confedentes tempoze se mozalibus/ceremontalibs/iudicialibs

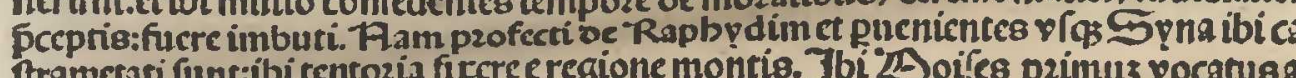
aco oe moncein mandatis accepic vebecfln̈s if el annunciare. Z 2 s vocatus vidiftis que fecerim egvp prya:quomodo poztaucrim ros fup alas aquilaz z affum pferim 108 mibi. Si ergo audieritis voecm mea et cuftodieritio pactum meñ: cris"

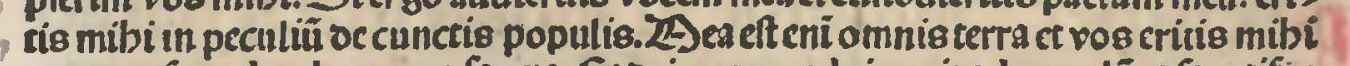

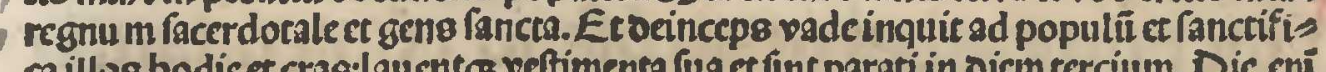

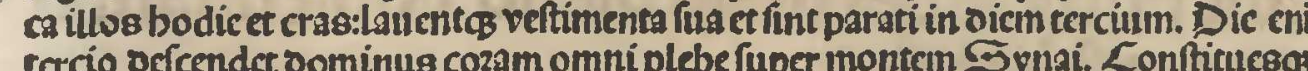
terminos populo per circuíicum z oices Cauetene afccndaris in montem nec tanges cis fincsillius. Dmnis cnim qui tetigeris montem mozictur. manus non tangent cum fed lapidibus oppzimetur:aut confodietur iaculis. Sille iumentum fuerit fis

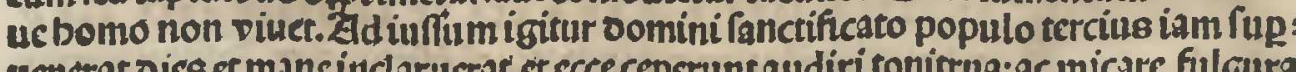

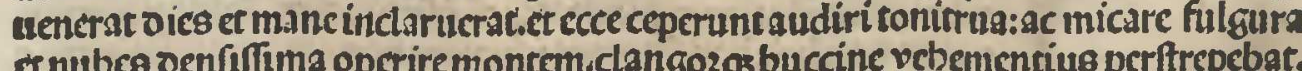
et rimur populus quicratin caftris. Cǘs edurif́ct 008 2Doifes in occurfum oc oe loco caftrozum:teterintad radices montis. Cotus autem mons Syna fumas

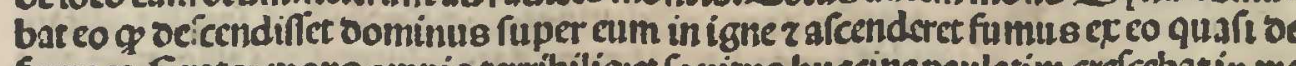

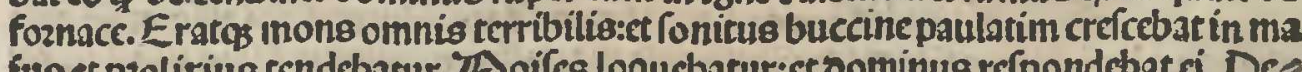
frend pzol titiug tendebant.

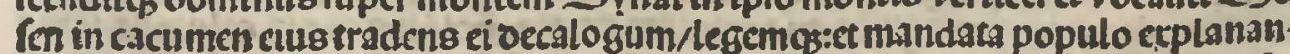

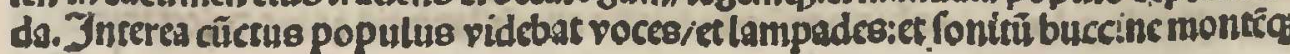

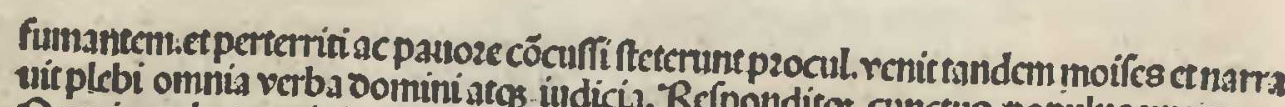
Dmnia verba oomini quel locurus ildicia. Refpondits cunctus populus rna roce.

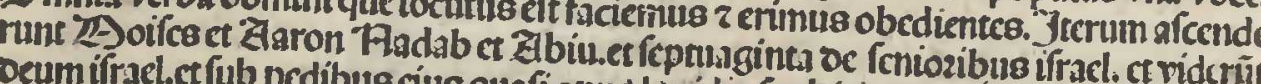

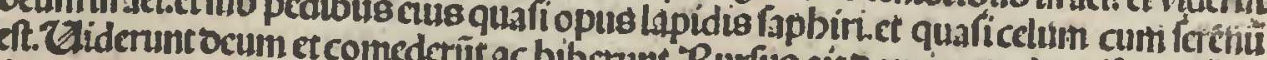

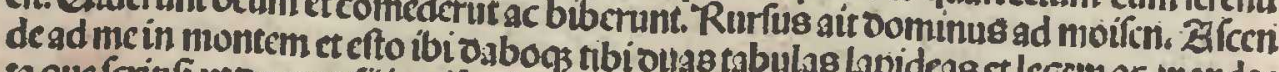

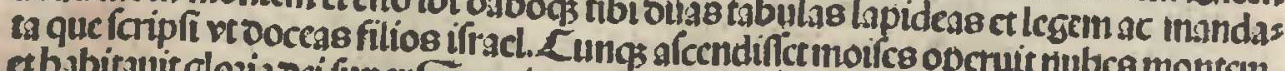
vocauit cum

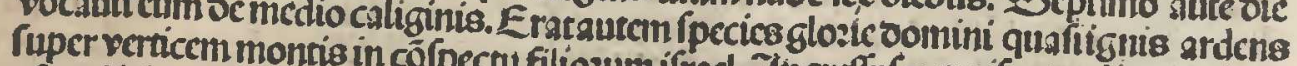
afcenditin montem:ct fuitibi quadragints oicbuse et quadrog moires medium ncbule

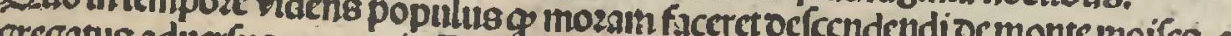
ic piro quinos a inauribus aurcis a populo fogpti gnozamus quid actiderit J Girur Zaron acceptis

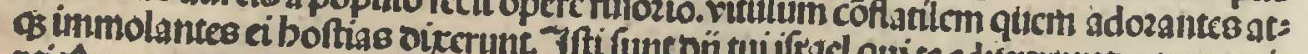

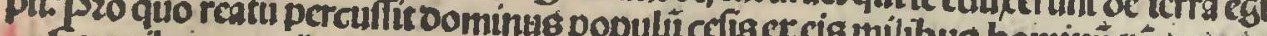

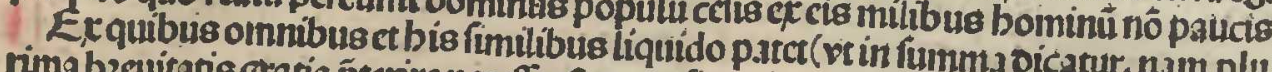
ille mös Diun mationis.mons nubis ct âliginis ctangelice frequêtrationis. mons luminise inflam

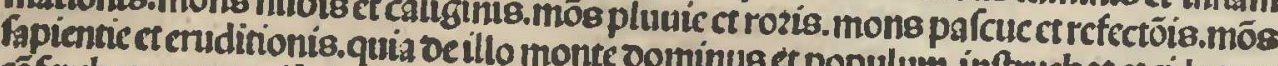

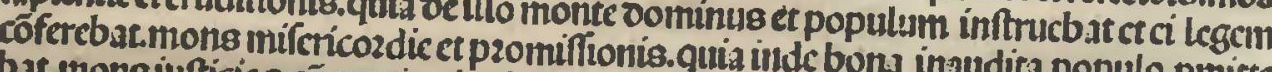

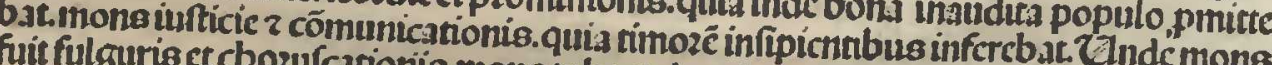

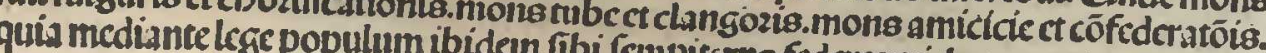

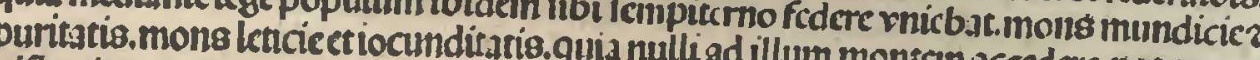

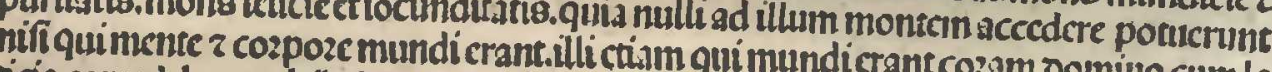

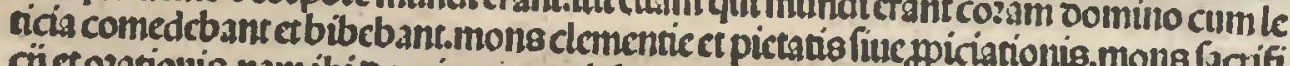

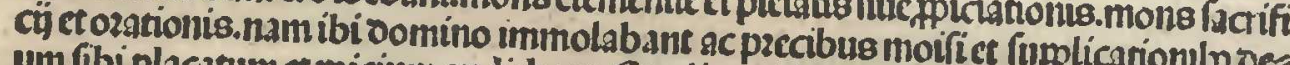

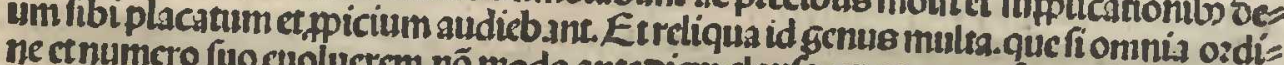
eroies et anni ine ocficerentet plurima carta

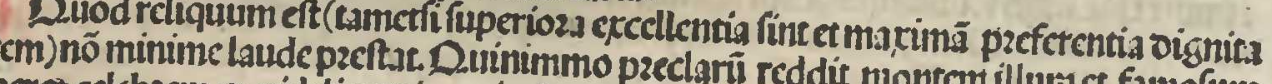

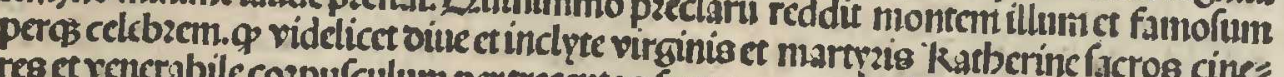
membzag virgincain co manibus gngelicis sepe annos tho babuitin verrice locatr. 300 biliter facto in ta quod oin croin ocuorifilim

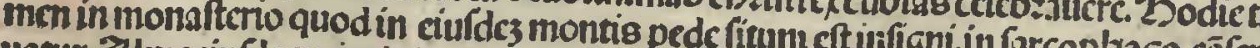

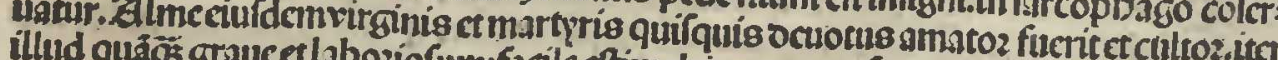

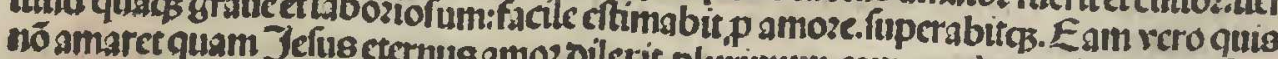

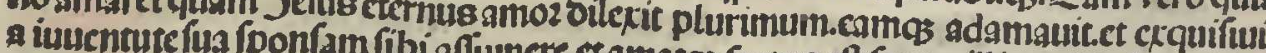

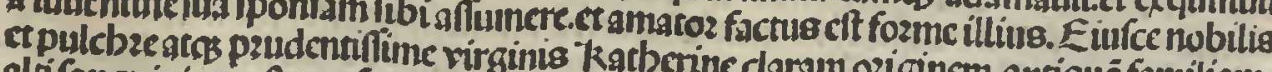
altianguinis oee quo ratificis

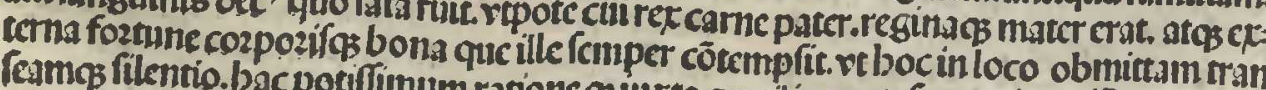
necclarum noméariozun

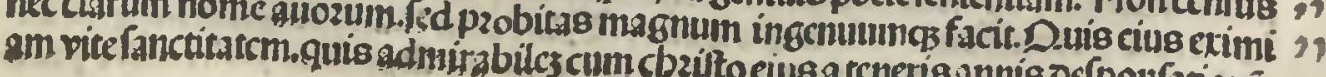

To. 


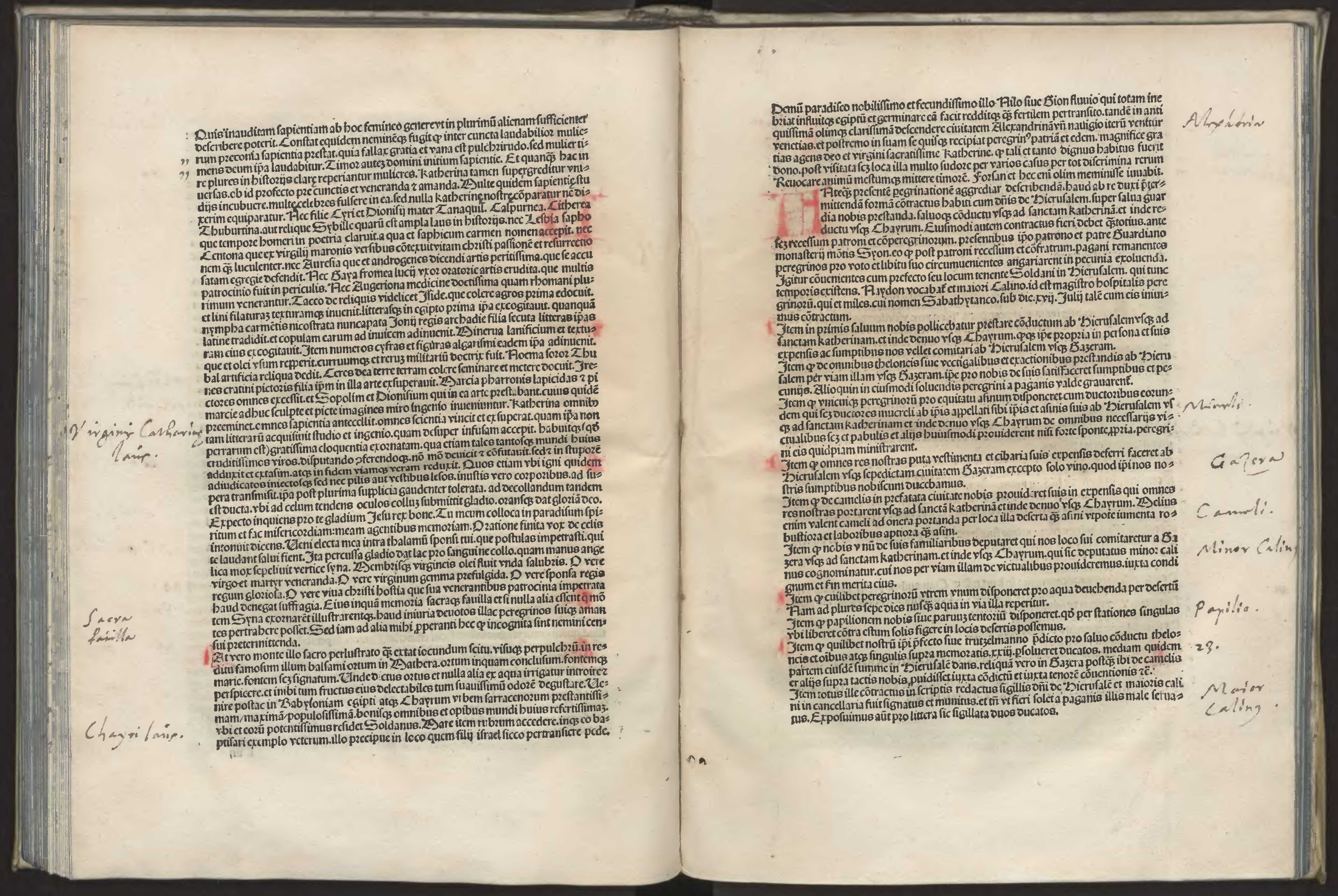




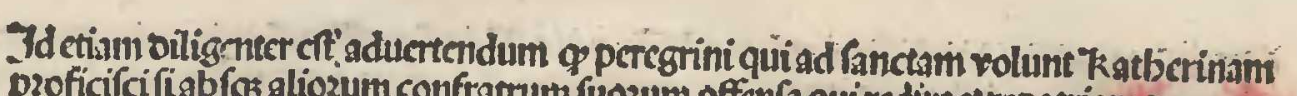
proficifcifis bfog aliozum confratrum friozum offenfa quircdireet trepatriare occrene

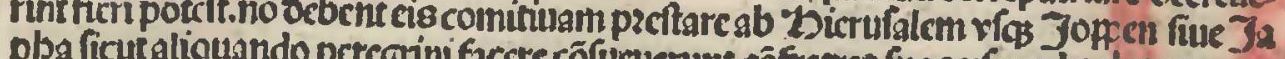

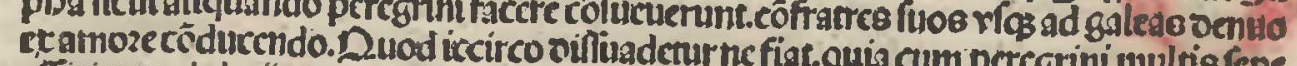

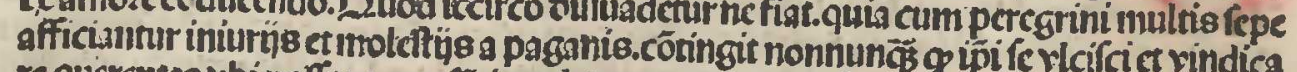

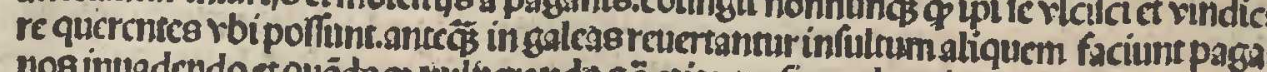

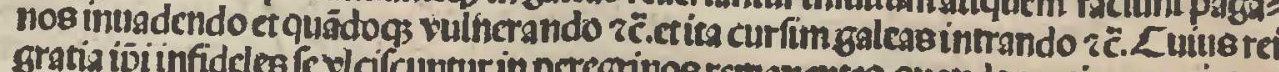

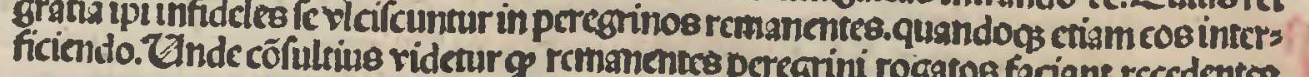

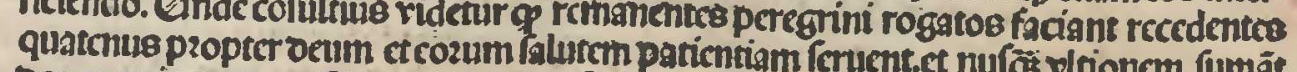

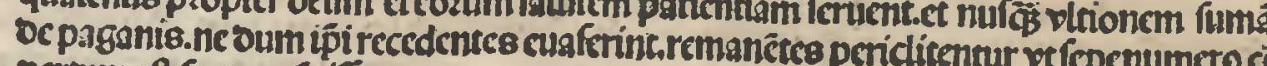
pertum eff factum fuifte

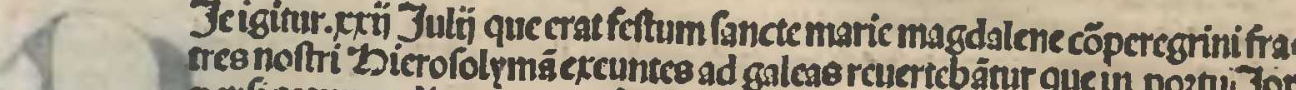

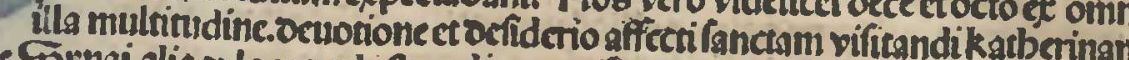 in mont s. frementuncomiporeprobibcas

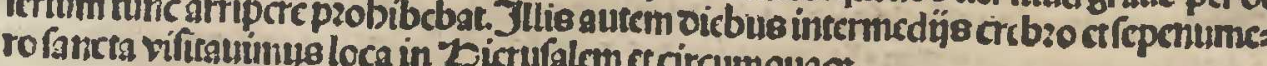

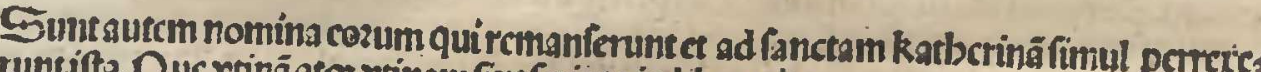 Domunus Jobannce L omes oc Coripta in libzo vite

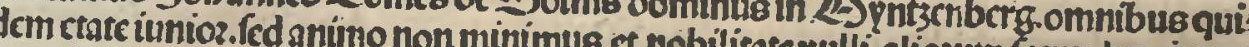 Dominus Bermardus or'

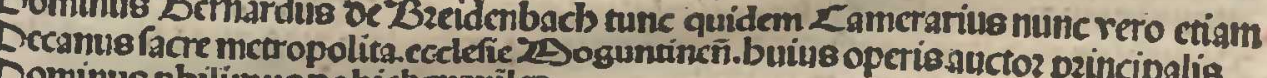 Cumbiserst inter cetcros cozum fam

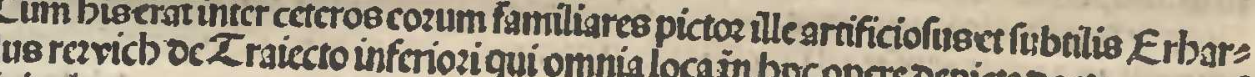 igiaut.

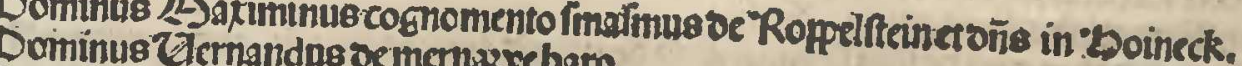 Domumis Cofparoebolach milese aro. \\ Dominne Scozgus mincmilce.

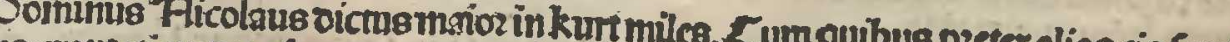 Dom

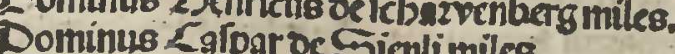 \\ Dominus \\ Domintre $\mathrm{f}$ ctrus velict miles.

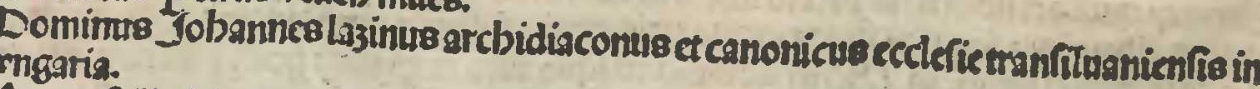 migaria. \\ Parer Felir fabridec ordincered

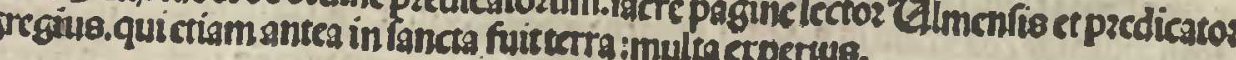

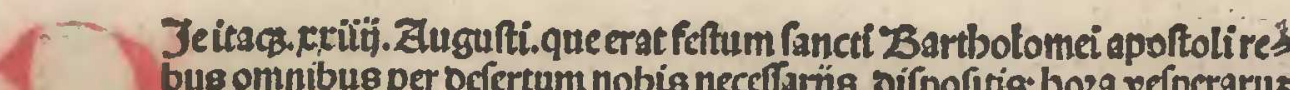

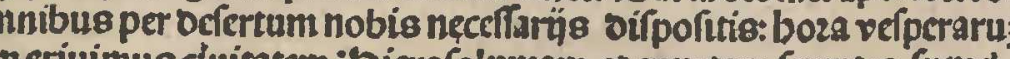
lectilem noftram in afinis:in 'Betblebem poft folis pernenimus oceflum Jn quantis autcm fuerimus anguttus inter viam it tam paruam faciendü p2oprer infídías paganoz et labozem babitus cam vino of recitarc.bono refpectu. ZSanfimus autem biduo in betblecm et omnia fancta vi Dieourcm illucercqubus fupza mentio et fact.

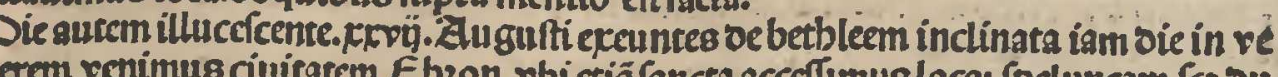

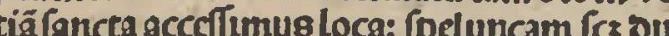

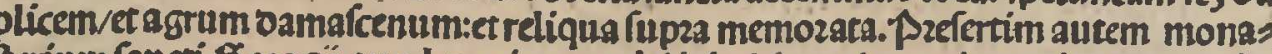

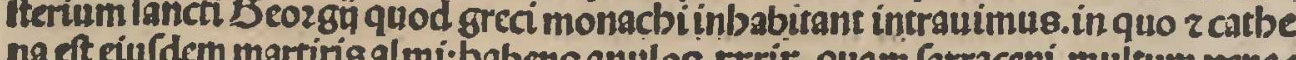
ng eft eiufdcm martins almi:babens anul o8. Kctic. quam farraceni multum vene sius contactu ftarim líbrells cozum qui a oentonty betantur et frencticis qui ex

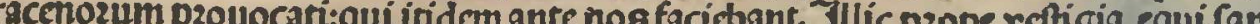

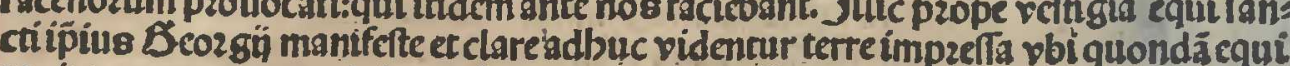

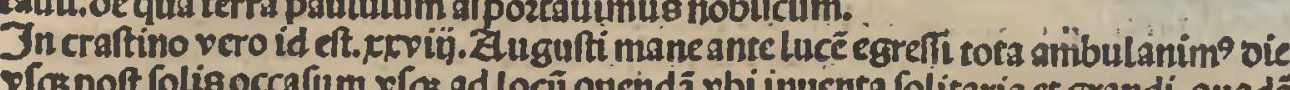

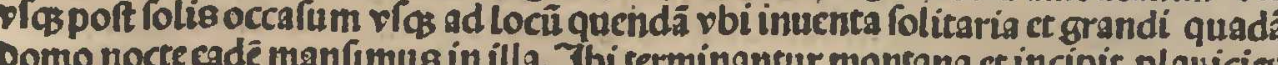

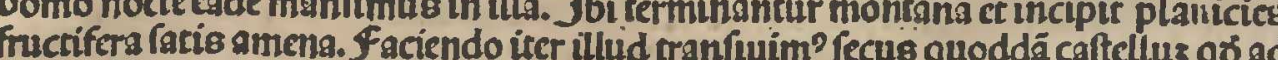

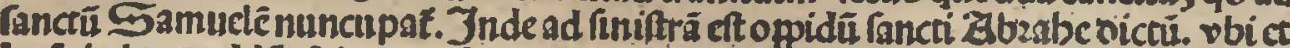

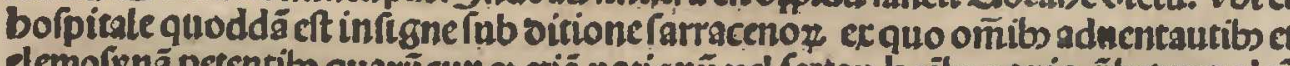

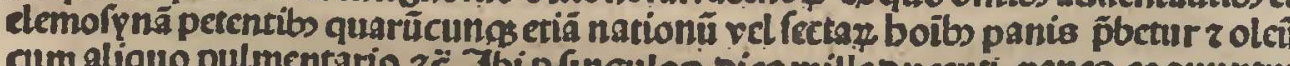
qui pauperibs crogantur. Expenie aüt que oles millesuincent panes coquuntur ad.xkiity.milia oucatoz cómuniter afcendunt.

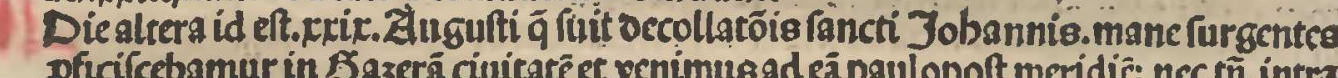

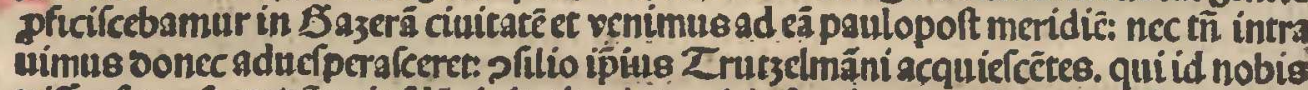

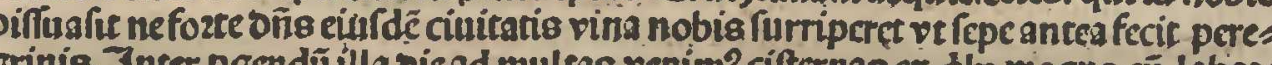

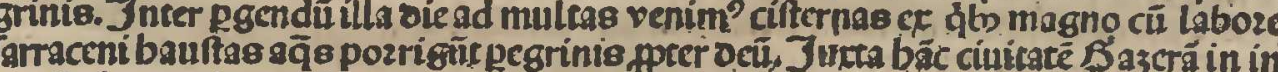

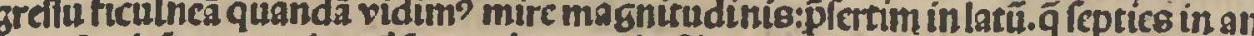
no pducitfictus:ct ticunt ficus pbaraonis. Jtags cil fol occidiffec ingrefi fumus

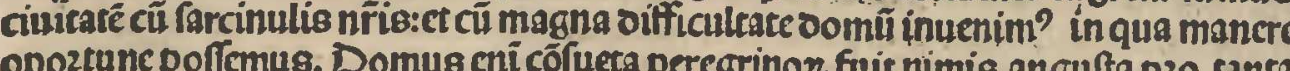
bortuntum et bettiarum mulcitudine capienda. Zanden in curiam angufta pro tants didamet plenam lateribs ex luto cöfectis setruft:nocte illa et fequentibs omnibs oo

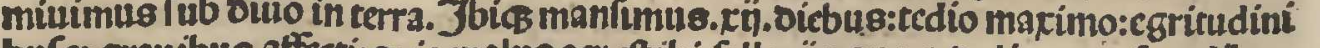
bufos grauibus affecti: quia malus acr eft tbi.fallacį̈s quog oolis atç fraudibus quibus longum effer fribere er fingula quibus longum effet cribereper fingula. Eftaurem Sazera ciuitas ouplo maio Et oiftat ad ouo oumtagat miliaria italica a mari. Erarơ quondā metropolis pbs

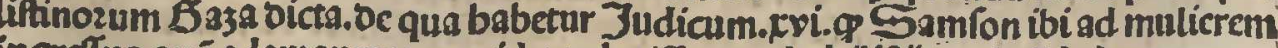

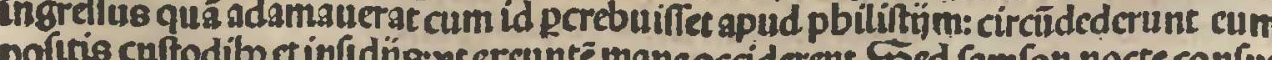

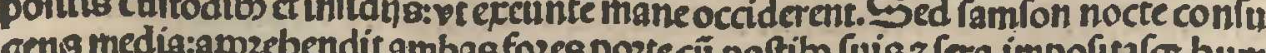

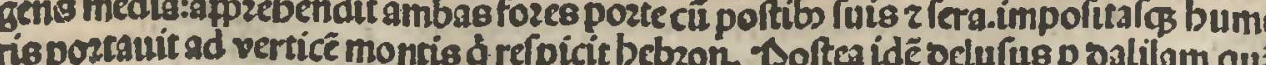
damauitque babirabas in valleforctb:captus, ct turis ribioculis ouctus fuicin
Morarimion - $20 \cos i x$

A. Srós Samming opridish $A$ hr

1200

24000

A rón proporion

fir, rhavio: i

$\cos 2$ mas

$4+2 \sim$ 
banc ciutrasem vbi cum pbiliftei vinctum catbenis ct claufum in carcere molercfes cerunt. Zandem adductiscozam pancipits pbilittym et omin frequentia ve lude retinthocaro oco hlo. nebat in quaches ramunutritud dinan que ibierar. Fam De tecto ct folario circiter tria milia bominus

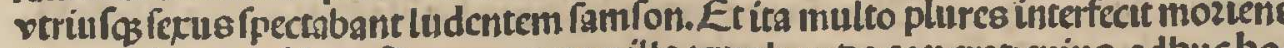
ons ante vinus occiderat. Do omus autem illa templum oagon crat:cuius adbuc bo die ibiccrnuntur ruine.

Dic tandem.ix. feptembzis extra cintitatem feceffimus in campum vbicamcli ftas

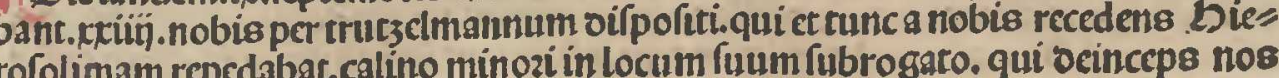

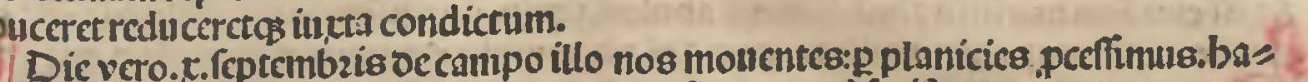
bentes mare magnum ad oecreram:ec terram fanctam ad finiltram. contra hos ve: ro ad pla gam meridionalem vefertum magnum ct raftam folitudincm in quameco

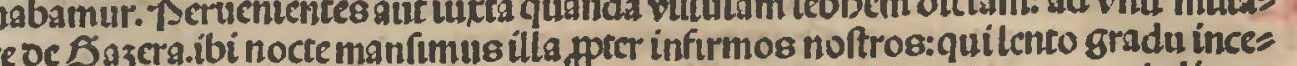
debant futisestentozus oifcurrimus per regionem ad colligendum paucula ligna. Fegs erí fruncillis in locis nifi arbulttule aridcequas radicitus cuellentes compoz tauimus in fomentin ignis p poctione ze. Eft quidem in loco illo pureus magnus

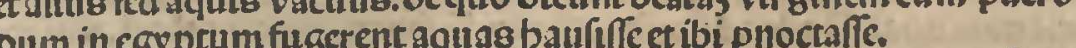

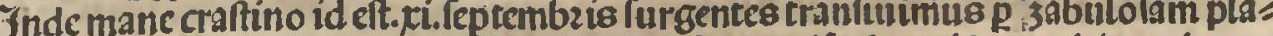
niciem adco f paciofam Q non vidimus eius finem nifi ab occidente rbi mari mas gmo cingebatur.tandem in reglonem Guandam venimus:Que lingua arabica $\mathcal{L}_{2}$

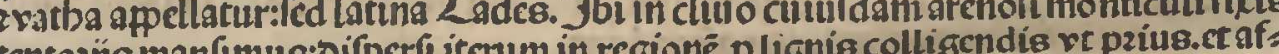

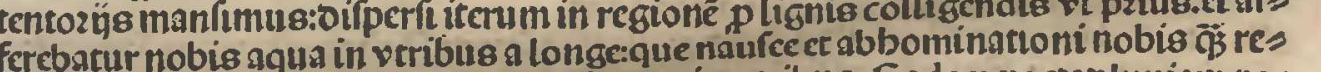
frigerio magis crat dpter eins corruptionem in veribus. Eadem nocteplutuiam par uain babuimus:quetamen fartentact ra magisac magis ocicta fect et bormibus endem oic in venis. vbierra necararip potet nec fert. per quam pgrediendo non vil lam/non ciuicatcm/non opidum/mmo nec oomum:aut tugurium vidinus. no agros/non vineas/non 02to8/non arbozce/non nemoza aur filtuas:fed cerram folis

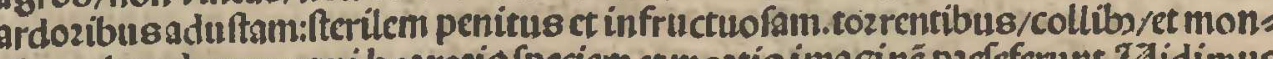
ribus abundantem:qui borrosis pecicm ct mottis inagine pzefecrunt. Zlidimus

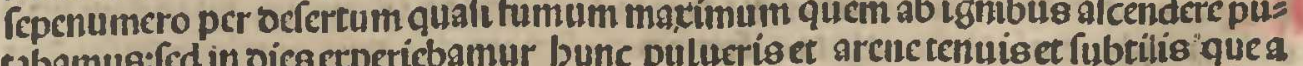
vento moucbatur effe elcuartionem. Fam et inde plerígs monticuli funt et vbi bos dic via eft plana:cras montecollecto/er arena vi rentozum agitara: mone eft et los cusinuilı factus

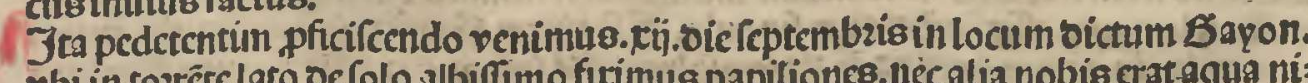

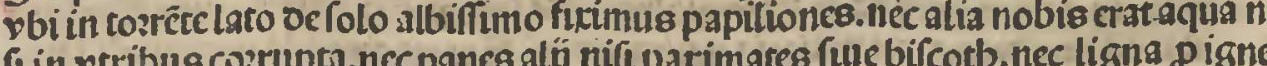
nif in vtribus co:rupta.nec panes aln nifl parimares fille bilcotb. neec ligna pigne Fero arabcs noltros vidimus fubcincricios coquere panes.

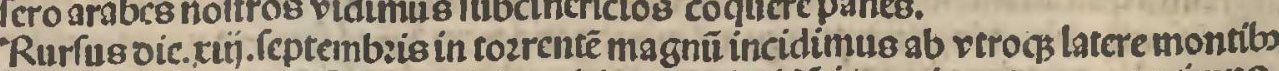

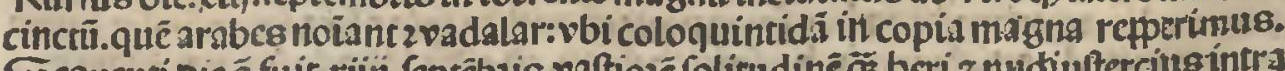

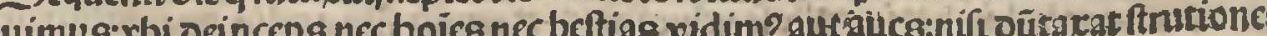

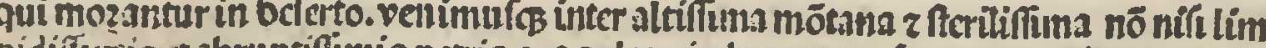
pidinisis (a) Onum venicntes:manfinus in loco sbi glcba rasalbifiung mozem ozientaliü regi

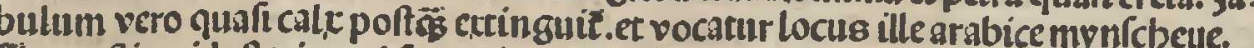
In craftino id clt oic, rvi.feptembzis pcefiumus p regionê nimis rigidam et atiffi mam que cotra ozientec fine no babet quã eques tranficns nố poteft in ouobs men (i)

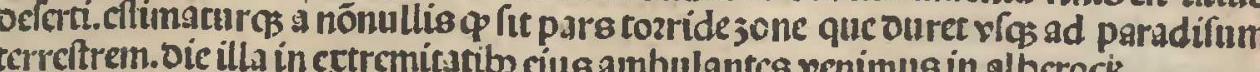

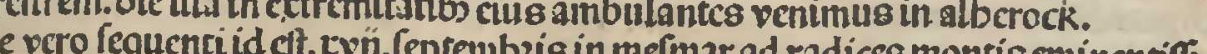
mi qué nominant calcb qui artificialiter videbatur factus.

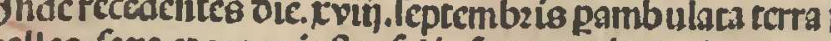
balles, faca ct arcna inftar falis funt. renimus in tosrentem qué aquofium, oiccbant Dicuero

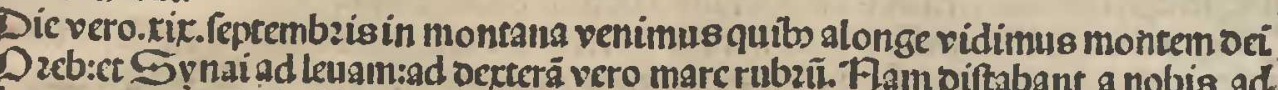

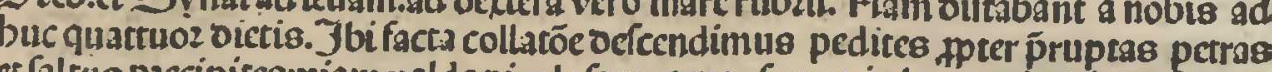
et falteils piccipitces: vian valde picul ofam:ct manfumus in loco qui oicirur Ramas rbyem per biem rnä er noctem, pper infirmos:in caucrnis fugpendenribs petris lati trantes.ucccrar ibi aqua nec arborcs aut pirgulte.

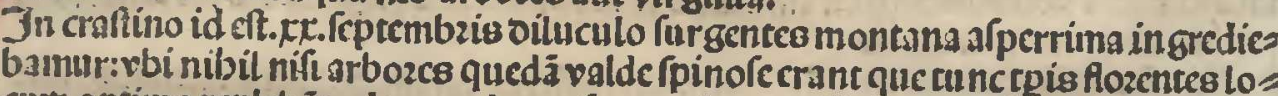
cum optimo repleteär odoze vndequafi recreabamur. Dicune quidä oc illis fpinis

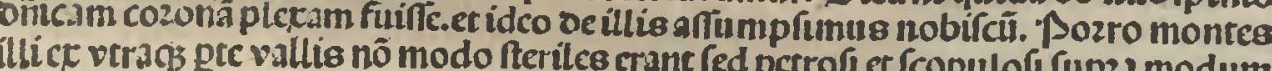
mietm colozis rubei et nigrict videbantur fara in folis reful

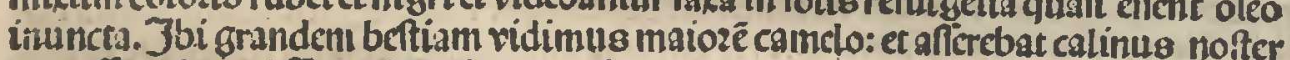

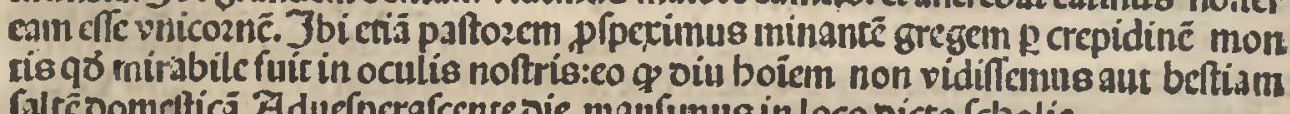

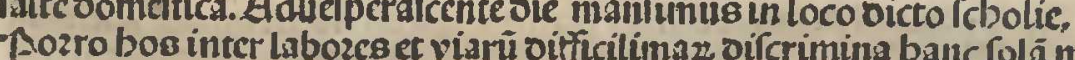

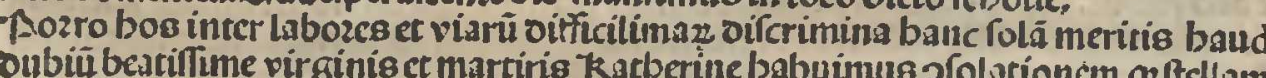

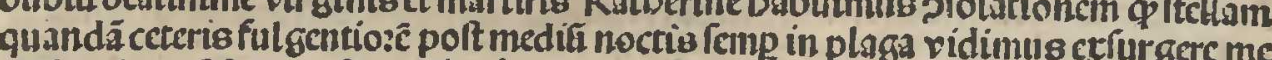

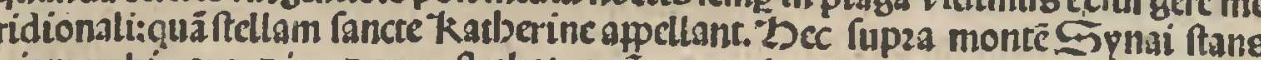
viam nobis ance oicm demonftrabat.et qnecullas ab ca beclinauimus viam noftras

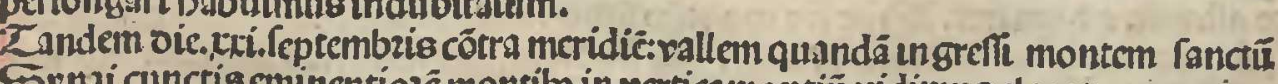

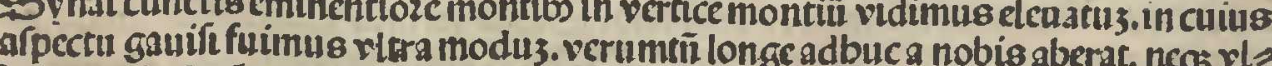

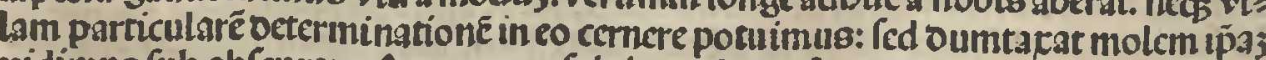

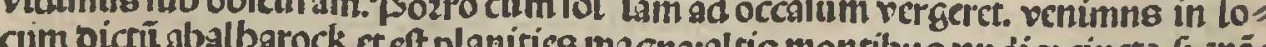

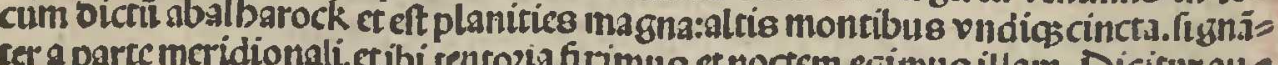

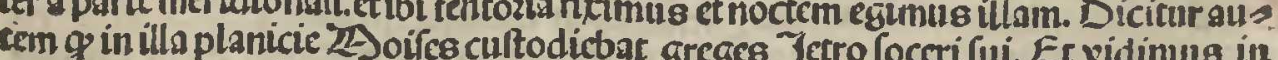
petra cauaturam in qua oicitur ipfe rir fanctus fed diffe frequenter er oozminiffe. De the cniloco rotam potcrat planicien facilc peripicere et creditum fibi gregem. S re

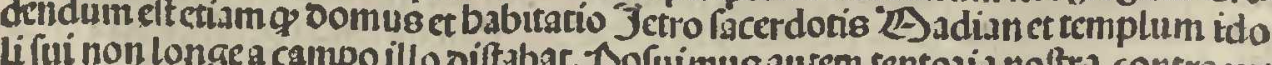
am que oucitinter altos mources per vallem ad interioze per quam in uinabat greges ad mpntē Sq̨nai.per quam et nos tranfituri is craltinũ crannus.
Myar

Farproveid

2. ney

Orut mis

thes

sing aniser

Divmoinge

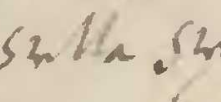

2.09íni 2

surmblimbar 
Altera bie queeft vicerimafecunda Teptembzis ralde mane furgentesad interioza am planicicm arenofam oeruffa arena ficut et montes rubei crantin regioneilla. etin bac vallc credutur filu ifracl babitaflect p tribus ctfamilias fuas tabernacula tispa

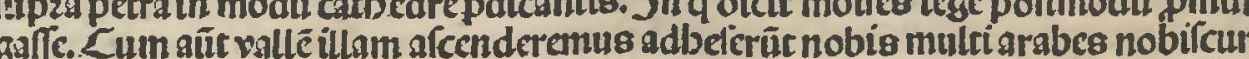

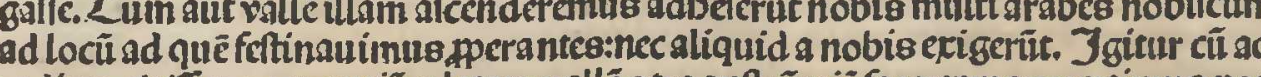
radices altifínoz montiü $p$ Latam vallé ptra auftrĩ viä faccremus:puertimus no

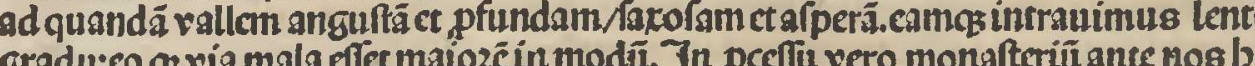
Griut

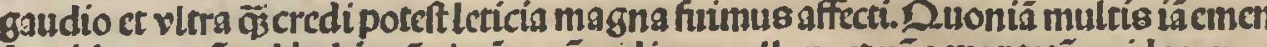

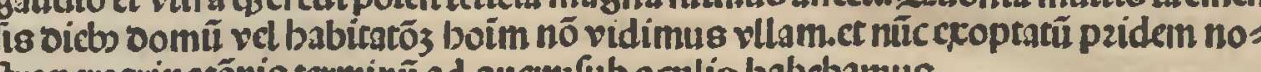

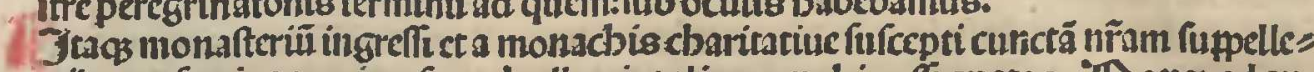
crilem et farcinas vnitcerfas ad cellas intulimus nobis affignaras. "Dopgs ad aus Reliquã illius dici parte in monafterio cregunus quictio operä neceffaria indul gen

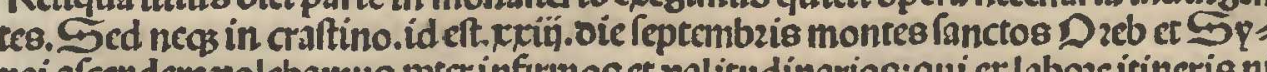
nai afcendere volebaanus pper infirmos et palitudinarios:qui cr laboze itinerio n

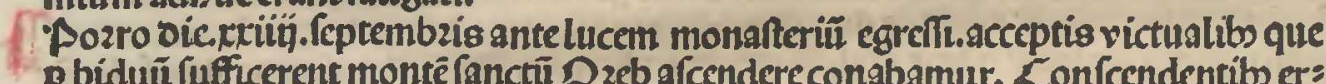
go nobis ppreceps fons llle riuts nobis mor obuius fuit.qui ab initio ibi mira

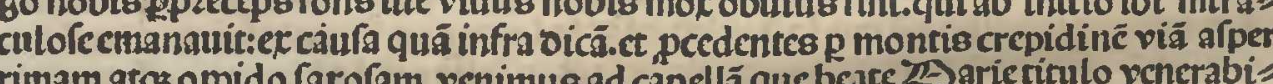

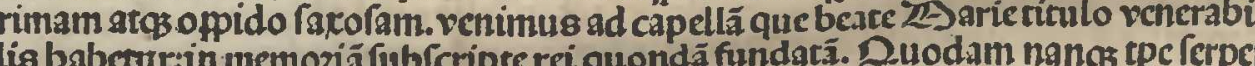
teB/vippere buffones/et alia anumatia venenofa adco in monafterio et p cius circüit

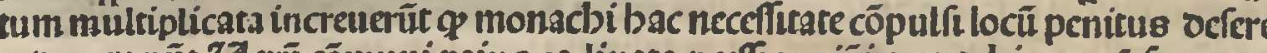

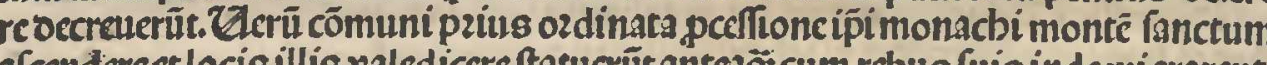

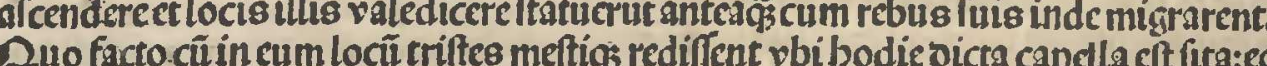

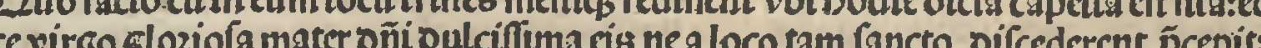

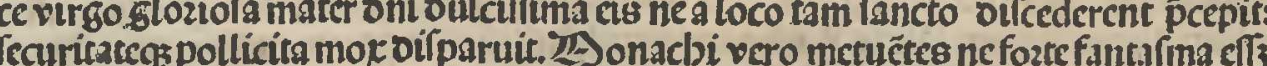

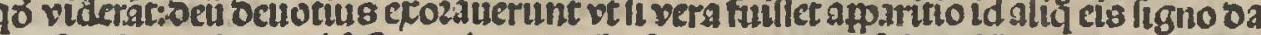

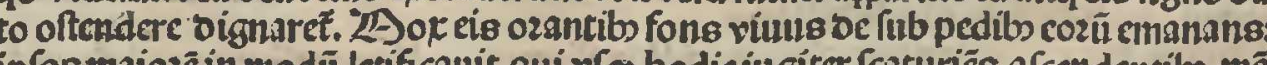

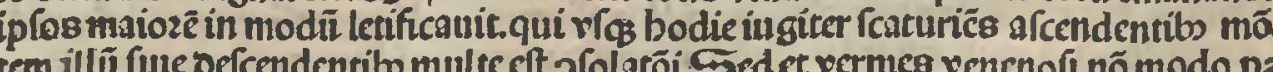
ail indegufugeñe

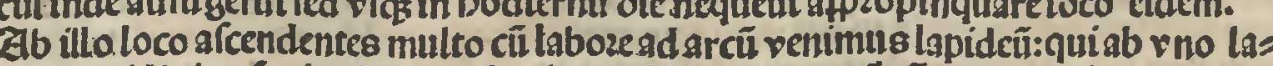

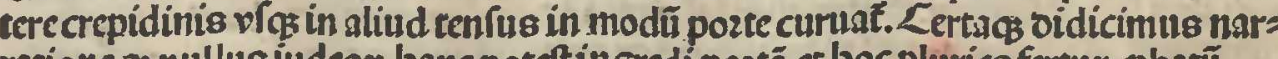
ratione g. nullus udeoz banc potect ingredi poztá.ct boc pluries fertur pbatî.

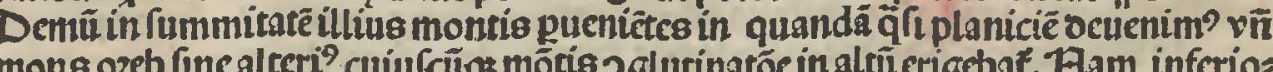

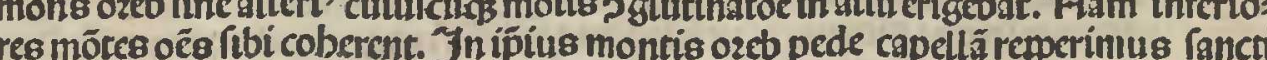

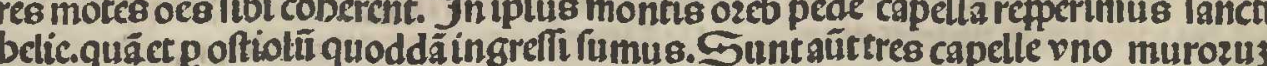

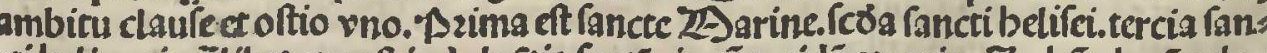

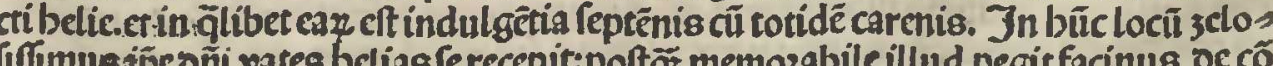

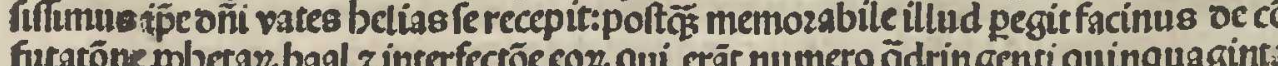

Viri.quos connictos et cópzchenfos ouxit ad tozrentem cy font et ibicos occidit ḋd vin.

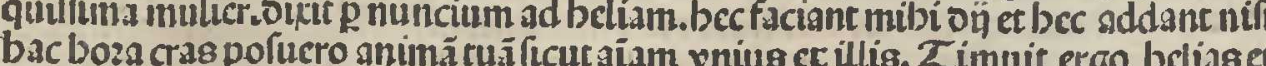

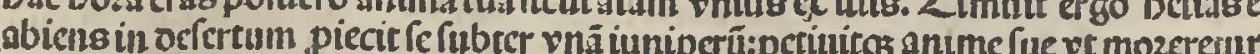
Cüßs obdozntuififet in vmbza iuniperi. Ecceangelus oñi tetigic enm oiritos illi.

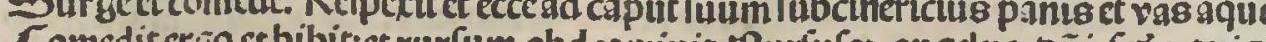

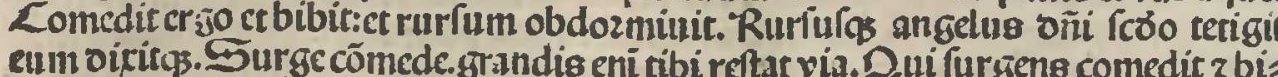
bir.ct ambentanit in foztitudine cibi illius quadraginta oiebus ec augdragint 2 bi ctibus bfos ad monté oci ozcb. Cunes veniffet illuc manfiut in fpel unca illa oe que

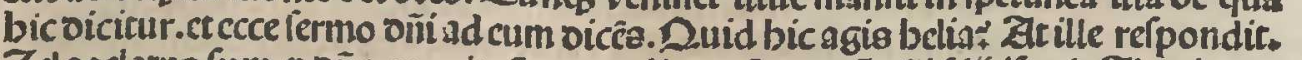

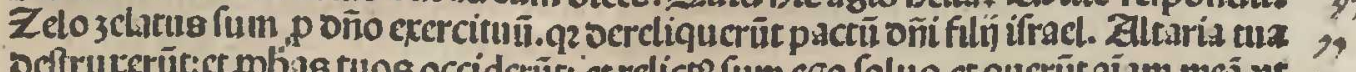

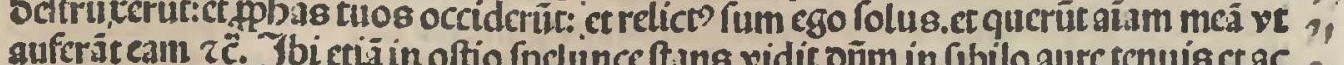

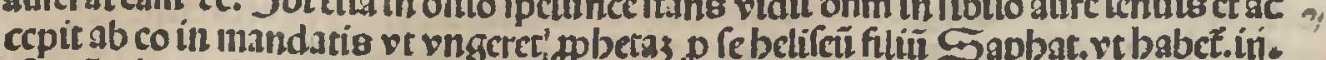

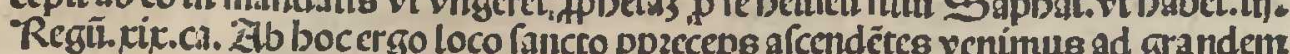
petra oc monteabicitain qucin via fertur cecidiffe oum beliss in verticem montis

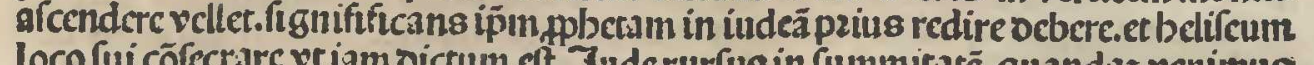
locolui côfectarc re Lam oictum eft. Jnderurfus in fummitate quandas venimuse

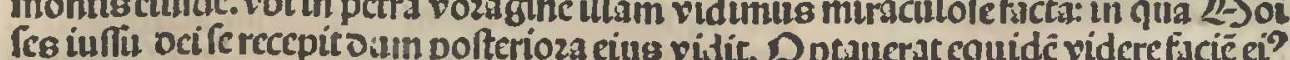

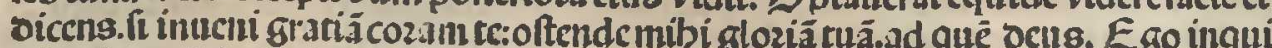
7 oltendam tibi ome bontuz zé. Rurfumgs nō poteris ait videré faciē meam!nō eni vis

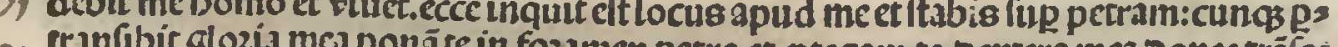

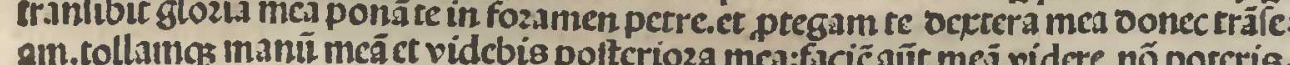

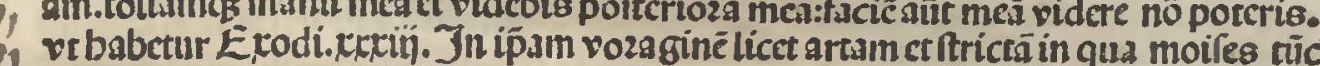

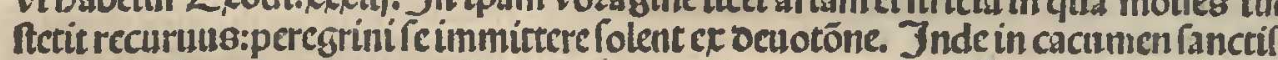
fumi montis ozeb pucnimus. J bi parua queda et pulcra eft edificata capella fer reo oltio firmiter claula:ftatos in eo loco rbi lanctus pzopbeta $2-$ oif cs occalogum in

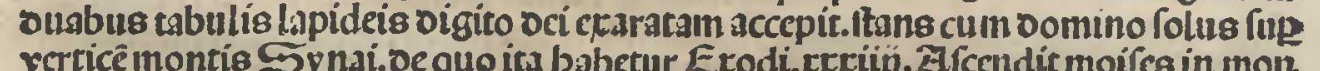
tem fynai ficur ei pieceperat octus. poztans feciñ ouas tabulas quas pzcf́idcras ins ftar pzioz. - Lln men onit.quo trancunte fét tinus moiles curuatus eft pnus in terta.etadozans aic. Dinatoz one ocus mifcricoze et clemens. pattês a c multemilcricozdie ac verus. qui

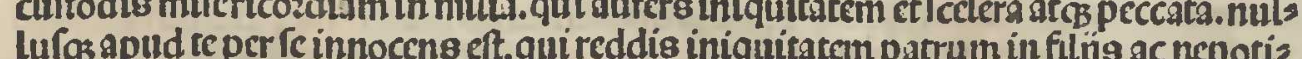
bus in tercian et quartas pzogeniem zë. Fuitcrgo ibi vir fanctifimus moifcs cum oomino quadraginta Dice ct quadra gutra noctes:panem nô comedenset aquã no

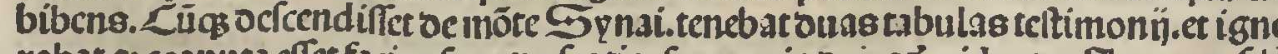
rif cos:vebabetur ibidem. nobifcũafcenderat:sifcalciaris nudí́as pedibo preuerentia loci intrauimus, noro

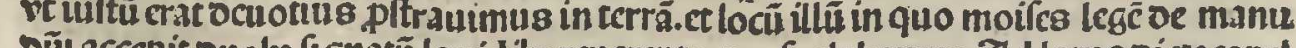
on accepit oulobs fignatu lapidibs reuerenter ocolcul abainur. Ad latus oictecapel

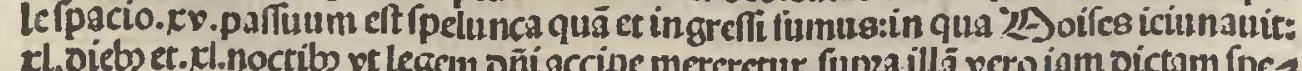
luncame cf quedam mufcbkea ad quam quoridie confluunt peregrini arabes z fars ter mayes notitiver intit at indmplas? orage ardio 
Four nloiri

7000

Sing Maicy

Grahtiónario.

40 Sanebre

Frigicangril as

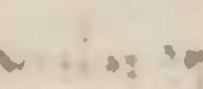

300 .
Drandis infup eft ibi cifterna bonä continens aquam z frigidam ato fanam valde

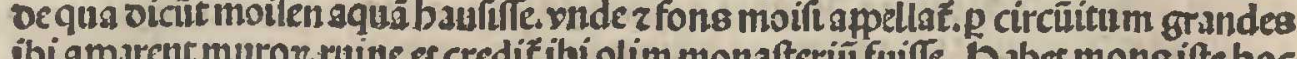

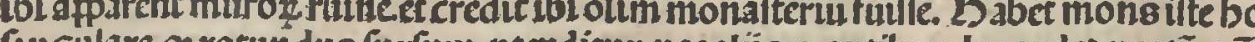

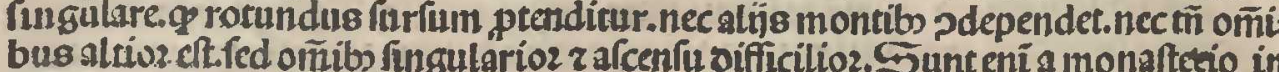

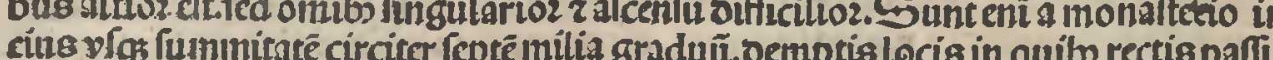

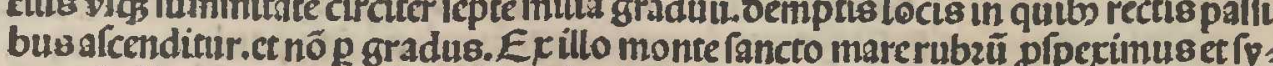

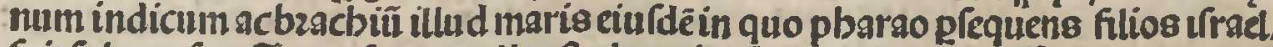
fuit finbmertus. In paefata capella eft plenaria oum peccatoz remifito.ex qua egref Explection

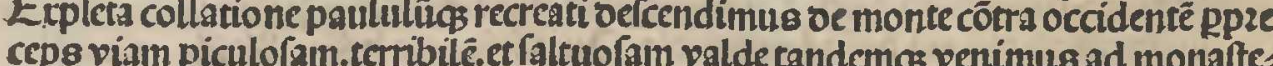

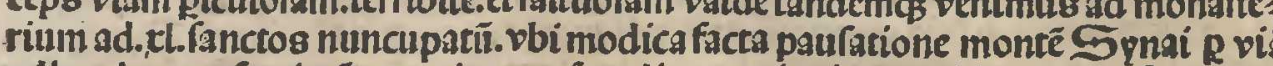
§dbuc longe a perioze et ouriozem alcendumus p inulas crepidinse. per feopulo

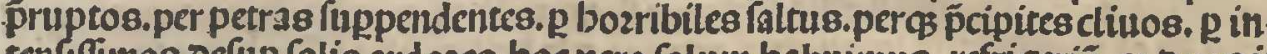
aliafcerfin foncerito Poaro cum iam pene in cacumen veniffemusareperínus collum montis adco tuofim aclongum q obltupuimus.nec alia erat nobis via. 3 teos p fcopulos nos

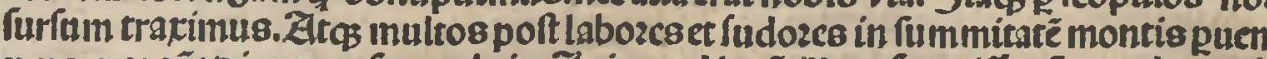

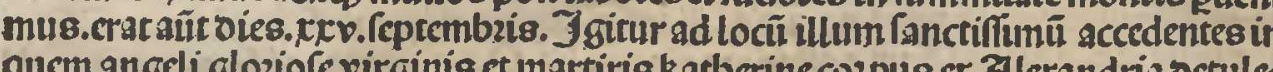
nuncin

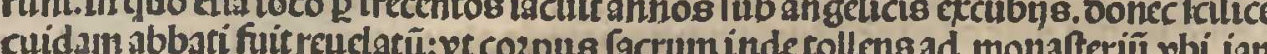

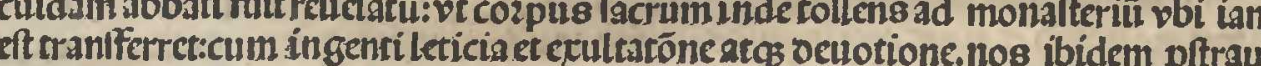

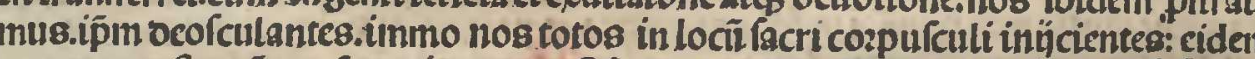

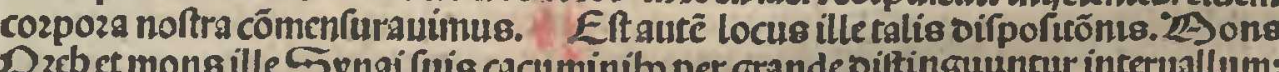

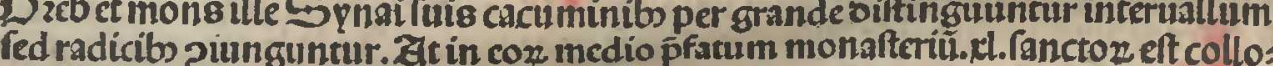

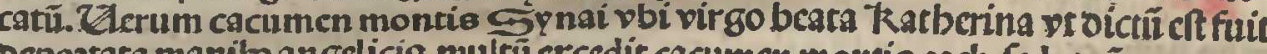
sepoztara manibs angelicis.multũ ercedit cacumen montis oreb. Fed et cǘcta mon

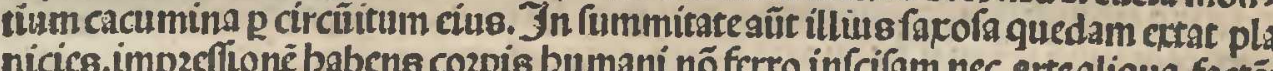

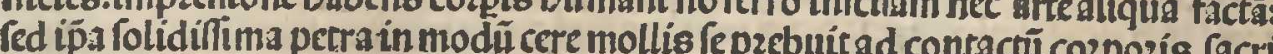

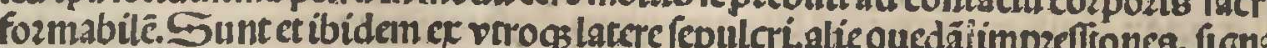

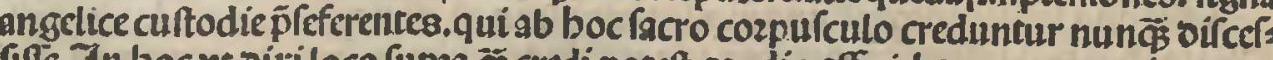

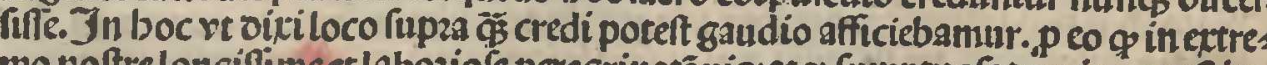

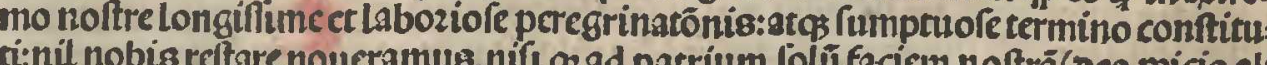

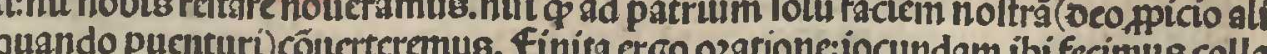
tionem comedentes et bibentes que apud nos erant. oco et virgini facre iocundifit mas gratias prolnentes. Inde circumiaccntes prpeximus regiones: et longelareos cerram วtemplabamur. Zlidebarur aút nobis $q$ sempto selerto $Q$ quod veninus

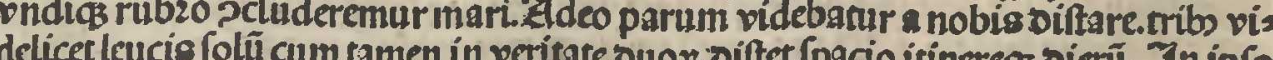

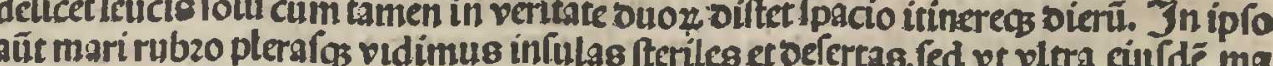
ris rubsi brachium.altifíma illa monsana a longe pipecimus in Z bebay da regio nc. voi (anctus Paulus. Anthonius et reliqui plurimi patres beremiricā viram ous

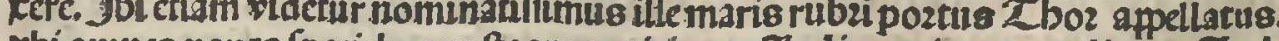

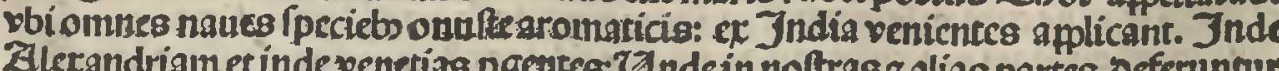
Jbietiam oefertum t) mari nubzo.caftra paimum metabantair. Inde ad radicem montis buius fanctiaplis

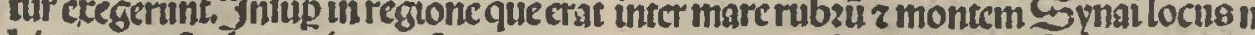
bis oemonftribatur.unquo /anctozum virozun monafteriu ferturefle.qnod moder:

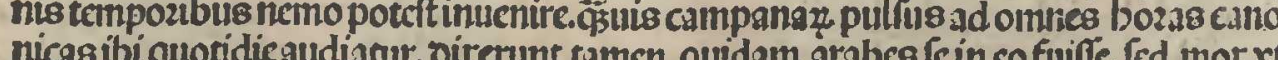

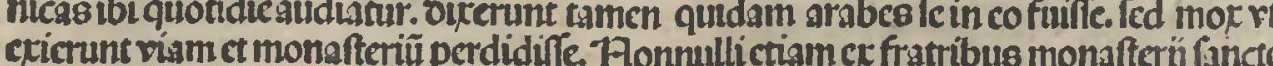

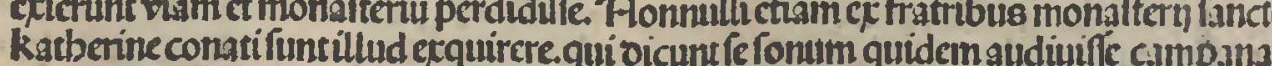

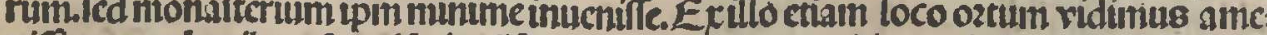

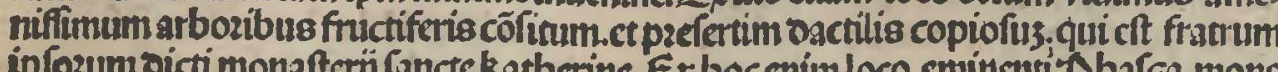

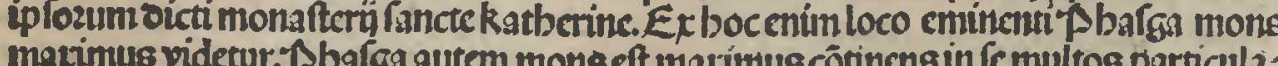

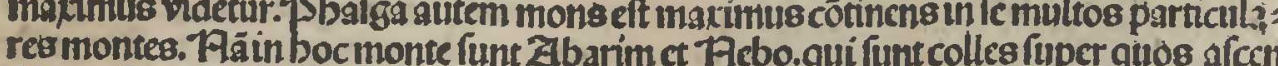

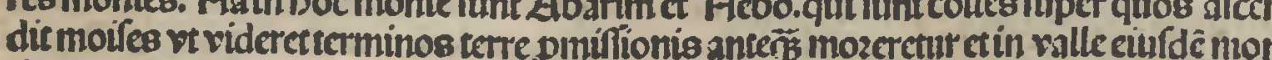

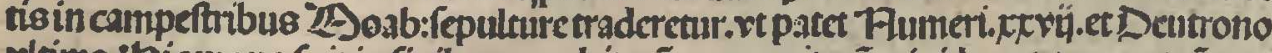

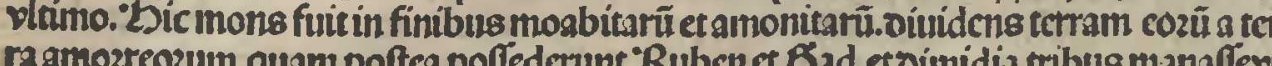

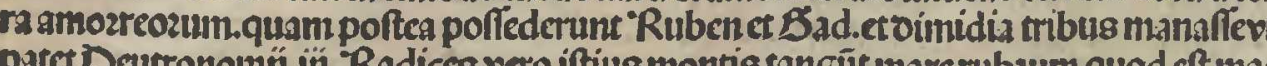

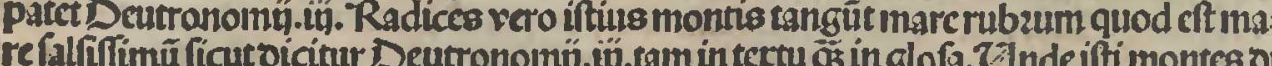
uidebant tecram quam inbabitauctunt moabicect ctians gmonitc a terra quam in pof

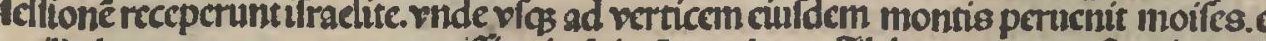

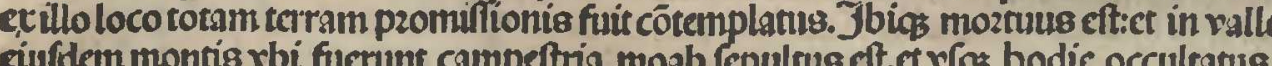
(a)

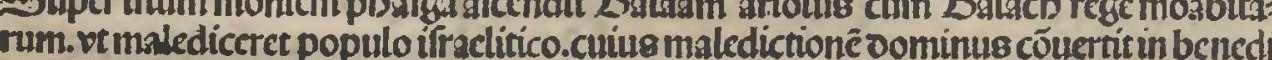

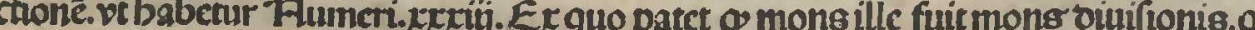

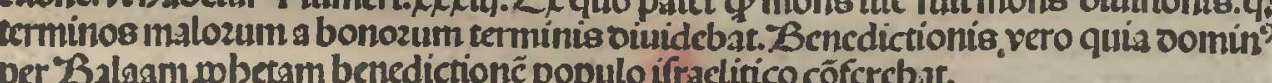

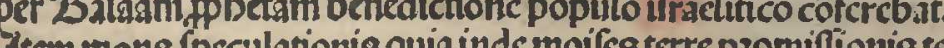

Fenos

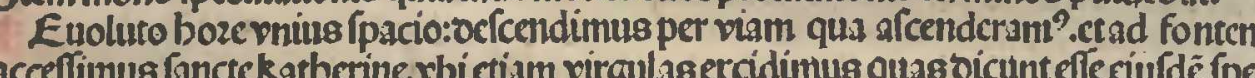

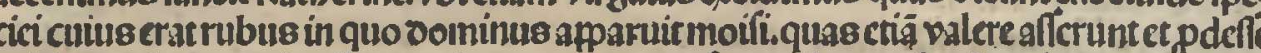
baldc mozbum paticntibus caducum. ourm eas fecum ferunt. "Poft multum tandem la

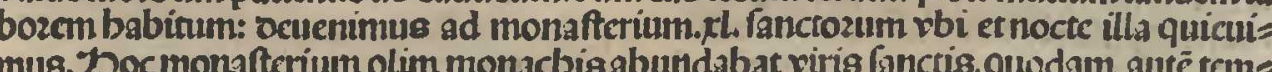

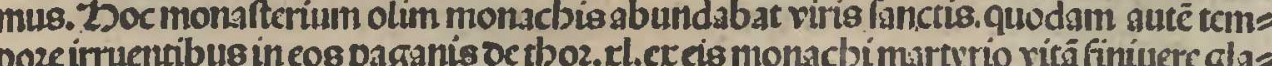
dij anuinaduerfione, qui ct ibidem funt fepultit. vnde ctian ad. pl. Fanctos nuncuparur.

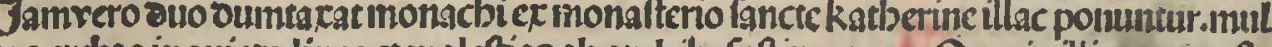

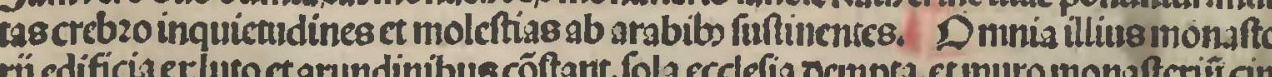

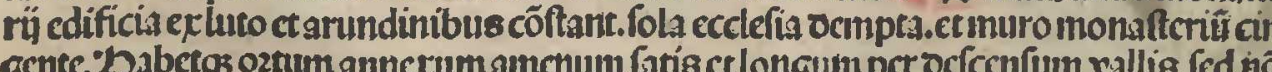

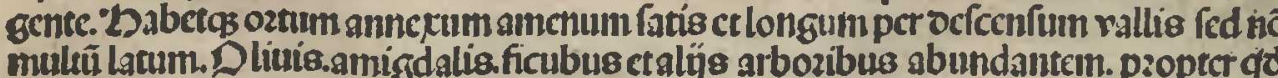
magnos babucrunt pzoucentus olim monacbifanctekarberinecr ozto illo.fed per los

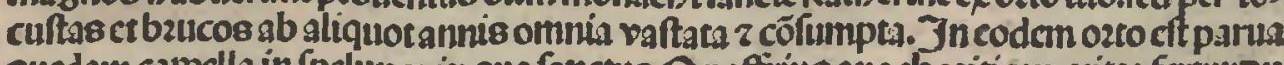
quedam cappella in 1 pelunca in qua Tanctus $D$ noffrius anachociticam ritas fertur on

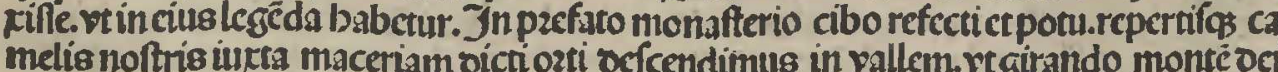
ozeb in monafterium fancte katberine reuereremur. Inuenimurfos ad radices montio

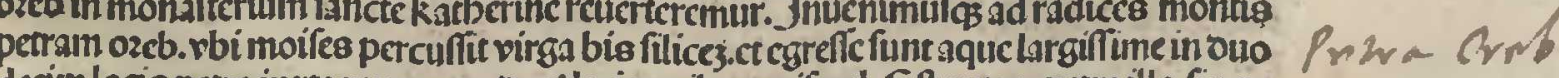

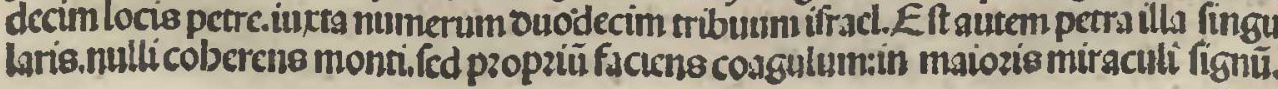




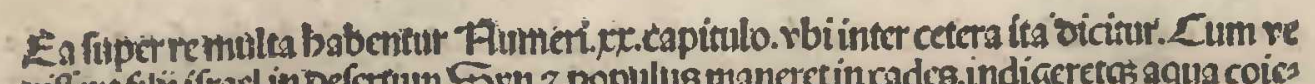

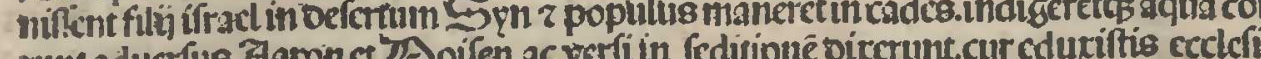

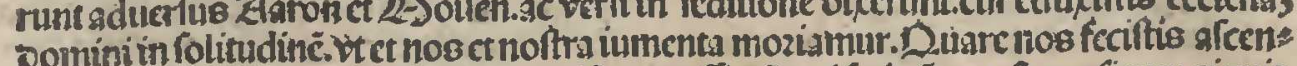

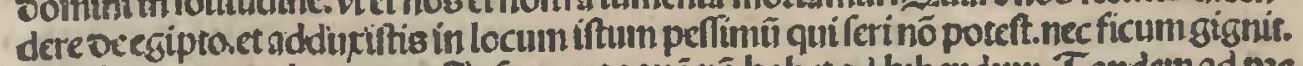
nec vincas nec malagranata. Jn nuper et aqua nê babet tad bibendum. Z andern ad pre

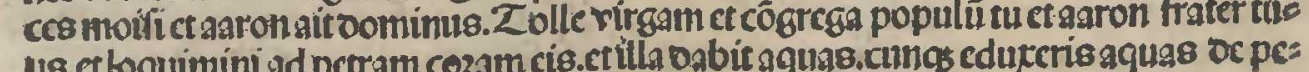

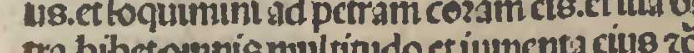

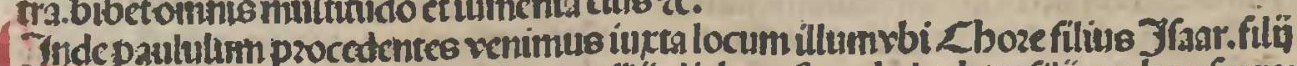

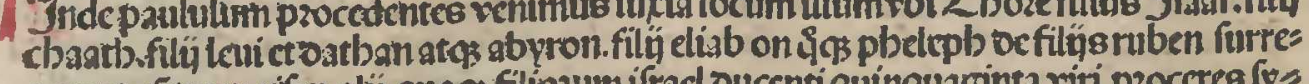

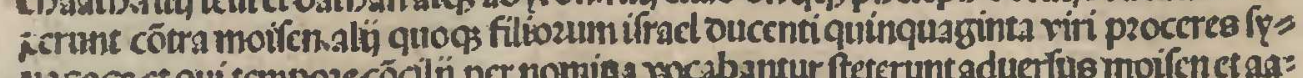

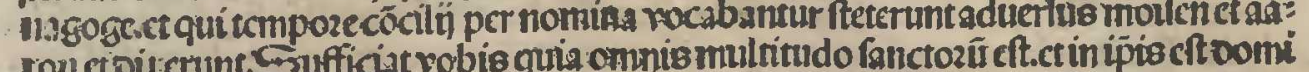

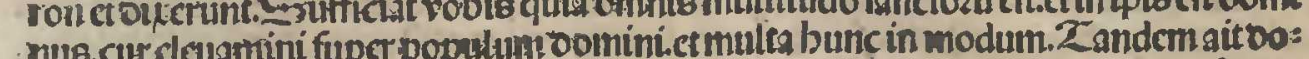

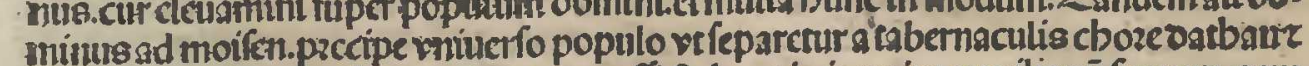
abyrom.quo facto oartban ct abyron cgrefi. ftabant in introitu paphlionu fuarum. cum

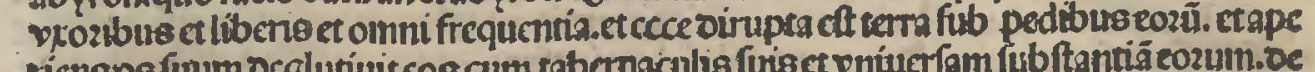

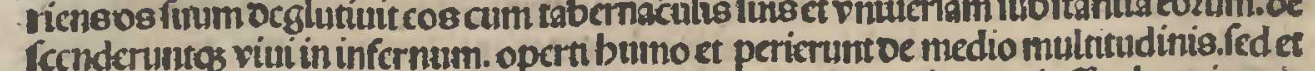

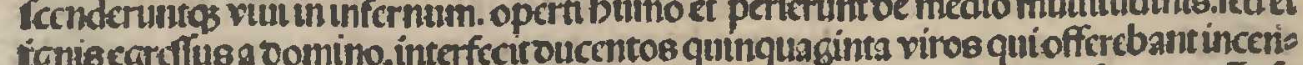

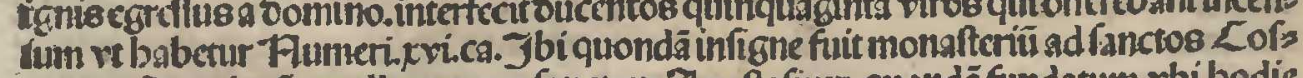

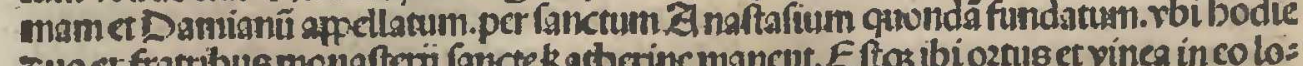

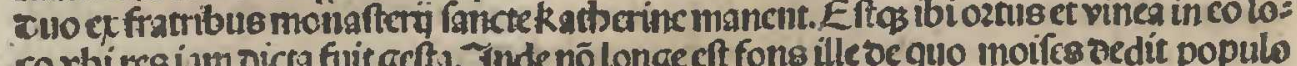

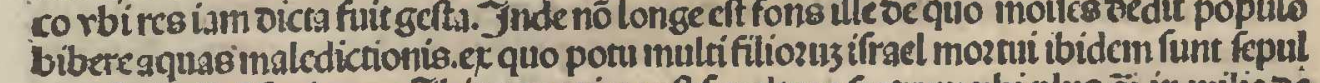

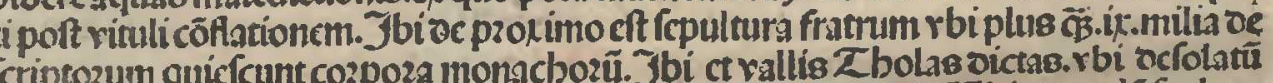

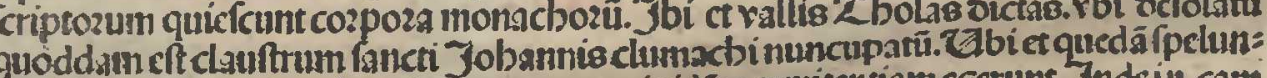

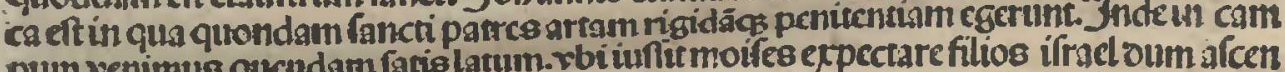

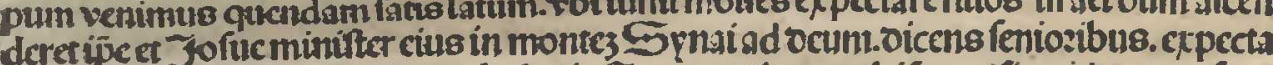

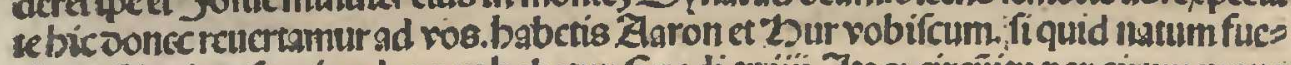

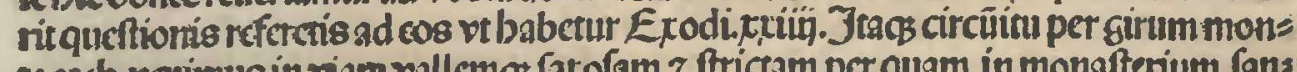

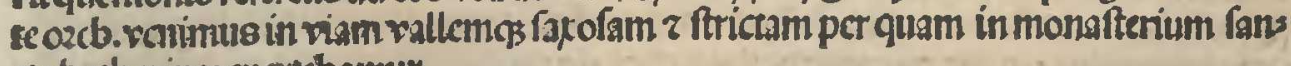

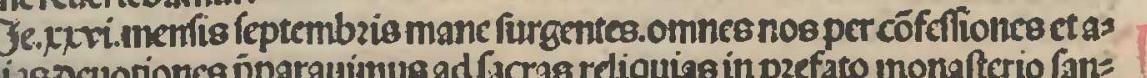
政

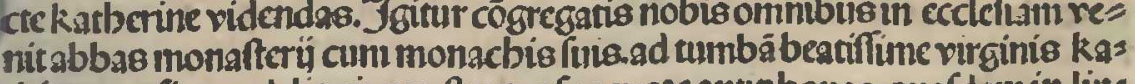
docrine multis accenfis candelis circumflatantes fuo moze antupbonas quafdam in lins gua gras occantaternts.

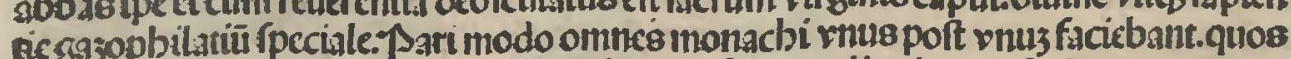

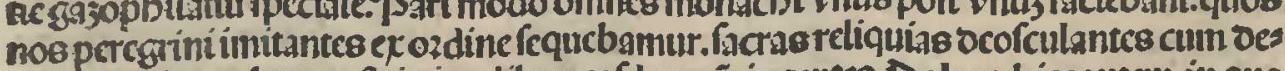

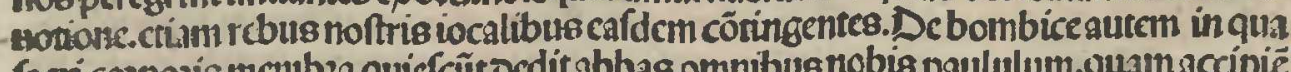

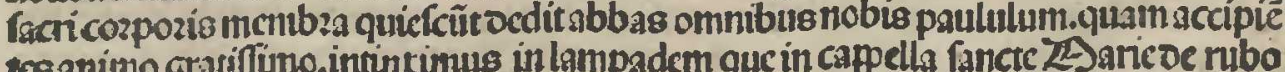
pendet.

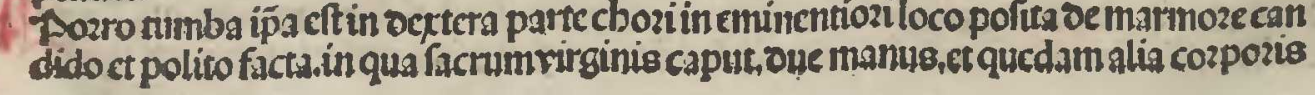

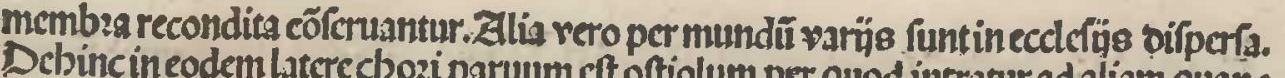
dam fancifinä cuaterecbosi paruum eft oftiolum per quod intratur ad aliam quans

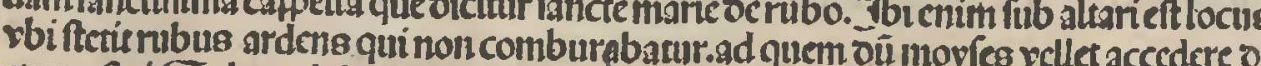
ctume eft ei. Soluecalciamentum oe pedibus tuis ä qö ct nobis monacbi in merno

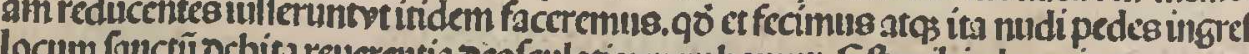

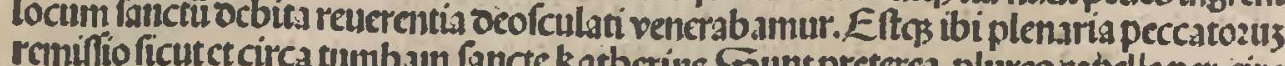

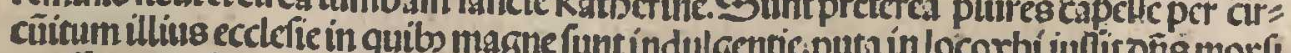

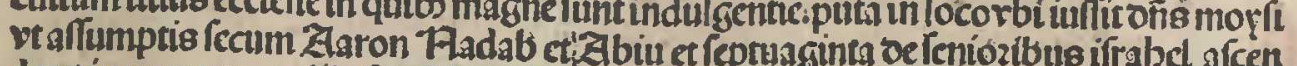

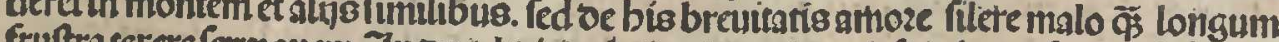

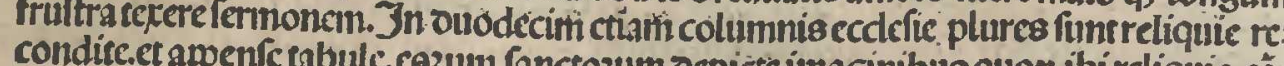

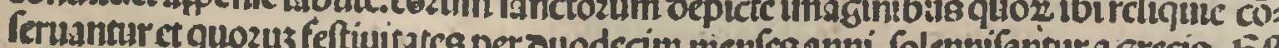

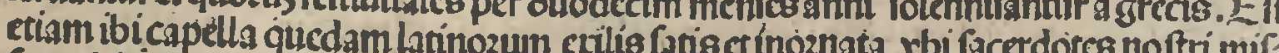

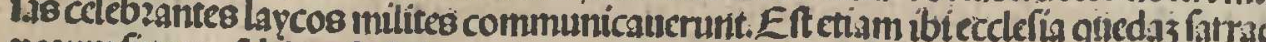
nozum itue mulcbkea fatis lats $z$ iuxta modum cozũ pulcbra.turrim quandaui babé nerantur

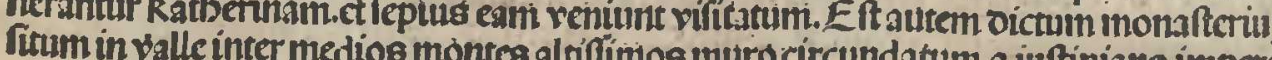
toze primitus fundatum. Eft os pzimum monafterium in grecangrioneordinio

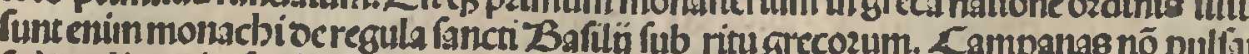

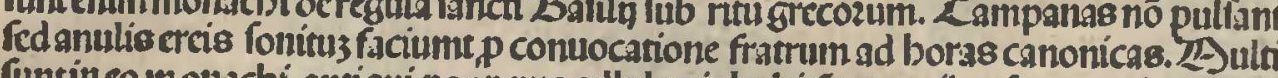

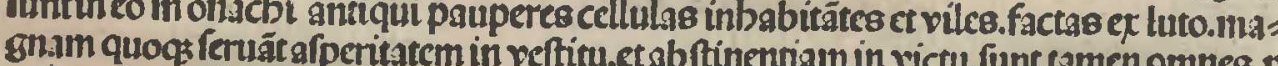

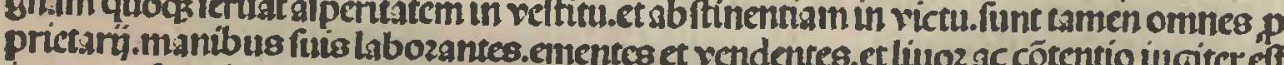
inter eo8. forte in penam et pltionem fue inobedientic. quis eccleffie 'Romanenon funt

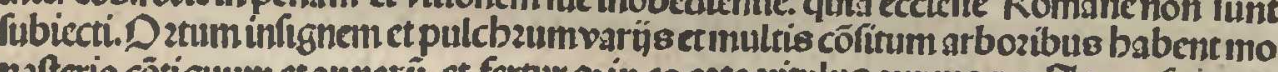
nalterio côtigume ctannetü. et fertur 9 in eo ozto ritulus gurcus per Aaron fuit con

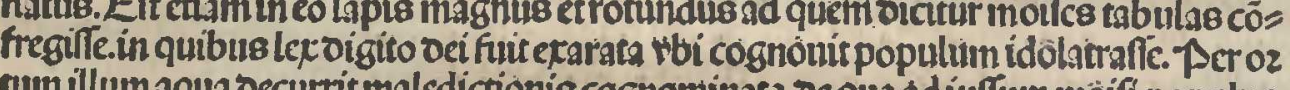

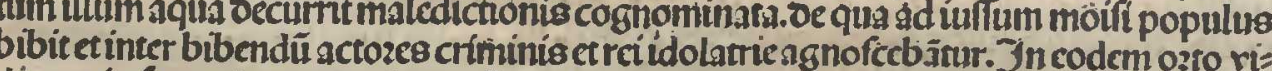
demus in fummitcasemiontis quafi vitulum quendain grandem lapideum in fupcrcilio

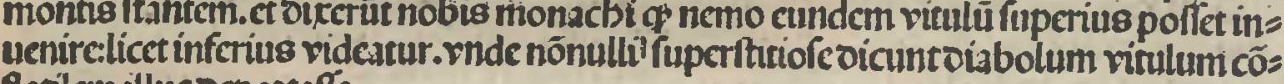
fartiem illuc ocpoztaffe.

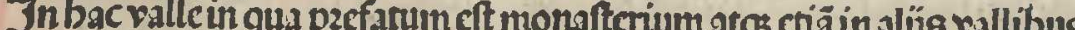
uspercircumis nacbicolligentes et Arabce:aduentantibus vendunt peregrinis. Eadit antem verfus

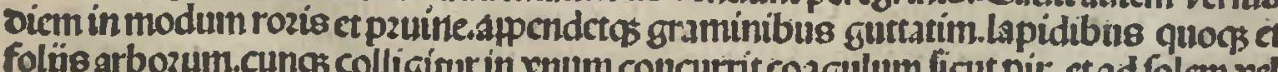
folty arbozum.cungs colligitur in rnum concurtitcoagulum ficut pic. et ad folcm rel oc co inultas cóparauimus gurts cum qua tanta fecit mirabilita in terra esipri in accem plagis.

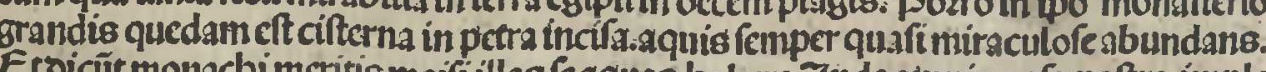

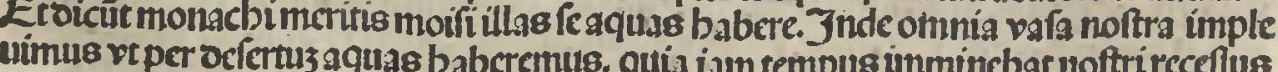

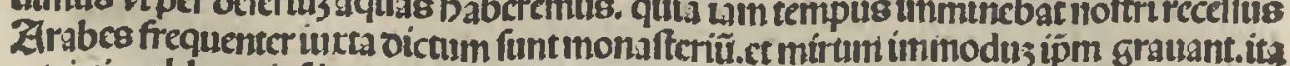

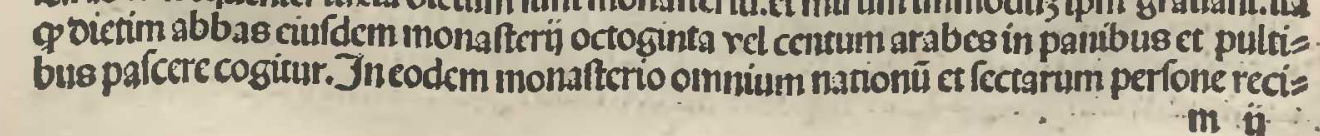

Comollo 54

llowes ab $R=60$.

Cigndiastiva Virist, anim, A Yisheraidri apioance 
Aration

Macionizm

Camplario.

Cim forim

$\underset{12}{12}$

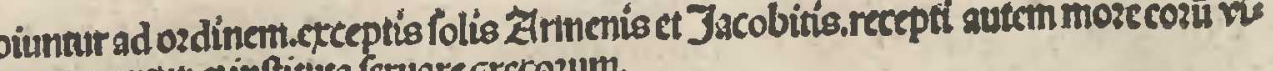

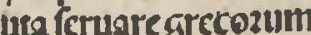

I Deregreltuocmonte Synai verfus Cbayrum.

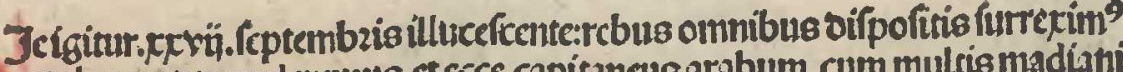

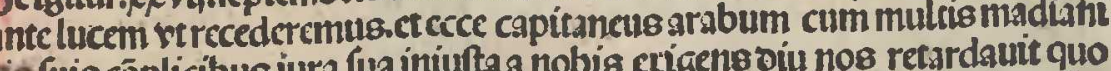
tis fnis cōplicibus iura fua iniufta a nobis erigente diu nos retardauit quo

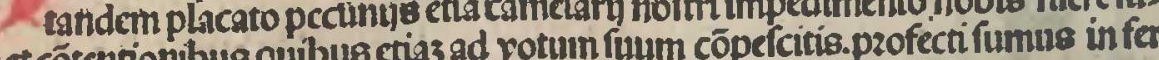

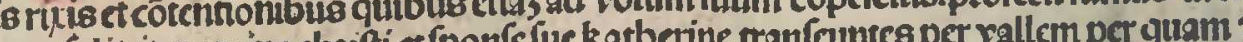

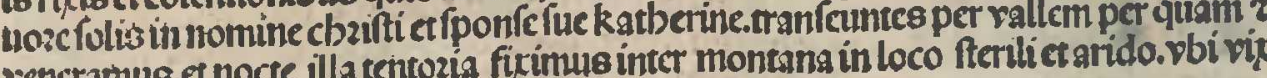

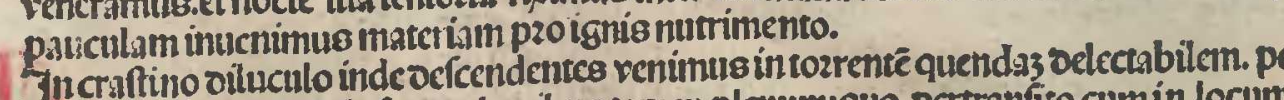
crofis montibus conclufum.arbozibus tamen plenum:quo pettrantito cum in locurn

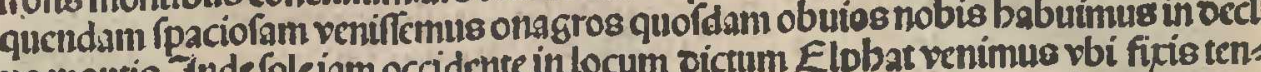
uo montis. Indefoletam occict mez therine per fingul os annos in feprembzi mitrcere zfueturt Soldano in $\angle$ baytum oe fru

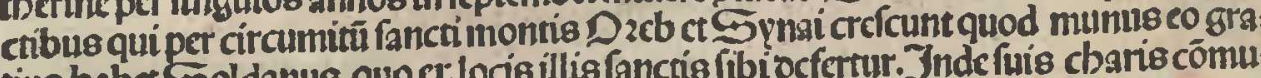
tius babet Soldanus.quto ca locis nicans amicis. Lum . Zandem oie paima ocrobžis renin" oe octing ocfertiad reglam et tritam riam. qua Bazcta oirccietran ont in

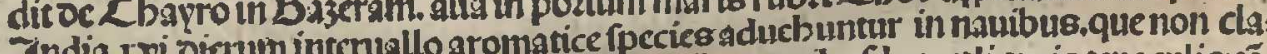
J.8.

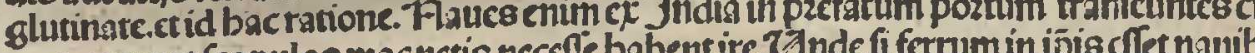
carmonicertropulos moxmagnes cas arto

. popres

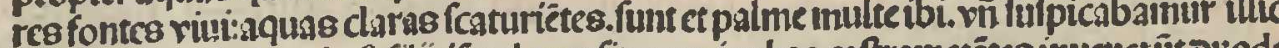

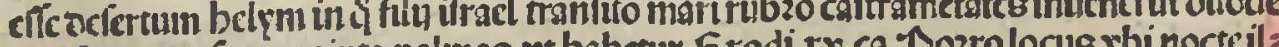

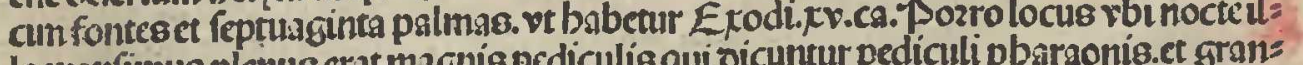
La thanfinus plenus crat magnis pedicullis qui vicuntur pediculi pbsiraonis.ct grams

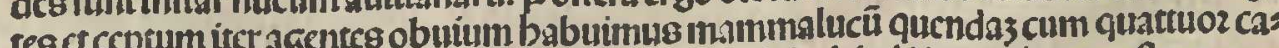

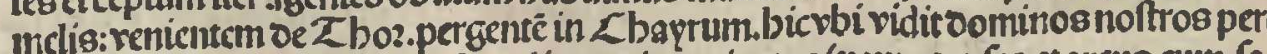

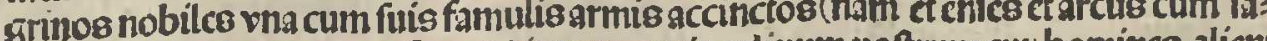

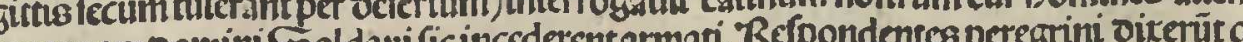
per tertam oontin cunnct

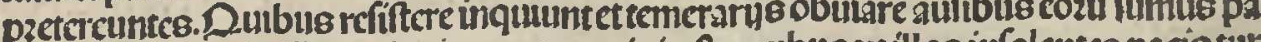

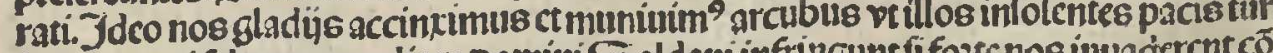
batozes qui

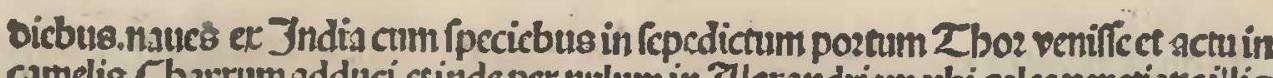

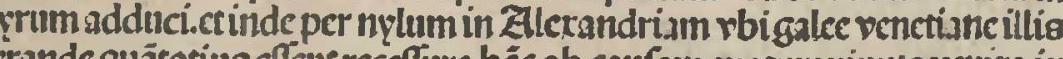

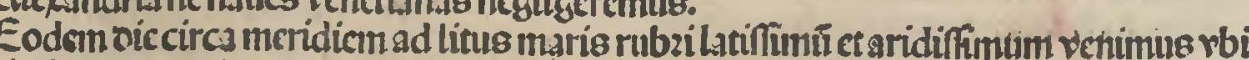

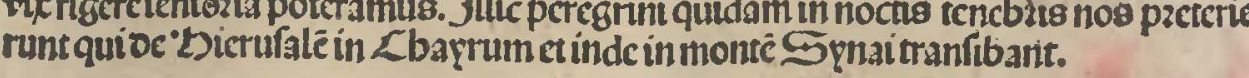

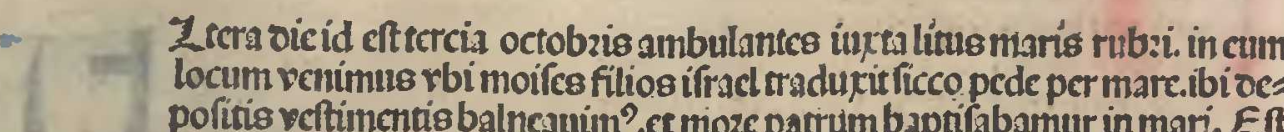

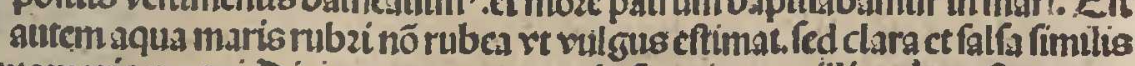

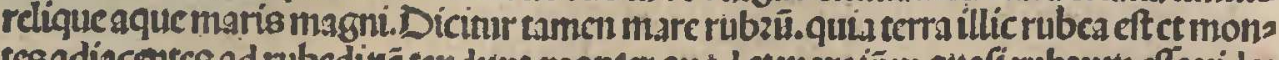

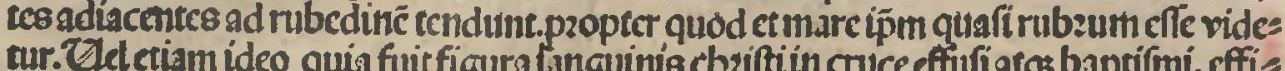

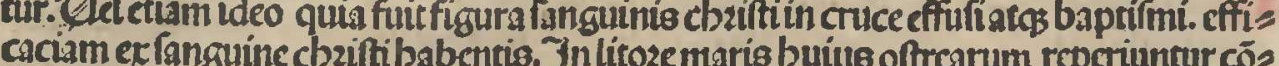

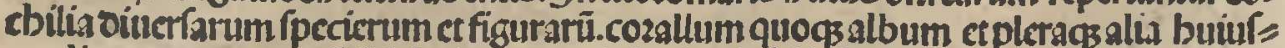
modigenera genmarni. J bi ctias in tharis butus intulis tignum crefcit robcum ad mo dumprefing. In politozecôtedentes in locunditate contred imus ct bibumus que babe (ricl (⿸丆口

Ane fequentis oicipecdentce perlinus maris renumus ad, montana rbi ma cegle lum. et ita ozicntem et occidensé colli

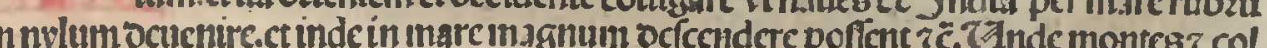
les petrasect rata pzo meatu marls taciendo infitt tantrodi.fed boc opte grande non

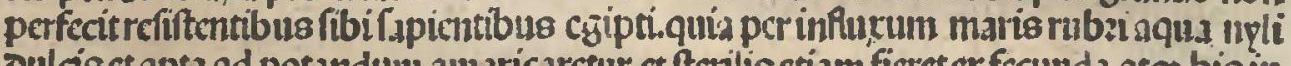

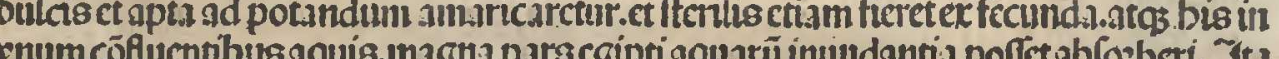
bisetaln̈ caufis ceptum negocím mufitunfectum.

(1) fiactis ligneis vafis noftris vacuis puta lagenisignes eretis accendimus.vi rel offam ynameoguctemus,

政 qquam reperimus.neqs tentosia ficere porceramus. piofa funumu claucs tenereno pofferes its fub oino dozmire opeztebar.

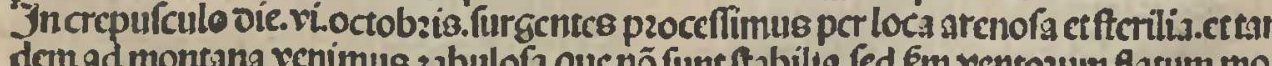

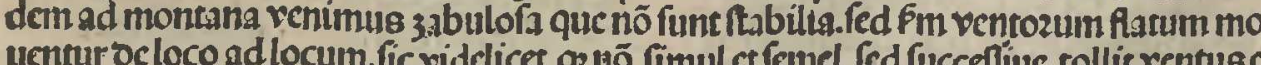

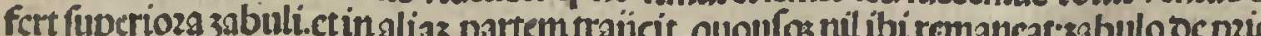

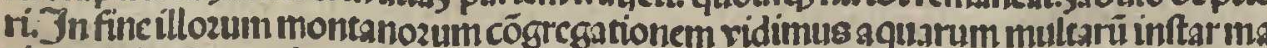

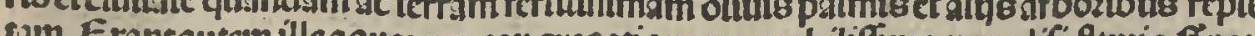
quinunc $\mathrm{F}$ lus

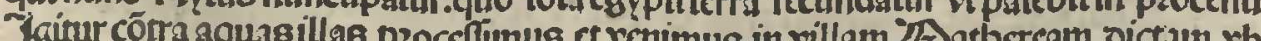

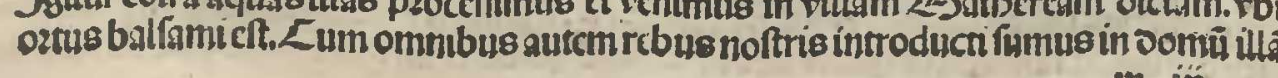

amrion

former Nor: 
In qua fons beare virginis emanat qui irrigat ottum ballami Domtnicidem gnnetum.

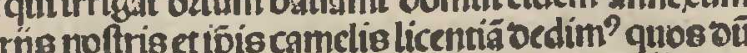

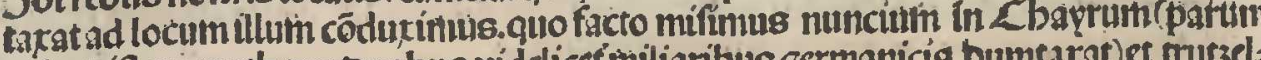

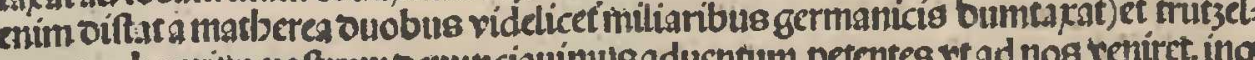

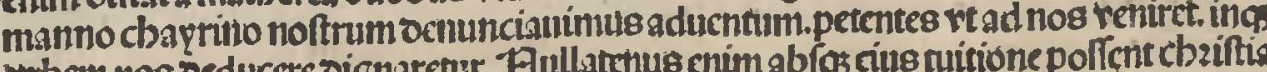
nT. cogduentu multra comisntus caterua clicntum ad nos venit rolens nos ftatim bab bylo

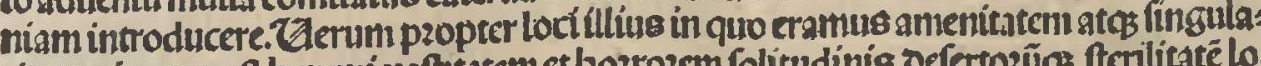

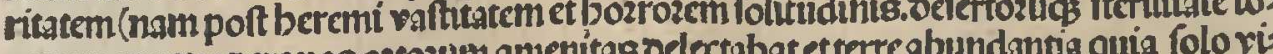

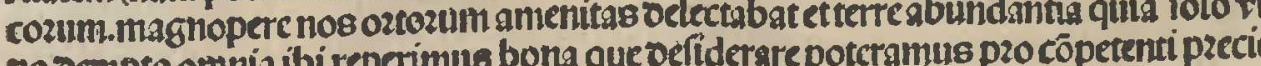
no damplo

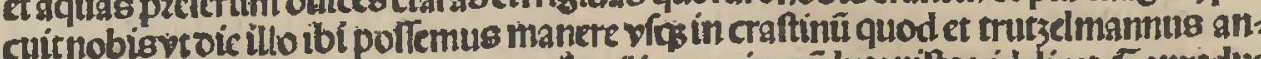

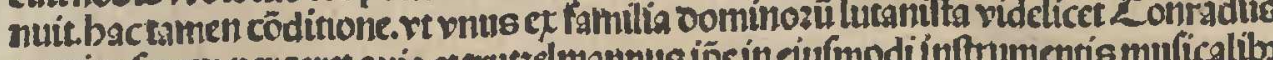
nomine fecum pergeter quia etrats erat pering. quam ad.cctain

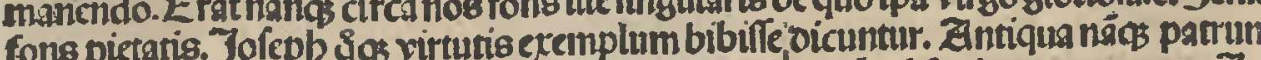

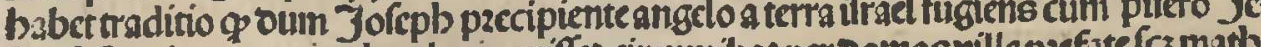

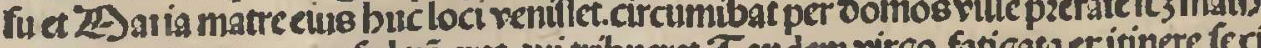

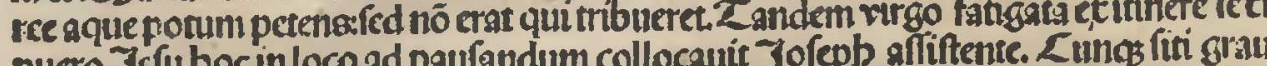
puero ctuarentrecce fons

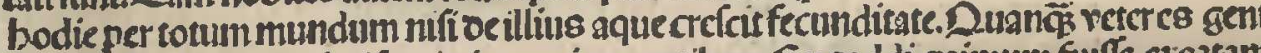

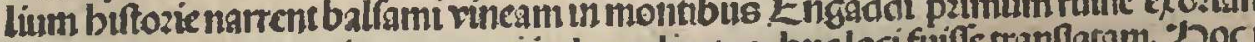

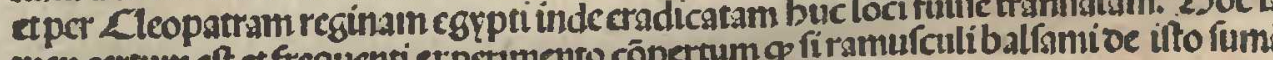

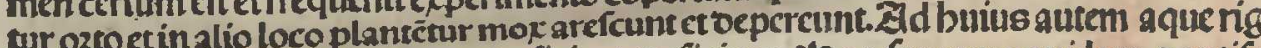

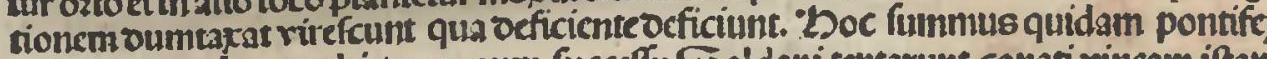

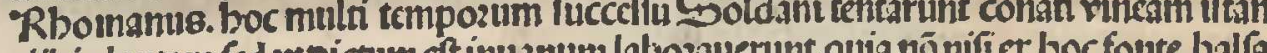
alibi plantare.ced rroictimn mun

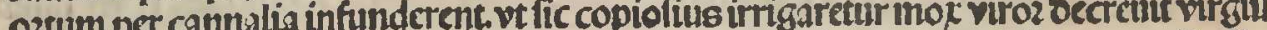

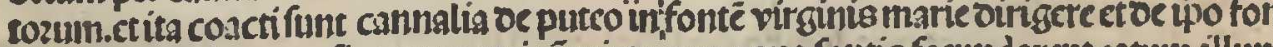

guvibs ct bodie irrigatur.

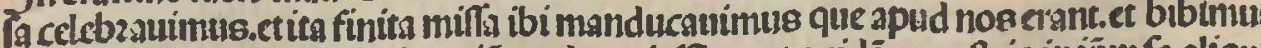

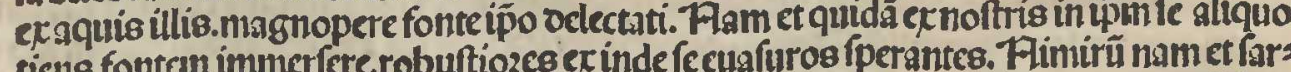

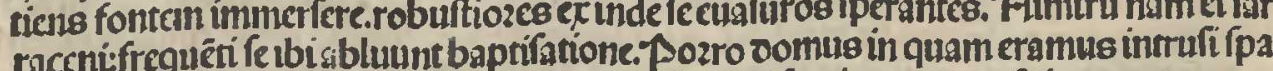

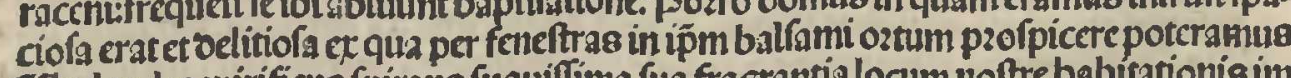

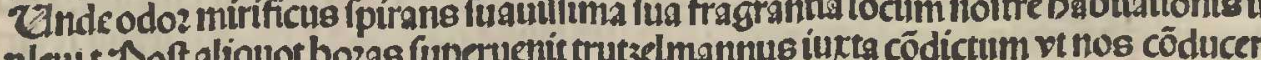
in Fab rlonem.quem nô modico oato guro crozatum fecimus.yt in oztum illum octe

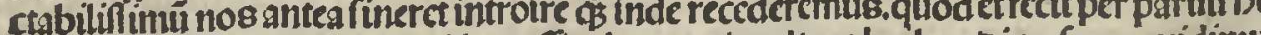

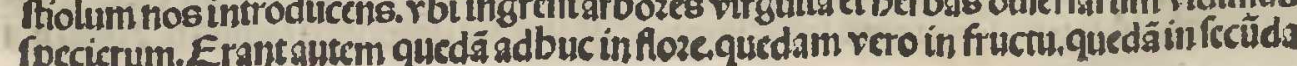

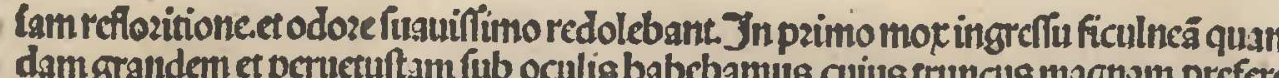

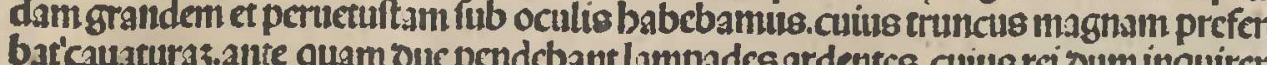

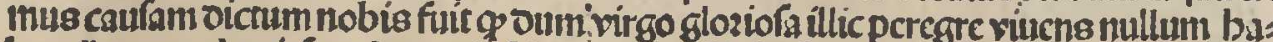
berefilocumad quicicendumet ipfacum puero Jefu fesd arbozem illant quictio gras a recer modocliffiumi fed to

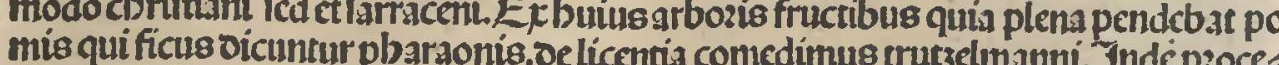
dentes ad aliam quandam nurabilen renimus arborem cuus folia rr. Juc

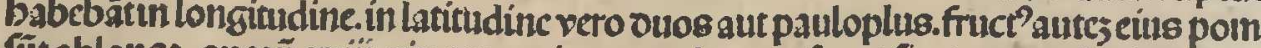

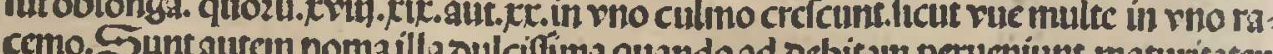

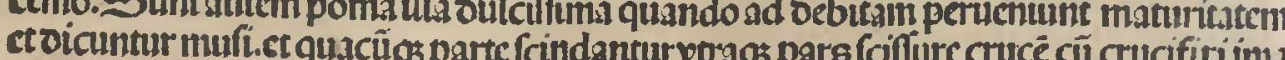
gine gerit impzeffam. Er fertur pzo certo o lignum picentic boni et malic cuius frucrie

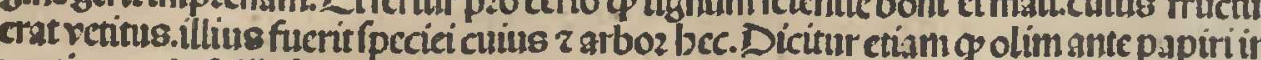

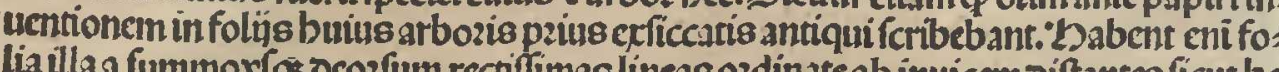

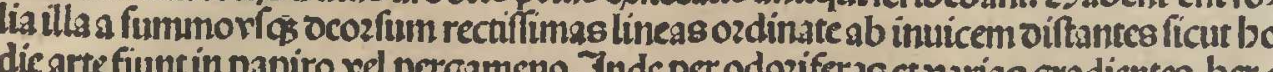

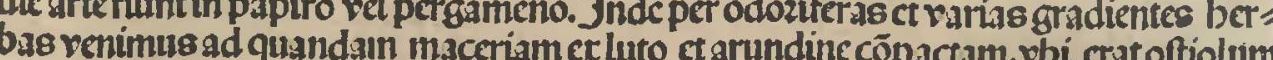
paruum Ied repagulis et feris ferteis finniter cloufum. Entequod cuftodes ftabant cum fuftibus ct lignis nobilifinmũ bal(ami oztum cuftodicntes et ob feruantes ne quis illumingredipoflit. 3 bi ixerum not1o dato argento cuftodibus boc nos o2dine intro

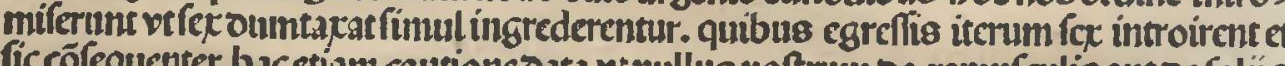

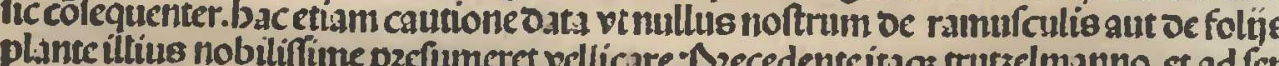
fum fingulis demonftranteridimus que longeante oc balfiamo legimus ct

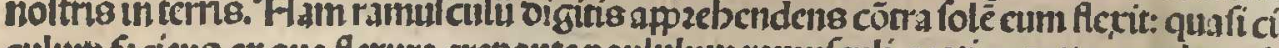

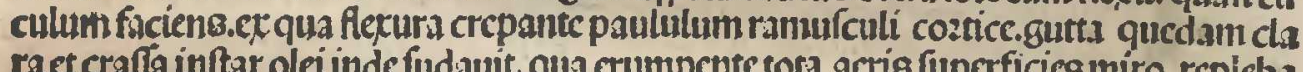

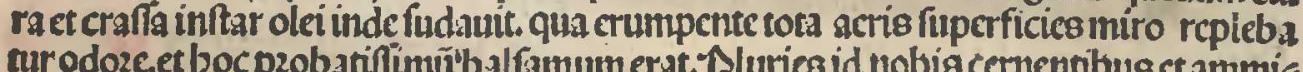
rantibus trutselmannu facical

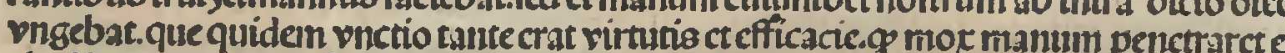
ab alia manus parte fentiretur. Folia criam balfami m..nibus pecfla et ramulfeuli oum

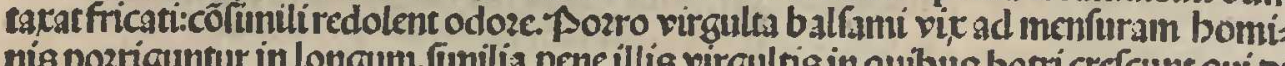
nis pozriguntur in longumn. frumlia pene illis virgultis in quibus botricrefeunt qui

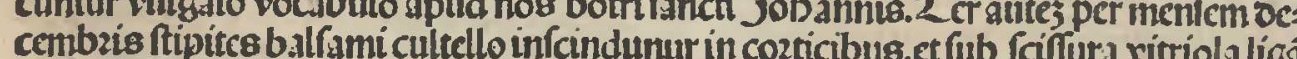
cur. in que guttatim oleumb ballami oe vulneref fillat et fuit.ct quod paimo indecolligis tur oomino oumtaxat Soldano releruatur.quis optimü ballaumum eft. oc quo fi quis

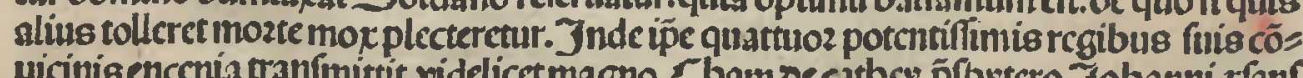

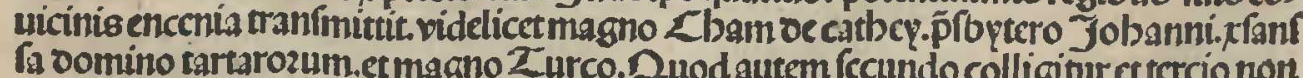

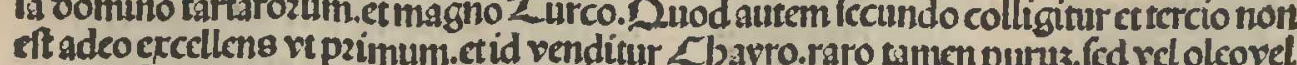

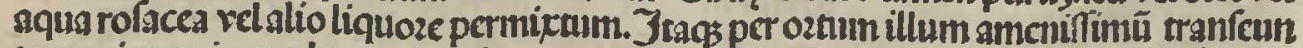
cs maiozem in modum oelectabamur varictare virgultozum.ct rapiebamur ex boc in quo vill aing

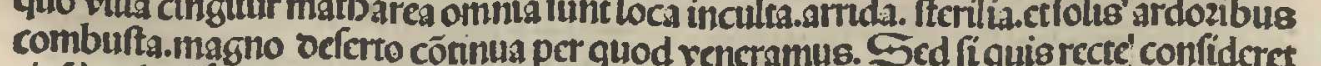

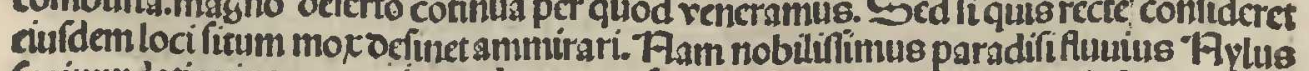
fueinundationis tempozein matbaream vf os contingitet totan cerram infundit. ctita thais phovenes mulif finctongin aimivafui" bat

Burishi: rectling formis zenor's bering ho As:ting 
eam reddiffecundam.nec oubinth quin fons beate virginis otumt irrigans ptedictum

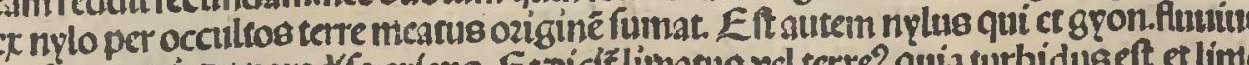

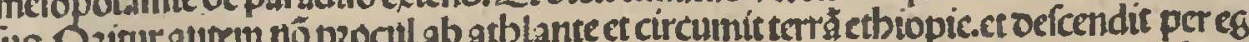
3. D.

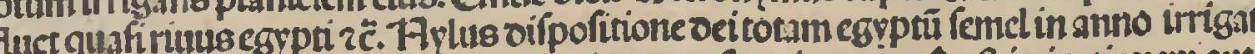

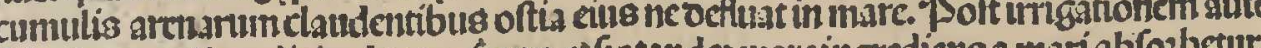

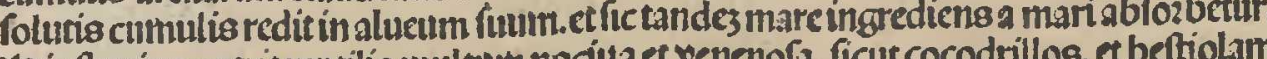

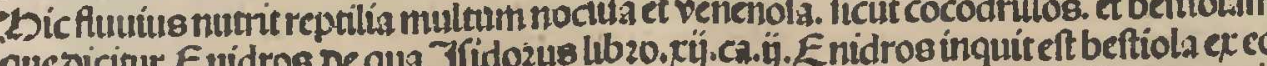
que cntem roluturfe in luto pzimo et intrat per 08 culls in rentrem et carpitomnia interioz

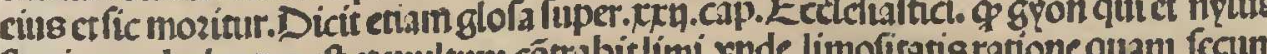

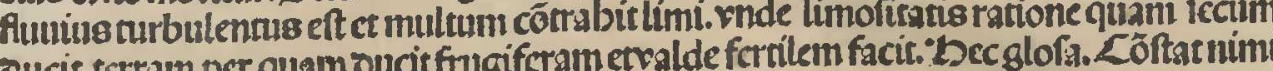

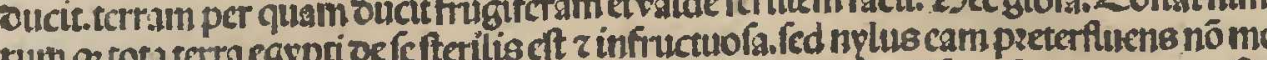
do

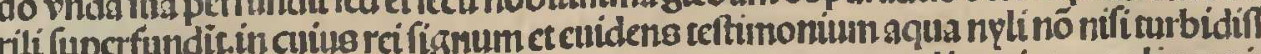

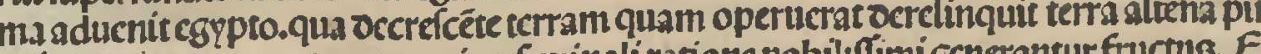

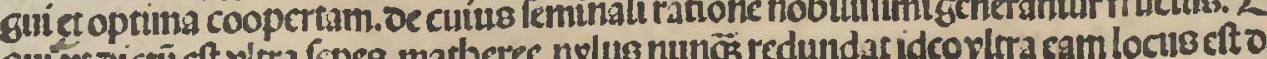

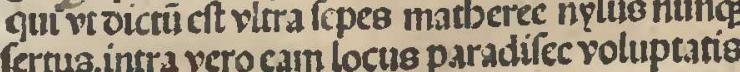

\section{Deingrefiuet 2 asbereain Lbayrum.}

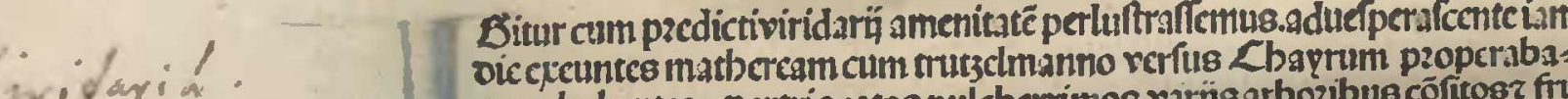
oicercuntes mathereamc cum trutzçmanno rertus Laynum pzopcraba cribuscumula

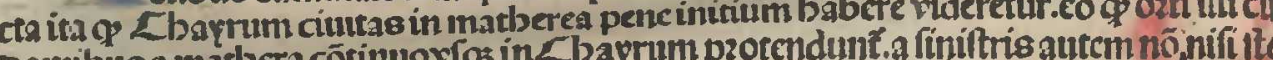

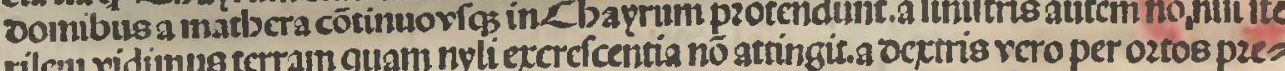
ritcill ridunus

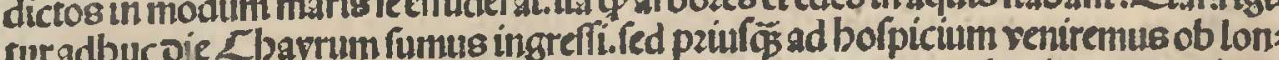

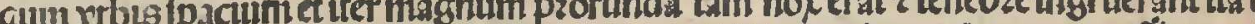

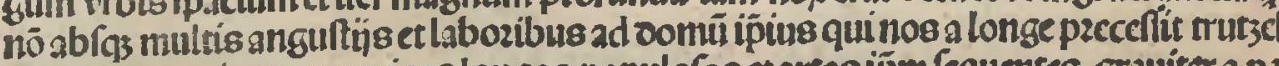

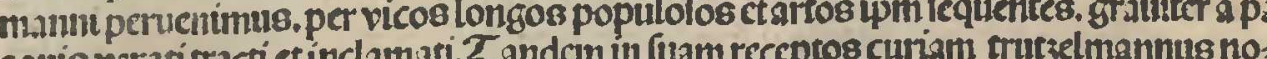
gantis recteidactiction noctine res noftras intulimus. Et quanös melius apud alios fuiffemus bolpitio fulfepti z tra

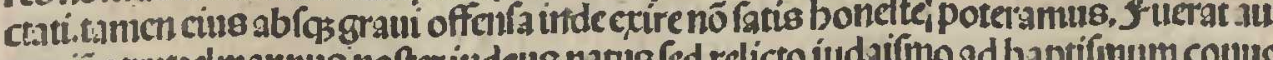

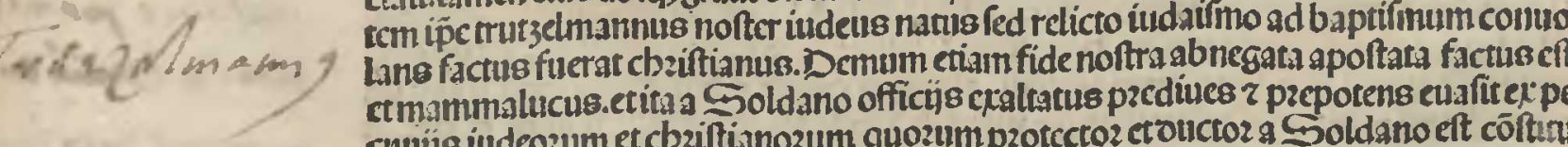

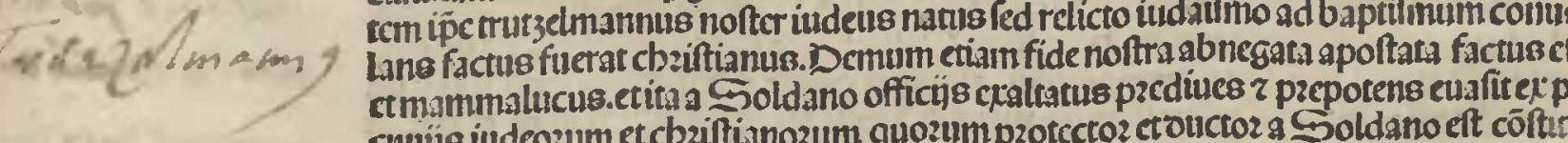
cuniug iudeozum et cbziftianozum quozum pzotcctozetouctoz a Soldanoeft cổt

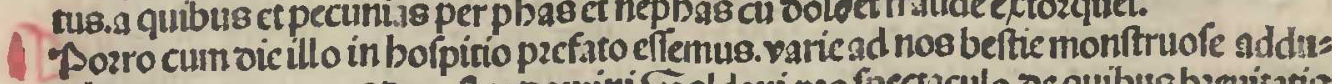
ccbantur per matroe de caftro oomini Soldani pzo fipectuculo.oc quibus bzenitat

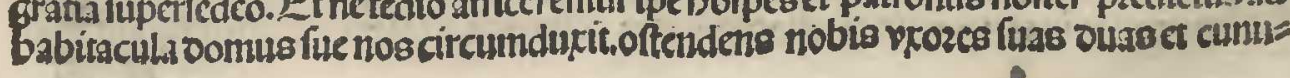

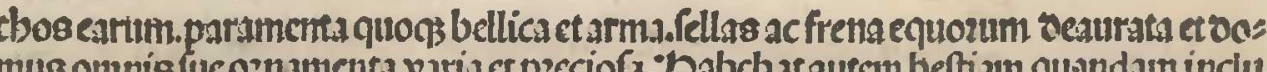

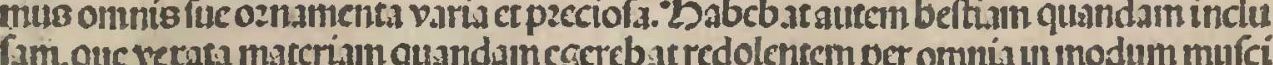

quenö renditurnific cquali pondere aturum remetiatur.

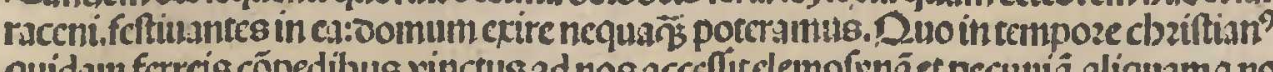
bis menticins of

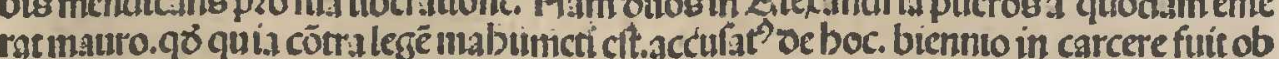

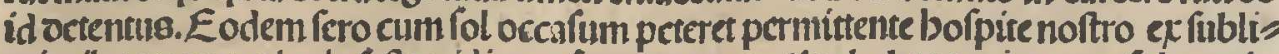

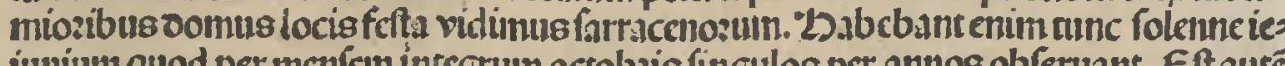
unium quod per menten integrum ocrobisis ingulos per annos obferuant. Eftauté

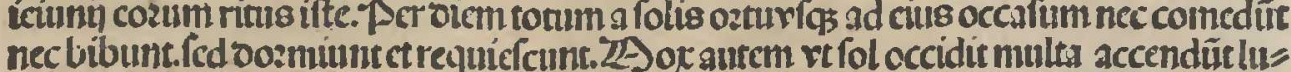
minaria in turribus mufcbk carum furẫ et clumozibuв ac vocibusaltrifonis fidei fure pronunciant profeflioné. quo facto incipunnt crapulls ct conclationibus fe replete.ct

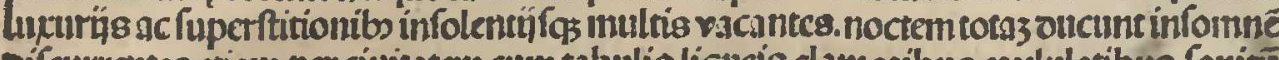
ginter

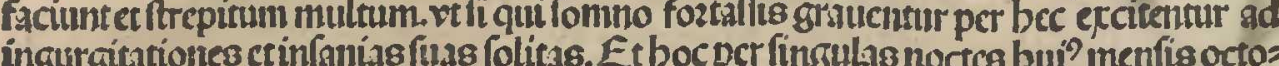

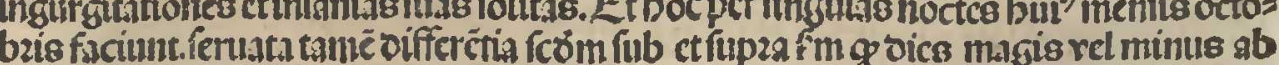
cis babentur folcmnes.quo enin feftum maius co nocturna oiffolutio maioz. 2) bec vis dentes anmmirabanur.pzecipuc oe tot mille eurnbus ct lampadibus innulneris quibs

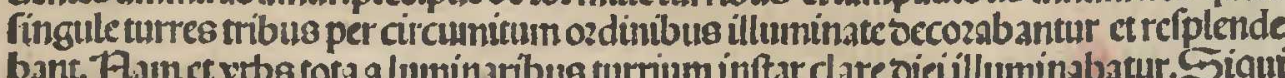
dem

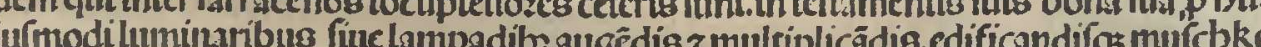

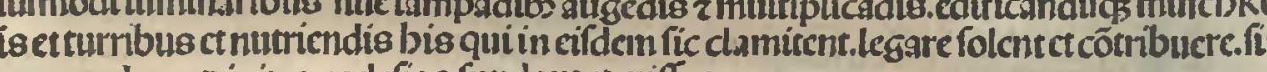

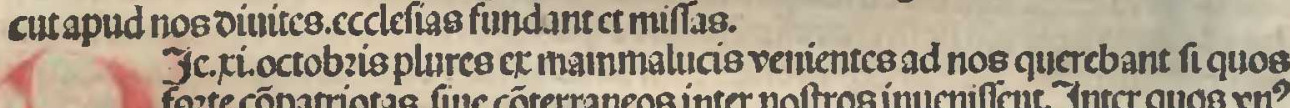
ount et perfonecgregie ad oominü Jobammem arcbidiaconú etcanonicume ecelefie Zranfí

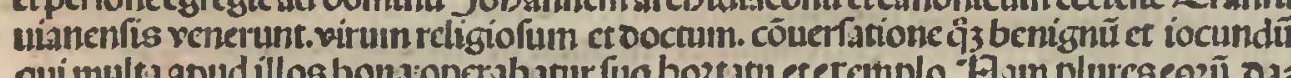

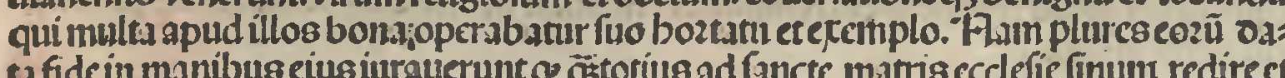

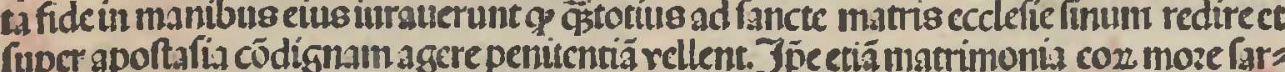

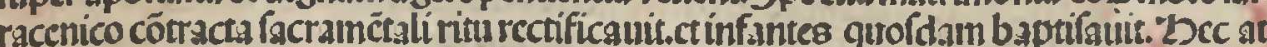

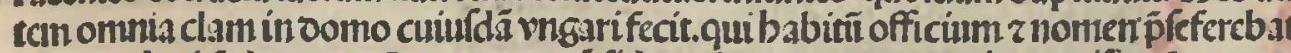

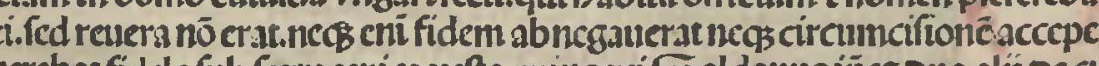

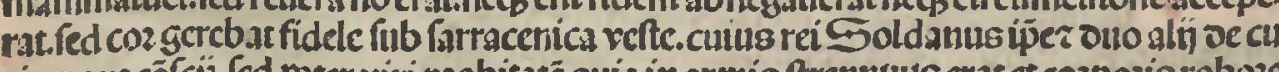

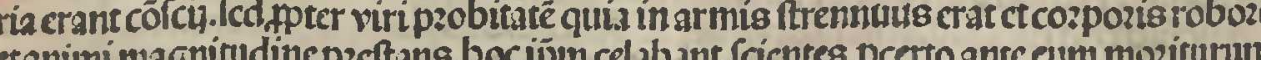

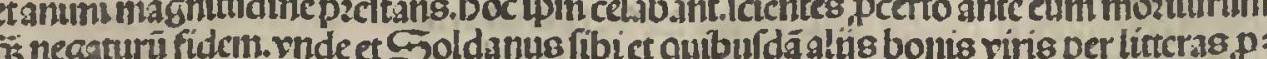

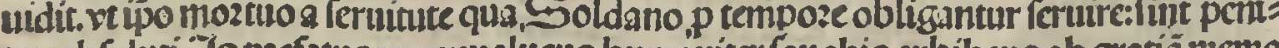
tus abfoluni. Is pecfatus inamumalucus bumaniter fe nobis crbibens ob gratiä memo

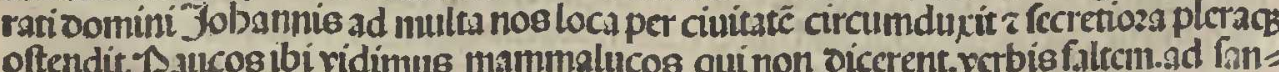

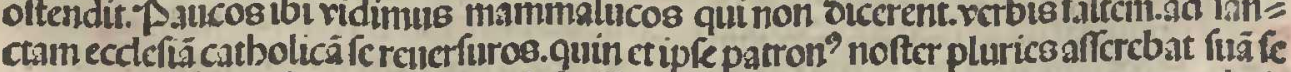

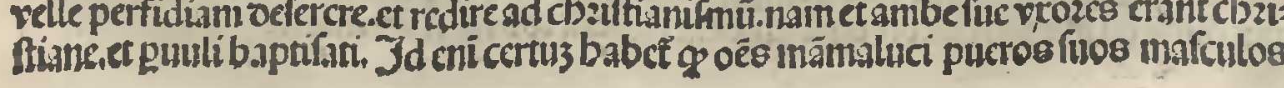


faciunt baptifarinob obid quidem (multozum opinione) vt puerifalutem cöfequantur

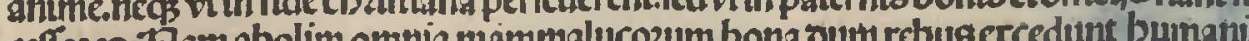
Cold

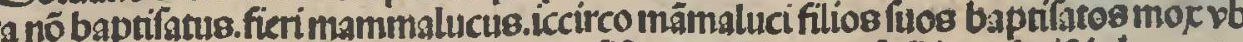

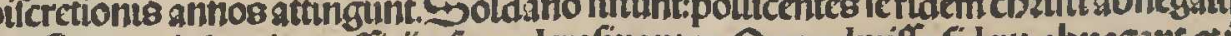

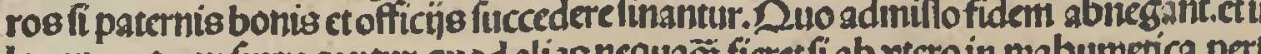
locum patrum turtrogantur.quod allas nequags ficretti ab vtcro in mabumetica per

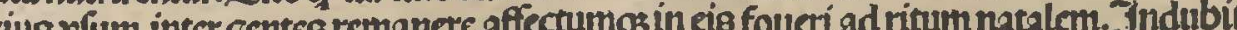

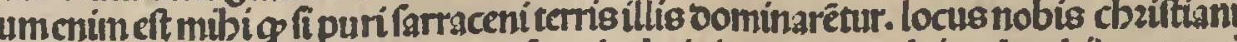

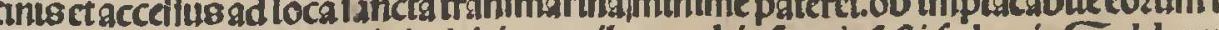
108 odium et incrozabiles inimicicias.quibus nobis lunt tinfeftitied quia Soldanu um funt manmaluci. pzoregic murab cis.

3exü.octobris andita miffa (HAam Dominica vicclima poft octauas fancte

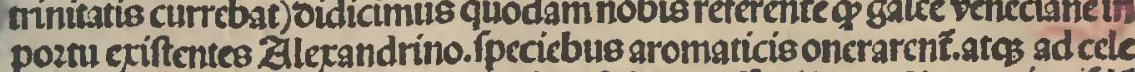
con pararentur receffum quo ecrumoze plurima fuimus affecti iocunditate. galeis repatriare fperabamus. Eodem oic poft cuinfdam adductebeftiequam copin galchrepsture

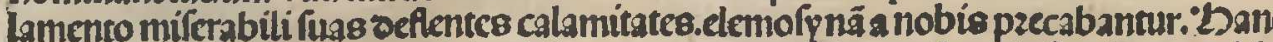
enim fartraceni oilcretionem fuls obleruant cum captuis.

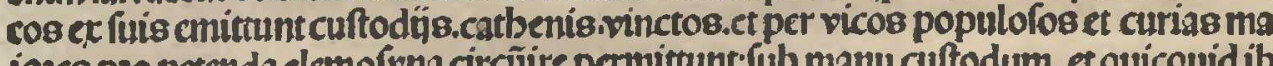

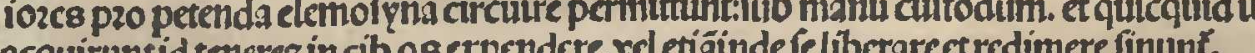

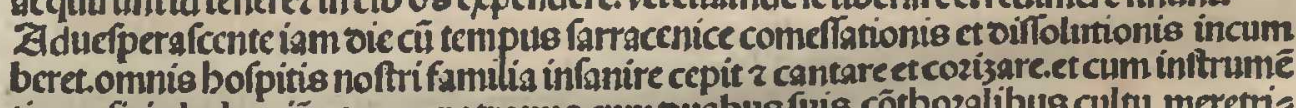
rismufficis ludere.ipe queos patronus cum ouabus fuis côtbozalibus cultu meretrit runt.verifis ctleticie materiam et caufain acciperentex nobis.vndeethoctem illamm buimus opido inquictam proptcr clamozes et infanias (arracenozun cum ftrepittuc umpectu per vicos et platcas currentium et blulantia in turribus. vtillo in tempozemo riseft fui vfitati.

- Jextï.fumpto pzandio in partes nos oinidentes.tres ant quattuoz fumul \& non plares. vicos intraulumus mercatozum. vbi tantram vidimus bominum in anno iubileo et tot penditozes etemptozes prifu mirü:et pene incredibile

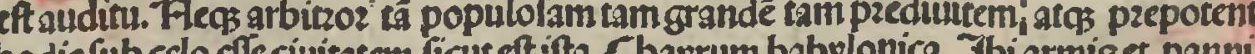

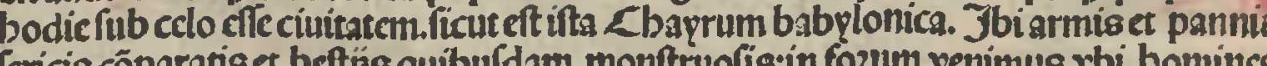
cricis coparatis ct bealio am renditionemexpectabant. Lunos mammalucus quiouctor nofter erar bincinde noscircumduceret. venit metcatoz quidam inquirens ab eo quant pzeç feblauíllief

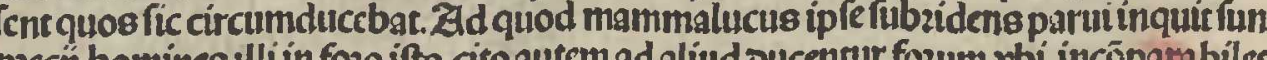

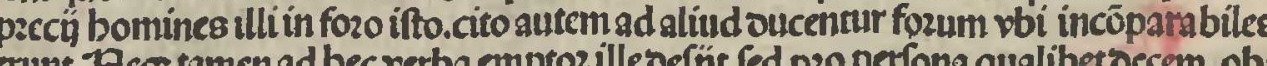
tulitoucatos mammaluco:oonec tandem cóperit cos nō ef́e venales.
Jevero.rilif. fummmo dilnculo venientce ono mammalucia patrono noftro (a) (a) am ferteam pozram.venimus ad populofífimas plateas:vbi poft nulles oerifionce

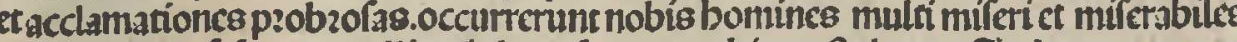

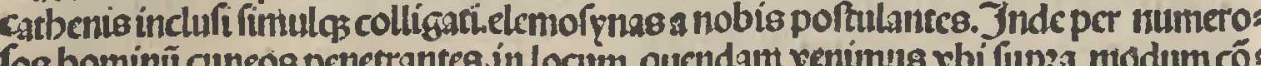

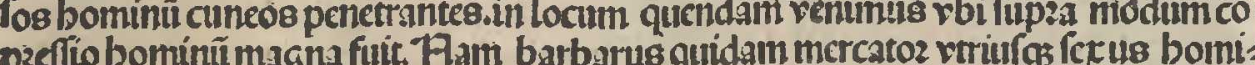
preplurion pro illis emendis tants côfluxerat bominû multiutudo. Inde pulcb3a quadam platece

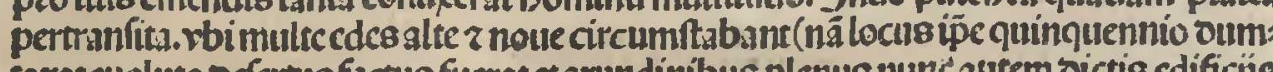

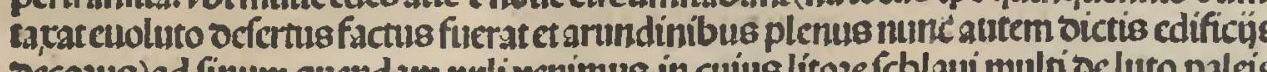
Decozug)ad finum quendam nnli venimus in cuius lítoze icblaui multi oc luto palces

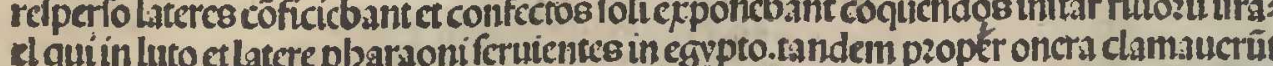
政

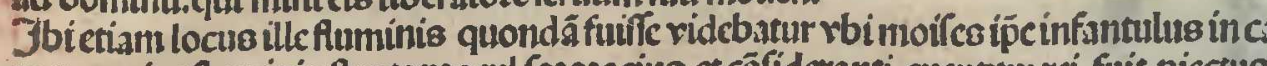

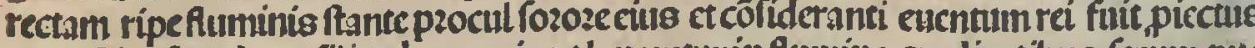

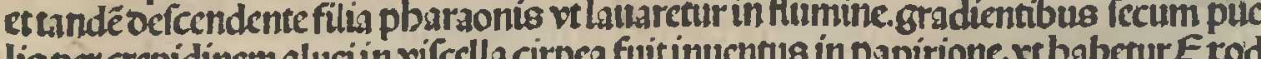
ig - bi me Indead maceriam ozt cuuldam alcendumus vbi quond am arboz ingens ftabat fub

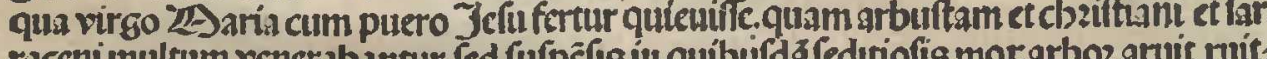

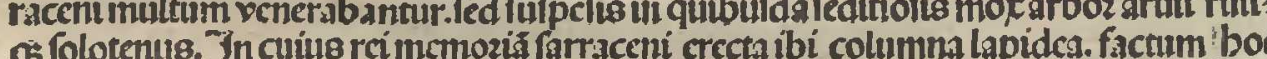

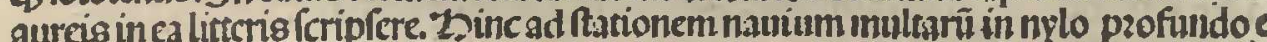
lato venicntcs, maximácirca lllas vidimusenegociatione,

De'Babỵlonia egypti.

Andem per quafdam rectuftifimas'ct ipiffas valde bozribilefos po:tas in

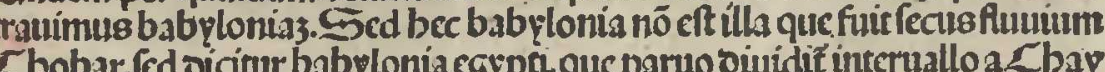
Cbobar.fed oicitur babylonia egypti. quc paruo oinidiliz interualloa $\mathcal{C}$ baq

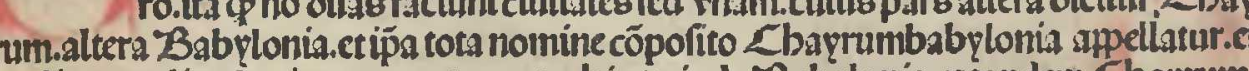

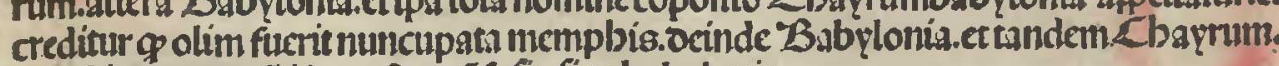
quod interpzetarũ idem eft $Q$ côfufito fitte baby lonia. In bac babplonia ancetctumpoza Pbarao rex egipti mozabatur in íngenti palatio.q

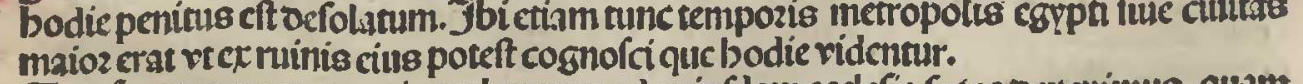

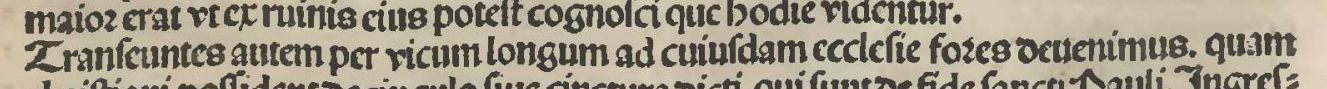

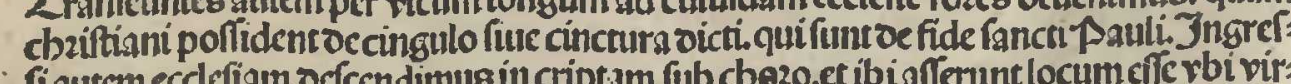

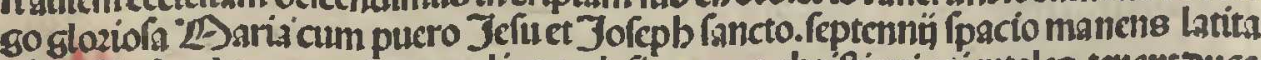
uit. Indead quartuoz lencas alias ecclefias quas chziftiani ozientalcs renenrouce bamur.in quibus aliqua fanctozum cozpoza requicfcunt.

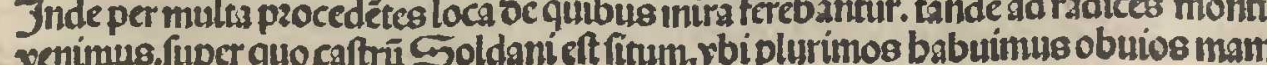




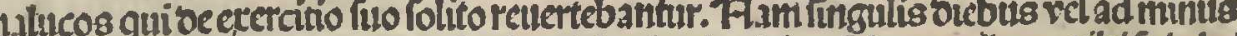

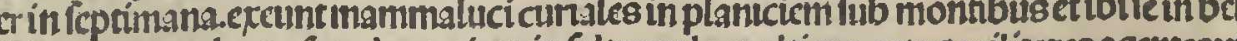
jo modo in Cich

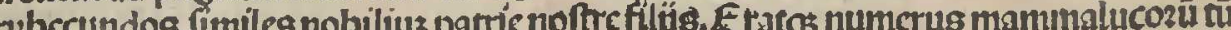

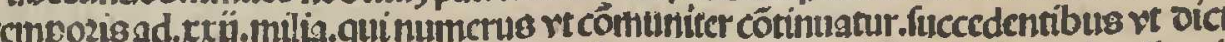

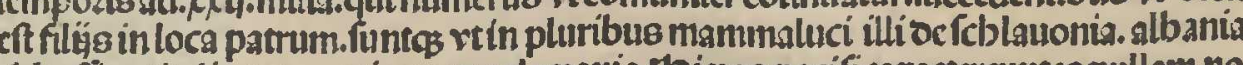
is molctiaminaulcrunt.

Decôtemplatione Cbay̨riexmonte.

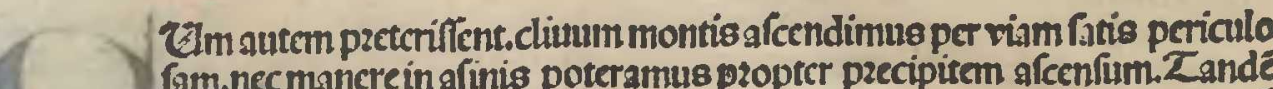
m.nec mancre in alinis poreramus propter preceptrem alcenfirm. Zand idergndame en uidam enin qui id certum babebantaffercbanteam in latitudine ouodecim mi

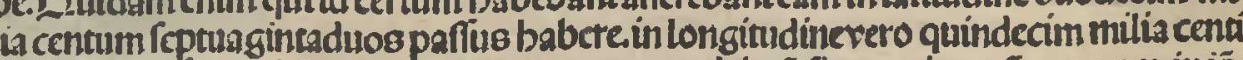

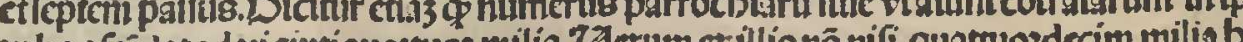

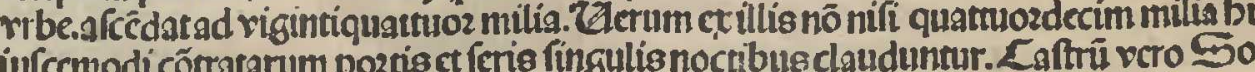
dice

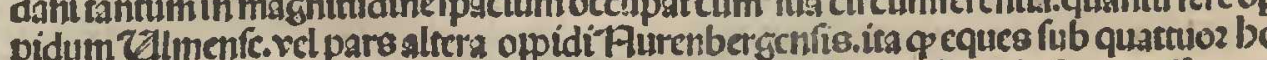

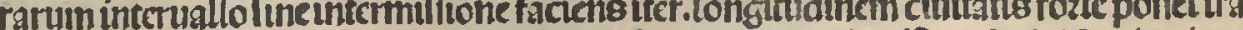

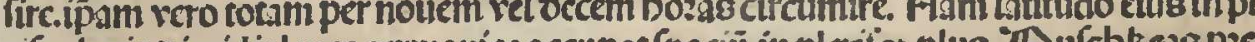

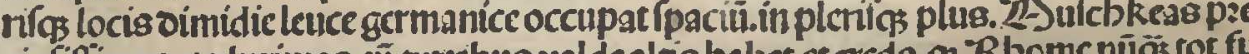

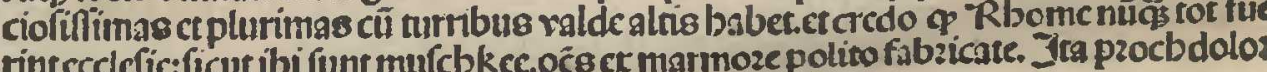
ratbanas fuas ibi fynagogas amplantrec mulriplicauti.

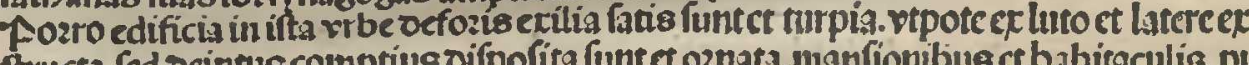

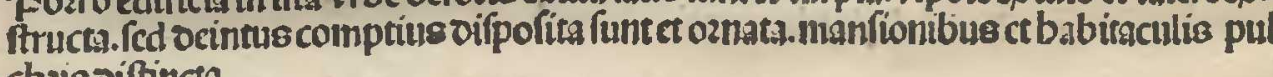
cbris oiftinct

2licisisa

5000

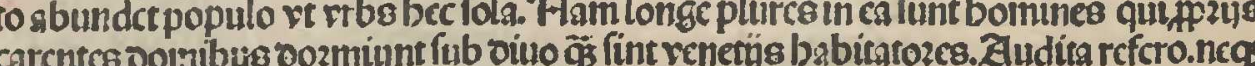

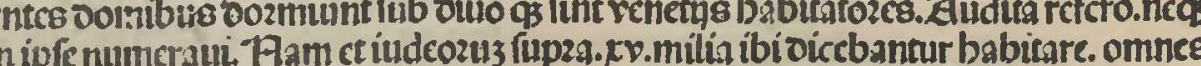

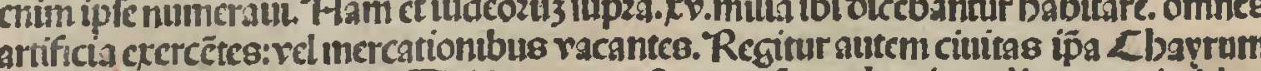
es admuniftratur per regem Soldanum. poft quem fecundus in ordine ammiraldus fcmper alpellatur.poft illum rero tercius oindarus.ct bitres toti pzefint paganifimo.

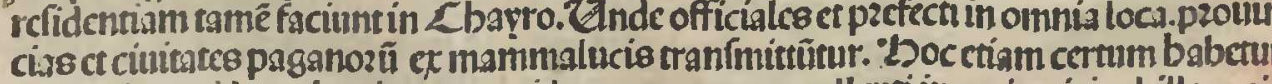
q abolim quidam ab osicnte ctoccidente magno etcxcellentri ingenio viniad illam ci uitarcm tpptalncre. Wi

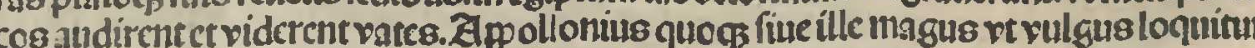
fiuc pbilofopbus vt pitagozicitradut.poft lultrang sandcm carptumn intraunt trt famofiftima folis menfam ridcretin 3 abulo que in Lbape * Ectediur intemplo folis thethe

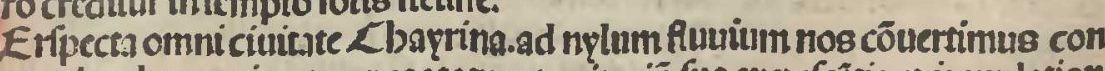

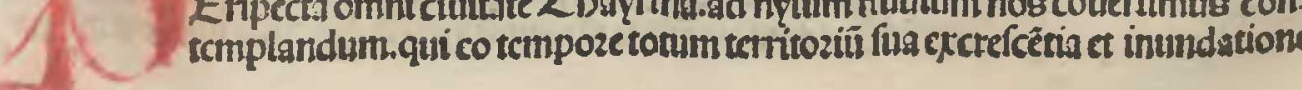

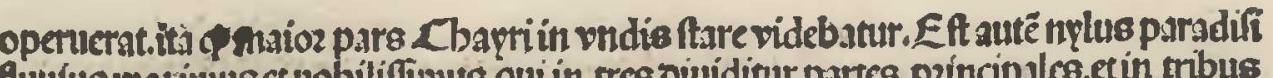

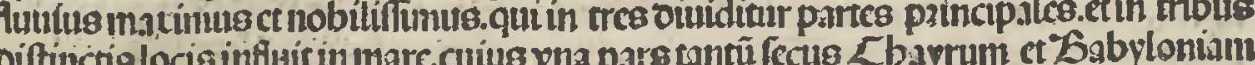

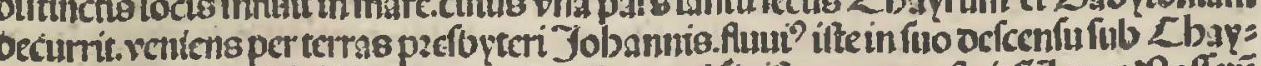

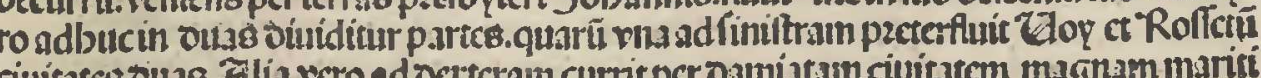

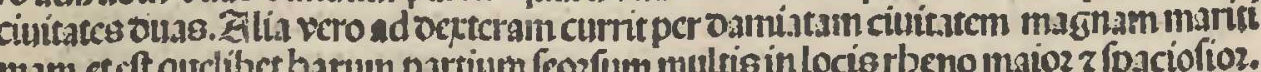
mank

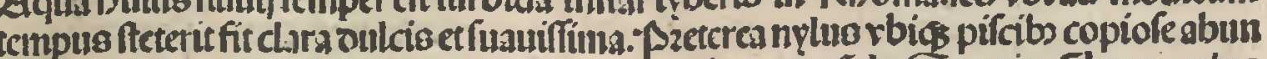
dar.ncc creditur in boc parcm babeataquam in toto múdo. Sunt in Clayro plus

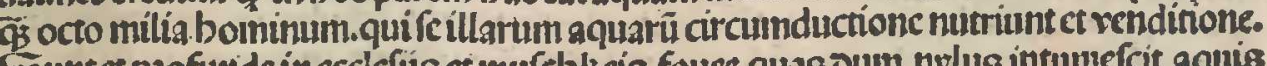

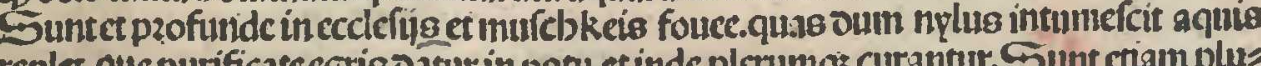

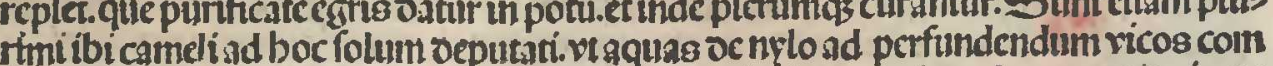
poztent.ne pultuis ex frequienti bominum ocambulatione numum elcuetur.ct ita impe

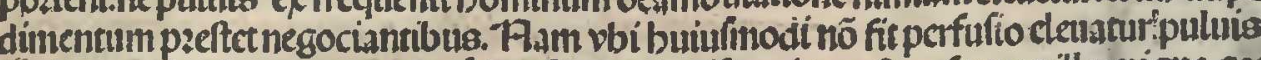

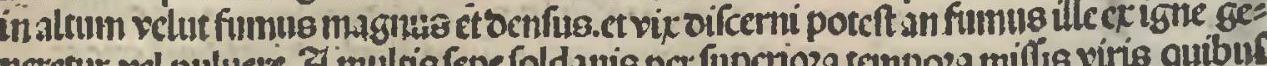

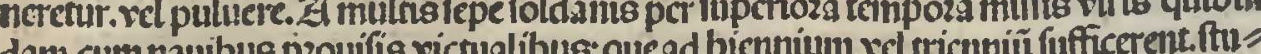

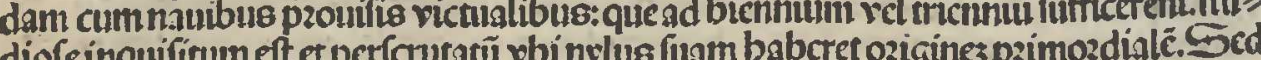
qui miffi fucrant poff ctactum bichnium rel mienniunn cum etism viltra indiam longc

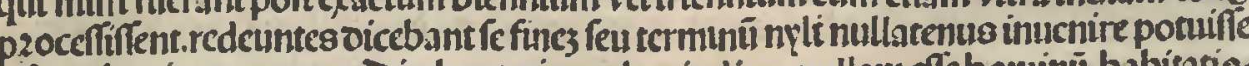

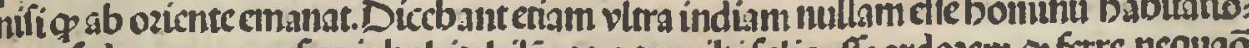
nem. portitctic

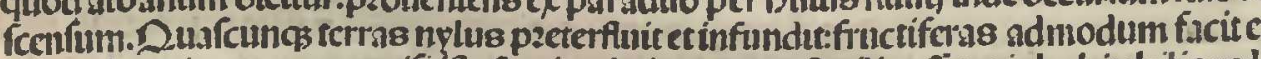

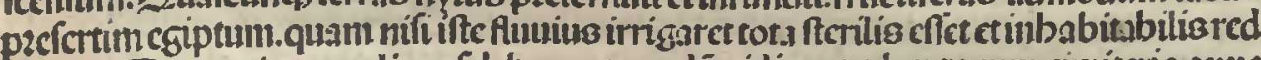

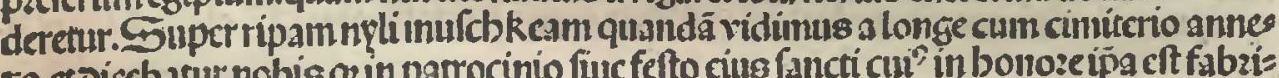
ro.ctolccbatur nobls

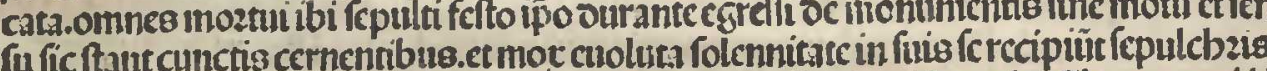
et boc fingulis annis fieti alcrebanc.nec eft adultus aliquis aicbantin Chayro quiid

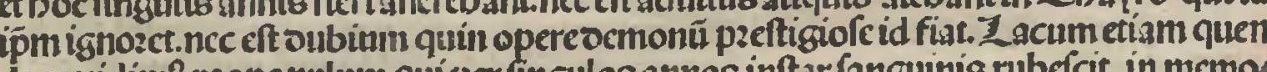

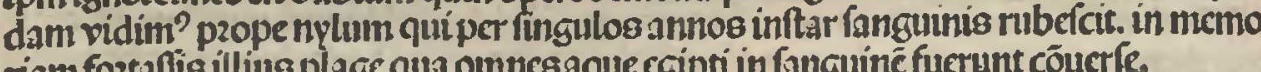
riam foztafits fupz in villa inarbarca. Flam quinos oumtarat leucis isalicis ouftart a cbayro vel pau

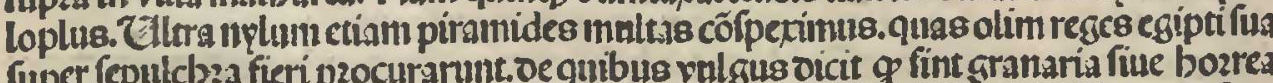

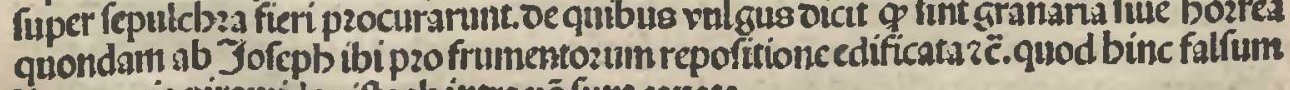

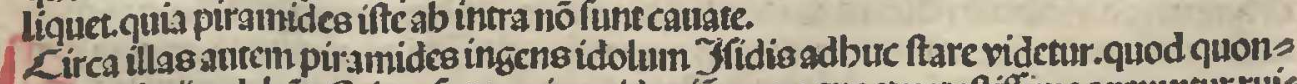

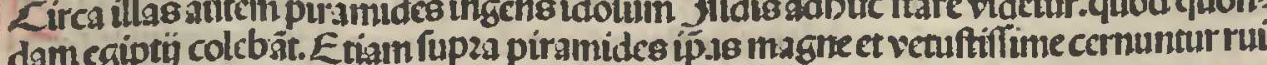
nc.rbi quondam illa porchnaffuma z nobiliffuma ciutras egiptiftetit.que centum poztas

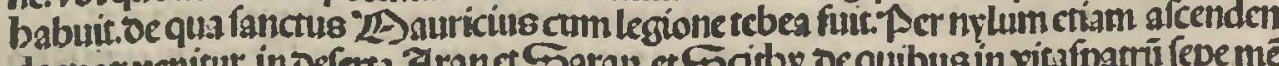

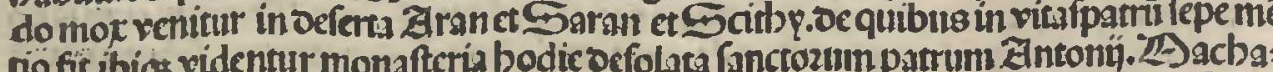

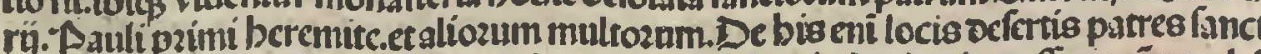

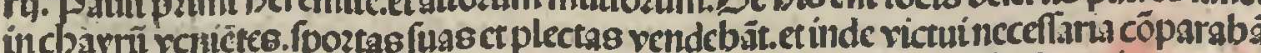
Jllisin locie fapertozibs annis multomonacbigrecimotabant.quicalogericommun 


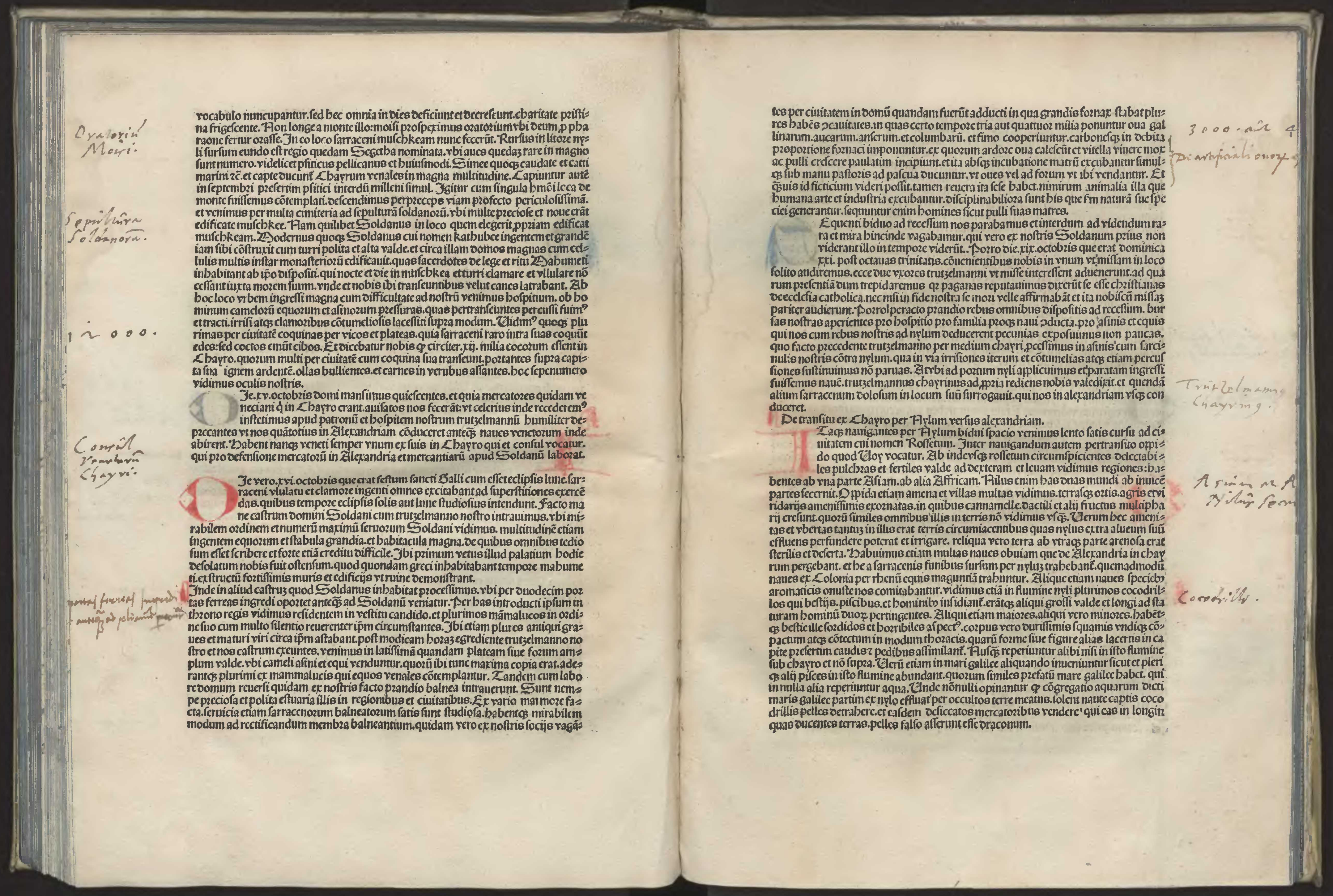




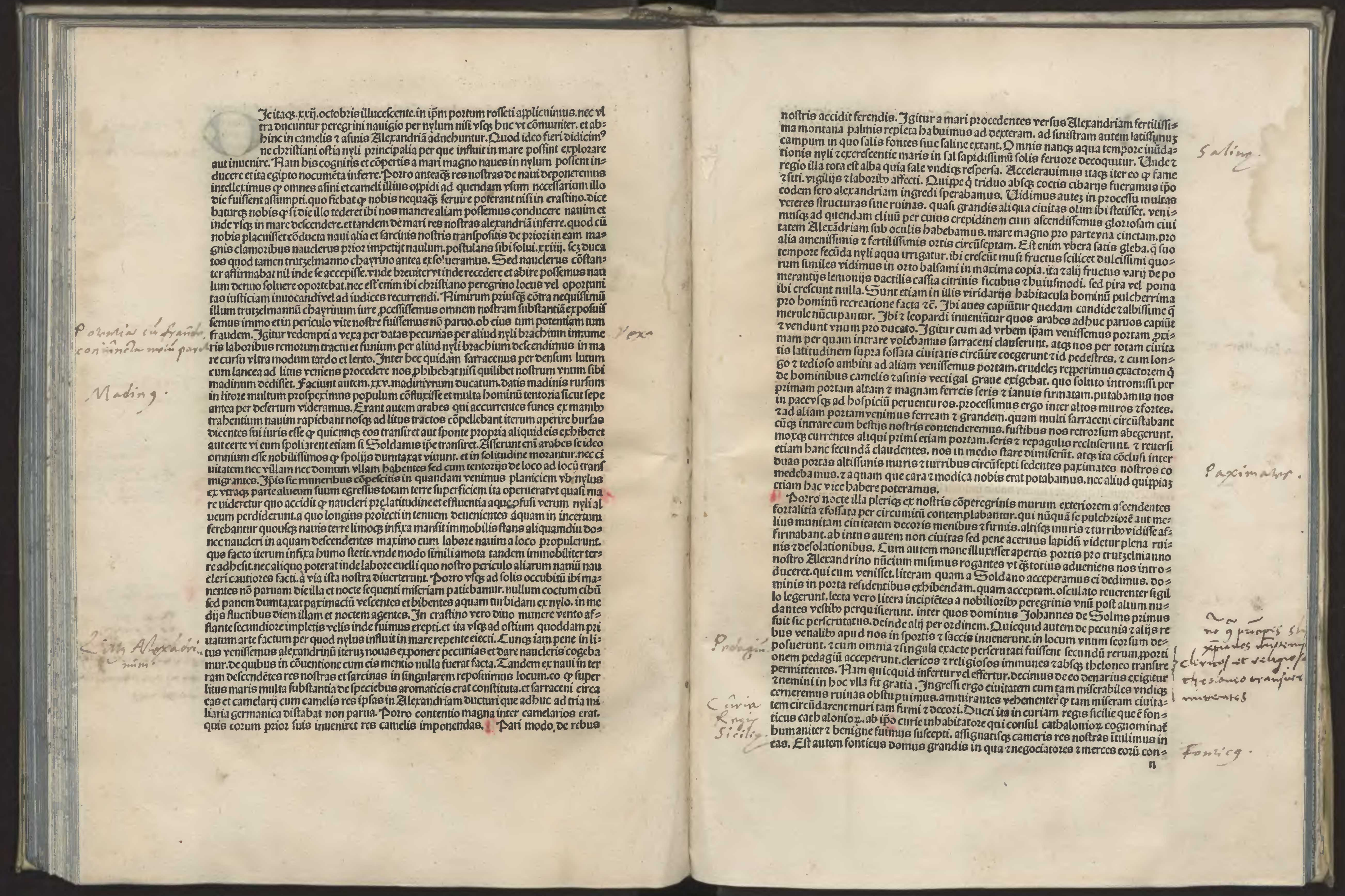


feruanturvbiz fozum rerum venthalum babẻt. Duăuls enim zveneti in alecrandria Onos babent fonticos zianuenfes vnum. peregrini tamen abolim in fontico carbs lonioz le recipereconfucucrit. manno zlectandruno lub

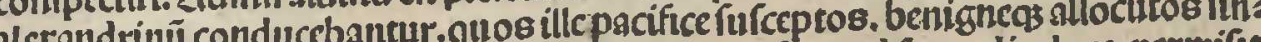
guloz nomina crquirens, in carclba tuffit cốfribi. ficę ad fuaredircloca pcrmifte. Dozro quidam ex nof tris cópercgrinis facta contsentione cum confule carbalo nicnfi.cömenfales cius fuerunt.cibo zportu perparco vtentce apud cundem.quit ta

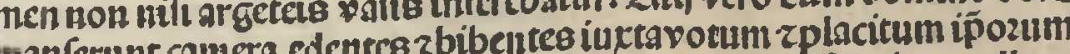

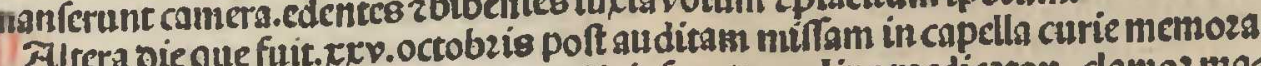
Bltcra bie que fuit. $x$ rev. octobrem religiofum be ozdine predicatoy. damoz mas

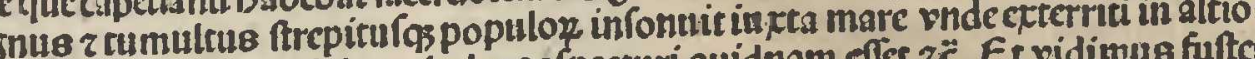

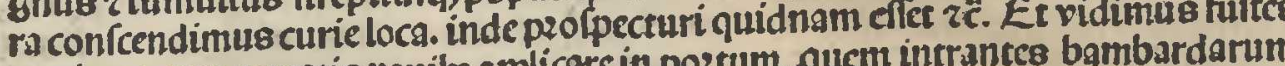

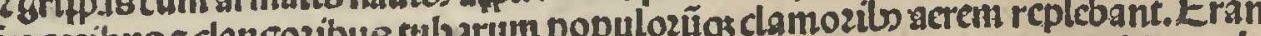

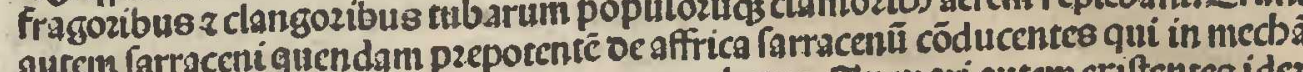
cepulchrum mabumeci vificacurus peregrinabatur. In mari autemecriltentes ides

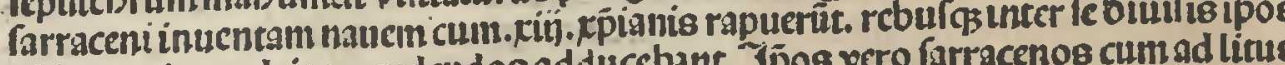
eptanos a leckandriantendendos ad applicuificnt maimaluci alctandrint zcagna ac multis clamozibus ciuitarć introdurc

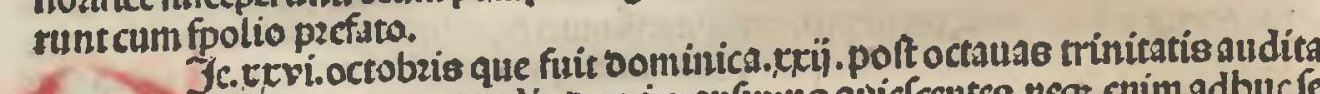

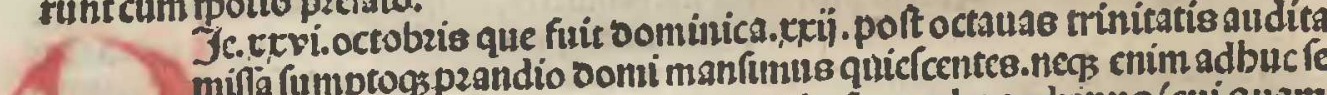
cure tutegserire potcramus. eo 9 truts clmänus alctrandris

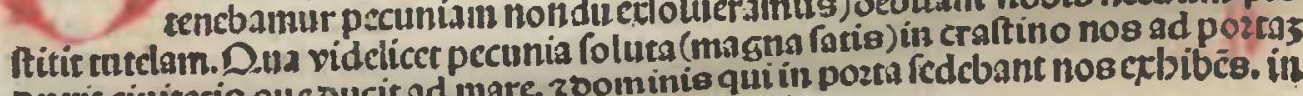

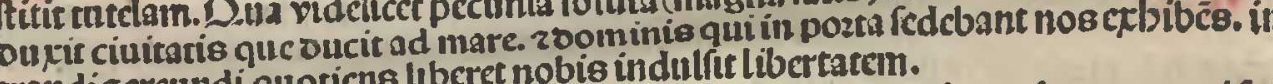
trandi zercundi quoticns ltberet nobis indule poft biuina celebzata. in cum oucti fui Gequenti oic que futit Try

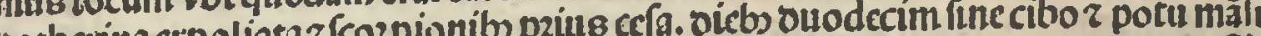

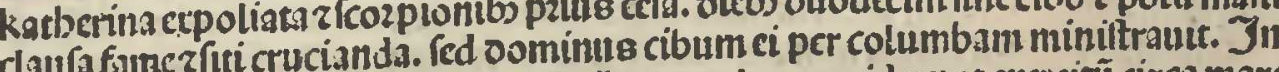

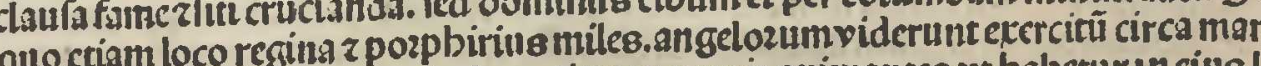

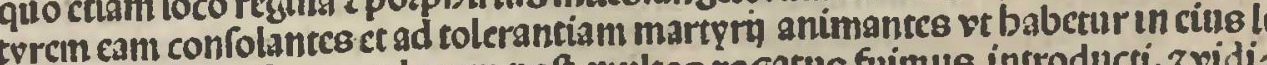

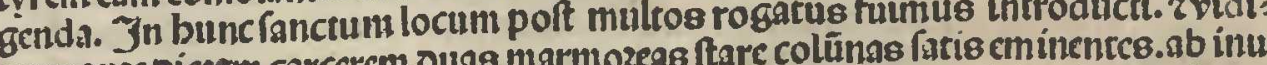
alts ante oictam carca cem paffibub. $x y$. quafi quatuozmilia Gearilitin inde necati. Eodem ectam inl loco quinquagintait

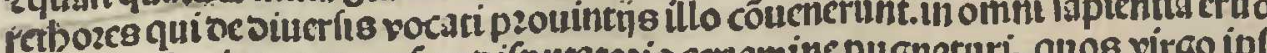
ti cuin kathertina cozam celaresilputar

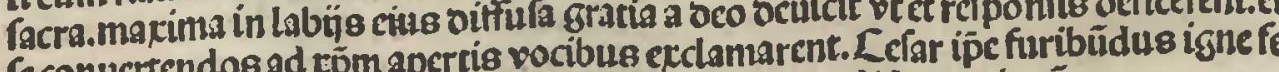
Ic conncrtendo vefibo cozum z capillisillefis multi credideruns in $x$ p̆m.

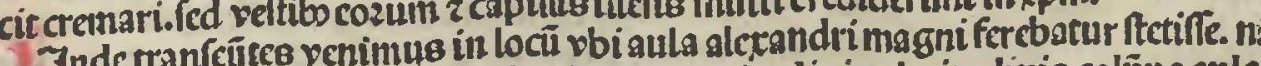

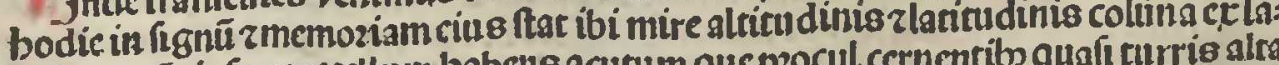

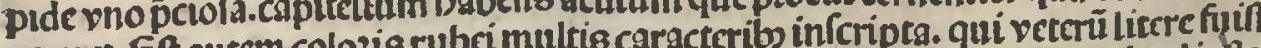
aparct. Ent a

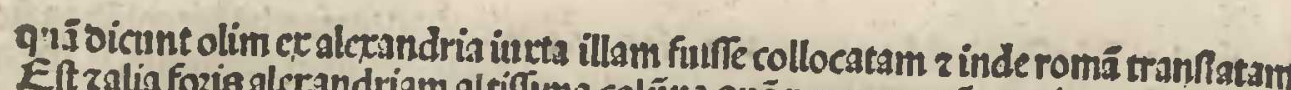
pompeius in fini memoziam fieri fccit colünt quäpompeyanā nominant. quia cam

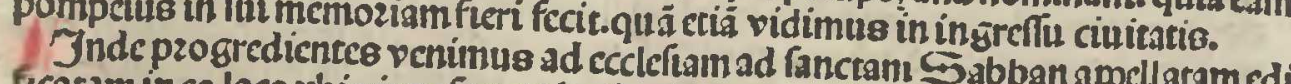
ficaramin co loco vbi virso fancte katbram ad anctam Sabban apellaramed batur regali.familiä fuan oefuncto patre Lofto rege cuivnica crat gubernans.bä́
ecclefiam 'greci monacbi inbabitant.

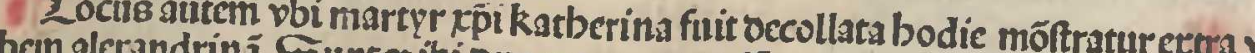
dam erecte fuito.

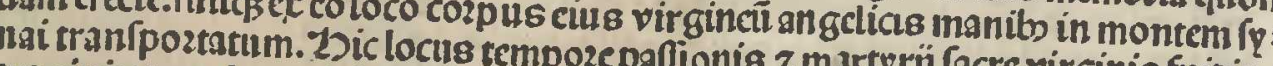

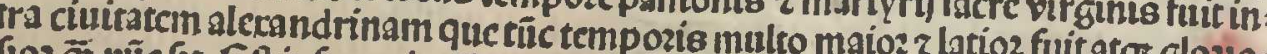
foz

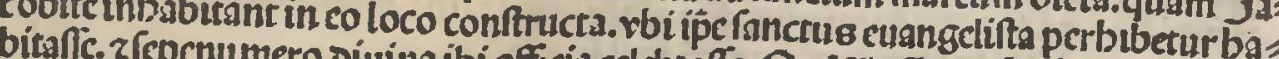

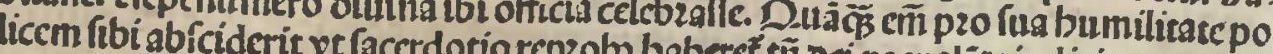

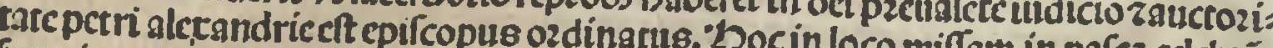

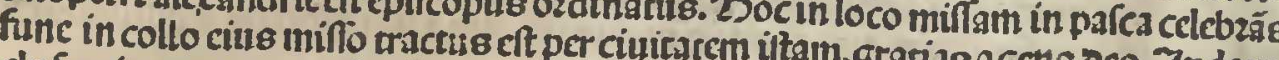
clufus in carcerem a $x$ po ibu zangetis fibiapparent tbo confoze agens oco. Jndere o mine comendo

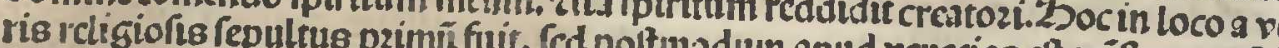

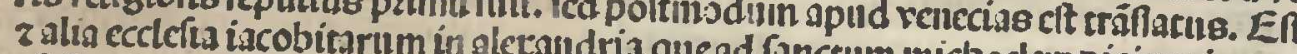

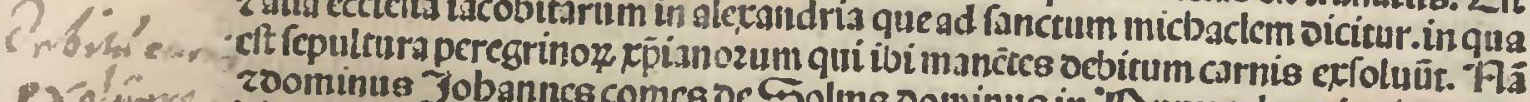

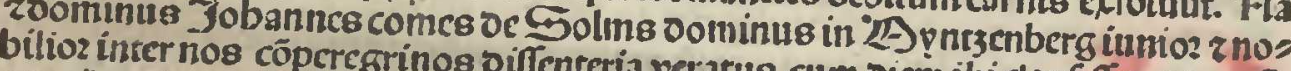

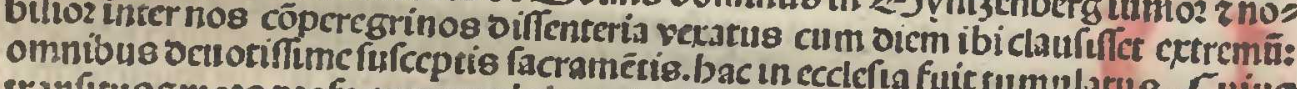
Iranfitus zmo:s pzofecto merozizinctui nobis multo fuit.

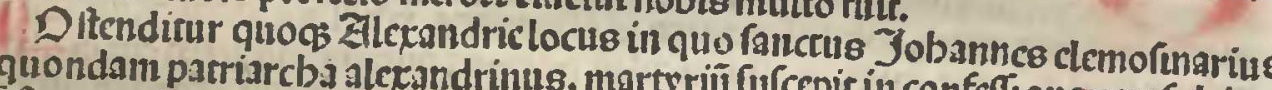
iftens. vt in culs legenda babetur. Sunt pzeteres in quatu In alexindria. pulcbrequedam zoznate capelleccapcllanos babentes

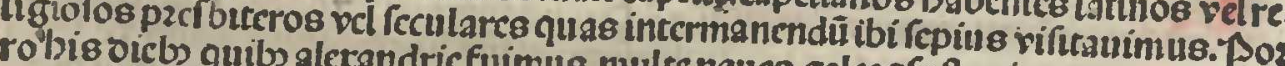
crunt. multeç ficbant acpsedationes. Flam veneri galec zfultcs in pozcum applict litanam que alexandrum properabat fooliaucrunc.co

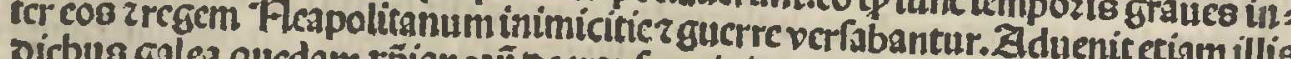
crant quizoircrunt nil slind in oina tranfmarinis partibus in qua alemani quidam

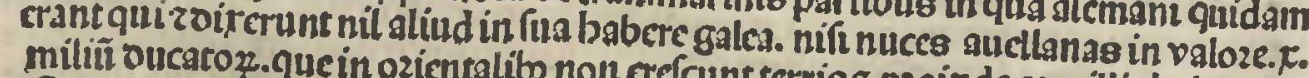
Fertures o vitra centumes a

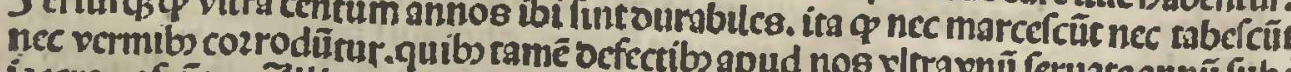

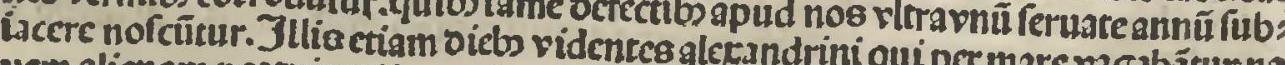

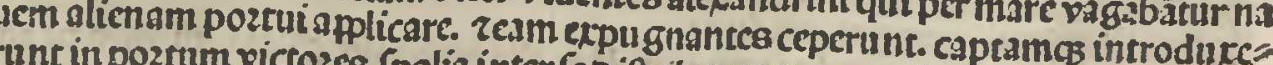
alecanidriam amlicarce

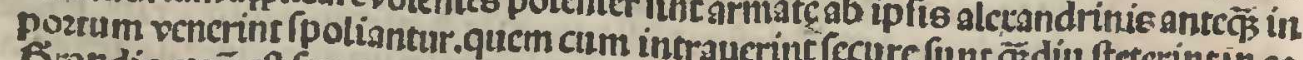
Crandis ctement larracentis cura pzo cuftodia poztusillius yt riscontem viderint Amiraldo oeñ quirtraducntantiü. Et
Colainne. 


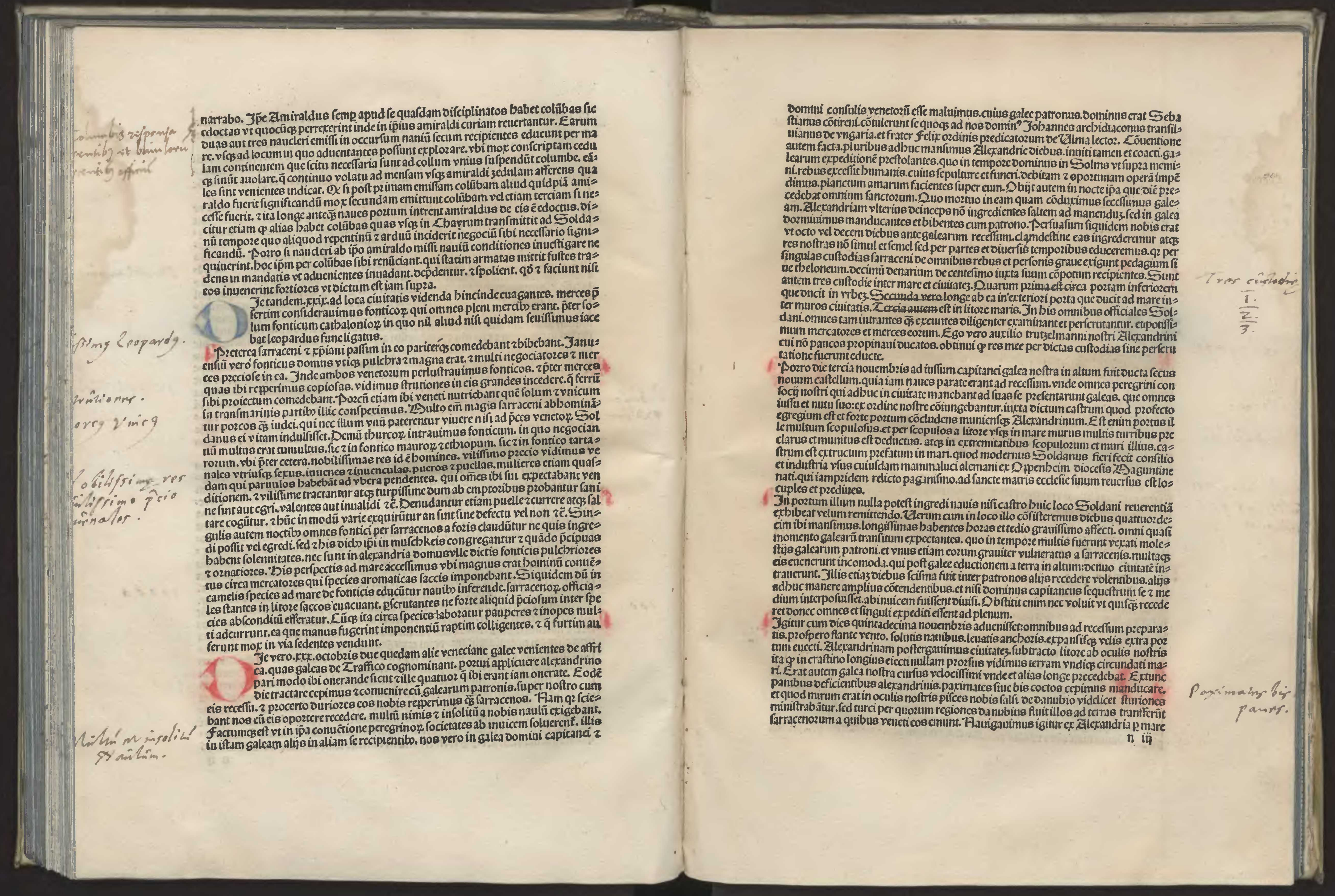




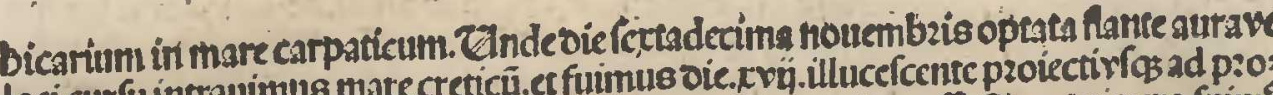

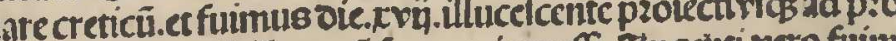

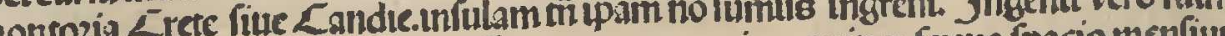

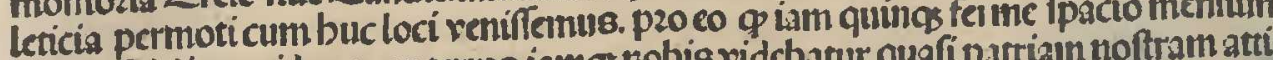
null las fidelium ridetramus terne. (inter

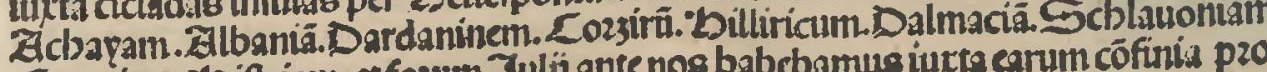

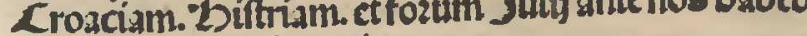
Cinciarum viam fracturi

Canośa

craluoifo

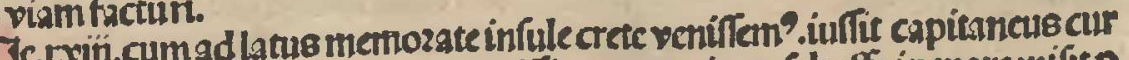

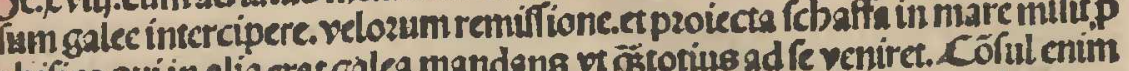

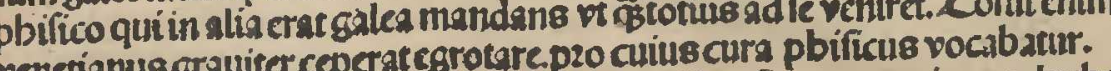

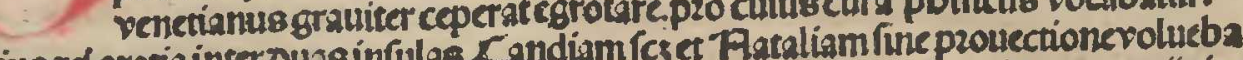

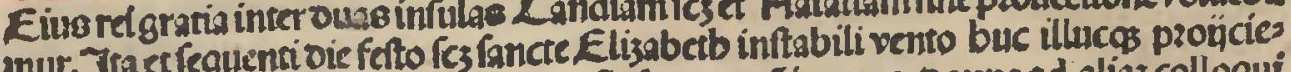

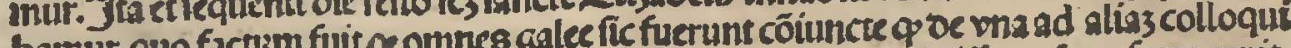
bamur.qno

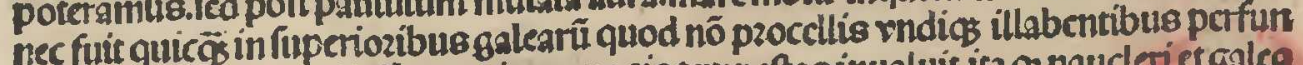

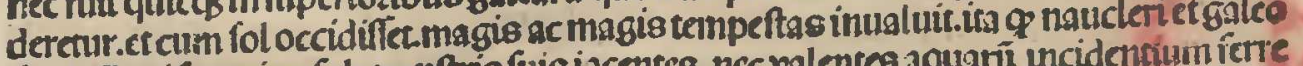

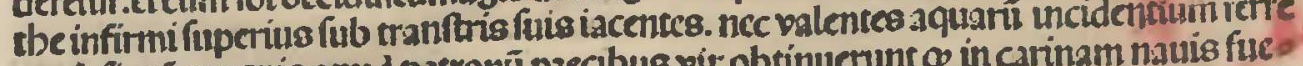

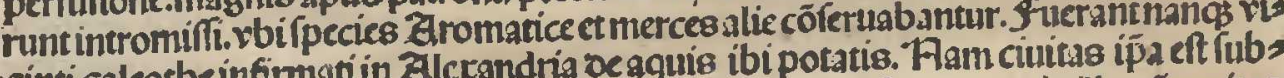
sintigalcona

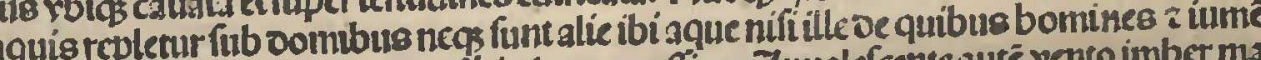

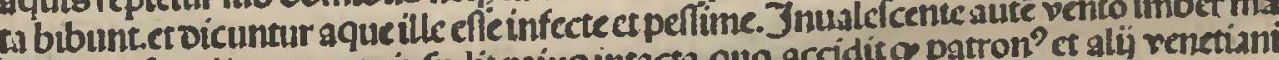
kimusockendic. gui bactenus

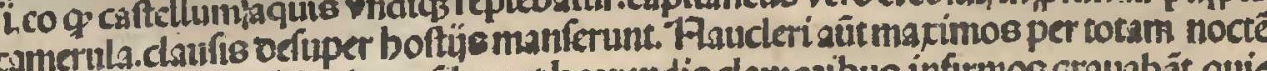

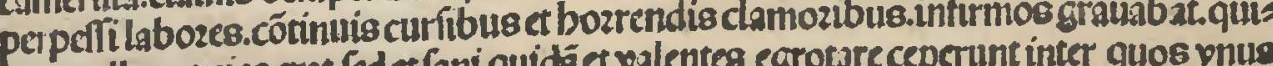

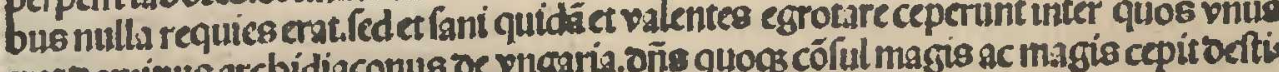

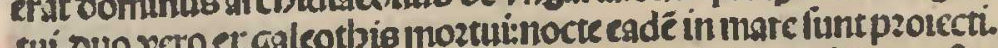

Sicd illucefcente oi que erat ricefima noucmbais cepit timpeftas ipa paulatim occre

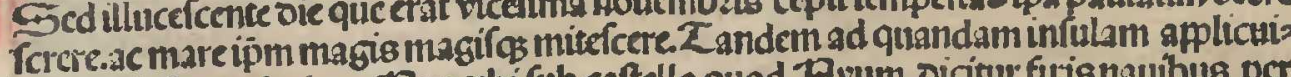
mus cuicef rocabulum 'Fro. rbi fitub caftello quod Fivum oicitur futisnaububs $\mathrm{pC}$ actas anclooraspecipirene

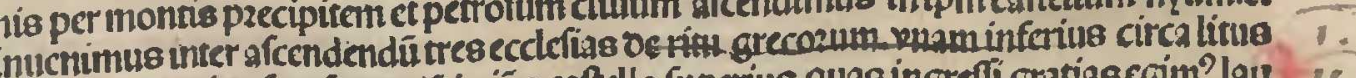

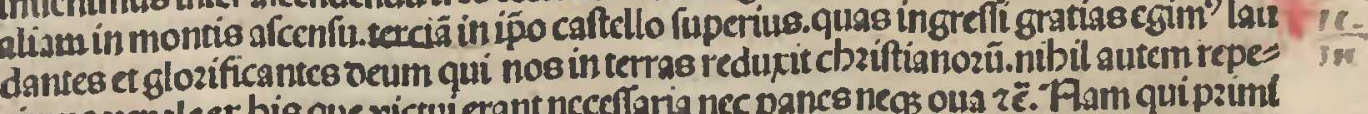

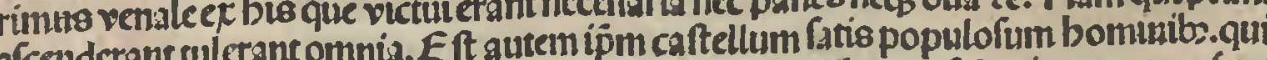
efenderenction in paruilation feralis frasect pinguis glebe. Eft quoßs caftellum ipm returttifumu a grects quonda

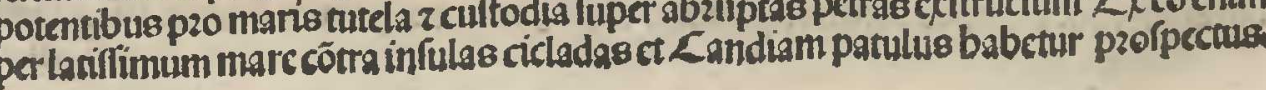

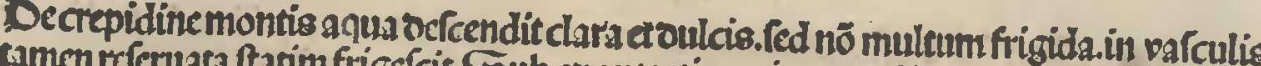

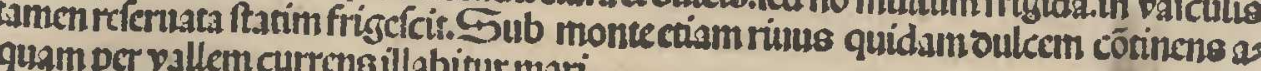
q̨am per vallem currens illabitur man

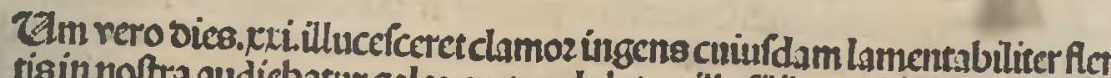

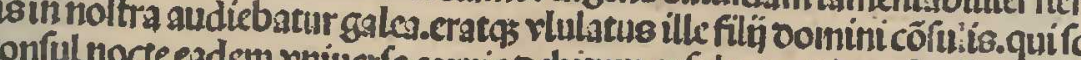

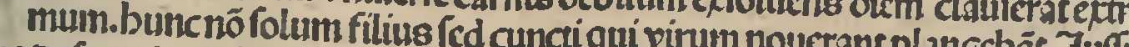

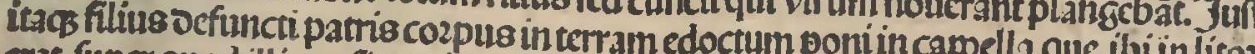

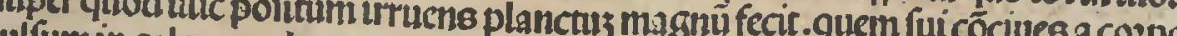

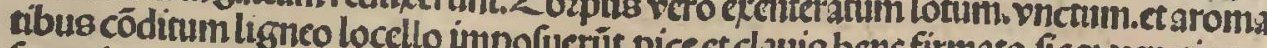

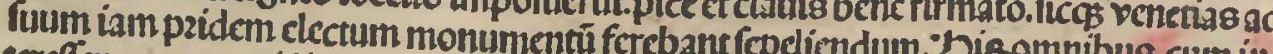

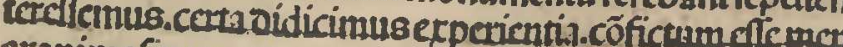

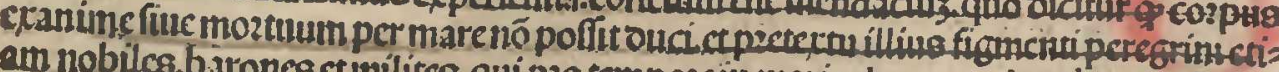
cim in demaic a

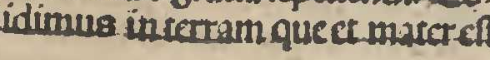

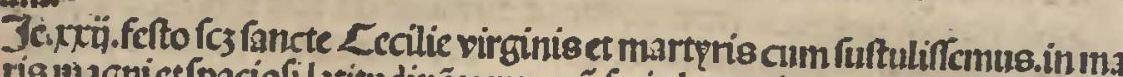

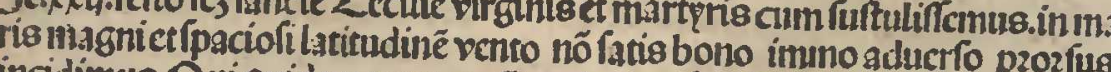
incidimus. Dui quidem rentus cỗtrarius.rectue Traciam nos impulit.at Gs fub pallcarum ipacio bozarum ficius fuiffemus impectum inficuti. Con

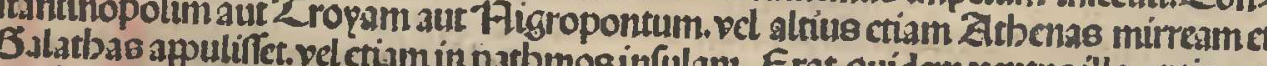

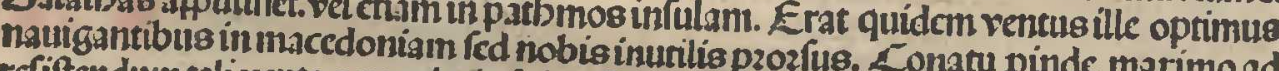

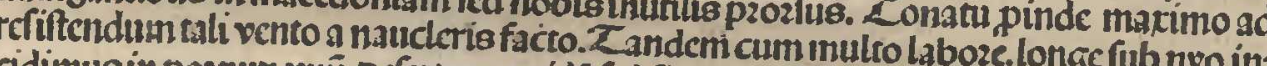
eidimus in poznum vnü.ocertum quidé fed firmum. Itepbanum nominect ibi nautes

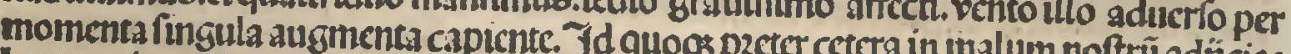

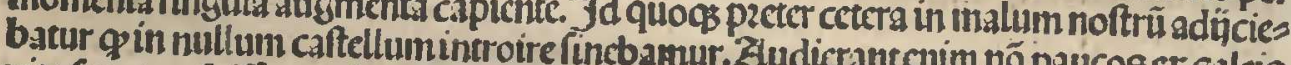
vita functos fuiffe.putzabantos galcas onnes pefte infectas iccirco nos ab bominab

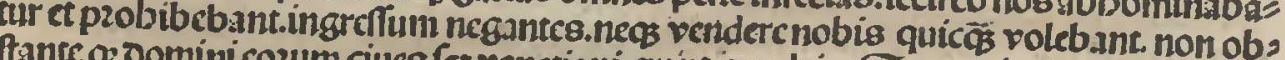

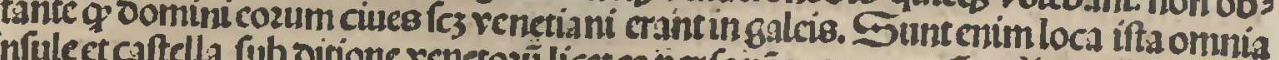

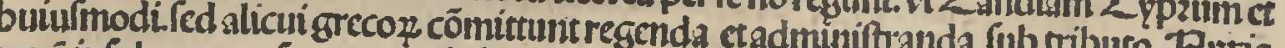
caune infula oc qua lupza mentio babita eff fub oominio of cuif

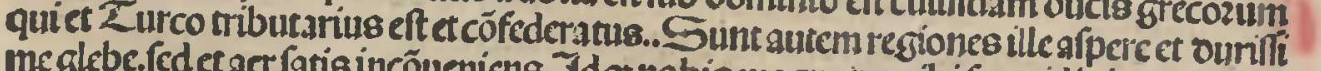
teibiointivemancre oporterne. Jd nobls magnopercibi fozmidini crat ne fors

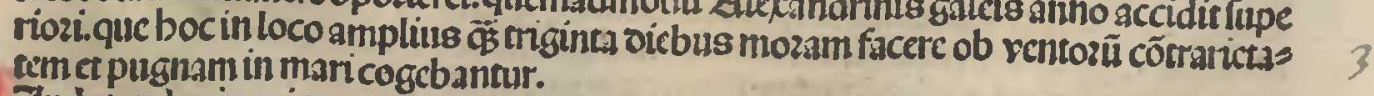

plurima pericula corect

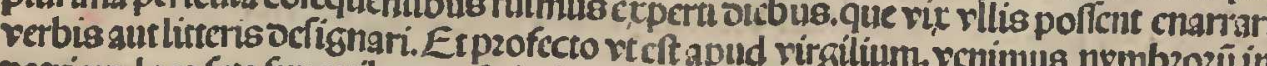

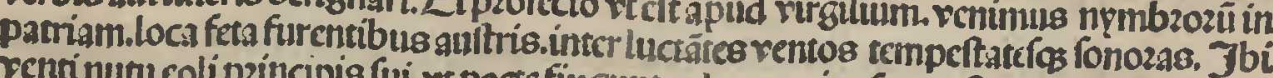

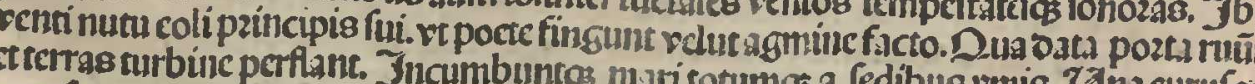

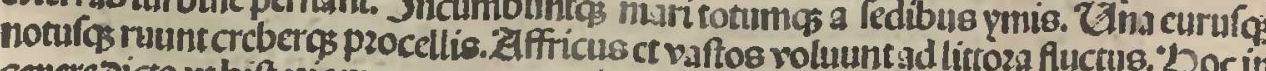
gencrescto visis pericul of periculotoz.ctaccuranus ct latius neceffect ve vexplicetur. 


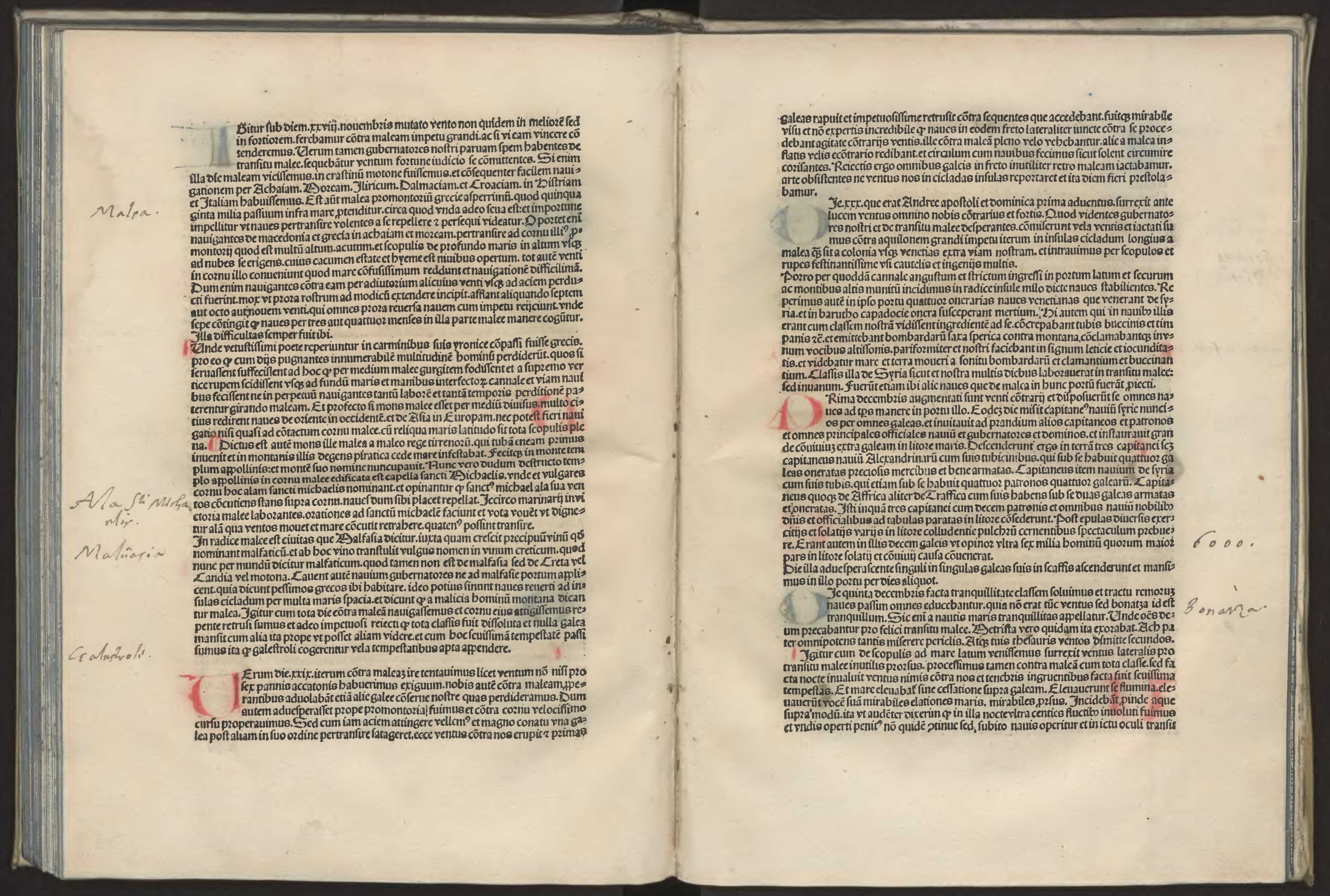




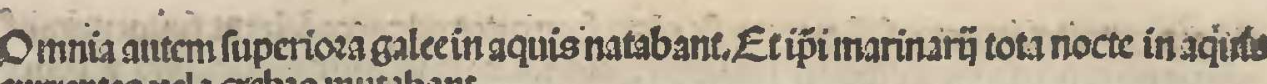
currentcs rela crebzo mutaban

ozro tempeftateilla ourantrecum naues abinuicen longius efícntfeperate vnawven

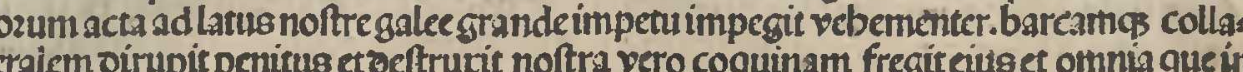
eacrant.

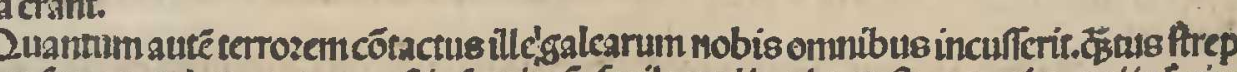
us fragoz ctclamoz et anguftia fuerit nó facilectplicari poreft. nos cním qui infertius

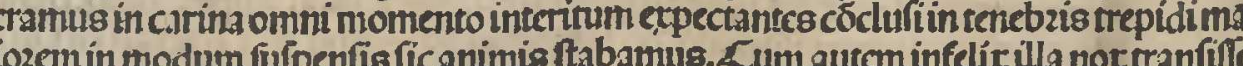

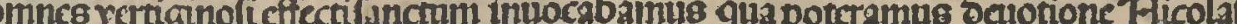

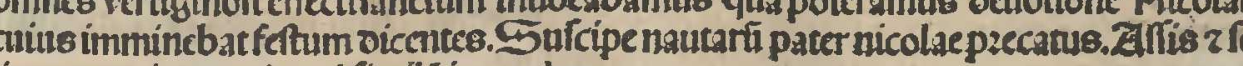
tranunc picateruis.rectumle in modum.

míoralio

Gifiroids

Pavromionaris

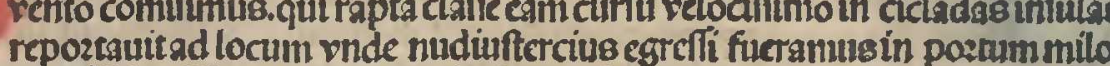
ibiog naures tonfillis alligatis etancbozis inumerisis byamare ibitimuununs.p20 200 in oicbus patronozt maris mare folito implacabilus babuimus. Suntenum fcom marnianosume

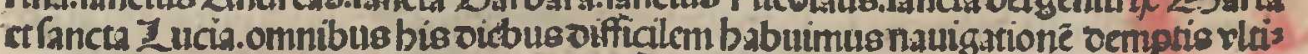

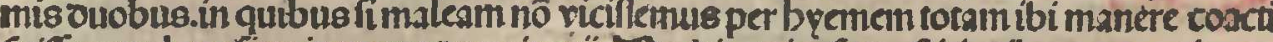
fuiffemus. boc firmiter tenent marinaru. LS Uultis cnim fuperftitionibus vacant in na

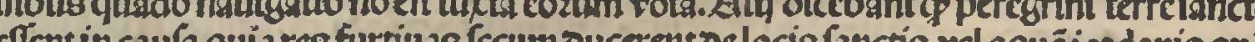
trenauigationem.

Jeicptina decembzis inutilem ventum babenres.eodem manfimus in loco polt piandium vero plures ozationis gratia ad litus nauigantes in capell

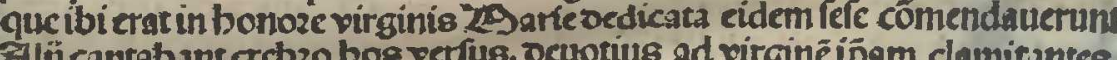

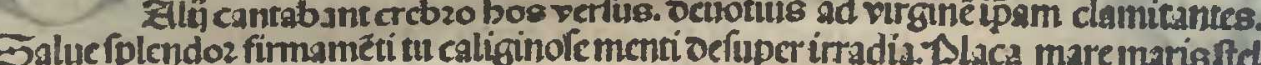
La nc inuoluar nos pzocella et tempeftas obuia. Zlidebatur surem nobis tanō̄ féfein

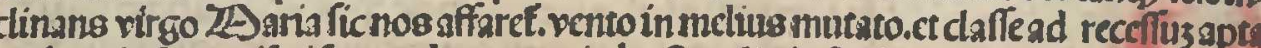

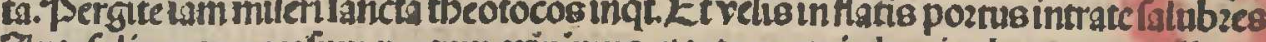
Antefoliscrgooccsirum poztum cxiuimus ettota 110cte in lumine lume tranqualla ne.

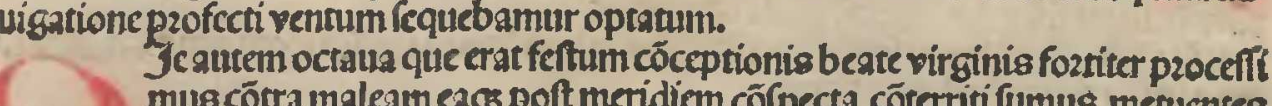
heratam repulfione. Sed aduéperafcente aic vento femper in meliue cons foztato repente cozlu illud feuif imũ malee finc laboze penc fuperantes fub

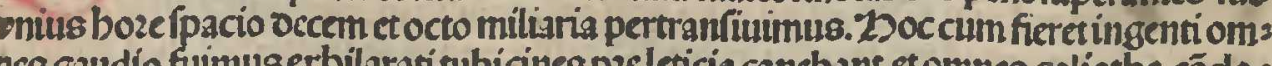

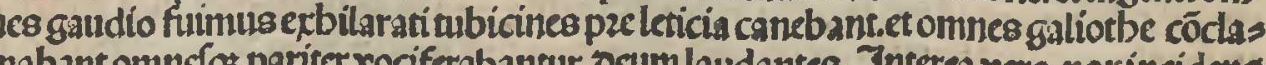

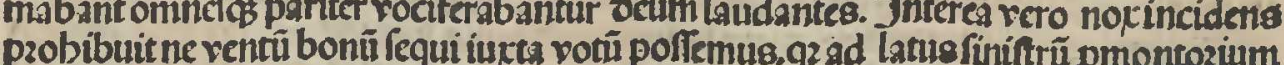

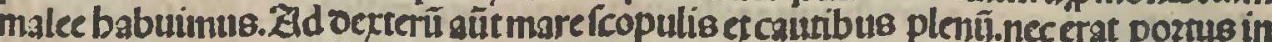
qué intrare poffemus. Jccirco remilerüt gubernatozesomnis rela.naues ad latiozalo

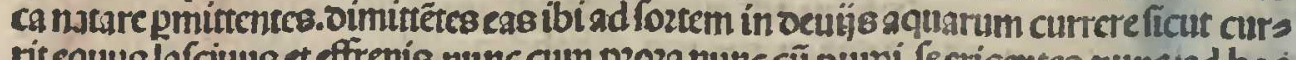

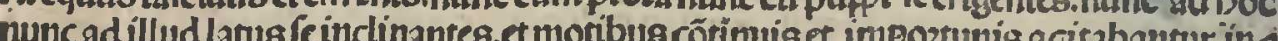

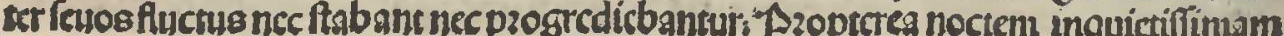

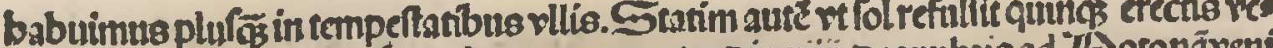

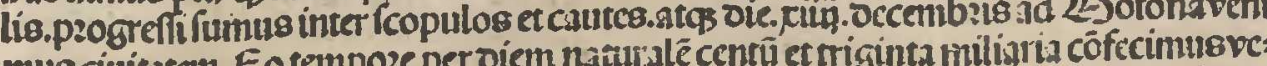

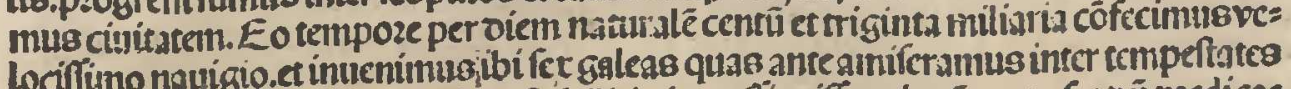

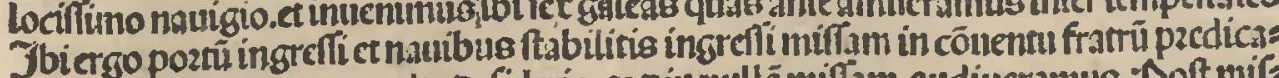

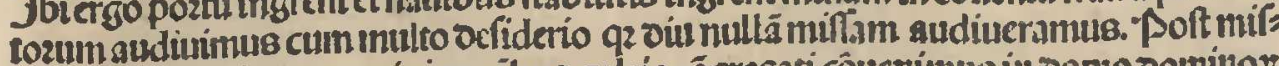

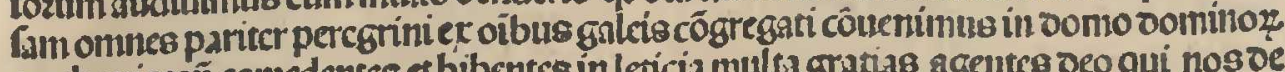
reurbonico:ii comedentes et bibentes in leticia multa gratias agentes deo qui nos magnis liberaros pericula miblua vithonefecithics

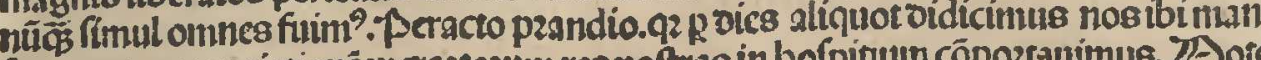
furef dinasebdomodalos illac cônenunt cun cbziftimis.

Galess ingreffiet claffe foluta ad infulam 'oppofirs oiftarpe

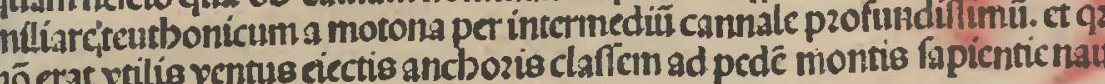
deriftabilienunt.

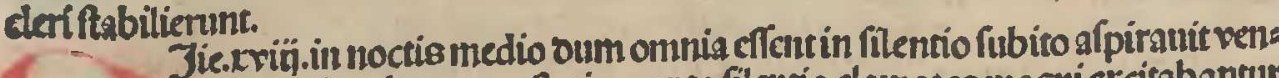
tus falubsis et bonnes, et ftatim rupto filentio clanozes magni cxcitabantu

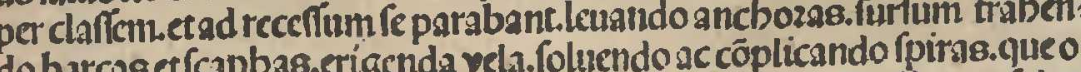
do barcasere

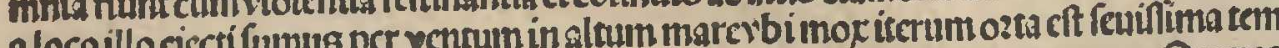

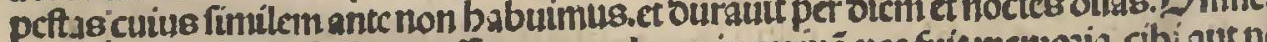
me bac tempeftascouranter. quia coquina crocin squis for.

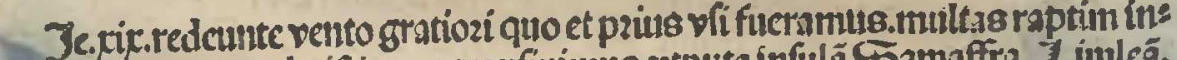

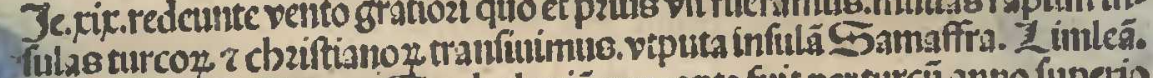

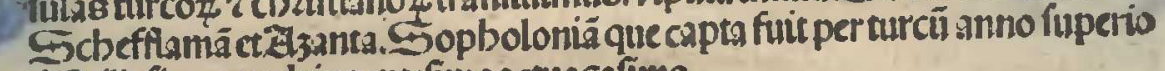

ević Latur bonam et fertilece. Et reperimus ibi claffem armatä.er magnam cbanifti am pantsz omnium neceflantozi. Jccirco capitancus claffis armate miale fe

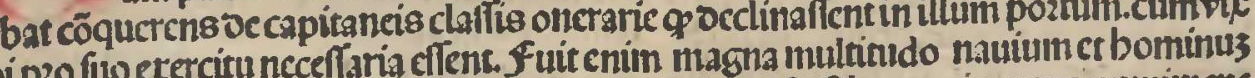

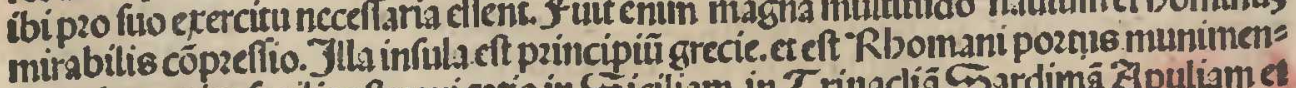
curn. Jatliam. Ex opporla

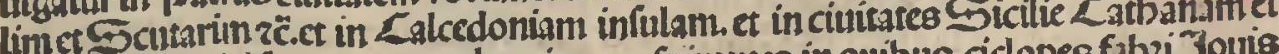
fulmina fabsicant fm poetse.

Tevicefumatcria fuftulenunt fimul onnes galee numero becé cuminna nant Humuscum bono rento

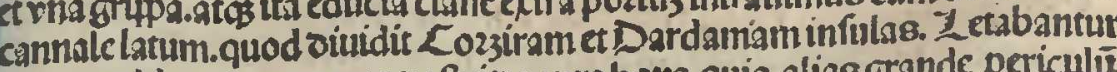
autem ralde gubernatozes noftri oe aura bongaguia alias grande pericultī

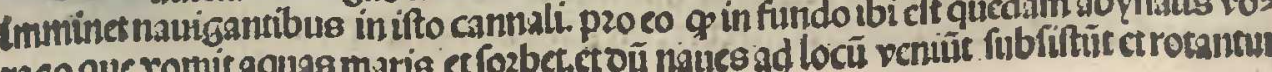

130

ogron lota

4 so. an

$\sin \cdot \sin$

Cximperfat

A For prah 


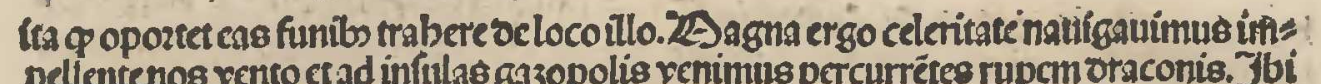

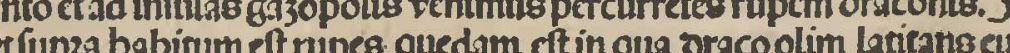

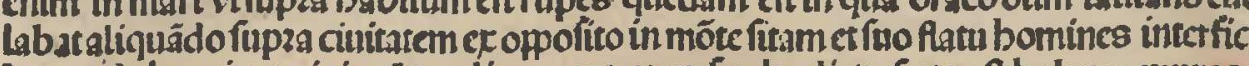

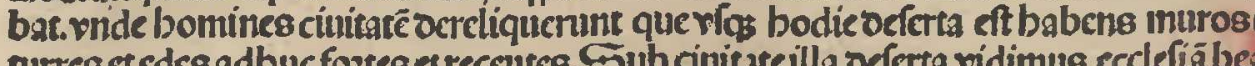

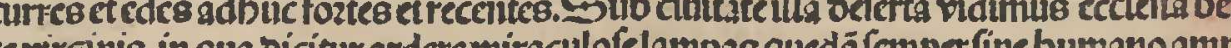
miculouis in qua dichir ardecenirsculo

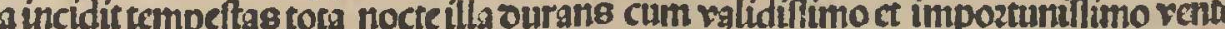

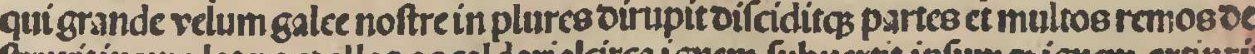

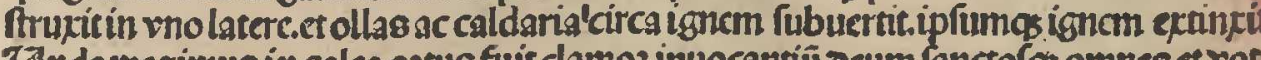

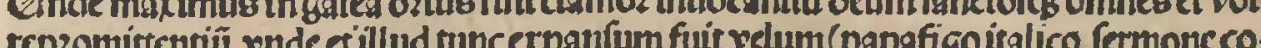
Grominarï) uigantes numio timoze percufit per fungula moments fubmeritionis oilcrimen expects

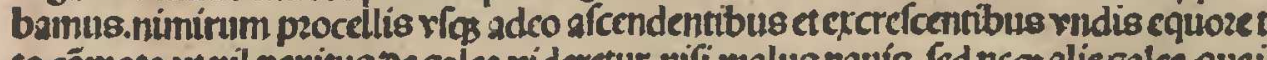

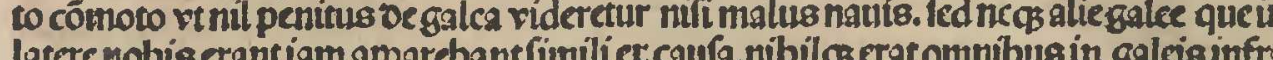
lacter nobis erant lam app arebanter.

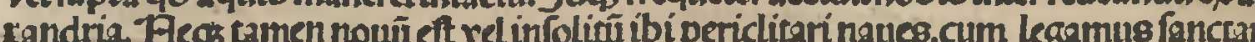

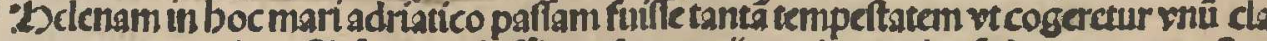

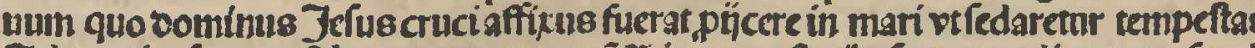

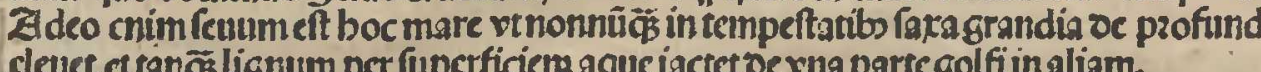

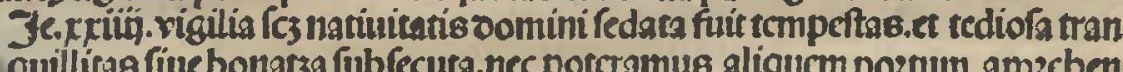
dere vt feftü inftans celebzarcmes.

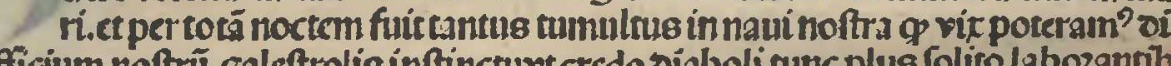

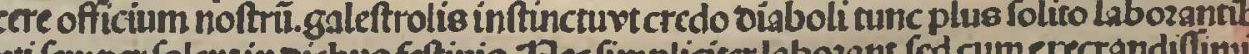
iuramparrio blarph paritcr et oic oominicinatalli excegerunt. Ode oic vidimus fine al baniexcl accbaye

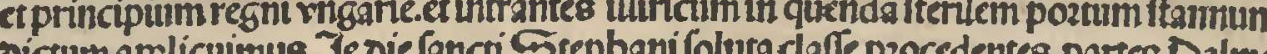

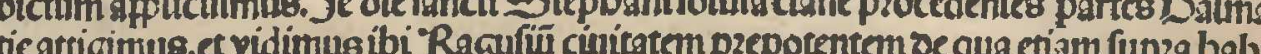

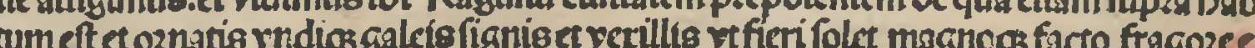

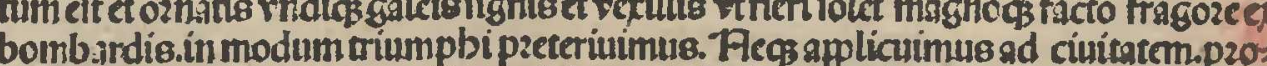

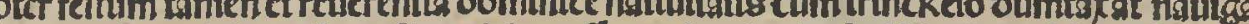

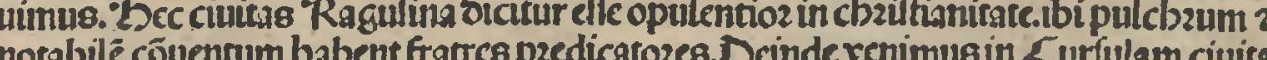
notabiectin

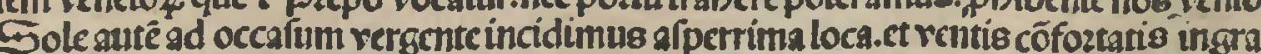
uatum ef t mare côtra nos nimis. Dus pzoprer gubermatozes noftriancï in oubio ftes

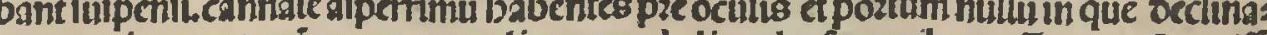
rent.oenuallerant eni a retra cannali per quod alie galee frcure bant. Cum auté ventife mus ad lañoze locion

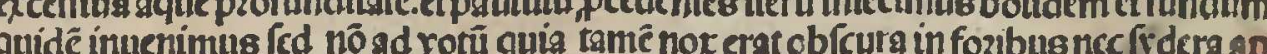

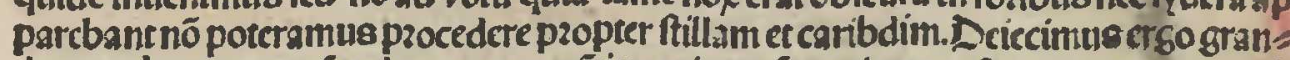
hav bofim bus mozdacemocntcminfigeret.fed currenté galeam fcquebatur arans terramoc qquo vilra modum to2qucbätur gubernatozes. Rurfumn e'go cum ingenti laboze retratá

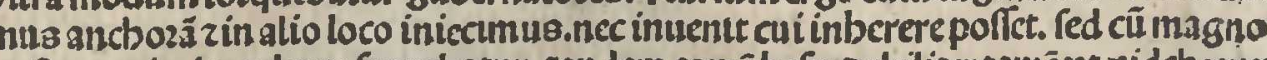

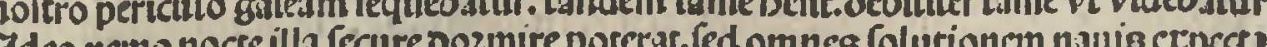

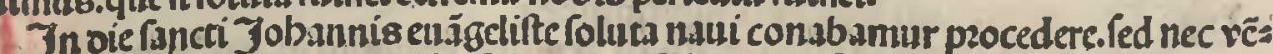
cosum nee fiderum adiutoziil affuir nobts. Fed aura obfcura que femp nauigantibs eft trolefta. Zandem inundante plutuia cónertimus nos in Ylliricí montana. zpoz: cumn toztnla oictum intrauimus. in quo alias ouas galeas noftre claffis repprimin? 3 oténnocétum clafferm folnimus z cum pzofpero vento pzofecti vidimus zd

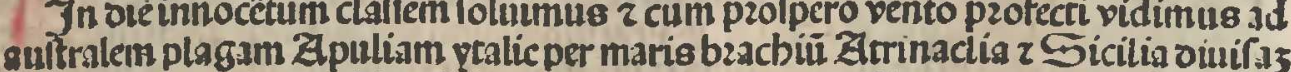
zin coznu spulie quod in adriacum mare extenditur vidínus monem 5 argani. In buins montis radicibb eft Sepontus ciuttas olim a Diontede inontis vicroze condits. Jbi fuit palladis templī in quo oiu feruata funteppi frumenta. iniquo criä gipennes eree $z$ arma oiomedis sepofita oftendebantur. In boc monte Anno onni

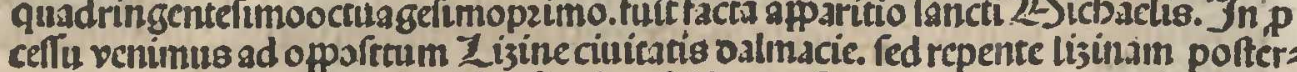

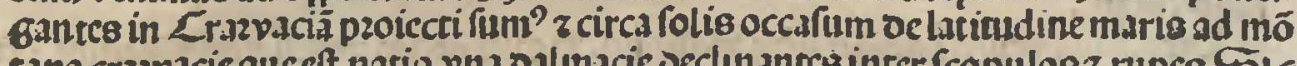

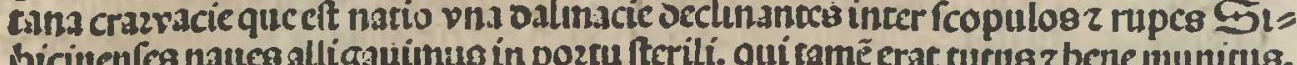

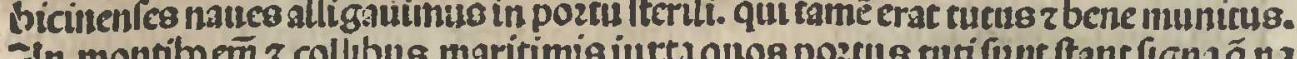

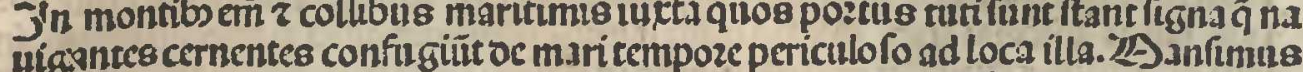
sureis in poztu illo vfos ad fectum diern cum tedLo maximo. Uerü in oie fanctifils

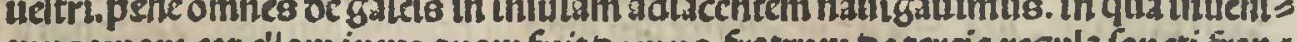
mus vnam capelam linta quam fuit oomus fratrum oe ecrcia regula lancti fran: circ. Capella bec oc gratia marie nuicupatur vbi pirgo ipla gloziofa in innumeria 

cbrauimuв. J pra ctiam die villam pers mane galcam excuntes in cadem capellace oe veceri mozezconfuetud dine id ibi obferranatur $\phi$ vbi coventum fuerit.quzfquis in Galcis znanibus altcri quidpiamioebet.mor foluere compellitur z fatiffacere p fen

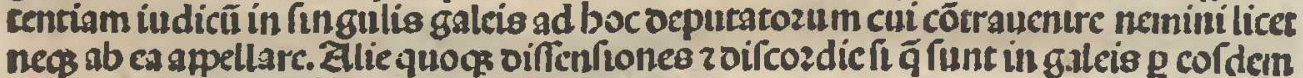
udices $z$ arbitros fedantur modis opoztanis.

Die fecunda Januarị rbi ventus conquieuiteduxerǘt claftem cetra poztum it= lum fed cum in latum veniffemus furrectit ventus contrantus repellés triremes co cra appuliam zidco magna vireduximus naues ad montana crazvacic.et in portĩ

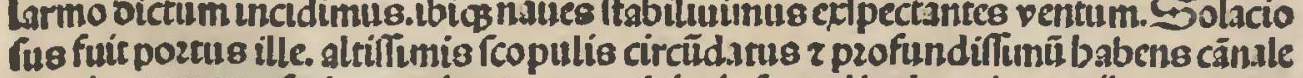
educi autem nos fecimus z in cacumen altiozis icopuli vel rupis manilon reptanses be rupead rupem afcendimus b/gad adgnum qo in fummitare oemonftrans poz

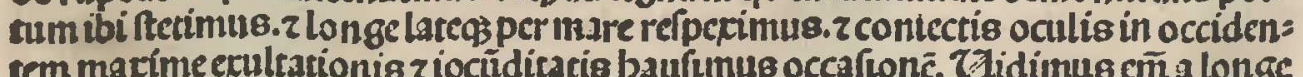
miucos noftros alpes fuiscandidis cauminits nubes atringence quif a longe

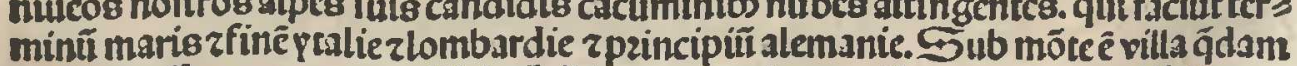
quä ingrefive cmeremus aliód nibil in ca reperimus nifi pauperrumos fcblauos. Die tercia Januarí cum bono vento poztin illī egreffi veloci curfua ad saram ci=

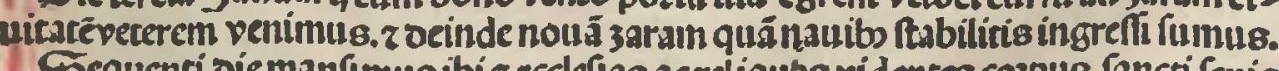
Sprmeonis in ecclefia metropolitang reperimus 
Focte fequenti incidimus in Eoznerum qui eft peffumms mario oiftrictus zpes

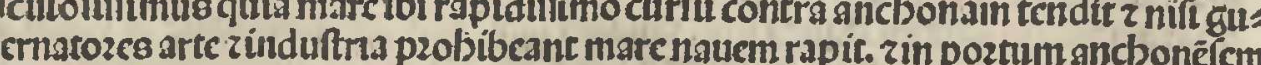
lunge pofitum oepoztar. In illo coznero grauifiumam noctem egimue z rempeftate valde bozribilempant hum zinquietudinern

Jlucefcette autem oie mirigatus eft ventus zventmus in biftriam que eft ng

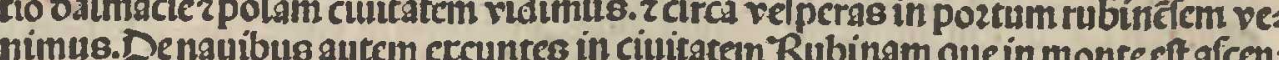
dumusgd velperas in ecclefiam fancte Eufemic virginis. Audítis vefperis ar cols

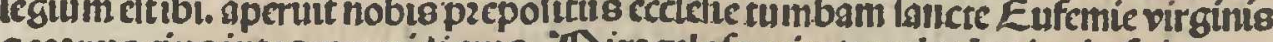
zcosphochla

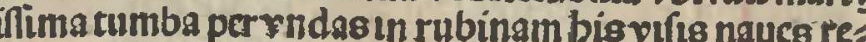

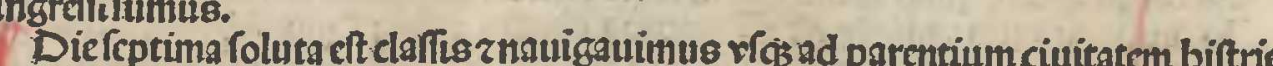

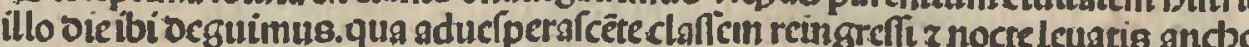

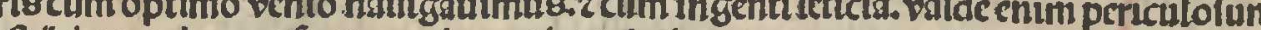

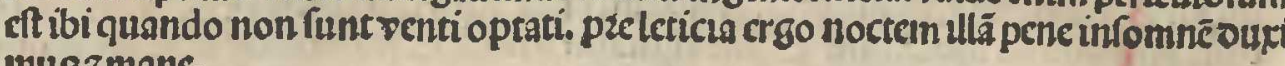

Dicoctaua venccias ciuitatem vidimus. คozro ö̈cito reneti nos viderunt. očs oliti runt fiutratis copulfaucrunt. zplurimi in barcis obutiam nobisvenerunt vit cum fancti fice in aduentu felici z fecundo natiii vel galcarum fuarum. Ante poz

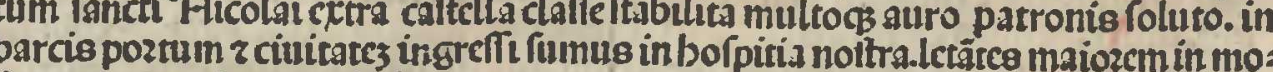

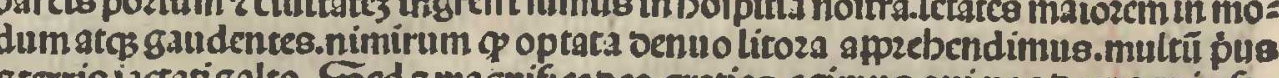

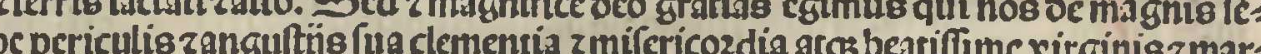

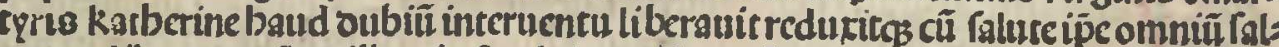
uatoz zliberatoz 5 pts ists in fecula benedictus amen.

f Sccundeperegrinationisad biutam virginem zmartyrem katberinam inmonicm Sormai proceflus sfinic feliciser. 1.te funt Jnfulca venetijs ivfä 'Rodum.

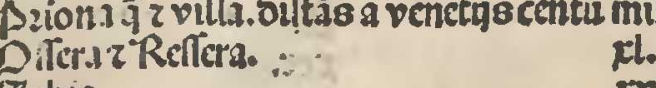

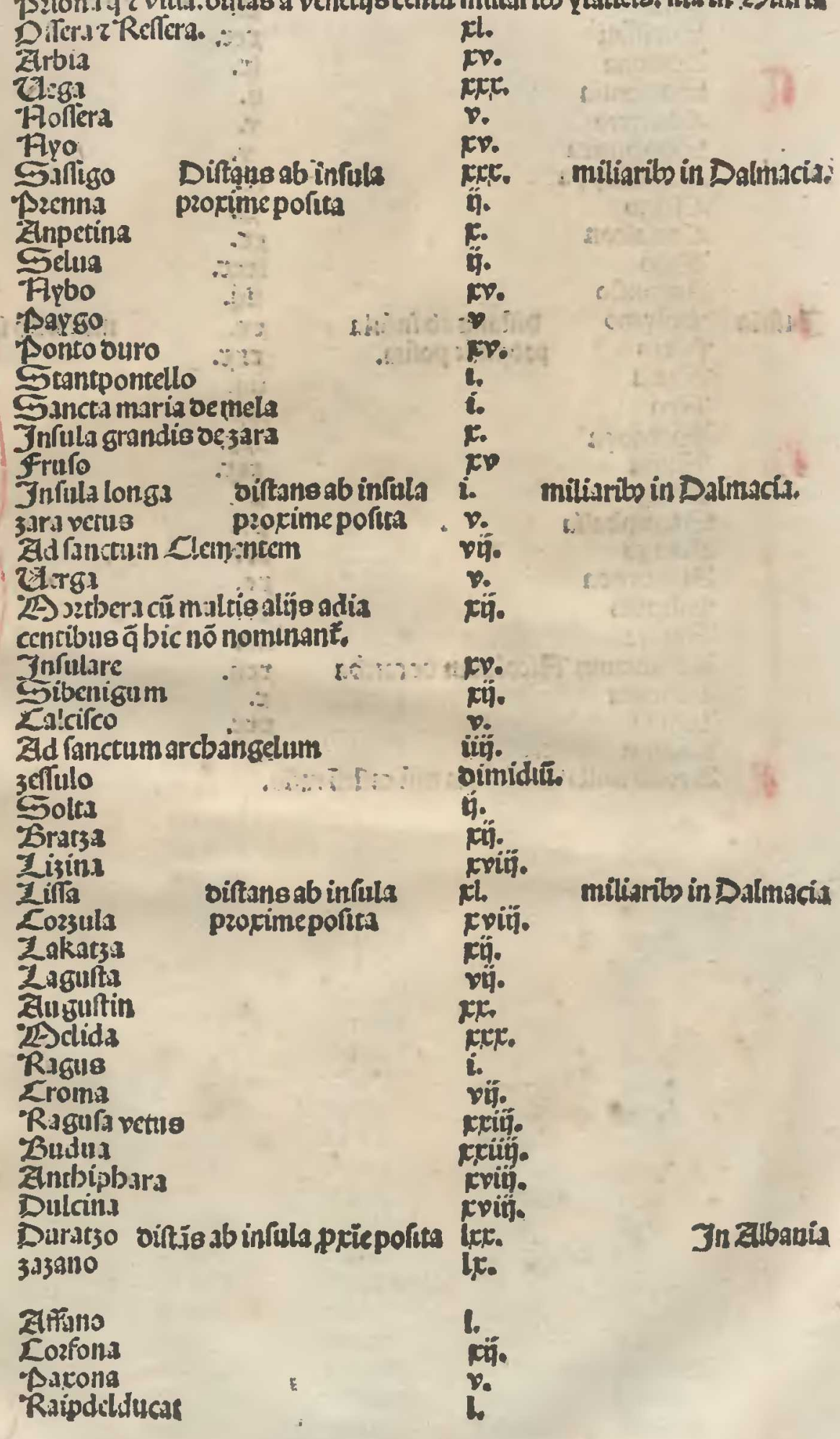




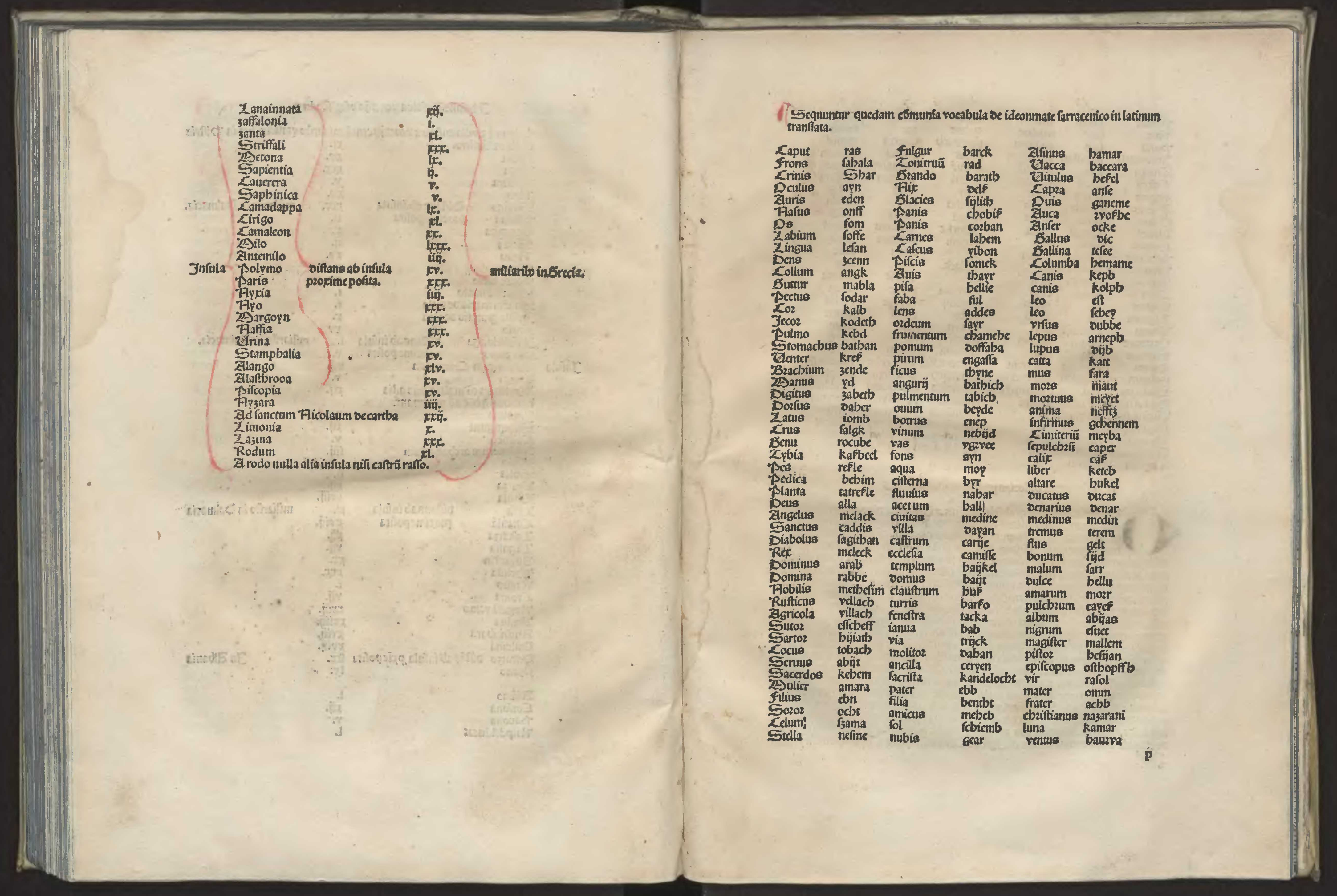




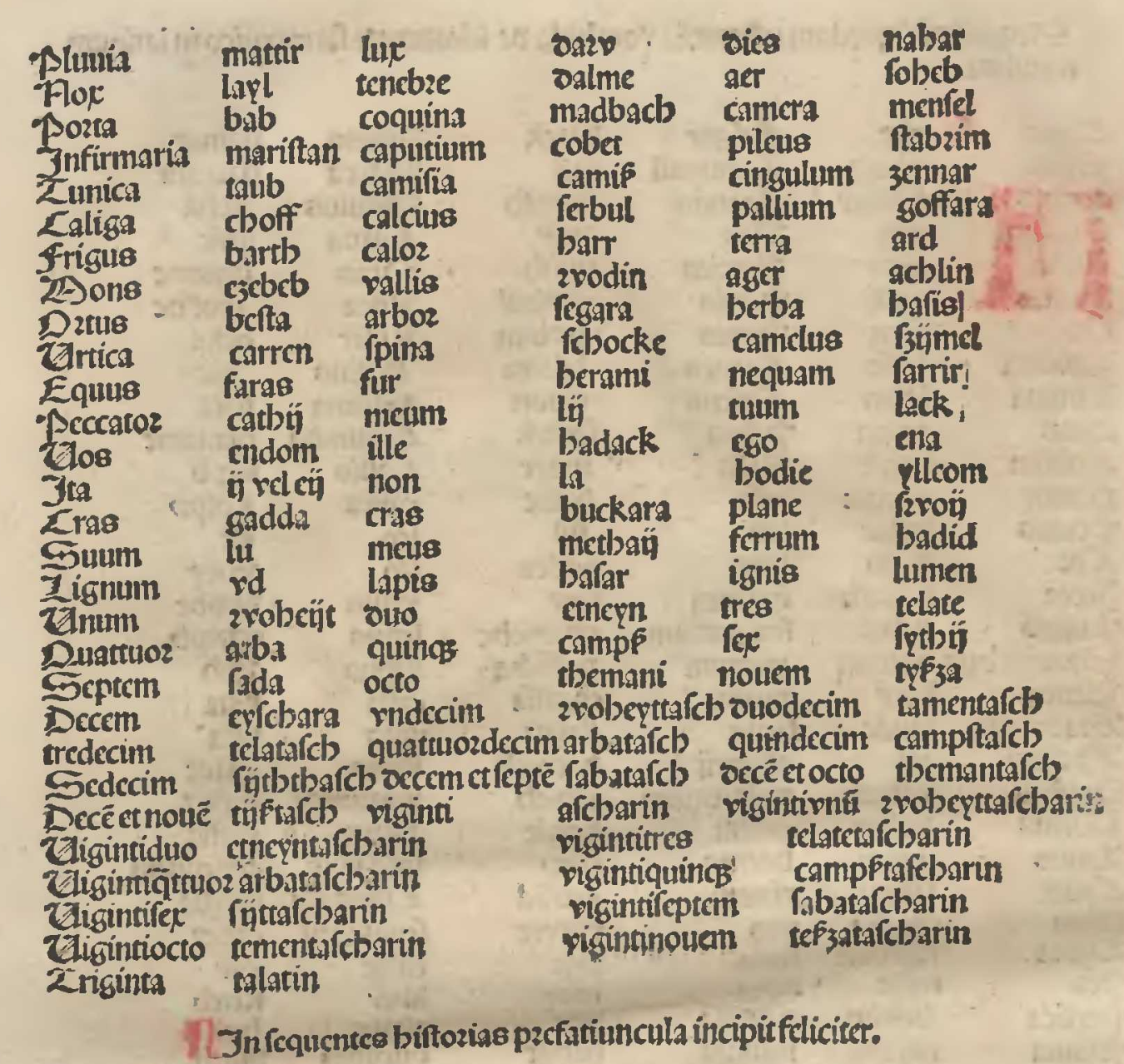

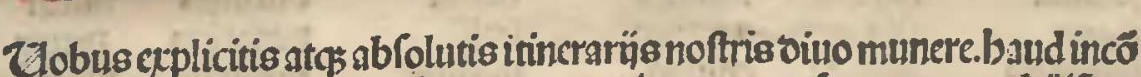

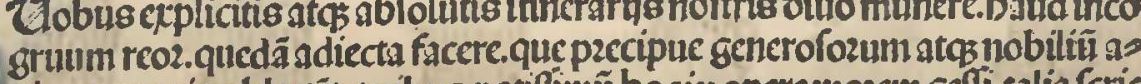

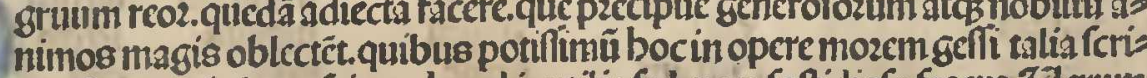
bendo.quecis lectu fcirugs baud inutrilia fed nces faftidiofa fozenc. Telerum vtiin pzinozdio buius libelli cômemo:atum fint.optarem vel beméter pzetcr cum que misi pzefituctas fuisem paincipale.ectiam bunc nilbilominus meo laboziaccedere frus

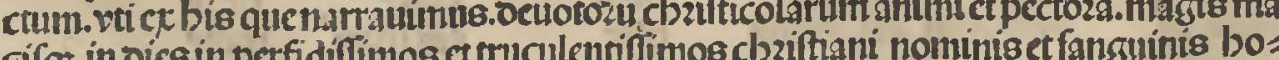

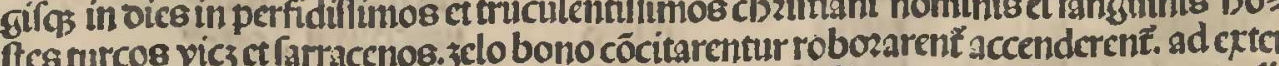
minandü cos oc finibus fuis rel faltem ad refíftendü cíddcm oum noftros occupare fí

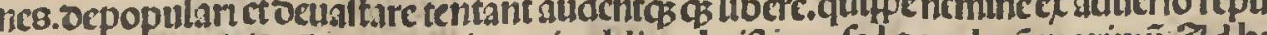

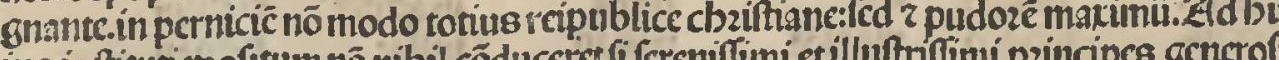

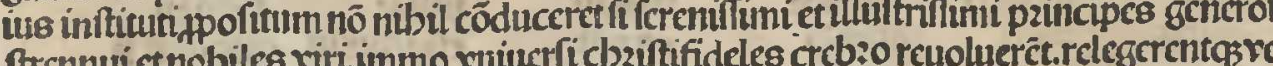
Galconn

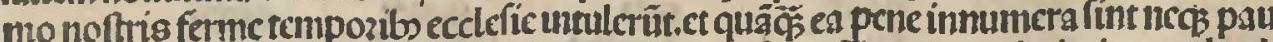

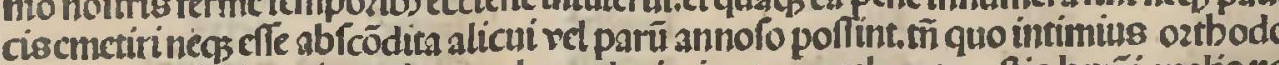

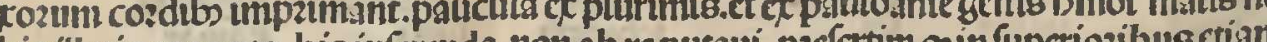
bis llatis cectrpta. bisinctenta expugnationebzeniffime.poftac oc 'Rbodie vrbis obfidionelatius. Acoemum idruns f De Conftantinopolitane vrbis expugnatione.

Pino omini 2Sillefimoquadringentecímoquinquagefimofccuindo. ciutitus Eumponficen 7 icolaum niarum. Quos nöecraudiuit Ficolaus, indignü oucensitaliam eet pecl

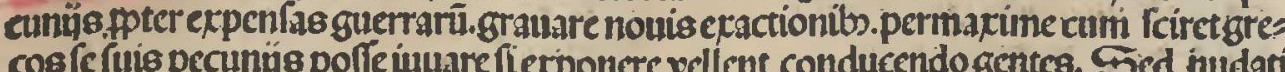
cos feftuis pecunins poffe uuluare fiexponere vellent conducendo gentes. Sed hudati

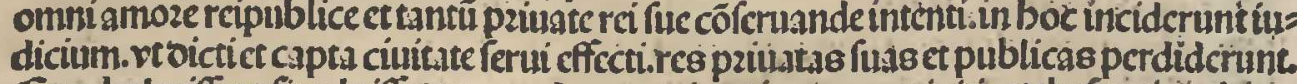

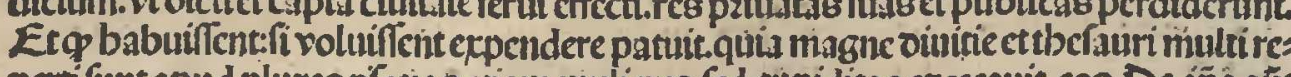
pertifintapud plures píonas etiam mulieres. (ed cupiditascecccautit cos. De ípa aŭt captione oictec ciututatis per turcos. Reuerendiffimus pater $z$ oñs cardinalis rutbenus cpilcopus S.abinenfis.grccus.noic Jfidozus.qui tuncerat ibíl legatus pape ficicribi

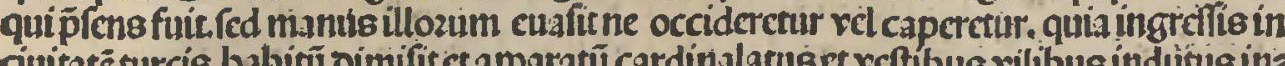

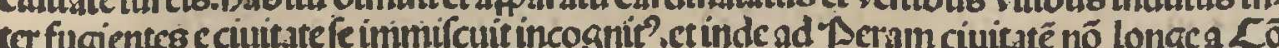

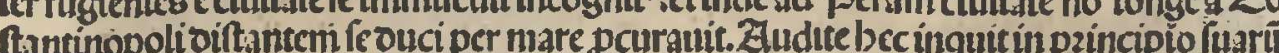

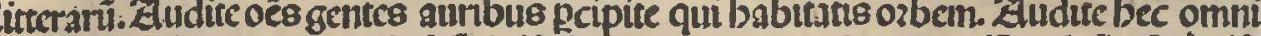

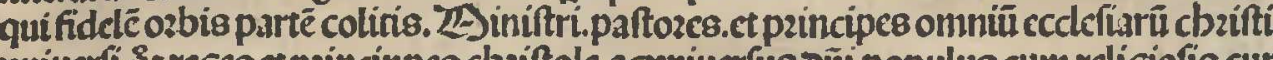

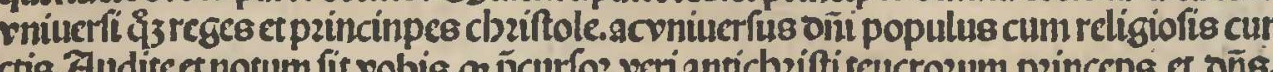
Geruug aure to

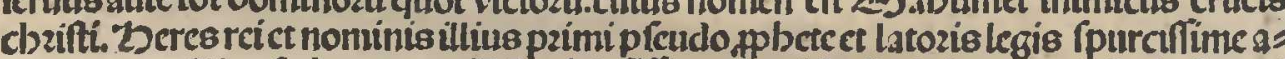

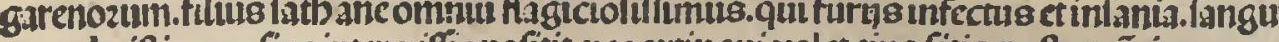

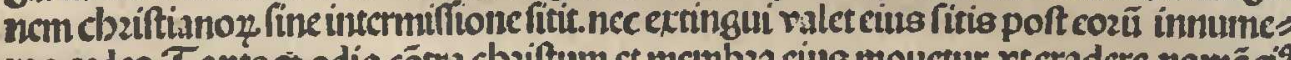

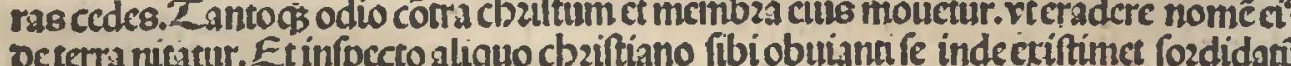

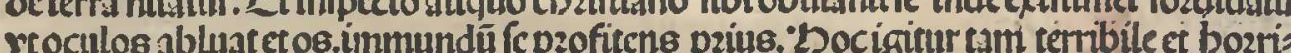

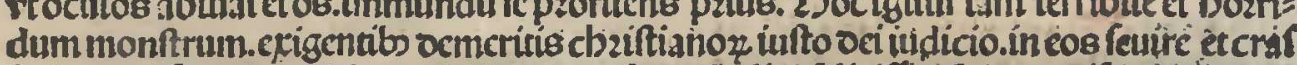

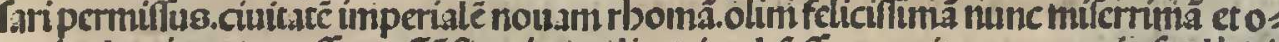

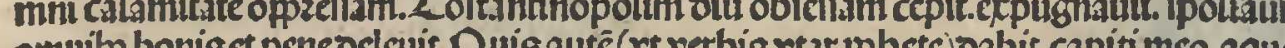

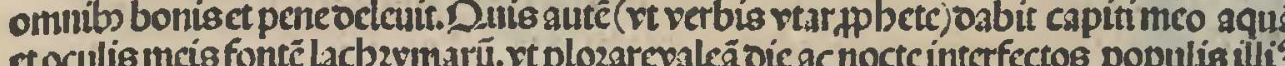
tocleftirin facrile

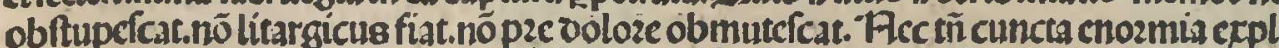

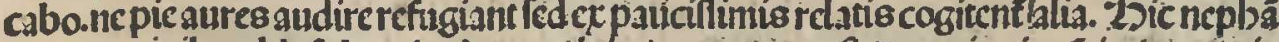
dus.nominibus blafpbemic plenus.cultate recepta poft occapitatione imperatozis.

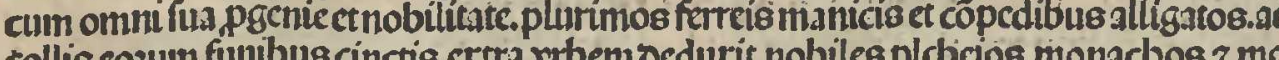

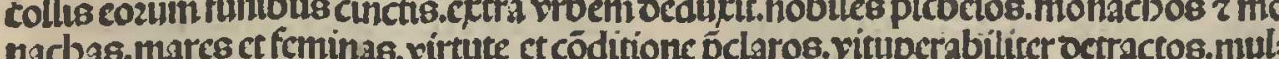

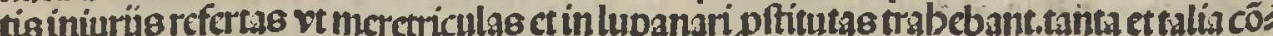

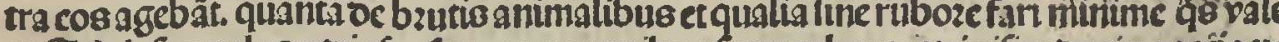

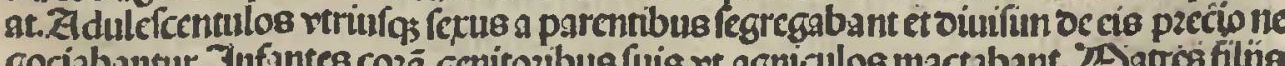

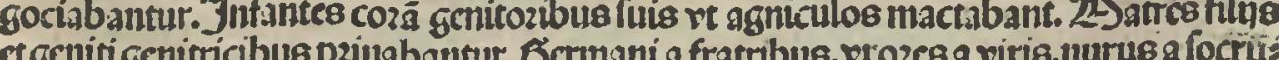

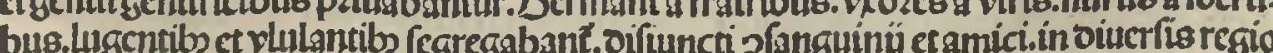

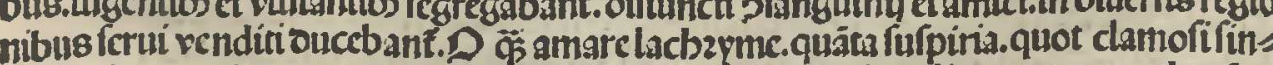
gultus inter amicos et rotos, que milcrabiles voces emitrebant inter tantes cedes. fer
$1+5$ 1.6 a mone V re is $r$ s. 5 . amm 106 Caromalos: ceosfory. Nona Coma 


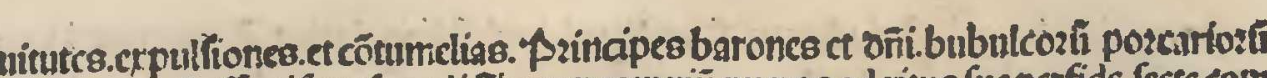

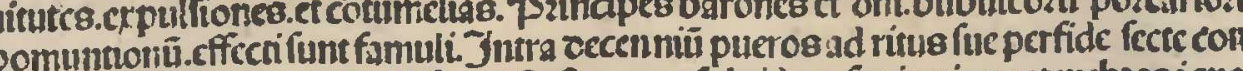

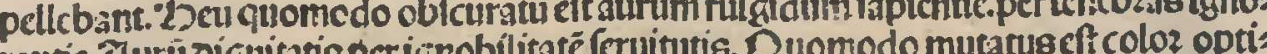
antic.2.

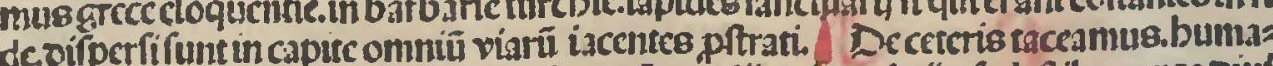

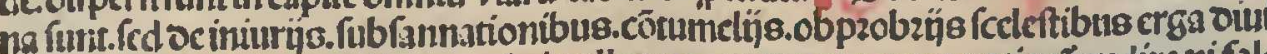

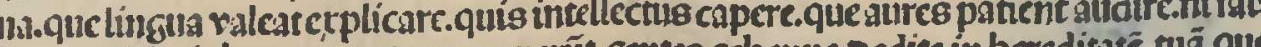

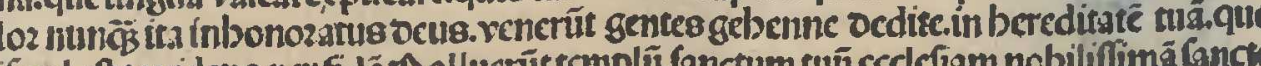

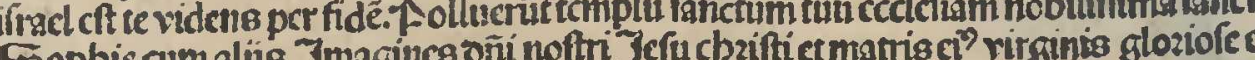
(G)

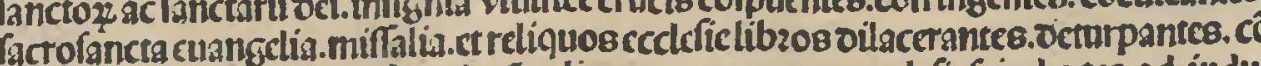

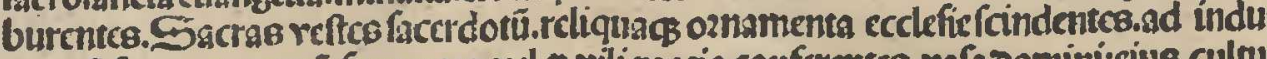
mentü futtim $\mathrm{k}$ o

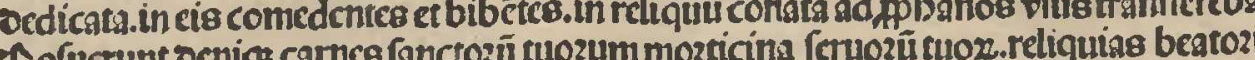
Porprum

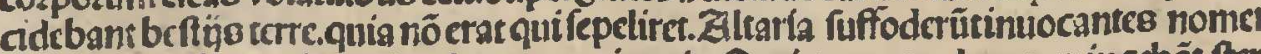
maledictimabumeti.eum laudantes oc victoria. D mittro prepudoze

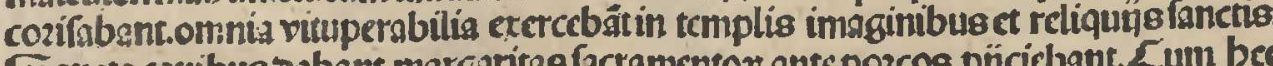
is

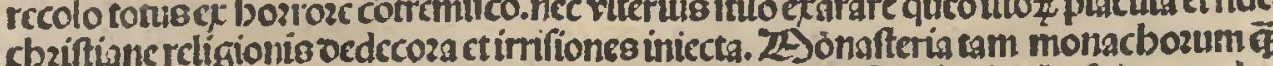

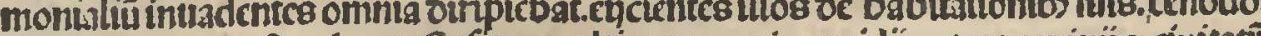

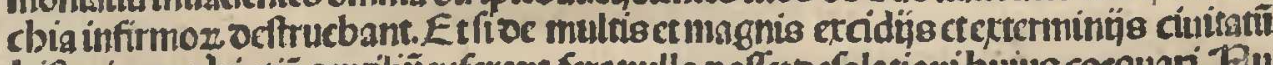

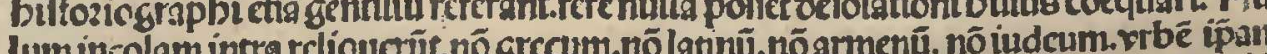
lumincolam intra r

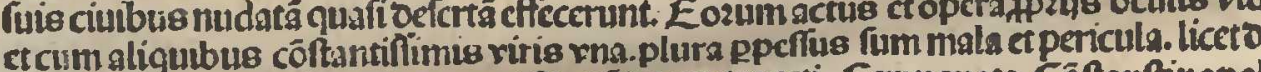
manibuscom me cripueris ocus vo Jonä oc rentreceti. Expughata Cöftanftinopol - Peram nô miltú oittanteccripa jo pallisccectian bom

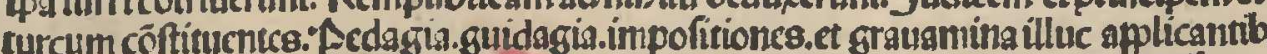
vel pmanentibs fuapte ftatuerūt. "bisatrocifímis rebus nō côtentus callídiffintus

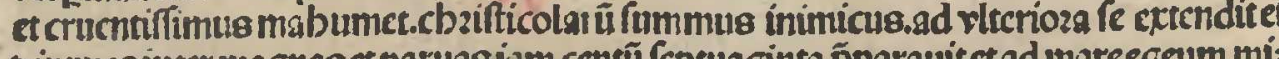
miremcesutcr nagnasect partage

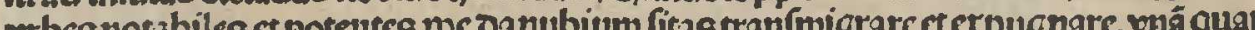

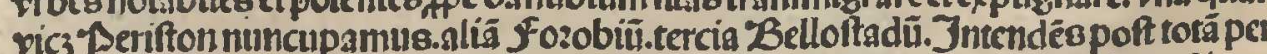

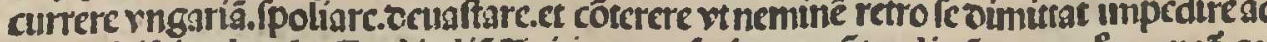

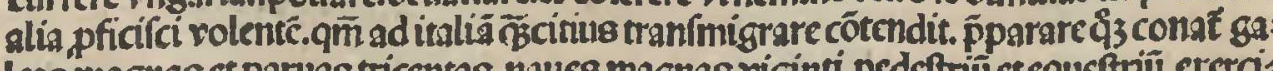

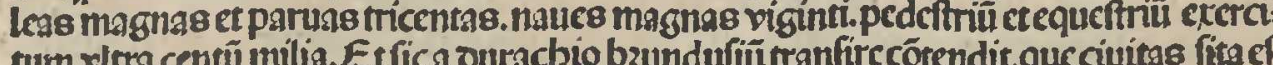

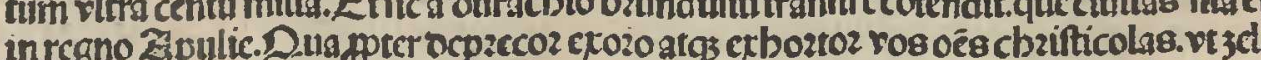
fidciet topianc religionis preftra libertarc gladio accingamini cốrra tä biros bofteses

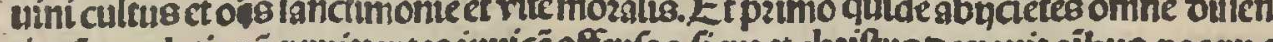

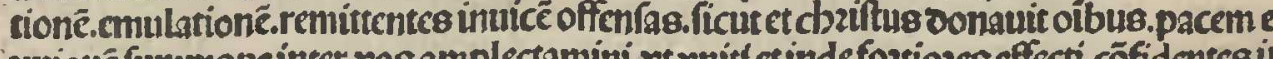

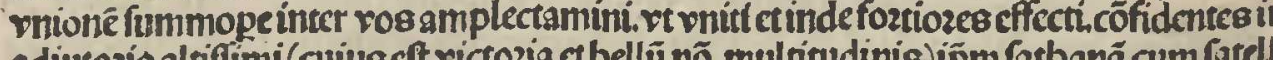

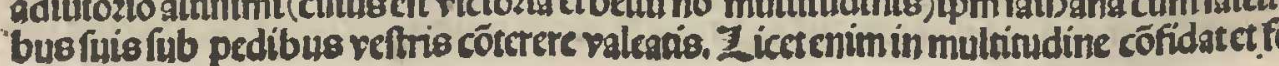

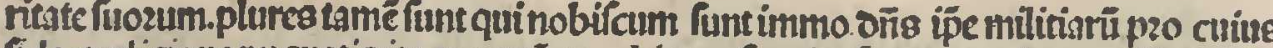

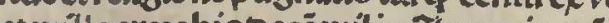

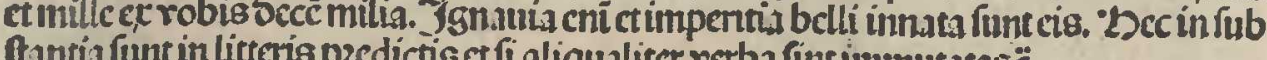

(Denigroponticaprione.

Jdelis quidem ctpzepotens wrbs - Figrnpontenfiscbziftianónominis tim

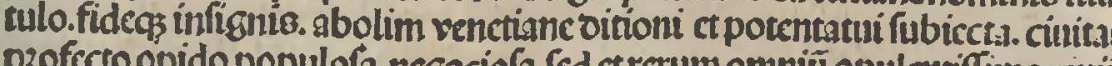
p2ofecto opido populofa.negociofa.

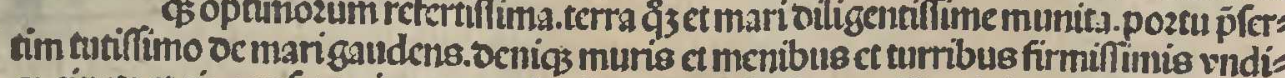
as cincta ct circumfepta.ita rt prosfirs inexpugnabilis omniui opinione crederet. Due çiam ciuitis 2 alcides alio rocsbulo cognominata oinofcitur. in infulla Enboia fits

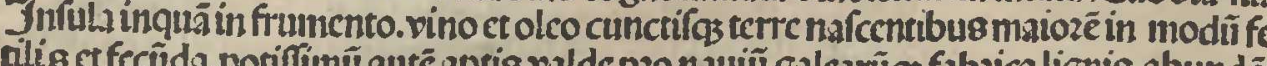

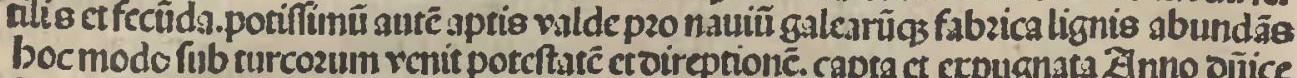

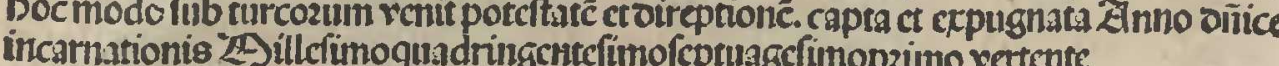
Clarífinimis quidè venctis femperabolim turcos opido fuulfeinfeftos, sum ob cozü in

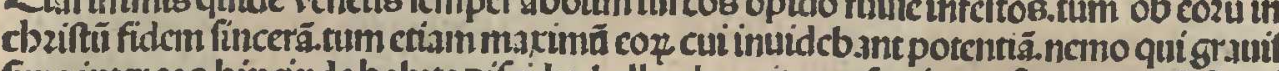

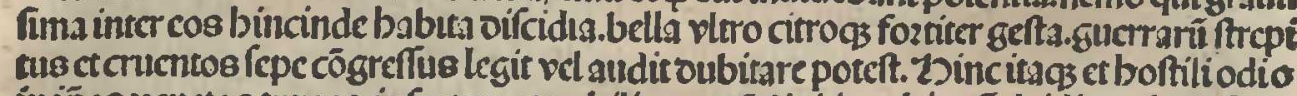

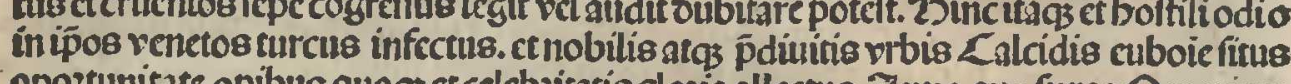

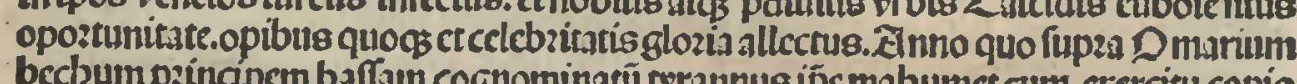
becbum p:incapem baffam cognominatũ ţrannus î́p mabumet cum cxercitul copio

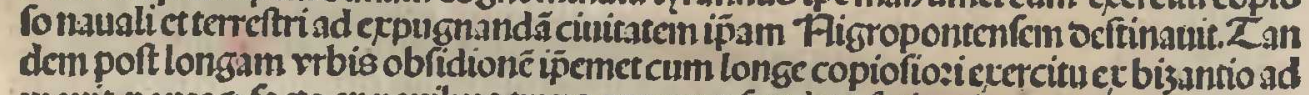

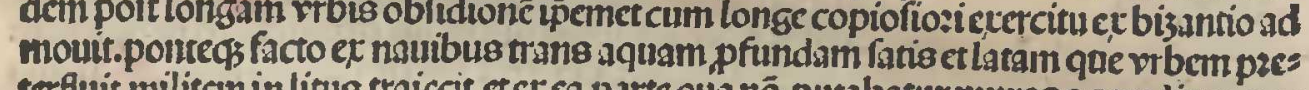

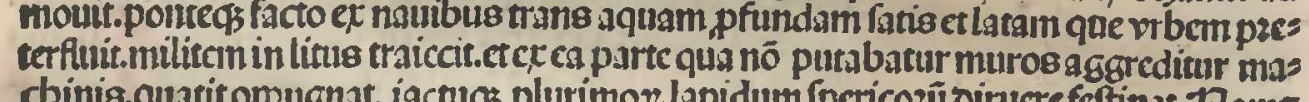

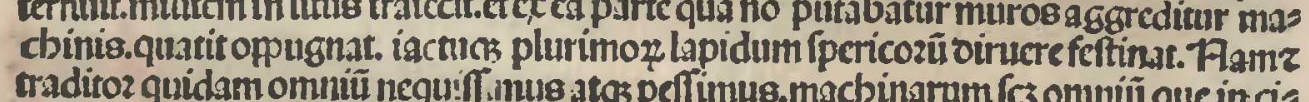

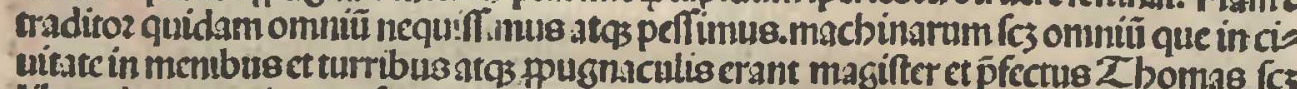

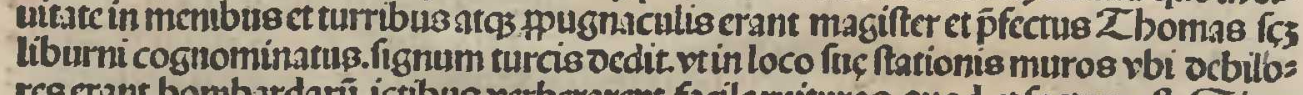

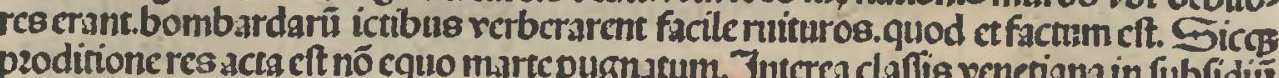

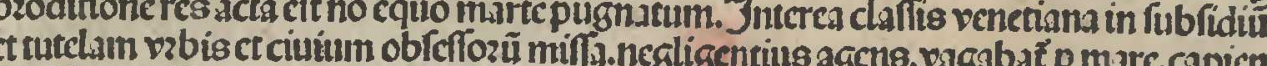

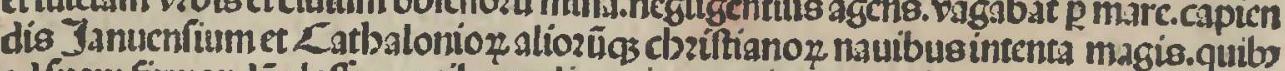

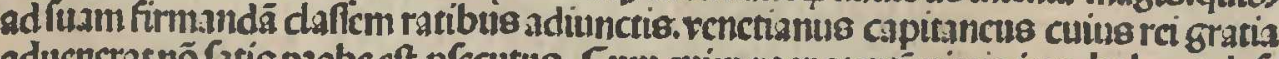

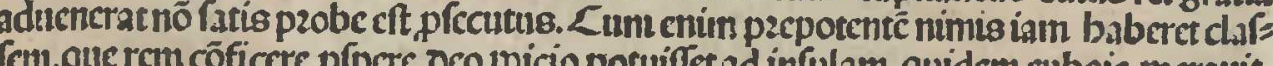

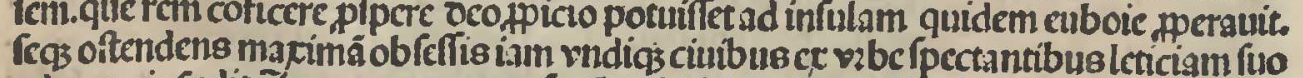

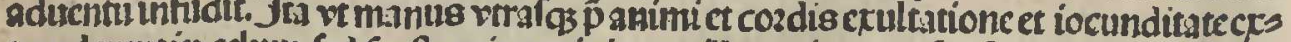
panderent in celum. Icd fruffrauit cos iniquus ille capitancus fpe fua opinionc foffllit.

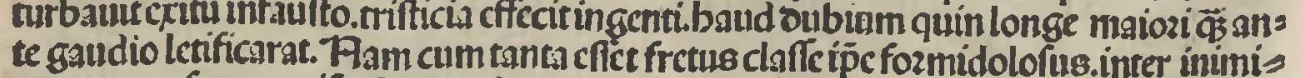

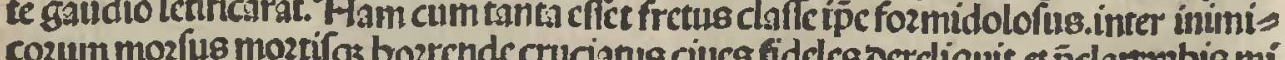

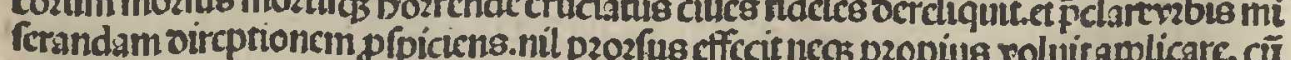

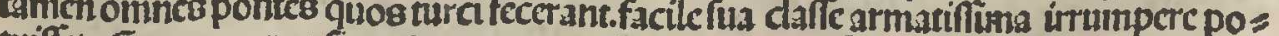

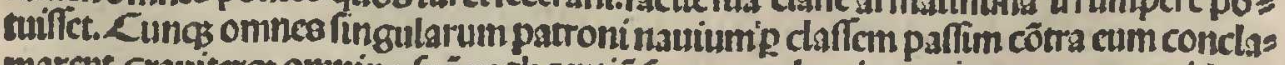

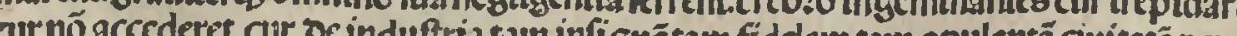
mumivi permitcret. Jile furda bec aurc bauricns in eodemloco immebilis inutilifo
Caloiáp

1471.

amo in 15

sor yma a mom

aing g.o8.

mquieor Cap 


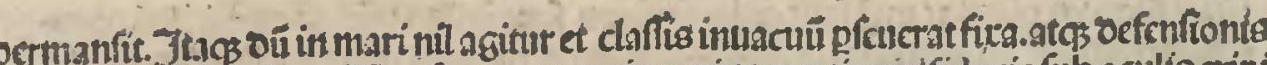
lgnu,

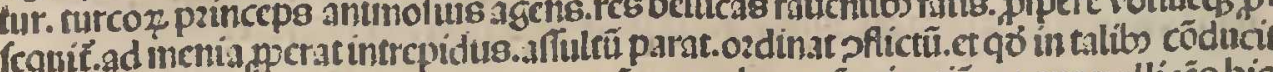

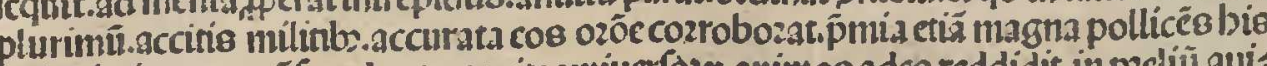

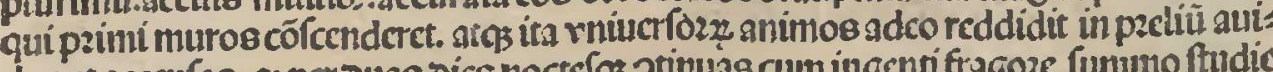

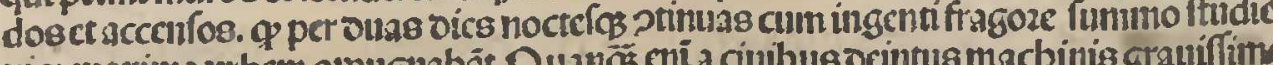
rigs mate tina we

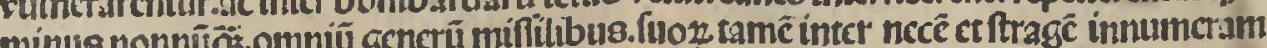

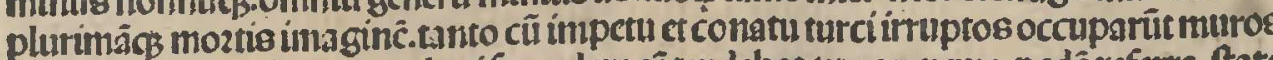
ummo tanta als nemo copertus itcuncti reco ant

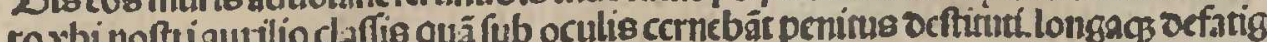

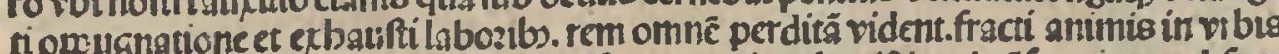

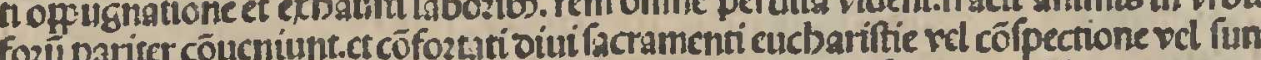

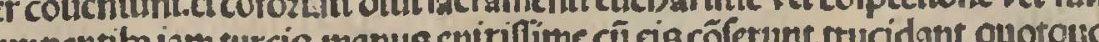

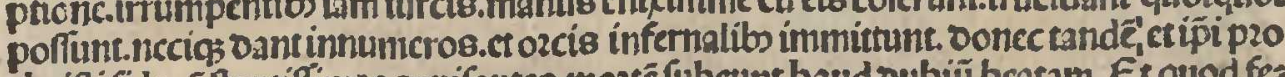

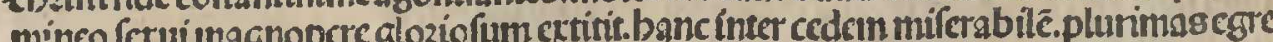
gia fosma fidcos feminas.tum nuptas tum virgincs.armis indutre ferunt mozcamsa

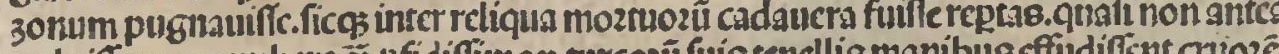

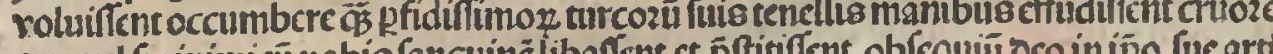

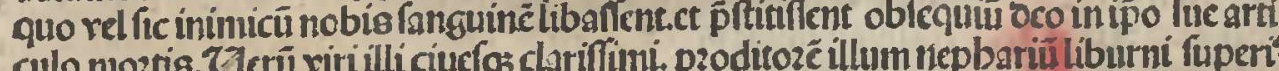
cello

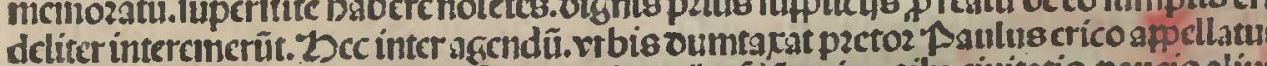

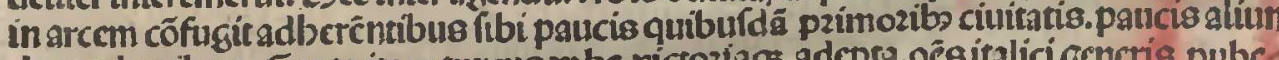

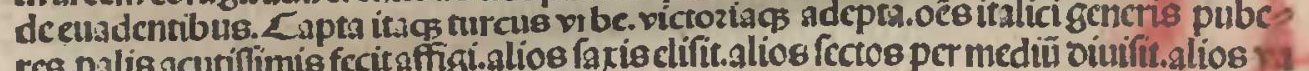

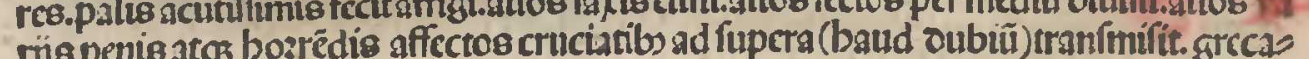

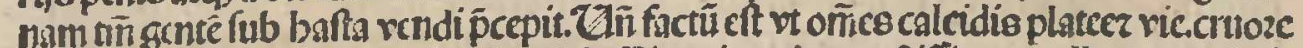
occifox replectnt. Interea ptozis rrbis filiarnica. virgo caftituma.puella occoza facie et renufto afpectu.turco ob fux phebsindine ingente adducta.cum turpitudint et fos decomittiont colchnire proz

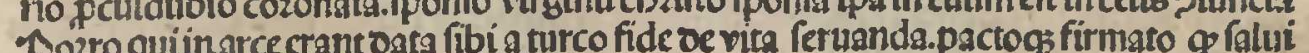
Pouderéf furcem libercin oeditionêt traderent.ctinde afidenter exictunt.ed mot egrof

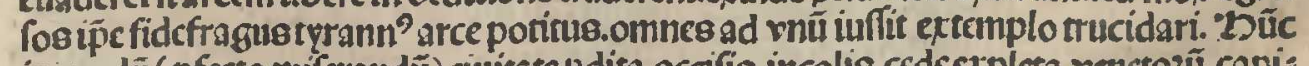
in modu (pfecto milcrand talnctusc

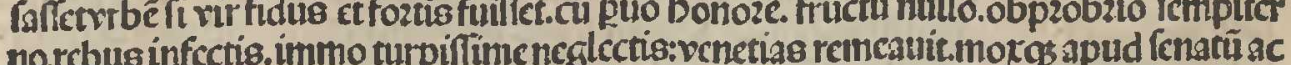
cứarus. Defcenfionis quidé locũacccpit.fed poft multas inuanü adductas falfas rönces

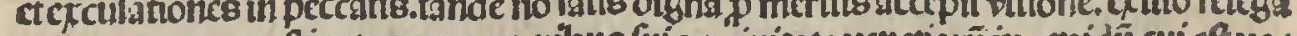

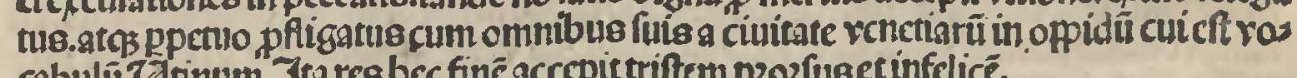

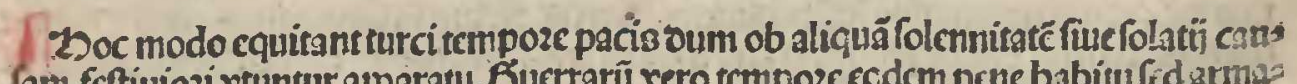
tura alia:cnfibue fçaccincti ż. in paclíi pergunt,

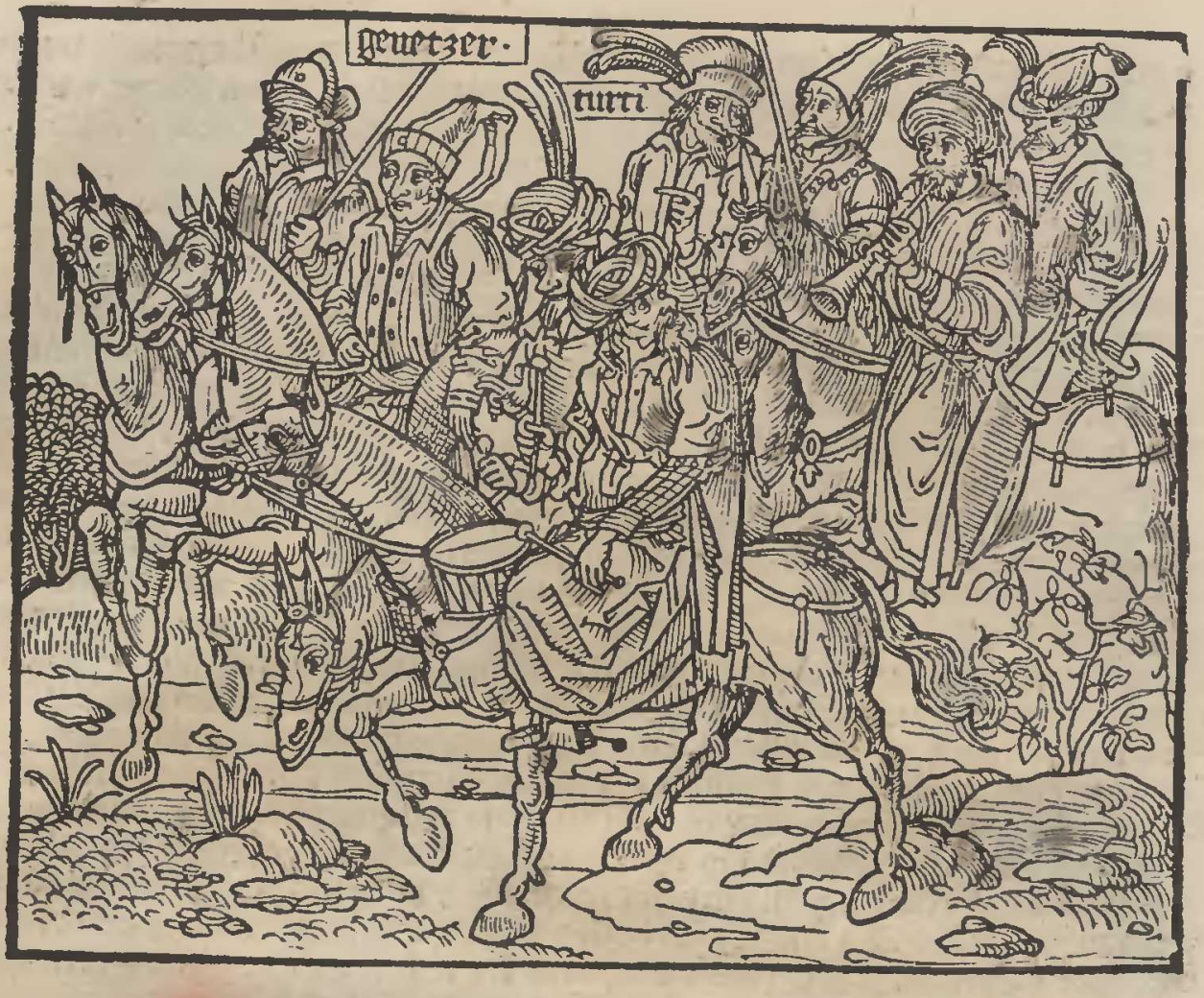

De'Rbodic vrbis obfidione.

bodic vrbis obfidioné Defcripturus caufas in peimis narrare inftítuli.que o a gredcre 7 tis olim. tamen cum co qui une imperat grauia gefécre bella. Tnimicus quidẽ vires in oice angens infolentioz reddirtur. nuartuoz et vigunti circulo annozí cōplures fibi vicinas ditiones fubegiffetanimo in hatuscgreadmodil egit rbodiam vrbent.equitügs bicrofolymozü ottionem finitimä infructis offella ctrodion gas res inu

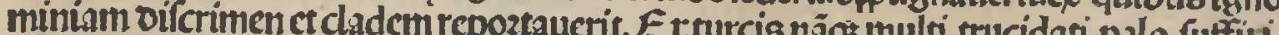

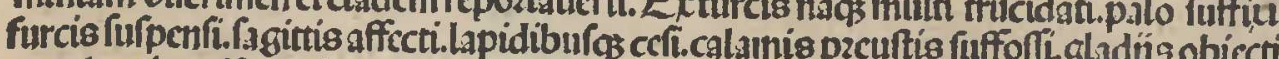

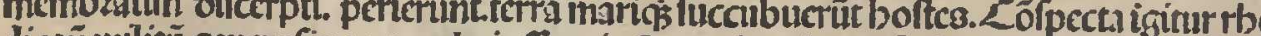

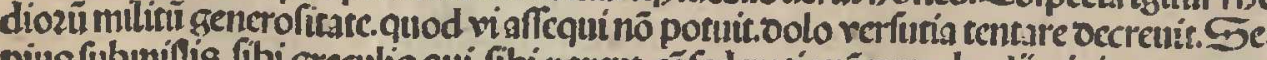

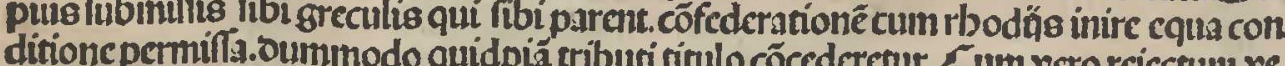
crigal frpenumero videre arte pacé tractat

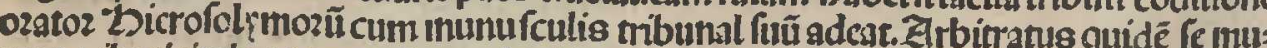

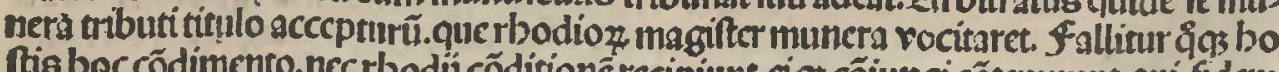
chzinti get

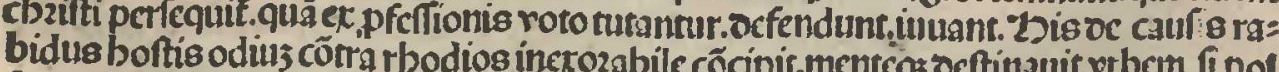

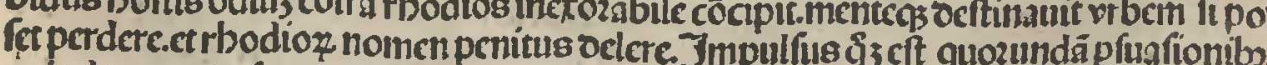

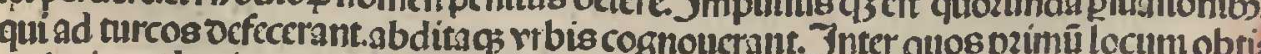
buit rirnepbariuset perditiingenuy Antonius meligabo Rbodios noninfimo loco 
nitus.quil rem oomefticăiam paidem oilapidaucrat. Jo rei ooncetice penuria onctus

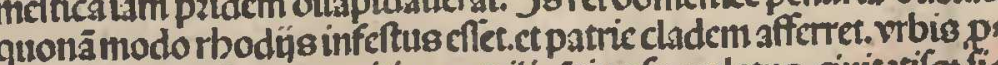

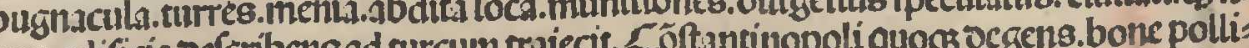
cum.cdificis occrion

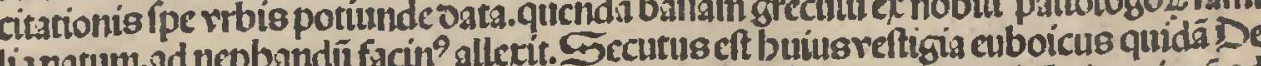
.

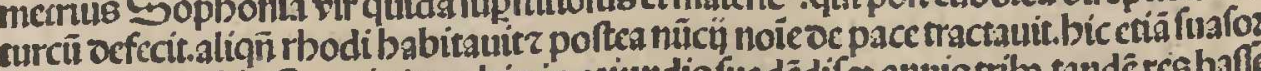
obfidion

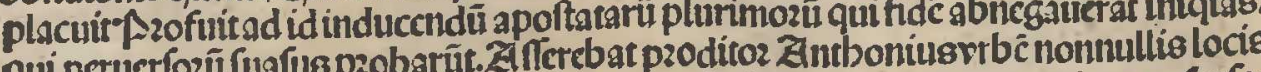

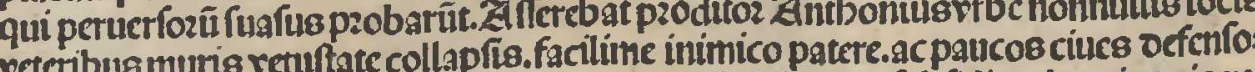

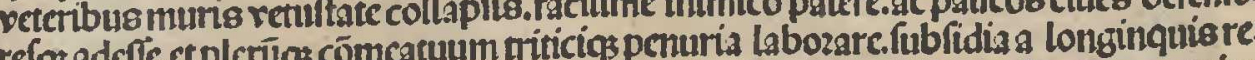

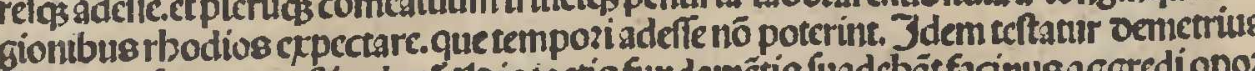

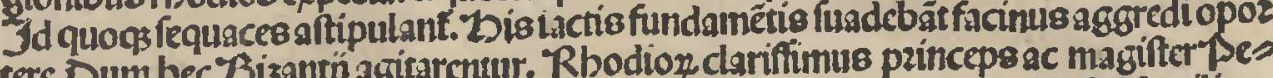

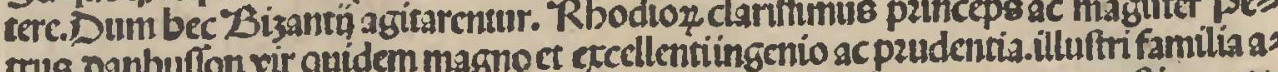

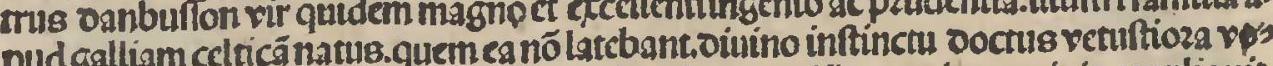
bis loca oelap a e tminus inuenta toto triennio imagnifice creceit. muntuit. ampliauit.

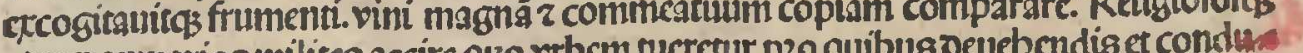

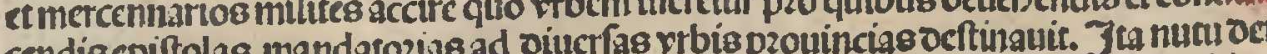
cendiseprio

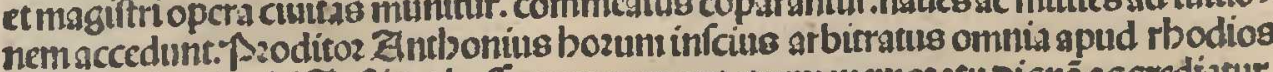

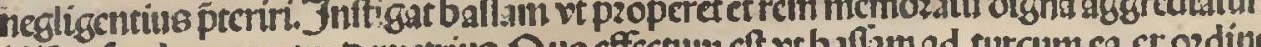

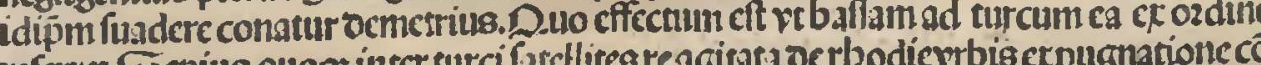

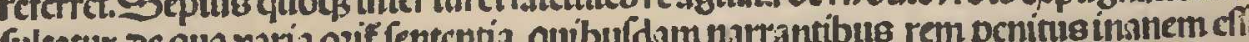

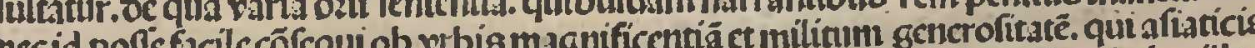

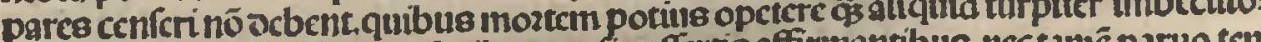

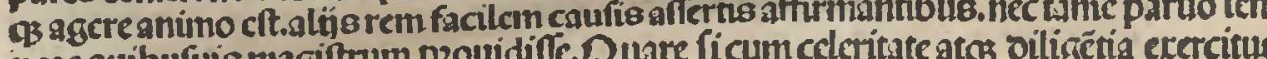
pooequiburtuin mager marn

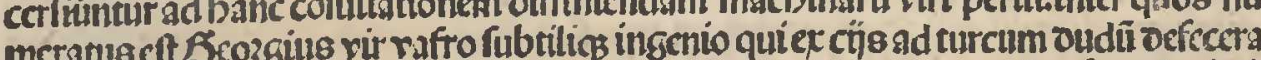

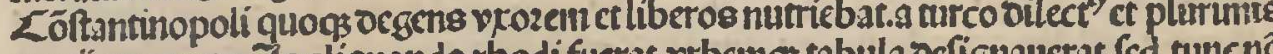

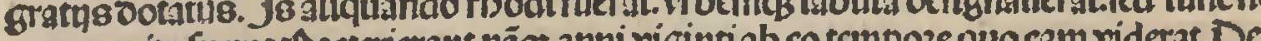

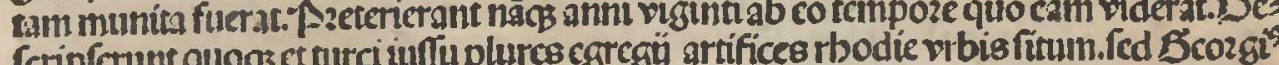

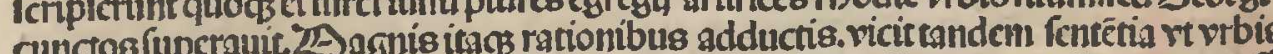

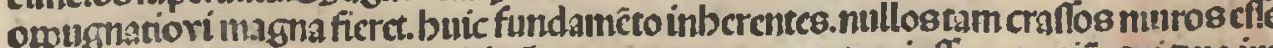

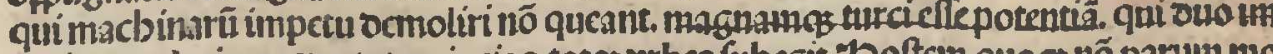

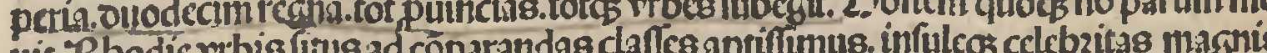

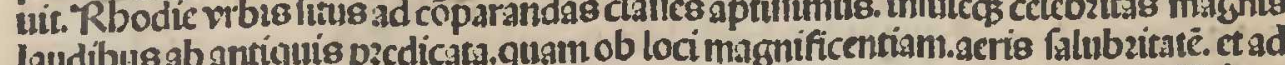

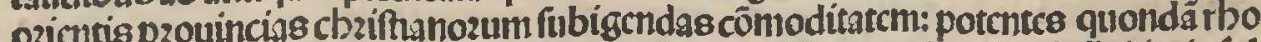

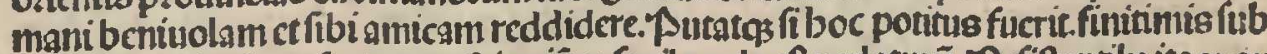

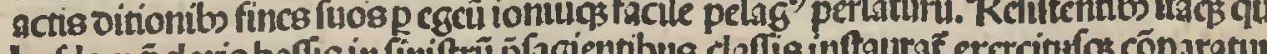
burdam pctaris baffle in inimf Decrếs

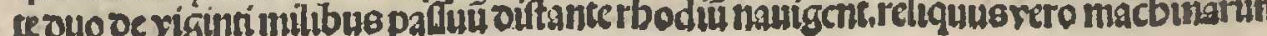

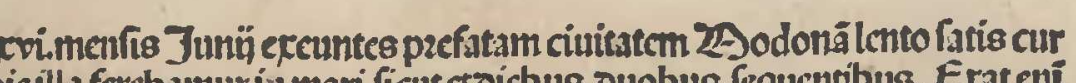

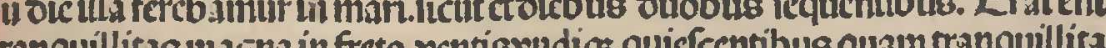

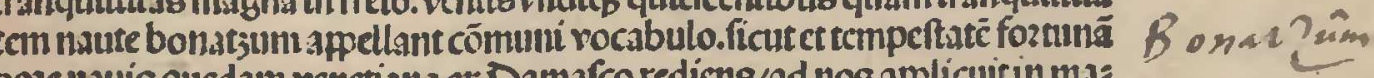
Eo in ecmpozenauis quedam renctiana er Damafco redicns/ad nos applicuitun maz

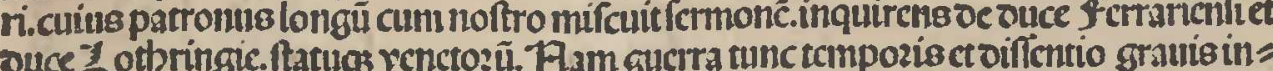

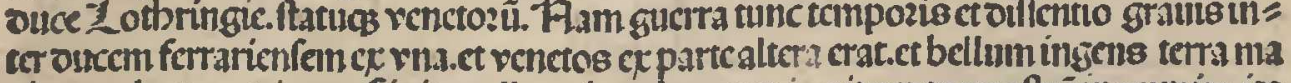

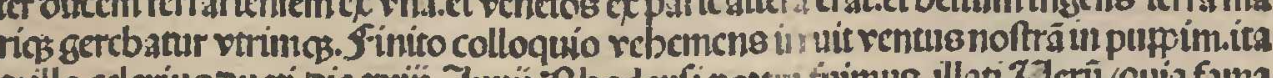

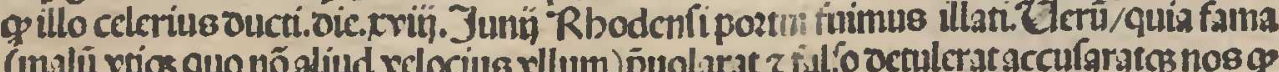

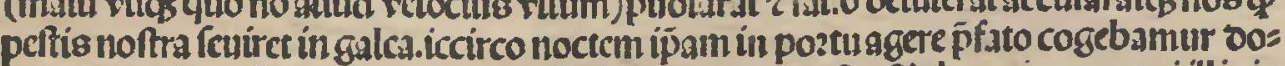

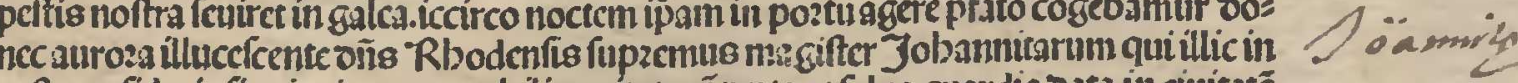

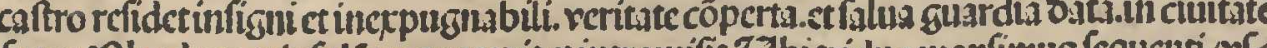

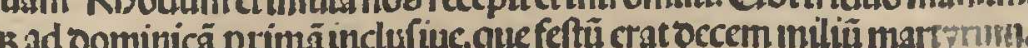

De reliquige que babentur Rbodí.

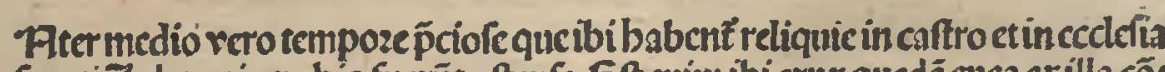

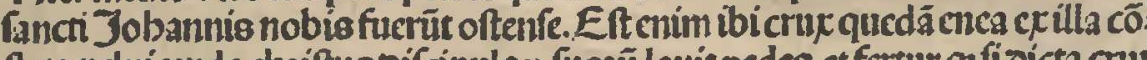
Alata pelui nnde car in ceram

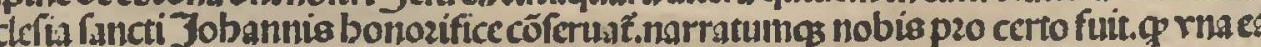

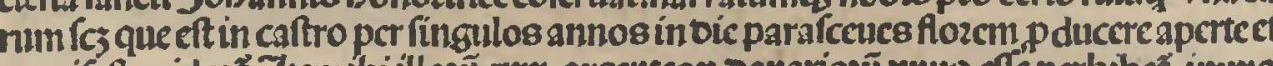

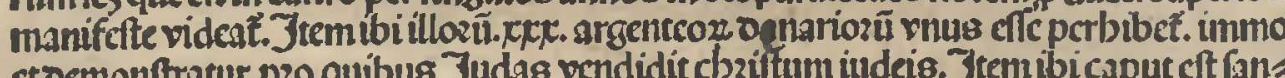

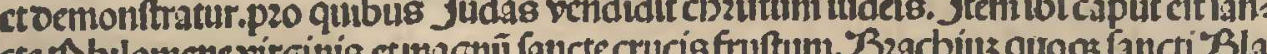

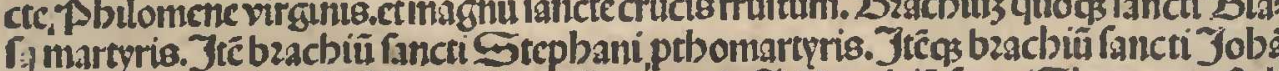

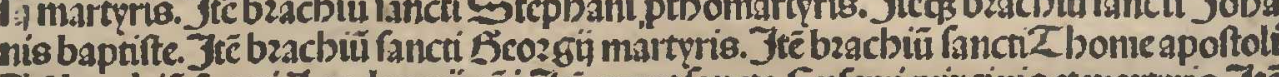

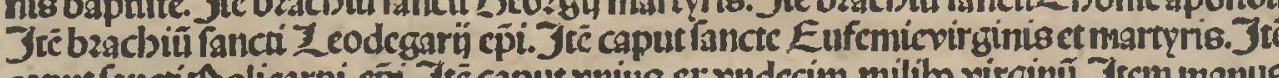

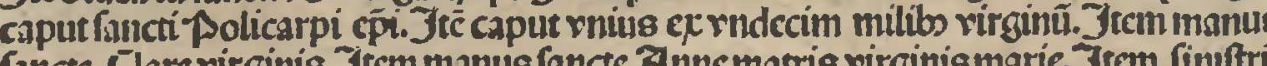
(a)

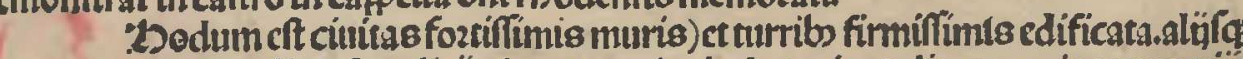
pugnaculis z foztalicüs bene munita. babctog intrta litus maris turres, ,iij.

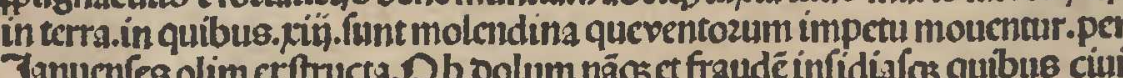
Januenfes olim cetfructa. D b oolum nāos ct frandé infídial

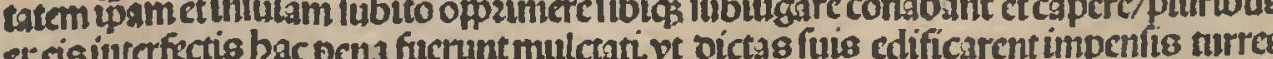

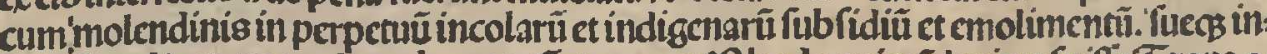
iufte fraudis memoztalc perbentuc. Cetcrumer R bodo pzimun legitur fuifle Tropa oc

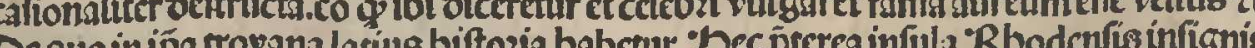
Dequoin ipa troyzana mare

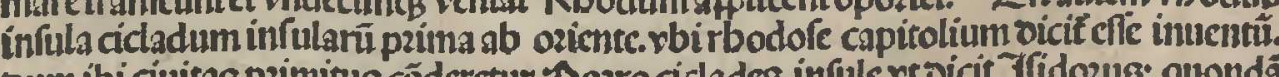

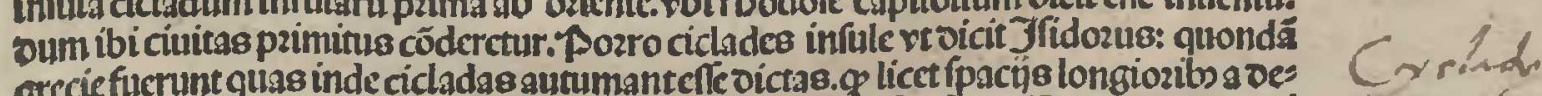

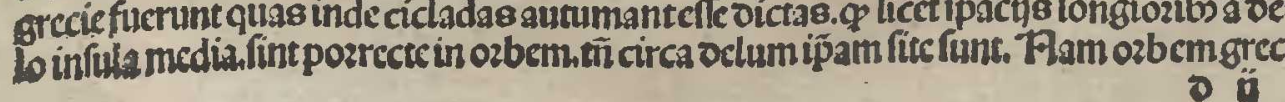




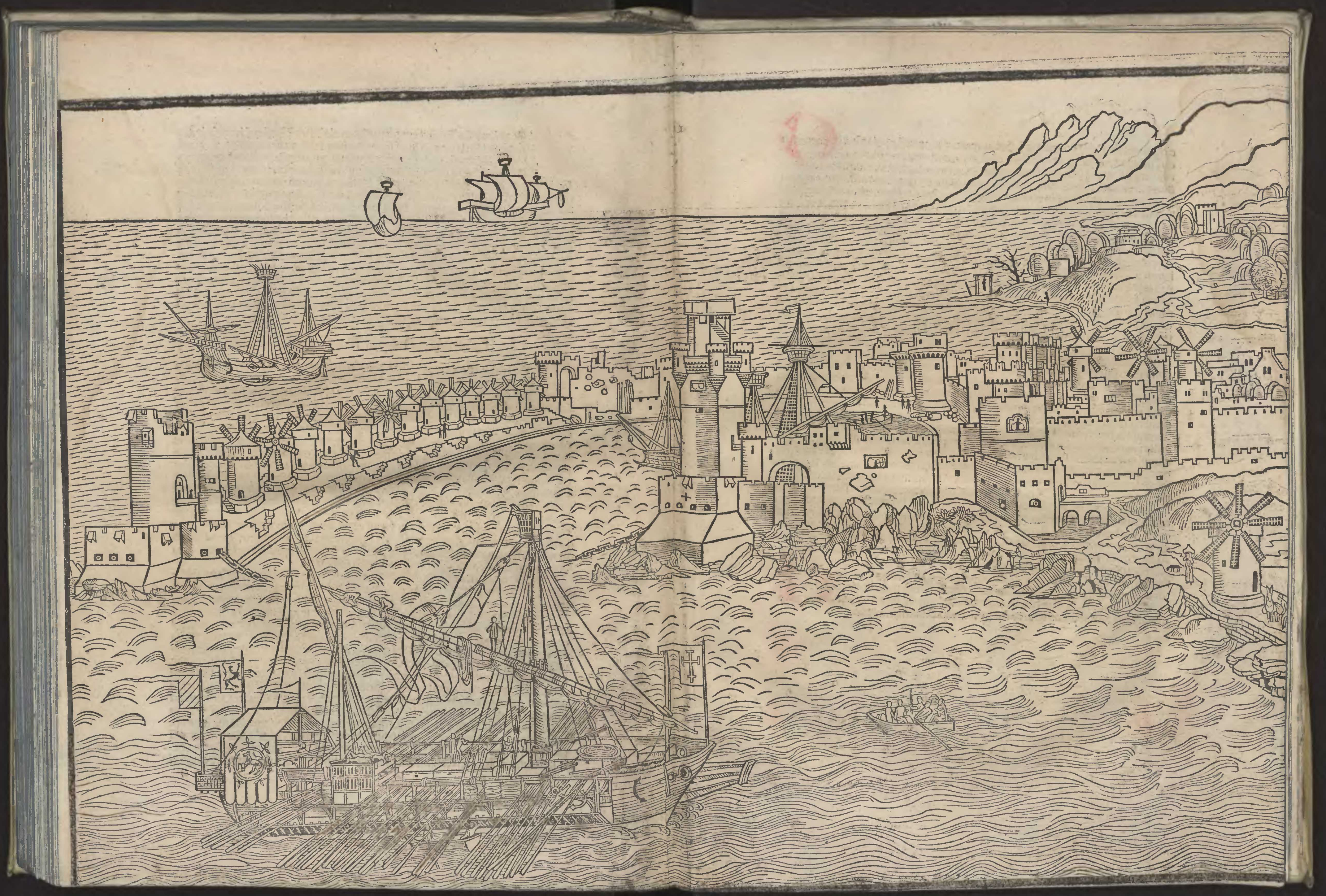




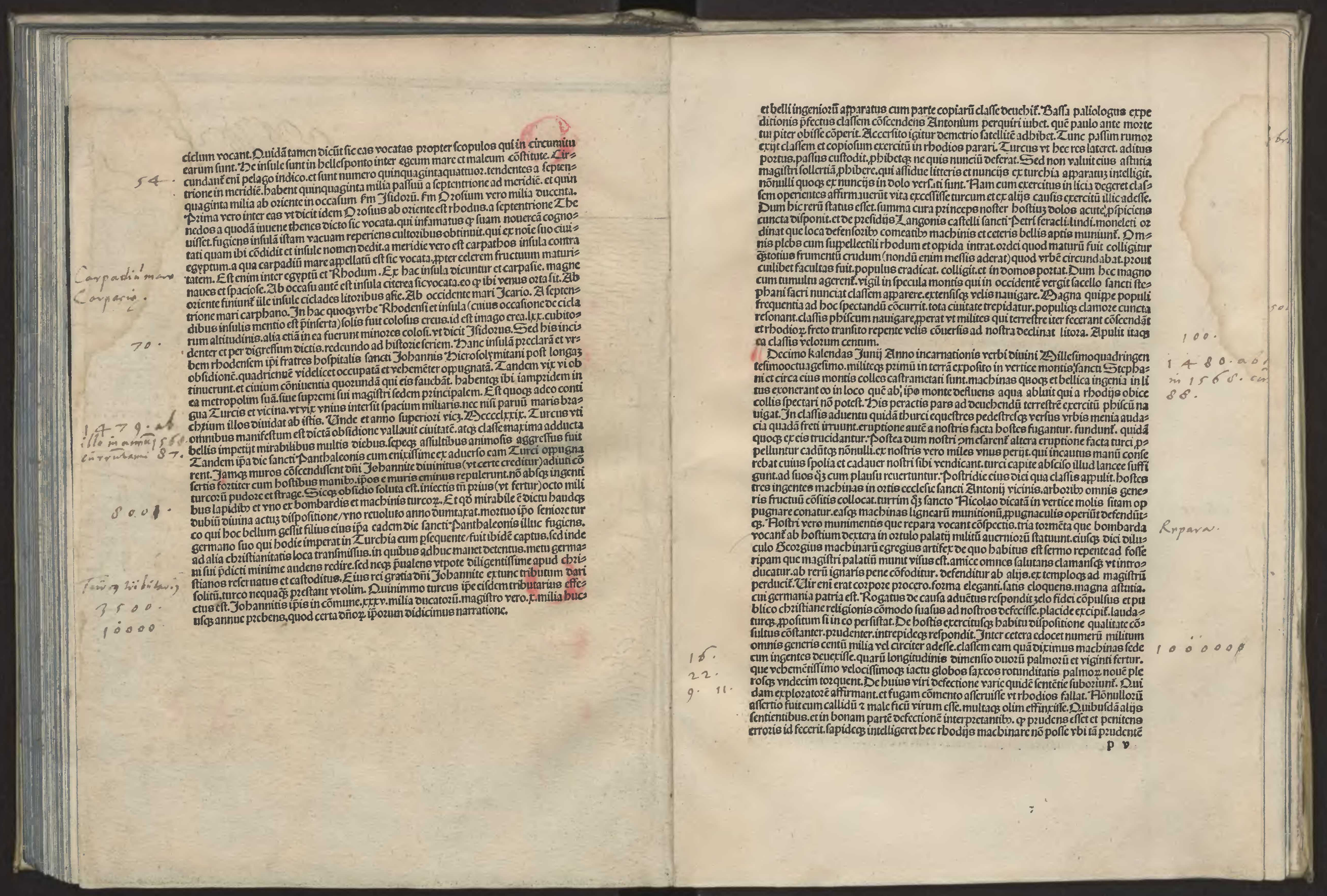




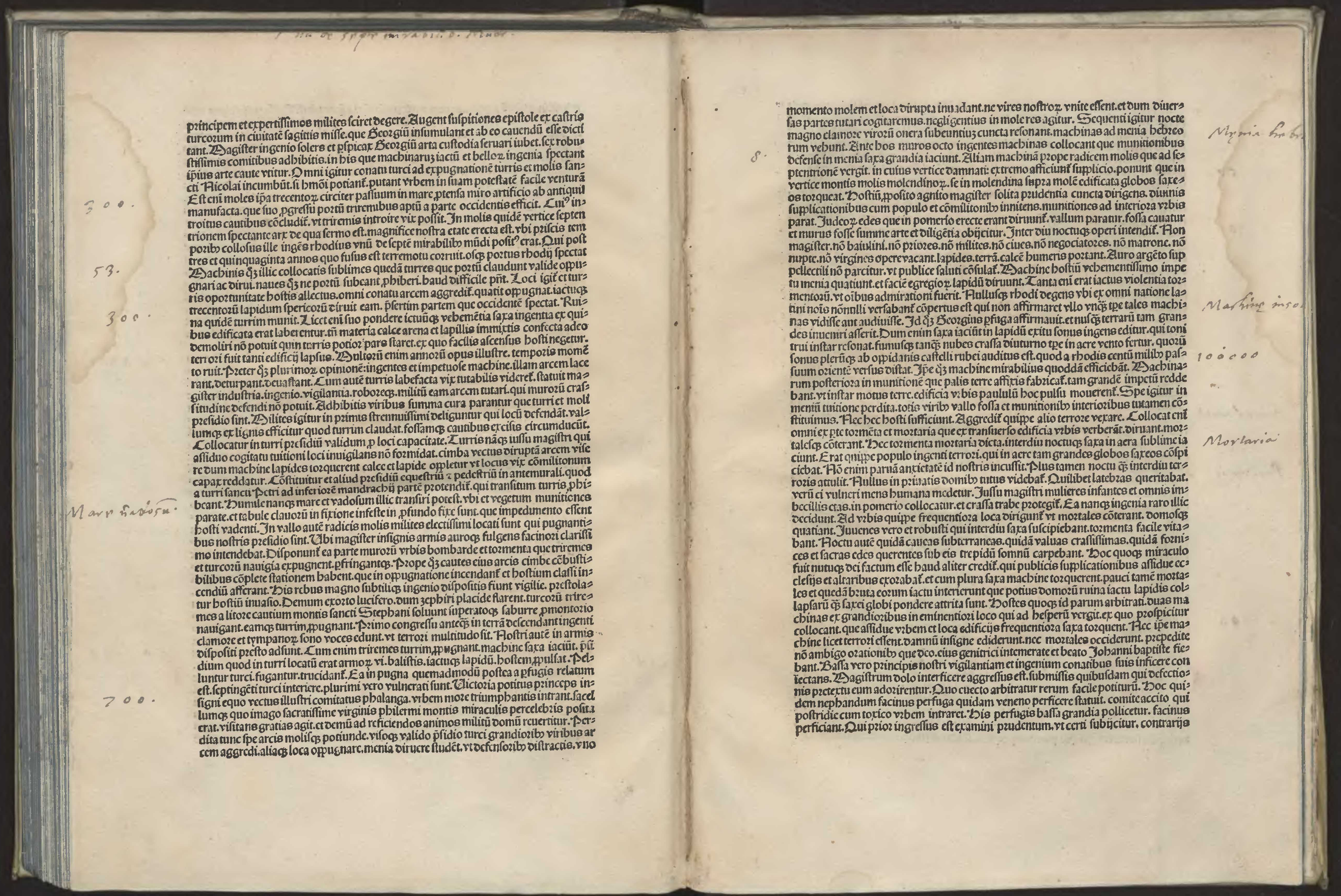




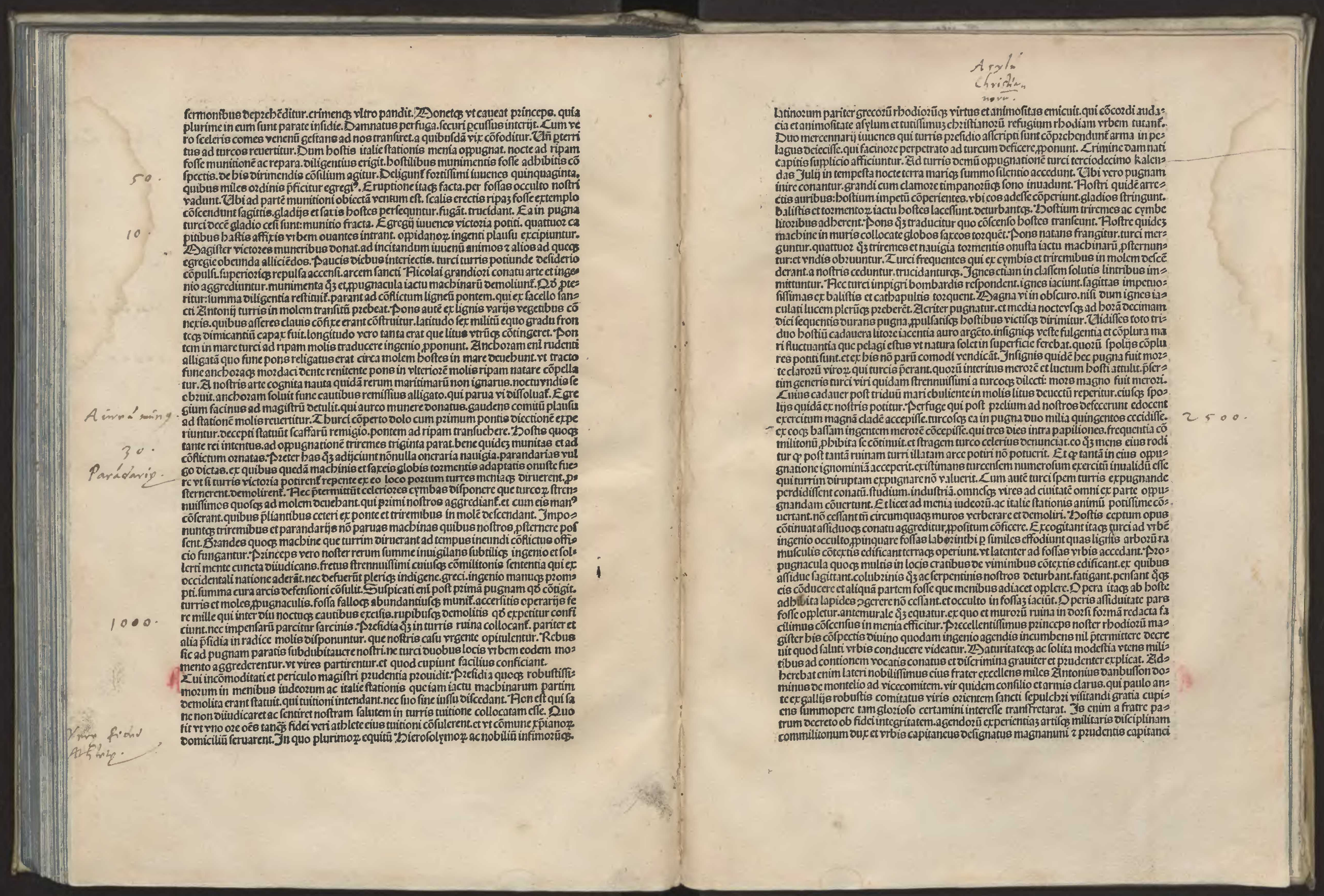




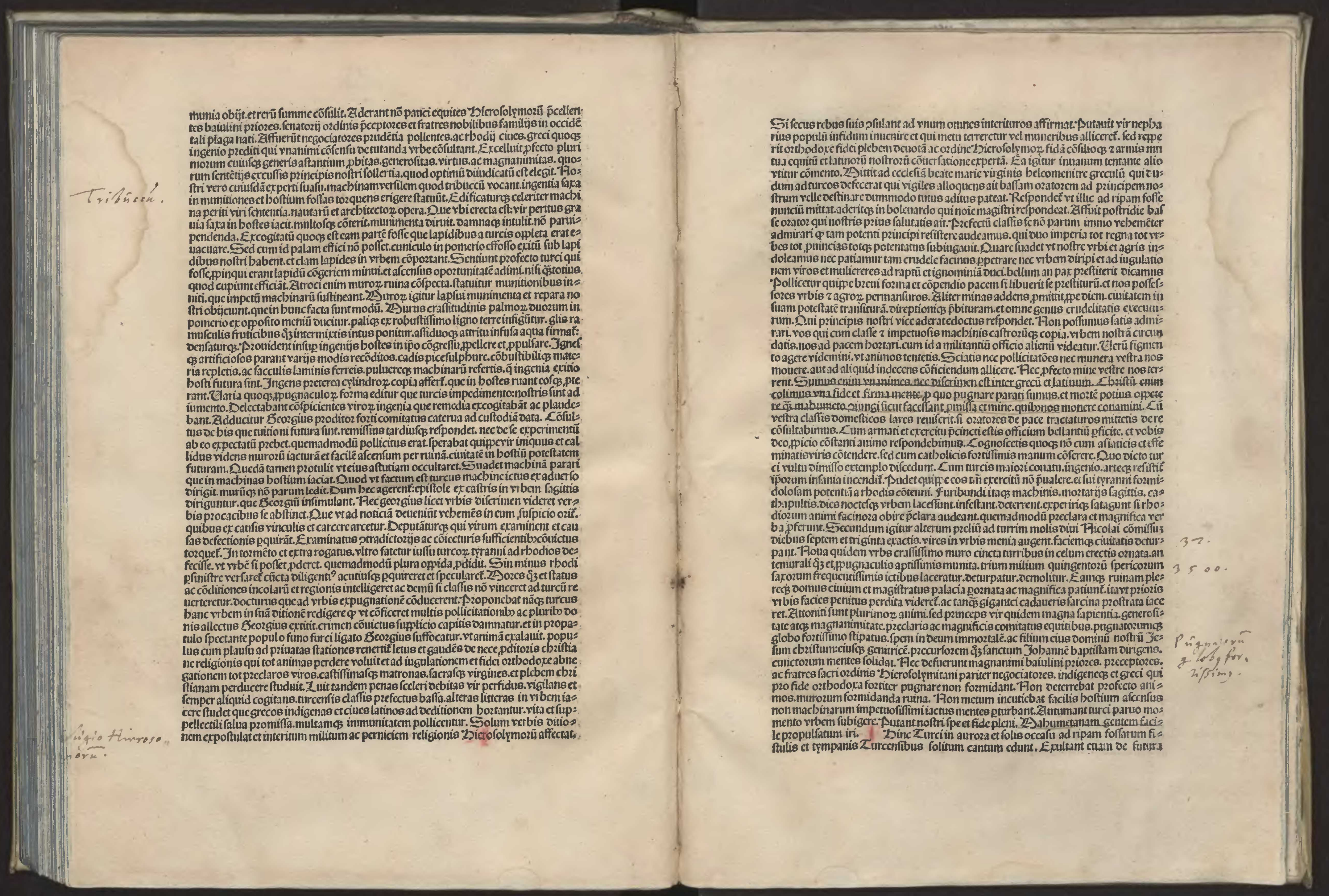




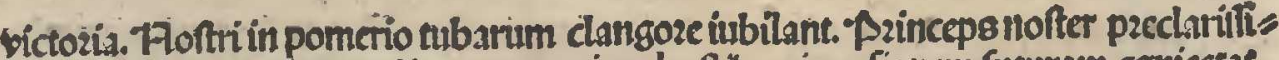
nuvs in gentiacuminc pzedífus pzope oiem boftitcm inuafionem futuram coniectat.

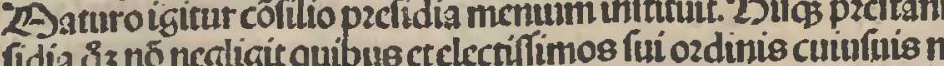

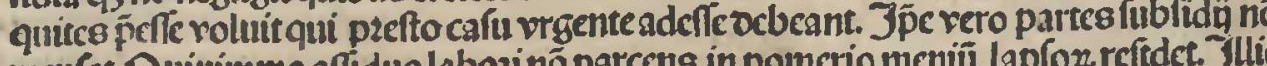

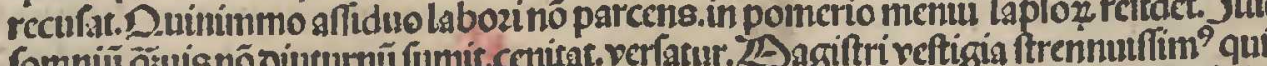
Tomnili

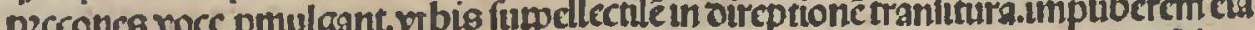

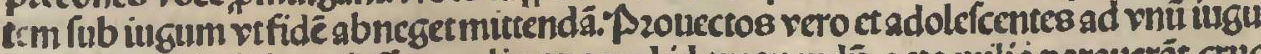

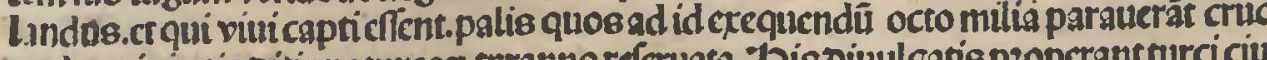
andos ciltratis oitioncturcozty s.m. cunt-

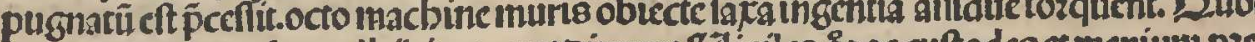

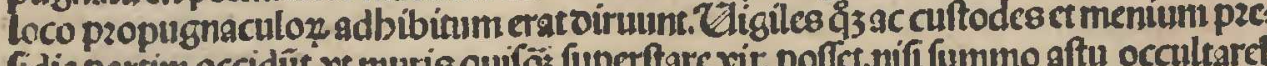
hedis partin

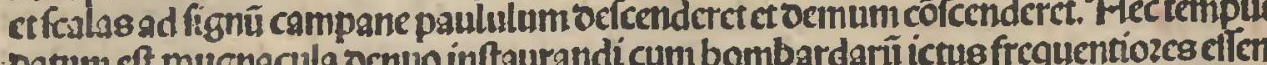
ita ve tum pano sempoze recenta vel circiter fara iacta funt. Zurci vero iactu macbi

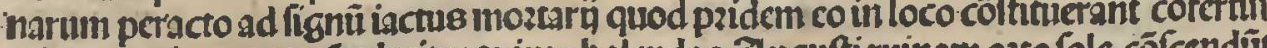
mi magno imperu qua celcritcr quinto kalendas augurt Eat rova as muros

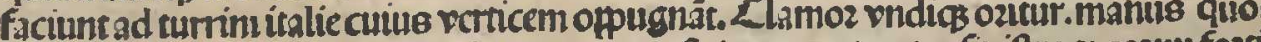

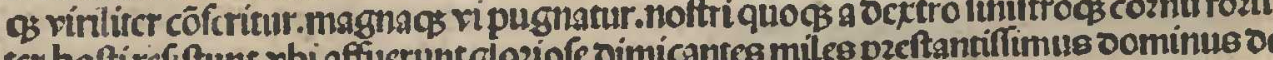

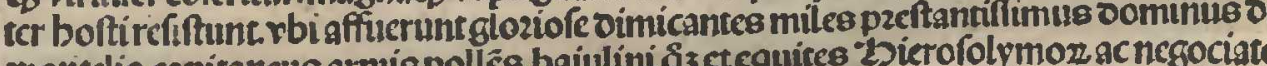
montectio capling

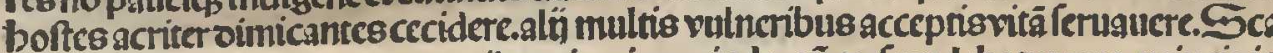

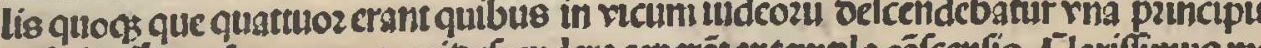

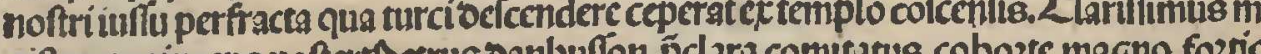

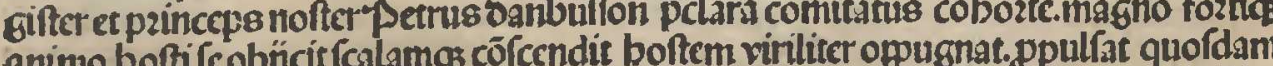

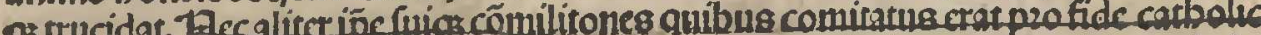
etrepublice coifion

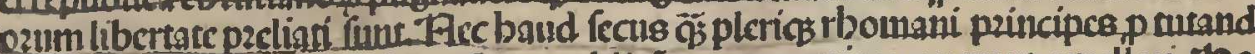
parria oincicas chim pcellctîh mos vir.

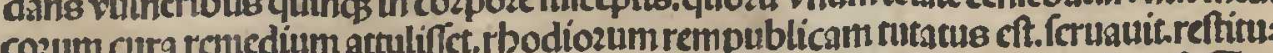
ii. O b quod pacclarum quidem facinus pater partic optimo iure appcllandusent. $\mathcal{X u}$

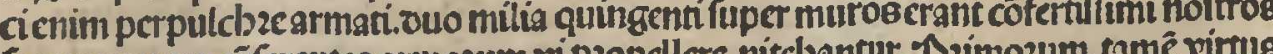

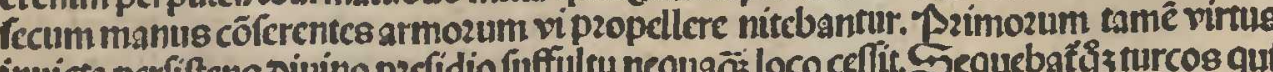

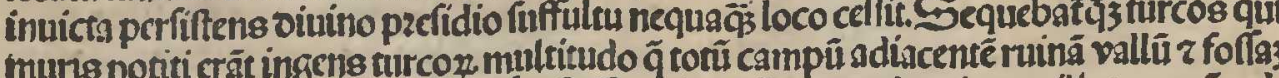
mule

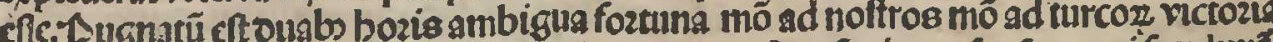
inciurante Z ande oiling

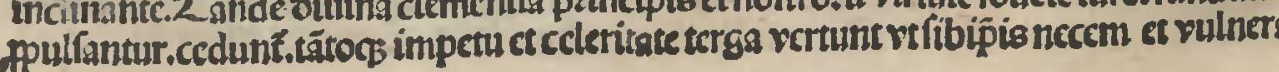

afferent. Spectacnlo nobis fuir quod a nottris inerpugnamdă geftum eft cöfpiccre icum

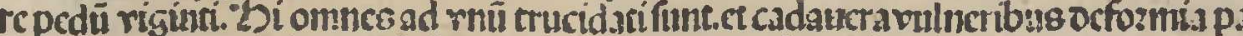

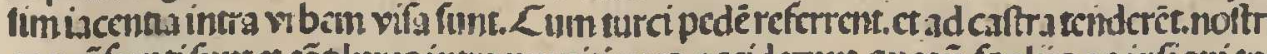

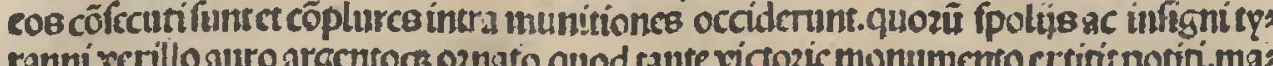
(a) (is quingentiquozū cadauera intravbem fuper meni fof

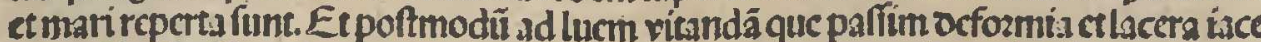

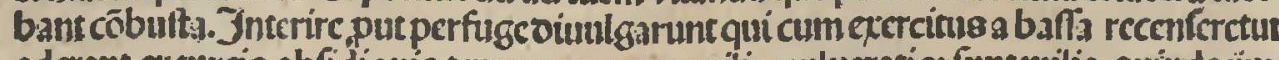

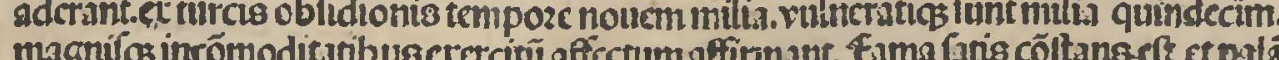
a peffugis vul

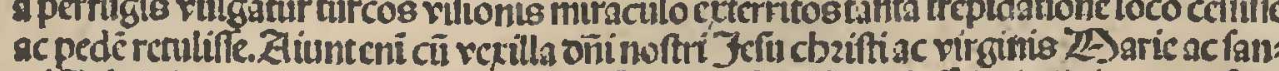
ca Jobänis baptiftcreligioni

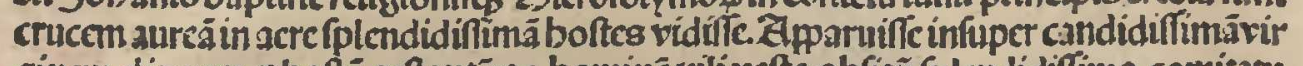
gintem clipcume ct baftã geftantế:ac bomine vili vefte obfitiu f plendidifitimo comitaru Tatum pridio ad

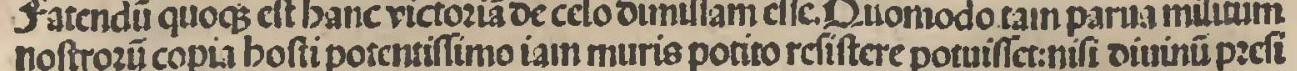

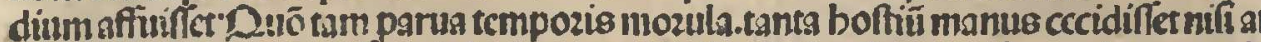

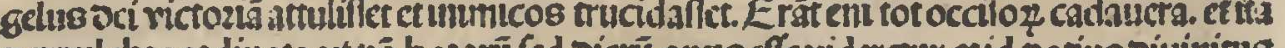

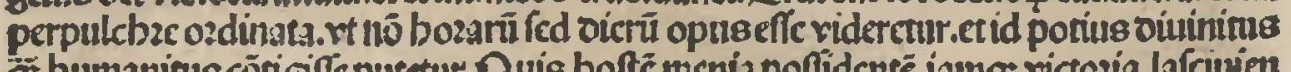
prestanter

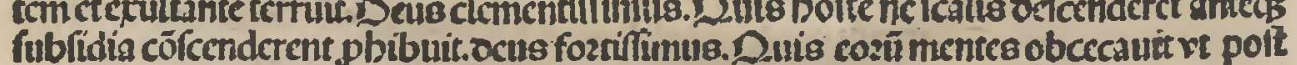
paimä pugnam no aggrcdiunt noftros.et millis vulncribus oppzeffoect nefarigatos

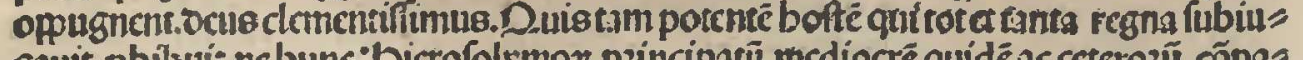

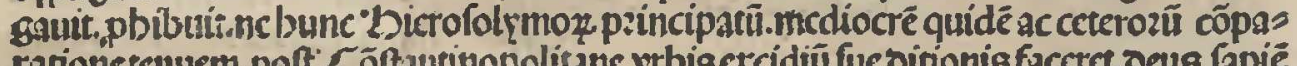
tifimut

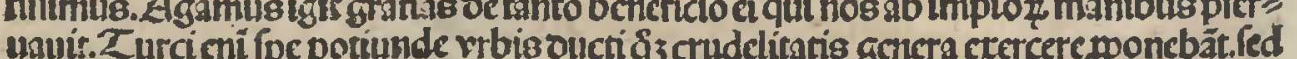
nep bando optatu frufratit tanös pecades mucidant. pullantur.vincuntur. Extcmplo pugna cómifta ad pzimun lapidé caftra $z$ papiliones turcicollocant.machinas ad lítus

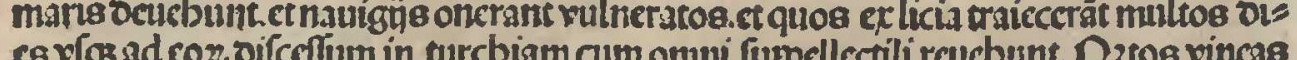

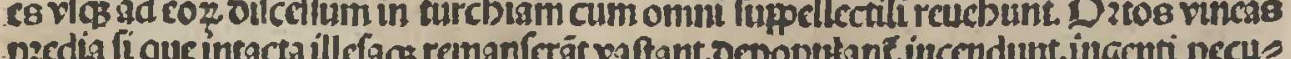

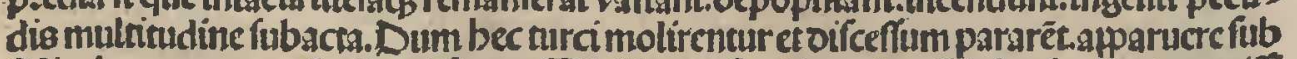

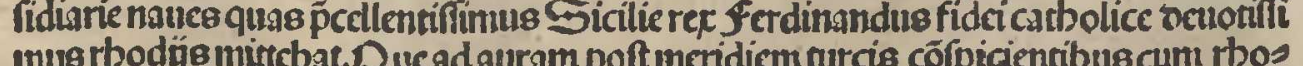
inus rbodgs mittebat. Q ue ad auram poft mendiem turcis corpicientibus cum thos

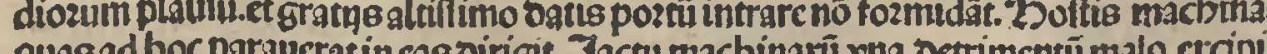
quasad boc parancrat incas oirigit. Jactu macbinarü vna oetrimentū malo crcipu:

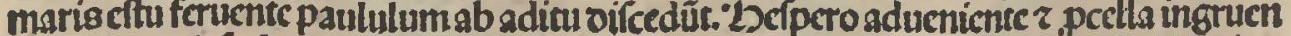
tensuis que léa fuctrat poztũ intrat.altera vela ventis oarccompellitur. Poftridic cins

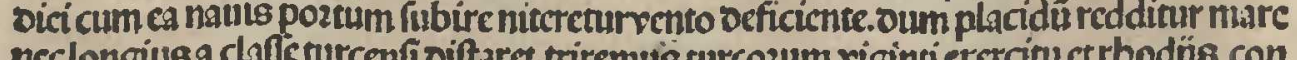

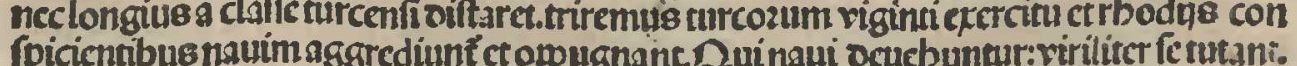

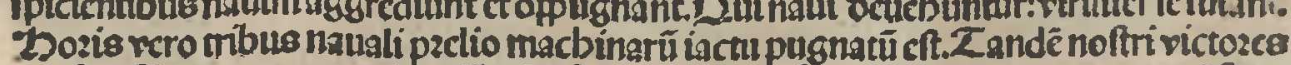

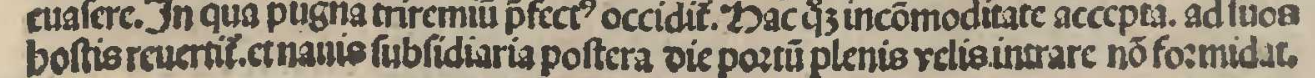

300. 
Attulemunt pzofecto benance nunciul quod 'Rbodios nō parua affecitleticia. Pontif cequipelitercrein

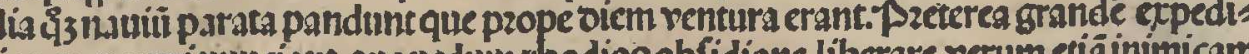
concm pararion (manum

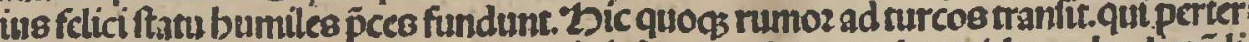

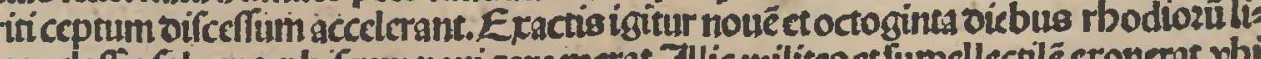

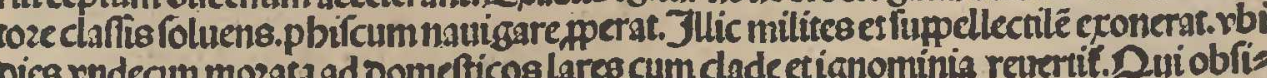
dics indecum morta Ptuncrelígionis eraltarionê etrbodioz gloziärerü gettarũ comentariú edidic

Decaptioneciuitatis 3druntine.

(1) Znepoftea ös infula rbodi oiuino potius miraculo ôs alias murisilliusp

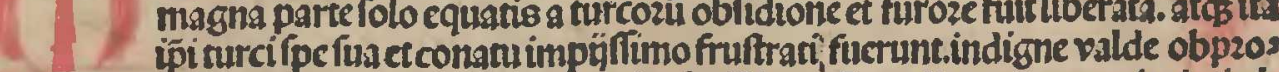
bzium illud ferentes,atcos plurinis quas ciscbziftianozü negligentia parauit elativis

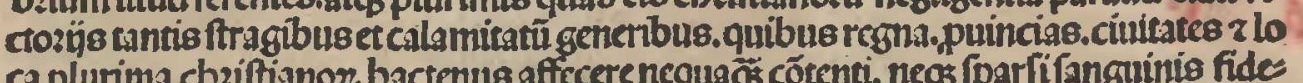

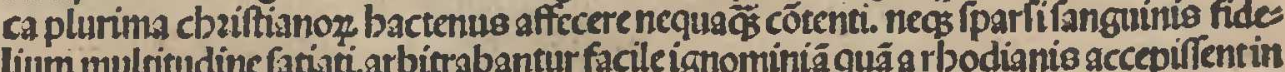

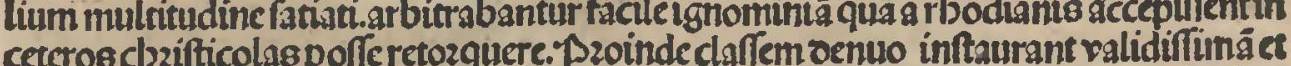
copiofum ckercitu parant.ptes italie.macime mariadriatico vicinas.terta marios ins

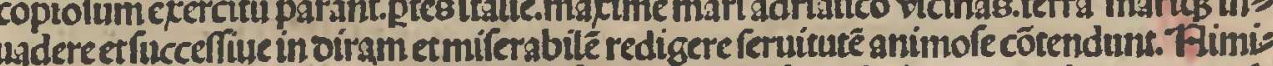

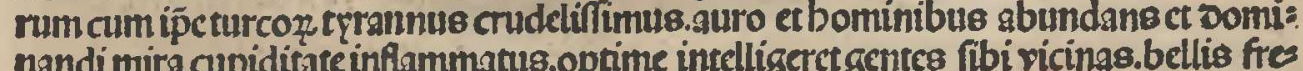

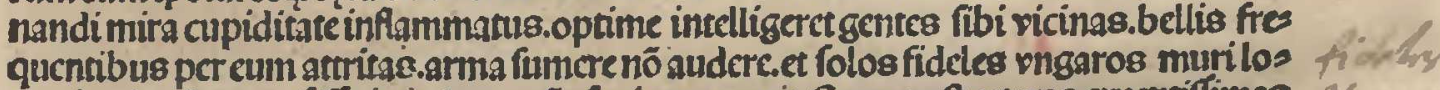

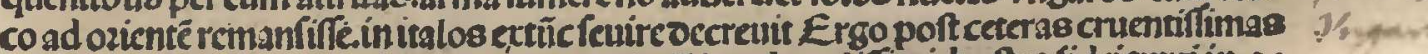

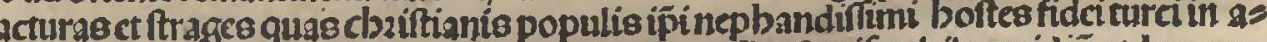

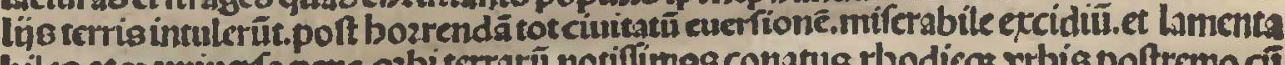

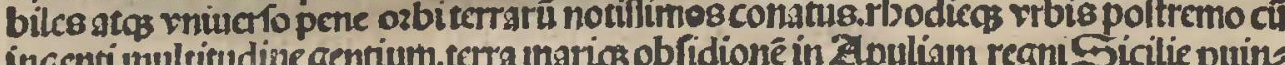

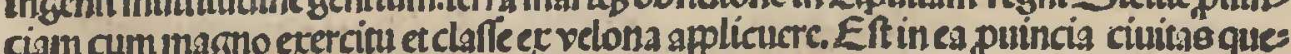
dain arcbicpifcopali fede infignis.stos populoz muleutudine trerum omniu opulens

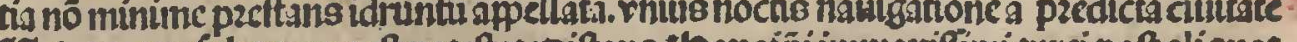

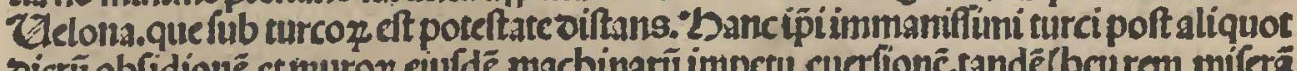
oicrin obrtione.ction

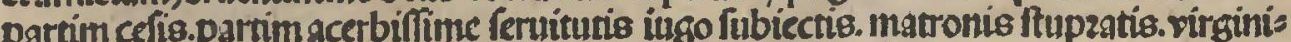
bus raptis.anctimonialibus fedatis,adolefentibs immaniter trucdativ. IEmibus bos

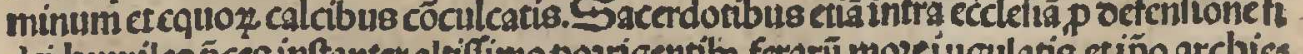

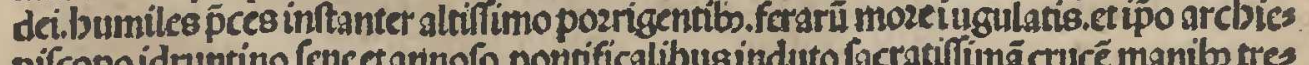
pirspoidn pers

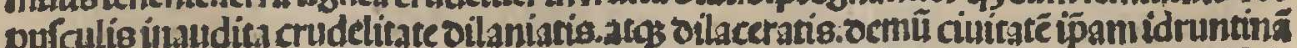
in fuä ditioné redactä. bominibus et numero copiolo.munz. victualibo.yallo. makbis

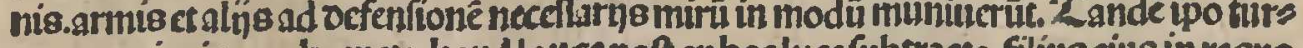
cozumpzincipemabumeto.baud longe poft ex bacluce fubtracto. filins gius in regno

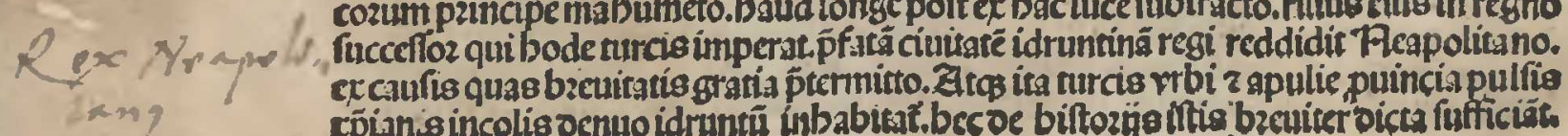

Dereginine peregnnantium tranfimarefctbit 'bali medicns in

Fimariperegrinantififubuctio ct romitus acciderit. Ztaturfirupo febes

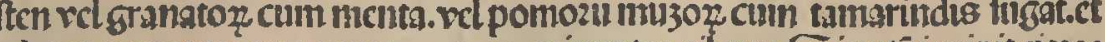

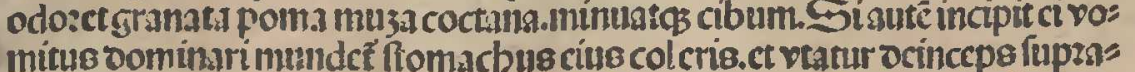

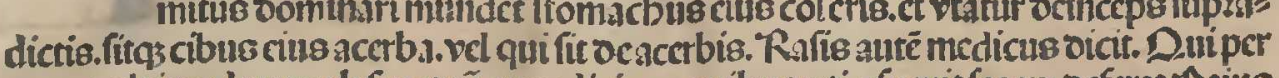

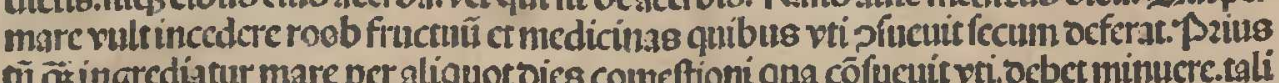

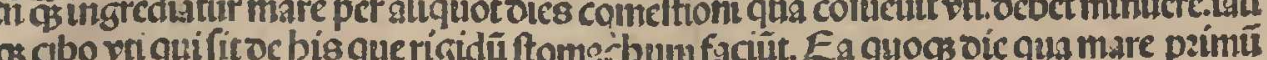

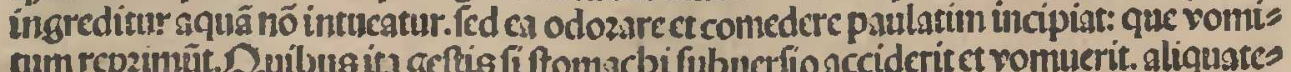

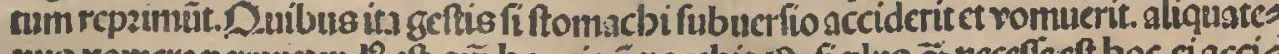

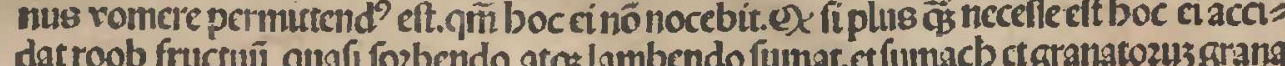

Decourda cótrapediculos ac pulices ctmufcss in mari

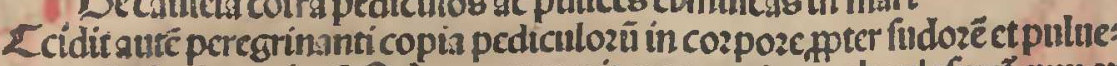

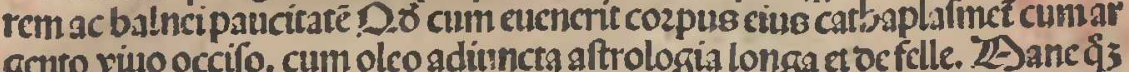

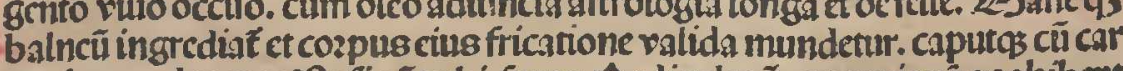

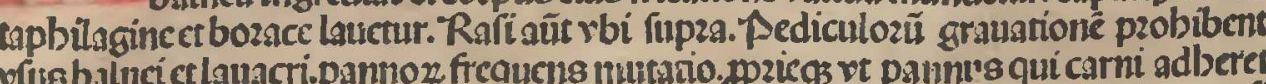

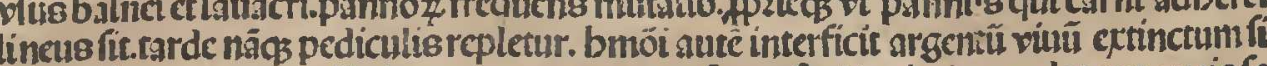

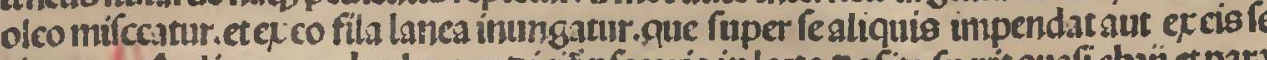

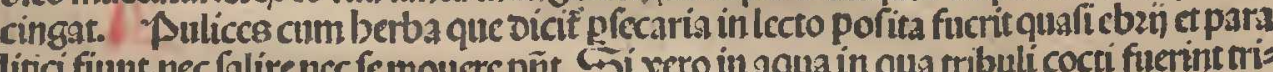

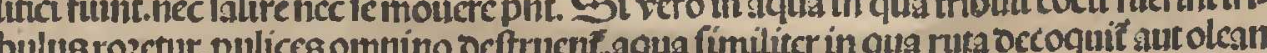

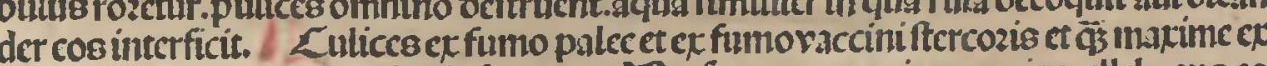

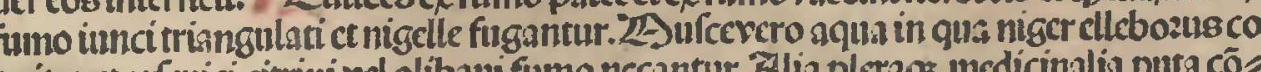

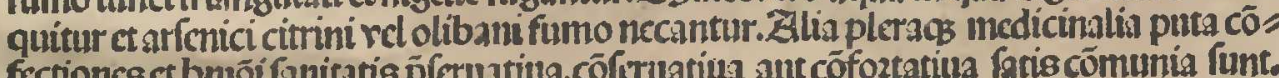

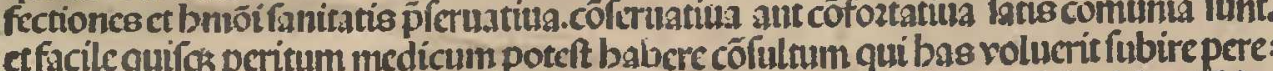
grinationcs fup bis rebus quas fibi inter peregrinandüncceffarisearbitrabitur. Jdeo

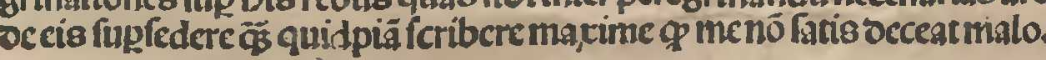

| Sanctarum peregrimationñ in montem SY̛on ad venerandũ cbzift fepulcbzum

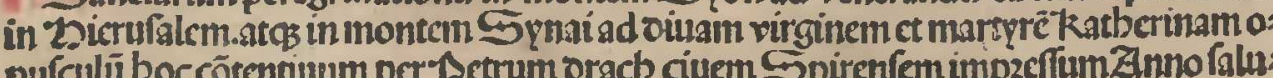

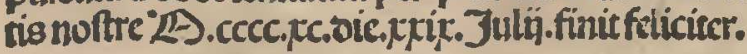






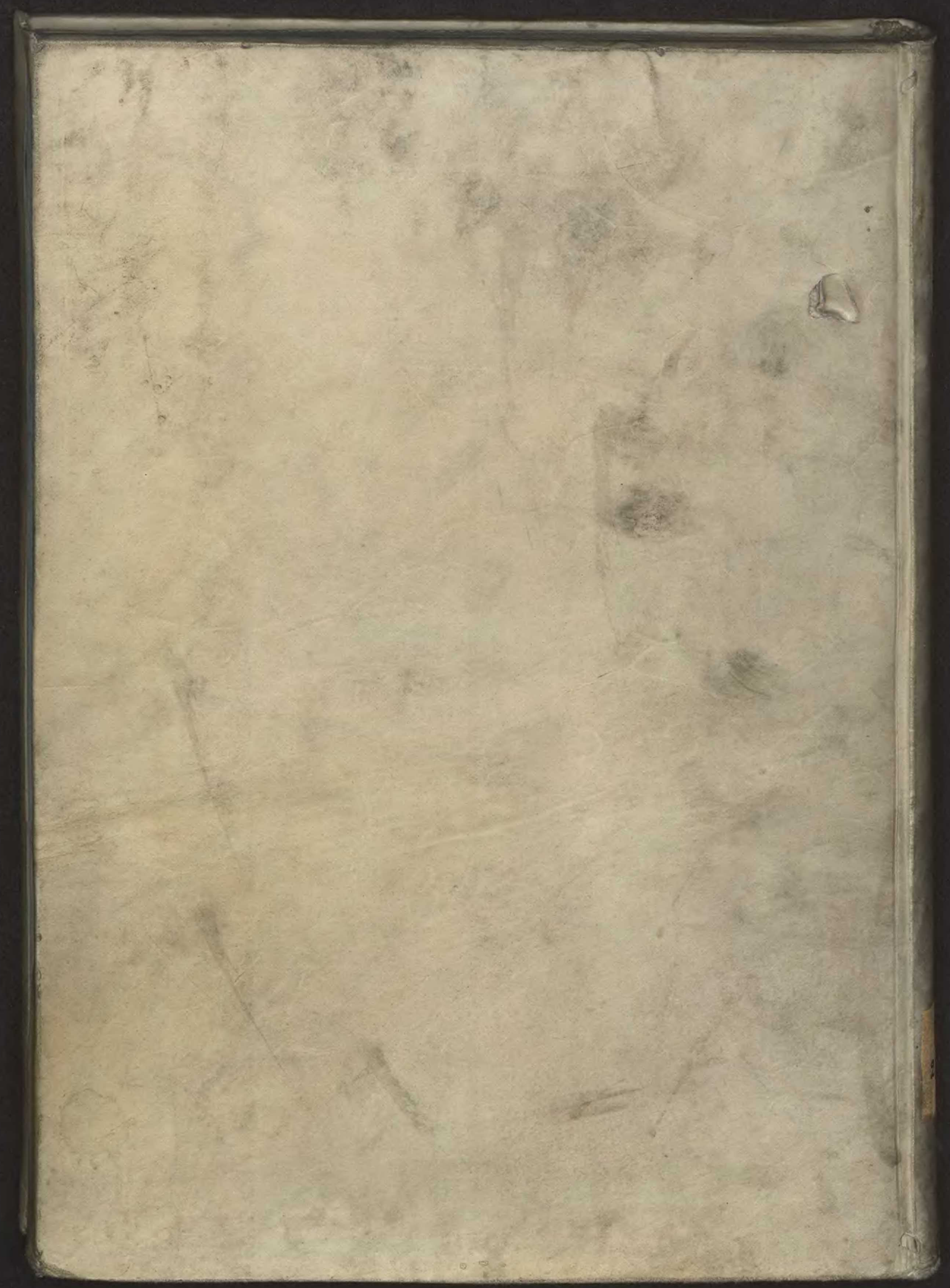




$$
\square
$$




$$
\text { [ }
$$




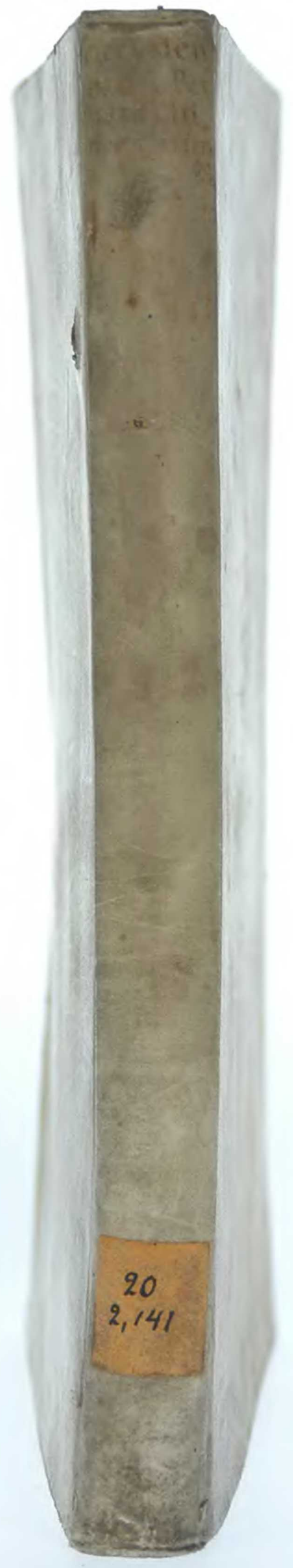

